\title{
Photochemical Stability of Organic Solar Cells: The Role of Electron Acceptors
}

\author{
Speller, Emily M.
}

How to cite:

Speller, Emily M. (2018) Photochemical Stability of Organic Solar Cells: The Role of Electron Acceptors. Doctoral thesis, Swansea University.

http://cronfa.swan.ac.uk/Record/cronfa50959

Use policy:

This item is brought to you by Swansea University. Any person downloading material is agreeing to abide by the terms of the repository licence: copies of full text items may be used or reproduced in any format or medium, without prior permission for personal research or study, educational or non-commercial purposes only. The copyright for any work remains with the original author unless otherwise specified. The full-text must not be sold in any format or medium without the formal permission of the copyright holder. Permission for multiple reproductions should be obtained from the original author.

Authors are personally responsible for adhering to copyright and publisher restrictions when uploading content to the repository.

Please link to the metadata record in the Swansea University repository, Cronfa (link given in the citation reference above.)

http://www.swansea.ac.uk/library/researchsupport/ris-support/ 


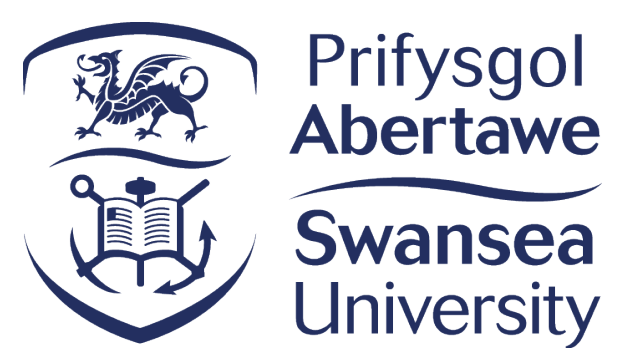

\title{
Photochemical Stability of Organic Solar Cells: The Role of Electron Acceptors
}

\author{
Emily Mae Speller
}

September 2018

Under the Supervision of

Dr. Wing Chung Tsoi \& Prof. James R. Durrant

Submitted to Swansea University in fulfilment of the requirements for the Degree of Doctor of Philosophy 


\section{DECLARATION}

This work has not previously been accepted in substance for any degree and is not being concurrently submitted in candidature for any degree.

Signed:

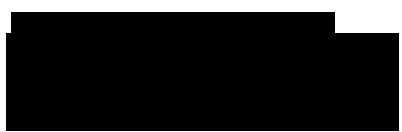

Date: $\quad 26 / 09 / 18$

\section{STATEMENT 1}

This thesis is the result of my own investigations, except where otherwise stated. Where correction services have been used, the extent and nature of the correction is clearly marked in a footnote(s). Other sources are acknowledged by footnotes giving explicit references. A bibliography is appended.

Signed:

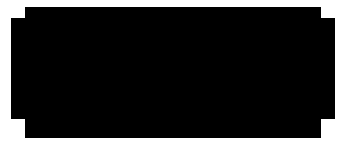

Date: $\quad 26 / 09 / 18$

\section{STATEMENT 2}

I hereby give consent for my thesis, if accepted, to be available for photocopying and for inter-library loan, and for the title and summary to be made available to outside organisations.

Signed:

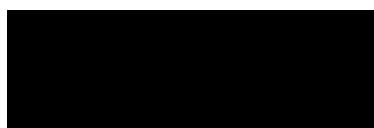

Date:

$26 / 09 / 18$ 


\section{SUMMARY}

Environmental stability remains to be a critical barrier for the commercialisation (along with cost) of organic solar cells, and understanding the roles of material degradation is the key to address this challenge. The thesis investigates the photochemical stability (namely under illumination and air) of well established fullerene-based acceptors and some cutting edge non-fullerene acceptors, and their impact upon organic solar cell performance.

Fullerene photo-oxidation was found to have a general and detrimental effect to polymer:fullerene organic cell performance for a range of device architectures, benchmark polymers and benchmark fullerenes. This photo-oxidation was found to correlate to the degree of aggregation, whereby the more aggregated the fullerene the more resistant it was to photo-oxidation. The photo-oxidation was found to substantially reduce electron mobility through the formation of trap states. In donor polymer:fullerene films the photochemical stability of both the polymer and fullerene were related to the degree of aggregation, and correlated with a decrease in device stability. Transient absorption spectroscopy (TAS) revealed fullerene photo-oxidation occurs primarily due to singlet oxygen generation via the fullerene triplet states for photo-inactive polymer:fullerene films and via the polymer triplet states for photo-active polymer:fullerene films.

An energetic origin of acceptor photochemical stability was also determined. Generally, it was found that fullerenes with a lower lowest unoccupied molecular orbital (LUMO) underwent less photo-oxidation and device performance degraded less. For both fullerene and non-fullerene acceptors, the lower the LUMO level of the acceptor, the more stable the polymer:acceptor film. This relationship was shown to be strongly mediated by the yield of superoxide formation via the acceptor LUMO level.

The work presented herein establishes relationships between electron acceptor structural/nanomorphological/electronic properties with material/device stability, thereby paving the way toward achieving long-term environmental stability of low-cost organic solar cells with minimal encapsulation. 


\section{ACKNOWLEDGEMENTS}

Firstly, I would like to acknowledge the National Research Network in Advanced Engineering and Materials for funding my doctorate and additional funding for travel. For additional funding for travel, I would like to thank the Welsh Assembly Government Sêr Solar funded project and the Materials an Manufacturing Academy (M2A). I would also like to acknowledge funding from the EPSRC funded project EP/M025020/1 for funding my research assistant position at Swansea University. Further to this, I would like to thank the European COST Action MP1307 Stablenextsol for their generous contributions for travel, including a short-term scientific mission to DTU and a summer school in Corsica which among the highlights of my $\mathrm{PhD}$ experience.

I need to express my gratitude to Dr Chung Tsoi for being such a well organised and supportive supervisor, especially when it came to writing up my thesis - your careful guidance has really got me through the PhD. Thank you also for the Research Assistant opportunity within your group. I also need to thank Zhe for all the mentoring, guidance and discussions over the years. Likewise to Harrison, thank you for all of your help, both in the lab and with discussions and for keeping everything experimental running smoothly. I would like to thank everyone at SPECIFIC for making it such a friendly and welcoming environment to work in. I think that Trystan and the sub-group leaders are doing a good job at creating a culture at SPECIFIC of inclusivity and mixing.

I also need to thank my other supervisor, Prof James Durrant, for his guidance and support through my PhD. I would also like to extend my thanks to the Durrant group have always been very welcoming when I have visited Imperial. I have been very fortunate to have collaborated with many different groups (external and internal) during my $\mathrm{PhD}$ who I would also like to thank.

I am very grateful for the friends that I have made along the way and have kept me going through the $\mathrm{PhD}$ process. To name a few but not all: Jeŕeḿy for those evening swims and beach lunches; Phil for bouncing off ideas for new hobbies and life-hacks/plans; Andy for those puncture-filled cycle trips; James for many delicious dinners and a safe place to stay; Stephen for going through that drama with me; Regina for providing an ear to bend; Amirah for lab company and general distractions; Barry for your Irish charm and keen eye; Seb for being my Imperial twin; Mike and James for a good dose of laddy chat and teasing; and my new friends in Milan for keeping me going whilst I was writing.

Finally but definitely not least, I am very grateful to parents who have always been 
there for me and are very supportive in my endeavours. Least to say, my achievements are testament to the upbringing they gave me and continued support. In the same vein, thanks to the rest of my family for their love and support. 
$\begin{array}{ll}\text { Summary } & 2\end{array}$

Acknowledgements $\quad 3$

$\begin{array}{ll}\text { List of Publications } & 10\end{array}$

List of Figures $\quad 12$

List of Tables $\quad 25$

$\begin{array}{lr}\text { Abbreviations } & 27\end{array}$

1 Introduction $\quad 29$

1.1 Introduction to organic solar cells . . . . . . . . . . . . . . . . . . . . 29

1.1.1 Motivation . . . . . . . . . . . . . . . . . . 29

1.1.2 Organic semiconductors . . . . . . . . . . . . . . 33

1.1.3 Polymer-based organic solar cells . . . . . . . . . . . . . . . . . 35

1.1.4 Exciton generation, excited states and exciton diffusion . . . . . . 37

1.1.5 Charge transfer state and charge separation . . . . . . . . . . . 39

1.1.6 Recombinative Photovoltaic loss mechanisms . . . . . . . . . . . . . 41

1.1.7 Device characterisation . . . . . . . . . . . . . . . . 42

1.2 Stability of organic solar cells . . . . . . . . . . . . . . . . . . . 44

1.2 .1 Introduction . . . . . . . . . . . . . . . . . . . 44

1.2.2 Photochemical stability of electron donors (polymers) . . . . . . . 46

1.2.3 Photochemical stability of fullerene electron acceptors . . . . . . . . 51 
1.2.4 Non-fullerene acceptors . . . . . . . . . . . . . . . . . 54

1.2.5 LUMO level of acceptors . . . . . . . . . . . . . . . 55

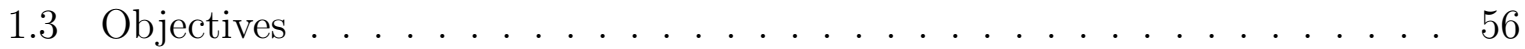

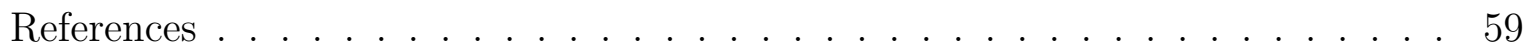

$\begin{array}{lll}2 & \text { Experimental methods } & 72\end{array}$

2.1 Materials . . . . . . . . . . . . . . . . . . 72

2.2 Film preparation and device fabrication . . . . . . . . . . . . 75

2.3 Optical characterisation . . . . . . . . . . . . . . 79

2.3.1 UV-Vis-NIR absorption spectroscopy . . . . . . . . . . . . . 79

2.3.2 Microsecond transient absorption spectroscopy . . . . . . . . . . . . 81

2.3.3 Photoluminescence spectroscopy . . . . . . . . . . . . . . . . . 84

2.4 Chemical/structural characterisation . . . . . . . . . . . . 86

2.4.1 Mass spectrometry . . . . . . . . . . . . . . 86

2.4.2 Fourier transform infrared spectroscopy . . . . . . . . . . . . 88

2.4.3 X-ray photoelectron spectroscopy . . . . . . . . . . . . . . 92

2.4.4 Grazing incidence wide-angle x-ray scattering . . . . . . . . . . 94

2.4.5 Atomic Force Microscopy . . . . . . . . . . . . . . . . . . . . . 95

2.5 (Opto-)electrical characterisation . . . . . . . . . . . . . 96

2.5.1 Cyclic voltammetry . . . . . . . . . . . . . . 96

2.5.2 Current density-voltage device characterisation . . . . . . . . . . . 97

2.5.3 Device stability testing . . . . . . . . . . . . . . . . . 97

2.5.4 Electroluminescence spectroscopy . . . . . . . . . . . . . . . . . . . 98

2.5.5 External quantum efficiency . . . . . . . . . . . . . . . 98 
2.6 Theory . . . . . . . . . . . . . . . . . . . . . . . . . . . . 99

2.6.1 (Time-dependent) density functional theory . . . . . . . . . . . 99

2.7 Comparison of the techniques used to probe photochemical degradation $\quad 99$

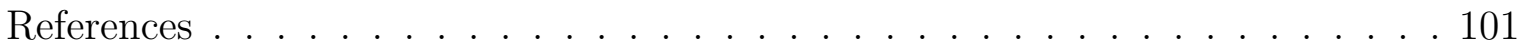

3 The role of photo-oxidation of fullerenes in the stability of polymer:fullerene $\begin{array}{ll}\text { solar cells } & 104\end{array}$

3.1 Introduction . . . . . . . . . . . . . . . . . . . . 105

3.2 Experimental details . . . . . . . . . . . . . . . . 105

3.3 Results and discussion f. . . . . . . . . . . . . . 106

3.3.1 Impact of fullerene photo-oxidation upon OSC devices . . . . . . 106

3.3.2 The effect of polymer photodegradation on device performance . . . 110

3.3.3 Generality of fullerene photo-oxidation to device degradation . . . . 112

3.4 Conclusions . . . . . . . . . . . . . . . . . . 116

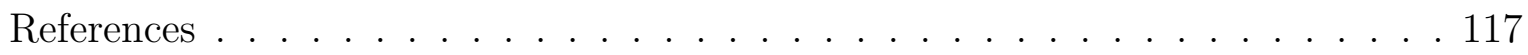

4 The Impact of aggregation to the stability of organic photovoltaic films $\begin{array}{ll}\text { and devices } & 119\end{array}$

4.1 Introduction . . . . . . . . . . . . . . . . . . 120

4.2 Experimental details . . . . . . . . . . . . . . . . . . 120

4.2.1 Part 1: Fullerene and PS:fullerene . . . . . . . . . . . . . 120

4.2.2 Part 2: PCDTBT:fullerene . . . . . . . . . . . . . 123

4.3 Results and discussion . . . . . . . . . . . . . . . . . . . 124

4.3.1 Effect of fullerene aggregation on the photostability of fullerene films in air . . . . . . . . . . . . . . . . . . . . . . . . 124

4.3.1.1 Spectroscopic studies . . . . . . . . . . . . . . . . . 124 
4.3.1.2 Investigation into the photo-oxidation mechanism . . . . . 129

4.3.1.3 Impact of photo-oxidation on electrical and optical properties of films and devices . . . . . . . . . . . . . 132

4.3.2 Control of fullerene aggregation in PCDTBT: $\mathrm{PC}_{61} \mathrm{BM}$ films and devices and the effect to their photostability in air . . . . . . . . . 136

4.3.2.1 Spectroscopic studies on thin films . . . . . . . . . . 136

4.3.2.2 Impact of photoaging in air on devices . . . . . . . . . 140

4.3.2.3 Investigation into the photo-oxidation mechanism . . . . . 143

4.3.2.4 Thickness dependent aggregation and stability . . . . . 147

4.4 Conclusions . . . . . . . . . . . . . . . . . . . . . . . 152

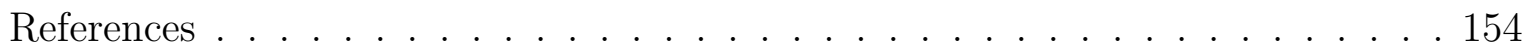

5 Effect of LUMO energy on the photochemical stability of fullerene ac$\begin{array}{lr}\text { ceptors in air } & 158\end{array}$

5.1 Introduction . . . . . . . . . . . . . . . . . . . . 159

5.2 Experimental details $\ldots \ldots \ldots$. . . . . . . . . . . . . . . . . . 159

5.3 Measurement of fullerene LUMO levels . . . . . . . . . . . . . . . . 160

5.4 Spectroscopic studies of LUMO dependence of photo-oxidation in fullerenes 162

5.5 Impact of LUMO dependent photo-oxidation on fullerene based films and devices . . . . . . . . . . . . . . . . . . . . . 171

5.6 PDCTBT:PC ${ }_{61}$ BM:fullerene ternary blend devices . . . . . . . . . . 176

5.7 Conclusion . . . . . . . . . . . . . . . . . . . . 178

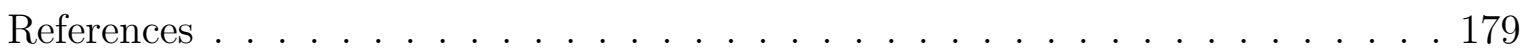

$6 \quad$ Stability of non-fullerene acceptors $\quad 181$

6.1 Introduction . . . . . . . . . . . . . . . . . . . . . 182

6.2 Experimental details . . . . . . . . . . . . . . . . . . 182 
6.3 Light-only stability of non-fullerene acceptors f . . . . . . . . . . 183

6.3.1 Impact on rhodanine flanked acceptor based devices . . . . . . . . . 183

6.3.2 Spectroscopic studies of rhodanine flanked acceptors . . . . . . . . . 185

6.3.3 Comparison of burn-in behaviour for different systems . . . . . . . . 190

6.4 Stability of rhodanine flanked acceptors under illumination and air . . . . . 191

6.4.1 Device behaviour . . . . . . . . . . . . . . . . . 191

6.4.2 Spectroscopic studies of photodegradation . . . . . . . . . . 195

6.4.3 Investigation into the photodegradation mechanism. . . . . . . . . 200

6.4.4 Correlation between acceptor energetics and photodegradation . . . 205

6.5 Conclusion . . . . . . . . . . . . . . . . . . . . . . 208

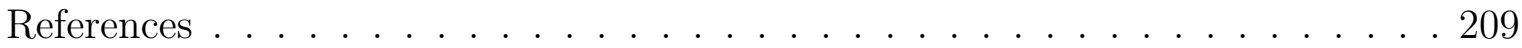

7 Conclusions and further work

B.0.1 Part 1: Fullerene and PS:Fullerene . . . . . . . . . . . 218

B.0.2 Part 2: PCDTBT:Fullerene . . . . . . . . . . . . 223 


\section{LIST OF PUBLICATIONS}

[1] A. J. Clarke, R. Meitzner, J. Luke, E. M. Speller, H. Cha, J. Wu, H. Bristow, Y. Wang, K. Hooper, A. D. Evans, F. Gao, H. Hoppe, J.-S. Kim, I. McCulloch, U. S. Schubert, T. M. Watson, J. R. Durrant, W. C. Tsoi, and Z. Li, "Influence of non-fullerene acceptors on the photostability of organic photovoltaics in inert atmospheres," In preparation.

[2] E. M. Speller, S. D. Dimitrov, A. J. Parnell, M. F. Wyatt, A. J. Clarke, A. D. F. Dunbar, J. R. Durrant, Z. Li, H. K. H. Lee, and W. C. Tsoi, "Morphological control of polymer:fullerene film and device photochemical stability," In preparation.

[3] E. M. Speller, A. J. Clarke, J. Luke, H. K. H. Lee, J. R. Durrant, N. Li, T. Wang, H. C. Wong, J.-S. Kim, W. C. Tsoi, and Z. Li, "From fullerene acceptors to nonfullerene acceptors: Prospects and challenges in the stability of organic solar cells," Submitted.

[4] E. M. Speller, A. J. Clarke, N. Aristidou, M. F. Wyatt, L. Francàs, G. Fish, H. Cha, H. K. H. Lee, J. Luke, A. Wadsworth, A. D. Evans, I. McCulloch, J.-S. Kim, S. Haque, J. R. Durrant, S. D. Dimitrov, W. C. Tsoi, and Z. Li, "Toward improved environmental stability of polymer:fullerene and polymer:non-fullerene organic solar cells: A common energetic origin of light and oxygen induced degradation," ACS Energy Lett., vol. 4, p. 846-852, 2019.

[5] J. Luke, E. M. Speller, A. Wadsworth, M. F. Wyatt, S. Dimitrov, H. K. H. Lee, Z. Li, W. C. Tsoi, I. McCulloch, D. Bagnis, J. R. Durrant, and J.-S. Kim, "Twist and degrade - impact of molecular structure on the photostability of nonfullerene acceptors and their photovoltaic blends," Adv. Energy Mater., vol. 9, p. 1803755, 2019.

[6] H. K. H. Lee, A. M. Telford, J. A. Rohr, M. F. Wyatt, J. Wu, A. De Castro Maciel, S. M. Tulandhar, E. Speller, J. McGettrick, J. R. Searle, S. Pont, T. Watson, T. Kirchartz, J. R. Durrant, W. C. Tsoi, J. Nelson, and Z. Li, "The Role of Fullerenes in The Environmental Stability of Polymer: Fullerene Solar Cells," Energy Environ. Sci, vol. 11, pp. 417-428, 2018.

[7] M. J. Newman, E. M. Speller, J. Barbe, J. Luke, M. Li, Z. Li, Z.-K. Wang, S. M. Jain, J.-S. Kim, H. K. H. Lee, and W. C. Tsoi, "Photo-stability study of a solutionprocessed small molecule solar cell system: correlation between molecular conformation and degradation," Sci. Technol. Adv. Mater, vol. 19, pp. 194-202, 2018.

[8] H. K. H. Lee, J. Wu, J. Barbe, S. M. Jain, S. Wood, E. M. Speller, Z. Li, F. A. Castro, J. R. Durrant, and W. C. Tsoi, "Organic photovoltaic cells - promising indoor light 
harvesters for self-sustainable electronics," J. Mater. Chem. A, vol. 6, pp. 5618-5626, 2018.

[9] E. M. Speller, J. D. McGettrick, B. Rice, A. M. Telford, H. K. H. Lee, C.-H. Tan, C. S. De Castro, M. L. Davies, T. M. Watson, J. Nelson, J. R. Durrant, Z. Li, and W. C. Tsoi, "The Impact of Aggregation on the Photochemistry of Fullerene Films: Correlating Stability to Triplet Exciton Kinetics," ACS Appl. Mater. Interfaces, vol. 9, pp. 22739-22747, 2017.

[10] J. D. McGettrick, E. M. Speller, Z. Li, W. C. Tsoi, J. R. Durrant, and T. M. Watson, "Use of gas cluster ion source depth profiling to study the oxidation of fullerene thin films by XPS," Organic Electronics, vol. 49, pp. 85-93, 2017.

[11] E. M. Speller, "The significance of fullerene electron acceptors in organic solar cell photo-oxidation," Materials Science and Technology, vol. 33, pp. 924-933, 2017. 


\section{LIST OF FIGURES}

1.1 Swanson effect showing the trend in the price of solar energy over the past

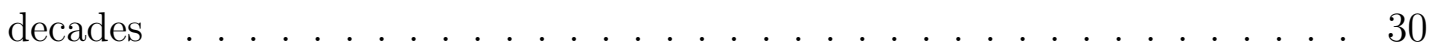

1.2 Illustrations to show (a) $\mathrm{sp}^{2} \mathrm{p}_{\mathrm{z}}$ hybridisation and (b) the chemical structure and $\mathrm{p}_{\mathrm{z}}$ orbitals in the $\pi$ system of a conjugated organic material. . . . . . . 33

1.3 Chemical structure of some typical donor polymers. . . . . . . . . . . . . . 34

1.4 Chemical structures of fullerenes and typical fullerene electron acceptors. . 34

1.5 Typical OSC architectures: (a) conventional structure and (b) inverted structure. . . . . . . . . . . . . . . . . . . . 35

1.6 Schematic diagram of the photovoltaic mechanism for OPV bulk heterojunctions. . . . . . . . . . . . . . . . . . 36

1.7 A Jablonski diagram showing electronic transitions in organic molecules and the timescales of these transitions. . . . . . . . . . . . . . . . 38

1.8 An energy diagram portraying the generation and fate of a charge transfer (CT) state. . . . . . . . . . . . . . . . . . . . . 40

1.9 Energy level diagram illustrating the trapping of charge carriers . . . . . . 41

1.10 A typical J-V curve measurement for OPV cells used to determine their device characteristics. . . . . . . . . . . . . . . . . . . . . . . 42

1.11 An illustration to show the lifetimes that can be quoted to define the stability of OPV devices. . . . . . . . . . . . . . . . . . . 43

1.12 A cross-sectional schematic of a conventional OSC illustrating some of the degradation processes. . . . . . . . . . . . . . . . . 44

1.13 TEM images of morphological changes in active layers. . . . . . . . . . . 45

1.14 Triplet formation mechanism in neat polymer films and in blend films. . . . 47

1.15 Proposed mechanisms for superoxide anion generation. . . . . . . . . . 47

1.16 Decrease in the peak ground state absorbance of PTB7 and DPP-TT-T films, both neat and blended with $\mathrm{PC}_{71} \mathrm{BM}$, during photoaging. . . . . . . 48 
1.17 Photochemical degradation measured via FTIR of MDMO-PPV, fullerene and blend. . . . . . . . . . . . . . . . . . . . . 50 50

1.18 Illustration of a photoexcitation and electron transport in a P3HT: $\mathrm{PC}_{61} \mathrm{BM}$ film, a) without photo-oxidation of $\mathrm{PC}_{61} \mathrm{BM}$ and b) with photo-oxidation of $\mathrm{PC}_{61} \mathrm{BM}$, showing that the deeper LUMO level of photo-oxidised $\mathrm{PC}_{61} \mathrm{BM}$ introduces trap states that would inhibit electron mobility in $\mathrm{PC}_{61} \mathrm{BM}$ domains. . . . . . . . . . . . . . . . . . . . . . . 51

1.19 The rate of photobleaching of polymers as a function of fullerene electron affinity in blend films. . . . . . . . . . . . . . . . . . . . . . 52

1.20 Chemical structures of fullerene acceptor SIMEF and when it is photooxidised to $\mathrm{SIMEF}_{-} \mathrm{O}_{2} \ldots \ldots \ldots \ldots \ldots$. . . . . . . . . . . . . . 53

2.1 Chemical structures and full names of the fullerene acceptors studied in this thesis. . . . . . . . . . . . . . . . . . . . . . 73

2.2 Chemical structures and full names (where known) of the non-fullerene acceptors studied in this thesis. . . . . . . . . . . . . . . . . . . 74

2.3 Chemical structures and full names of the polymers studied in this thesis. . 75

2.4 Schematic diagram and photos of layout of devices fabricated in this thesis 76

2.5 Methods for selective photodegradation of the active layer. . . . . . . . . . 78

2.6 Illustration of the fate of incident light on a sample. . . . . . . . . . . . . 79

2.7 Illustration of the Beer-Lambert Law. . . . . . . . . . . . . . . . . . 80

2.8 Schematic of a UV-vis spectrometer. . . . . . . . . . . . . . . . 81

2.9 Schematic of a microsecond TAS set-up. . . . . . . . . . . . . . . 83

2.10 Schematic of a photoluminescence concept. . . . . . . . . . . . . . . . 84

2.11 Schematic of superoxide fluorescence probe sensitisation set-up. . . . . . . 85

2.12 Chemical structures of hydroethidine (HE) and ethidium $\left(\mathrm{E}^{+}\right) \ldots \ldots$. . . 85

2.13 Schematic of a MALDI-TOF set-up. . . . . . . . . . . . . . . 87

2.14 Schematic of a FTIR system. . . . . . . . . . . . . . . . 89 
2.15 Energy level diagram which shows the vibrational energy levels, rotational energy levels and electronic energy levels of a material. . . . . . . . . . . 89

2.16 Schematic showing the working principle of an ATR system. . . . . . . . 91

2.17 Schematic showing the photo-excitation of a core electron by a photon. . . 92

2.18 Schematic showing an example layout of an XPS system. . . . . . . . . . 93

2.19 Illustration of an AFM set-up. . . . . . . . . . . . . . . . . 95

2.20 Schematic representation of an electrochemical cell for CV experiments. . . 96

2.21 A photograph of the environmental chamber used for measuring the stability of devices. . . . . . . . . . . . . . . . . . . . . . . . . . 98

3.1 J-V characteristics of conventional 1:2 PCDTBT:PC ${ }_{61} \mathrm{BM}$ devices where the BHJ films were exposed to simulated AM1.5G illumination in air prior to back contact deposition. . . . . . . . . . . . . . . . . . . . 106

3.2 UV-vis spectra of PCDTBT:PC ${ }_{61} \mathrm{BM}$ blend films without and with 60 minutes of exposure to AM1.5G conditions in dry air. . . . . . . . . . . . . 107

3.3 MALDI-TOF measurements of 1:2 PCDTBT:PC ${ }_{61} \mathrm{BM}$ blend films a) fresh, b)photoaged for 1 hour and c) photoaged for 10 hours and d) $\mathrm{PC}_{61} \mathrm{BM}$ solution photoaged for 72 hours in air under 1sun AM1.5G illumination. . . 108

3.4 (a) J-V characteristics of devices made with different fractions of solution degraded $\mathrm{PC}_{61} \mathrm{BM}$, the percentages indicate the mass fraction of $\mathrm{O}$ $\mathrm{P}_{61} \mathrm{CBM}$ in the acceptor phase. (b) $\mathrm{PCE}$ as a function of $\mathrm{O}-\mathrm{PC}_{61} \mathrm{BM}$ fraction for degraded PCDTBT: $\mathrm{PC}_{61} \mathrm{BM}$ devices and pre-degraded $\mathrm{PC}_{61} \mathrm{BM}$ devices.

3.5 Normalised (a) PCE, (b) $\mathrm{V}_{\mathrm{oc}},(\mathrm{c}) \mathrm{J}_{\mathrm{sc}}$, and (d) FF of 1:2 PCDTBT: $\mathrm{PC}_{61} \mathrm{BM}$ devices as a function of the percentage of degraded PCDTBT solution employed to form the active layer. . . . . . . . . . . . . . . . . . 111

3.6 JV characteristics of (a) P3HT:PC ${ }_{61} \mathrm{BM}$ and (b) PTB7:PC ${ }_{61} \mathrm{BM}$ made with aged and unaged polymer donor solution. . . . . . . . . . . . . . . . . . 112

3.7 J-V characteristics of devices using different OPV benchmark polymers (a) P3HT, (b) PTB7, and (c) PCE10 in blend with fresh $\mathrm{PC}_{61} \mathrm{BM}$ and selectively degraded $\mathrm{PC}_{61} \mathrm{BM} \ldots \ldots \ldots . \ldots \ldots . \ldots \ldots$ 
3.8 Normalised PCE of PCDTBT:PC ${ }_{61} \mathrm{BM}$ devices with active layers photoaged on different interlayers prior to electrode deposition. . . . . . . . . . . 114

$3.9 \mathrm{~J}-\mathrm{V}$ characteristics of devices using P3HT blended with different OPV benchmark fullerene acceptors. . . . . . . . . . . . . . . . . . . . . 115

4.1 UV-Vis absorbance spectra of (a) neat $\mathrm{PC}_{61} \mathrm{BM}$ and $\mathrm{PC}_{61} \mathrm{BM}: \mathrm{PS}$ films without photoaging (normalised at $336 \mathrm{~nm}$ ), and of (b) neat and (c) 50 wt\% $\mathrm{PC}_{61} \mathrm{BM}$ films, normalised to $\sim 265 \mathrm{~nm}$, with increasing photoaging time under AM1.5 irradiation in air. . . . . . . . . . . . . . . . . 125

4.2 Normalised ATR-FTIR absorbance spectra of (a) neat and $50 \mathrm{wt} \% \mathrm{PC}_{61} \mathrm{BM}$ films respectively, with increasing photoaging times. . . . . . . . . . . . . 126

4.3 (a) XPS spectrum (oxygen 1s envelope) of neat and $40 \mathrm{wt} \% \mathrm{PC}_{61} \mathrm{BM}$ films, before and after 920 minutes of photoaging; XPS spectra (carbon 1s envelope fitted with peaks for different carbon environments) of (b) neat $\mathrm{PC}_{61} \mathrm{BM}$ and (c) $40 \mathrm{wt} \% \mathrm{PC}_{61} \mathrm{BM}$ films, both after 920 minutes of photoaging; (d) atomic percentage of oxygen as a function of depth of neat and $40 \mathrm{wt} \% \mathrm{PC}_{61} \mathrm{BM}$ films, before and after 920 minutes of photoaging, respectively.

4.4 (a) Transient absorption decays of $\mathrm{PC}_{61} \mathrm{BM}$ triplet excitons of a $60 \mathrm{wt} \%$ $\mathrm{PC}_{61} \mathrm{BM}$ film in nitrogen and oxygen environments, (b) fractional loss of UV-Vis absorbance, relative growth of $\mathrm{C}=\mathrm{O}$ FTIR signal and relative yield of triplet states quenched by oxygen, all normalised and as a function of wt $\% \mathrm{PC}_{61} \mathrm{BM}$, following photoaging. . . . . . . . . . . . . . . . . 129

4.5 Model used to describe the possible degradation mechanism of $\mathrm{PC}_{61} \mathrm{BM}$ via triplet-mediated singlet oxygen generation . . . . . . . . . . . . . . . 131

4.6 Current density versus electric field of electron-only devices using $\mathrm{PC}_{61} \mathrm{BM}$ as the active layer with different photoaging times.

4.7 Simulated mobility in $\mathrm{PC}_{61} \mathrm{BM}$ doped with different volume fractions of oxidised defects. . . . . . . . . . . . . . . . . . . . . . . . . . 134

4.8 (a) Normalised PL and EL spectra of $\mathrm{PC}_{61} \mathrm{BM}$ films and devices, respectively, before and after the photoaging, and (b) calculated PL spectra (normalised) of a $\mathrm{PC}_{61} \mathrm{BM}$ molecule with and without oxidative defects. . . . . 135 
4.9 (a) GIWAXS spectra for the PCDTBT:PC ${ }_{61} \mathrm{BM}$ films with different blend ratios. (b) Mean size of ordered domains and (c) d-spacings corresponding to the peaks observed as a function of $\mathrm{PC}_{61} \mathrm{BM}$ content. (d) normalised mean size of ordered domains as a function of $\mathrm{PC}_{61} \mathrm{BM}$ content. . . . . . . 137

4.10 Photobleaching of PCDTBT:PC ${ }_{61} \mathrm{BM}$ films with wt rations of (a) 8:1 (b) 1:1 (c) 1:8 films, and (d) fractional loss of absorbance against the wt\% of $\mathrm{PC}_{61} \mathrm{BM}$ in films (photoaged for 120 minutes). . . . . . . . . . . . . . . 139

4.11 (a) Percentage of fullerenes oxidised and (b) intensity of oxidised fullerene peaks as a function of $\mathrm{PC}_{61} \mathrm{BM}$ content within PCDTBT: $\mathrm{PC}_{61} \mathrm{BM}$ films after 120 minutes of exposure to AM1.5G solar simulator exposure in air, obtained via MALDI TOF. . . . . . . . . . . . . . . . . . . . 140

4.12 Device characteristics (a) PCE (b) $\mathrm{V}_{\mathrm{oc}}$ (c) $\mathrm{J}_{\mathrm{sc}}$ (d) FF of PCDTBT: PC ${ }_{61}$ BM devices with different blend ratios as a function of the wt $\%$ of $\mathrm{PC}_{61} \mathrm{BM}$ after 10 and 30 minutes of photoaging of the active layer. . . . . . . . . . . . . 141

4.13 EQE spectra of PCDTBT:PC ${ }_{61} \mathrm{BM}$ (a) 8:1, (b) 1:1 (c) 1:8 devices after 0, 10, and 30 minutes of photoaging and (d) normalised EQE at $350 \mathrm{~nm}$ $\left(\mathrm{PC}_{61} \mathrm{BM}\right.$ peak) and $570 \mathrm{~nm}$ (PCDTBT peak) after 10 and 30 minutes of photoaging as a function of $\mathrm{PC}_{61} \mathrm{BM}$ wt\% . . . . . . . . . . . . . 142

4.14 Transient absorption spectra of neat PCDTBT and blend film with $67 \mathrm{wt} \%$ $\mathrm{PC}_{61} \mathrm{BM} \ldots \ldots \ldots \ldots \ldots \ldots \ldots \ldots$

4.15 TAS measurements: a) Representative TAS decay kinetics of a 4:1 PCDTBT: $\mathrm{PC}_{61} \mathrm{BM}$ film measured under nitrogen and oxygen environments. b) The change in optical density $(\Delta \mathrm{OD})$ between transient absorption kinetics measured under nitrogen and oxygen for 4:1, 2:1, 1:2 and 1:4 PCDTBT: $\mathrm{PC}_{61} \mathrm{BM}$ blend films. . . . . . . . . . . . . . . . . . . . . . 144

4.16 Normalised yields of: polymer triplet states quenched by oxygen as calculated from TAS measurements, percentage of $\mathrm{PC}_{61} \mathrm{BM}$ acceptor $(\mathrm{A})$ molecules photo-oxidised after 8 hours of degradation as measured by MALDITOF, fractional loss of UV-vis absorbance of $\mathrm{PC}_{61} \mathrm{BM}$ acceptor $(\mathrm{A})$ and PCDTBT donor (D) after 8 hours of photoaging, as a function of $\mathrm{PC}_{61} \mathrm{BM}$ wt\% . . . . . . . . . . . . . . . . . . . . 145

4.17 Model used to explain photodegradation of PCDTBT: $\mathrm{PC}_{61} \mathrm{BM}$ via polymertriplet mediated singlet oxygen generation . . . . . . . . . . . . . . . . 146 
4.18 (a) GIWAXS spectra for the 1:2 PCDTBT:PC ${ }_{61} \mathrm{BM}$ films with different film thicknesses. (b) Mean size of ordered domains and (c) d-spacings corresponding to the peaks observed as a function of film thickness. . . . . 147

4.19 Absorbance of PCDTBT (581 nm) and $\mathrm{PC}_{61} \mathrm{BM}(265 \mathrm{~nm})$ peaks in 1:2 PCDTBT:PC ${ }_{61}$ BM films, after 8 hours of photoaging under 1 sun AM1.5G solar simulator radiation in air, normalised to the unaged spectra, as a function of film thickness. . . . . . . . . . . . . . . . . . . . . . . . . 148

4.20 Percentage of $\mathrm{PC}_{61} \mathrm{BM}$ photo-oxidised in the films, after 2 hours of photoaging under 1 sun AM1.5G solar simulator radiation in air, as a function of film thickness. . . . . . . . . . . . . . . . . . . . . . . . . . . . . . 149

4.21 Normalised device characteristics (a) PCE (b) $\mathrm{V}_{\text {oc }}$ (c) $\mathrm{J}_{\mathrm{sc}}$ (d) FF PCDTBT: $\mathrm{PC}_{61} \mathrm{BM}$ devices with different film thicknesses as a function of photoaging time. . . . . . . . . . . . . . . . . . . . 150

4.22 Normalised device characteristics (a) PCE (b) $\mathrm{V}_{\text {oc }}$ (c) $\mathrm{J}_{\mathrm{sc}}$ (d) FF of 10 and 30 minutes photoaged PCDTBT:PC ${ }_{61} \mathrm{BM}$ devices as a function of PCDTBT:PC ${ }_{61}$ BM film thickness. . . . . . . . . . . . . . . . . . . 151

5.1 Cyclic voltammetry scans for: (a) Kloc-6 (b) $\mathrm{PC}_{71} \mathrm{BM}$ (c) $\mathrm{PC}_{61} \mathrm{BM}$ (d) ICBA (e) BisPCBM (f) ICTA, with the onset of the first oxidation potential fitted.

5.2 Normalised UV-vis absorption spectra of fullerene acceptor neat films under 1 Sun AM1.5G radiation in air, as a function of irradiation time: (a) Kloc-6 (b) $\mathrm{PC}_{71} \mathrm{BM}$ (c) $\mathrm{PC}_{61} \mathrm{BM}$ (d) ICBA (e) BisPCBM (f) ICTA . . . . . . . . . 163

5.3 Trends in fullerene acceptor photobleaching: (a) normalised UV-vis absorbance as a function of phtoaging time, and (b) normalised absorbance after 32 hours of photoaging as a function of fullerene acceptor LUMO level.164

5.4 Normalised FTIR spectra of: (a) $\mathrm{PC}_{61} \mathrm{BM}$ (b) $\mathrm{PC}_{71} \mathrm{BM}$ (c) Kloc-6 neat films fresh and photoaged degraded for 2, 4 and 8 hours in air under 1 Sun AM1.5G illumination.

5.5 Normalised absorbance of the carbonyl peak at $\sim 1780 \mathrm{~cm}^{-1}$ after 8 hours of degradation for different fullerene acceptors as a function of LUMO level. 165 
5.6 MALDI-TOF spectra of: (a) Kloc-6 (b) $\mathrm{PC}_{71} \mathrm{BM}$ (c) $\mathrm{PC}_{61} \mathrm{BM}$ (d) ICBA (e) BisPCBM (f) ICTA neat films degraded for 32 hours in air under 1 Sun AM1.5G illumination. . . . . . . . . . . . . . . . . . . . 167

5.7 MALDI-TOF spectra of: ICBA (a) degraded for 8 hours and (b) 16 hours and ICTA (c) degraded for 8 hours and (d) 16 hours in air under 1 Sun AM1.5G. illumination.

5.8 Mass spectroscopy (a) percentage of fullerenes photo-oxidised and (b) the number of additional oxygens per molecule as a function of photoaging time for neat fullerene acceptor films after up to 32 hours of degradation to 1 Sun AM1.5G illumination in air. (c) Percentage of fullerenes photo-oxidised and d) the number of additional oxygens per photo-oxidised molecule after 32 hours of photoaging as a function of LUMO level.

5.9 Proposed degradation mechanism via superoxide formation.

5.10 Fractional loss of (a) the P3HT and (b) acceptor peaks in P3HT: fullerene blend films after photoaging under 1 Sun irradiation in air for 8 hours as a function of acceptor LUMO level. . . . . . . . . . . . . . . . . . . . . 171

5.11 MALDI-TOF spectra of: (a) Kloc-6 (b) $\mathrm{PC}_{71} \mathrm{BM}$ (c) $\mathrm{PC}_{61} \mathrm{BM}$ (d) ICBA (e) BisPCBM solutions degraded and stirred for 24 hours in air under 1 Sun AM1.5G illumination. . . . . . . . . . . . . . . . . . . . . . . 173

5.12 Normalised device characteristics of P3HT: fullerene devices (a) PCE, (b) $\mathrm{V}_{\mathrm{oc}}$, (c) $\mathrm{J}_{\mathrm{sc}}$, and (d) FF as a function of degraded fullerene solution content.174

5.13 Normalised device characteristics (a) PCE, (b) $\mathrm{V}_{\mathrm{oc}}$, (c) $\mathrm{J}_{\mathrm{sc}}$, and (d) FF of solution degraded devices as a function of fullerene acceptor LUMO level. . 175

5.14 Normalised device characteristics (a) PCE, (b) $\mathrm{V}_{\text {oc }}$, (c) $\mathrm{J}_{\mathrm{sc}}$, and (d) FF as a function of BHJ film degradation time. . . . . . . . . . . . . . . 177

6.1 Normalised device characteristics (a) PCE, (b) $\mathrm{J}_{\mathrm{sc}}$, (c) $\mathrm{V}_{\mathrm{oc}}$ and (d) FF as a function of device photoaging time under dry nitrogen. . . . . . . . . . . 184

6.2 Normalised UV-Vis absorbance of (a) IDFBR and (b) IDTBR annealed neat films under 1 sun AM1.5G illumination under dry nitrogen. . . . . . . 185

6.3 Normalised UV-Vis absorbance of (a) Eh-IDTBR and (b) P3HT annealed neat films under 1 sun AM1.5G illumination under dry nitrogen. . . . . . . 186 
6.4 Normalised UV-Vis absorbance of (a) $\mathrm{PC}_{61} \mathrm{BM}$ and (b) P3HT: $\mathrm{PC}_{61} \mathrm{BM}$ annealed films under 1 sun AM1.5G illumination under dry nitrogen. . . . 186

6.5 Normalised UV-Vis absorbance of (a) P3HT:IDFBR and (b) P3HT: IDTBR annealed films under 1 sun AM1.5G illumination under dry nitrogen. . . . 187

6.6 Normalised absorbance at specified wavelengths of annealed a) $\mathrm{PC}_{61} \mathrm{BM}$, P3HT and NFA films and b) P3HT:acceptor films (donor contribution) and c) P3HT:acceptor films (acceptor contribution) as a function of photoaging time under AM1.5G illumination in dry nitrogen. . . . . . . . . . . . . 188

6.7 Negative ion mode MALDI-TOF measurements of: a) IDTBR and b) IDFBR neat films after 16 hours of photoaging under AM1.5G illumination in nitrogen.

6.8 Normalised device characteristics (a) PCE, (b) $\mathrm{V}_{\text {oc }}$ which is enlarged in the inset, (c) $\mathrm{J}_{\mathrm{sc}}$ and (d) FF as a function of device photoaging time under 1 Sun equivalent white LED light in dry nitrogen. . . . . . . . . . . . . . . . 190

6.9 Normalised device characteristics (a) PCE (b) $\mathrm{V}_{\mathrm{oc}}$, (c) $\mathrm{J}_{\mathrm{sc}}$, (d) FF as a function of photoaging time under white LED illumination in air. . . . . . 192

6.10 Topography images of annealed (a) P3HT:IDTBR and (b) P3HT: IDFBR films. . . . . . . . . . . . . . . . . . . . . . 193

6.11 Normalised device characteristics (a) PCE (b) $\mathrm{V}_{\text {oc }}$ (c) $\mathrm{J}_{\mathrm{sc}}$ (d) FF of P3HT: PC $_{61}$ BM, P3HT:IDTBR, P3HT:Eh-IDTBR and P3HT:IDFBR devices, as a function of active layer photoaging time in air. . . . . . . . . . . . . . . . 194

6.12 Photobleaching of (a) IDFBR and (b) IDTBR neat films under 1 sun illumination in air.

6.13 Photobleaching of (left) neat annealed $\mathrm{PC}_{61} \mathrm{BM}$ and (right) an annealed P3HT:PC ${ }_{61}$ BM blend films under 1 sun illumination in air. . . . . . . . . . 196

6.14 Photobleaching of a P3HT neat film under 1 sun illumination in air. . . . . 196

6.15 Photobleaching of (left) P3HT:IDFBR and (right) P3HT:IDTBR under 1 sun illumination in air.

6.16 Photobleaching of (a) Eh-IDTBR and (b) $\mathrm{PC}_{61} \mathrm{BM}$ neat films under 1 sun illumination in air. 
6.17 Normalised absorbance at specified wavelengths of annealed a) $\mathrm{PC}_{61} \mathrm{BM}$, P3HT and NFA films and b) P3HT:acceptor films (donor contribution) and c) P3HT:acceptor films (acceptor contribution) as a function of photoaging time under AM1.5G illumination and air. . . . . . . . . . . . . . . . . . . 198

6.18 Negative ion mode MALDI-TOF measurements of: a) IDTBR after 8 hours of degradation and b) zoomed-in on oxidation. . . . . . . . . . . . . . . 199

6.19 Negative ion mode MALDI-TOF measurements of IDFBR photoaged as a thin film under AM1.5G illumination and air for: a) 1 hour, b) 4 hours and c) 8 hours. . . . . . . . . . . . . . . . . . . . . . . . . 199

6.20 Transient absorption spectra under dry nitrogen for (a) IDFBR (ex 520

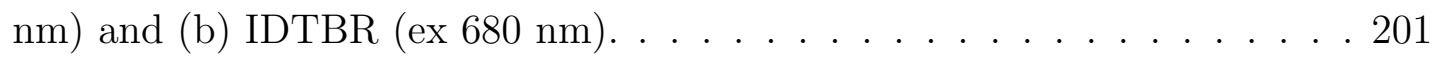

6.21 Transient decay kinetics under dry nitrogen and oxygen atmospheres for (a) IDFBR and (b) IDTBR. . . . . . . . . . . . . . . . . . . . . . 201

6.22 Transient absorption spectra under nitrogen for (a) P3HT:IDFBR (ex 520

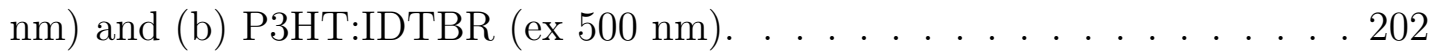

6.23 Transient decay kinetics under nitrogen and oxygen atmospheres for (a) P3HT:IDFBR and (b) P3HT:IDTBR. . . . . . . . . . . . . . . . . . 202

6.24 Fits of transient decay kinetics under nitrogen and oxygen atmospheres for (a) P3HT:IDFBR and (b) P3HT:IDTBR. . . . . . . . . . . . . 203

6.25 (a) fluorescence spectra for P3HT:IDFBR as a function of illumination time (b) normalised fluorescence intensity increase of the HE probe. . . . . . . . 204

6.26 Fractional loss of (a) the P3HT and (b) acceptor peaks in P3HT blends with fullerene and non-fullerene acceptors after degrading under 1 Sun irradiation in air for 8 hours as a function of acceptor LUMO level. . . . . 206

6.27 Proposed degradation mechanism via superoxide formation. . . . . . . . . 207

A.1 Summary of normalised device parameters of (a) 1:2 PCDTBT:PC ${ }_{61}$ BM devices with different degradation times under simulated AM1.5G illumination in air, before top electrode deposition and (b) PCDTBT: PC $_{61} \mathrm{BM}$ blend devices made with different fractions of degraded $\mathrm{PC}_{61} \mathrm{BM}$. 
A.2 J-V characteristics of 1:2 PCDTBT:PC ${ }_{61} \mathrm{BM}$ devices exposed to air for 60 minutes in the dark and under 1000 lux of compact fluorescent lamp. . . . 215

A.3 Device characteristics (a) PCE, (b) $\mathrm{V}_{\mathrm{oc}}$, (c) $\mathrm{J}_{\mathrm{sc}}$, and (d) FF of 1:2 PCDTBT: $\mathrm{PC}_{61} \mathrm{BM}$ devices as a function of the proportion of degraded PCDTBT solution employed to form the active layer. . . . . . . . . . . . . . 216

A.4 J-V characteristics of (a) $\mathrm{MoO}_{3}$ and (b) $\mathrm{ZnO}$ based devices where the 1:2 PCDTBT:PC ${ }_{61} \mathrm{BM}$ film was exposed to air and 1sun AM1.5G illuminaition prior to top electrode $\left(\mathrm{Ca} / \mathrm{Al}\right.$ and $\mathrm{MoO}_{3} / \mathrm{Ag}$ respectively) deposition. . . . 216

A.5 PCE as a function of BHJ degradation time of 1:2 PCDTBT:PC ${ }_{61} \mathrm{BM}$ devices utilising PEDOT:PSS and $\mathrm{MoO}_{3}$ interlayers with a conventional architecture with $\mathrm{Ca} / \mathrm{Al}$ top electrodes and $\mathrm{ZnO}$ for the inverted architecture devices with $\mathrm{MoO}_{3} / \mathrm{Ag}$ top electrodes. . . . . . . . . . . . . . . 217

B.1 UV-Vis absorbance spectra of neat PS before and after photoaging under AM1.5 irradiation, in air. . . . . . . . . . . . . . . . . . . . . . 218

B.2 UV-Vis absorbance spectra of (a) $80 \mathrm{wt} \%$ and (b) $20 \mathrm{wt} \% \mathrm{PC}_{61}$ :PS films (normalised at $265 \mathrm{~nm}$ ), with increasing photoaging time under AM1.5 irradiation and air

B.3 Normalised ATR-FTIR absorbance spectra of (a) 95 wt\% (b) 90 wt\% (c) 80 wt\% and (d) $20 \mathrm{wt} \% \mathrm{PC}_{61} \mathrm{BM}$ films respectively, with increasing photoaging times. . . . . . . . . . . . . . . . . . . . . 219

B.4 ATR-FTIR spectra of fresh and photoaged PS. . . . . . . . . . . . . . 219

B.5 XPS spectrum (carbon 1s envelope) of (a) neat and (b) $40 \mathrm{wt} \% \mathrm{PC}_{61} \mathrm{BM}$ films before photoaging (in counts per second (CPS)) . . . . . . . . . 220

B.6 XPS spectrum (a) (carbon (1s) envelope) of $\mathrm{PC}_{61} \mathrm{BM}$ film after 1 hour of photoaging and (b) (oxygen (1s) envelope) of $\mathrm{PC}_{61} \mathrm{BM}$ films fresh and after 1 hour of photoaging. . . . . . . . . . . . . . . . . . . . . . 220

B.7 Possible photo-oxidation mechanism of $\mathrm{PC}_{61} \mathrm{BM}$ : Sequential formation of epoxides, diols and carbonyls. . . . . . . . . . . . . . . . . . . . 221 
B.8 Epoxide:carbonyl ratio $(\mathrm{C}-\mathrm{O}: \mathrm{C}=\mathrm{O})$ ratio as estimated from the $\mathrm{C}(1 \mathrm{~s})$ envelope and total measured atomic percentage of oxygen as a function of etch depth for photoaged $40 \mathrm{wt} \% \mathrm{PC}_{61} \mathrm{BM}$ film. Note that the data at the surface $(0 \mathrm{~nm})$ is less reliable as it is subject to surface contamination as often observed in the field. . . . . . . . . . . . . . . . . . . . . . . . 221

B.9 TAS measurements: (a) Transient absorption spectra (with different time delays) of an $80 \mathrm{wt} \% \mathrm{PC}_{61} \mathrm{BM}$ :PS film, showing the spectral region where triplet-triplet absorption occurs. TAS decay kinetics (excited at $355 \mathrm{~nm}$ and probed at $700 \mathrm{~nm}$ ) of different wt\% $\mathrm{PC}_{61} \mathrm{BM}$ films under (a) nitrogen and (b) oxygen environments, fitted with biexponential decays. . . . . . . 222

B.10 UV-Vis absorbance spectra of (a) 4:1 (b) 2:1 (c) 1:2 (d) 1:4 PCDTBT: $\mathrm{PC}_{61} \mathrm{BM}$ films with up to 120 minutes of photoaging. . . . . . . . . 223

B.11 Normalised absorbance of the (a) PCDTBT (579 nm) and (b) $\mathrm{PC}_{61} \mathrm{BM}$ $(266 \mathrm{~nm})$ peaks of the PCDTBT:PC ${ }_{61} \mathrm{BM}$ films with varying wt\% ratios with up to 120 minutes of photoaging.

B.12 MALDI-TOF measurements of PCDTBT:PC ${ }_{61} \mathrm{BM}$ blend films with varying wt\% ratios photoaged for 2 hours in air under 1 sun AM1.5G illumination.

B.13 Device characteristics (a) PCE (b) $\mathrm{V}_{\mathrm{oc}}$ (c) $\mathrm{J}_{\mathrm{sc}}$ (d) FF of PCDTBT: $\mathrm{PC}_{61} \mathrm{BM}$ devices with different wt\% ratios as a function of photoaging time. . . . . . 225

B.14 EQE spectra of (a) 4:1, (b) 2:1 (c) 1:2 and (d) 1:4 PCDTBT:PC ${ }_{61}$ BM devices after 0,10 , and 30 minutes of photoaging. . . . . . . . . . 226

B.15 Normalised EQE at (a) PCDTBT (570 nm) and (b) $\mathrm{PC}_{61} \mathrm{BM}(350 \mathrm{~nm})$ of PCDTBT: $\mathrm{PC}_{61} \mathrm{BM}$ devices with different wt $\%$ ratios, as a function of photoaging time.

B.16 TAS decay kinetics spectra of a) neat PCDTBT (with exponential fit), b) 2:1, c) 1:2 and d) 1:4 PCDTBT:PC ${ }_{61} \mathrm{BM}$ films measured under nitrogen and oxygen environments.

B.17 Photobleaching of 1:2 PCDTBT:PC ${ }_{61} \mathrm{BM}$ films, varying in thickness from $93-35 \mathrm{~nm}$, with up to 8 hours of photoaging under 1 sun AM1.5G illumination in air. 
B.18 Normalised UV-Vis absorbance at (a) $581 \mathrm{~nm}$ (PCDTBT) and (b) $265 \mathrm{~nm}$ $\left(\mathrm{PC}_{61} \mathrm{BM}\right)$ of 1:2 PCDTBT: $\mathrm{PC}_{61} \mathrm{BM}$ films with different thicknesses, as a function of photoaging time.

B.19 MALDI-TOF measurements of 1:2 PCDTBT:PC ${ }_{61} \mathrm{BM}$ blend films with different thicknesses, photoaged for 2 hours in air under 1 sun AM1.5G illumination. $\mathrm{Mw} \mathrm{PC}_{61} \mathrm{BM}=910.90 \mathrm{gmol}^{-1}$. . . . . . . . . . . . . . . 230

B.20 Device characteristics (a) PCE (b) $\mathrm{V}_{\text {oc }}$ (c) $\mathrm{J}_{\mathrm{sc}}$ (d) FF of PCDTBT: $\mathrm{PC}_{61} \mathrm{BM}$ devices with different film thicknesses as a function of degradation time. . . 231

B.21 Device characteristics (a) PCE (b) $\mathrm{V}_{\text {oc }}$ (c) $\mathrm{J}_{\mathrm{sc}}$ (d) FF of 1:2 PCDTBT: $\mathrm{PC}_{61} \mathrm{BM}$ devices photaged for 0,10 and 30 minutes, as a function of active layer film thickness. . . . . . . . . . . . . . . . . . . . . . 232

C.1 UV-vis absorption spectra of (a) Kloc-6 (b) PC $\mathrm{PC}_{71} \mathrm{BM}$ (c) $\mathrm{PC}_{61} \mathrm{BM}$ (d) ICBA (e) BisPCBM (f) ICTA neat films for up to 32 hours under 1 sun AM1.5G illumination in air. . . . . . . . . . . . . . . . . . . 233

C.2 FTIR spectra of: (a) Kloc-6 (b) $\mathrm{PC}_{71} \mathrm{BM}$ (c) $\mathrm{PC}_{61} \mathrm{BM}$ (d) ICBA (e) BisPCBM (f) ICTA neat films fresh and photoaged degraded for 2, 4 and 8 hours in air under 1 Sun AM1.5G illumination. . . . . . . . . . . . . . . 235

C.3 UV-vis absorption spectra of blend films with up to 8 hours under 1 sun AM1.5G illumination in air. P3HT blended with: (a) Kloc-6 (b) $\mathrm{PC}_{71} \mathrm{BM}$

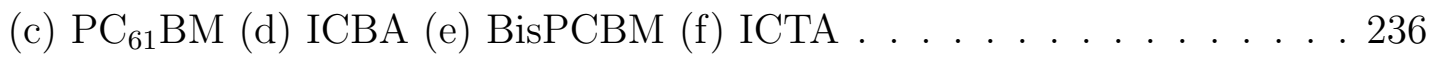

C.4 Device characteristics of P3HT:fullerene devices as a function of degraded solution content: (a) PCE, (b) $\mathrm{V}_{\mathrm{oc}}$, (c) $\mathrm{J}_{\mathrm{sc}}$, and (d) FF. . . . . . . . . . . 237

C.5 Device characteristics (a) PCE, (b) $\mathrm{V}_{\mathrm{oc}}$, (c) $\mathrm{J}_{\mathrm{sc}}$, and (d) FF as a function of BHJ film photoaging time.

D.1 Device characteristics (a) PCE (b) $\mathrm{V}_{\mathrm{oc}}$, (c) $\mathrm{J}_{\mathrm{sc}}$, (d) FF as a function of device photoaging time under dry nitrogen. . . . . . . . . . . . . . . 238

D.2 Photobleaching of (a) IDFBR (b) IDTBR films under 1 sun illumination with a UV-cutting filter, in nitrogen. . . . . . . . . . . . . . . . 239

D.3 Device characteristics (a) PCE (b) $\mathrm{V}_{\mathrm{oc}}$ (c) J $\mathrm{J}_{\mathrm{sc}}$ (d) FF of P3HT devices blended with different various acceptors, degraded prior to electrode deposition, as a function of degradation time. 
D.4 Photobleaching of (a) IDFBR (b) IDTBR films under 1 sun illumination with a UV-cutting filter, in air. . . . . . . . . . . . . . . . 240

D.5 Photobleaching of unannealed (a) IDFBR and (b) IDTBR neat films under 1 sun illumination in air. . . . . . . . . . . . . . . . . . . . . . . 240

D.6 Photobleaching of (a) IDFBR (b) IDTBR annealed films under 1 sun equivalent white LED illumination in air. . . . . . . . . . . . . . . . . . . 241

D.7 Negative ion mode MALDI-TOF measurements of: a) IDTBR and b) IDFBR fresh neat films . . . . . . . . . . . . . . . . . . . . . 241

D.8 Device characteristics (a) PCE (b) $\mathrm{J}_{\mathrm{sc}}$, (c) $\mathrm{V}_{\mathrm{oc}}$, (d) FF as a function of device degradation time (as completed devices) in air. . . . . . . . . . . 242

D.9 Cyclic voltammetry scans of neat NFA films. . . . . . . . . . . . 243 


\section{LIST OF TABLES}

1.1 Comparison on silicon and OSCs in terms of efficiency, lifetime and upscaled power density. . . . . . . . . . . . . . . . . . . . . . . . . 32

2.1 Purities and molecular weights $\left(\mathrm{M}_{\mathrm{w}}\right)$ of the fullerene acceptors. . . . . . . 72

2.2 Supplier, purity and molecular weight $\left(\mathrm{M}_{\mathrm{w}}\right)$ of non-fullerene acceptors.(I.M. denotes that the material was synthesised in-house by Iain McCulloch's group.) . . . . . . . . . . . . . . . . . 73

2.3 Supplier, molecular weights $\left(\mathrm{M}_{\mathrm{w}}\right)$, polydispersity $(\mathrm{PD})$ and regioregularity (RR) of the polymers.

3.1 Concentration of Donor (D) and acceptor (A) in CB, spin speed/ acceleration, DIO added to prepare the solutions for active layers. For P3HT, it was blended with various kinds of fullerene acceptors (FA). . . . . . . . . 105

3.2 Fullerene acceptors used in P3HT:fullerene devices and the initial PCE, PCE obtained when using fullerene solutions that had been aged for 72 hours in air under 1sun AM1.5G illumination and the percentage of PCE remaining when using the photo-aged solution compared to the fresh. . . . 115

4.1 Concentration of PCDTBT (D) and $\mathrm{PC}_{61} \mathrm{BM}(\mathrm{A})$ in $\mathrm{DCB}$ for solutions of different blend wt ratios and the spin speeds employed to make thin films with thicknesses $\approx 70 \mathrm{~nm}$. . . . . . . . . . . . . . . . . . 123

4.2 PCDTBT:PC ${ }_{61} \mathrm{BM}$ films (1:2 wt\% ratio) with decreasing thicknesses which are controlled by reducing the concentration of PCDTBT and $\mathrm{PC}_{61} \mathrm{BM}$ (in $\mathrm{CB})$ in solutions.

4.3 Properties of the triplet kinetics in blend $\mathrm{PC}_{61} \mathrm{BM}$ films . . . . . . . . . 129

4.4 Calculated effect of different defect types on the LUMO energy of oxidised $\mathrm{PC}_{61}$ BM. . . . . . . . . . . . . . . . . . . . . . . 133

4.5 GIWAXS peak positions of the PCDTBT:PC ${ }_{61} \mathrm{BM}$ films with different blend ratios. . . . . . . . . . . . . . . . . . . . . 137 
6.1 Excitation wavelengths $\left(\lambda_{\text {pump }}\right)$, approximately at the ground state absorption maximum, and probe wavelengths $\left(\lambda_{\text {probe }}\right)$, around the maximum of transient absorption spectrum, used in the TAS studies. . . . . . . . . 183

6.2 Properties of triplets and oxygen quenching for neat IDFBR and IDTBR films under nitrogen and oxygen atmospheres. . . . . . . . . . . . . . 201

B.1 XPS peak assignments and typical bulk compositions of neat $(\mathrm{N})$ and 40 wt\% (B) $\mathrm{PC}_{61} \mathrm{BM}$ films, before and after photoaging for 920 minutes. . . . 220

C.1 IR peak position and assignment for photo-oxidised fullerenes. . . . . . . . 234

D.1 $\mathrm{T}_{80}$ lifetimes of devices aged under white LED illumination in nitrogen. A dash indicates that the device had not reached its $\mathrm{T}_{80}$ in the 120 hours of measuring. . . . . . . . . . . . . . . . . . . . . . . . . . 240

D.2 $\mathrm{T}_{80}$ lifetimes of devices aged under white LED illumination in nitrogen. . . 241

D.3 $\mathrm{T}_{80}$ lifetimes of devices aged under white LED illumination in air. . . . . . 242 


\section{ABBREVIATIONS}

AFM Atomic Force Microscopy

ATR Attenuated total reflectance

BisPCBM $\operatorname{Bis(1-[3-(methoxycarbonyl)propyl]-1-phenyl)-[6,6]C62~}$

CS Charge separation

CT Charge transfer

CV Cyclic voltammetry

DCTB Trans-2-[3-(4-tert-butylphenyl)-2-methyl-2-propenylidene]malononitril

$\triangle O D \quad$ Change in optical density

$\mathrm{E}_{\mathrm{g}} \quad$ optical bandgap

EL Electroluminescence

EQE External quantum efficiency

$\mathrm{E}^{+} \quad$ Ethidium

FF Fill factor

FTIR Fourier Transform Infrared Spectroscopy

FWHM Full width half maximum

GIWAXS Grazing incidence wide-angle x-ray scattering

HE Hydroethidine

HOMO Highest occupied molecular orbital

ICBA Indene- $\mathrm{C}_{60}$-bis-adduct

ICTA Indene- $\mathrm{C}_{60}$-tris-adduct

IR Infrared

ISC Intersystem crossing

ITO Indium tin oxide

$\mathrm{J}_{\mathrm{SC}} \quad$ Short-circuit current density

Kloc-6 -

LUMO Lowest unoccupied molecular orbital

MALDI Matrix-assisted laser desorption/ionisation

MW Molecular weight

NIR Near infrared

OFET Organic field effect transistor

OSC Organic solar cell

$\mathrm{PC}_{61} \mathrm{BM} \quad$ Phenyl- $\mathrm{C}_{61}$-butyric acid methyl ester

$\mathrm{PC}_{71} \mathrm{BM} \quad$ Phenyl- $\mathrm{C}_{71}$-butyric acid methyl ester

PCDTBT Poly[N-9'-heptadecanyl-2,7-carbazole-alt-5,5-(4',7'-di-2-thienyl-2',1', 3'-benzothiadiazole)]

PCE Power conversion efficiency 
PCE10 Poly[4,8-bis(5-(2-ethylhexyl)thiophen-2-yl)benzo[1,2-b;4,5-b']dithiophene2,6-diyl-alt-(4-(2-ethylhexyl)-3-fluorothieno[3,4-b]thiophene-)-

2-carboxylate-2-6-diyl)]

P3HT Poly(3-hexylthiophene-2,5-diyl)

PL Photoluminescence

PTB7 Poly[2-methoxy-5-(2-ethylhexyloxy)-1,4-phenylenevinylene]

TAS Transient Absorption Spectroscopy

TBAHFP Tetrabutylammonium hexauorophosphate

TD-DFT Time-dependent density functional theory

TOF Time of flight

UV Ultraviolet

Vis Visible

$\mathrm{V}_{\mathrm{oc}} \quad$ Open circuit current

XPS X-ray Photoelectron Spectroscopy 


\section{INTRODUCTION}

\subsection{Introduction to organic solar cells}

\subsubsection{Motivation}

The Industrial Revolution in the $18^{\text {th }}$ Century saw massive technological advances driven by fossil fuels which have since been the main source of mankind's energy. However, there are two major problems with burning fossil fuels for energy. Firstly, the depleting fossil fuel reserves are struggling to keep up with the world's growing energy demands and it is thought they will be completely exhausted in the next century.[1] Secondly, the combustion of fossil fuels is known to emit greenhouse gases and other pollutants, namely carbon dioxide. The increase in carbon dioxide emissions has been shown to have an effect on the climate to include an increase of global temperature, rising sea levels, changes in extreme weather and climate events and increased acidity of the oceans, thus impacting on human lives and threatening a number of animals with extinction. [2] With the global energy demand growing, fossil fuel reserves depleting and the increasing need to tackle climate change, there is an ever growing need to develop alternative clean energies. As a result, in December 2015196 countries signed the Paris Climate Accord, with each agreeing on a plan to mitigate their contribution to climate change. For instance, the UK aims to generate $15 \%$ of its national energy consumption through renewable energy by 2020.[3] However, in 2013, only 5.2\% of the UK's energy came from renewable energies so there is still some way to go before this target is met.[4]

Renewable energy is energy derived from natural sources that are constantly replenished such as solar, wind and tidal.[5] Among renewable energy resources, solar energy is by far the largest exploitable resource, providing more energy in one hour to the earth than all of the energy currently used in an entire year by mankind.[6] Solar energy can be harvested in three ways. Firstly, solar thermal directly heats water and buildings. Solar 
photovoltaics covert the solar energy into electricity. Solar fuels which store the solar energy chemically to be released later, e.g. biofuels, fuel cells and hyrdogen storage.[7]

\section{The Swanson effect}

Price of crystalline silicon photovoltaic cells, \$ per watt

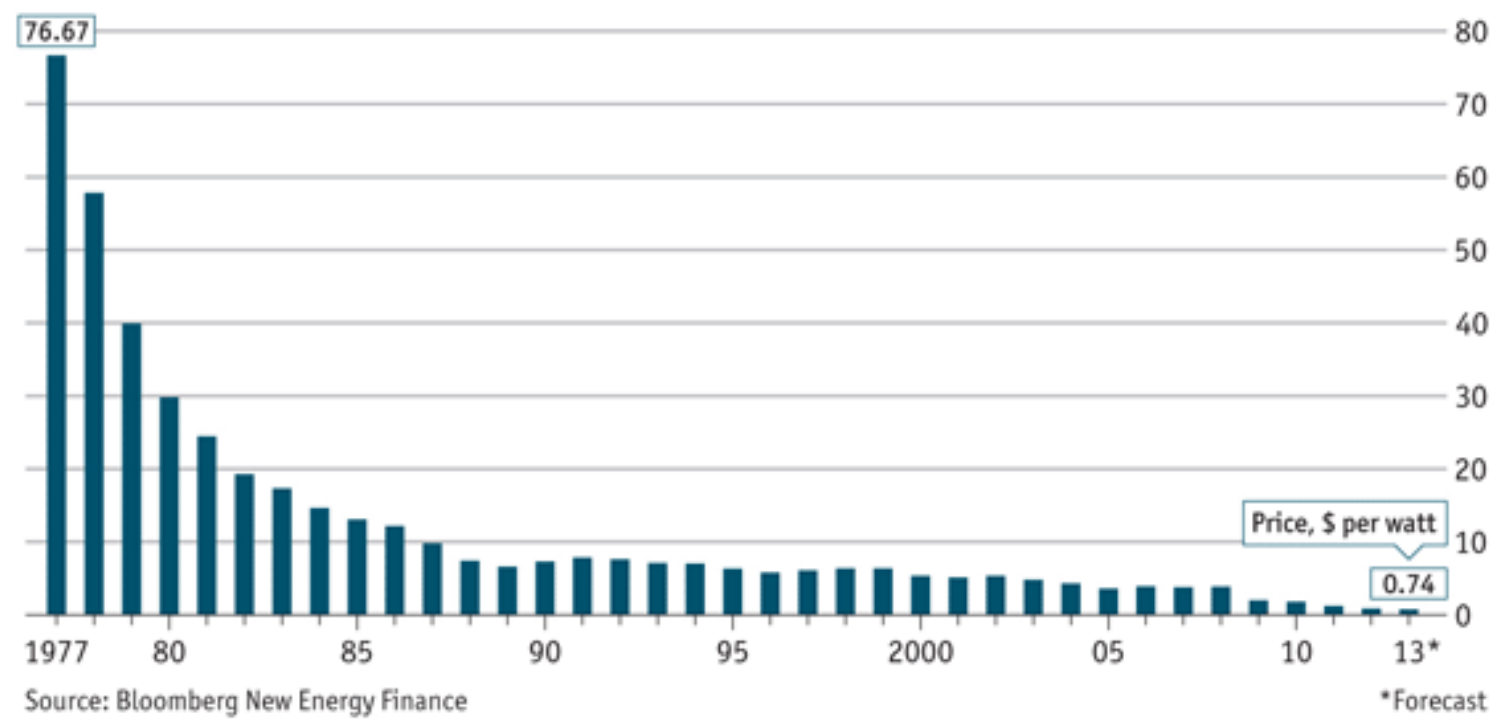

Economist.com/graphicdetail.

Figure 1.1: Swanson effect showing the trend in the price of solar energy over the past decades.[8]

Photovoltaics are the branch of technology involving the production of an electric current upon absorption of light as described by the Photovoltaic Effect. This effect was first observed by Becquerel in 1839, with "the production of an electric current when two plates of platinum or gold immersed in an acid, neutral, or alkaline solution are exposed in an uneven way to solar radiation". $[9,10]$ Devices that can directly convert light energy into electricity are called photovoltaic cells. An integrated group of connected photovoltic cells orientated in one plane constitutes a module. Currently, commercialised (and the first generation of photovoltaic technology) solar cells are mainly based on crystalline silicon, an inorganic semiconductor, which, despite a steady (and recently sharp) price reduction of modules, are still not competitive against fossil fuels. [8, 11-13] This trend is described by the Swanson Effect, a $20 \%$ price reduction for each doubling of cumulative production, shown in Figure 1, and is a similar type of forecast as Moore's law for the number of transistors that can be fitted onto an integrated circuit.[14] Silicon is a highly demanded raw material which requires high temperature and vacuum processing to make it of suitable quality to be used as a semiconducting material, thus making silicon solar cells expensive.

The second generation of photovoltaic is classed as thin film solar cells because they are based on films just a few micrometers thick of inorganic semiconducting materials 
such as amorphous silicon, cadmium telluride or copper indium gallium selenide.[15] The lower material consumption mean that production costs are reduced compared to the first generation. However, high temperature and vacuum processing is still used and the utilisation of rare elements means that the price that can be achieved is limited.

Third generation photovoltaics utilise solution processable semiconducting materials. Organic solar cells (OSCs) are one member of this generation of photovoltaic which have potential to be more versatile and cheaper than their silicon counterparts for the following reasons: 1) The polymers and some small molecules are soluble in organic solvents, enabling them to be produced with low temperature solution processing such as roll-toroll processing, reducing the price and making the manufacture of OSCs far easier than that of silicon systems. 2) The semiconducting layers of OSCs are composed of organic polymers and/or small molecules, deposited in thin films (100 - $200 \mathrm{~nm})$, which with development could be cheaper than silicon, which is an expensive material to process to get it to the necessary quality for semiconductors. This means that OSCs will make solar more accessible both on a large scale, for use in business, and for deployment in developing countries, where electricity has yet to be introduced or where the supply is unreliable. 3) The chemical structures of these photoactive materials can be varied so that their properties can be tuned, including the wavelengths at which they absorb thus giving them the capability to maximise their absorption by matching the solar spectrum or making them semi-transparent - a property that silicon solar cells do not possess. 4) These coating methods allow for OSCs to be fabricated onto a vast range of substrates, including flexible plastic, making them light weight and highly mechanically flexible. These properties increase the versatility of OSCs and could lead to novel applications such as: Building Integrated Photovoltaics where photovoltaics replace some building materials in the roof or facade; power generating windows; or solar textiles. These factors mean that OSCs have the potential to be a more cost-effective and versatile method than silicon solar cells to harness the power of the Sun.

However, there are a few aspects of OSCs that need to be developed before they are able to compete with their silicon counterparts, summarised in Table 1.1. Firstly, despite a recent rapid increase in power conversion efficiencies, OSCs have only reached $14.2 \%$ for single junction cells [16] efficiency which falls short of the 15-20\% of commercially available crystalline silicon modules and $25 \%$ for lab scale devices.[17, 18] Secondly, stability of OSCs is less than half silicon PV's 25 year lifetime [19](10 years under AM1.5G LED lamp in ambient conditions [20] and 7 years tested under AM1.5 sulphur plasma lamp in air on a hotplate at $(37 \pm 2)^{\circ} \mathrm{C}[21]$ respectively) due to their sensitivity to numerous environmental factors which will be explained later. However, these OSC stabilities are not an accurate measure of how long a solar cell can last outside under real weathering conditions as there were no high humidity conditions included and the solar spectra used 
do not match that of the Sun (sulpher plasma lamp similar but LED lamp is dissimilar, with no UV light). Finally, the ability to upscale OSCs also needs to be improved as they are still generally being developed on the lab-scale. Larger devices have been produced but with reduced efficiencies $\left(5.7 \%\right.$ with $0.1 \mathrm{~cm}^{2}$ active area[22] and $3.3 \%$ with 29 $\mathrm{cm}^{2}$ active area[23]) but these do not compare to Silicon systems which are already on rooftops, typically with an power density of $150-200 \mathrm{Wm}^{-2}$ for modules.[18] Due to the low cost potential means that OSCs would not have to be as efficient or as stable to contend with silicon based systems. The levelised electricity cost (cost of kWh produced from a system including all factors such as capital, discount rates, maintenance and costs of continuous operation) has been predicted to be competitive if organic solar modules could be produced with a $7 \%$ efficiency and a 5 year lifetime.[24] This also implies that with further development, OSCs could match the performance of silicon based systems and compete with fossil fuels. Further than this, the versatility of OSCs allow them to be used for applications where they outperform silicon, such as for indoor or space applications, where the light intensity, spectrum and environment.[25, 26]

Table 1.1: Comparison on silicon and OSCs in terms of efficiency, lifetime and upscaled power density.

\begin{tabular}{||c|c|c||}
\hline \hline & Silicon solar cells & OSCs \\
\hline Efficiency (\%) & $\begin{array}{c}25 \text { (labscale) }[17] \\
\text { (commercially available) }[18]\end{array}$ & 14.2 (labscale) $[16]$ \\
\hline Lifetime (years) & $25[19]$ & $7-10[20,21]$ \\
\hline $\begin{array}{l}\text { Upscaled power } \\
\text { density }\left(\mathrm{Wm}^{-2}\right)\end{array}$ & $150-200[18]$ & 10 \\
\hline \hline
\end{tabular}

Although all three areas need improvement to make OSCs commercially viable, it can be argued that their stability is the most pressing of these issues. This is due to the fact that the testing of stability is not so established and has only recently had some testing protocols been outlined by the community.[27] Before these guidelines were established, much of the research was carried out under different testing conditions which sometimes did not simulate real life weathering conditions. For example, the two values stated earlier for the best OSC lifetimes were obtained without lifelike weathering conditions such as high humidity taken into account and the LED lamp source is far from the solar spectrum, with no UV radiation. As stability seems to be the area where the most development is required, this was chosen to be the focus of this thesis. 


\subsubsection{Organic semiconductors}

The electronic properties of organic semiconducting materials originate from their conjugation: the successive alternating of double and single bonds. Here, the overlapping of the $\mathrm{p}_{\mathrm{z}}$ orbitals of the $\pi$-conjugated carbon atoms resulting from the $\mathrm{sp}^{2} \mathrm{p}_{\mathrm{z}}$ hybridisation (Figure 1.2a), allows the delocalisation of electrons, enhancing charge transport along the conjugated backbone of these organic molecles (Figure 1.2b).[28, 29] Charge mobility can reach the range of $0.1-20 \mathrm{~cm}^{2} / \mathrm{Vs}$ for crystalline organic semiconductors, and drops to below w $0.1 \mathrm{~cm}^{2} / \mathrm{Vs}$ for amorphous. [30] The energy bandgap $\left(\mathrm{E}_{\mathrm{g}}\right)$ of the organic semiconductor is determined by the energy difference between the highest occupied molecular orbital (HOMO) and lowest unoccupied molecular orbital (LUMO). When a molecule absorbs a photon with energy equal or greater than the optical band gap, an electron is excited from the HOMO to the LUMO, forming a bound electron-hole pair, called an exciton. A semiconductor is defined as a material with $\mathrm{E}_{\mathrm{g}} \leq 3 \mathrm{eV}$ (e.g. the $\mathrm{E}_{\mathrm{g}}$ of silicon is $\sim 1.1 \mathrm{eV}$ ), which is small enough that electrons can be excited to the conduction band (or LUMO) upon absorption of a photon with sufficient energy (or thermal energy).
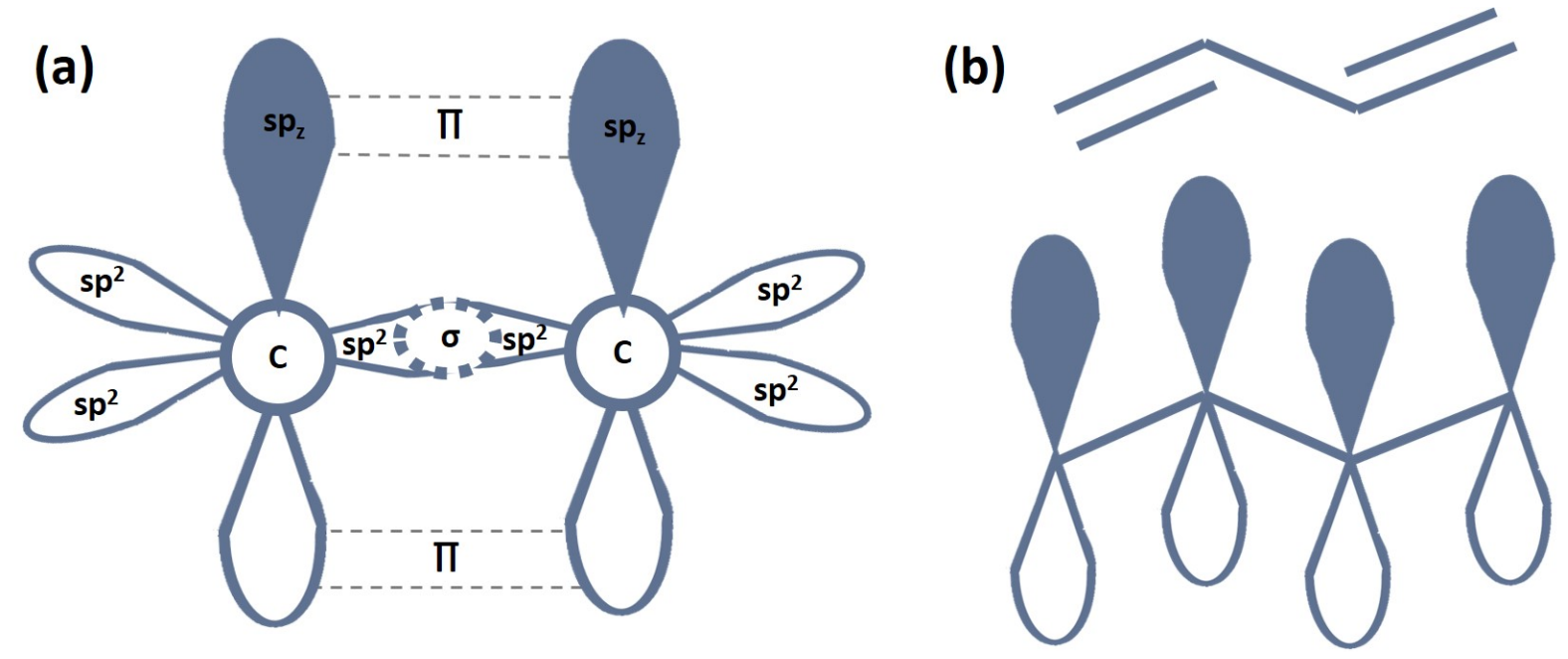

Figure 1.2: Illustrations to show (a) $\mathrm{sp}^{2} \mathrm{p}_{\mathrm{z}}$ hybridisation and (b) the chemical structure and $\mathrm{p}_{\mathrm{z}}$ orbitals in the $\pi$ system of a conjugated organic material.

The photoactive layer in OSCs is made up of a donor polymer/small molecule and an electron acceptor which form different domains, optimising the interface between the two materials. The most commonly used electron acceptor for OSCs is Phenyl-C61-butyric acid methyl ester which will be referred to as $\mathrm{PC}_{61} \mathrm{BM}$ herein. This acceptor contains a fullerene $\left(\mathrm{C}_{60}\right)$ ball, however, other fullerenes can be used; for example, $\mathrm{PC}_{71} \mathrm{BM}$ which contains the $\mathrm{C}_{70}$ fullerene. Commonly used donor polymers are shown in Figure 1.3 and electron acceptors in Figure 1.4. 


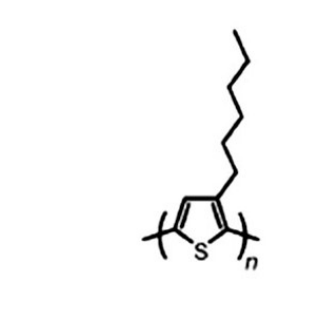

P3HT

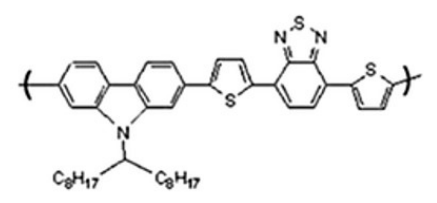

PCDTBT
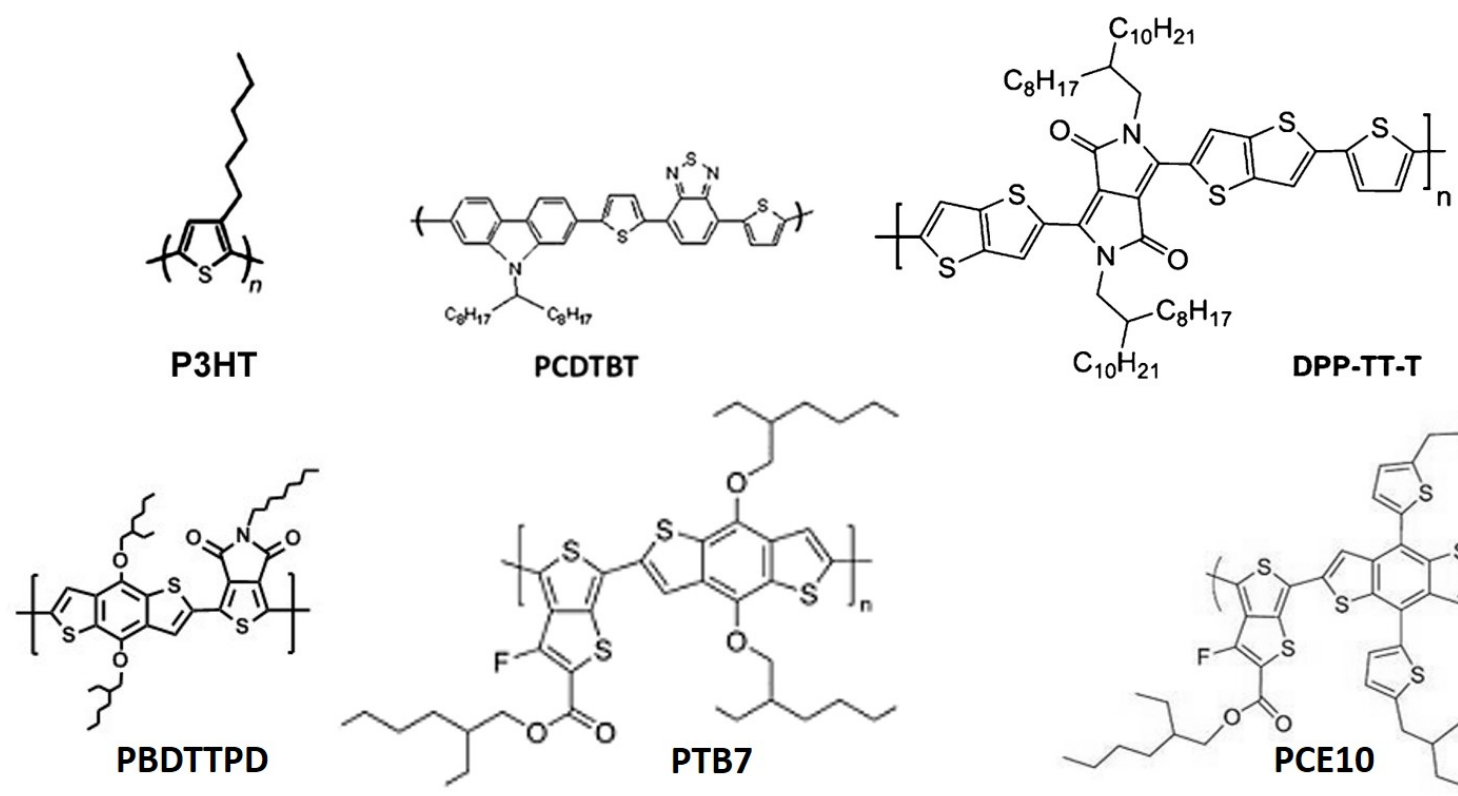

Figure 1.3: Chemical structure of some typical donor polymers. From left to right, Top: $\quad$ Poly(3-hexylthiophene-2,5-diyl) (P3HT); Poly[N-9'-heptadecanyl-2,7-carbazolealt-5,5-(4',7'-di-2-thienyl-2',1',3'-benzothiadiazole)] (PCDTBT); diketopyrrolopyrrole - thieno[3,2-b]thiophene thiophene (DPP-TT-T). Bottom: Poly[[5-(2-ethylhexyl)-5,6dihydro-4,6-dioxo-4H-thieno[3,4-c]pyrrole-1,3-diyl][4,8-bis[(2-ethylhexyl)oxy] benzo[1,2b:4,5-b']dithiophene-2,6-diyl]] (PBDTTPD); Poly[2-methoxy-5-(2-ethylhexyloxy)-1,4phenylenevinylene] (PTB7); Poly[4,8-bis(5-(2-ethylhexyl)thiophen-2-yl)benzo[1,2b;4,5-b'] dithiophene-2,6-diyl-alt-(4-(2-ethylhexyl)-3-fluorothieno[3,4-b]thiophene-)-2carboxylate-2-6-diyl)] (PCE10).

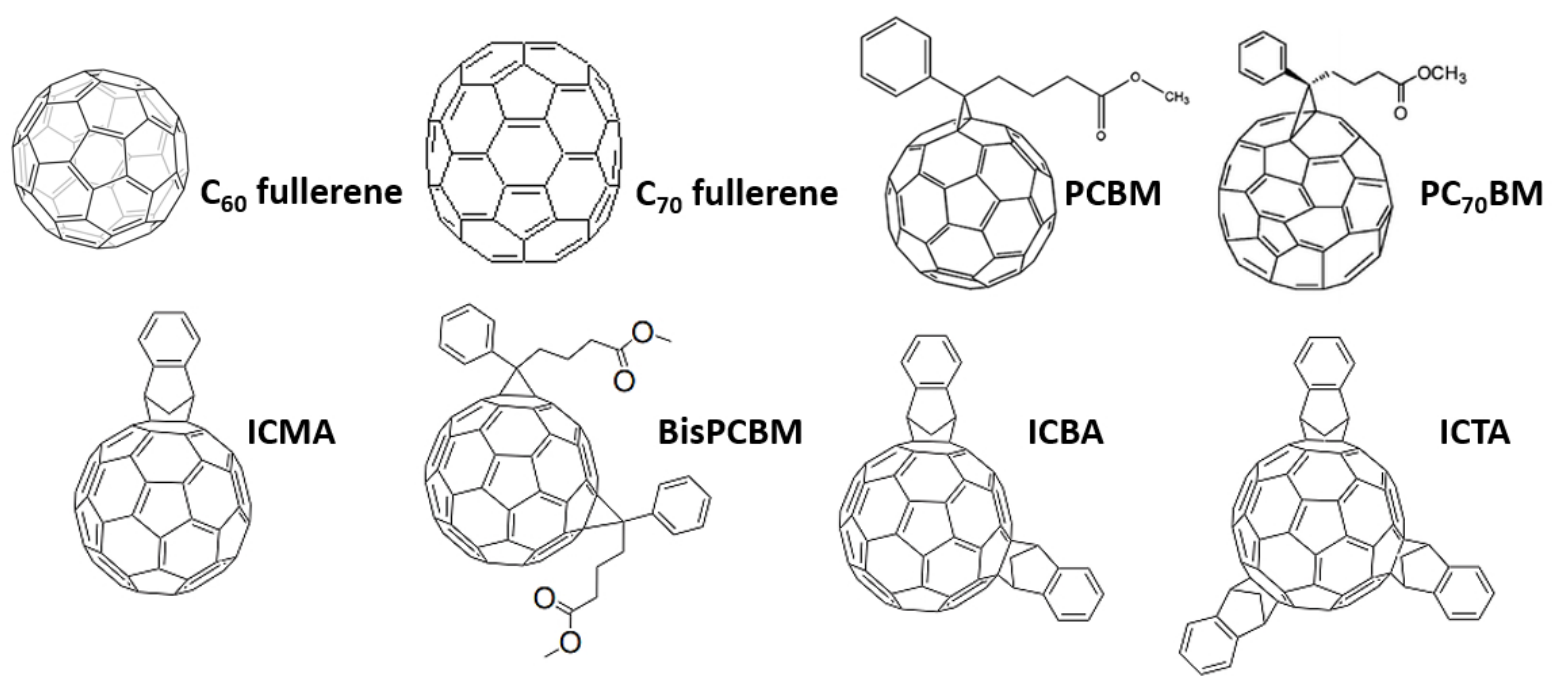

Figure 1.4: Chemical structures of fullerenes and typical fullerene electron acceptors. From left to right, Top: $\mathrm{C}_{60}$ fullerene; $\mathrm{C}_{70}$ fullerene; Phenyl-C61-butyric acid methyl ester $\left(\mathrm{PC}_{61} \mathrm{BM}\right)$; Phenyl-C71-butyric acid methyl ester $\left(\mathrm{PC}_{70} \mathrm{BM}\right)$. Bottom: $\quad 1^{\prime}, 4^{\prime}$-Dihydro-naphtho[2',3':1,2][5,6]fullerene-C60 (ICMA); Bis(1-[3(methoxycarbonyl)propyl]-1-phenyl)-[6,6]C62 (BisPCBM); $\quad 1^{\prime}, 1^{\prime \prime}, 4^{\prime}, 4^{\prime \prime}$-Tetrahydrodi[1,4] methanonaphthaleno[1,2:2',3',56,60:2"',3'"][5,6]fullerene-C60 (ICBA); indene-C60tris-adduct (ICTA). 


\subsubsection{Polymer-based organic solar cells}

OSCs are composed of a number of thin film layers. Typically the active layer, a combination of the donor polymer and acceptor materials, is sandwiched between two electrodes, one of which needs to be transparent so that the active layer can be illuminated. Thermally evaporated small molecules can also be used for the active layer, but this thesis will concentrate on the solution processable polymer-based solar cells. Interlayers (or charge transport layer) can be inserted between the active layer and the electrodes to improve charge transport and collection. Figure 1.5 depicts typical OPV device architectures.

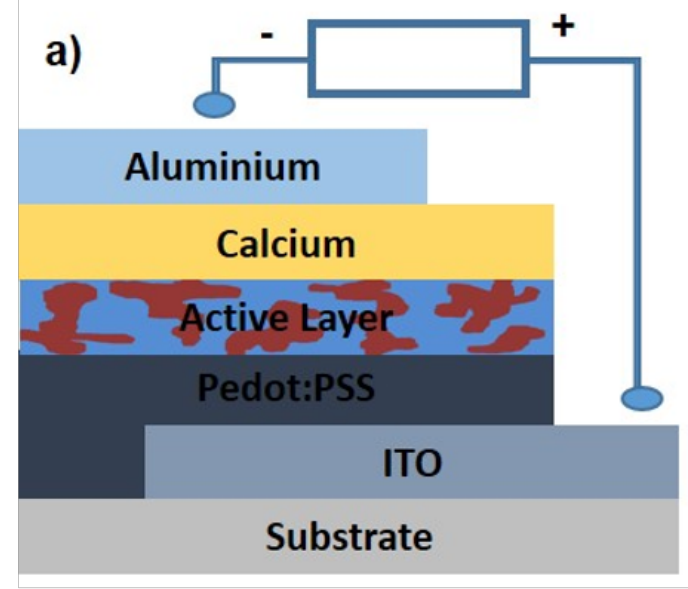

Conventional

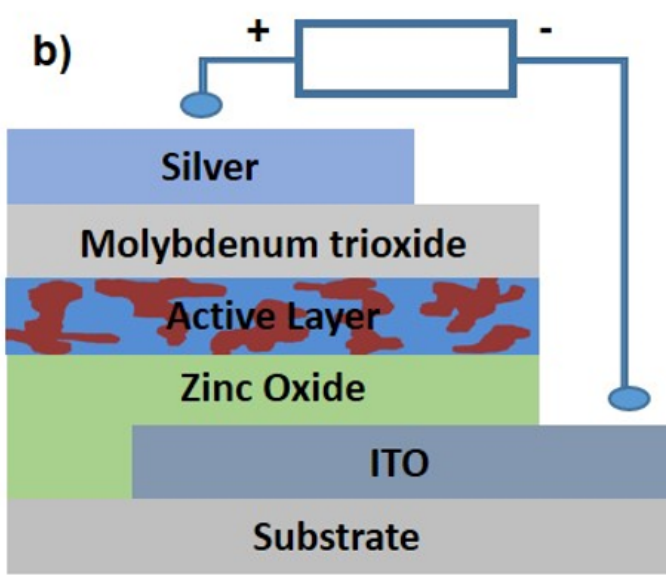

Inverted

Figure 1.5: Typical OSC architectures: (a) conventional structure and (b) inverted structure.

The photoactive layer is where the photoelectric effect occurs, generating a photocurrent, and have taken three different forms: single layer; bi-layer; and bulk heterojunction. The first OPVs were made with a single layer active layer sandwiched between two electrodes of different work functions which created a built-in potential to the device. However, this method of charge separation is inefficient leading to low efficiencies of $<0.1$ \%.[31] Efficiencies were drastically increased to $\sim 1 \%$ after introducing donor/acceptor bilayer structures where these layers are brought together to form a planar heterojunction. [32] However, only excitons generated within a diffusion length (typically $\sim 10 \mathrm{~nm}$ ) of the donor-acceptor interface can successfully diffuse to the interface and separate into free carriers. Subsequently, only light absorbed by a very thin layer next to the interface can contribute to the photocurrent - the rest being lost to recombination processes (typical active layer thicknesses $\sim 100 \mathrm{~nm}$. A breakthrough was achieved with the introduction of the bulk heterojunction, where the donor and acceptor materials are blended forming a nanoscale phase separation throughout the active layer.[33] This morphology means the interface is distributed throughout the active layer, allowing for more excitions to 
successfully dissociate. Additionally, the bicontinuous network of the active layer blend allows for the efficient percolation of charge carriers to the electrodes. Consequently, the charge separation of bulk heterojunction devices are vastly improved and efficiencies are now over $13 \%$.[34-36]

The working principle for bulk heterojunction solar cells is shown in Figure 1.6.

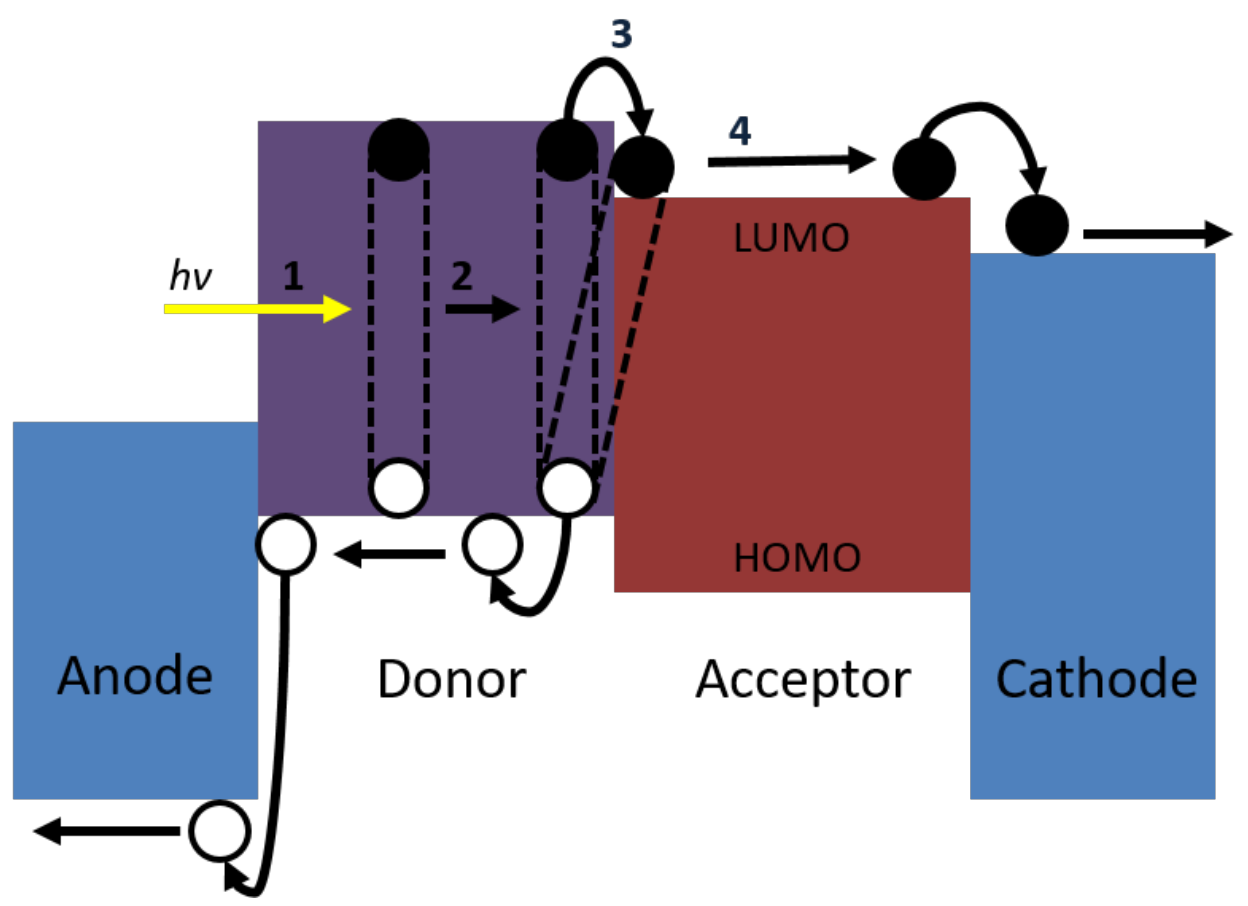

Figure 1.6: Schematic diagram of the photovoltaic mechanism for OPV bulk heterojunctions.

1. Photon absorption and exciton formation: A photon of incident light, with an energy equal or greater than the energy gap of the polymer, can be absorbed and excites an electron from the HOMO to the LUMO of the polymer, leaving behind a positively charged hole in the HOMO of the polymer, where the electron resided. Likewise, when an electron acceptor absorbs a photon, a hole is transferred to the HOMO of the donor, leaving behind an electron. The electron and hole are attracted to each other via the Coulomb force, resulting in a bound but mobile state called an exciton. The solar spectrum is AM $1.5 \mathrm{G}$ which is the solar radiation after attenuation by the Earth's atmosphere when the sun is $48.2^{\circ}$ off from being directly overhead. Section 1.1.4 describes exciton generation in more detail along with photoexcited states.

2. Exciton diffusion: The exciton diffuses along the donor or acceptor and reaches the donor-acceptor interface (as long as recombination does not take place). See Section 1.1.4 for more details.

3. Charge dissociation: At the interface between the donor and acceptor, the exciton dissociates if the exciton binding energy ( $\sim 1 \mathrm{eV}$ for organic electronics) can be overcome. 
The declination of the energy offset drives the electron in the LUMO of the donor is transferred to the LUMO of the acceptor, and the hole in the HOMO of the acceptor are transferred to the HOMO of the donor. Despite residing on different molecules, the electron and hole can still be attracted to each other via their Coulomb attraction. This is called a charge transfer or a bound polaron pair state and is described in more detail in a Section 1.1.5.

4. Charge separation, transport and collection: The electron-hole pairs that escape from their Coulomb attraction become free charges (or polarons). These free charges are transported through the active layer and interlayers and can be collected at the electrodes, generating the photocurrent in the external circuit. The photovoltage is determined by the LUMO-LUMO offset of the donor-acceptor.[37] Charges are transported via hopping transport $\left(0.01 \mathrm{~cm}^{2} / \mathrm{Vs}\right)$ for molecular solids (intermolecular materials) (Frenkle-type small radius excitons) and by band transport $\left(1 \mathrm{~cm}^{2} / \mathrm{Vs}\right)$ for crystalline intramolecular materials (Mott-Warnier-type large radius excitons). The corresponding energy levels of the interlayers and electrodes must be at lower energies (above the HOMO for the holetransport side and below the LUMO for the electron-transport side) than the layer before it such that the charge carriers can cascade through the device. Charge separation is discussed more in Section 1.1.5 and the photovoltaic losses that reduce the charge extraction are discussed in Section 1.1.6.

\subsubsection{Exciton generation, excited states and exciton diffusion}

Organic semiconducting materials have low dielectric constants (typically $\sim 2-4$, much lower than their inorganic counterparts $(\sim 12)$. [38-40] This means that the Coulombic attraction between opposite charges is screened less effectively in OSC devices than in conventional inorganic PV, making charge recombination more likely and efficient charge collection a challenge for OSC. Hence in exciton theory, photoexcitation generates bound electron-hole pairs rather than separated free charges. In the case of inorganic semiconductors, the thermal energy at room temperature is enough to create free charges. 


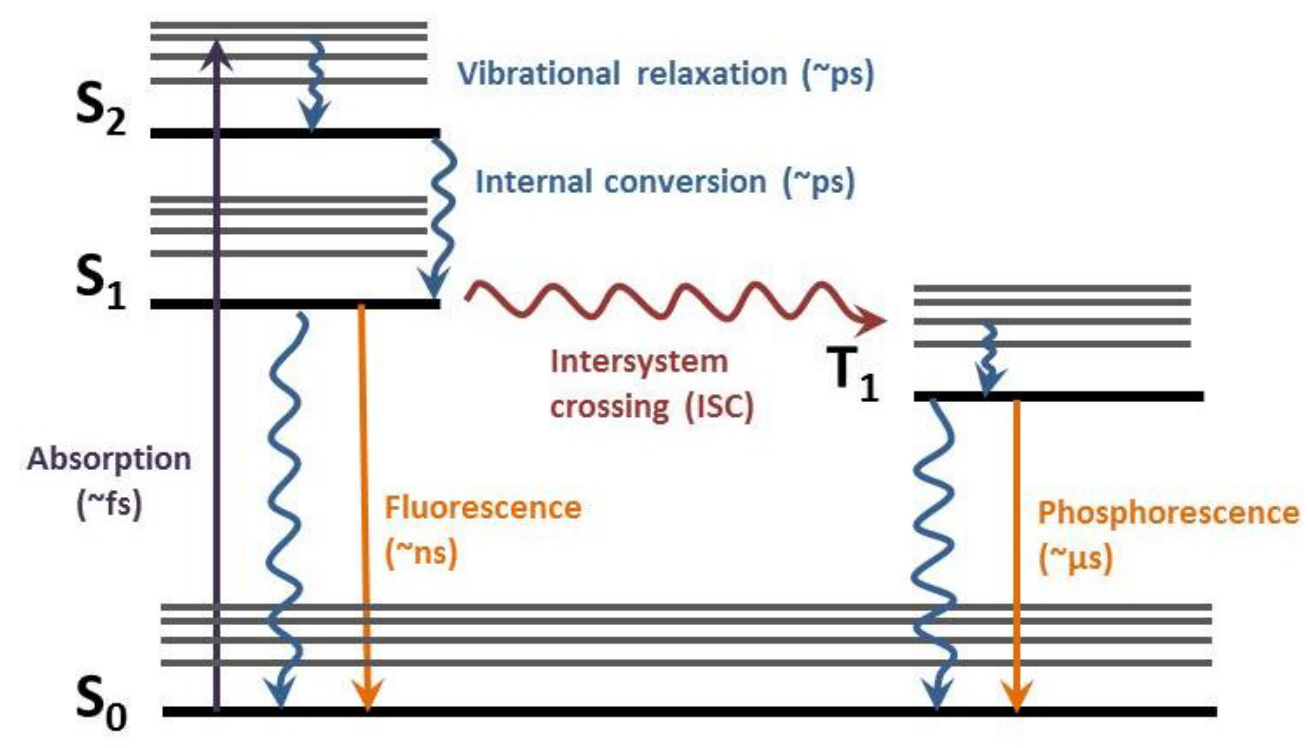

Figure 1.7: A Jablonski diagram showing electronic transitions in organic molecules and the timescales of these transitions. Straight lines indicate transitions involving photons and wavy lines indicate non-radiative transitions.[41]

When a photon is absorbed by an organic semiconducting material, the photophysical processes can take place, illustrated in Figure 1.7. When a photon of an appropriate energy $\left(\geq E_{g}\right)$ is absorbed, photoexcitation of an electron in the HOMO of the molecule will occur from the ground state $S_{0}$ to an excited singlet state $S_{n}(n=1,2 \ldots)$. Each excited state has multiple vibrational states, allowing for a range of photon energies to be absorbed. If an electron is excited to a higher vibrational energy level $(\nu>0)$, it can rapidly undergo vibrational relaxation to the lowest vibrational level $(\nu=0)$ of the same excited state.[42] Likewise, electrons excited to higher lying excited states $\mathrm{S}_{\mathrm{n}+1}(\mathrm{n}=$ $1,2 \ldots$ ) can undergo rapid internal conversion to the lowest excited state $\mathrm{S}_{1}$. Vibrational relaxation and internal conversion are both non-radiative decays.

An electron can also return to the ground state by emitting a photon of the same energy as the difference between the two states. This process is termed fluorescence. Due to vibrational relaxation and internal conversion, the energy of the photon emitted is less than the photons absorbed. This leads to a red-shift in energy of the emission band compared to the absorption band, known as Stokes shift.

The excited state formed directly from light absorption is a singlet, an anti-parallel spin state, due to spin conservation. Intersystem crossing (ISC) is a non-radiative process which allows electrons in the singlet states $\left(S_{n}\right)$ to transfer to the equivalent triplet state $\left(\mathrm{T}_{\mathrm{n}}\right)$ with parallel spin (Figure 1.7). Spin selection rules forbid the decay of this triplet state to the ground state and can only take place when there is an efficient spin-orbit coup- 
ling. Organic semiconductors generally have a weak spin-orbit coupling, meaning that the radiative emission of the triplet state via phosphorescence (a type of photoluminescence where electrons slowly decay down to the ground state from a metastable state) is a weak and slow process. Therefore, triplet states are in general longer-lived than singlet states.

Although the exciton theory is widely accepted as the standard model for charge generation in organic photovoltaics, a second theory called ultrafast charge generation has been challenging this in recent years. Here, a charge pair is formed directly after photon absorption in an extremely fast timescale $(<100 \mathrm{fs})$, which may not involve the formation of an exciton.[43-45]

An exciton typically has a binding energy of $0.3-0.5 \mathrm{eV}$, much higher than the thermal energy at room temperature.[46-48] So an energy input is required for the exciton to be dissociated into free charges. This energy is provided by downhill driving energy at the interface of the donor and acceptor through the difference in LUMO levels. If the exciton is formed away from the donor-acceptor interface, it must diffuse in order for charge dissociation to occur. The diffusion length, the distance an exciton can travel before recombining, is about $10 \mathrm{~nm}$ for an organic semiconductor. Excitons can diffuse via two ways: a) Direct exchange interactions between molecules adjacent to the donor or acceptor. This could be hopping or excitation diffusion between overlapping electron clouds of adjacent donor and acceptor molecules. This gives rise to a short range (0.3 $2 \mathrm{~nm}$ ) transfer what is called a Dexter transfer. Only the total spin of the system must be conserved and an exciton retains its spin configuration during this transfer.[49] b) Coulombic interactions, mainly due to dipole-dipole interactions, giving rise to long range (3 - $10 \mathrm{~nm})$ Förster transfers. Spins of the donor and acceptor molecules are conserved during these transfers due to a condition imposed by allowed dipolar transitions. [49]

\subsubsection{Charge transfer state and charge separation}

There is still debate over the exact mechanism of charge separation. However, charge generation may happen through an intermediate charge transfer (CT) state, after exciton dissociation at the donor-acceptor interface. This is where the electron is localised on the acceptor LUMO and the hole on the HOMO of the donor, forming a partially bound electron-hole pair, spatially separated but still under the influence of a mutual Coulomb attraction. Therefore, the CT state can be considered as an intermediate species between tightly bound excitons and fully dissociated free charges. CT states can also be referred to as a "bound polaron pair", "geminate pair" or "exciplex" depending on the nomenclature. $[50,51]$ Here the electron is on the acceptor and the hole on the donor, but they are still coulombically bound. 


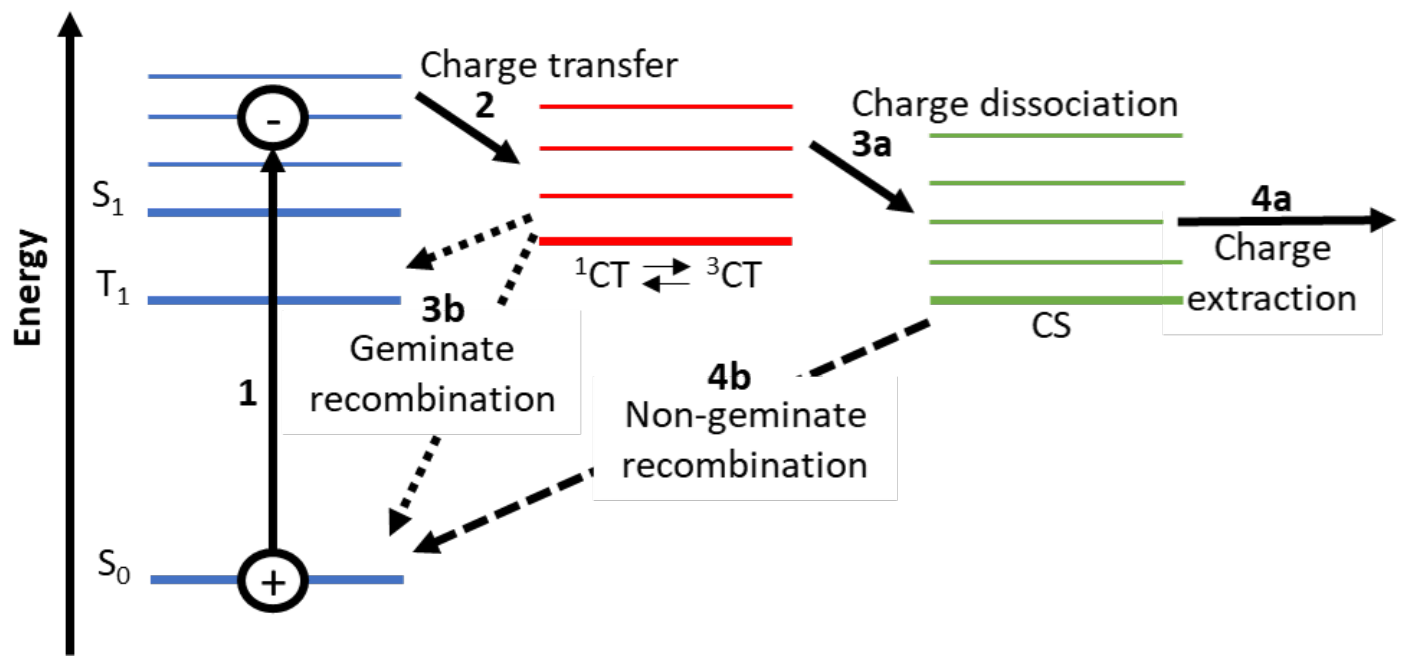

Figure 1.8: An energy diagram portraying the generation and fate of a charge transfer (CT) state. (1) Excitation of an electron from the ground state $\left(\mathrm{S}_{0}\right.$ to an excited state $\left(\mathrm{S}_{1}\right.$, creates an exciton. (2) The electron can be transferred to form a singlet CT state $\left({ }^{1} \mathrm{CT}\right)$ that can (3a) dissociate to form charge separated states (CS). Or they can undergo (3b) geminate recombination to the ground state or the triplet exciton $\left(\mathrm{T}_{1}\right)$ after intersystem crossing to the triplet charge transfer state $\left({ }^{3} \mathrm{CT}\right)$. CS can either $(4 \mathrm{a})$ be extracted or $(4 \mathrm{~b})$ recombine non-geminately.

The attraction between the hole and the electron in the CT state must be overcome in order to form free charges. The Onsager theory is one model proposed to describe the separation of the CT state (Figure 1.8 illustrates the processes which are discussed below).[52, 53, 38] It suggests that the efficiency of charge separation is dependent on a combination of the spatial charge dissociation and the energetic relaxation of the CT state. A CT state generated from a high energy exciton has more thermal or electronic energy, and is referred to as a "hot" CT state; thus helping the physical charge separation exceed the Coulomb capture radius for formation of charge separated states (CS). If the hot CT state does not separate, it will relax to lower energy states. These thermally relaxed CT states will have less energy to dissociate, and are more likely to geminately recombine to the ground state. Additionally, these low energy CT states, which are in the singlet state $\left({ }^{1} \mathrm{CT}\right)$, can also intersystem cross to the triplet state $\left({ }^{3} \mathrm{CT}\right)$ which can then recombine geminately to the triplet excited state $\left(\mathrm{T}_{1}\right)$. The separates charges can also undergo non-geminate recombination to the ground state. Recombination will be explained in the next section.

It has been shown that the CT state can contribute to the $\mathrm{V}_{\mathrm{OC}}$ (the maximum voltage available from a solar cell, occurring when there is no current) of OSCs, not the electronic coupling (interaction between p orbitals) between the donor and acceptor materials. [54] The $\mathrm{V}_{\mathrm{OC}}$ can be enhanced by increasing the energy of the CT state which itself is influenced by the optical band gap, crystallinity, donor-acceptor orientation and interfacial dipoles.[55] 


\subsubsection{Recombinative Photovoltaic loss mechanisms}

The OPV photocurrent is reduced when the number and rate of charge carriers reaching the electrodes is reduced. Impurities and dislocations (atoms out of position) can trap charge carriers, which can then recombine with a charge carrier of the opposite polarity, removing them from the photocurrent as shown by Figure 1.9. An electron becomes trapped when the energy level of the defect is lower than the LUMO of the acceptor molecules. Similarly, a hole is trapped when the energy level of the defect is higher than the HOMO of the donor molecules.
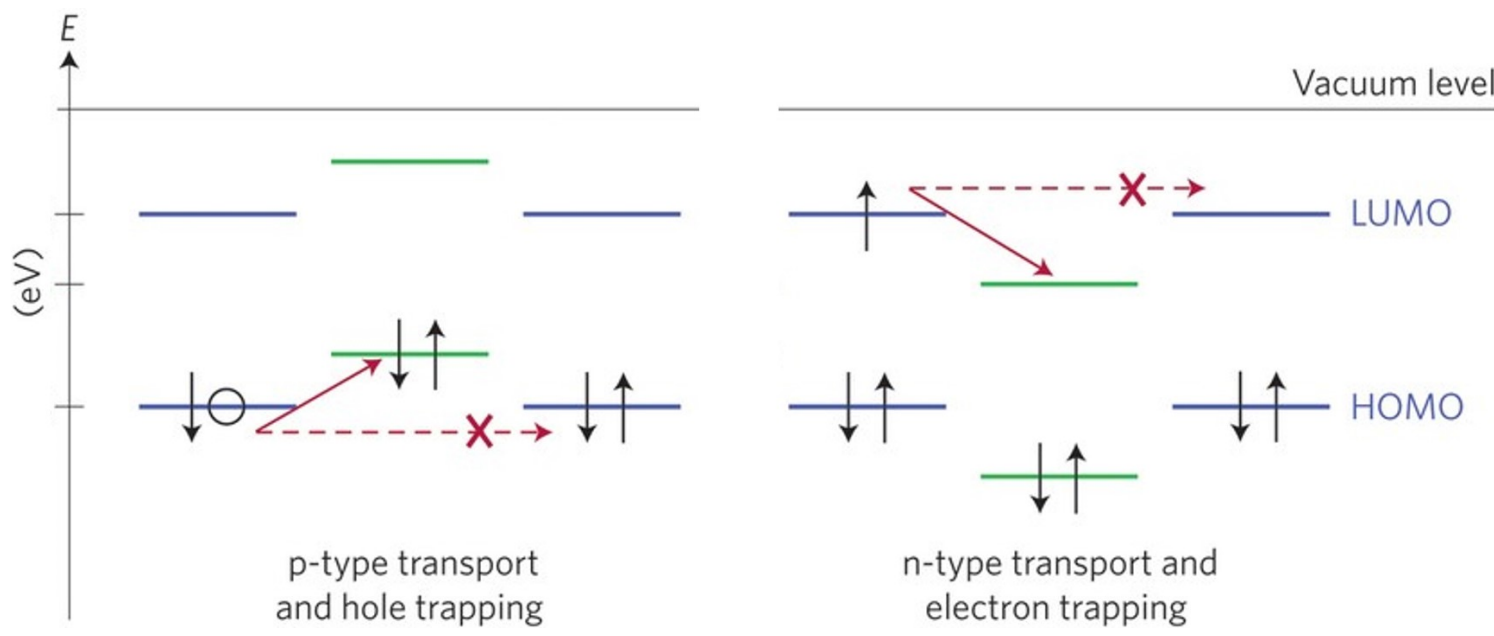

Figure 1.9: Energy level diagram illustrating the trapping of charge carriers. Left: a hole (circle) is trapped by a higher-lying defect level (green) instead of being transferred between HOMO's of adjacent molecules (blue). Right: an electron (black arrow) is trapped by a lower-lying defect level instead of being transferred between adjacent LUMO levels.[56]

There are two types of recombination that can reduce the yield of charge generation - geminate and non-geminate, as previously mentioned. Geminate (monomolecular) recombination involves an electron and a hole that originate from the same photogenerated exciton. These typically are still coulombically bound in a CT state. In addition, dissociated charges that are still in their respective domains such that the same electron and hole recombine is also geminate recombination. This process occurs on short timescales (ps - ns) and therefore can compete with charge dissociation.[57, 58, 38] Geminate recombination can be fitted with a monomolecular exponential decay function when the transient absorption decay kinetics are measured.[59, 60]

Non-geminate recombination involves the recombination of fully-dissociated charge carriers that did not originate from the same exciton. This process differs from geminate recombination in that the charge carriers need to diffuse to within their coulomb capture radius (the separation where the coulomb attraction is approx. the thermal energy at room temperature, $\sim \mathrm{kT}$ ) before they can recombine. As the charge carriers in non- 
geminate recombination need to diffuse before recombination, the times scale is longer - nanoseconds to milliseconds.[38, 61, 62, 60] Non-geminate recombination can be fitted with a power law decay function when the transient absorption decay kinetics are measured and is reportedly affected by the presence of charge trapping. [59, 62, 63]

\subsubsection{Device characterisation}

Figure 1.10 shows the typical current density-voltage (J-V) curve for a solar cell. There are some important points on the graph that helps in determining the performance of solar cells. Firstly, the intersection of the curve with the x-axis is called the open-circuit voltage $\left(\mathrm{V}_{\mathrm{OC}}\right)$ and is the maximum output voltage from the cell. The intersection of the curve with the $\mathrm{y}$-axis is the short-circuit current density $\left(\mathrm{J}_{\mathrm{SC}}\right)$ and is the maximum current density from the cell under illumination without an externally applied voltage. The point on the $\mathrm{J}-\mathrm{V}$ curve which gives the maximum output power is called maximum power point $\left(\mathrm{P}_{\mathrm{M}}\right)$ and the corresponding voltage and current density at this point are named $V_{M}$ and $J_{M}$. The maximum power output is $V_{M} \times J_{M}$ and is given by the shaded region on Figure 1.10. The fill factor $(\mathrm{FF})$ is defined as the ratio between $\mathrm{P}_{\mathrm{M}}$ and the rectangle that would be given with the intersection of the $\mathrm{V}_{\mathrm{OC}}$ and $\mathrm{J}_{\mathrm{SC}}$ which gives the "squareness" of the curve equation. It reflects how closely a device performs to an ideal device and is limited by charge recombination and resistive losses within the device.

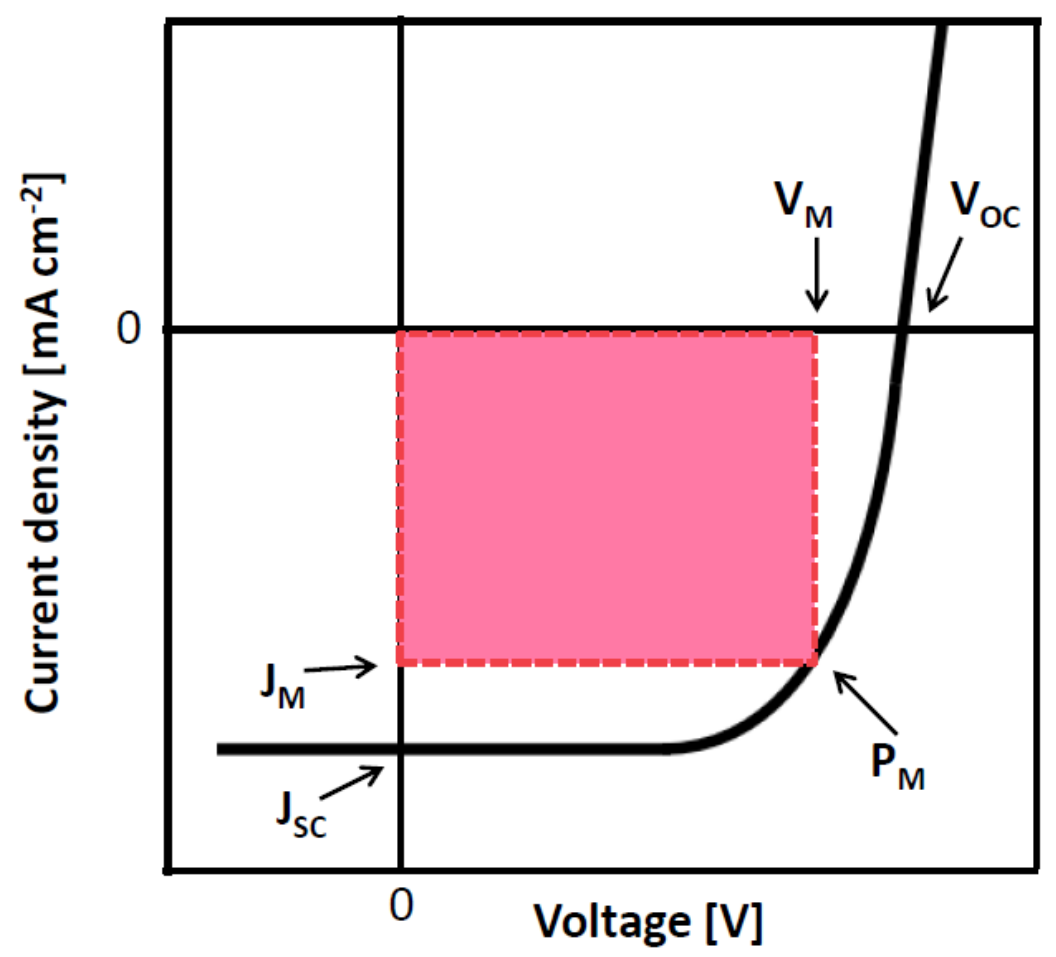

Figure 1.10: A typical J-V curve measurement for OPV cells used to determine their device characteristics. 


$$
\begin{gathered}
\mathrm{P}=\mathrm{I} \times \mathrm{V} \\
\mathrm{FF}=\frac{\mathrm{I}_{\mathrm{M}} \cdot \mathrm{V}_{\mathrm{M}}}{\mathrm{I}_{\mathrm{SC}} \cdot \mathrm{V}_{\mathrm{OC}}}
\end{gathered}
$$

The power conversion efficiency (PCE) of a solar cell, is defined as the maximum power produced by the cell divided by the power of the incident light (Pin).

$$
P C E=\frac{\mathrm{J}_{\mathrm{SC}} \cdot \mathrm{V}_{\mathrm{OC}} \cdot \mathrm{FF}}{\mathrm{P}_{\mathrm{IN}} \times 100}
$$

There are two types of resistive losses in solar cells: series resistance and shunt resistance. Series resistance $\left(R_{s}\right)$ originates from bulk resistances of the active layer and electrodes, and the contact resistances between the active layer and electrodes. [64] It can be calculated by taking the inverse gradient of the JV curve at $V_{\text {oc }}$. Shunt resistance $\left(R_{\mathrm{sh}}\right)$ indicates current losses in the cells, such as the current leakage from the edge of the cell, from pinholes or by the charge traps.[64] It can be calculated by taking the inverse gradient of the JV curve at $\mathrm{J}_{\mathrm{sc}}$.

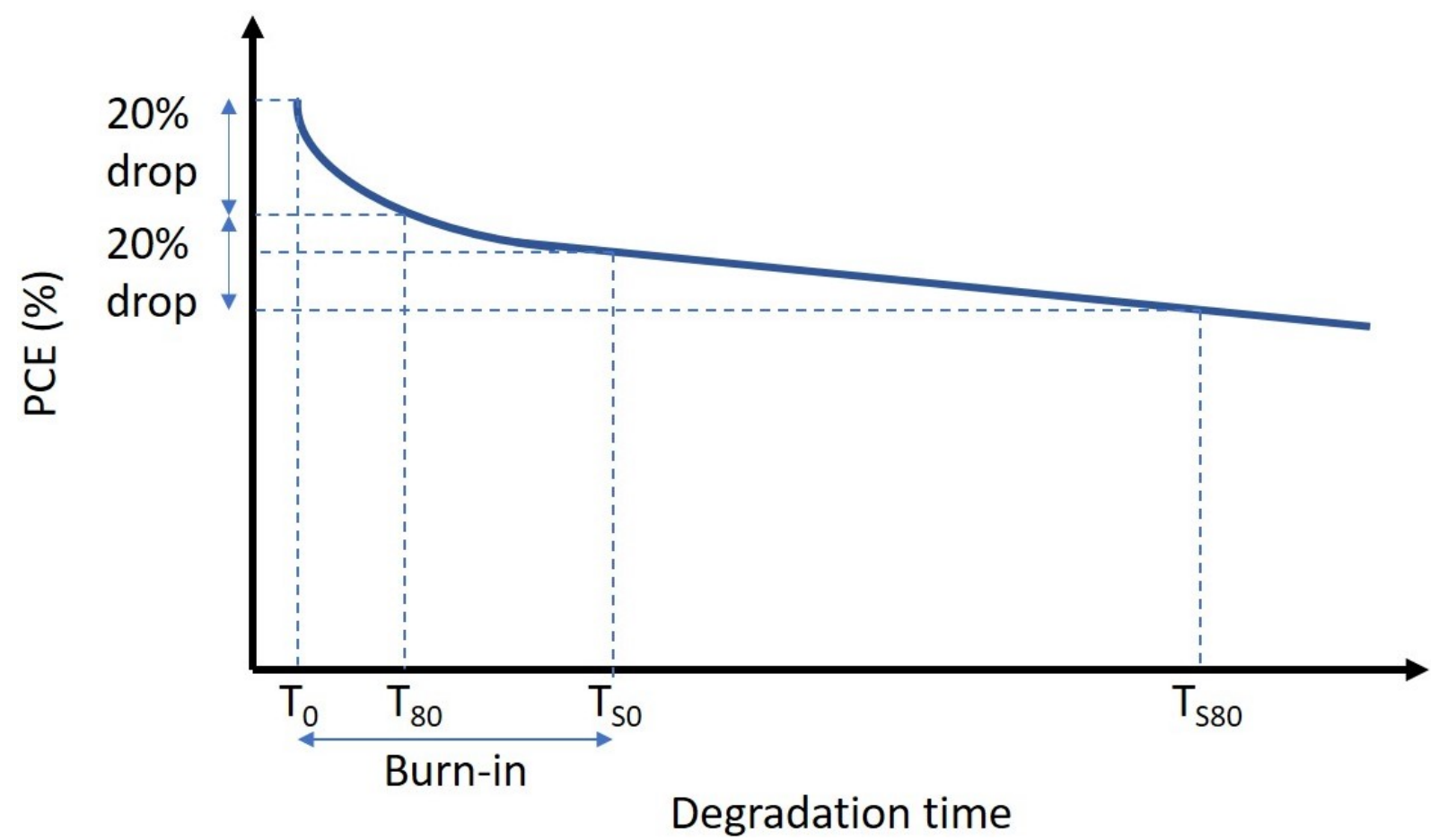

Figure 1.11: An illustration to show the lifetimes that can be quoted to define the stability of OPV devices.

Although the issue of stability in OSCs has been receiving more attention over recent years, the measurement conditions would often vary significantly from site to site in terms 
of environmental conditions, spectral distribution and intensity. This made comparing results within the community difficult and led to the formation of international summits on OPV stabilty (ISOS-1, 2 \& 3) which provided recommended procedures on OSC lifetime measurements and reporting. [27] The decay in OSC performance over time can be quantified in a number of ways. Figure 1.11 shows how to define the OPV lifetimes: Firstly, if the device is tested immediately after fabrication, the PV characteristics are taken from the point where the PCE has degraded to $80 \%$ of the initial value, this lifetime is called $\mathrm{T}_{80}$. If the device is allowed to stabilise before beginning the stability testing, the time for the PCE to degrade to $80 \%$ of its initial value is then named $\mathrm{T}_{\mathrm{S} 80}$. Interestingly, it is observed under a nitrogen atmosphere, there is an initial fast decay before a stabilisation to a slower decay. This initial exponential decay is called the "burn-in" period for the device.

\subsection{Stability of organic solar cells}

\subsubsection{Introduction}

The limited device stability of OSCs is one of the main issues that needs addressing before commercialisation is possible.[65, 66] This is a complex issue where numerous stress factors are known to have an effect: oxygen, light, humidity, thermal stress and physical stress. These stress factors either cause the device materials to undergo a chemical reaction and/or change their morphology, classified as (photo)chemical or morphological degradation respectively.

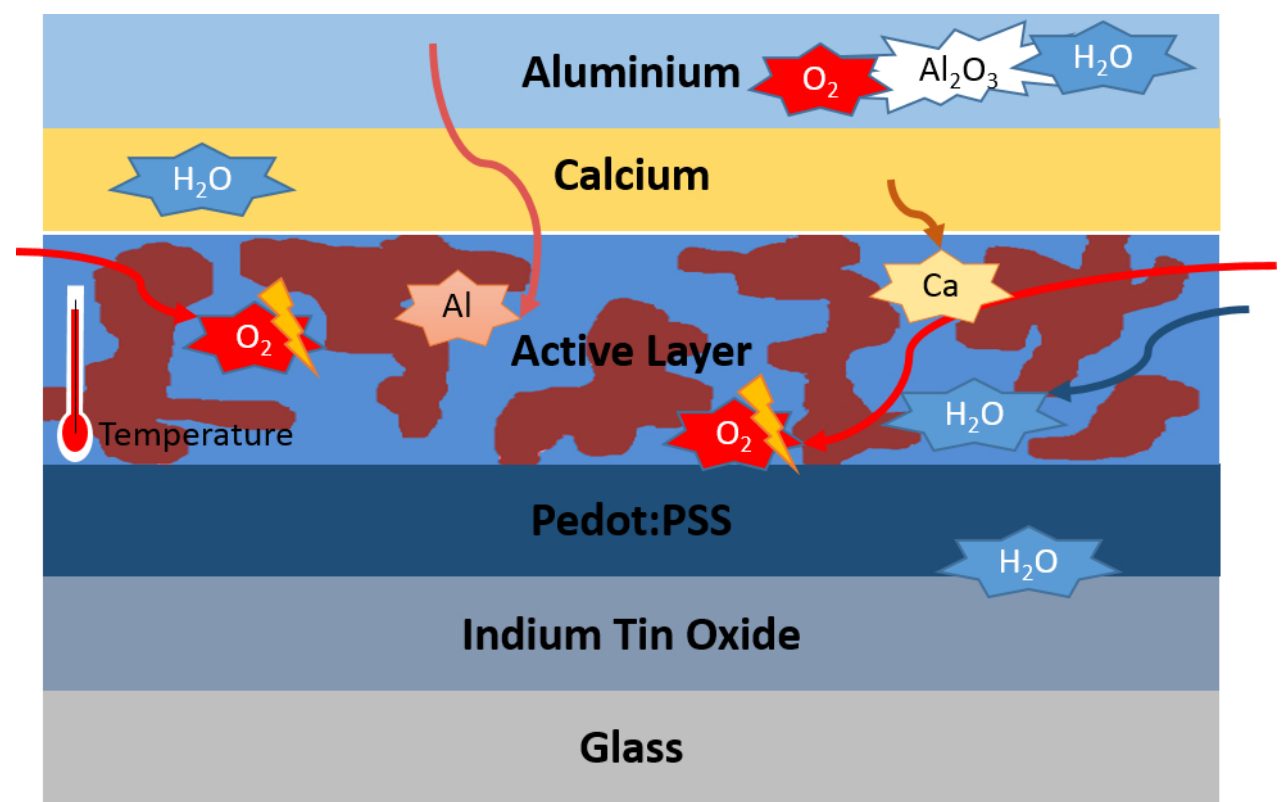

Figure 1.12: A cross-sectional schematic of a conventional OSC illustrating some of the degradation processes. 
Figure 1.12 summarises the degradations which can happen in each layer of the OSC. The photoactive layer is prone to (photo)chemical and morphological degradation. The device can reach high temperatures $\left(\sim 85^{\circ} \mathrm{C}\right)$ after long exposure to the Sun which can cause the change of nanomorphology of the active layer, affecting charge generation, recombination and transport. $[27,67,68]$ An example of this is shown in Figure 1.13, where two polymers Poly[2-methoxy-5-(3',7'-dimethyloctyloxy)-1,4-phenylenevinylene] (MDMOPPV) and Poly(p-phenylene vinylene) with a high glass transition temperature ('High Tg PPV') are annealed at $110^{\circ} \mathrm{C}$ for up to 16 hours, yielding the formation of large $\mathrm{PC}_{61}$ BM-clusters for MDMO-PPV but a more stable morphology for 'High Tg PPV', indicating that this morphological stability is polymer dependent.[69] Photolysis (or photodissociation) of the organic molecules can occur upon exposure to illumination, where sufficiently energetic photons can cause the chemical bonds to break.[70] Additionally, the organic semiconducting materials can also undergo photo-oxidation under ambient conditions. [71, 72] There are two pathways for the photodegradation that studies focus on: the generation of singlet oxygen (high energy quantum state of oxygen) or superoxide radical anions $\left(\mathrm{O}_{2}^{-}\right)$, both of which react with the OPV materials and therefore change the electrical and optical properties of the active layer materials, creating charge traps and will be described further in the next section. [71] This thesis will focus on the photochemical stability of the active layer materials, therefore only a short summary of other degradation mechanisms within OSCs is given here.

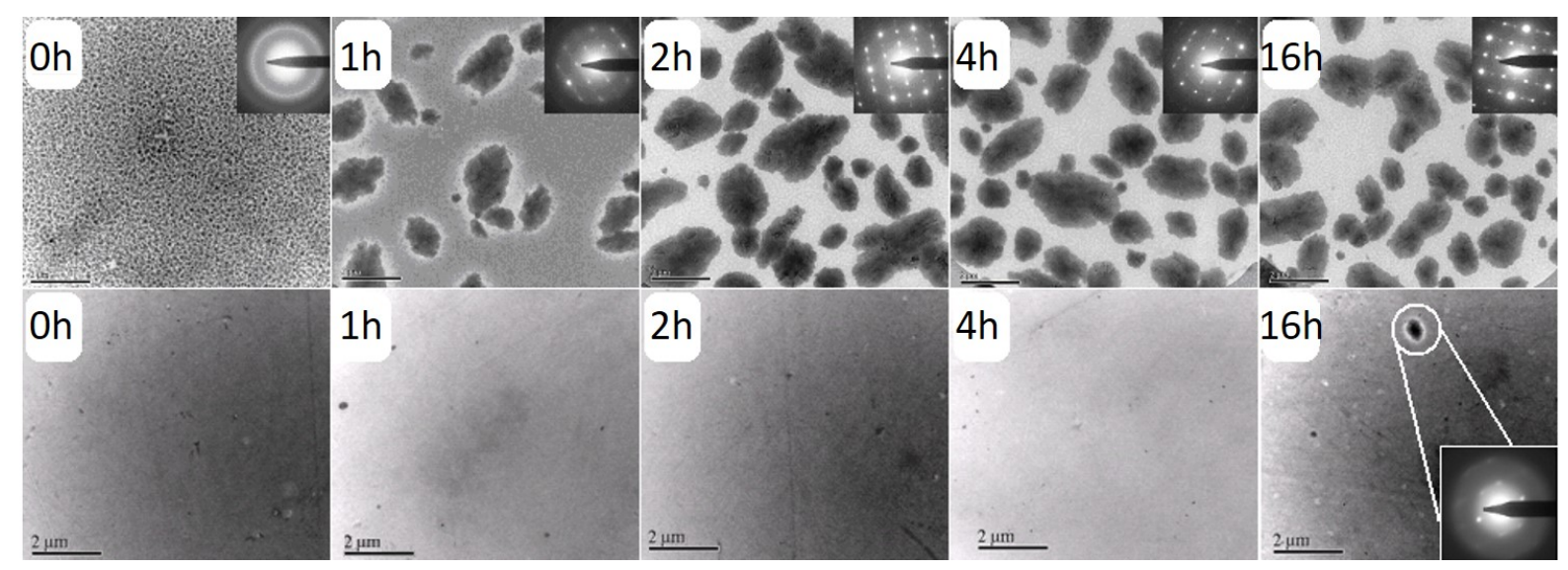

Figure 1.13: TEM images of morphological changes in the active layer of: top MDMOPPV:PC ${ }_{61} \mathrm{BM} 1: 4$ and bottom 'High Tg PPV':PC ${ }_{61} \mathrm{BM} 1: 4$, as a result of annealed at 110 ${ }^{\circ} \mathrm{C}$ for up to 16 hours.[69]

In a conventional OSC device, the aluminium electrode, calcium interlayer and other low work function metals can easily be oxidised in the presence of oxygen and water to form electrically insulating metal oxides at the metal/organic interface.[71, 73] Furthermore, aluminium can diffuse through the device structure, explained by a phenomenon known as interlayer diffusion, which then when are oxidised, further deteriorates device performance. $[71,74]$ The indium in the indium tin oxide electrode has also been shown 
to diffuse all the way through the device structure, although this has not yet been correlated to the degradation of device performance.[74] The hole-transport layer PEDOT:PSS is also highly susceptible to water and can corrode the anode (indium tin oxide) layer due to its acidic and hydrophilic properties.[71, 73] These degradations at the cathode, anode and hole transporting layer hinder charge collection, resulting in poorer device performance.

As demonstrated, each layer of OSCs has its own stability issues but the photochemical stability of the photoactive layer seems to be the most complex. Further complication is added when the interfacial properties are considered as these affect the processing of devices, which in turn could affect the stability. For example, benchmark OSCs stability under thermal stress was substantially increased in cells deposited on a zinc oxide substrate in an inverted structure compared to those using a PEDOT:PSS substrate in conventional structure cells correlated to $\mathrm{PC}_{61} \mathrm{BM}$ nucleation.[75] It should also be noted that encapsulation can be used to hinder the diffusion of oxygen and water through the device, however, a high quality encapsulation will add significant cost to the OSC. The photochemical stability of electron acceptors will be the focus of this thesis, however, the photochemical stability of polymer donors will be introduced in the next section, which could provide some insight on the machanisms of photochemical degradation of electron acceptors which are not yet well-studied.

\subsubsection{Photochemical stability of electron donors (polymers)}

Photochemical degradation in the presence of light and oxygen has been observed for a wide range of benchmark photovoltaic polymers, with the degradation rates varying significantly. The origin of the polymer photochemical degradation is not fully understood. Literature suggests it could be related to: chemical structure; formation of singlet oxygen and superoxide radical anions $\left(\mathrm{O}_{2}^{-}\right) \cdot[71,76,77]$ 


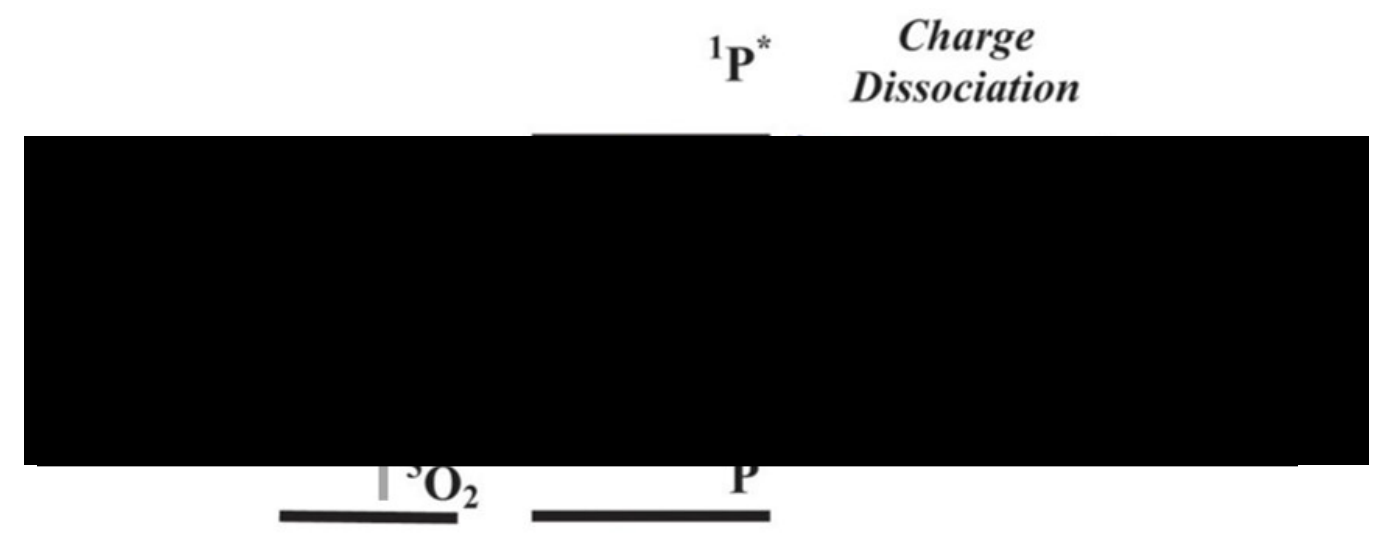

Figure 1.14: Triplet formation mechanism in neat polymer films (blue solid arrow) and in blend films (blue dashed arrow) which can lead to the generation of singlet oxygen and polymer photo-oxidation. Adapted from ref [72].

The generation of singlet oxygen, a high energy quantum state of molecular oxygen, is one of the mechanisms for photo-oxidation of polymer donors.[72] Singlet oxygen is highly reactive and can react with the polymers to cause degradation. Figure 1.14 illustrates the triplet mediated photo-oxidation pathways which can result in singlet oxygen formation in blend films. Triplet formation in a neat polymer film occurs via intersystem crossing from the polymer singlet state $\left({ }^{1} \mathrm{P}^{*}\right)$ to the lower energy triplet state $\left({ }^{3} \mathrm{P}^{*}\right)$ (blue solid arrow) $(1)$. In blended polymer: $\mathrm{PC}_{61} \mathrm{BM}$ films, triplet formation occurs via charge recombination of dissociated charges $\left(\mathrm{P}^{+}\right.$and $\left.\mathrm{PC}_{61} \mathrm{BM}^{-}\right)$(dashed blue arrows)(2). Oxygen quenching of the polymer triplet state may occur depending on the triplet's energy and lifetime. The quenching occurs by energy transfer from the polymer triplet state to the triplet ground state of molecular oxygen, exciting it to the singlet state (3), reducing the number of triplet state electrons that will decay via phosphorescence to the ground state (P). Singlet oxygen is highly reactive and so can react with the polymer, degrading it.

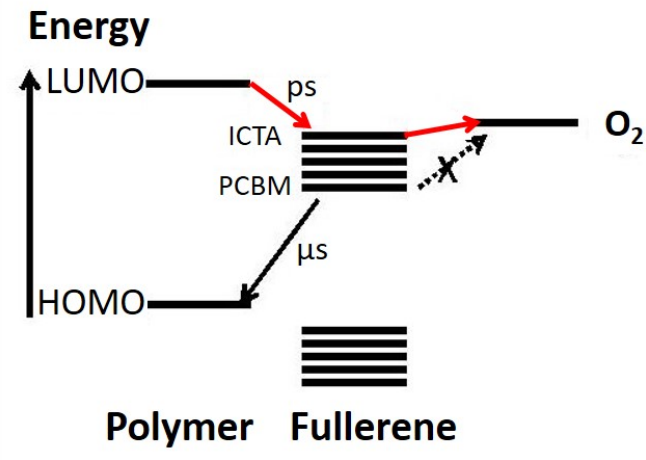

(a)

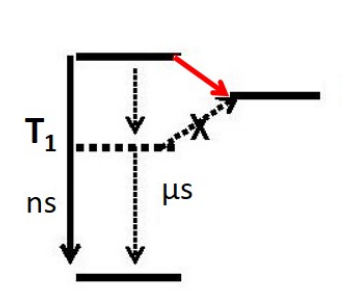

Polymer CT

(b)

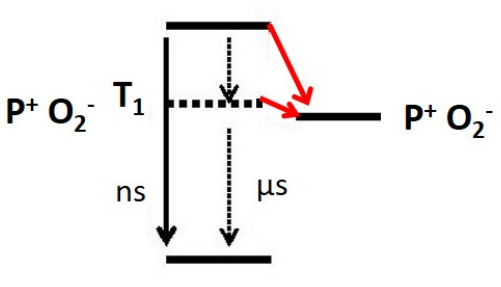

Polymer CT

(c)

Figure 1.15: Proposed mechanisms for superoxide anion generation from a) polymer:fullerene blends b) a pure polymer film and c) a pure polymer film with a high triplet energy and significant intersystem crossing yields.[77]

The generation of superoxide anions $\left(\mathrm{O}_{2}{ }^{-}\right)$, an oxygen molecule with an unpaired valence 
electron which makes it highly chemically reactive, is another mechanism for polymer photodegradation of OSCs.[77] The higher lying the LUMO level of the fullerene, the more energetically likely photogenerated electrons on the fullerene (generated by electron transfer from polymer or direct photo-excitation of fullerene) will transfer to oxygen to form a superoxide anion which will degrade the organic materials. Figure 1.15a illustrates the generation of superoxide anions from polymer:fullerene blends in the presence of oxygen. Microsecond charge recombination from the electron on the fullerene LUMO with the hole in the HOMO of the polymer competes with the electron transfer from the fullerene LUMO to molecular oxygen (electron affinity of $3.75 \mathrm{eV}$, where electron affinity $\approx$ LUMO). The discrete acceptor energy levels represent increasingly electronegative fullerene derivatives, from ICTA $(\mathrm{LUMO}=3.77 \mathrm{eV})$ to $\mathrm{PC}_{61} \mathrm{BM}(\mathrm{LUMO}=4.07 \mathrm{eV})$. Figure $1.15 \mathrm{~b}$ depicts nanosecond recombination of the singlet exciton competing with electron transfer from the excited polymer state to oxygen to form a polymer-oxygen charge transfer (CT) state complex. Figure 1.15c shows how polymers with significant intersystem crossing yields and high energy triplet excitons and significant intersystem crossing yields may also undergo electron transfer from the triplet state to oxygen, which could form substantial yield of superoxide anions.

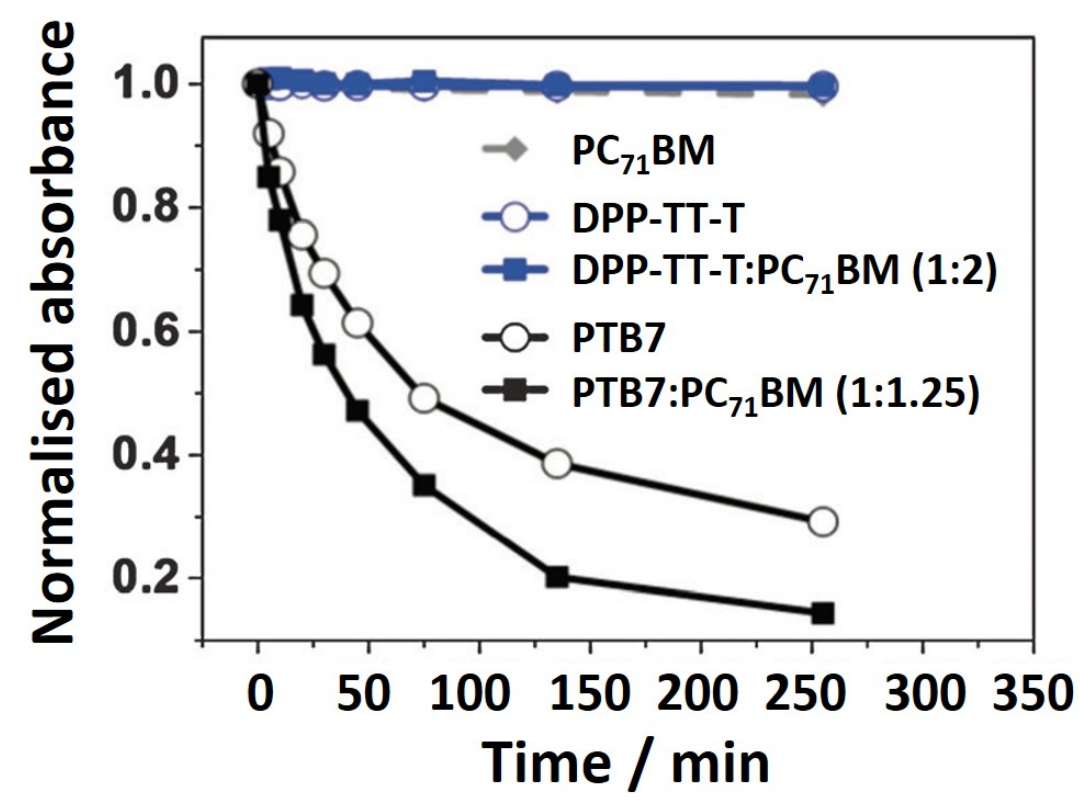

Figure 1.16: Decrease in the peak ground state absorbance of PTB7 and DPP-TT-T films, both neat and blended with $\mathrm{PC}_{71} \mathrm{BM}$, during photoaging under pure oxygen and $80 \mathrm{mWcm}^{-2}$ illumination.[72]

Two polymers: Poly (\{4,8-bis[(2-ethylhexyl)oxy]benzo[1,2-b:4,5-b']dithiophene-2,6-diyl $\}$ $\{(3$-fluoro-2-[(2-ethylhexyl)carbonyl] thieno[3,4-b]thiophenediyl $\})$ (PTB7) and diketopyrrolopyrrole - thieno[3,2-b] thiophene (DPP-TT-T) had their photochemical stabilities studied in neat and blend films with $\mathrm{PC}_{71} \mathrm{BM}$. PTB7 neat and blend films severely photobleach 
(loss of UV-vis absorbance) in 250 hours whereas there is little or no change in the UVVis absorbance of DPP-TT-T (Figure 1.16).[72] The relative instability of PTB7 is due to generation of singlet oxygen via triplet excitons, as described above. These triplet excitons in PTB7 blend films are generated via non-geminate charge recombination. No transient states were observed for DPP-TT-T but if triplet states were formed, these would not be energetically sufficient to generate singlet oxygen.[78] Interestingly, PTB7 is destabilised upon blending with $\mathrm{PC}_{71} \mathrm{BM}$. This study does not investigate the reason behind this destabilisation, but it could either be due to oxygen being able to penetrate the film more readily or an increase in the yield of PTB7 triplets.

The structure of a polymer is another factor that can affect its photochemical stability. For example introducing a silicon atom to bridge solubilising alkyl side-chains in a polymer backbone has shown to improve the photochemical stability of the resultant polymer. This is due to the silicon atom blocking the unpaired electron from moving toward the $\mathrm{C}_{5}$ ring, forming a reactive macroradical. The photochemical stability of over 25 different $\pi$-conjugated polymers were observed in a study and five crucial trends in structure that affected polymer photostability were identified.[76] Firstly, exocyclic double bonds in the main backbone - such as in Poly[2-methoxy-5-(2-ethylhexyloxy)-1,4-phenylenevinylene] (MEH-PPV) and MDMO-PPV - leads to poor stability. Secondly, moieties that contain a quaternary site - part of the molecule with one centralised positive atom bound to four side chains - are very unstable as these sites are easily oxidised, for example fluorene or cyclopentadithiophene. Stability is also restricted by the presence of readily cleavable bonds - such as C-N or C-O. Side-chains are also an important factor in conjugated polymer stability as removing them generally improves stability and keeping their number low as possible - no matter of their chemical nature - is a good rule to follow. Finally, good photochemical stability is generally displayed by aromatic polycyclic units - hydrocarbons composed of numerous rings in which the electrons are delocalised. These rules could be implemented to attempt to find a photochemically polymer.

There have been some studies on how the planarity and crystallinity of a polymer affect its triplet exciton dynamics. It has been reported that the conjugation length (length of electron delocalisation along backbone) of $\alpha$-oligothiophene polymers influences the triplet photophysics, longer conjugation red-shifts the transtion energy.[79, 80] Furthermore, thermally annealing poly(9,9dioctyl)fluorine (PFO) films significantly lower yield of triplet excitons and there was an observed presence of triplet states in polythiophene films when amorphous but not when crystalline. [81, 59] Furthermore, a study of broad range of polymers showed that the more crystalline a polymer, the triplet states had shorter lifetime and lower oxygen quenching rates.[82] This is due to triplet exciton mobility and the oxygen diffusion through the film being reduced with decreasing polymer crystallinity. This quenching generates singlet oxygen which would degrade the organic materials in 
the film. Hence, employing more crystalline donor polymers, which generally have triplet lifetimes too short to generate singlet oxygen and a slower rate of oxygen diffusion into the film, and thus have a better the photochemical stability.
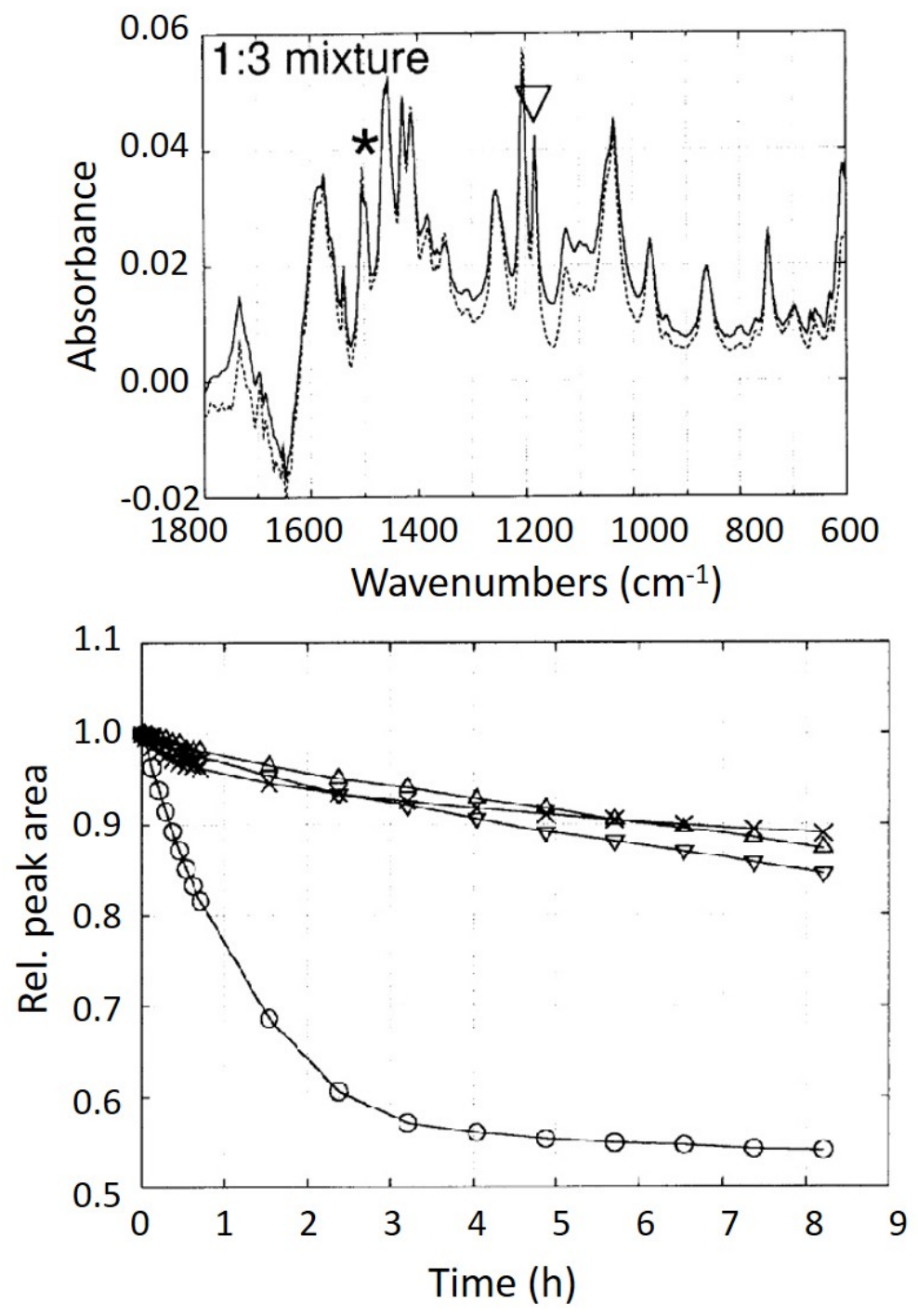

Figure 1.17: Photochemical degradation in oxygen and laser light for up to 8 hours, measured via FTIR of: MDMO-PPV $1506 \mathrm{~cm}^{-1}$ (o), fullerene $1182 \mathrm{~cm}^{-1}$ (x), blend 1506 $\mathrm{cm}^{-1}(\triangle)$, blend $1182 \mathrm{~cm}^{-1}(\nabla) \cdot[83]$

Blending a polymer with fullerene or $\mathrm{PC}_{61} \mathrm{BM}$ can alter its rate of photo-oxidation.[8486] The photo-oxidation is shown by the loss in the specific absorbance bands at 1182 $\mathrm{cm}^{-1}\left(\mathrm{C}_{60}\right)$ and $1506 \mathrm{~cm}^{-1}$ (MDMO-PPV) (Figure 1.17). The rate of degradation of the MDMO-PPV under $30 \mathrm{~mW} / \mathrm{cm}^{2}$ argon ion laser light (at $488 \mathrm{~nm}$ ) and pure oxygen is reduced greatly with the addition of the fullerene which can be seen when comparing the loss of the specific absorbance bands for the fullerene, polymer and blend as a function of degradation time. By adding the fullerene to the polymer, the polymer triplet state is quenched and therefore singlet oxygen formation is supressed, reducing the photooxidation of the polymer. On the otherhand, the fullerene is destabilised, albeit by a 
small amount, upon blending with the polymer.[86]

In this section, it has been shown that the photochemical degradation of donor polymers can be affected by their chemical structure, crystallinity, triplet exciton yield, in addition to whether it is blended with a fullerene and on the rate of oxygen diffusion through the film. In the next section, the studies into the photochemical stability of fullerene acceptors will be introduced.

\subsubsection{Photochemical stability of fullerene electron acceptors}

Fullerene electron acceptors are widely used in organic electronics and $\mathrm{PC}_{61} \mathrm{BM}$ is the most commonly employed electron acceptor for OSCs. This is down to the low lying LUMO of the fullerene, promoting fast charge separation, high electron mobility and its high solubility in organic solvents, making solvent processing possible.[87] The majority of studies use $\mathrm{PC}_{61} \mathrm{BM}$ as an acceptor when testing new polymers, architectures or upscaling methods. However, despite being widely used, the photo-oxidation of fullerene electron acceptors is far less studied than that of polymers.
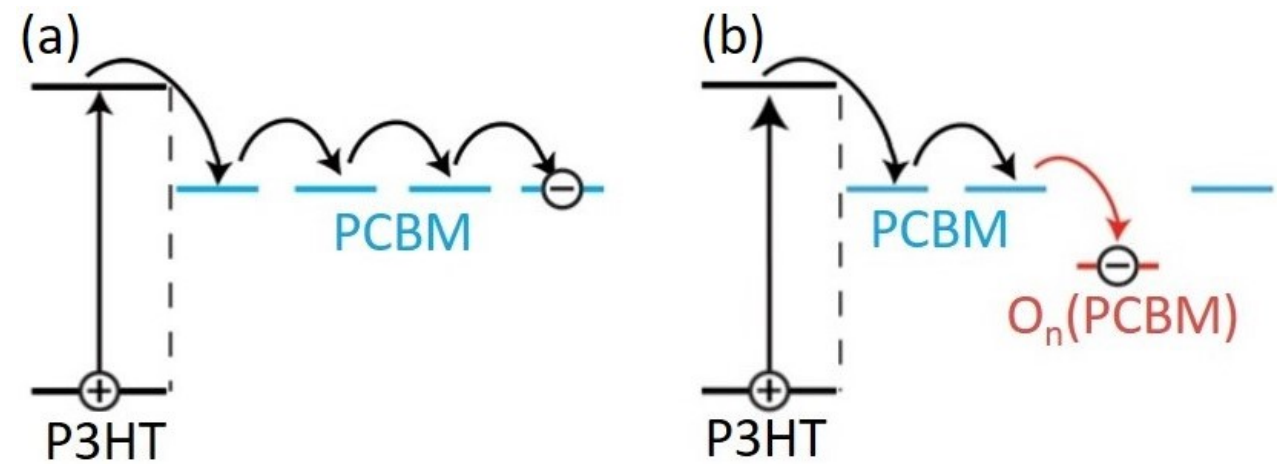

Figure 1.18: Illustration of a photoexcitation and electron transport in a $\mathrm{P} 3 \mathrm{HT}: \mathrm{PC}_{61} \mathrm{BM}$ film, a) without photo-oxidation of $\mathrm{PC}_{61} \mathrm{BM}$ and b) with photo-oxidation of $\mathrm{PC}_{61} \mathrm{BM}$, showing that the deeper LUMO level of photo-oxidised $\mathrm{PC}_{61} \mathrm{BM}$ introduces trap states that would inhibit electron mobility in $\mathrm{PC}_{61} \mathrm{BM}$ domains.[88]

It has been shown via FTIR that $\mathrm{C}_{60}$ and $\mathrm{PC}_{61} \mathrm{BM}$ in neat and blend films degrade under harsh-medium accelerated photo-aging conditions (an oxygen environment with argon laser light or filtered mercury lamp illumination in air). [85, 86] The reduction of this degradation kinetic of the polymer is attributed to the scavenging effect of the fullerene whereby it reacts with radicals that would otherwise react with the polymer, suggesting that the fullerene can be photo-oxidised.[89, 90] It has been shown that the oxidation of the fullerene cage leads to the deterioration of the photoconductivity of the blend film. [88] The oxidised $\mathrm{PC}_{61} \mathrm{BM}$ has a deeper LUMO level than fresh $\mathrm{PC}_{61} \mathrm{BM}$, and therefore act as electron traps in the $\mathrm{PC}_{61} \mathrm{BM}$ domains (Figure 1.18).[88] 


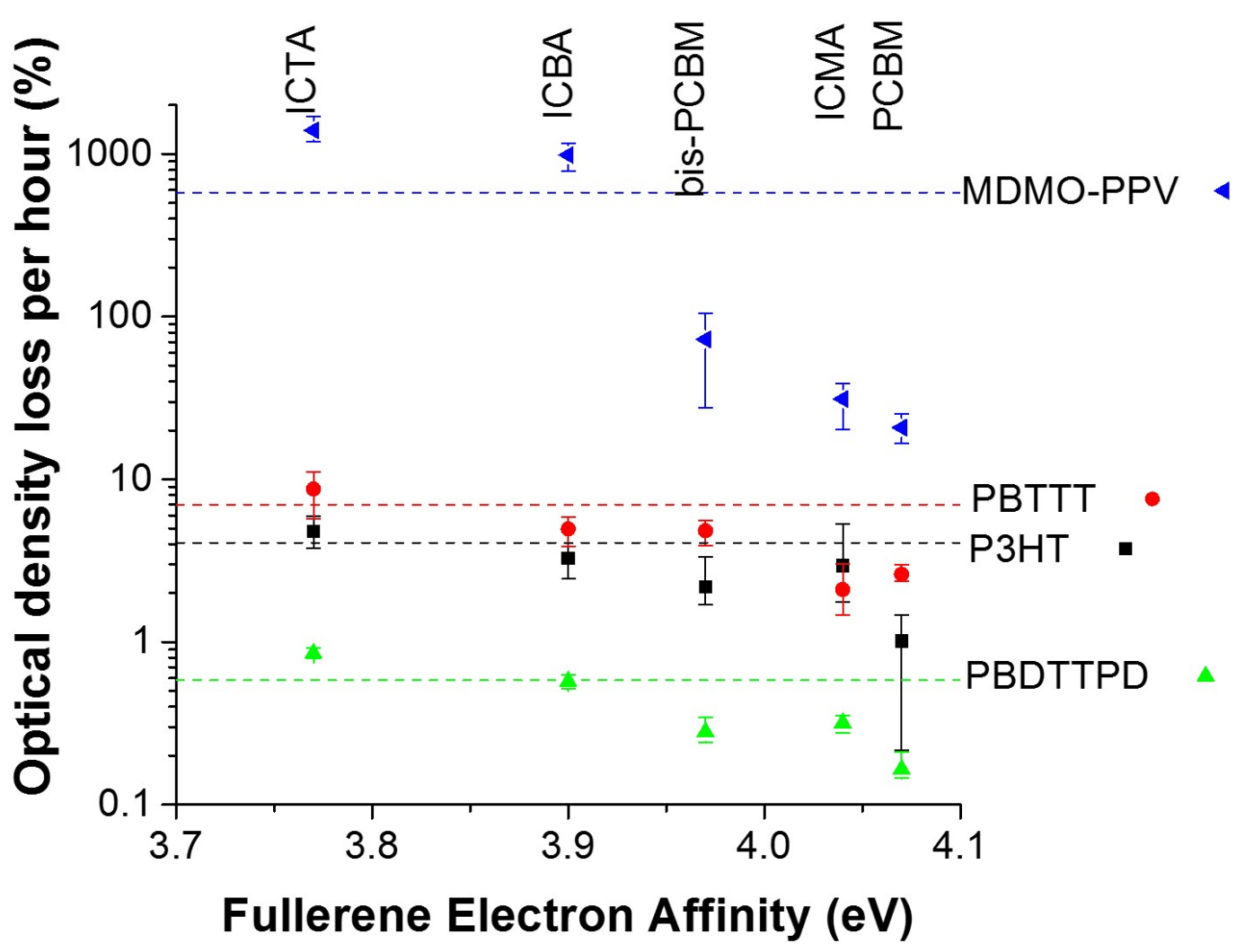

Figure 1.19: The rate of photobleaching of polymers as a function of fullerene electron affinity in blend films. Dashed lines denote the photobleaching rate of pure polymer films. The error bars show the minimum and maximum measured values for different equivalent samples \pm a standard error in the fitted rate.[77]

Not only does the addition of fullerene to the polymer can slow its rate of photodegradation, the electron affinity (the change in energy of a neutral atom when an electron is added to form a negative ion, a good indicator of the LUMO level[91-93]) of the fullerene acceptor can also have a strong effect to the rate of photo-oxidation of the polymer in a polymer:fullerene blends.[77] Polymers blended with a fullerene with a lower electron affinity, or a higher lying LUMO level, had decreased photochemical stability upon light and oxygen exposure (Figure 1.19). This is consistent with increased generation of highly reactive superoxide radical anions $\left(\mathrm{O}_{2}^{-}\right)$for fullerenes with higher lying LUMOs, which can then degrade the polymer (Figure 1.15). 


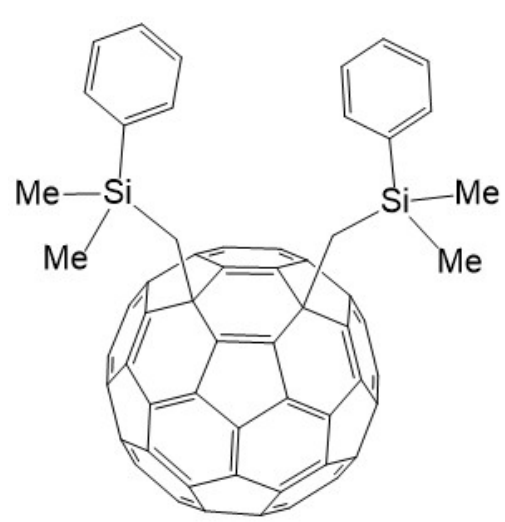

SIMEF

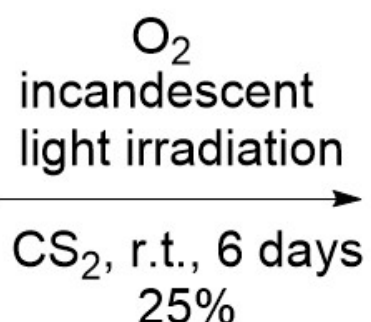

$25 \%$

Figure 1.20: Chemical structures of fullerene acceptor SIMEF and when it is photooxidised to SIMEF-O $2 \cdot[94]$

A fullerene acceptor, 1,4-bis(dimethylphenylsilylmethyl [60]fullerene (SIMEF), has also been shown to react with molecular oxygen when in an oxygen saturated environment, under a $60 \mathrm{~W}$ incandescent lamp to give SIMEF-O $\mathrm{O}_{2}$ and other more heavily photo-oxidised products (Figure 1.20).[94] Moreover, OSCs were fabricated with various amounts of SIMEF-O $\mathrm{O}_{2}$ added to the fresh SIMEF and device performance swiftly decreased as the amount of SIMEF-O $\mathrm{O}_{2}$ was increased (with a slight recovery when the composition became $100 \%$ SIMEF-O $\mathrm{S}_{2}$ ). It should be emphasised that even an addition of 1 wt $\%$ SIMEF-O was enough to almost half the efficiency of the OSC (from $3.2 \%$ to 1.9\%). This study suggests that a minute amount of a oxidised fullerene acceptor can drastically reduce the efficiency of the OSC.

As discussed in the previous section, crystallisation of polymers can affect the degree of its photodegradation. A similar effect could be possible for fullerene electron acceptors where the crystallinity or aggregation level could affect photo-oxidation, as it would be physically more difficult for the oxygen to diffuse through the film (in addition to affecting the polymer triplet yield). However, if the domain size is too large, the efficiency of charge transfer may be reduced, so the domain size will have to be optimised in order to balance these effects. It has been shown that fullerene solution after light exposure can form fullerene epoxides, where oxygen binds onto the fullerene ball, straining the ring to which it binds. [95] This subsequently leads to the fullerenes clustering, caused by the large dipole moment of the $\mathrm{C}_{60} \mathrm{O}$ localised around the oxygen which allows these oxidised fullerenes to coulombically bind, in the solution. These clusters are expected to have a negative impact on the optoelectronic properties of the films.

Fullerene derivatives are also used as electron transport layers in organic field effect transistors (OFETs).[96-98] It is well known that the performance of n-type organic field effect transistors are far inferior to that of the p-type.[99] This is due to the susceptibility of charge trapping in n-type transistors when exposed to environmental oxidants (oxygen 
and water), reducing electron carrier mobility.[100, 101] The air stability (in the dark) of fullerene electron transport layers in n-type organic field effect transistors was found to depend upon the electron affinity of the fullerene and energetic disorder (distribution of the energy levels) in the semiconducting layer.[102] There have been attempts to address this problem, e.g. by changing the chemical structure of the electron transport material such that it has less of a tendency to react with oxygen or water.[101, 103] For example, the addition of a solubilising group to a fullerene created fullerene monoadducts with relatively long stability in air in the dark (OXCMA- and ICMA-based OFETs drop by 1 order of magnitude after 6 weeks, 8 orders of magnitude for $\left.\mathrm{PC}_{61} \mathrm{BM}\right)$.[103] Additionally, it has been proposed that lowering the redox potential (electron affinity) of the n-type materials below the trap energy level of environmental oxidants would make it stable against charge trapping.[100-102, 104] Considerable research has been undertaken by the organic field effect transistor community on the oxidation of fullerene based electron transporting materials (but without illumination). However, some of the methods to improve the stability of these materials in OFETs could be trialled with OSCs (under illumination).

\subsubsection{Non-fullerene acceptors}

Fullerene acceptors have dominated OSCs for $\sim$ two decades, but it could be argued that developmental progress (in terms of efficiency) is slow or has reached a bottleneck. Nonfullerene acceptors are a new class of electron acceptor providing promising new research avenues and have yielded device efficiencies over $14 \%$ in just 2-3 years, surpassing the best performing fullerene-based devices (11.7\%).[36, 16] Unlike fullerenes, their optical, morphological and electronic properties, such as absorption, crystallinity, planarity and electron energy levels, can be readily tuned.[105]. Furthermore, the efficiency limit of OPV devices employing NFAs that dominate the absorption in the near-IR has been estimated to be close to $20 \%$.[106] In addition to increased tunability, NFAs have few syntheic steps meaning they can have low synthetic costs, making them highly attractive compared to the traditional fullerene-based acceptors.[107, 108] Furthermore, it has been demonstrated that some of these NFAs have excellent thermal stability (ITIC) and burn-in free behaviour (Eh-IDTBR).[107, 109]

The NFA families studied in this thesis have indacenodithiophene (IDT)-type core structures, which have been widely used in many donor polymers that exhibit good hole mobilities and strong and broad absorption due to the rigid and coplanar structure. IDTBR, a small molecule with a bandgap of $1.63 \mathrm{eV}$, achieved the highest PCE $(6.3 \%)$ with the donor polymer P3HT, which is still believed to be the most upscaleable 
polymer.[110] When IDTBR was blended with IDFBR, in a ternary blend with P3HT, a PCE of $7.6 \%$ was achieved and above $5 \%$ maintained for thick film devices.[111] ITIC, first published in 2015, is another medium bandgap (1.6 eV) NFA, with a high device performance $(11.22 \%)$. When an electron-rich methyl group is incorporated (M-ITIC), the LUMO is lowered, which leads to a higher obtained $\mathrm{V}_{\mathrm{oc}}$ and enhanced efficiency (12.05\%).[112] When electron deficient $\mathrm{F}$ atoms are incorporated into the end-capping groups instead, 3,9-bis(2-methylene-((3-(1,1-dicyanomethylene)-6,7-difluoro)-indanone))5,5,11,11-tetrakis(4-hexylphenyl)-dithieno[2,3-d:2',3'-d']-s-indaceno[1,2-b:5,6-b'] dithiophene (IT-4F) has a stronger intramolecular charge transfer which leads to a downshifting of its molecular energy levels, red-shifted absorption and enhanced extinction coefficient.[36] The highest PCE for a single junction OSC was achieved by employing this NFA (14.2\%). [16] Significantly, some NFA-based devices have been shown to be "burn-in" free, even with thick active layers $(\sim 300 \mathrm{~nm})$, and hence have superior stability under dry nitrogen compared to fullerene-based devices, but the reasons behind this burn-in free behavior is not well understood.[109, 113] Furthermore, the stability of NFA-based devices and the NFA materials themselves when exposed to light and air is not well studied.

\subsubsection{LUMO level of acceptors}

The LUMO level of electron acceptors has been shown to control the $\mathrm{V}_{\text {oc }}$ of OSCs. It has been shown that fullerenes acceptors with a shallower LUMO level raise the $V_{\text {oc }}$ of bulk heterojunction solar cells linearly.[114, 115, 37] In addition, it has also been reported that a sufficient energy offset (the difference between the LUMO of the donor and the acceptor) $(0.3-0.6 \mathrm{eV})$ is essential for creating a large driving force for efficient electron transfer.[66, 116, 117] However, for fullerene-free devices it has been shown that electron transfer could still be efficient, resulting in devices with a $V_{\text {oc }}$ of $>1 \mathrm{~V}$ with a small LUMO offset.[118]

Early fullerene acceptors with shallower LUMO levels than $\mathrm{PC}_{61} \mathrm{BM}$ were trialled in OPV devices but suffered from insufficient charge transport, inefficient charge-dissociation or morphological problems.[115] Then in 2008, Lennes et al. reported a novel fullerene derivative, bisPCBM, with a LUMO $\sim 100 \mathrm{meV}$ shallower than $\mathrm{PC}_{61} \mathrm{BM}$ but little negative impact on the charge carrier properties and yielded enhanced $\mathrm{V}_{\mathrm{oc}}$ and overall cell performance.[119] ICBA with a LUMO $170 \mathrm{mV}$ higher than $\mathrm{PC}_{61} \mathrm{BM}$ yielded even higher device performance with P3HT (6.48 \%).[120, 121] The addition of another side chain to form ICTA, to further raise the LUMO and $\mathrm{V}_{\mathrm{oc}}$, resulted in a deterioration of electron transport in the fullerene phase and heavily reduced device performance $(1.56 \%)$, explained through lower light absorption and electron mobility.[122] The $\mathrm{C}_{71}$ analogue 
$\mathrm{PC}_{71} \mathrm{BM}$ addressed the problem of poor light absorption by fullerenes in the visible region, enhancing the $\mathrm{J}_{\mathrm{sc}}$ of cells.[123] Many OSCs employing $\mathrm{PC}_{71} \mathrm{BM}$ yielded PCEs $10 \%$ higher than with $\mathrm{PC}_{61} \mathrm{BM}$.[124] $\mathrm{PC}_{71} \mathrm{BM}$ has a similar LUMO level $\mathrm{PC}_{61} \mathrm{BM}$, however, its relatively low electron mobility and its relatively big molecular size influences the charge transport and phase separation in devices.[125] Kloc-6 is a fullerene ketolactam produced by Solenne BV, Groningen, Netherlands, with a LUMO level $\sim 0.2 \mathrm{eV}$ lower than that of $\mathrm{PC}_{61} \mathrm{BM}$.

Hoke et al. have showed that the level of photodegradation (in air) undergone by a polymer in blend films is determined by the LUMO level of the fullerene electron acceptor, proposing this is due to degradation via superoxide anion genration through the fullerenes.[77] However, a couple of points were not covered in this study: i) There was no data on how the LUMO level affected the photostability of fullerene acceptor itself nor the device stability. ii) Fullerenes with a deeper LUMO than $\mathrm{PC}_{61} \mathrm{BM}$ were neglected from the study.

\section{$1.3 \quad$ Objectives}

This thesis focuses on the photochemical stability of the electron accepting materials employed in OSC devices. A wide range of well established fullerene based materials are studied along with some cutting edge non-fullerene acceptors. Neat electron acceptor and blended films are studied using various thin film characterisation techniques in order to identify the photodegradation mechanisms observed in the devices fabricated which were photoaged under controlled environmental conditions. The techniques employed in these studies include UV-Vis absorption and fourier transform infrared spectroscopies to study the photobleaching and photochemcial degradation. Mass and x-ray photoelectron spectroscopies allowed the chemical cause of the observed degradations to be identified quantatively. Microsecond transient absorption spectroscopy was applied to probe the excited states in the organic materials to be probed, which could provide important information to deduce possible degradation mechanisms. The sensitisation of a fluorescent probe that reacted with the superoxide radical allowed confirmation of a photodegradation pathway. Devices were fabricated and tested to investigate how these degradation phenomena manifested and impacted device performance. An important objective for these studies is to establish relationships between acceptor structural/nanomorphological/electronic properties and material/device stability. Ultimately, the aim of this thesis is to provide some guidelines for the design of stable electron acceptors in OSCs. All the structures of materials studied and experimental techniques involved are given in Chapter 2. 
The role of the common fullerene derivative $\mathrm{PC}_{61} \mathrm{BM}$ in limiting the photostability of benchmark OSCs was quantitatively studied in Chapter 3. Photo-oxidation of $\mathrm{PC}_{61} \mathrm{BM}$ was shown to result in a depression in LUMO level, which would cause electron trapping in the fullerene domains. This photodegradation was observed for OSCs made using both standard and inverted device architectures, and a range of benchmark polymers and benchmark fullerenes, where the fullerenes were selectively photoaged. These results demonstrate that the photo-oxidation of fullerenes have a general and detrimental effect to the stability of polymer:fullerene OSCs exposed to light and air.

In Chapter 4, the photochemical stability of $\mathrm{PS}: \mathrm{PC}_{61} \mathrm{BM}$ blend films were found to be dependent upon the degree of $\mathrm{PC}_{61} \mathrm{BM}$ aggregation, correlating with the observed photo-oxidation of $\mathrm{PC}_{61} \mathrm{BM}$ and oxygen quenching of its triplet states. Based on these findings, it is proposed that $\mathrm{PC}_{61} \mathrm{BM}$ photo-oxidation is primarily due to triplet-mediated singlet oxygen generation. The implications of fullerene photo-oxidation on the optoelectronic properties of films and devices were also investigated, with the electron mobility and PL/El spectra affected and consistent with TD-DFT calculations of defect generation. Then $\mathrm{PC}_{61} \mathrm{BM}$ aggregation was shown to affect the stability of donor polymer (PCDTBT):fullerene blend films and devices, which correlated with the yield of oxygen quenching of the polymer triplet state. Here, it is proposed that the dominate photodegradation pathway is triplet-mediated singlet oxygen generation via the polymer triplet state.

Six fullerene acceptors with different LUMO levels were studied in Chapter 5 in order to investigate how energetics could affect film and device photochemical stability. The degree of photobleaching of neat and blend films and photo-oxidation via carbonyl formation, correlated well with the fullerene LUMO levels obtained via cyclic voltammetry. A good correlation was observed between the level of photo-oxidation in neat films with the photodegradation of devices (PCE and $\mathrm{J}_{\mathrm{sc}}$ ) made with selectively degraded fullerenes, while the reduction in $\mathrm{V}_{\text {oc }}$ correlated well with the fullerene LUMO. Furthermore, the large drop in PCE shows the significance of fullerene photo-oxidation in its role on the stability of OSCs.

The photostability of NFAs is investigated in Chapter 6. Firstly, the burn-in behaviour of NFAs and fullerene acceptors are compared and one NFA family is found to exhibit superior burn-in free behaviour. Then a range of optical and chemical measurements were utilised to investigate the photodegradation (in air) of benchmark fullerene-based and NFA-based OSC blend films, and a critical correlation was established between the electron acceptor LUMO level and the photochemical stability of both the polymer donor and electron acceptor in the active layer films. This correlation can be explained by a reduction in acceptor LUMO level mediated formation of superoxide. 
Finally, all significant results from Chapters 3-6 are summarised in Chapter 7 and implications are discussed. Additionally, some future investigations regarding TD-DFT modelling of Kloc-6 photo-oxidation, the photochemical stability of polymer: $\mathrm{PC}_{61} \mathrm{BM}$ :fullerene ternary blend devices and burn-in behaviour of both fullerene and NFA based devices have also been suggested. 


\section{REFERENCES}

[1] S. Shafiee and E. Topal, "When will fossil fuel reserves be diminished?," Energy Policy, vol. 37, pp. 181-189, 2009.

[2] "IPCC, 2013: Summary for Policymakers. In: Climate Change 2013: The Physical Science Basis. Contribution of Working Group I to the Fifth Assessment Report of the Intergovernmental Panel on Climate Change."

[3] U. R. E. R. Department of Energy \& Climate Change, "UK Renewable Energy Roadmap," 2011.

[4] Department of Energy and Climate Change, "Digest of United Kingdom energy statistics (DUKES) - Chapter 6: Renewable sources of energy," 2013.

[5] I. R. E. W. Party, "Renewable Energy... into the mainstream (2002)."

[6] N. S. Lewis and D. G. Nocera, "Powering the planet: chemical challenges in solar energy utilization.," Proceedings of the National Academy of Sciences of the United States of America, vol. 103, pp. 15729-35, 2006.

[7] D. J. MacKay, Sustainability - without the hot air. No. 3.5.2., Cambridge: UIT Cambridge, 2009.

[8] R. M. Swanson, "A vision for crystalline silicon photovoltaics," Progress in Photovoltaics: Research and Applications, vol. 14, pp. 443-453, 2006.

[9] A. Becquerel, "Recherches sur les effets de la radiation chimique de la lumiere solaire au moyen des courants electriques," Comptes Rendus de L'Academie des Sciences, vol. 9, pp. 145-149, 1839.

[10] A. Becquerel, "Memoire sur les effects electriques produits sous I'influence des rayons solaires," Annalen der Physick und Chemie, vol. 54, pp. 35-42, 1841.

[11] "Daily chart: Pricing sunshine - The Economist." http://www.economist.com/blogs/graphicdetail/2012/12/daily-chart-19, Accessed: 2015-03-04.

[12] "World Energy Perspective: Cost of Energy Technologies," 2013.

[13] U.S. Energy Information Administration (EIA), "Levelized Cost and Levelized Avoided Cost of New Generation Resources in the Annual Energy Outlook 2015.”

[14] Cramming More Components onto Integrated Circuits, vol. 86, 1998. 
[15] S. Hegedus, "Thin film solar modules: the low cost, high throughput and versatile alternative to Si wafers," Progress in Photovoltaics: Research and Applications, vol. 14, pp. 393-411, 2006.

[16] S. Li, L. Ye, W. Zhao, H. Yan, B. Yang, D. Liu, W. Li, H. Ade, and J. Hou, "A Wide Band Gap Polymer with a Deep Highest Occupied Molecular Orbital Level Enables 14.2\% Efficiency in Polymer Solar Cells," Journal of the American Chemical Society, vol. 140, pp. 7159-7167, 2018.

[17] M. A. Green, "The path to $25 \%$ silicon solar cell efficiency: History of silicon cell evolution," Progress in Photovoltaics: Research and Applications, vol. 17, pp. 183$189,2009$.

[18] "How Much Electricity Can I Generate with Solar Panels?." http://www.theecoexperts.co.uk/how-much-electricity-can-i-generate-solar-panels, Accessed: 2016-01-27.

[19] M. A. Maehlum, "The Real Lifespan of Solar Panels - Energy Informative," 2014.

[20] J. Adams, G. D. Spyropoulos, M. Salvador, N. Li, S. Strohm, L. Lucera, S. Langner, F. Machui, H. Zhang, T. Ameri, M. M. Voigt, F. C. Krebs, and C. J. Brabec, "Air-processed organic tandem solar cells on glass: toward competitive operating lifetimes," Energy \& Environmental Science, vol. 8, pp. 169-176, 2015.

[21] C. H. Peters, I. T. Sachs-Quintana, J. P. Kastrop, S. Beaupré, M. Leclerc, and M. D. McGehee, "High Efficiency Polymer Solar Cells with Long Operating Lifetimes," Advanced Energy Materials, vol. 1, pp. 491-494, 2011.

[22] G. D. Spyropoulos, P. Kubis, N. Li, D. Baran, L. Lucera, M. Salvador, T. Ameri, M. Voigt, F. C. Krebs, and C. J. Brabec, "Flexible Organic Tandem Solar Modules with 6\% efficiency: Combining Roll-to-Roll Compatible Processing with High Geometric Fill Factors," Energy Environ. Sci., vol. 7, pp. 3284-3290, 2014.

[23] M. Helgesen, J. E. Carlé, G. A. dos Reis Benatto, R. R. Søndergaard, M. Jørgensen, E. Bundgaard, and F. C. Krebs, "Making Ends Meet: Flow Synthesis as the Answer to Reproducible High-Performance Conjugated Polymers on the Scale that Roll-toRoll Processing Demands," Advanced Energy Materials, vol. 5, p. 1401996, 2015.

[24] B. Azzopardi, C. J. M. Emmott, A. Urbina, F. C. Krebs, J. Mutale, and J. Nelson, "Economic assessment of solar electricity production from organic-based photovoltaic modules in a domestic environment," Energy \& Environmental Science, vol. 4, p. 3741, 2011. 
[25] H. K. H. Lee, Z. Li, J. R. Durrant, and W. C. Tsoi, "Is organic photovoltaics promising for indoor applications?," Applied Physics Letters, vol. 108, p. 253301, 2016 .

[26] S. Guo, C. Brandt, T. Andreev, E. Metwalli, W. Wang, J. Perlich, and P. MüllerBuschbaum, "First Step into Space: Performance and Morphological Evolution of P3HT:PCBM Bulk Heterojunction Solar Cells under AM0 Illumination," ACS Applied Materials \& Interfaces, vol. 6, pp. 17902-17910, 2014.

[27] M. O. Reese, S. A. Gevorgyan, M. Jørgensen, E. Bundgaard, S. R. Kurtz, D. S. Ginley, D. C. Olson, M. T. Lloyd, P. Morvillo, E. A. Katz, A. Elschner, O. Haillant, T. R. Currier, V. Shrotriya, M. Hermenau, M. Riede, K. R. Kirov, G. Trimmel, T. Rath, O. Inganäs, F. Zhang, M. Andersson, K. Tvingstedt, M. Lira-Cantu, D. Laird, C. McGuiness, S. J. Gowrisanker, M. Pannone, M. Xiao, J. Hauch, R. Steim, D. M. DeLongchamp, R. Rösch, H. Hoppe, N. Espinosa, A. Urbina, G. Yaman-Uzunoglu, J.-B. Bonekamp, A. J. van Breemen, C. Girotto, E. Voroshazi, and F. C. Krebs, "Consensus stability testing protocols for organic photovoltaic materials and devices," Solar Energy Materials and Solar Cells, vol. 95, pp. 1253-1267, 2011 .

[28] A. J. Heeger, S. Kivelson, J. R. Schrieffer, and W. P. Su, "Solitons in conducting polymers," Reviews of Modern Physics, vol. 60, pp. 781-850, 1988.

[29] A. J. Heeger, "Semiconducting and Metallic Polymers: The Fourth Generation of Polymeric Materials (Nobel Lecture)," Angewandte Chemie, vol. 40, no. 14, pp. 2591-2611, 2001.

[30] V. Coropceanu, J. Cornil, D. A. da Silva Filho, Y. Olivier, R. Silbey, and J.L. Brédas, "Charge Transport in Organic Semiconductors," Chemical Reviews, vol. 107, pp. 926-952, 2007.

[31] S. Karg, W. Riess, V. Dyakonov, and S. M., "Electrical and optical characterization of poly(phenylene-vinylene) light emitting diodes," Synthetic Metals, vol. 54, pp. 427-433, 1993.

[32] C. W. Tang, "Two-layer organic photovoltaic cell," Applied Physics Letters, vol. 48, p. $183,1986$.

[33] G. Yu, J. Gao, J. C. Hummelen, F. Wudl, and A. J. Heeger, "Polymer photovoltaic cells: Enhanced efficiencies via a network of internal donor-acceptor heterojunctions," Science, vol. 270, pp. 1789-1791, 1995. 
[34] S.-H. Liao, H.-J. Jhuo, P.-N. Yeh, Y.-S. Cheng, Y.-L. Li, Y.-H. Lee, S. Sharma, and S.-A. Chen, "Single junction inverted polymer solar cell reaching power conversion efficiency $10.31 \%$ by employing dual-doped zinc oxide nano-film as cathode interlayer.," Scientific reports, vol. 4, 2014.

[35] Y. Liu, J. Zhao, Z. Li, C. Mu, W. Ma, H. Hu, K. Jiang, H. Lin, H. Ade, and H. Yan, "Aggregation and morphology control enables multiple cases of high-efficiency polymer solar cells.," Nature communications, vol. 5, p. 5293, 2014.

[36] W. Zhao, S. Li, H. Yao, S. Zhang, Y. Zhang, B. Yang, and J. Hou, "Molecular Optimization Enables over 13\% Efficiency in Organic Solar Cells," Journal of the American Chemical Society, vol. 139, pp. 7148-7151, 2017.

[37] G. T. Mola and N. Abera, "Correlation between LUMO offset of donor/acceptor molecules to an open circuit voltage in bulk heterojunction solar cell," Physica B: Condensed Matter, vol. 445, pp. 56-59, 2014.

[38] T. Clarke and J. Durrant, "Charge Photogeneration in Organic Solar Cells," Chemical Reviews, vol. 110, pp. 6736-6767, 2010.

[39] L. J. A. Koster, S. E. Shaheen, and J. C. Hummelen, "Pathways to a New Efficiency Regime for Organic Solar Cells," Advanced Energy Materials, vol. 2, pp. 1246-1253, 2012 .

[40] S. M. Sze and K. K. Ng, Physics of semiconductor devices. Wiley-Interscience, 2007.

[41] J. W. Lichtman and J.-A. Conchello, "Fluorescence microscopy.," Nature methods, vol. 2, pp. 910-9, 2005.

[42] I. G. Scheblykin, A. Yartsev, T. Pullerits, V. Gulbinas, and V. Sundström, "Excited state and charge photogeneration dynamics in conjugated polymers.," Journal of Physical Chemistry B, vol. 111, pp. 6303-21, 2007.

[43] I. A. Howard, R. Mauer, M. Meister, and F. Laquai, "Effect of Morphology on Ultrafast Free Carrier Generation in Polythiophene:Fullerene Organic Solar Cells," Journal of the American Chemical Society, vol. 132, pp. 14866-14876, 2010.

[44] S. L. Smith and A. W. Chin, "Ultrafast charge separation and nongeminate electron-hole recombination in organic photovoltaics," Physical Chemistry Chemical Physics, vol. 16, pp. 20305-20309, 2014.

[45] H. Tamura and I. Burghardt, "Ultrafast Charge Separation in Organic Photovoltaics Enhanced by Charge Delocalization and Vibronically Hot Exciton Dissociation," Journal of the American Chemical Society, vol. 135, pp. 16364-16367, 2013. 
[46] M. Scheidler, U. Lemmer, R. Kersting, S. Karg, W. Riess, B. Cleve, R. F. Mahrt, H. Kurz, H. Bässler, E. O. Göbel, and P. Thomas, "Monte Carlo study of picosecond exciton relaxation and dissociation in poly(phenylenevinylene)," Physical Review B, vol. 54, pp. 5536-5544, 1996.

[47] A. Ruini, M. J. Caldas, G. Bussi, and E. Molinari, "Solid State Effects on Exciton States and Optical Properties of PPV," Physical Review Letters, vol. 88, p. 206403, 2002 .

[48] J.-L. Brédas, J. E. Norton, J. Cornil, and V. Coropceanu, "Molecular Understanding of Organic Solar Cells: The Challenges," Accounts of Chemical Research, vol. 42, pp. 1691-1699, 2009.

[49] A. Moliton and J.-M. Nunzi, "How to model the behaviour of organic photovoltaic cells," Polymer International, vol. 55, pp. 583-600, 2006.

[50] J. Guo, H. Ohkita, H. Benten, and S. Ito, "Charge generation and recombination dynamics in poly(3-hexylthiophene)/fullerene blend films with different regioregularities and morphologies.," Journal of the American Chemical Society, vol. 132, pp. 6154-64, 2010.

[51] P. Peumans and S. R. Forrest, "Separation of geminate charge-pairs at donor-acceptor interfaces in disordered solids," Chemical Physics Letters, vol. 398, pp. 27-31, 2004.

[52] L. Onsager, "Initial Recombination of Ions," Physical Review, vol. 54, pp. 554-557, 1938.

[53] S. D. Dimitrov and J. R. Durrant, "Materials Design Considerations for Charge Generation in Organic Solar Cells," Chemistry of Materials, vol. 26, pp. 616-630, 2014.

[54] K. Graham, A. Amassian, R. Lee, G. O. N. Ndjawa, P. Erwin, M. Thompson, D. Nordlund, K. Vandewal, E. Hoke, A. Salleo, and M. McGehee, "Charge-transfer state energy determines open-circuit voltage in organic photovoltaics," 2013.

[55] C.-C. Lee, W.-C. Su, W.-C. Chang, B.-Y. Huang, and S.-W. Liu, "The effect of charge transfer state on the open-circuit voltage of small-molecular organic photovoltaic devices: A comparison between the planar and bulk heterojunctions using electroluminescence characterization," Organic Electronics, vol. 16, pp. 1-8, 2015.

[56] A. Köhler, "Organic semiconductors: No more breaks for electrons.," Nature materials, vol. 11, pp. 836-7, 2012. 
[57] I.-W. Hwang, D. Moses, and A. J. Heeger, "Photoinduced Carrier Generation in P3HT/PCBM Bulk Heterojunction Materials," The Journal of Physical Chemistry C, vol. 112, pp. 4350-4354, 2008.

[58] S. De, T. Pascher, M. Maiti, K. G. Jespersen, T. Kesti, F. Zhang, O. Inganäs, A. Yartsev, and V. Sundström, "Geminate charge recombination in alternating polyfluorene copolymer/fullerene blends.," Journal of the American Chemical Society, vol. 129, pp. 8466-72, 2007.

[59] H. Ohkita, S. Cook, Y. Astuti, W. Duffy, S. Tierney, W. Zhang, M. Heeney, I. McCulloch, J. Nelson, D. D. C. Bradley, and J. R. Durrant, "Charge carrier formation in polythiophene/fullerene blend films studied by transient absorption spectroscopy.," Journal of the American Chemical Society, vol. 130, pp. 3030-42, 2008.

[60] F. Etzold, I. A. Howard, N. Forler, D. M. Cho, M. Meister, H. Mangold, J. Shu, M. R. Hansen, K. Müllen, and F. Laquai, "The effect of solvent additives on morphology and excited-state dynamics in PCPDTBT:PCBM photovoltaic blends.," Journal of the American Chemical Society, vol. 134, pp. 10569-83, 2012.

[61] I. Montanari, A. F. Nogueira, J. Nelson, J. R. Durrant, C. Winder, M. A. Loi, N. S. Sariciftci, and C. Brabec, "Transient optical studies of charge recombination dynamics in a polymer/fullerene composite at room temperature," Applied Physics Letters, vol. 81, p. 3001, 2002.

[62] A. F. Nogueira, I. Montanari, J. Nelson, J. R. Durrant, C. Winder, N. S. Sariciftci, and C. Brabec, "Charge Recombination in Conjugated Polymer/Fullerene Blended Films Studied by Transient Absorption Spectroscopy," The Journal of Physical Chemistry B, vol. 107, pp. 1567-1573, 2003.

[63] T. Kirchartz, B. E. Pieters, J. Kirkpatrick, U. Rau, and J. Nelson, "Recombination via tail states in polythiophene:fullerene solar cells," Physical Review B, vol. 83, p. $115209,2011$.

[64] B. Qi and J. Wang, "Fill factor in organic solar cells," Physical Chemistry Chemical Physics, p. 8972, 2013.

[65] R. Po, A. Bernardi, A. Calabrese, C. Carbonera, G. Corso, and A. Pellegrino, "From lab to fab: how must the polymer solar cell materials design change? - an industrial perspective," Energy \& Environmental Science, vol. 7, p. 925, 2014.

[66] G. Dennler, M. C. Scharber, and C. J. Brabec, "Polymer-Fullerene BulkHeterojunction Solar Cells," Advanced Materials, vol. 21, pp. 1323-1338, 2009. 
[67] F. C. Krebs, ed., Stability and Degradation of Organic and Polymer Solar Cells. John Wiley \& Sons, 2012.

[68] J. U. Lee, J. W. Jung, J. W. Jo, and W. H. Jo, "Degradation and stability of polymer-based solar cells," Journal of Materials Chemistry, vol. 22, p. 24265, 2012.

[69] S. Bertho, G. Janssen, T. J. Cleij, B. Conings, W. Moons, A. Gadisa, J. D'Haen, E. Goovaerts, L. Lutsen, J. Manca, and D. Vanderzande, "Effect of temperature on the morphological and photovoltaic stability of bulk heterojunction polymer:fullerene solar cells," Solar Energy Materials and Solar Cells, vol. 92, pp. 753$760,2008$.

[70] A. Burrows, J. S. J. S. Holman, A. F. Parsons, G. Pilling, and G. J. Price, Chemistry $^{3}$ : introducing inorganic, organic, and physical chemistry. Oxford University Press, 2017.

[71] M. Jørgensen, K. Norrman, and F. C. Krebs, "Stability/degradation of polymer solar cells," Solar Energy Materials and Solar Cells, vol. 92, pp. 686-714, 2008.

[72] Y. W. Soon, H. Cho, J. Low, H. Bronstein, I. McCulloch, and J. R. Durrant, "Correlating triplet yield, singlet oxygen generation and photochemical stability in polymer/fullerene blend films.," Chemical communications, vol. 49, pp. 1291-3, 2013.

[73] M. Jørgensen, K. Norrman, S. A. Gevorgyan, T. Tromholt, B. Andreasen, and F. C. Krebs, "Stability of polymer solar cells.," Advanced materials, vol. 24, pp. 580-612, 2012 .

[74] F. C. Krebs and K. Norrman, "Analysis of the failure mechanism for a stable organic photovoltaic during $10000 \mathrm{~h}$ of testing," Progress in Photovoltaics: Research and Applications, vol. 15, pp. 697-712, 2007.

[75] Z. Li, K. H. Chiu, R. S. Ashraf, S. Fearn, R. Dattani, H. C. Wong, C.-H. Tan, J. Wu, J. T. Cabral, and J. R. Durrant, "Toward Improved Lifetimes of Organic Solar Cells under Thermal Stress: Substrate-Dependent Morphological Stability of PCDTBT:PCBM Films and Devices," Scientific Reports, vol. 5, p. 15149, 2015.

[76] M. Manceau, E. Bundgaard, J. E. Carlé, O. Hagemann, M. Helgesen, R. Søndergaard, M. Jørgensen, and F. C. Krebs, "Photochemical stability of $\pi$ conjugated polymers for polymer solar cells: a rule of thumb," Journal of Materials Chemistry, vol. 21, p. 4132, 2011.

[77] E. T. Hoke, I. T. Sachs-Quintana, M. T. Lloyd, I. Kauvar, W. R. Mateker, A. M. Nardes, C. H. Peters, N. Kopidakis, and M. D. McGehee, "The Role of Electron 
Affinity in Determining Whether Fullerenes Catalyze or Inhibit Photooxidation of Polymers for Solar Cells," Advanced Energy Materials, vol. 2, pp. 1351-1357, 2012.

[78] Y. W. Soon, Charge Separation, Triplets and Photochemical stability in Polymer/Fullerene Solar Cells. PhD thesis, Imperial College London, 2013.

[79] R. A. J. Janssen, L. Smilowitz, N. S. Sariciftci, and D. Moses, "Triplet-state photoexcitations of oligothiophene films and solutions," The Journal of Chemical Physics, vol. 101, p. 1787, 1994.

[80] R. S. Becker, J. Seixas de Melo, A. L. Maçanita, and F. Elisei, "Comprehensive Evaluation of the Absorption, Photophysical, Energy Transfer, Structural, and Theoretical Properties of $\alpha$-Oligothiophenes with One to Seven Rings," The Journal of Physical Chemistry, vol. 100, pp. 18683-18695, 1996.

[81] A. J. Cadby, P. A. Lane, H. Mellor, S. J. Martin, M. Grell, C. Giebeler, D. D. C. Bradley, M. Wohlgenannt, C. An, and Z. V. Vardeny, "Film morphology and photophysics of polyfluorene," Physical Review B, vol. 62, pp. 15604-15609, 2000.

[82] Y. W. Soon, S. Shoaee, R. S. Ashraf, H. Bronstein, B. C. Schroeder, W. Zhang, Z. Fei, M. Heeney, I. McCulloch, and J. R. Durrant, "Material Crystallinity as a Determinant of Triplet Dynamics and Oxygen Quenching in Donor Polymers for Organic Photovoltaic Devices," Advanced Functional Materials, vol. 24, pp. 1474$1482,2014$.

[83] H. Neugebauer, C. Brabec, J. Hummelen, and N. Sariciftci, "Stability and photodegradation mechanisms of conjugated polymer/fullerene plastic solar cells," Solar Energy Materials and Solar Cells, vol. 61, pp. 35-42, 2000.

[84] T. Endale, E. Sovernigo, A. Radivo, S. Dal Zilio, A. Pozzato, T. Yohannes, L. Vaccari, and M. Tormen, "Investigation of photodegradation in polymer solar cells blended with different fullerenes derivatives," Solar Energy Materials and Solar Cells, vol. 123, pp. 150-158, 2014.

[85] H. Neugebauer, C. Brabec, J. Hummelen, R. Janssen, and N. Sariciftci, "Stability studies and degradation analysis of plastic solar cell materials by FTIR spectroscopy," Synthetic Metals, vol. 102, pp. 1002-1003, 1999.

[86] S. Chambon, A. Rivaton, J. L. Gardette, and M. Firon, "Photo- and thermal degradation of MDMO-PPV:PCBM blends," Solar Energy Materials and Solar Cells, vol. 91, pp. 394-398, 2007. 
[87] T. Liu and A. Troisi, "What makes fullerene acceptors special as electron acceptors in organic solar cells and how to replace them.," Advanced materials (Deerfield Beach, Fla.), vol. 25, pp. 1038-41, 2013.

[88] M. O. Reese, A. M. Nardes, B. L. Rupert, R. E. Larsen, D. C. Olson, M. T. Lloyd, S. E. Shaheen, D. S. Ginley, G. Rumbles, and N. Kopidakis, "Photoinduced Degradation of Polymer and Polymer-Fullerene Active Layers: Experiment and Theory," Advanced Functional Materials, vol. 20, pp. 3476-3483, 2010.

[89] M. Seno, M. Maeda, and T. Sato, "Effect of fullerene on radical polymerization of vinyl acetate," Journal of Polymer Science Part A: Polymer Chemistry, vol. 38, pp. 2572-2578, 2000.

[90] R. Borghi, L. Lunazzi, G. Placucci, G. Cerioni, and A. Plumitallo, "Photolysis of Dialkoxy Disulfides: A Convenient Source of Alkoxy Radicals for Addition to the Sphere of Fullerene C 60," The Journal of Organic Chemistry, vol. 61, pp. 33273331, 1996.

[91] C.-G. Zhan, J. Nichols, and D. Dixon, "Ionization Potential, Electron Affinity, Electronegativity, Hardness, and Electron Excitation Energy: Molecular Properties from Density Functional Theory Orbital Energies," Journal of Physical Chemistry A, vol. 107, pp. 4184-4195, 2003.

[92] H. A. Kurtz, "LUMO energies and negative electron affinities," Journal of Chemical Education, vol. 61, p. 580, 1984.

[93] A. M. Teale, F. De Proft, and D. J. Tozer, "Orbital energies and negative electron affinities from density functional theory: Insight from the integer discontinuity," The Journal of Chemical Physics, vol. 129, p. 044110, 2008.

[94] Y. Matsuo, A. Ozu, N. Obata, N. Fukuda, H. Tanaka, and E. Nakamura, "Deterioration of bulk heterojunction organic photovoltaic devices by a minute amount of oxidized fullerene.," Chemical communications, vol. 48, pp. 3878-80, 2012.

[95] R. Dattani, K. F. Gibson, S. Few, A. J. Borg, P. A. DiMaggio, J. Nelson, S. G. Kazarian, and J. T. Cabral, "Fullerene oxidation and clustering in solution induced by light," Journal of Colloid and Interface Science, vol. 446, pp. 24-30, 2015.

[96] R. Ahmed, M. Sams, C. Simbrunner, M. Ullah, K. Rehman, G. Schwabegger, H. Sitter, and T. Ostermann, "Reproducibility and stability of C60 based organic field effect transistor," Synthetic Metals, vol. 161, p. 2562, 2012. 
[97] C. Waldauf, P. Schilinsky, M. Perisutti, J. Hauch, and C. Brabec, "SolutionProcessed Organic n-Type Thin-Film Transistors," Advanced Materials, vol. 15, pp. 2084-2088, 2003.

[98] M. Woebkenberg, P. H., Bradley, D. D. C., Kronholm, D., Hummelen, J. C., de Leeuw, D. M., Coelle, M., Anthopoulos, T. D., H. Wobkenberg, P. \& Colle, "High mobility n-channel organic field-effect transistors based on soluble C-60 and C70 fullerene derivatives - Research database - University of Groningen," Synthetic Metals., vol. 158, pp. 468-472, 2008.

[99] D. K. Hwang, C. Fuentes-Hernandez, M. Fenoll, M. Yun, J. Park, J. W. Shim, K. A. Knauer, A. Dindar, H. Kim, Y. Kim, J. Kim, H. Cheun, M. M. Payne, S. Graham, S. Im, J. E. Anthony, and B. Kippelen, "Systematic reliability study of top-gate pand n-channel organic field-effect transistors.," ACS applied materials $\&$ interfaces, vol. 6, pp. 3378-86, 2014.

[100] T. D. Anthopoulos, G. C. Anyfantis, G. C. Papavassiliou, and D. M. de Leeuw, "Airstable ambipolar organic transistors," Applied Physics Letters, vol. 90, p. 122105, 2007.

[101] T. J. M. Brooks A. Jones, Antonio Facchetti, Michael R. Wasielewski, "Tuning Orbital Energetics in Arylene Diimide Semiconductors. Materials Design for Ambient Stability of n-Type Charge Transport," Journal of American Chemical Society, vol. 129, pp. 15259-15278, 2007.

[102] J. M. Ball, R. K. M. Bouwer, F. B. Kooistra, J. M. Frost, Y. Qi, E. B. Domingo, J. Smith, D. M. de Leeuw, J. C. Hummelen, J. Nelson, A. Kahn, N. Stingelin, D. D. C. Bradley, and T. D. Anthopoulos, "Soluble fullerene derivatives: The effect of electronic structure on transistor performance and air stability," Journal of Applied Physics, vol. 110, p. 014506, 2011.

[103] J. H. O. Hojeong Yu, Han-Hee Cho, Chul-Hee Cho, Ki-Hyun Kim, Dong Yeong Kim, Bumjoon J. Kim, "Polarity and Air-Stability Transitions in Field-Effect Transistors Based on Fullerenes with Different Solubilizing Groups," ACS applied materials \& interfaces, vol. 5, pp. 4865-4871, 2013.

[104] H. Usta, C. Risko, Z. Wang, H. Huang, M. K. Deliomeroglu, A. Zhukhovitskiy, A. Facchetti, and T. J. Marks, "Design, synthesis, and characterization of laddertype molecules and polymers. Air-stable, solution-processable n-channel and ambipolar semiconductors for thin-film transistors via experiment and theory.," Journal of the American Chemical Society, vol. 131, pp. 5586-608, 2009. 
[105] P. Cheng, G. Li, X. Zhan, and Y. Yang, "Next-generation organic photovoltaics based on non-fullerene acceptors," Nature Photonics, vol. 12, pp. 131-142, 2018.

[106] N. Li, I. McCulloch, and C. J. Brabec, "Analyzing the efficiency, stability and cost potential for fullerene-free organic photovoltaics in one figure of merit," Energy 8 Environmental Science, vol. 11, pp. 1355-1361, 2018.

[107] W. Zhao, D. Qian, S. Zhang, S. Li, O. Inganäs, F. Gao, and J. Hou, "Fullerene-Free Polymer Solar Cells with over 11\% Efficiency and Excellent Thermal Stability," Advanced Materials, vol. 28, pp. 4734-4739, 2016.

[108] S. Holliday, R. S. Ashraf, C. B. Nielsen, M. Kirkus, J. A. Röhr, C.-H. Tan, E. Collado-Fregoso, A.-C. Knall, J. R. Durrant, J. Nelson, and I. McCulloch, "A Rhodanine Flanked Nonfullerene Acceptor for Solution-Processed Organic Photovoltaics," Journal of the American Chemical Society, vol. 137, pp. 898-904, 2015.

[109] H. Cha, J. Wu, A. Wadsworth, J. Nagitta, S. Limbu, S. Pont, Z. Li, J. Searle, M. F. Wyatt, D. Baran, J.-S. Kim, I. McCulloch, and J. R. Durrant, "An Efficient, "Burn in" Free Organic Solar Cell Employing a Nonfullerene Electron Acceptor," Advanced Materials, vol. 29, p. 1701156, 2017.

[110] S. Holliday, R. S. Ashraf, A. Wadsworth, D. Baran, S. A. Yousaf, C. B. Nielsen, C.-H. Tan, S. D. Dimitrov, Z. Shang, N. Gasparini, M. Alamoudi, F. Laquai, C. J. Brabec, A. Salleo, J. R. Durrant, and I. McCulloch, "High-efficiency and air-stable P3HT-based polymer solar cells with a new non-fullerene acceptor," Nature Communications, vol. 7, p. 11585, 2016.

[111] D. Baran, R. S. Ashraf, D. A. Hanifi, M. Abdelsamie, N. Gasparini, J. A. Röhr, S. Holliday, A. Wadsworth, S. Lockett, M. Neophytou, C. J. M. Emmott, J. Nelson, C. J. Brabec, A. Amassian, A. Salleo, T. Kirchartz, J. R. Durrant, and I. McCulloch, "Reducing the efficiency-stability-cost gap of organic photovoltaics with highly efficient and stable small molecule acceptor ternary solar cells," Nature Materials, vol. 16, pp. 363-369, 2017.

[112] S. Li, L. Ye, W. Zhao, S. Zhang, S. Mukherjee, H. Ade, and J. Hou, "Energy-Level Modulation of Small-Molecule Electron Acceptors to Achieve over 12\% Efficiency in Polymer Solar Cells," Advanced Materials, vol. 28, pp. 9423-9429, 2016.

[113] N. Gasparini, M. Salvador, S. Strohm, T. Heumueller, I. Levchuk, A. Wadsworth, J. H. Bannock, J. C. de Mello, H.-J. Egelhaaf, D. Baran, I. McCulloch, and C. J. Brabec, "Burn-in Free Nonfullerene-Based Organic Solar Cells," Advanced Energy Materials, vol. 7, p. 1700770, 2017. 
[114] C. J. Brabec, A. Cravino, D. Meissner, N. S. Sariciftci, T. Fromherz, M. T. Rispens, L. Sanchez, and J. C. Hummelen, "Origin of the Open Circuit Voltage of Plastic Solar Cells," Advanced Functional Materials, vol. 11, pp. 374-380, 2001.

[115] F. B. Kooistra, J. Knol, F. Kastenberg, L. M. Popescu, W. J. H. Verhees, J. M. Kroon, and J. C. Hummelen, "Increasing the Open Circuit Voltage of BulkHeterojunction Solar Cells by Raising the LUMO Level of the Acceptor," Organic Letters, pp. 551-554, 2007.

[116] M. A. Faist, S. Shoaee, S. Tuladhar, G. F. A. Dibb, S. Foster, W. Gong, T. Kirchartz, D. D. C. Bradley, J. R. Durrant, and J. Nelson, "Understanding the Reduced Efficiencies of Organic Solar Cells Employing Fullerene Multiadducts as Acceptors," Advanced Energy Materials, vol. 3, pp. 744-752, 2013.

[117] J. J. M. Halls, J. Cornil, D. A. dos Santos, R. Silbey, D.-H. Hwang, A. B. Holmes, J. L. Brédas, and R. H. Friend, "Charge- and energy-transfer processes at polymer/polymer interfaces: A joint experimental and theoretical study," Physical Review $B$, vol. 60, pp. 5721-5727, 1999.

[118] D. Baran, T. Kirchartz, S. Wheeler, S. Dimitrov, M. Abdelsamie, J. Gorman, R. S. Ashraf, S. Holliday, A. Wadsworth, N. Gasparini, P. Kaienburg, H. Yan, A. Amassian, C. J. Brabec, J. R. Durrant, and I. McCulloch, "Reduced voltage losses yield $10 \%$ efficient fullerene free organic solar cells with $>1 \mathrm{~V}$ open circuit voltages," Energy $\&$ Environmental Science, vol. 9, pp. 3783-3793, 2016.

[119] M. Lenes, G.-J. A. H. Wetzelaer, F. B. Kooistra, S. C. Veenstra, J. C. Hummelen, and P. W. M. Blom, "Fullerene Bisadducts for Enhanced Open-Circuit Voltages and Efficiencies in Polymer Solar Cells," Advanced Materials, vol. 20, pp. 2116$2119,2008$.

[120] Y. He, H.-Y. Chen, J. Hou, and Y. Li, "Indene-C 60 Bisadduct: A New Acceptor for High-Performance Polymer Solar Cells," Journal of the American Chemical Society, vol. 132, pp. 1377-1382, 2010.

[121] G. Zhao, Y. He, and Y. Li, "6.5\% Efficiency of Polymer Solar Cells Based on poly(3-hexylthiophene) and Indene-C60 Bisadduct by Device Optimization," $A d$ vanced Materials, vol. 22, pp. 4355-4358, 2010.

[122] H. Kang, C.-H. Cho, H.-H. Cho, T. E. Kang, H. J. Kim, K.-H. Kim, S. C. Yoon, and B. J. Kim, "Controlling Number of Indene Solubilizing Groups in Multiadduct Fullerenes for Tuning Optoelectronic Properties and Open-Circuit Voltage in Organic Solar Cells," ACS Applied Materials \& Interfaces, vol. 4, pp. 110-116, 2012. 
[123] M. M. Wienk, J. M. Kroon, W. J. H. Verhees, J. Knol, J. C. Hummelen, P. A. van Hal, and R. A. J. Janssen, "Efficient Methano[70]fullerene/MDMO-PPV Bulk Heterojunction Photovoltaic Cells," Angewandte Chemie International Edition, vol. 42, pp. 3371-3375, 2003.

[124] Y. He and Y. Li, "Fullerene derivative acceptors for high performance polymer solar cells," Physical Chemistry Chemical Physics, vol. 13, pp. 1970-1983, 2011.

[125] F. Zhang, Z. Zhuo, J. Zhang, X. Wang, X. Xu, Z. Wang, Y. Xin, J. Wang, J. Wang, W. Tang, Z. $\mathrm{Xu}$, and $\mathrm{Y}$. Wang, "Influence of $\mathrm{PC}_{60} \mathrm{BM}$ or $\mathrm{PC}_{70} \mathrm{BM}$ as electron acceptor on the performance of polymer solar cells," Solar Energy Materials and Solar Cells, vol. 97, pp. 71-77, 2012. 
CHAPTER

TWO

\section{EXPERIMENTAL METHODS}

\subsection{Materials}

The fullerene acceptors used in this thesis were all purchased from Solenne BV. Their characteristics are outlined in Table 2.1 and their structures and full chemical names are given in Figure 2.1.

Table 2.1: Purities and molecular weights $\left(\mathrm{M}_{\mathrm{w}}\right)$ of the fullerene acceptors.

\begin{tabular}{||c|c|c||}
\hline \hline Name & Purity $(\%)$ & $\mathrm{M}_{\mathrm{w}}(\mathrm{g} / \mathrm{mol})$ \\
\hline $\mathrm{PC}_{61} \mathrm{BM}$ & 99.5 & 910.9 \\
\hline $\mathrm{PC}_{71} \mathrm{BM}$ & 99.0 & 1031.0 \\
\hline BisPCBM & 99.5 & 1101.1 \\
\hline ICBA & 99.0 & 953.0 \\
\hline ICTA & 99.0 & 1068.2 \\
\hline Kloc-6 & $>99.0$ & 988.0 \\
\hline \hline
\end{tabular}




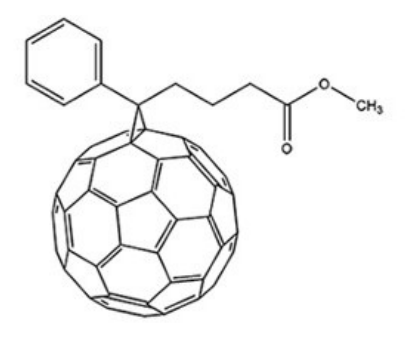

$\mathrm{PC}_{61} \mathrm{BM}$

$[6,6]$-phenyl- $\mathrm{C}_{61}$-butyric acid methyl ester

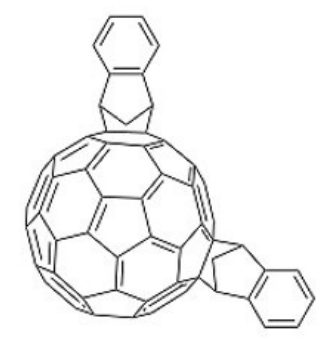

ICBA

indene- $\mathrm{C}_{60}$ bisadduct

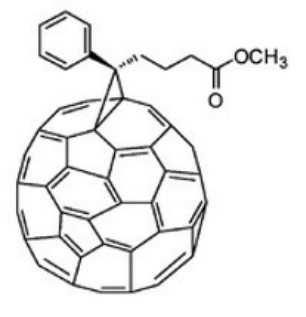

PC $_{71}$ BM

$[6,6]-P h e n y l-C 71-$ butyric acid methyl ester.

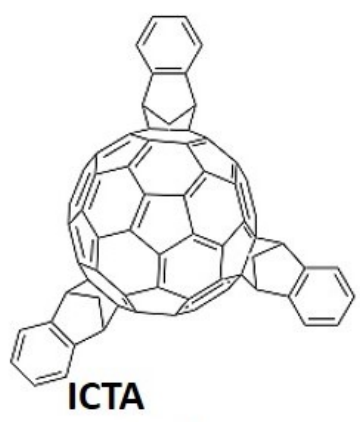

C60-indene tris-adduct

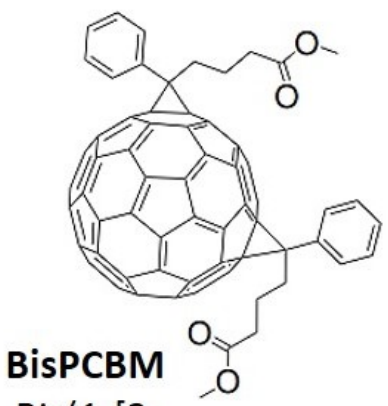

Bis(1-[3-

(methoxycarbonyl)prop

yl]-1-phenyl)-[6,6]C62

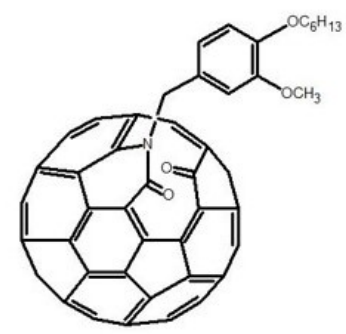

Kloc-6

2a-Aza-1,2(2a)-homo-1,9-

seco[5,6]fullerene-C60-Ih-1,9-dione,

2a-[(4- hexyloxy)-3-

methoxyphenyl]methyl]

Figure 2.1: Chemical structures and full names of the fullerene acceptors studied in this thesis.

The details for the non-fullerene acceptors studied in this thesis are shown in Table 2.2 and their chemical structures and full chemical names are given in Figure 2.2.

Table 2.2: Supplier, purity and molecular weight $\left(\mathrm{M}_{\mathrm{w}}\right)$ of non-fullerene acceptors.(I.M. denotes that the material was synthesised in-house by Iain McCulloch's group.)

\begin{tabular}{||c|c|c|c||}
\hline \hline Name & Supplier & Purity (\%) & $\mathrm{M}_{\mathrm{w}}(\mathrm{g} / \mathrm{mol})$ \\
\hline O-IDFBR & I.M. & & 1314.0 \\
\hline O-IDTBR & I.M. & & 1326.0 \\
\hline Eh-IDTBR & I.M. & & 1326.0 \\
\hline ITIC & 1-Material & 99 & 1427.9 \\
\hline M-ITIC & 1-Material & 98 & 1456.0 \\
\hline \hline
\end{tabular}




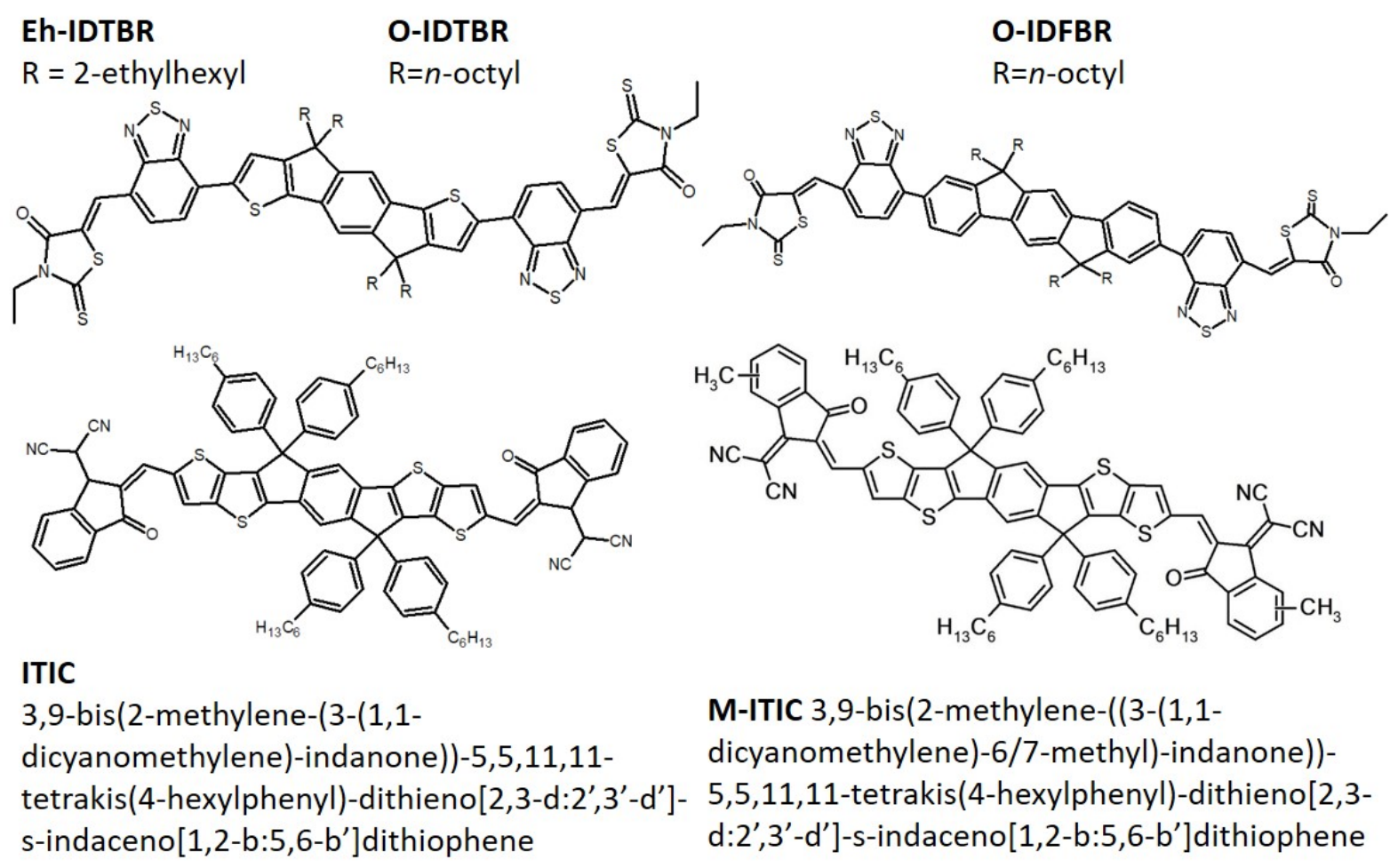

Figure 2.2: Chemical structures and full names (where known) of the non-fullerene acceptors studied in this thesis.

The details for the polymers studied in this thesis are outlined in Table 2.3 and their chemical structures and names are given in Figure 2.3. The polydispersity (PD) describes the distribution of the molecular weight of a polymer and is given by

$$
P D=\frac{M_{\mathrm{w}}}{M_{\mathrm{n}}}
$$

where $\mathrm{M}_{\mathrm{w}}$ is the weight average molecular weight and $\mathrm{M}_{\mathrm{n}}$ is the number average molecular weight. The closer the PD is to 1 , the more uniform the polymer chain length.

Table 2.3: Supplier, molecular weights $\left(\mathrm{M}_{\mathrm{w}}\right)$, polydispersity $(\mathrm{PD})$ and regioregularity (RR) of the polymers.

\begin{tabular}{||c|c|c|c|c||}
\hline \hline Name & Supplier & $\mathrm{M}_{\mathrm{w}}(\mathrm{g} / \mathrm{mol})$ & $\mathrm{PD}$ & $\mathrm{RR}(\%)$ \\
\hline Polystyrene & Scientific Polymer Inc & $210 \mathrm{~K}$ & $\sim 2.0$ & - \\
\hline P3HT \#1 & Rieke Metals & $53 \mathrm{~K}$ & $\sim 2.3$ & 93 \\
\hline P3HT \#2 & Rieke Metals & $69 \mathrm{~K}$ & 2.3 & $\geq 96$ \\
\hline PCDTBT & 1-Material & $50-60 \mathrm{~K}$ & $2-3$ & - \\
\hline PTB7 & 1-Material & $100 \mathrm{~K}$ & $2-3$ & - \\
\hline PCE10 & 1-Material & $\sim 130 \mathrm{~K}$ & $\sim 2.5$ & - \\
\hline PCE11 & Ossila & $117.5 \mathrm{~K}$ & 2.14 & - \\
\hline PCE12 & 1-Material & $80-100 \mathrm{~K}$ & 2.4 & - \\
\hline \hline
\end{tabular}




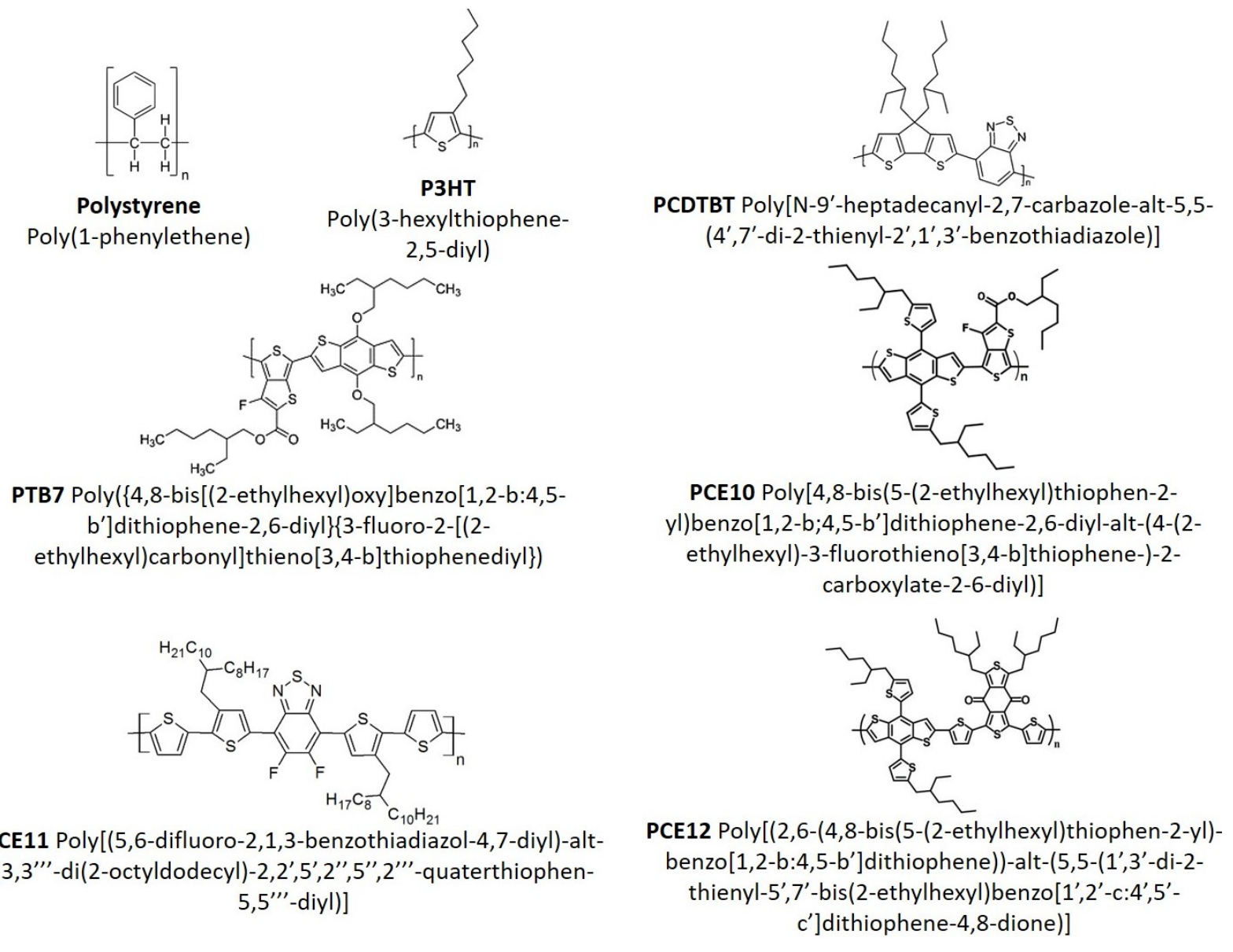

Figure 2.3: Chemical structures and full names of the polymers studied in this thesis.

The solvents chlorobenzene $(\mathrm{CB})$, dichlorobenzene $(\mathrm{DCB})$ and chloroform $(\mathrm{CF})$ were purchased from Sigma Aldrich.

\subsection{Film preparation and device fabrication}

Solutions were prepared with CB or DCB for polymer:acceptor blends and CF for neat acceptor solutions at the stated concentration in each chapter. CF was chosen for neat acceptor solutions due to its higher solubility limit for the acceptor materials, lower boiling point and hence capability to yield better film homogeneity compared to CB. For blend solutions, the polymer:acceptor ratio was chosen to match those optimised for device performance. All solutions were heated and stirred at $60{ }^{\circ} \mathrm{C}$ overnight, unless otherwise stated, to ensure that the materials had properly dissolved. The oxygen sensitive solutions were stirred in a nitrogen-filled glovebox.

Quartz or glass substrates were used for UV-Vis absorption, Transient Absorption and Photoluminescence Spectroscopies, depending on the wavelengths the samples were measured/probed. For photoluminescence measurements where a fluorscent probe to detect 
superoxide generation was measured $1 \mathrm{~cm}^{2}$ glass substrates were used and submerged in the fluorescent probe. For Matrix-assisted laser desorption/ionisation (MALDI-TOF) mass spectroscopy, samples were made by preparing thin films on glass substrates, photoaging for the time specified for each experiment in later chapters, and redissolved. Aluminium (Al) substrates were used for Attenuated Total Reflectance Fourier Transform Infra-red (ATR-FTIR) measurements (or glass was used and the film was scraped off to create a powder for the measurement). Samples for X-ray Photoelectron Spectroscopy (XPS) and cyclic voltammetry were spun onto $1 \mathrm{~cm}^{2}$ and $2 \mathrm{~cm}^{2} 15 \Omega / \mathrm{sq}$ indium tin oxide (ITO) conductive glass, respectively. For Grazing Incidence Wide-Angle X-ray Scattering (GIWAXS) measurements, double side polished silicon wafers were used. Glass substrates were used for atomic force microscopy. All substrates were cleaned by sequential sonication in acetone once for 10 minutes, twice for 5 minutes and then twice in isopropanol for 5 minutes. The substrates were then allowed to air dry in a fume hood before being blown with air, and air plasma treated prior to spincoating. Films were spincoated with parameters described in each chapter in air or in a dry nitrogen-filled glovebox depending on oxygen sensitivity of the films. Some films (where stated in text) were thermally annealed after spincoating in a nitrogen-filled glovebox to increase the film crystallinity. Films were photoaged under a AM1.5G solar simulator in either air or in a Linkham THMS600 environmental chamber purged with dry nitrogen.

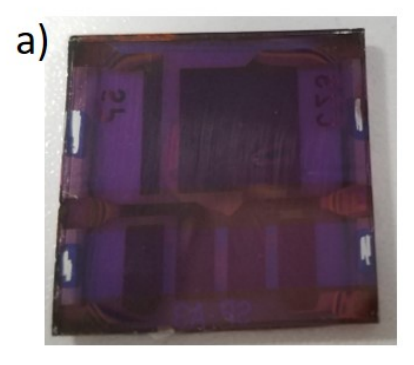

b)

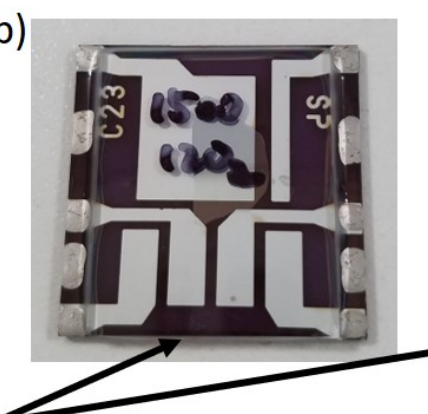

c)

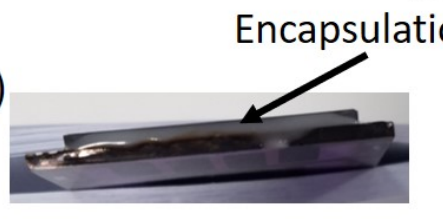

Ag contacts (anode) for cells 1 - 6

d)

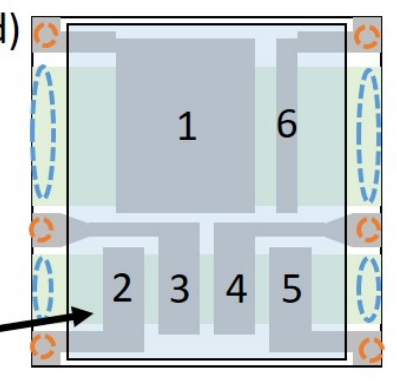

ITO contacts (cathode) for cells $1 \& 6$ i,

ITO contacts (Cathode) for cells 2 - 5

Figure 2.4: Device layout: a) ITO side (light shines through this side), b) encapsulation glass side, c) side view and d) a schematic of the encapsulation glass side labelled for an inverted device. If conventional, then ITO is the anode and silver $(\mathrm{Ag})$ is the cathode. Photo and schematic credit: Dr. Harrison Lee (Swansea University).

The device layout is shown in Figure 2.4, where the ITO substrate is $2.5 \times 2.5 \mathrm{~cm}^{2}$, cell 1 has a pixel area of $1 \mathrm{~cm}^{2}$ and cells $2-6$ are $0.15 \mathrm{~cm}^{2}$. The majority of devices studied were fabricated following the inverted architecture: ITO glass / zinc oxide $(\mathrm{ZnO}) /$ Active Layer / molybdenum trioxide $\left(\mathrm{MoO}_{3}\right)$ / silver $(\mathrm{Ag})$. The ITO glass used was patterned 
with two stripes with $15 \Omega /$ sq sheet resistance. Firstly, the ITO glass substrates were sonicated in soap water for 10 minutes and three times in deionised water for 5 minutes, before following the same cleaning procedure as that used for substrates for thin film characterisation samples (see previous paragraph). The $\mathrm{ZnO}$ solution was prepared by dissolving $109.75 \mathrm{mg}$ zinc acetate dehydrate per $1 \mathrm{ml}$ of 2-methoxythanol and $30.2 \mu \mathrm{l}$ (mono)ethanolamine. This solution was stirred overnight at room temperature. The ZnO precursor layer was spincoated onto the ITO substrate in air at $4000 \mathrm{rpm}$, with $7500 \mathrm{rpm} / \mathrm{s}$ acceleration for $60 \mathrm{~s}$. Two stripes of the $\mathrm{ZnO}$ precursor were then wiped off with ethanol using a cottonbud to expose the ITO contacts and then the film was annealed at $150{ }^{\circ} \mathrm{C}$ on a hotplate for 10 minutes to form $\mathrm{ZnO}(40 \mathrm{~nm})$. The active layer was then spincoated in the dry nitrogen filled glovebox (or in air depending on the oxygen sensitivity of the film) using spin parameters described in each chapter. Finally $10 \mathrm{~nm}$ of $\mathrm{MoO}_{3}$ and 100 $\mathrm{nm}$ of Ag were thermally evaporated to complete the devices. Devices were encapsulated with glass slides and epoxy, unless they were to be tested for air stability in which case they were kept unencapsulated.

In some cases the conventional architecture was utilised for devices: ITO glass / Poly(3,4-ethylenedioxythiophene)-poly(styrenesulfonate) (PEDOT:PSS) / Active Layer / Calcium (Ca) / Aluminium (Al). PEDOT:PSS (Heraeus Clevios P VP AI 4083) was spincoated onto the ITO substrate in air at $4000 \mathrm{rpm}$, with $7500 \mathrm{rpm} / \mathrm{s}$ acceleration for $60 \mathrm{~s}$ and thermally annealed for 10 minutes at $150{ }^{\circ} \mathrm{C} .30 \mathrm{~nm}$ of calcium and $100 \mathrm{~nm}$ of aluminium were thermally evaporated to complete the devices. Some of these devices were fabricated by Dr. Zhe Li and Dr. Harrison Lee at Swansea University. 
a)

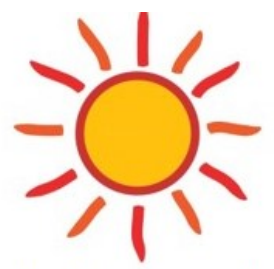

Degraded in air under 1 sun
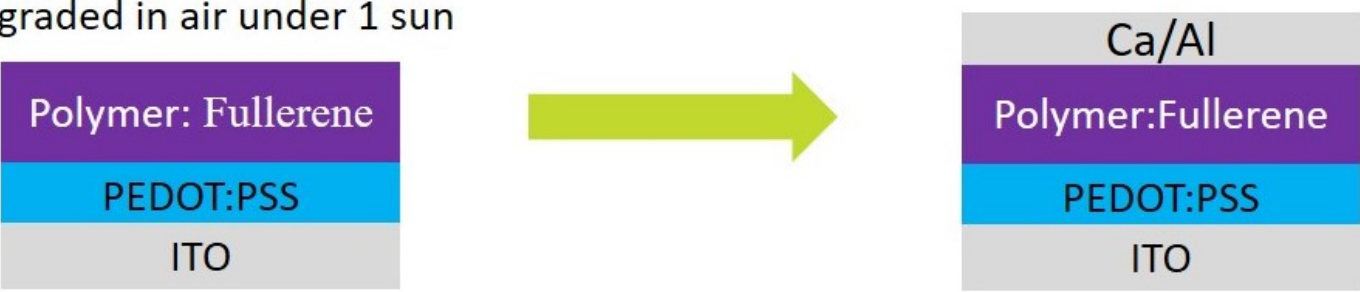

b)
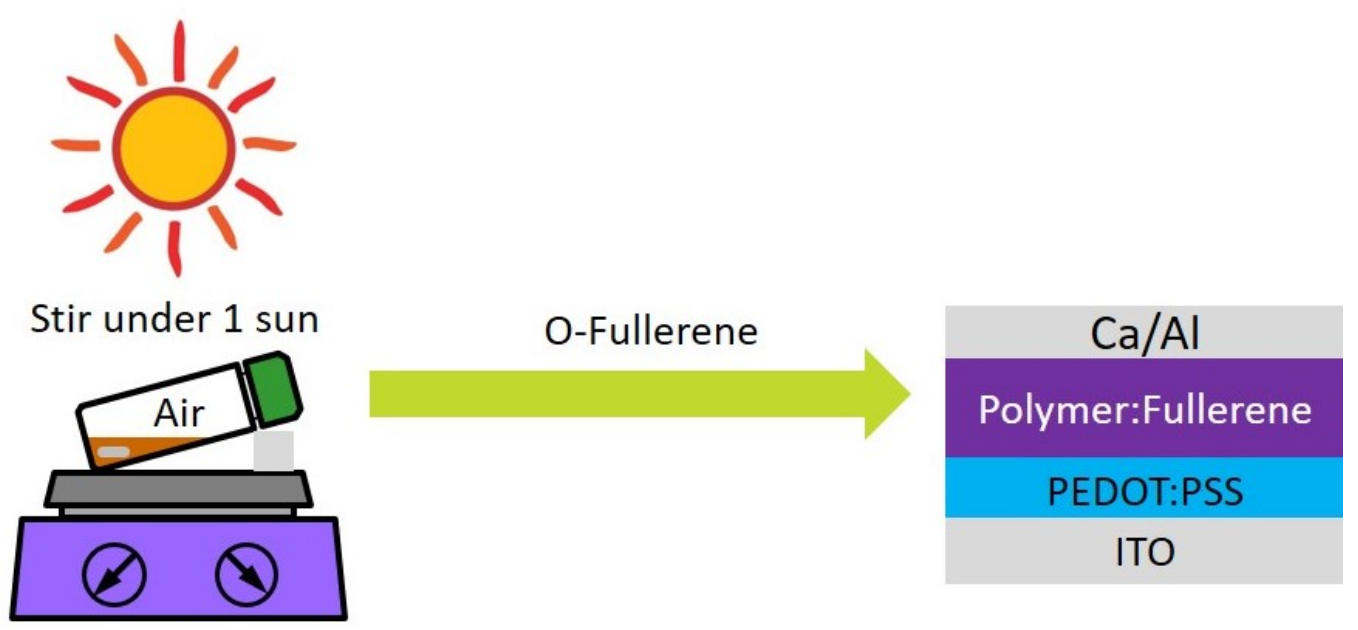

Figure 2.5: Methods for selective photodegradation of the active layer: (a) where the incomplete device is photoaged prior to electrode deposition, and b) where a constituent solution is photoaged and then used to make the active layer solution for device fabrication. The illustration shows the conventional structure and selective degradation of fullerene solution but the inverted structure and selectively degraded polymer solutions were also used.

For some stability studies, the photodegradation of the active layer, without the complications of oxygen diffusion and electrode degradation, were undertaken by photoaging the active layer prior to electrode deposition, using the following two methods. The first method (as shown in Figure 2.5a) was to fabricate the devices until having deposited the active layer film, then these incomplete devices were photoaged under 1 sun AM1.5G solar simulator radiation, before completing the devices as normal (deposition of top interlayer/electrode). This method exposes both the polymer and acceptor components of the active layer film to the same level of illumination so it is difficult to distinguish which material has photodegraded the most and which has the most effect to the device performance. To tackle this problem, a second approach (as shown in Figure 2.5b) was applied. Here, the degradation of the polymer and the acceptor can be further deconvoluted by exposing one component separately to 1 sun AM1.5G illumination as a solution in a clear vial filled with air. However, the drawback for this second method is that the photodegradation in solution of an individual component may not exactly reflect the 
photodegradation in blend films quantitatively.

\subsection{Optical characterisation}

\subsubsection{UV-Vis-NIR absorption spectroscopy}

UV-Vis-NIR Absorption Spectroscopy is a fundamental characterisation technique that measures the absorption of light by films or solutions for the UV, visible and near infra-red ranges (200 - $1000 \mathrm{~nm}$ ). Electronic/morphological properties such as polymer conjugation length, crystallinity and aggregation of small molecules can be deduced from the optical absorbance spectra. A blueshift in the absorption spectra indicates a loss of conjugation length of conjugated molecules.[1] The formation of a shoulder of a peak can indicate an increase in polymer crystallinity caused by improved packing of predominantly planar polymer chains.[2] A heightened absorption in the visible region indicates an increases in $\mathrm{PC}_{61} \mathrm{BM}$ aggregation due to the formation of charge transfer excited states bewteen fullerene molecules.[3]

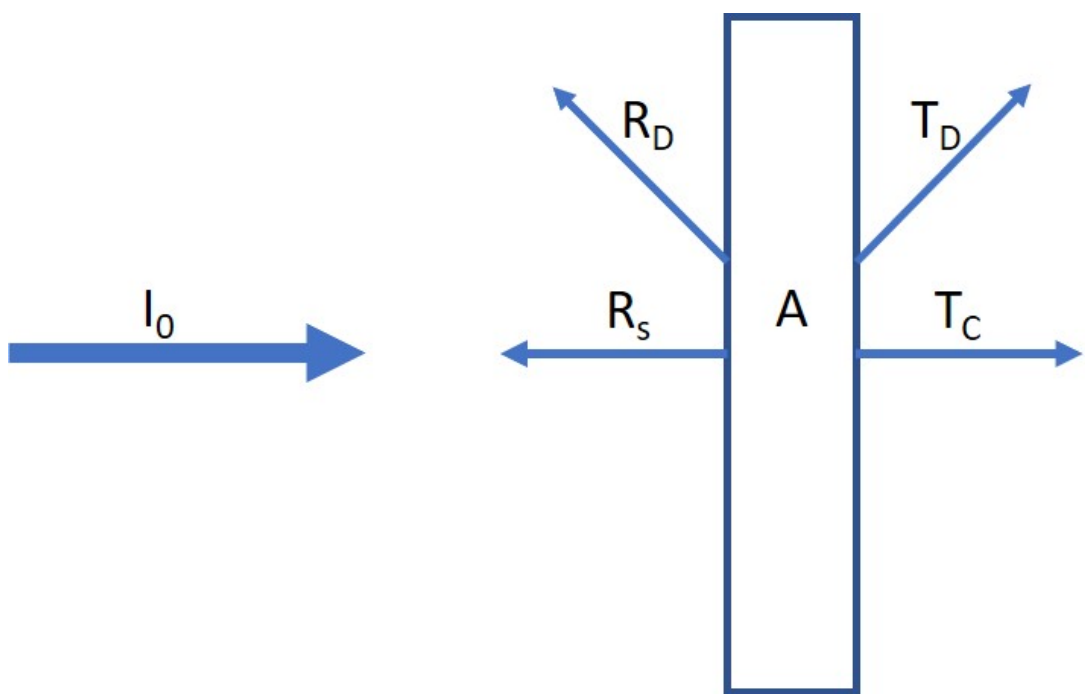

Figure 2.6: Schematic to show how an incident beam of light $\left(\mathrm{I}_{0}\right)$ is absorbed $(A)$ by a sample, reflected off the sample surface at the same $\left(R_{S}\right)$ or at a different angle $\left(R_{D}\right)$ or transmitted through the sample at the same $\left(\mathrm{T}_{\mathrm{C}}\right)$ or a different angle $\left(\mathrm{T}_{\mathrm{D}}\right)$.

As illustrated by Figure 2.6, when an incident beam of light hits a sample $\left(\mathrm{I}_{0}\right)$, a number of processes can occur which can be described by Equation 2.2:

$$
I_{0}=A+R+T=A+R_{\mathrm{S}}+R_{\mathrm{D}}+T_{\mathrm{C}}+T_{\mathrm{D}}
$$


1. It can be absorbed by the sample, where the amount of light absorbed is referred to as absorption $(\mathrm{A})$.

2. It can be reflected at the air/sample interfaces, where the amount of light reflected is known as reflection $(\mathrm{R})$. If this reflection is in the same direction as the incident beam, the reflection is mirror-like and known as specular reflection $\left(R_{S}\right)$. The beam can also be reflected in different angles to the incident beam, it is known as diffuse reflection $\left(R_{D}\right)$ and is caused by scattering from the surface roughness of the sample.

3. It can pass through the sample, known as transmission (T). Again, this can occur where the transmitted beam is at the same angle as the incident beam, known as collimated transmission $\left(\mathrm{T}_{\mathrm{C}}\right)$, or transmitted diffusely $\left(\mathrm{T}_{\mathrm{D}}\right)$.

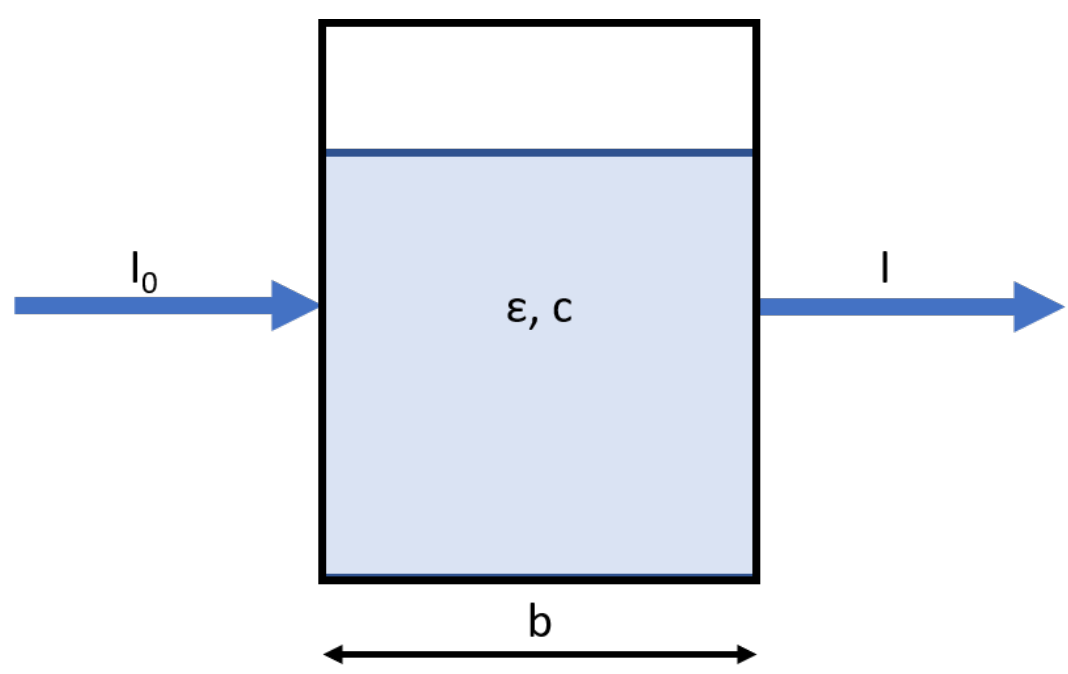

Figure 2.7: Illustration of the Beer-Lambert Law.

Figure 2.7 illustrates the Beer-Lambert law. This describes the linear correlation between absorbance (A) and the concentration (c) of absorbing species in a sample solution by the following equation:

$$
A=\varepsilon \cdot b \cdot c
$$

where $\varepsilon$ is the wavelength-dependent molar absorptivity coefficient $\left(\mathrm{M}^{-1} \mathrm{~cm}^{-1}\right)$ and $\mathrm{b}$ is the pathlength of light through the solution. Experimental measurements are usually taken in terms of transmittance:

$$
T=\frac{I}{I_{0}}
$$

and the relation between absorbance and transmission is

$$
A=-\log _{10} T
$$


The Beer Lambert law assumes that there is no scattering from the sample and the same law can be applied when measuring films when the reflectance is negligible.
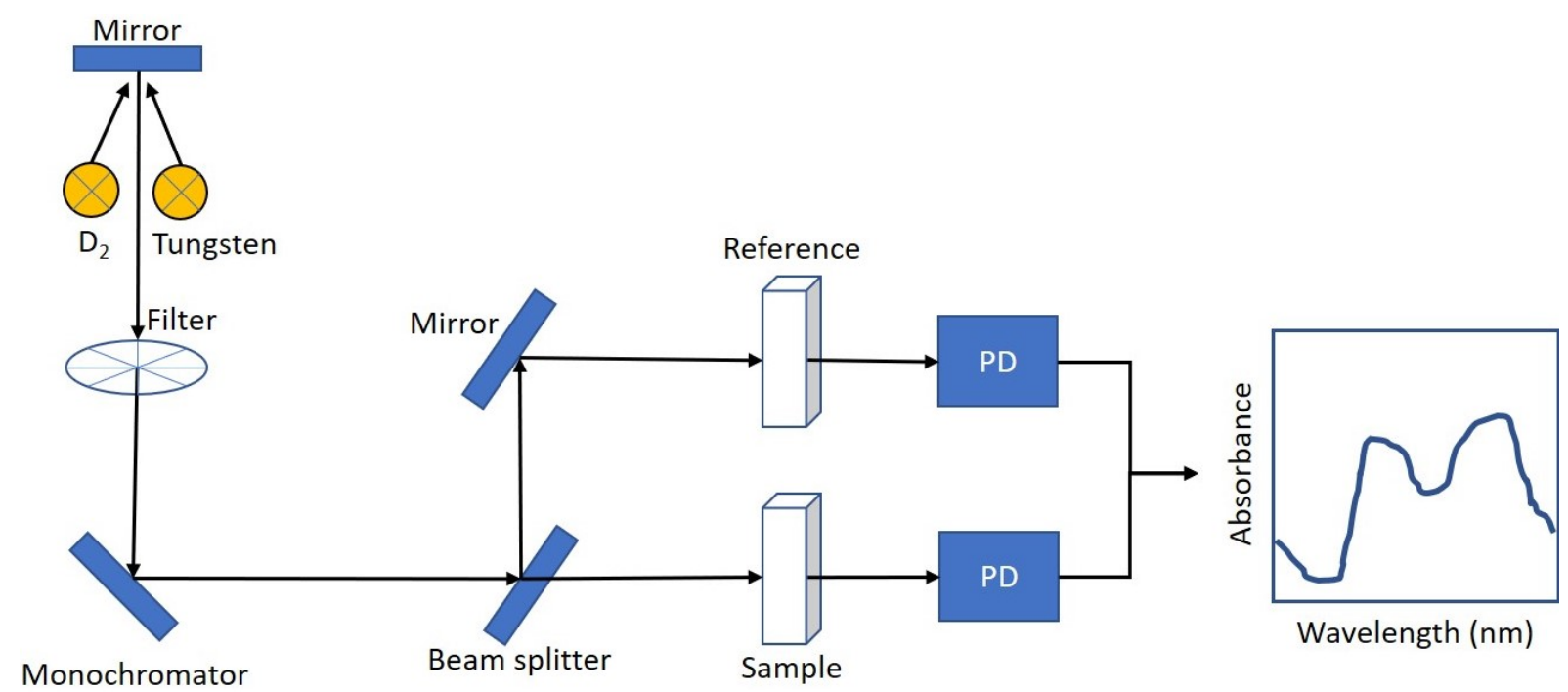

Figure 2.8: Schematic of a UV-vis spectrometer composed of deuterium $\left(\mathrm{D}_{2}\right)$ and tungsten light sources, filter, monochromator, beam splitter, reference, sample, and photodetectors $(\mathrm{PD})$.

Figure 2.8 shows the layout of a UV-vis spectrometer. A deuterium lamp $\left(\mathrm{D}_{2}\right)$ is used as the light source for the UV range and for $\geq 319 \mathrm{~nm}$, a tungsten lamp is used. The light is filtered and passed through a monochromator, where the wavelength of light is selected and changed with user-defined intervals. The light is then split by a beam splitter and with one beam passing through the sample, and one through a reference sample (so that the absorbance of the substrate can be subtracted). High sensitivity R928 photomultiplier and $\mathrm{PbS}$ detectors (range 190 - $3300 \mathrm{~nm}$ ) measure the light from both beams, and this is then analysed to produce an absorbance against wavelength spectrum.

In this thesis the absorbance spectra were measured using either a Perkin Elmer Lambda 750 (Swansea University) or UV-2600 Shimadzu UV-vis spectrophotometer with integrating sphere attachment (Imperial College London) when reflectance measurements were required.

\subsubsection{Microsecond transient absorption spectroscopy}

Transient absorption spectroscopy (TAS) is used to probe species formed after photoexcitation and their absorption dynamics. It is a pump-probe technique, where photoexcited states are generated by a laser pulse, then their absorbance is probed with a white light probe. The absorption by photoinduced species induces a change in optical density 
$(\triangle O D)$, a unit of absorption, given by:

$$
O D(\lambda, t)=\log _{10}\left(\frac{I_{i}(\lambda)}{I(\lambda, t)}\right)
$$

where $I_{i}(\lambda)$ is the intensity of the incident beam from the probe light, $I(\lambda, t)$ is the intensity of the light after passing through the sample, measured by a photodetector at a time $t .[4]$

After laser excitation, the optical density of the sample can change due to a change $\triangle O D(\lambda, t)$ (or the transient absorbance), induced by the absorption of the photoinduced species. Therefore the resultant $O D$ is [5]:

$$
O D(\lambda, t)=O D_{0}\left(\lambda, t_{0}\right)+\Delta O D(\lambda, t)
$$

where $O D_{0}\left(\lambda, t_{0}\right)$ is the initial optical density obtained by measuring the intensity of the probe light after passing through the sample $I(\lambda, t)$ without any laser excitation, and $\triangle O D(\lambda, t)$ is measured by measuring this beam after the sample has been pumped by the laser.

The laser pump wavelength for TAS studies is generally chosen to be around the absorbance maxima of the ground state of the films. Transient absorption spectra $(\triangle O D$ vs $\lambda$ ) were measured by recording the absorption of the sample, after an amount of time after the photo-excitation (set by changing the time difference between the laser and probe pulses), as a function of probe wavelength. From the transient absorption spectra, the photoinduced absorption maxima can be found. The transient decay kinetics $(\triangle O D$ vs time) are probed at the photoinduced absorption maxima over several $\mu \mathrm{s}$, in order to identify the photoexcited species.

On the $\mu$ s timescale, the decay (mono/biexponential) of long-lived triplet excitons $[6,7]$ and the decay of polarons (power law decay) (ns - ms) [6, 8, 9] can be observed. Singlets have a shorter lifetime (ns) than triplets $(\mu \mathrm{s})$ so are unobservable on this timescale. The majority of TAS measurements were taken under an inert atmosphere by continuously purging the sample cuvette with dry nitrogen, unless stated otherwise. Measurements were sometimes taken under an oxygen atmosphere instead, to investigate how the photoexcited species present interact with oxygen, as these can be rapidly quenched by molecular oxygen. 


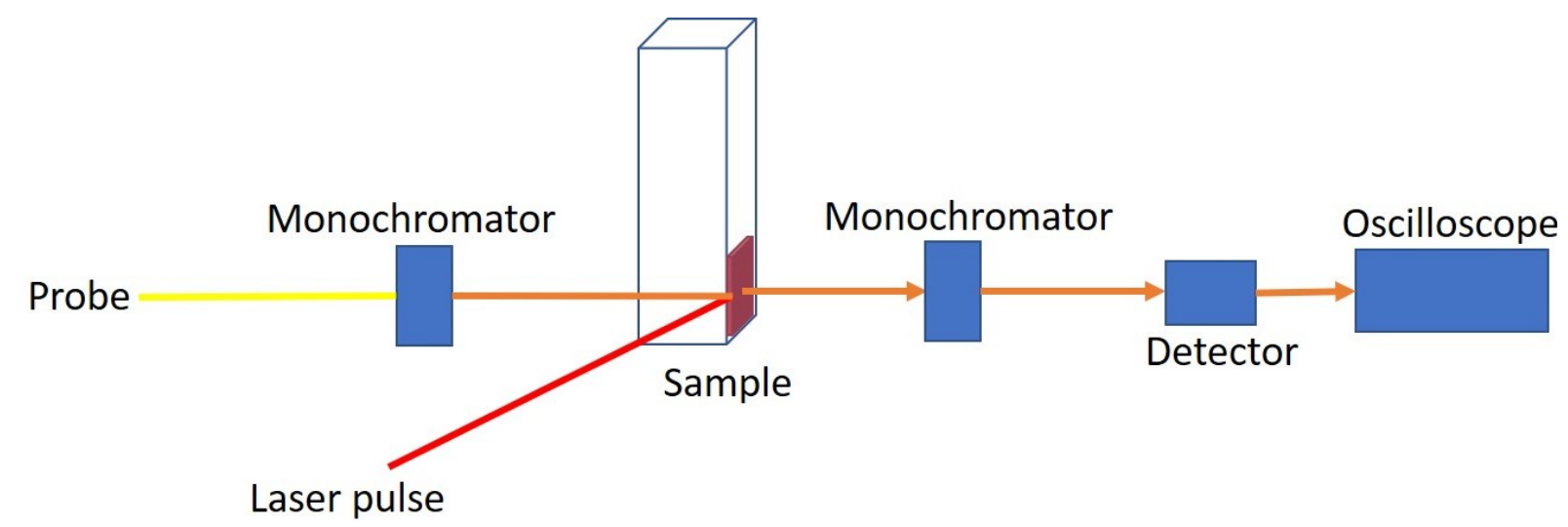

Figure 2.9: Schematic of a microsecond TAS set-up.

The TAS system used for the measurements in this thesis (Figure 2.9) can monitor decay kinetics on a timescale of $\sim 100 \mathrm{~ns}$ to ms range. The sample was excited with a laser pulse from a tunable Nd:Yag laser with an optical parametric oscillator (Oppolette 355). Each laser pulse has a duration of $20 \mathrm{~ns}$ and these were pulsed with a repetition rate of $20 \mathrm{~Hz}$. The intensity of the pump pulse could be measured with an energy meter, and altered using neutral density filters. The pump pulse was focussed with a light guide onto the sample which was inside a quartz cuvette purged with either dry nitrogen or oxygen. A $100 \mathrm{~W}$ quartz halogen lamp (Bentham, IL 1) (350 nm - $2.5 \mathrm{~mm}$ ) with a stabilised power supply (Bentham, 605) provided the probe light. This light beam was passed through a monochromator, before the sample, so that the probe wavelength could be selected and then through a second monochromator after the sample to minimise any emission or laser scatter from reaching the $\mathrm{Si}$ and InGaAs photodiode detectors. The detected signals were then preamplified, filtered (Costronics Electronics), recorded by a digital oscilloscope (Tektronics, TDS220) and the data was transferred to the computer.

It is possible to ascertain the reactivity of the photoinduced species with molecular oxygen by performing measurements under both nitrogen $\left(\mathrm{N}_{2}\right)$ and oxygen $\left(\mathrm{O}_{2}\right)$ environments. The obtained spectra were normalised for photons absorbed. The relative fraction of excited states quenched by oxygen $\left(\Phi_{\mathrm{Q}}\right)$ was calculated by:

$$
\Phi_{\mathrm{Q}}=Y_{\mathrm{N}_{2}} \Phi_{\mathrm{O}_{2}}
$$

where $Y_{\mathrm{N}_{2}}$ is the relative triplet yield in a nitrogen environment (determined from the $\mathrm{T}_{1} \rightarrow \mathrm{T}_{\mathrm{n}}$ absorption amplitude) and $\Phi_{\mathrm{O}_{2}}$ is the efficiency of oxygen quenching given by

$$
\Phi_{\mathrm{O}_{2}}=\left(1-\frac{\tau_{\mathrm{O}_{2}}}{\tau_{\mathrm{N}_{2}}}\right)
$$

Where $\tau_{\mathrm{O}_{2}}$ and $\tau_{\mathrm{N}_{2}}$ are the lifetimes of the triplets under nitrogen and oxygen environments, respectively. Lifetimes were extracted from the $\mathrm{T}_{1} \rightarrow \mathrm{T}_{\mathrm{n}}$ absorption fit. 
The rate constant of oxygen quenching of the triplets $\left(k_{\mathrm{O}_{2}}\right)$ was calculated by:

$$
k_{\mathrm{O}_{2}}=\frac{1}{\tau_{\mathrm{O}_{2}}}-\frac{1}{\tau_{\mathrm{N}_{2}}}
$$

\subsubsection{Photoluminescence spectroscopy}

Luminescence is the emission of a photon that occurs with the transition of an electron from an excited electronic state to a lower unoccupied electronic state. When the excitation of an electron is caused by the absorption of a photon, the luminescence is called photoluminescence (PL). PL spectroscopy, also referred to as fluorescence spectroscopy, involves using a beam of light to excite electrons in molecules, causing them to emit light via fluorescence and/or phosphorescence.

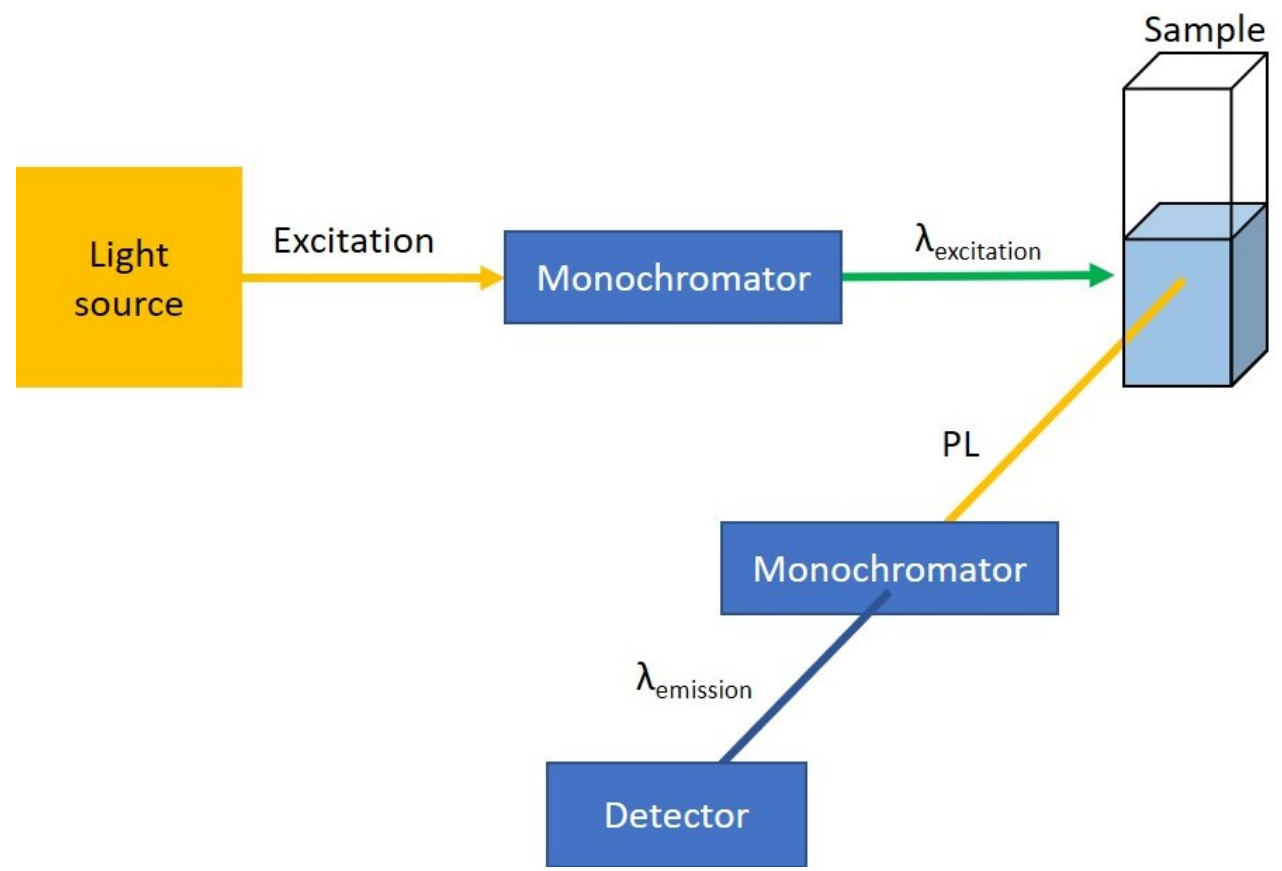

Figure 2.10: Schematic of a photoluminescence (PL) concept. Light from an excitation light source is monochromated to select a single wavelength $\left(\lambda_{\text {excitation }}\right)$ to excite a sample. The PL fro the sample is monochromated to probe the intensity from a specific wavelength of emission $\left(\lambda_{\text {emission }}\right)$ by the detector. The light intensity versus $\lambda_{\text {emission }}$ (i.e. a PL spectrum) is obtained by scanning a grating in the monochromator.

The working principle of obtaining a PL emission spectrum is illustrated in Figure 2.10. An excitation light passes through a monochromator to select a particular excitation wavelength, which the sample absorbs (to excite the sample), and the PL from the sample passes through another monochromator to be probed by a photodetector. By scanning the grating inside the emission monochromator, the intensity of PL versus wavelength (i.e. PL spectrum) can be obtained.[10] 
PL spectra shown in this thesis were measured by Andrew Telford at Imperial College London, using a spectrograph (Andor Shamrock 303) combined with an InGaAs photodiode array (Andor iDUS 491) cooled to $-90^{\circ} \mathrm{C}$, calibrated with a Bentham CL2 quartz halogen lamp with known emission spectrum. The data analysis was carried out by myself.

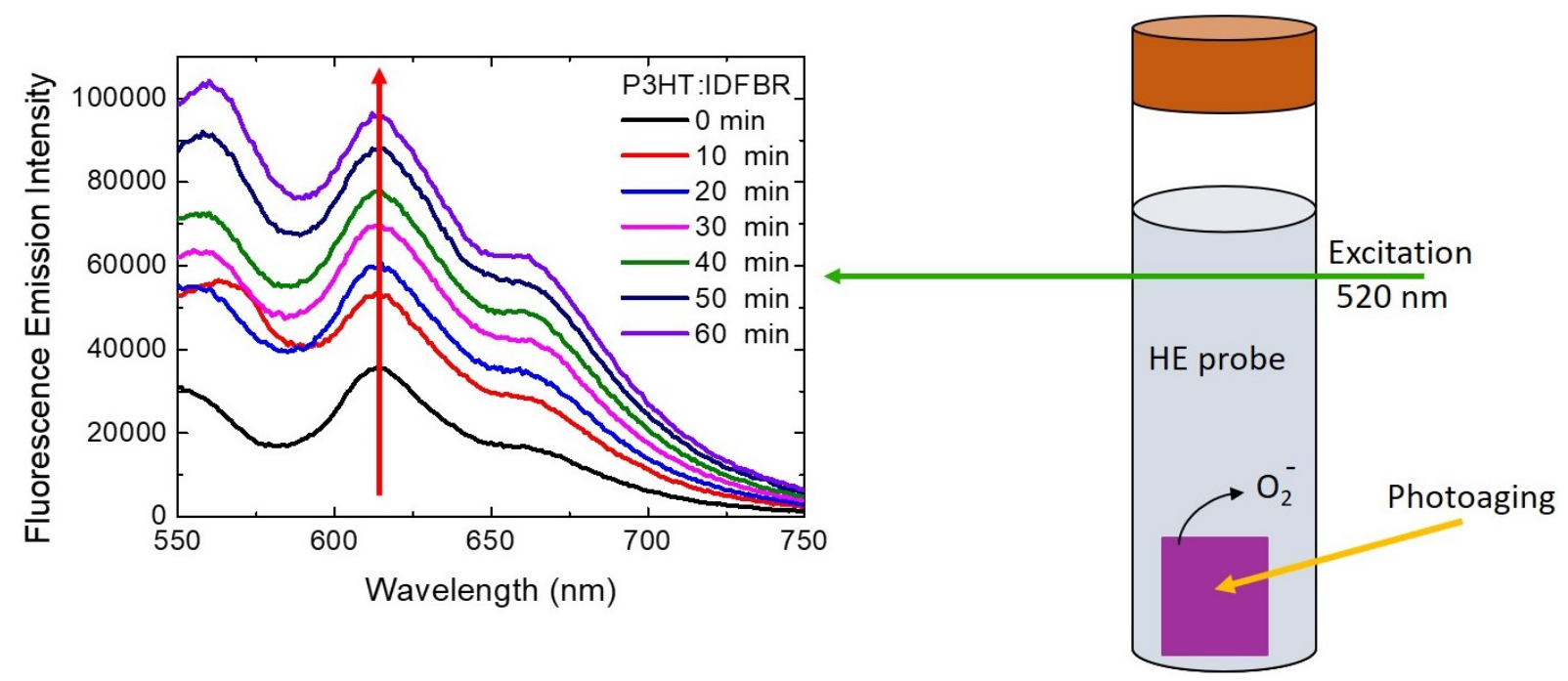

Figure 2.11: Schematic of superoxide fluorescence probe sensitisation set-up.

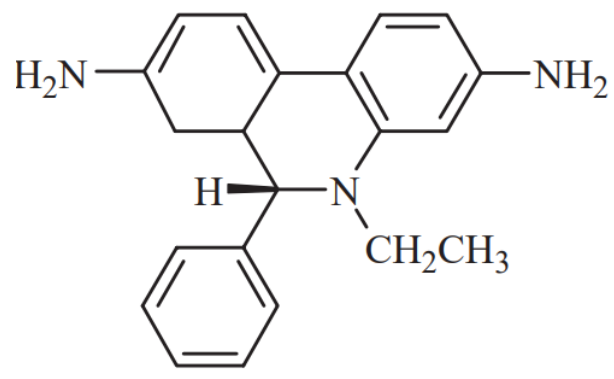

HE

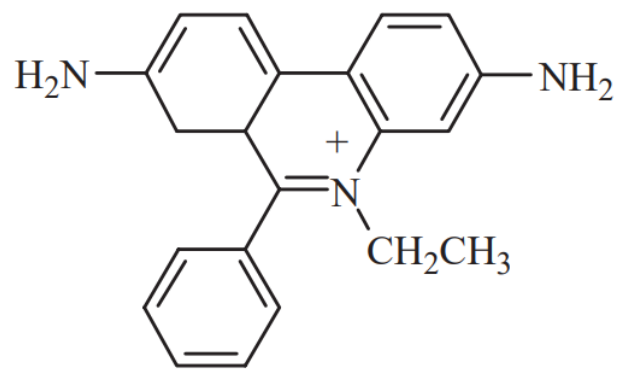

$\mathrm{E}^{+}$

$$
\begin{aligned}
& \lambda_{\text {excitation }}=520 \mathrm{~nm} \\
& \lambda_{\text {emission }}=610 \mathrm{~nm}
\end{aligned}
$$

Figure 2.12: Chemical structures of hydroethidine (HE) and ethidium $\left(\mathrm{E}^{+}\right) .[11]$

PL spectroscopy can also be used to probe the generation of reactive chemical species (such as superoxide anions $[12,13]$ or singlet oxygen [14] which can degrade the OPV films) by thin film samples by immersing them in a probe solution that fluoresces in the presence of the chemical species under investigation. Figure 2.11 shows a thin film submerged in the fluorescent molecular probe hydroethidine (HE). HE oxidises when exposed to the superoxide radical anion to form ethidium $\left(\mathrm{E}^{+}\right)$(Figure 2.12), a fluorescent compound $\left(\lambda_{\text {excitation }}=520 \mathrm{~nm} ; \lambda_{\text {emission }}=610 \mathrm{~nm}\right)$ (Figure 2.11).[11] The more superoxides generated by the film with photoaging, the more the $\mathrm{HE}$ will be oxidised to $\mathrm{E}^{+}$and the higher the intensity of the emission peak at $620 \mathrm{~nm}$ of the PL spectrum.

Sensitisation tests to detect the generation of superoxide anions were carried out with 
Nicholas Aristidou at Imperial College London, using a Horiba Jobin-Yvon Fluorolog-3 spectrofluorometer and a HE probe solution. Data analysis was carried out myself. Films were sealed in a controlled environment, where dry air was gassed through for the duration of the degradation and illumination was provided by a tungsten lamp equipped with a UV-blocking filter (to prevent degradation of the HE probe).

\subsection{Chemical/structural characterisation}

\subsubsection{Mass spectrometry}

Mass spectrometry is an analytical technique that can quantify the mass of chemical species and their relative amounts within a sample. From the measured mass, the chemical species can be deduced. The sample is bombarded by an ionisation source to form gaseous ions. These are accelerated by electric and/or magnetic fields in a vacuum system. The ions are separated according to their mass-to-charge ratio in a mass analyser following the Lorentz force law (Equation 2.11) and Newton's second law of motion (Equation 2.12):

$$
\vec{F}=q(\vec{E}+\vec{v} \times \vec{B})
$$

where $\vec{F}=$ the force applied to the ion, $q=$ charge of the ion, $\vec{E}=$ applied electric field and $\vec{v} \times \vec{B}=$ vector cross product of the ion velocity and the applied magnetic field.

$$
\vec{F}=m \vec{a}
$$

where $\vec{F}=$ the force applied to the ion, $m=$ the mass of the ion and $\vec{a}=$ the acceleration.

The lighter and/or more charged an ion is, the more acceleration it experiences when the sample force is applied to it whilst passing through the electric and/or magnetic fields in the mass analyser. The higher the mass-to-charge ratio of an ion, the more its trajectory is deflected, hence the ions are sorted and their abundance can be measured with a detector. 


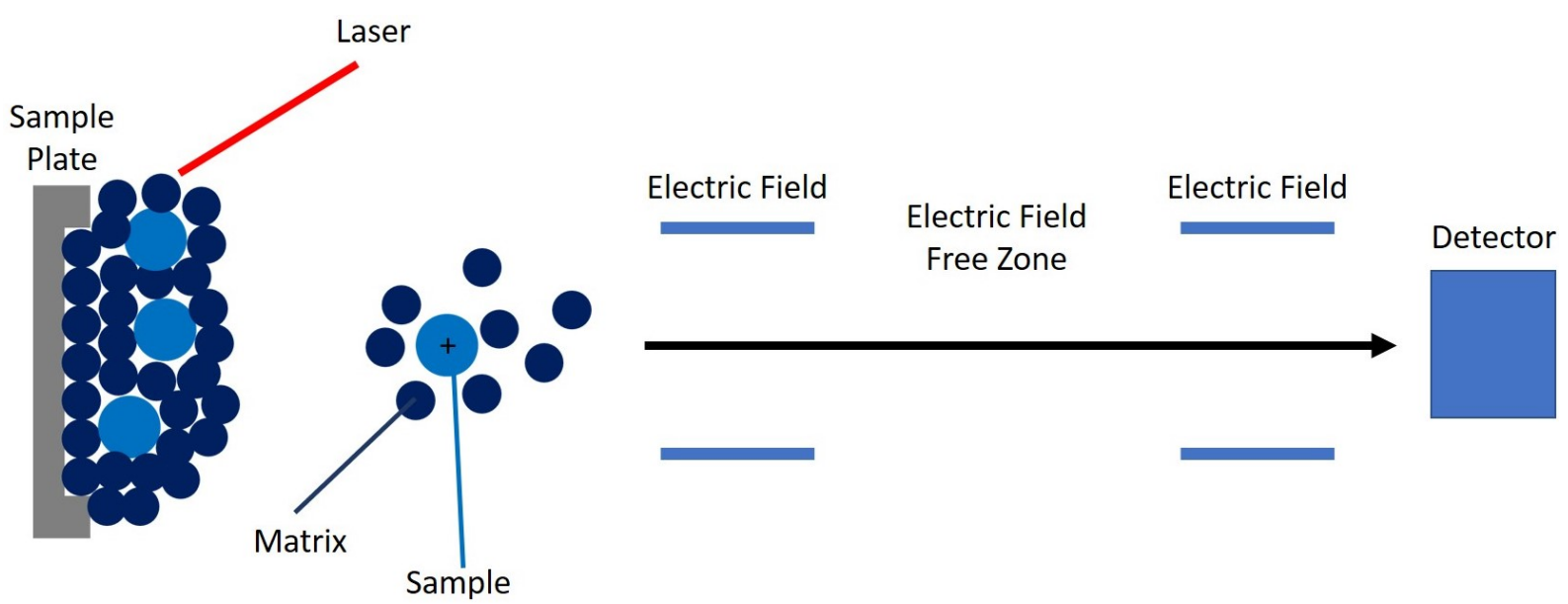

Figure 2.13: Schematic of a MALDI-TOF set-up.

Matrix-assisted laser desorption/ionisation (MALDI) is a method used to create the ions for mass spectrometry. This method involves dissolving the sample in a solvent which contains a solution with small organic molecules, called a matrix, which are a strong absorber of laser energy. This mixture (in the solvent) is then dried, leaving the molecules of the sample embedded throughout the matrix completely separated from one other, in a "solid solution". Next, under a vacuum, intense laser pulses ablate portions of the solid solution by exciting the matrix molecules. The hot plume created contains sample molecules which are thought to be ionised by proton transfer from the matrix.[15] MALDI can be coupled with a time of flight (TOF) analyser to make a mass spectrometer. Figure 2.13 shows the working principle of a MALDI-TOF mass spectrometer: a TOF analyser, separates ions, after their initial acceleration by the electric field, according to their velocities as they drift in the electric field free zone. The ions are accelerated towards the analyser by the potential difference between the charged sample plate and the first electric field (or extraction grid). As the ions all gain the same amount of kinetic energy, ions of different masses travel towards the detector with different velocities; the heavier the ion, the slower the velocity. The detector then records how many ions and the duration of their travel through the electric field free zone, from which the mass of the sample molecules and their abundance within the sample can be deduced.[15]

All mass spectrometry measurements were taken with MALDI-TOF by Dr Mark Wyatt at the EPSRC UK National Mass Spectrometry Facility. All samples were dissolved in chlorobenzene at an approximate concentration of $1 \mathrm{mg} / \mathrm{mL}$. MALDI matrix trans2-[3-(4-tert-butylphenyl)-2-methyl-2-propenylidene]malononitrile (DCTB) was purchased from Insight Biotechnology Ltd. (Wembley, U.K.) was used for fullerene acceptors, and anthracene (Sigma-Aldrich Company Ltd., Gillingham, UK) was used for non-fullerene acceptors. These were dissolved in chlorobenzene at $20 \mathrm{mg} / \mathrm{mL}$ and $10 \mathrm{mg} / \mathrm{mL}$ respectively. Solutions of sample and matrix were mixed together so the matrix is in $\sim 250$ 1000 fold molar excess, typically $1 \mu \mathrm{L}$ of sample to $49 \mu \mathrm{L}$ of matrix. $0.5 \mu \mathrm{L}$ of each of 
these mixture solutions for each sample was spotted onto the MALDI plate and dried in air. MALDI-TOF spectra were acquired in negative-reflectron mode using an ultrafleXtreme mass spectrometer (Bruker Daltonics, Bremen, Germany), which is equipped with a Smartbeam-II Nd:YAG laser $(\lambda=355 \mathrm{~nm})$. Data was acquired using flexControl software v3.4, while post-acquisition processing of data was performed by flexAnalysis software v3.4. PCBM and oxidised analogues were observed as negative radical ion species $\left(\mathrm{M}^{-} \cdot\right)$. Relative quantitative analyses were done myself via Microsoft Excel. The percentage of molecules oxidised $\left(m_{o}\right.$ can be calculated by:

$$
m_{o}=\frac{n_{o}}{n_{f}+n_{o}} x 100
$$

where $n_{o}$ is the number of oxidised molecules and $n_{f}$ is the number of remaining fresh molecules. The average number of additional oxygens $O$ on each oxidised molecule can be given by:

$$
O=\frac{o}{n_{o}}
$$

where $o$ is the total number of additional oxygen atoms.

\subsubsection{Fourier transform infrared spectroscopy}

Fourier Transform Infrared Spectroscopy (FTIR) is a chemical analysis technique that can be used to identify the vibrational modes of materials in order to identify molecular bonds and assist to identify chemicals. It can also be applied to determine the concentration/amount of the chemicals, and therefore make quantitative comparisons between samples. It is almost a universal technique, with many molecules absorbing in the midinfrared (IR) range $(2.5-25 \mu \mathrm{m})$ and gives information rich spectra with the peak positions giving information of the bonds in the molecules (each chemical bond between two atoms will vibrate at a different frequency so each bond will have a unique IR peak position), the peak intensity giving the relative abundance of these bonds and the peak widths being sensitive to the bond's surroundings including hydrogen bonding and pH.[16] 


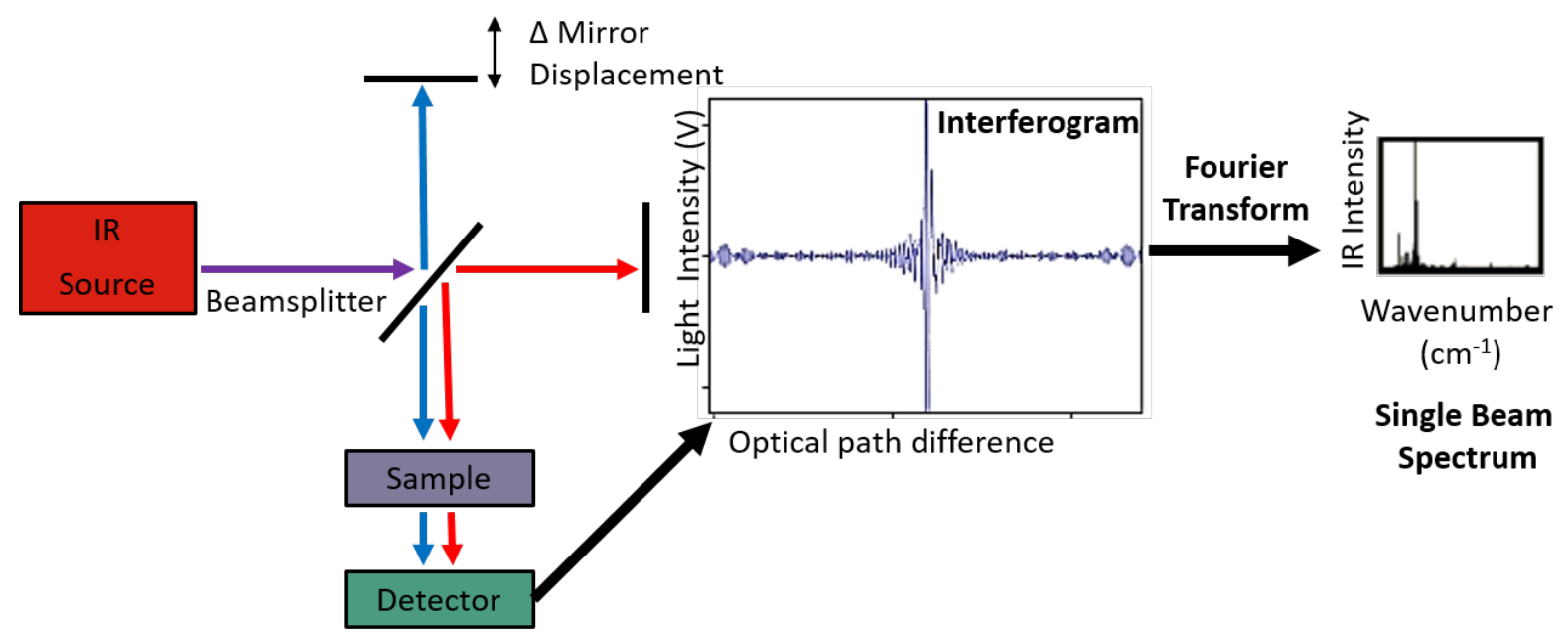

\section{Interferometer}

Figure 2.14: Schematic of a FTIR system.

Figure 2.14 illustrates the working principle of FTIR spectroscopy. Collimated IR radiation passes into an interferometer, where it is split into two beams by a beamsplitter. One of the beams hits a fixed mirror, the other beam hits a moving mirror. The reflected beams recombine at the beamsplitter, undergoing superposition, and leave the interferometer where it interacts with the sample and strikes a detector. The square of the amplitude of the measured beam is proportional to its intensity. As the displacement of the moving mirror changes, the optical path difference of the two beams varies, causing the interference pattern between the two beams to alter. The moving mirror is moved backwards and forwards once to form one scan to cover the whole range of wavenumbers. A plot of measured light intensity as a function of optical path difference and is called an interferogram. The interferograms obtained whilst scanning are Fourier transformed to yield a spectrum of IR intensity as a function of wavenumber (the inverse of wavelength), giving this technique its name.[16]

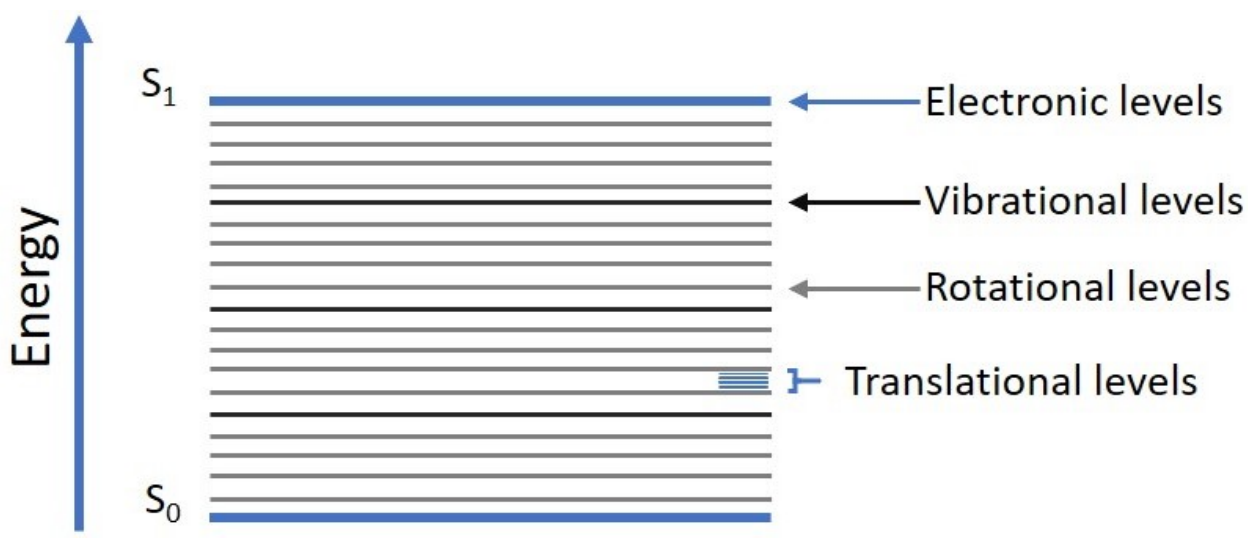

Figure 2.15: Energy level diagram which shows the vibrational energy levels, rotational energy levels and electronic energy levels of a material. 
The IR spectrum is formed as a consequence of the absorption of IR radiation by molecules at energies that correspond to the vibrational energies of specific sets of chemical bonds. The vibrational energy is just one component of the energy possessed by a molecule; the others being electronic, rotational and translational energies (Figure 2.15). The requirement for the absorption of IR radiation is that there must be a net change of the permanent dipole moment (product of the separation of the ends of the dipole and the magnitude of the charges) during the vibration. This requirement means that asymmetrical bonds (generally ionic) could present strongly in IR spectra.[17] Conversely, symmetrical bonds do not have a strong signal in the IR, so complementary methods, such as Raman spectroscopy, are needed to probe these.

The vibronic frequency can be modelled with Hooke's Law (equation 2.15). This links the frequency of the bond vibration with the strength of a covalent bond between two atoms and the mass of the atoms.

$$
\nu=\frac{1}{2 \pi c} \sqrt{\frac{\kappa}{\mu}}
$$

where $\nu=$ vibronic frequency, $\mathrm{c}=$ speed of light in a vacuum, $\kappa=$ force constant of the bond, and $\mu=$ reduced mass. The reduced mass $\mu$ of a chemical bond between atoms with masses $m_{1}$ and $m_{2}$ is given by:

$$
\mu=\frac{m_{1} m_{2}}{m_{1}+m_{2}}
$$

The number of vibrational degrees of freedom (or ways it can vibrate) possessed by a molecule depends on the number of atoms $N$ it contains:

$$
\text { No. vibrational modes }=3 N-6 \text { (for a non-linear molecule) }
$$

or

$$
\text { No. vibrational modes }=3 N-5(\text { for a linear molecule })
$$




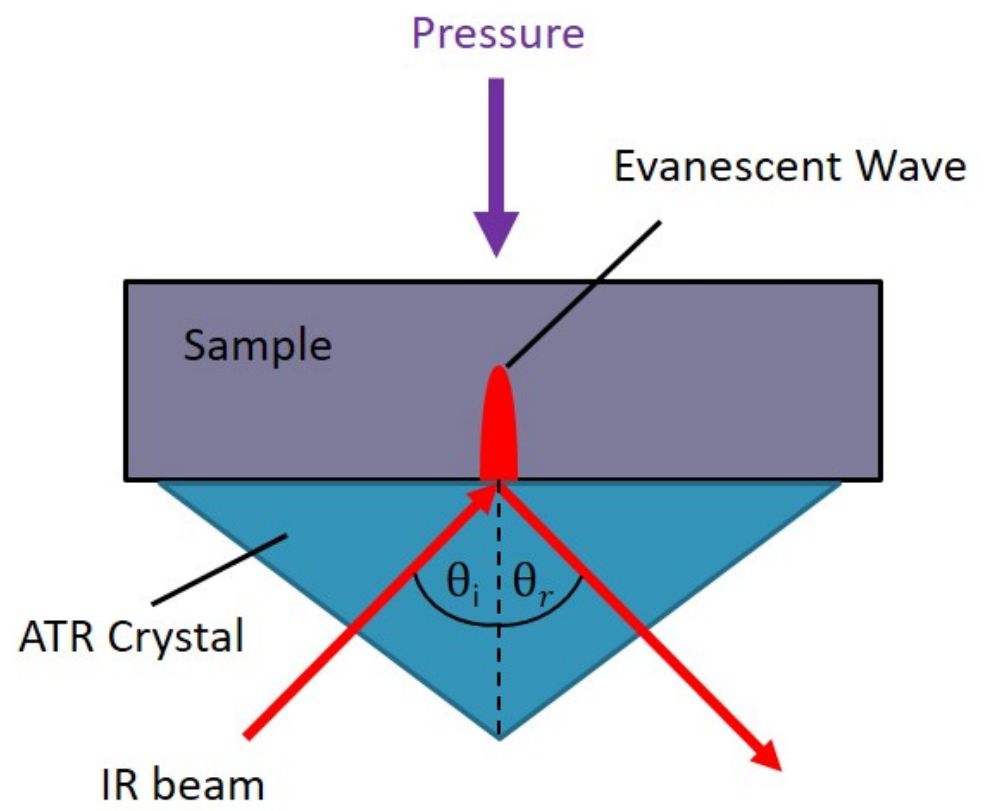

Figure 2.16: Schematic showing the working principle of an ATR system.

Attenuated total reflectance (ATR) is a commonly used FTIR measurement technique which enables solid or liquid samples to be measured without further preparation. A schematic of a single-pass ATR is shown in Figure 2.16, where an IR beam is passed through a crystal which has a sample placed above and a pressure is applied to ensure good contact between the sample and the crystal. An IR beam travels through the crystal of high refractive index, and encounters the interface between the crystal and a sample with a lower refractive index. If the angle of incidence of the IR beam is equal to or greater than a critical angle, then the beam undergoes total internal reflectance, where the whole beam is reflected back into the crystal. The critical angle is dependent upon the refractive indices of the crystal and sample, given by Snell's Law.

$$
\theta_{c}=\sin ^{-1}\left(\frac{n_{\mathrm{s}}}{n_{\mathrm{c}}}\right)
$$

where $\theta_{c}$ is the critical angle, $n_{\mathrm{s}}$ is the refractive index of the sample and $n_{\mathrm{c}}$ is the refractive index of the crystal.

At the point of internal reflectance, the incoming and outcoming IR beams occupy the same space so can undergo constructive interference, causing an evanescent wave of enhanced IR light to protrude above the crystal and into the sample. An evanescent wave is an oscillating electric and/or magnetic field that does not propagate as a wave, but whose energy is concentrated close to its source. There is no net flow of energy in this region over a complete oscillation cycle. Some of the IR beam is absorbed by the sample, and the reflected beam is then focussed onto the detector. Due to the absorbance of 
the sample which attenuates the intensity of the totally reflected IR beam, the technique gains its name.[16] ATR-FTIR is able to produce absorbance spectra with a better signal than FTIR transmission as more of the IR beam passes through more of the sample.

A Perkin Elmer Frontier FTIR with ATR accessory was used to obtain the FTIR spectra shown in this thesis. The ATR accessory had a germanium crystal with a diameter of 1.3 $\mathrm{mm}$ and refractive index of 4.0. It uses two deuterated triglycine sulfate detectors; one optimised for the mid-infrared $(2.5-25 \mu \mathrm{m})$ and one for the far-infrared $(25-1000 \mu \mathrm{m})$ regions. Each measurement consisted $1 \mathrm{~cm}^{-1}$ spectral resolution, 4 scan accumulation and baseline corrected in the Spectrum 10 software. Three spectra were taken in different sample locations and averaged to obtain FTIR spectra shown.

\subsubsection{X-ray photoelectron spectroscopy}

X-ray Photoelectron Spectroscopy (XPS) is the most commonly used electron spectroscopies for defining the elemental composition of a solid's surface ( first $10 \mathrm{~nm})$. It is able to identify and quantify any element between lithium to uranium to an accuracy of 0.1 atomic wt\% with minimal sample preparation.[18]

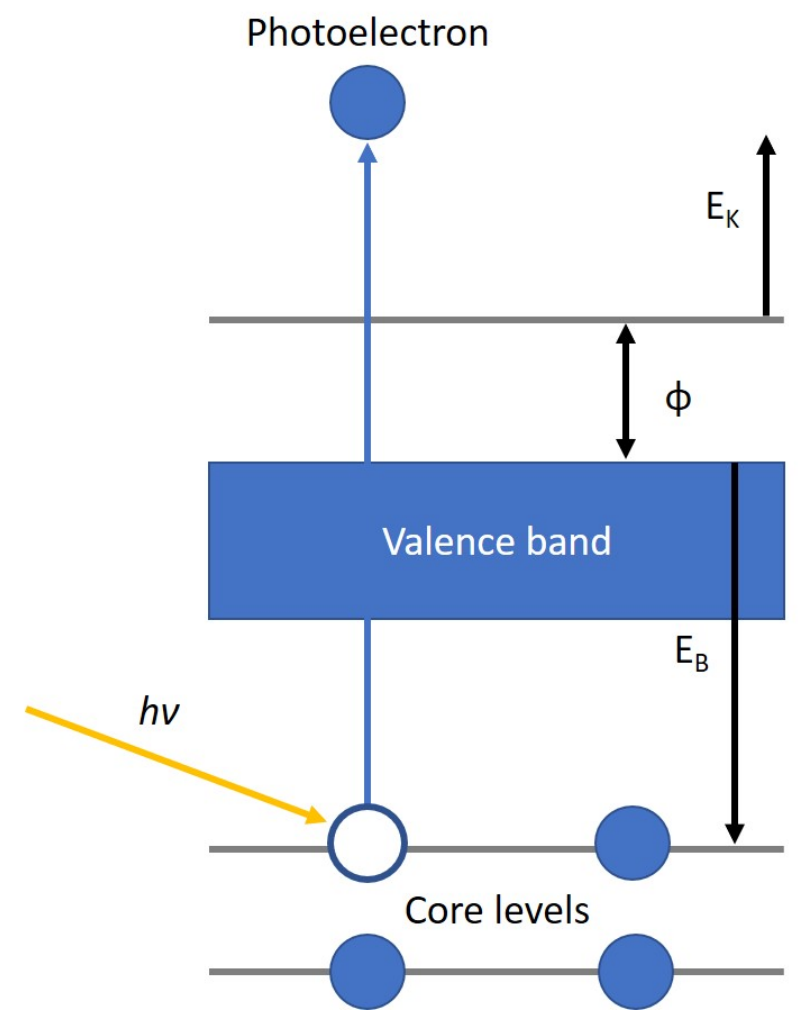

Figure 2.17: Schematic showing the photo-excitation of a core electron by a photon with energy $h \nu$. This photon energy is equal to the electron binding energy $\left(\mathrm{E}_{\mathrm{B}}\right)$ plus the work function $(\phi)$ of the spectrometer plus electron kinetic energy $\left(\mathrm{E}_{\mathrm{K}}\right)$. 
The working principle of XPS is illustrated in Figure 2.17. XPS obtains information by directing an energetic photon beam of x-rays at a sample, transferring energy to core level (non-valence) electrons. If the energy transferred is sufficient, the electrons will be emitted from the atom/ion of the solid sample with a kinetic energy $\left(\mathrm{E}_{\mathrm{K}}\right)$ that can then be measured which is dependent on the electron binding energy $\left(E_{B}\right)$, which is specific to chemical element and environment (i.e. the other atoms to which it is bound).[18]

$$
E_{\mathrm{k}}=h \nu-E_{\mathrm{B}}-\phi
$$

where $h$ is Planck's constant and $\nu$ is the photon frequency, together giving the energy of the photon. $\phi$ is the work function of the spectrometer.

Since the energy of incident x-ray photons and the work function of the spectrometer are known, measuring the kinetic energy of the photoelectrons allows the binding energy of the electrons to be calculated (illustrated in Figure 2.17).[19]

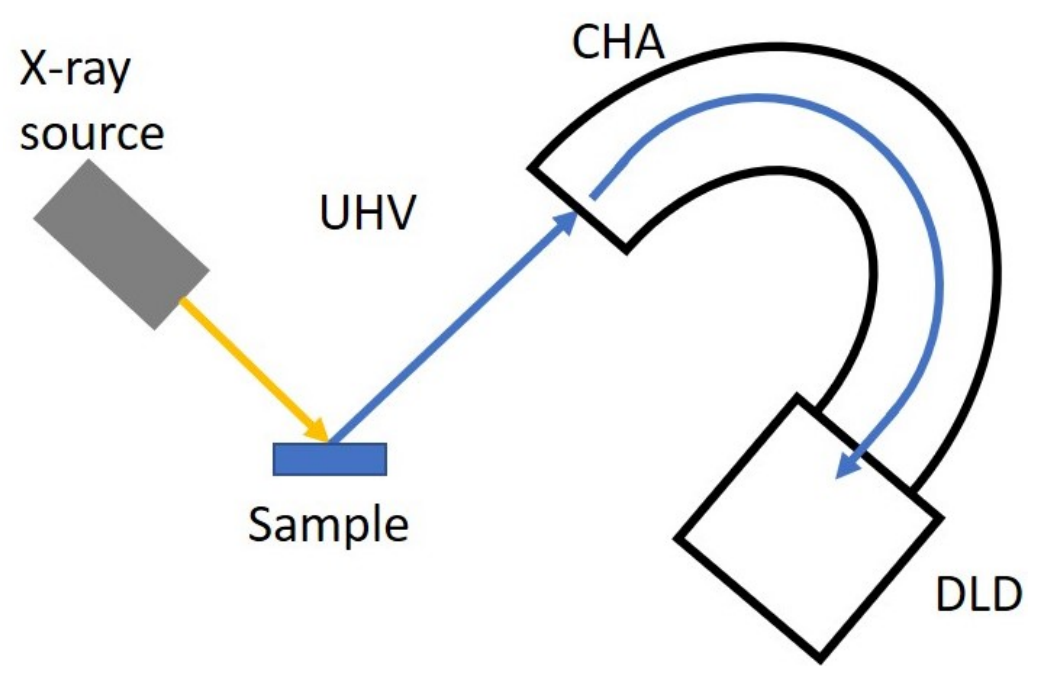

Figure 2.18: Schematic showing an example layout of an XPS system. X-ray photons (yellow arrow) from the x-ray source hit the sample and excite electrons in the sample which are emitted (blue arrows) and pass through the concentric hemispherical analyser (CHA), before being detected by a delay line detector (DLD).

The composition of an XPS system is illustrated by Figure 2.18. An x-ray source fires high energy photons at the sample, from which electrons are excited and emitted, according to the Photoelectric Effect. The electrons pass into a concentric hemispherical analyser (CHA) which retards the electrons by applying a potential in order to improve the resolution, before being detected by a delay line detector (DLD). Measurements are undertaken in an ultrahigh vacuum $\left(\sim 1 \times 10^{-7}-1 \times 10^{-10}\right.$ Torr $)$ to reduce the adsorption of gaseous molecules to the sample (in addition to allowing the emitted electrons to pass to the detector without being blocked by gaseous molecules).

XPS can be coupled with an ion beam to etch layers of the surface to obtain subsurface 
information. This combination of XPS analysis and etch cycles provides elemental composition information at known depths (confirmed with a Dektak 150 stylus profilometer) through a sample. The ion beam can be tuned, through trial and error, such that the sample experiences minimal damage, whilst the quality of the spectra are still good (in terms of signal-to-noise ratio and spectral resolution).

The XPS data shown in this thesis was acquired by Dr. James McGettrick (Swansea University) using a Kratos Axis Supra XPS with a monochromated Al Ka X-ray source (15 mA emission current, total power of $225 \mathrm{~W}$, giving $\sim 300 \times 700 \mu \mathrm{m}$ analysis area). Measurements were undertaken in an ultrahigh vacuum $\left(\sim 5 \times 10^{-9}\right.$ Torr $)$. Etching for depth profiles were performed using an argon gas cluster ion source gun (Kratos Minibeam 6 Gas Cluster Ion Source). Data analysis and interpretation was done myself.

\subsubsection{Grazing incidence wide-angle x-ray scattering}

Grazing incidence wide-angle x-ray scattering (GIWAXS) is an x-ray scattering surface technique used to probe molecular length scales. In this technique, an x-ray beam is directed to a thin film and the diffuse reflections are collected to form an x-ray scattering pattern. Grazing incidence means that the x-ray beam impinges the sample surface at a very shallow angle $\left(<1^{\circ}\right)$. And the wide angle means the sample-to-detector distance is small $(10-50 \mathrm{~cm})$ such that the reflected x-rays measured are making a wide angle with the sample surface.[20] The geometries of GIWAXS means that the x-ray passes through more of a thin film sample than more standard x-ray diffraction methods, meaning that it is more sensitive to smaller length-scale features (molecular).

GIWAXS measurements were performed with Dr. Andrew Parnell on a Xeuss 2.0 SAXS/WAXS laboratory beamline using a liquid Gallium MetalJet (Excillum) X-ray source $(9.2 \mathrm{keV}, 1.34 \AA$ ) , with scattered x-rays detected using a Pilatus3R $1 \mathrm{M}$ detector. The data was analysed by myself. The Scherrer equation was employed to analyse the spectra.

$$
\tau=\frac{K \lambda}{\beta \cos (\theta)}
$$

Where $\tau$ the mean size of ordered (crystalline) domains, $K$ is a dimensionless shape factor $\sim 0.93, \lambda$ is the x-ray wavelength, $\beta$ is the full width half maximum (FWHM)(in radian) of the GIWAXS peak, and $\theta$ the peak angle (in radian). Here the FWHM and peak angle are extracted from the GIWAXS spectra. The bigger the mean size ordered domain, the more crystalline the material. 
Another parameter that can be extracted from the peak positions on GIWAXS spectra is the d-spacing which is the spacing between adjacent lattice planes. The smaller the d-spacing, the closer together the planes, and the more crystalline the material.

\subsubsection{Atomic Force Microscopy}

Atomic Force Microscopy (AFM) is a technique that probes the surface morphology of a thin film with nm resolution. There is a non-contact "tapping" mode of AFM that can be utilised to measure soft materials such as organic films - so not to damage them. Whilst scanning in tapping mode, the tip is oscillated at a frequency near its resonant frequency at an amplitude smaller than the oscillation amplitude, such that the tip strikes the sample surface once each oscillation.[21] Intermolecular forces between the tip and the sample are highly dependent upon the distance between them, and as the height of the sample fluctuates, the amplitude and resonant frequency of the tip vibrations are affected. The tip is connected to a micro-cantilever which directs a laser to a photodiode. When the amplitude of the cantilever is deflected away from its equilibrium position, this is detected by the photodiode and converts it to an electrical signal whose intensity is proportional to the cantilever displacement, and an image can be produced. The AFM measurements were taken by Joel Luke at Imperial College London using a Park NX10 AFM system and SmartScan ${ }^{\top M}$ software with Park silicon PPP-NCHR tips. The image was analysed with the XEI software package. Data interpretation was undertaken by myself.

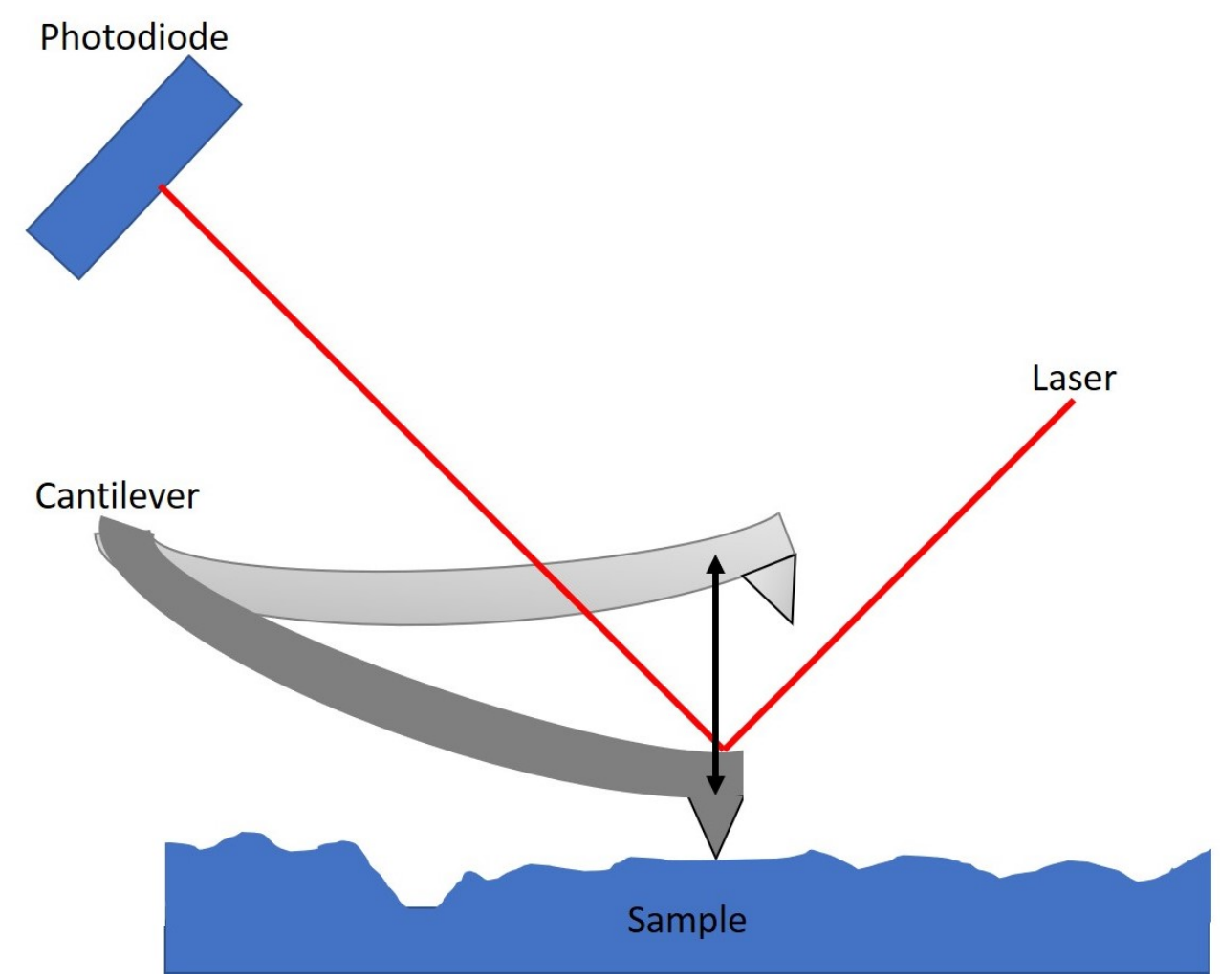

Figure 2.19: Illustration of an AFM set-up. 


\section{5 (Opto-)electrical characterisation}

\subsubsection{Cyclic voltammetry}

Cyclic voltammetry $(\mathrm{CV})$ is a popular technique to investigate the reduction and oxidation processes of molecular species. The potentials measured from a voltammogram can estimate the LUMO and HOMO levels of an organic semiconducting material.

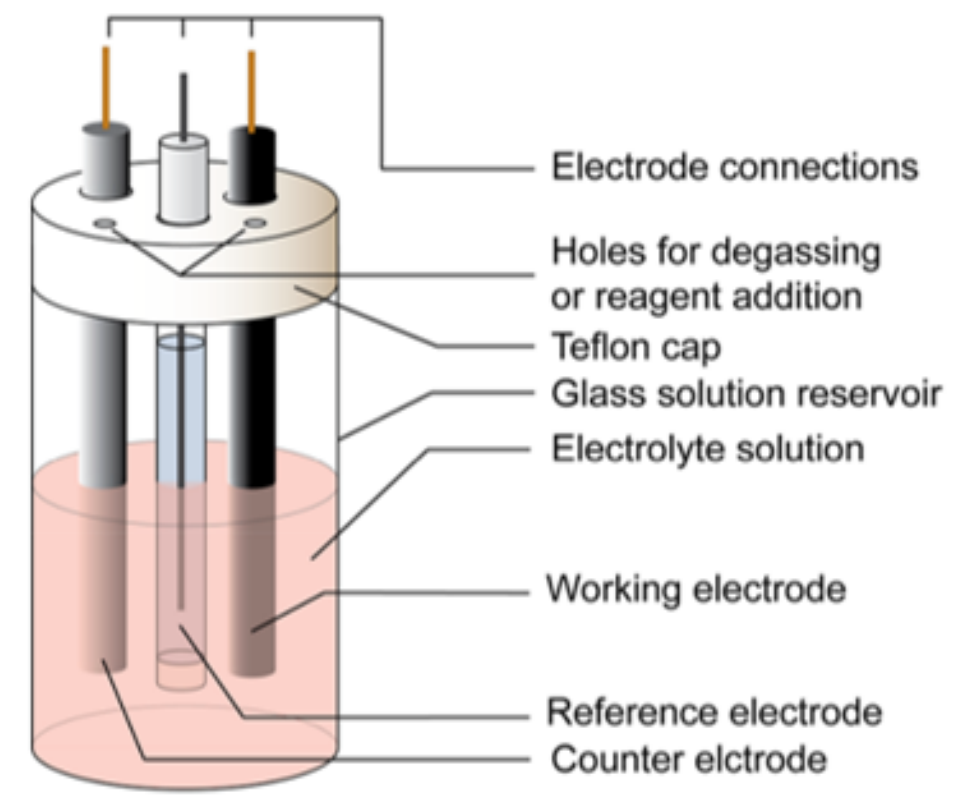

Figure 2.20: Schematic representation of an electrochemical cell for CV experiments.[22]

Figure 2.20 shows a schematic of an electrochemical cell used for CV, composed of three electrodes and an electrolyte solution. The potential of the working electrode is varied with time, whilst the reference electrode maintains a constant potential. The counter electrode conducts electricity from the signal source to the working electrode. The electrolyte solution provides ions to the electrodes during oxidation and reduction. The potentiostat uses a DC power source to produce a potential which can be accurately determined and maintained, while allowing small currents to be drawn into the system without changing the voltage. The current-to-voltage converter measures the resulting current, and the data acquisition system produces the resulting voltammogram (current as a function of applied potential).

For the CV measurements in this thesis, the working electrode was an ITO substrate coated with analyte as a thin film, prepared as described in Section 2.2, unlike the traditional procedure where analytes were dissolved in the electrolyte. The counter electrode was a platinum gauze wrapped around a platinum wire and the reference electrode was a $\mathrm{Ag} / \mathrm{AgCl}$ electrode which was standardised against Ferrocene/Ferrocenium $\left(F c / F c^{+}\right)$ which was found to be $-4.8 \mathrm{eV}$ against vacuum, and this value was used to calibrate all 
the CV measurements. The electrolyte employed in this project is $0.1 \mathrm{M}$ of tetrabutylammonium hexauorophosphate (TBAHFP) in anhydrous acetonitrile. The electrolyte solution was degassed with dry nitrogen for over 30 minutes before any measurement. The potential difference between counter and working electrodes was controlled with a Metrohm Autolab potentiostat. During measurement, small amount of nitrogen was injected into the electrolyte, in order to prevent any oxygen from dissolving in. The CV measurements in this thesis were undertaken at Imperial College London with the help of George Fish, Dr. Hyojung Cha and Dr. Laia Francis Forcada. The data was analysed by myself.

\subsubsection{Current density-voltage device characterisation}

J-V curves were obtained using a Newport 92193A-1000 solar simulator, providing AM1.5G illumination. The intensity of the solar simulator radiation was calibrated and measured using a silicon photodiode. A Keithley 2400331 sourcemeter was used to apply an external bias and a computer recorded the current density as a function of applied voltage. Multiple devices were tested for each condition and a representative pixel was chosen to determine trends.

\subsubsection{Device stability testing}

The stability of devices was tested under 1 sun equivalent white light illumination, provided by an array of LED's. Up to six devices at a time could be tested inside an environmental chamber (made in-house) (Figure 2.21) which could be purged with a gas, in the case of this thesis either dry air or dry nitrogen. The chamber was placed upon a cooling stage which kept the devices at $\sim 25^{\circ} \mathrm{C}$ and the relative humidity within the chamber was $\sim 40 \%$ (these parameters were checked with a thermocouple and humidity sensor within the environmental chamber). The chamber was purged with dry air/nitrogen before the test was started. J-V curves of the devices were measured at regular intervals as they photoaged. From these J-V curves, the device parameters were obtained and plotted as a function of photoaging time. Again, a representative pixel was chosen to determine stability trends of devices with different photoactive layers. White LEDs are chosen as the light source for this stability set-up as LEDs have a long lifespan and are relatively low cost. However, the white LED light sources do not contain UV or IR light, unlike the solar spectrum, so this could provide a spectral mismatch for devices where a component absorbs strongly at that range, and hence could undergo reduced degradation. 


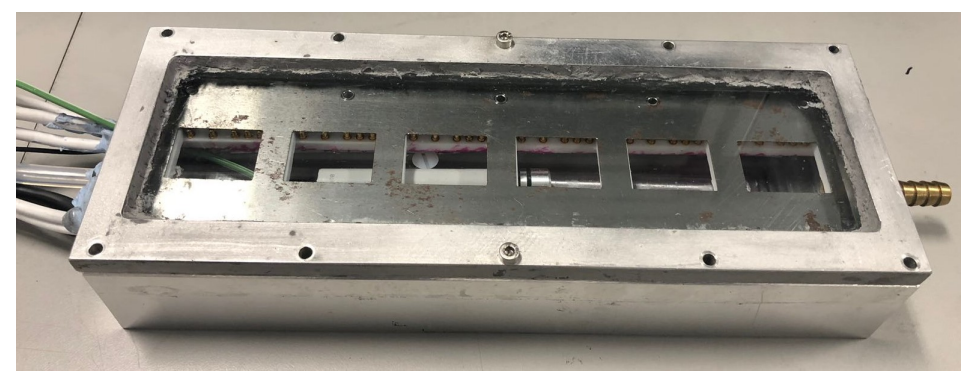

Figure 2.21: A photograph of the environmental chamber used for measuring the stability of devices. Photo credit: Andrew Clarke.

\subsubsection{Electroluminescence spectroscopy}

Electroluminescence (EL) is the emission of light as a result of the application of a forward bias on a diode and is complimentary PL. EL is more sensitive than PL to trap/electronic states, as it involves charge injection, transport and recombination. Furthermore, EL is more likely to be dominated by the emission from charge transfer states in the blend film of OPV devices. The electrical bias injects electrons and holes into the active layer of the solar cell, which can recombine radiatively via fluorescence and/or phosphorescence, emitting photons. An EL spectrum is obtained by measuring the intensity of emitted photons as a function of wavelength of the emitted light when a bias is applied to a device.[23]

EL spectra were measured by Dr. Andrew Telford at Imperial College London, using the same set-up as for obtaining the PL spectra (Section 2.3.3). Data was analysed and interpreted by myself.

\subsubsection{External quantum efficiency}

The external quantum efficiency (EQE) is another measurement that can assess device performance, including losses through transmission and reflection of the photons. It is defined as the ratio of the number of charge carriers collected at the electrodes to the number of incident photons at a particular wavelength. The integration of the area under an EQE spectrum is in principle the same as the $\mathrm{J}_{\mathrm{sc}}$ obtained in a device under AM 1.5 illumination. A (scanning) monochromatic light beam illuminates the solar cell and the photocurrent generated is recorded as a function of wavelength. The EQE measurements in this thesis were taken by Dr. Harrison Lee (Swansea University) using a QEX10 Quantum Efficiency Measurement System with 300 - 1100 nm monochromatic probe light. Data was interpreted by myself. 


\subsection{Theory}

\subsection{1 (Time-dependent) density functional theory}

Density functional theory (DFT) is a method of obtaining an approximate solution to the Schrödinger equation of a many-body system (atom or molecule). The solution, a quantum mechanical wavefunction describes the behaviour of electrons in this system to include the energy of the system, quantised energy levels and the electron density distribution. [24] Time-dependent density functional theory (TD-DFT) is an extension of DFT to time dependent systems, mainly concerning electronic excitation/recombination processes and the associated time evolution away from equilibrium.[25]

Calculations using DFT and TD-DFT were undertaken by Beth Rice at Imperial College London. All calculations were done at the B3LYP level of theory using the 6-31g* basis set. For the optical spectra calculations, the lowest energy structures for each defect (photooxidised $\mathrm{PC}_{61} \mathrm{BM}$ ) were chosen. TD-DFT calculations were performed on the structures with Gaussian09 in order to extract the oscillator strength and transition energy of the first 100 transitions. These were used to obtain an emission spectrum using:

$$
\varepsilon(E)=\frac{N}{\sigma} \sum_{i}^{N} f_{\mathrm{i}} D\left(E, E_{\mathrm{i}}\right) E_{\mathrm{i}}^{2} \exp \left(-\frac{E_{\mathrm{i}}}{k T}\right)
$$

where $N$ is the number of transitions, $\sigma$ is a width defined by the energy, and $f_{\mathrm{i}}$ and $E_{\mathrm{i}}$ are the oscillator strength and the energy of the ith excitation, respectively. $D\left(E, E_{\mathrm{i}}\right)$ is a lineshape function defined by:

$$
D\left(E, E_{\mathrm{i}}\right)=\exp \left[\left(\frac{E-E_{\mathrm{i}}}{\sigma}\right)^{2}\right]
$$

The results of these calculations were interpreted by myself.

\subsection{Comparison of the techniques used to probe pho- tochemical degradation}

There are a number of techniques that can be used to measure the levels of photodegradation in organic materials. Firstly, as shown above, FTIR is a useful technique that can probe the addition of oxygen atoms to the cage of fullerenes through the observation 
of of more $\mathrm{C}=\mathrm{O}$ vibrations (at $1782 \mathrm{~cm}^{-1}$ ). A draw-back to this technique is that the polymer donor may have a similar photo-oxidation product (leading to $\mathrm{C}=\mathrm{O}$ vibrations) and hence have similar peak positions on a FTIR spectrum, making them difficult to be distinguished from fullerene photo-oxidation when blended. However, the fullerene ball has a characteristic FTIR peak (at $526 \mathrm{~cm}^{-1}$ ) whose intensity decreases significantly with oxidation and so could be a good indicator of photo-oxidation.[26, 27] Mass spectrometry could also measure the chemical composition of a material and give a more quantitative measurement of photo-oxidation.[28] However, as the film is redissolved to prepare the sample, it can only give an average chemical composition (mass) of the whole film, without any information on depth or chemical structure. XPS is another technique that can quantitively measure the chemical composition of a film,[29] and (when coupled with an etching ion source) create depth profiles of chemical composition/photo-oxidation as a function of etch depth, which can provide useful insight such as the vertical propagation of photo-oxidation through a film. A drawback for this method is the difficulty in distinguishing between polymer and fullerene photo-oxidation, as it is likely the binding energies of products will be similar. Therefore, multiple complementary characterisation techniques are applied in this thesis to obtain a comprehensive study of the photodegradation. 


\section{REFERENCES}

[1] H. Hintz, H.-J. Egelhaaf, H. Peisert, and T. Chassé, "Photo-oxidation and ozonization of poly(3-hexylthiophene) thin films as studied by UV/VIS and photoelectron spectroscopy," Polymer Degradation and Stability, vol. 95, pp. 818-825, 2010.

[2] T. J. Savenije, J. E. Kroeze, X. Yang, and J. Loos, "The formation of crystalline P3HT fibrils upon annealing of a PCBM:P3HT bulk heterojunction," Thin Solid Films, vol. 511-512, pp. 2-6, 2006.

[3] S. Cook, H. Ohkita, Y. Kim, J. J. Benson-Smith, D. D. Bradley, and J. R. Durrant, "A photophysical study of PCBM thin films," Chemical Physics Letters, vol. 445, pp. 276-280, 2007.

[4] A. Maciejewski, R. Naskrecki, M. Lorenc, M. Ziolek, J. Karolczak, J. Kubicki, M. Matysiak, and M. Szymanski, "Transient absorption experimental set-up with femtosecond time resolution. Femto-and picosecond study of DCM molecule in cyclohexane and methanol solution," Journal of Molecular Structure, vol. 555, pp. 1-13, 2000.

[5] S. Monti and C. Chiorboli, "Transient Absorption Spectroscopy," in The Exploration of Supramolecular Systems and Nanostructures by Photochemical Techniques, pp. 185-207, Springer, Dordrecht, 2012.

[6] H. Ohkita, S. Cook, Y. Astuti, W. Duffy, S. Tierney, W. Zhang, M. Heeney, I. McCulloch, J. Nelson, D. D. C. Bradley, and J. R. Durrant, "Charge carrier formation in polythiophene/fullerene blend films studied by transient absorption spectroscopy.," Journal of the American Chemical Society, vol. 130, pp. 3030-42, 2008.

[7] F. Etzold, I. A. Howard, N. Forler, D. M. Cho, M. Meister, H. Mangold, J. Shu, M. R. Hansen, K. Müllen, and F. Laquai, "The effect of solvent additives on morphology and excited-state dynamics in PCPDTBT:PCBM photovoltaic blends.," Journal of the American Chemical Society, vol. 134, pp. 10569-83, 2012.

[8] T. Kirchartz, B. E. Pieters, J. Kirkpatrick, U. Rau, and J. Nelson, "Recombination via tail states in polythiophene:fullerene solar cells," Physical Review B, vol. 83, p. 115209, 2011.

[9] A. F. Nogueira, I. Montanari, J. Nelson, J. R. Durrant, C. Winder, N. S. Sariciftci, and C. Brabec, "Charge Recombination in Conjugated Polymer/Fullerene Blended Films Studied by Transient Absorption Spectroscopy," The Journal of Physical Chemistry B, vol. 107, pp. 1567-1573, 2003. 
[10] L. Prodi and A. Credi, "Spectrofluorimetry," in The Exploration of Supramolecular Systems and Nanostructures by Photochemical Techniques, pp. 97-129, Springer, Dordrecht, 2012.

[11] A. Gomes, E. Fernandes, and J. L. Lima, "Fluorescence probes used for detection of reactive oxygen species," Journal of Biochemical and Biophysical Methods, vol. 65, pp. $45-80,2005$.

[12] N. Aristidou, I. Sanchez-Molina, T. Chotchuangchutchaval, M. Brown, L. Martinez, T. Rath, and S. A. Haque, "The Role of Oxygen in the Degradation of Methylammonium Lead Trihalide Perovskite Photoactive Layers," Angewandte Chemie International Edition, vol. 54, pp. 8208-8212, 2015.

[13] D. Bryant, N. Aristidou, S. Pont, I. Sanchez-Molina, T. Chotchunangatchaval, S. Wheeler, J. R. Durrant, and S. A. Haque, "Light and oxygen induced degradation limits the operational stability of methylammonium lead triiodide perovskite solar cells," Energy $\&$ Environmental Science, vol. 9, pp. 1655-1660, 2016.

[14] Y. W. Soon, H. Cho, J. Low, H. Bronstein, I. McCulloch, and J. R. Durrant, "Correlating triplet yield, singlet oxygen generation and photochemical stability in polymer/fullerene blend films.," Chemical communications, vol. 49, pp. 1291-3, 2013.

[15] E. de Hoffmann and V. Stroobant, Mass Spectrometry: Principles and Applications. Wiley, 3rd ed., 2007.

[16] B. C. Smith, Fundamentals of Fourier Transform Infrared Spectroscopy. CRC Press, 2nd ed., 2011.

[17] J. Coates, Coates, and John, "Interpretation of Infrared Spectra, A Practical Approach," in Encyclopedia of Analytical Chemistry, Chichester, UK: John Wiley \& Sons, Ltd, 2006.

[18] P. van der Heide, X-ray Photoelectron Spectroscopy : An introduction to Principles and Practices. Wiley, 2011.

[19] J. M. Wagner, X-ray photoelectron spectroscopy. Nova Science Publishers, 2011.

[20] A. Hexemer and P. Müller-Buschbaum, "Advanced grazing-incidence techniques for modern soft-matter materials analysis.," IUCrJ, vol. 2, pp. 106-25, 2015.

[21] J. Tamayo and R. García*, "Deformation, Contact Time, and Phase Contrast in Tapping Mode Scanning Force Microscopy," Langmuir, vol. 12, pp. 4430-4435, 1996. 
[22] N. Elgrishi, K. J. Rountree, B. D. McCarthy, E. S. Rountree, T. T. Eisenhart, and J. L. Dempsey, "A Practical Beginner's Guide to Cyclic Voltammetry," Journal of Chemical Education, vol. 95, pp. 197-206, 2018.

[23] D. Abou-Ras, T. Kirchartz, and U. Rau, Advanced Characterization Techniques for Thin Film Solar Cells. Wiley-VCH, 2016.

[24] R. G. Parr and W. Yang, Density-Functional Theory of Atoms and Molecules. Oxford University Press, 1987.

[25] C. A. Ullrich, Time-Dependent Density-Functional Theory : Concepts and Applications. OUP Oxford, 2011.

[26] H. Neugebauer, C. Brabec, J. Hummelen, R. Janssen, and N. Sariciftci, "Stability studies and degradation analysis of plastic solar cell materials by FTIR spectroscopy," Synthetic Metals, vol. 102, pp. 1002-1003, 1999.

[27] S. Chambon, A. Rivaton, J. L. Gardette, and M. Firon, "Photo- and thermal degradation of MDMO-PPV:PCBM blends," Solar Energy Materials and Solar Cells, vol. 91, pp. 394-398, 2007.

[28] J.-N. Audinot, P. Lévêque, R. Bechara, N. Leclerc, J. Guillot, H.-N. Migeon, G. Hadziioannou, and T. Heiser, "Characterization of P3HT/PCBM bulk heterojunction photovoltaic devices using advanced secondary ion mass spectrometry techniques," Surface and Interface Analysis, vol. 42, pp. 1010-1013, 2010.

[29] J. Kettle, Z. Ding, M. Horie, and G. Smith, "XPS analysis of the chemical degradation of PTB7 polymers for organic photovoltaics," Organic Electronics, vol. 39, pp. 222$228,2016$. 


\section{THE ROLE OF PHOTO-OXIDATION OF FULLERENES IN THE STABILITY OF POLYMER:FULLERENE SOLAR CELLS}

This chapter studies the severe effect of fullerene photo-oxidation upon polymer:fullerene OSCs. Here, the role of the common fullerene derivative $\mathrm{PC}_{61} \mathrm{BM}$ in limiting the photostability of benchmark organic solar cells is quantitatively studied. It is shown that just a minor fraction $(<1 \%)$ of photo-oxidised $\mathrm{PC}_{61} \mathrm{BM}$, induced by short exposure to either solar or ambient laboratory lighting conditions in air, consistent with typical processing and operating conditions, is sufficient to severely compromise device performance. Photo-oxidation of $\mathrm{PC}_{61} \mathrm{BM}$ was shown to result in a decrease in LUMO level, which would cause electron trapping in the fullerene domains. This photodegradation was observed for OSCs made using both standard and inverted device architectures, and a range of benchmark polymers and benchmark fullerenes, where the fullerenes were selectively photoaged. These results show that the photo-oxidation of fullerenes have a general and detrimental effect to the stability of polymer:fullerene OSCs exposed to light and air. 


\subsection{Introduction}

This main objective of this chapter is to investigate the role of fullerene photo-oxidation to device performance. A novel approach is applied here to isolate the photo-degradation of fullerenes from that of the polymer in the active layer. Then, the studies were extended to a wider range of polymer donors, device architectures, and various kinds of fullerene acceptors. The results herein show that in general fullerene photo-oxidation plays an important role to degradation of OSCs.

\subsection{Experimental details}

\section{Film and device fabrication and photoaging:}

Devices were fabricated with the architectures ITO $/ \mathrm{ZnO} / \mathrm{AL} / \mathrm{MoO}_{3} / \mathrm{Ag}$, $\mathrm{ITO} / \mathrm{MoO}_{3} / \mathrm{AL} / \mathrm{Ca} / \mathrm{Al}$ or ITO/Pedot:PSS/AL/Ca/Al were fabricated and characterised with Dr. Zhe Li and Dr Harrison Lee (Swansea University) following the method stated in Chapter 2. Data analysis and interpretation was conducted by myself.

Table 3.1: Concentration of Donor (D) and acceptor (A) in CB, spin speed/acceleration, DIO added to prepare the solutions for active layers. For P3HT, it was blended with various kinds of fullerene acceptors (FA).

\begin{tabular}{|c|c|c|c|c|}
\hline & $\mathrm{D}(\mathrm{mg} / \mathrm{ml})$ & $\mathrm{A}(\mathrm{mg} / \mathrm{ml})$ & $\mathrm{rpm} / \mathrm{rpms}^{-1}$ & DIO (wt\%) \\
\hline PCDTBT:PC ${ }_{61} \mathrm{BM}$ & 6 & 12 & $800 / 7500$ & 0 \\
\hline PTB7:PC ${ }_{61} \mathrm{BM}$ & 10 & 15 & $1500 / 2500$ & 3 \\
\hline PCE10:PC 61 BM & 10 & 15 & $1500 / 2500$ & 3 \\
\hline P3HT:FA & 24 & 24 & $2000 / 2500$ & 0 \\
\hline
\end{tabular}

Table 3.1 summarises the concentration (in CB), spin speed/acceleration, and DIO added to prepare the solutions for active layers (AL) for thin films and devices characterised. For the P3HT:fullerenes films/devices, the films were thermally annealed at $130^{\circ} \mathrm{C}$ for 10 minutes.

Solutions for selective degradation studies were prepared by dissolving the material as above to make $1 \mathrm{ml}$ of solution in a $15 \mathrm{ml}$ vial in the dry nitrogen glovebox. The vials of fullerene or polymer solutions were then filled with air (with stir speed of $300 \mathrm{rpm}$ ) and photoaged under AM 1.5G illumination for the aging time stated in the text. See Chapter 2 for further explanation. Photoaging of the films and devices was performed 
under $\sim 1$ Sun unfiltered AM1.5G radiation in ambient conditions and temperature using a solar simulator for the aging time specified in the text.

Characterisation and modelling The UV-vis absorbance spectra were measured with a Perkin Elmer Lambda 750 spectrophotometer (Swansea University). MALDITOF measurements on redissolved thin film samples were carried out by Dr. Mark Wyatt (Swansea University) and data analysis was undertaken by myself as described in Chapter 2. DFT calulations were undertaken by Ms Beth Rice (Imperial College London) following the method in Chapter 2 and ref [1]. These calculations were interpreted myself.

\subsection{Results and discussion}

\subsubsection{Impact of fullerene photo-oxidation upon OSC devices}

Firstly, the effect of light and air exposure on OPV device performance was investigated. Devices with a conventional structure were made where the active layer (1:2 PCDTBT:PC ${ }_{61} \mathrm{BM}$ ) was photoaged for up to 600 minutes under AM1.5G illumination in air prior to the deposition of the back contacts. Exposing the active layer to illumination before electrode deposition allowed for the complication of oxygen diffusion through the top interlayer/electrode (and around) and the possible photodegradation of the top interlayer/electrode to be ruled out. The lateral diffusion of oxygen for devices with a top electrode that blocks oxygen have previously been shown to extend over days.[2, 3]

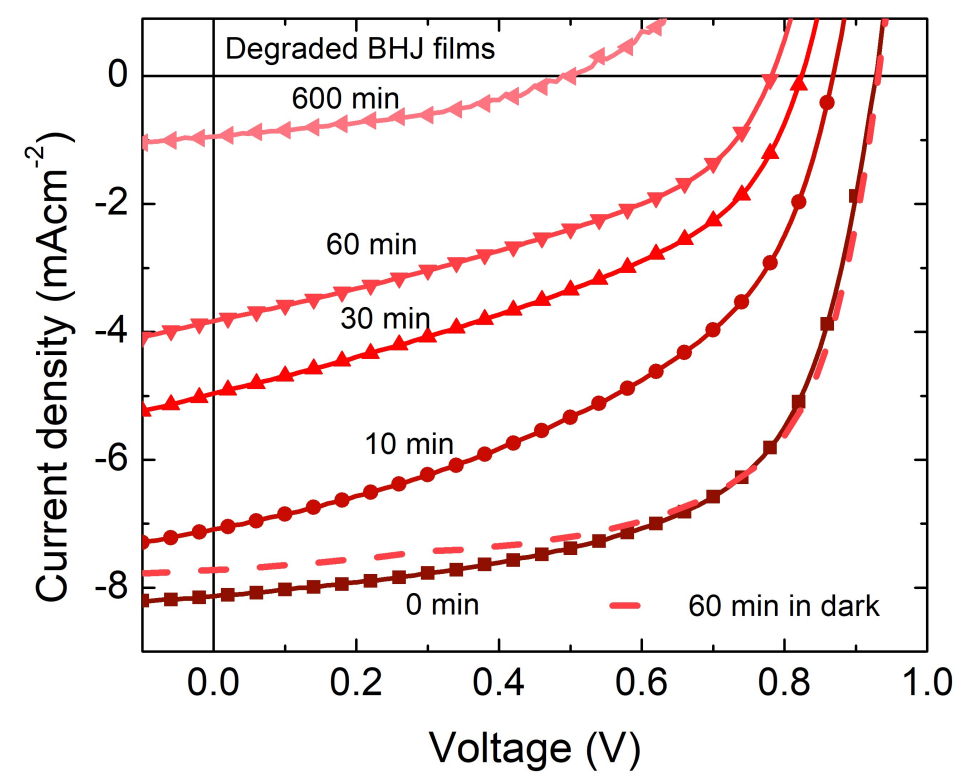

Figure 3.1: J-V characteristics of conventional 1:2 PCDTBT:PC ${ }_{61} \mathrm{BM}$ devices where the BHJ films were exposed to simulated AM1.5G illumination in air prior to back contact deposition. 
Figure 3.1 shows the evolution of the J-V curves of devices where the bulk heterojunction (BHJ) active layers were photoaged for the time specified. Notably, just 10 minutes of light exposure in air resulted in a loss of $36 \%$ of PCE $(5.01 \%$ to $3.19 \%$, due to a simultaneous reduction in $\mathrm{J}_{\mathrm{sc}}, \mathrm{V}_{\mathrm{oc}}$, and $\mathrm{FF}$ (Figure A.1). With 60 minutes of illumination in air underwent a loss of $73 \%$ PCE (to $1.35 \%$ ). In contrast, devices exposed to the air for 60 minutes but without any illumination exhibited a similar performance to the fresh devices $(5.18 \%$ ) (Figure A.2), signifying that it is the combination of light and oxygen that drives the device degradation. The photodegradation continues to develop, maintaining only 4 $\%$ of initial PCE (0.20 \%) after 600 minutes of exposure to air and illumination. Critically, even exposure to low-light levels (1000 lux fluorescent illumination), typical of laboratory environments, was sufficient to drive the same photodegradation effect in devices (Figure A.2), reiterating the need to reduce exposure to oxygen and light when preparing samples and devices.

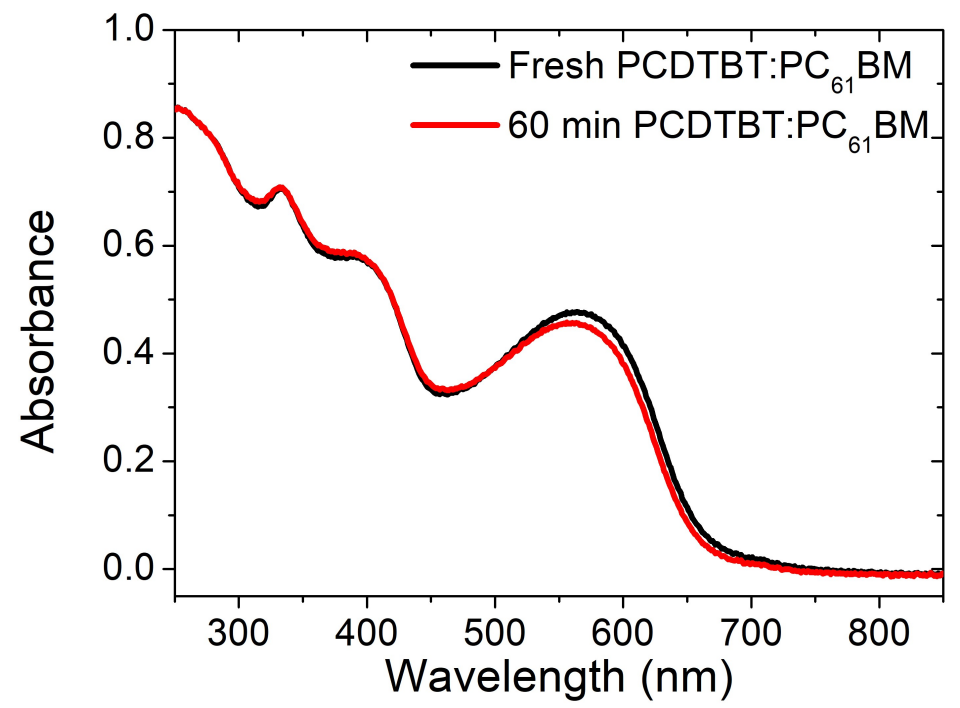

Figure 3.2: UV-vis spectra of PCDTBT:PC ${ }_{61} \mathrm{BM}$ blend films without and with 60 minutes of exposure to AM1.5G conditions in dry air.

To investigate the origin of the device degradation, UV-vis absorbance spectroscopy was used to study photobleaching of the blend films which were made and photoaged in the same way as the blend films used for the devices in Figure 3.1. Figure 3.2 shows the UV-vis absorbance spectra for a fresh blend film and a blend film that was photoaged under AM1.5G illumination in air for 60 minutes. There is was no obvious photobleaching of the absorbance peak at $332 \mathrm{~nm}$ due to the $\mathrm{PC}_{61} \mathrm{BM}$, while the absorbance peak at 564 nm due to the PCDTBT drops slightly by $4.4 \%$ (absorbance reduced from 0.48 to 0.46). This result seems to show that the device photo-degradation could be related to the photo-degradation of PCDTBT, without significant contribution from $\mathrm{PC}_{61} \mathrm{BM}$, similar to previous studies comparing the optical degradation of polymers and fullerenes. [4-6]. However, as shown in next section, $\mathrm{PC}_{61} \mathrm{BM}$ is also photo-degraded, and photobleaching is not a sensitive technique to probe degradation of $\mathrm{PC}_{61} \mathrm{BM}$ (charge transport can be 
degraded significantly without significant change in UV-vis absorbance, see chapter 4).
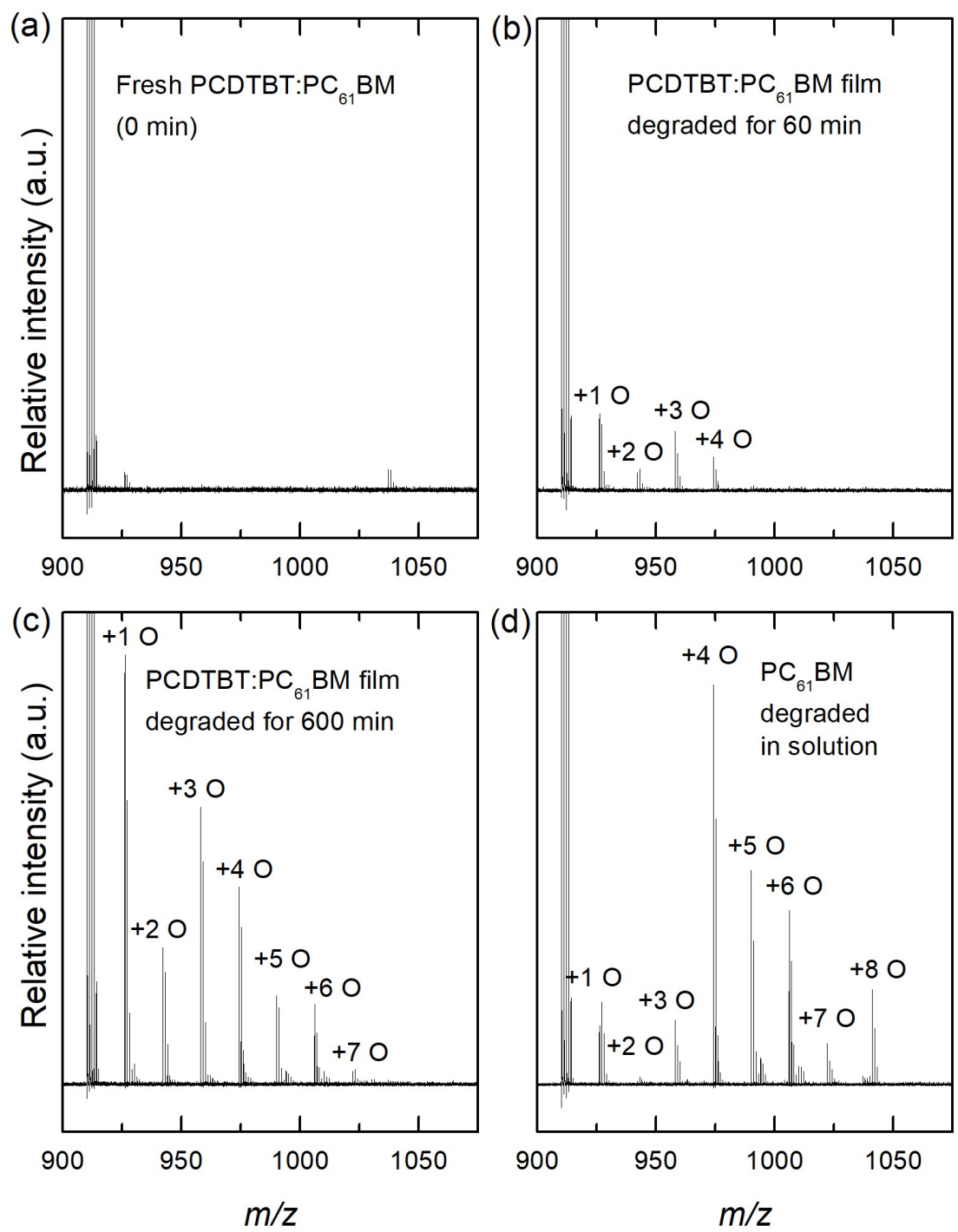

Figure 3.3: MALDI-TOF measurements of 1:2 PCDTBT: $\mathrm{PC}_{61} \mathrm{BM}$ blend films a) fresh, b)photoaged for 1 hour and c) photoaged for 10 hours and d) $\mathrm{PC}_{61} \mathrm{BM}$ solution photoaged for 72 hours in air under 1sun AM1.5G illumination.

Next, MALDI-TOF measurements were taken in order to identify any chemical cause for the degradation observed in the devices. The samples for MALDI-TOF measurements were prepared by re-dissolving the BHJ films after photochemical degradation. By comparing Figure 3.3 (a) with (b) it is seen that photo-oxidised $\mathrm{PC}_{61} \mathrm{BM}$ species (with up to 4 oxygen atoms) were formed upon 1 hour of AM1.5G illumination in air in the BHJ film. The degree of oxidation is further increased with length of exposure (up to 7 oxygen atoms after 10 hours) as seen in Figure 3.3 (c). Upon analysing the 12C-isotopic peak areas, it is revealed that a total of $0.8 \%$ of the $\mathrm{PC}_{61} \mathrm{BM}$ molecules have been oxidised in the BHJ film after 1 hour of aging. This is further increased to $4.3 \%$ after 10 hours of aging. Thus it is apparent that $\mathrm{PC}_{61} \mathrm{BM}$ can be readily photo-oxidised upon exposure to light and oxygen in unprotected BHJ films with conjugated donor polymers. 
In order to decouple the effect of fullerene photo-oxidation from polymer photodegradation, upon OSC device performance, 1:2 PCDTBT:PC ${ }_{61} \mathrm{BM}$ devices were fabricated using $\mathrm{PC}_{61} \mathrm{BM}$ solution that had been photo-oxidised separately, so that any degradation observed should have originated from the $\mathrm{PC}_{61} \mathrm{BM}$ alone. The aged $\mathrm{PC}_{61} \mathrm{BM}$ solution was analysed via MALDI-TOF to quantify the percentage of oxidised species within the solution (Figure $3.3(\mathrm{~d})$ ). It was found that $\mathrm{PC}_{61} \mathrm{BM}$ solution that had been illuminated for 72 hours under a AM1.5G solar simulator in air comprised $3.6 \%$ oxidised $\mathrm{PC}_{61} \mathrm{BM}$ species (herein referred to as $\mathrm{O}-\mathrm{PC}_{61} \mathrm{BM}$ ). Increasing amounts of photodegraded $\mathrm{PC}_{61} \mathrm{BM}$ solution were added to a fresh $\mathrm{PC}_{61} \mathrm{BM}$ solution (keeping the total concentration of $\mathrm{PC}_{61} \mathrm{BM}$ constant), to control the fraction of $\mathrm{O}-\mathrm{PC}_{61} \mathrm{BM}$ in the fullerene phase of the blend films in the OPV devices.
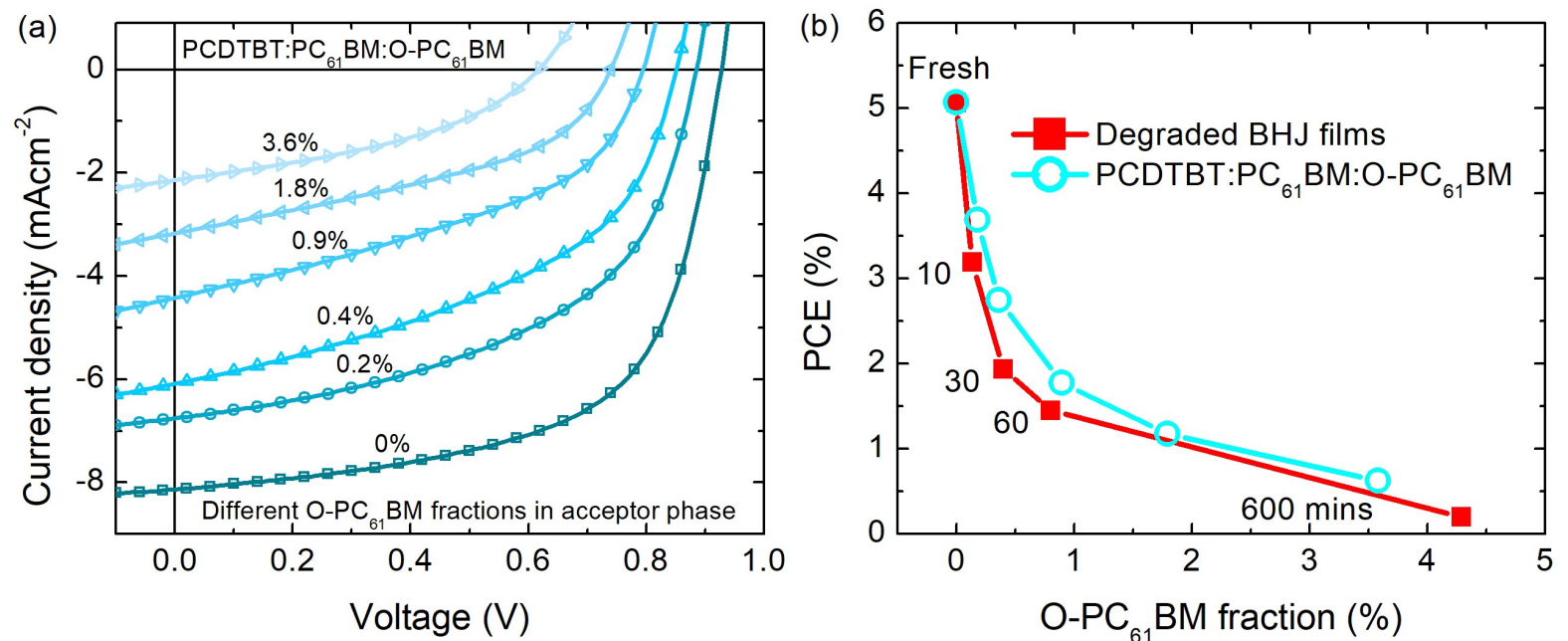

Figure 3.4: (a) J-V characteristics of devices made with different fractions of solution degraded $\mathrm{PC}_{61} \mathrm{BM}$, the percentages indicate the mass fraction of $\mathrm{O}-\mathrm{P}_{61} \mathrm{CBM}$ in the acceptor phase. (b) $\mathrm{PCE}$ as a function of $\mathrm{O}-\mathrm{PC}_{61} \mathrm{BM}$ fraction for degraded $\mathrm{PCDTBT}: \mathrm{PC}_{61} \mathrm{BM}$ devices and pre-degraded $\mathrm{PC}_{61} \mathrm{BM}$ devices.

Figures $3.4 \mathrm{a}$ and A.1b show the $\mathrm{J}-\mathrm{V}$ characteristics of these devices with increasing fractions of photodegraded $\mathrm{PC}_{61} \mathrm{BM}$ added. Remarkably, a $65 \%$ loss of PCE was observed for devices containing only $1 \%$ of $\mathrm{O}-\mathrm{PC}_{61} \mathrm{BM}$ in the fullerene phase, increasing to $\mathrm{a} \sim$ $90 \%$ PCE loss for devices with $3.6 \%$ O-PC ${ }_{61}$ BM. Again, this loss of PCE was due to a combined loss in $\mathrm{J}_{\mathrm{sc}}, \mathrm{V}_{\mathrm{oc}}$ and $\mathrm{FF}$. This trend of performance loss in PCDTBT:PC ${ }_{61} \mathrm{BM}$ devices with added photo-oxidised $\mathrm{PC}_{61} \mathrm{BM}$ correlates well with a previous study where addition of just $1 \%$ of an impurity $\left(\mathrm{PC}_{84} \mathrm{BM}\right)$ with a lower LUMO level severely affected the device performance by increasing trap-induced recombination.[7, 8] This could explain why a small amount of photo-oxidation can have such a large effect to device performance, despite $\mathrm{PC}_{61} \mathrm{BM}$ not significantly photobleaching (Figure 3.2).

Figure 3.4b gives a comparison of the PCE of devices where the blend films have been photodegraded and those made with controlled fractions $\mathrm{O}-\mathrm{PC}_{61} \mathrm{BM}$. The device $\mathrm{PCE}$ is 
plotted as a function of $\mathrm{O}-\mathrm{PC}_{61} \mathrm{BM}$ in the blend film, measured by MALDI-TOF after redissolving and analysing the active layers. The drop in device performance of the photooxidised $\mathrm{BHJ}$ films with increasing $\mathrm{O}-\mathrm{PC}_{61} \mathrm{BM}$ content coheres with the trend observed for devices made using $\mathrm{O}-\mathrm{PC}_{61} \mathrm{BM}$ solution. This clear correlation between device performance reduction with increasing $\mathrm{O}-\mathrm{PC}_{61} \mathrm{BM}$ for both film degraded and solution degraded devices makes it apparent that $\mathrm{PC}_{61} \mathrm{BM}$ photo-oxidation has a detrimental impact on device performance.

\subsubsection{The effect of polymer photodegradation on device per- formance}

To study the contribution of PCDTBT photodegradation (as observed in photobleaching) to the loss of performance for the devices where the blend films were exposed to illumination, devices were fabricated with selectively photoaged PCDTBT solution (which was then blended with fresh $\mathrm{PC}_{61} \mathrm{BM}$ solution). The solution was only degraded for 2 hours as longer illumination times led to cross-linking of the polymer. (Visible solid lumps appeared on the sides of the vial after $4 \mathrm{hr}$ of illumination.) Figure 3.5 shows the normalised characteristics of these devices as a function of degraded PCDTBT solution content, normalised to the characteristics of a fresh device. Absolute device characteristics show a substantial degradation of $72.2 \%$ (5.07 to $3.66 \%$ ) upon using the PCDTBT solution that had been degraded for just 2 hours (Figure A.3). It can be seen that PCDTBT photodegradation leads to a reduction in $\mathrm{PCE}$, here mainly through a drop in FF with a smaller drop in $\mathrm{J}_{\mathrm{sc}}$. Interestingly, the $\mathrm{V}_{\mathrm{oc}}$ remains stable implying that the reduction of $\mathrm{V}_{\mathrm{oc}}$ observed for devices where the blend films were photoaged prior to electrode deposition (Figure A.1 a) is dominated by $\mathrm{PC}_{61} \mathrm{BM}$ photodegradation (whch causes a significant drop in $\mathrm{V}_{\mathrm{oc}}$ ). This is consistent the concept of the photo-oxidised $\mathrm{PC}_{61} \mathrm{BM}$ being an defect and acting as charge traps which would lead to the observed drop in $\mathrm{V}_{\text {oc. }}$ [7-9] 
(a)
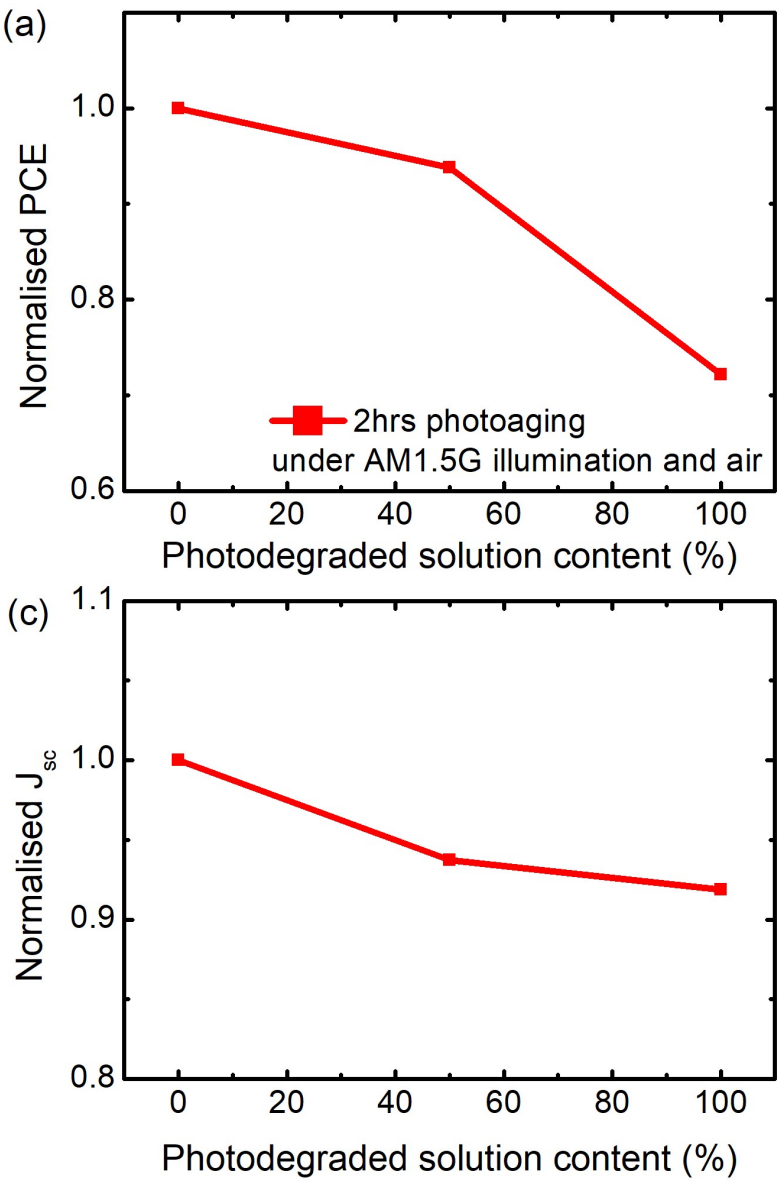
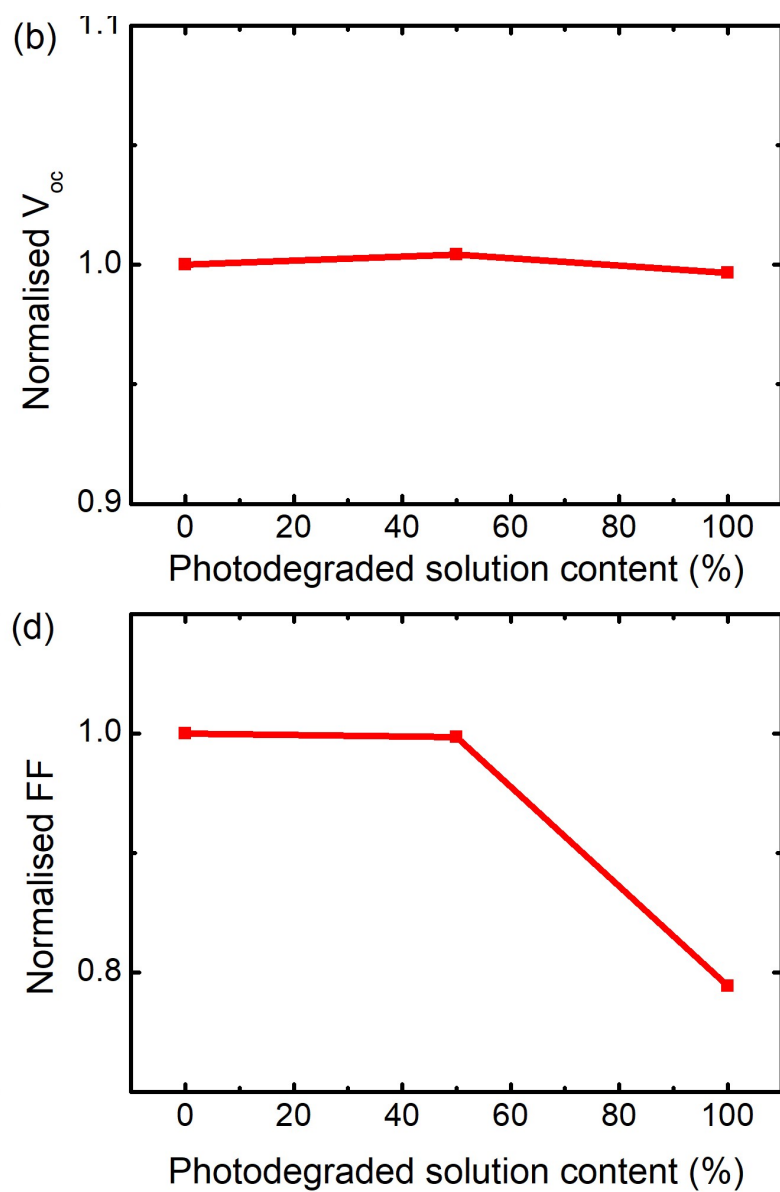

Figure 3.5: Normalised (a) PCE, (b) $\mathrm{V}_{\mathrm{oc}}$, (c) $\mathrm{J}_{\mathrm{sc}}$, and (d) FF of 1:2 PCDTBT:PC ${ }_{61} \mathrm{BM}$ devices as a function of the percentage of degraded PCDTBT solution employed to form the active layer. The photoaged PCDTBT solution was photoaged for 2 hours.

It has been shown that through quantum chemical calculations that electron traps can form in the fullerene domains of blend films via the oxidation of the fullerene cage where the resultant $\mathrm{PC}_{61} \mathrm{BM}$ oxides have a deeper LUMO level than fresh $\mathrm{PC}_{61} \mathrm{BM}$.[10] The effect of photo-oxidation on the HOMO and LUMO levels of $\mathrm{PC}_{61} \mathrm{BM}$ was explored using electronic structure calculations by Ms Beth Rice (Imperial College London) of the oxidation products, namely $\mathrm{PC}_{61} \mathrm{BM}$ bearing epoxide, diol and carbonyl defects, following the oxidation mechanism reported by Xiao et al.[11] For each defect type, the average HOMO and LUMO energies were calculated by averaging over different possible isomers weighted in proportion to their thermodynamic probability of occurrence. The LUMO was depressed for almost all oxidised $\mathrm{PC}_{61} \mathrm{BM}$ species, especially in the case of carbonyl defects. In contrast, the effect on the HOMO was small in all cases, except for the diol defect. Therefore, it is expected that the oxidation of $\mathrm{PC}_{61} \mathrm{BM}$ to affect electronic transport by introducing trap states for electrons, and to have a stronger effect on electron transport than on hole transport in the fullerene phase. This would cause electron tapping which could lead to the observed drop in $\mathrm{V}_{\text {oc }}$ in devices where $\mathrm{PC}_{61} \mathrm{BM}$ has been photooxidised. For further details and discussion on these calculations, please refer to ref [1]. The effect of $\mathrm{PC}_{61} \mathrm{BM}$ oxidation is further explored in Chapter 4. 

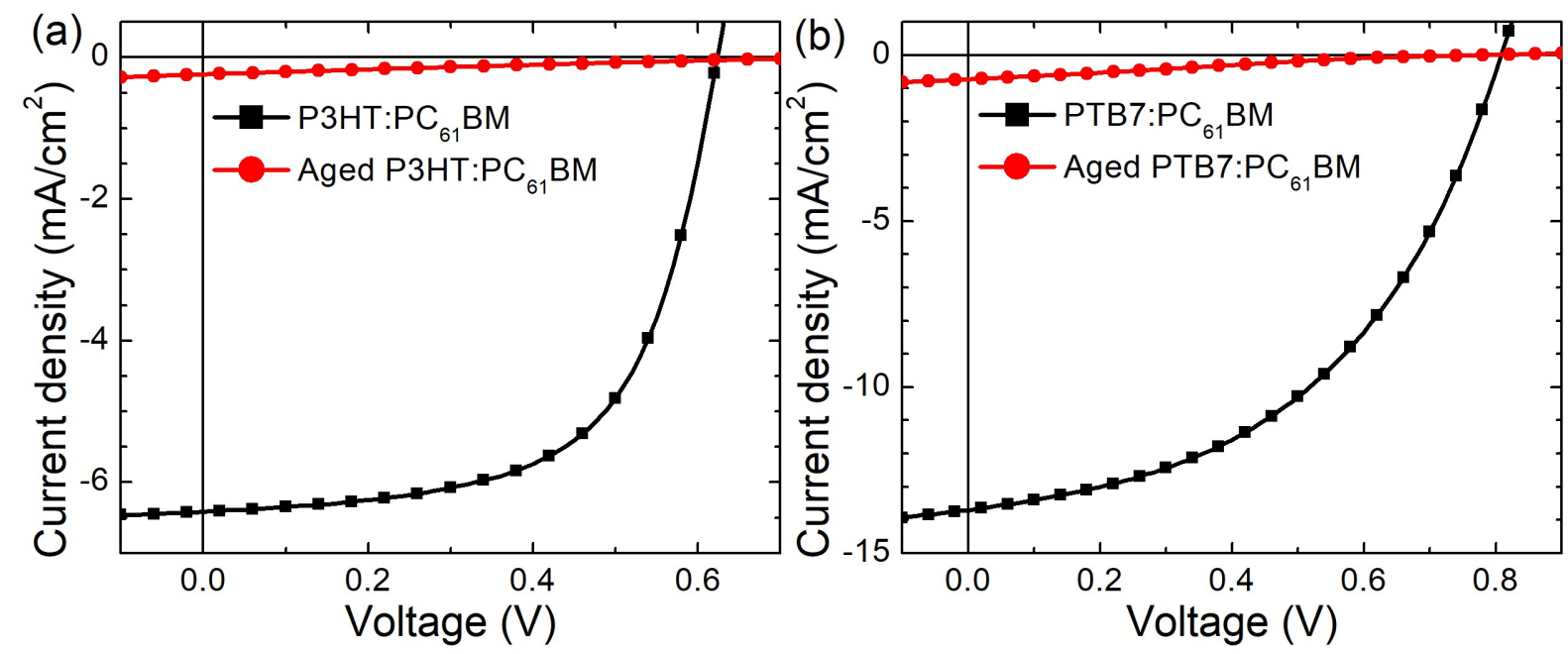

Figure 3.6: JV characteristics of (a) P3HT:PC ${ }_{61} \mathrm{BM}$ and (b) PTB7:PC ${ }_{61} \mathrm{BM}$ made with aged and unaged polymer donor solution.

The effect of the degradation of other polymers (P3HT and PTB7) on device performance was also studied. These polymers were degraded as solutions for 72 hours under 1sun AM1.5G illumination in air. Figure 3.6 shows the JV characteristics for both polymers with and without photoaging. It can be seen that both systems degrade similarly when utilising this degraded polymer solution, both dropping to 0.02 normalised PCE. This was predominantly due to a severe drop in $\mathrm{J}_{\mathrm{sc}}\left(0.05 \mathrm{P} 3 \mathrm{HT}: \mathrm{PC}_{61} \mathrm{BM}\right.$ and $\left.0.04 \mathrm{PTB} 7: \mathrm{PC}_{61} \mathrm{BM}\right)$ and a smaller drop in FF (0.37 P3HT:PC ${ }_{61} \mathrm{BM}$ and $\left.0.48 \mathrm{PTB} 7: \mathrm{PC}_{61} \mathrm{BM}\right)$. The $\mathrm{V}_{\text {oc }}$ was less affected (1.24 P3HT:PC ${ }_{61} \mathrm{BM}$ and $\left.0.95 \mathrm{PTB} 7: \mathrm{PC}_{61} \mathrm{BM}\right)$ ). These results imply that the photodegradation of polymer donors in OSC devices generally lead to a significant reduce in $\mathrm{J}_{\mathrm{sc}}$ and $\mathrm{FF}$, with little/no reduction in $\mathrm{V}_{\mathrm{oc}}$.

\subsubsection{Generality of fullerene photo-oxidation to device degrad- ation}

Finally, the impact of fullerene photo-oxidation on device performance was investigated for devices with different polymers, architectures and fullerene acceptors. This was done to test how general this effect was and whether it was a universal phenomenon for all fullerene-based BHJ OPV devices. 

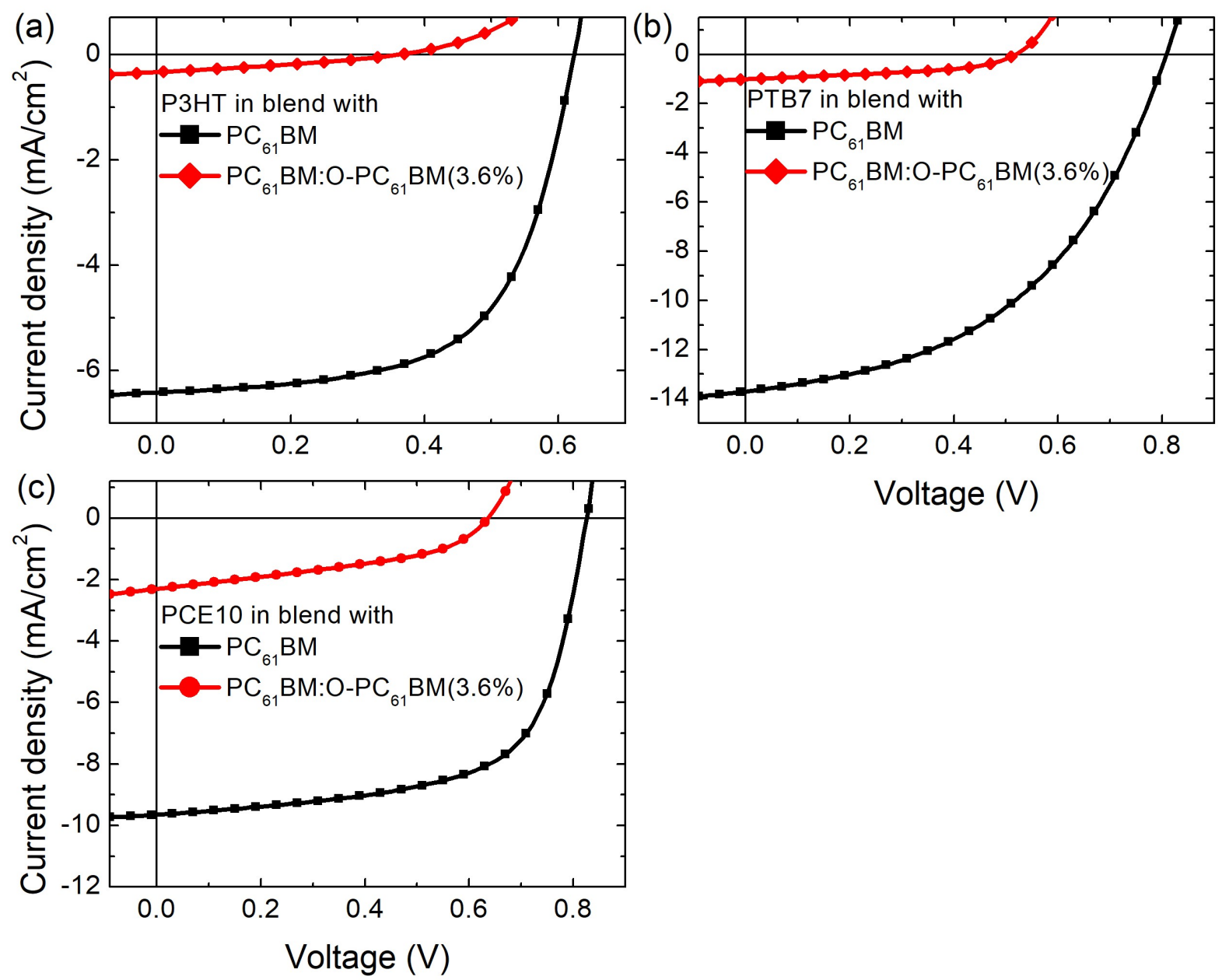

Voltage (V)

Figure 3.7: J-V characteristics of devices using different OPV benchmark polymers (a) P3HT, (b) PTB7, and (c) PCE10 in blend with fresh $\mathrm{PC}_{61} \mathrm{BM}$ (black) and selectively degraded $\mathrm{PC}_{61} \mathrm{BM}$ (red) containing $3.6 \%$ of $\mathrm{O}-\mathrm{PC}_{61} \mathrm{BM}$.

Figure 3.7 shows the JV characteristics of devices employing different donor polymers (P3HT, PTB7 and PCE10) with $\mathrm{PC}_{61} \mathrm{BM}$ and selectively degraded $\mathrm{PC}_{61} \mathrm{BM}$ containing $3.6 \%$ photo-oxidised $\mathrm{PC}_{61} \mathrm{BM}$. The same severe reduction of the device performance could be qualitatively observed for these devices as with using PCDTBT as the donor polymer (Figure 3.4), with the simultaneous drop of $\mathrm{J}_{\mathrm{sc}}, \mathrm{V}_{\mathrm{oc}}$ and FF. Note that again, with the photo-oxidation of $\mathrm{PC}_{61} \mathrm{BM}$, the $\mathrm{V}_{\text {oc }}$ drops considerably. The PCE10 system was the least affected by this inclusion of $\mathrm{O}-\mathrm{PC}_{61} \mathrm{BM}$ within the devices, with the $\mathrm{PCE}$ dropping to $11.9 \%$ of its initial value (from $6.51 \%$ to $0.77 \%$ ), followed by PTB7-based devices where the PCE dropped to $4.6 \%$ of its initial value (from $5.85 \%$ to $0.267 \%$ ). The inclusion of $\mathrm{O}-\mathrm{PC}_{61} \mathrm{BM}$ in P3HT devices caused the strongest reduction in PCE which fell to $1.6 \%$ of its initial value $(2.75 \%$ to $0.04 \%)$. This severe reduction of device performance with different polymer donors upon $3.6 \%$ photo-oxidised $\mathrm{PC}_{61} \mathrm{BM}$ solution makes it apparent that $\mathrm{PC}_{61} \mathrm{BM}$ photo-oxidation has a drastic impact on the photodegradation of these benchmark OPV systems. 


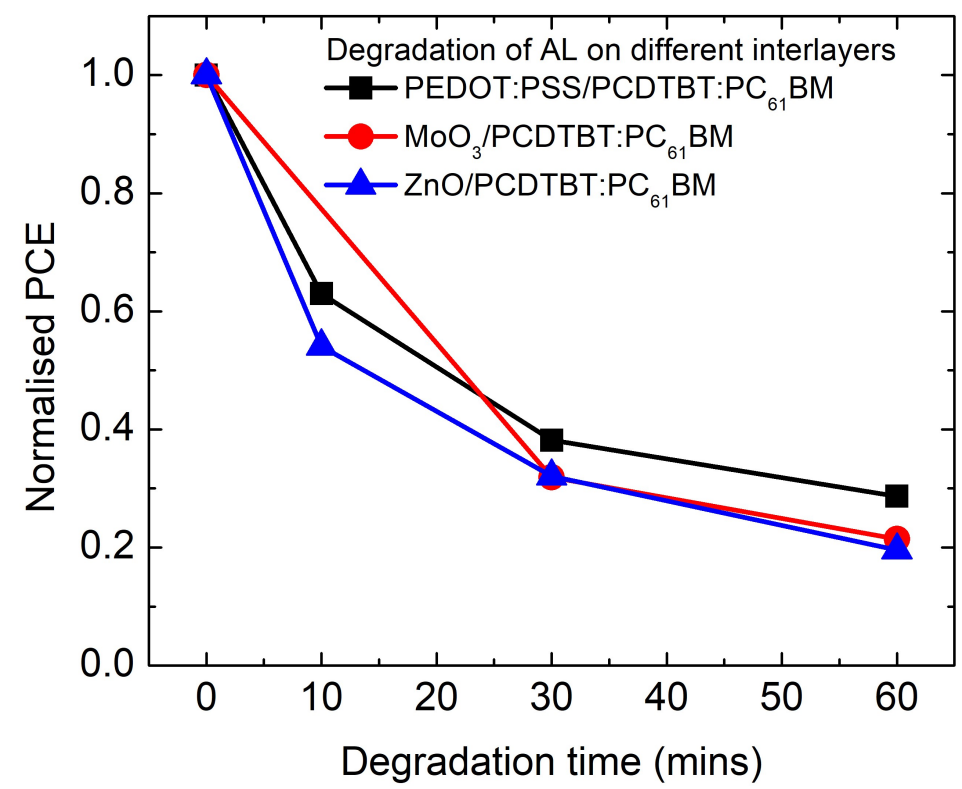

Figure 3.8: Normalised PCE of 1:2 PCDTBT:PC ${ }_{61} \mathrm{BM}$ devices with active layers photoaged on PEDOT:PSS, $\mathrm{MoO}_{3}$, and $\mathrm{ZnO}$ interlayers, prior to top electrode deposition. A Ca/Al as top electrode was used for PEDOT:PSS and $\mathrm{MoO}_{3}$ based devices and a $\mathrm{MoO}_{3} / \mathrm{Ag}$ top electrode was used for $\mathrm{ZnO}$ based devices.

To gain broader picture of the effect of $\mathrm{PC}_{61} \mathrm{BM}$ photo-oxidation on device performance, the photo-degradation study was also performed on a variety of device architectures with different interlayers. The PCDTBT: $\mathrm{PC}_{61} \mathrm{BM}$ active layer on top of either $\mathrm{MoO}_{3}$ hole transporting layer or $\mathrm{ZnO}$ electron transporting layer were photoaged prior to electrode deposition $\left(\mathrm{Ca} / \mathrm{Al}\right.$ and $\mathrm{MoO}_{3} / \mathrm{Ag}$ respectively) to give conventional and inverted devices respectively (Figure A.4). These devices have a similar trend in the reduction of device performance upon exposure to 1 sun AM1.5G illumination in air as those with the PEDOT:PSS based conventional device structure (PEDOT:PSS/PCDTBT:PC ${ }_{61} \mathrm{BM} / \mathrm{Ca} / \mathrm{Al}$ ) (Figures 3.8). The PCE of the fresh devices using PEDOT:PSS, $\mathrm{MoO}_{3}$, and $\mathrm{ZnO}$ are 5.01 $\%, 4.73 \%$, and $4.43 \%$, respectively (Figure A.5) and the percentage of PCE remaining after 60 minutes of photoaging were $29 \%, 21 \%$ and $20 \%$, respectively. This similarity in photodegradation between devices using different architectures and interlayers, shows that the interlayer beneath the active layer does not affect the amount of photo-oxidation of the $\mathrm{PC}_{61} \mathrm{BM}$ in the active layer. It also shows that the effect of photo-oxidation of $\mathrm{PC}_{61} \mathrm{BM}$ to device performance is fairly general, likely independent of the device architecture and interlayers employed. 

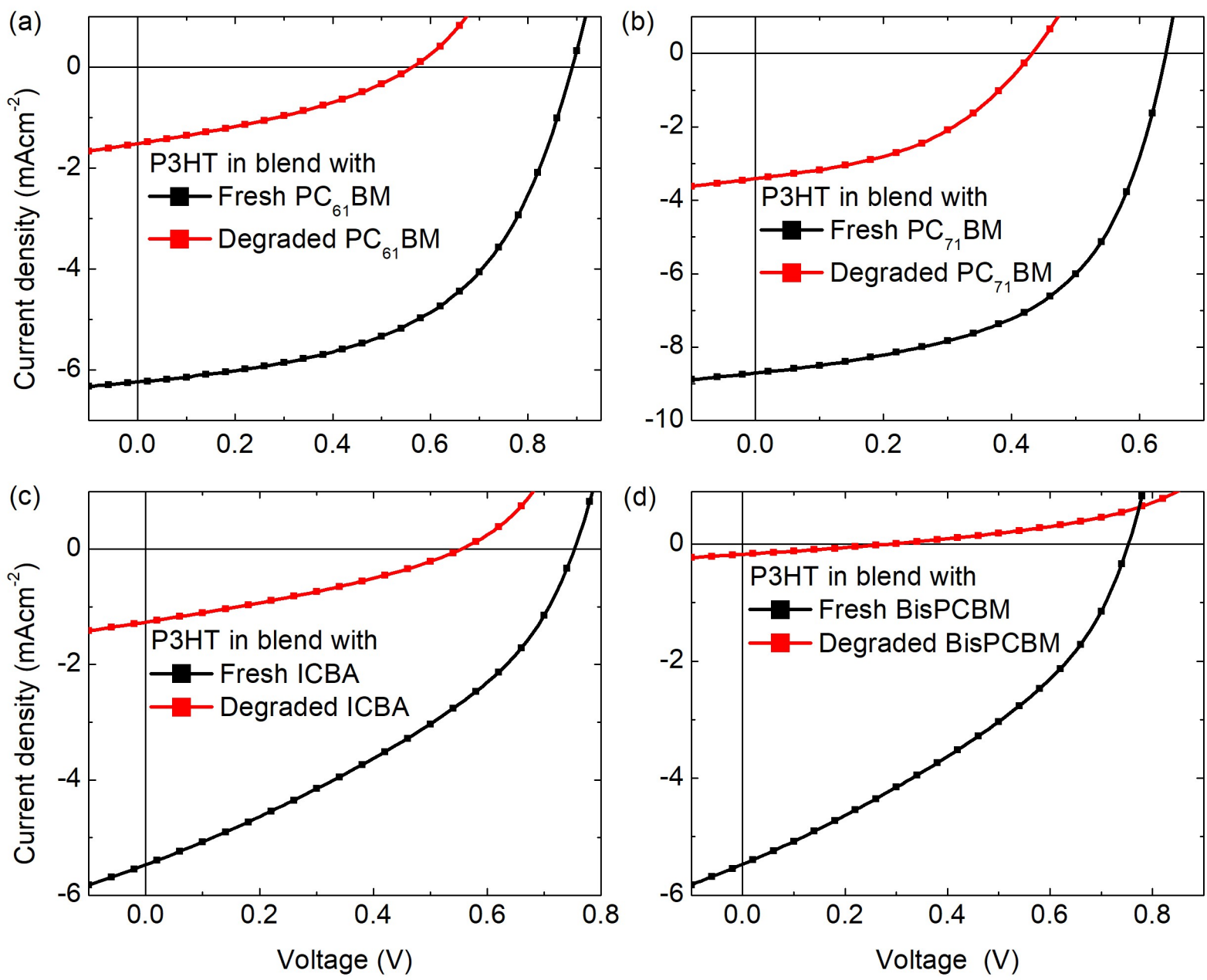

Figure 3.9: J-V characteristics of devices using P3HT blended with different OPV benchmark fullerene acceptors (a) $\mathrm{PC}_{61} \mathrm{BM}$, (b) $\mathrm{PC}_{71} \mathrm{BM}$ (c) ICBA and (d) BisPCBM which was fresh or had been degraded in air under 1 Sun AM1.5G illumination for 72 hours.

Table 3.2: Fullerene acceptors used in P3HT:fullerene devices and the initial PCE, PCE obtained when using fullerene solutions that had been aged for 72 hours in air under 1sun AM1.5G illumination and the percentage of PCE remaining when using the photo-aged solution compared to the fresh.

\begin{tabular}{||c|c|c|c||}
\hline \hline Fullerene & Initial PCE (\%) & Aged PCE (\%) & Percentage of PCE remaining (\%) \\
\hline $\mathrm{PC}_{71} \mathrm{BM}$ & 3.05 & 0.63 & 20.7 \\
$\mathrm{PC}_{61} \mathrm{BM}$ & 2.95 & 0.29 & 10.0 \\
$\mathrm{ICBA}$ & 2.94 & 0.23 & 7.8 \\
$\mathrm{BisPCBM}$ & 1.52 & 0.01 & 0.1 \\
\hline \hline
\end{tabular}

Finally, a range of benchmark fullerene acceptors were tested in devices to investigate whether the photo-oxidation was a general trait of fullerene acceptors. These fullerene acceptors were degraded as solutions for 72 hours under 1sun AM1.5G illumination in air. Inverted P3HT:fullerene devices were fabricated with (100\% content) and without these selectively degraded fullerene solutions and the resultant JV-curves are compared 
in Figure 3.9. It can be seen that each fullerene undergoes the same degradation process, with a severe effect to the device performance. When using the selectively degraded solution, $\mathrm{PC}_{71} \mathrm{BM}$ retained the most of its initial PCE, followed by $\mathrm{PC}_{61} \mathrm{BM}$, then ICBA, and BisPCBM completely degraded (Table 3.2). These results show that photo-oxidation is a problem for these benchmark fullerene acceptors in OPV and likely other fullerene based materials in optoelectronic devices.

\subsection{Conclusions}

The results in this chapter show the severe photodegradation of polymer:fullerene OSCs upon exposure to 1 sun AM1.5G illumination in air, either where the active layer is photoaged before top electrode deposition or the fullerene acceptors are selectively photoaged before blending with the polymer. The significant reduction in device performance correlates well with the photodegradation of the fullerenes which can undergo strong photo-oxidation. Remarkably, if just $1 \%$ of $\mathrm{PC}_{61} \mathrm{BM}$ within a device active layer were photo-oxidised, the device PCE was $\sim 65 \%$ lower than a fresh device. While polymer photodegradation did not have significant effect to the $\mathrm{V}_{\mathrm{oc}}$, photo-oxidation of $\mathrm{PC}_{61} \mathrm{BM}$ causes a significant drop in $\mathrm{V}_{\mathrm{oc}}$, hence the $\mathrm{V}_{\text {oc }}$ can be used as an indicator to identify if fullerenes have photodegraded in devices. This drop in $\mathrm{V}_{\mathrm{oc}}$ upon photo-oxidation of $\mathrm{PC}_{61} \mathrm{BM}$ was shown to have been caused by oxidised $\mathrm{PC}_{61} \mathrm{BM}$ having a deepened LUMO level with respect to fresh $\mathrm{PC}_{61} \mathrm{BM}$, causing electron trapping in the fullerene domains. Similar photodegradation of $\mathrm{V}_{\mathrm{oc}}, \mathrm{J}_{\mathrm{sc}}$ and FF was also observed for OCSs with standard and inverted device architectures, and different benchmark polymers and benchmark fullerenes, where the fullerenes were photoaged. These results show that the photoaging (photo-oxidation) of fullerenes have a general and detrimental effect to the photodegradation of polymer:fullerene OSCs. Therefore, in the remaining chapters, the photostability of fullerene acceptors is further studied, including the effect of aggregation (chapter 4) and LUMO energy (chapter 5), in addition to using non-fullerene acceptors (chapter 6). 


\section{REFERENCES}

[1] H. K. H. Lee, A. M. Telford, J. A. Rohr, M. F. Wyatt, J. Wu, A. De Castro Maciel, S. M. Tulandhar, E. Speller, J. McGettrick, J. R. Searle, S. Pont, T. Watson, T. Kirchartz, J. R. Durrant, W. C. Tsoi, J. Nelson, and Z. Li, "The Role of Fullerenes in The Environmental Stability of Polymer: Fullerene Solar Cells," Energy Environ. Sci, 2018.

[2] S. Shoaee and J. R. Durrant, "Oxygen diffusion dynamics in organic semiconductor films," J. Mater. Chem. C, vol. 3, no. 3, pp. 10079-10084, 2015.

[3] T. Jeranko, H. Tributsch, N. S. Sariciftci, and J. C. Hummelen, "Patterns of efficiency and degradation of composite polymer solar cells," Solar Energy Materials \&s Solar Cells, vol. 83, pp. 247-262, 2004.

[4] M. Jørgensen, K. Norrman, and F. C. Krebs, "Stability/degradation of polymer solar cells," Solar Energy Materials and Solar Cells, vol. 92, pp. 686-714, 2008.

[5] M. Jørgensen, K. Norrman, S. A. Gevorgyan, T. Tromholt, B. Andreasen, and F. C. Krebs, "Stability of polymer solar cells.," Advanced materials, vol. 24, pp. 580-612, 2012.

[6] Y. W. Soon, H. Cho, J. Low, H. Bronstein, I. McCulloch, and J. R. Durrant, "Correlating triplet yield, singlet oxygen generation and photochemical stability in polymer/fullerene blend films.," Chemical communications, vol. 49, pp. 1291-3, 2013.

[7] S. R. Cowan, W. L. Leong, N. Banerji, G. Dennler, and A. J. Heeger, "Identifying a Threshold Impurity Level for Organic Solar Cells: Enhanced First-Order Recombination Via Well-Defined PC84BM Traps in Organic Bulk Heterojunction Solar Cells," Advanced Functional Materials, vol. 21, pp. 3083-3092, 2011.

[8] A. J. Heeger, "25th Anniversary Article: Bulk Heterojunction Solar Cells: Understanding the Mechanism of Operation," Advanced Materials, vol. 26, pp. 10-28, 2013.

[9] J. C. Blakesley and D. Neher, "Relationship between energetic disorder and opencircuit voltage in bulk heterojunction organic solar cells," Physical Review B, vol. 84, p. $075210,2011$.

[10] M. O. Reese, A. M. Nardes, B. L. Rupert, R. E. Larsen, D. C. Olson, M. T. Lloyd, S. E. Shaheen, D. S. Ginley, G. Rumbles, and N. Kopidakis, "Photoinduced Degradation of Polymer and Polymer-Fullerene Active Layers: Experiment and Theory," Advanced Functional Materials, vol. 20, pp. 3476-3483, 2010. 
[11] Z. Xiao, J. Yao, D. Yang, F. Wang, S. Huang, L. Gan, Z. Jia, Z. Jiang, X. Yang, B. Zheng, G. Yuan, S. Zhang, and Z. Wang, "Synthesis of [59]Fullerenones through Peroxide-Mediated Stepwise Cleavage of Fullerene Skeleton Bonds and X-ray Structures of Their Water-Encapsulated Open-Cage Complexes," Journal of the American Chemical Society, vol. 129, pp. 16149-16162, 2007. 


\section{THE IMPACT OF AGGREGATION TO THE STABILITY OF ORGANIC PHOTOVOLTAIC FILMS AND DEVICES}

This chapter finds the photochemistry and stability of fullerene films and devices is strongly dependent upon film nanomorphology. In particular, PS: $\mathrm{PC}_{61} \mathrm{BM}$ blend films were found to be more photochemically unstable than more aggregated neat films, correlating with an increase in observed photo-oxidation and increased oxygen quenching of $\mathrm{PC}_{61} \mathrm{BM}$ triplet states, suggesting $\mathrm{PC}_{61} \mathrm{BM}$ photo-oxidation is primarily due to tripletmediated singlet oxygen generation (i.e. via energy transfer from the fullerene triplet states to molecular oxygen, forming highly reactive singlet oxygen). The electron mobility degraded substantially with fullerene photo-oxidation, indicative of electron trap formation which was supported by observation of red shifts in PL and EL with photo-oxidation, and TD-DFT calculations of defect generation. The study was then extended to investigate how $\mathrm{PC}_{61} \mathrm{BM}$ aggregation affects the stability of donor polymer (PCDTBT):fullerene blend films and devices. Less aggregated $\mathrm{PC}_{61} \mathrm{BM}$ were more photochemically unstable for both $\mathrm{PC}_{61} \mathrm{BM}$ and $\mathrm{PCDTBT}$, correlating with an increase in the amount of $\mathrm{PC}_{61} \mathrm{BM}$ photo-oxidation as measured by mass spectrometry, and a decrease in device stability, particularly a significant drop in $\mathrm{V}_{\mathrm{oc}}$. This correlated with an increase in oxygen quenching of the polymer triplet state, suggesting again the dominate degradation pathway is triplet-mediated singlet oxygen generation but this time via the polymer triplet state. 


\subsection{Introduction}

The first part of this chapter presents a detailed study of the photodegradation in air of neat (aggregated) $\mathrm{PC}_{61} \mathrm{BM}$ films and films where $\mathrm{PC}_{61} \mathrm{BM}$ is dispersed in a polystyrene (PS) matrix. PS has previously been shown to be an effective matrix to disperse $\mathrm{PC}_{61}$ BM.[1-3] PS has also been used as an additive to improve the performance of organic small molecule:fullerene $\mathrm{OPV},[4]$ and blended with $\mathrm{PC}_{61} \mathrm{BM}$ to form the electron transport layer in "OPV type" perovskite solar cells with improved homogeneity and suppressed electron recombination.[5] Firstly, the photo-oxidation of fullerene neat and blend films was investigated using UV-vis, FTIR and XPS spectroscopies. This photodegradation was then correlated to the triplet exciton kinetics via TAS measurements, from which a degradation mechanism could be suggested. The optoelectronic properties of the fullerene films/devices were then investigated by characterising electron-only devices with current density vs electric field measurements and measuring the PL/EL which could be compared with TD-DFT models.

The second part of this chapter extends the the fullerene aggregation-related air photostability studies from PS:PC ${ }_{61} \mathrm{BM}$ films to photoactive polymer (PCDTBT) : $\mathrm{PC}_{61} \mathrm{BM}$ films, which approximates the effect of aggregation to device stability. GIWAXS measurements quantified the degree of crystallisation (aggregation) of the fullerene in blend films with different wt\% blend ratios. Then, UV-vis and mass spectroscopies were used to probe the photodegradation and quantify the photo-oxidation. Transient absorption kinetics were measured to help determine a degradation pathway for this system and OPV devices were measured to determine how this photo-oxidation affected device stability.

\subsection{Experimental details}

\subsubsection{Part 1: Fullerene and PS:fullerene}

Film preparation: Solutions for films were prepared by dissolving PS in CB at $8 \mathrm{mg} / \mathrm{ml}$, and the relevant amount of $\mathrm{PC}_{61} \mathrm{BM}$ powder was added to form the different wt\% blend solutions. The concentration of the $\mathrm{PC}_{61} \mathrm{BM}$ in the solution was kept below the solubility limit of CB $(24 \mathrm{mg} / \mathrm{ml})$ by diluting any solution which surpassed this limit with extra CB. For neat films, the $\mathrm{PC}_{61} \mathrm{BM}$ powders were dissolved in $\mathrm{CF}$ at $24 \mathrm{mg} / \mathrm{ml}$ [and for ATRFTIR: it was $27 \mathrm{mg} / \mathrm{ml}$ ] as the neat $\mathrm{PC}_{61} \mathrm{BM}$ solutions yielded better film homogeneity with $\mathrm{CF}$ rather than $\mathrm{CB}$. The solutions were then spin-coated onto the substrates to form $\approx 100 \mathrm{~nm}$ thick films. Quartz (UV-Vis absorbance), aluminium (ATR-FTIR), indium tin 
oxide (ITO) (XPS)) and glass (PL and TAS) substrates were used. All the substrates were cleaned as described in Chapter 2.

Device preparation and testing: Electron-only devices (devices where only the electrons are collected) were fabricated by Dr. Harrison K.H. Lee (Swansea University) and characterised by myself. They were fabricated with a structure of ITO/ aluminium $(\mathrm{Al}) / \mathrm{PC}_{61} \mathrm{BM} /$ calcium $(\mathrm{Ca}) / \mathrm{Al} .50 \mathrm{~nm} \mathrm{Al}$ was thermally evaporated onto cleaned ITO glass substrates at $2 \times 10^{-5}$ mbar. $\mathrm{CB}$ was first spin-coated on the ITO/Al substrates to help with the wetting, followed by the $\mathrm{PC}_{61} \mathrm{BM}$ solution, with concentration of $50 \mathrm{mg} / \mathrm{ml}$ (to ensure thick films) in $\mathrm{CB}$ (heated and stirred overnight at $60{ }^{\circ} \mathrm{C}$ and filtered with a $0.45 \mu \mathrm{m}$ PTFE), forming a $\mathrm{PC}_{61} \mathrm{BM}$ layer with thickness $\sim 170 \mathrm{~nm}$. The films were then photoaged, after which $30 \mathrm{~nm}$ Ca and $100 \mathrm{~nm}$ Al were sequentially thermally evaporated onto the $\mathrm{PC}_{61} \mathrm{BM}$ layers to complete the devices with an active area of $0.15 \mathrm{~cm}^{2}$. All the devices were encapsulated with glass slides and epoxy. Devices for EL were fabricated by spincoating $\mathrm{PC} 6{ }_{61} \mathrm{BM}$ solution $(24 \mathrm{mg} / \mathrm{ml}$ in $\mathrm{CF}$ ) onto PEDOT:PSS coated ITO substrates (thickness of $\mathrm{PC}_{61} \mathrm{BM}$ film $\sim 100 \mathrm{~nm}$ ), followed by the same thermal evaporation of the top electrode and encapsulation. Electron-only devices were characterised with a Keithley 2400 source meter where electrons were injected from the $\mathrm{Ca} / \mathrm{Al}$ electrodes.

Photoaging: Photoaging of the films and devices was performed under $\sim 0.9-1$ Sun AM1.5G illumination in ambient conditions and temperature using a solar simulator for the aging time specified in the text.

Characterisation: The UV-Vis absorbance was calculated from the transmission and diffuse reflectance measurements performed on a UV-2600 Shimadzu UV-Vis spectrophotometer with an integrating sphere attachment.

ATIR-FTIR spectra were acquired using the set-up at Swansea University as described in Chapter 2. ATR-FTIR spectra were processed from measurements that consisted of 4 scans and $1 \mathrm{~cm}^{-1}$ resolution. The spectra were baseline corrected in the Spectrum 10 software. Three spectra were taken from different regions on the thin film and averaged. The FTIR absorbance of the $\mathrm{C}=\mathrm{O}$ stretch of the side chain of $\mathrm{PC}_{61} \mathrm{BM}$ was used to normalise the data, as it is not that sensitive to the photoaging.

XPS spectra were recorded using the set-up described in Chapter 2 by Dr. James McGettrick (Swansea University) using a charge neutralizer to limit differential charging. As no adventitious carbon peak is present in the bulk, the main carbon peak is charge referenced to $284.5 \mathrm{eV}$.[6, 7] Depth profiles were generated by rastering a $2.5 \mathrm{kV}$ Ar500+ beam over a $2 \times 2 \mathrm{~mm}$ area. The etching conditions were carefully tuned, so that the chemistry of the films was not significantly altered by the etching. During depth profiles, a $110 \mu \mathrm{m}$ aperture was used to limit any influence of crater edges. Wide scans were 
recorded with a pass energy of $160 \mathrm{eV}$ and dwell time of $198 \mathrm{~ms}$, high resolution data at a pass energy of $40 \mathrm{eV}$. The data was fitted using CASA XPS with Shirley backgrounds. Film thicknesses, and hence etch rates, were confirmed after each etch by measurement with a Dektak 150 stylus profilometer. Data was analysed and interpreted by myself.

The microsecond TAS measurements were done at Imperial College London with the assistance of Dr. Ching-Hong Tan. The set-up, methodology and data analysis are described in Chapter 2. The films were selectively pumped at $355 \mathrm{~nm}$, with an intensity of $7 \mu \mathrm{Jcm}^{-2}$ and a repetition rate of $20 \mathrm{~Hz}$. The transient data were probed at $700 \mathrm{~nm}$. Measurements were undertaken under both dry oxygen and dry nitrogen environments. The data analysis was undertaken by myself.

PL and EL spectra were measured by Dr. Andrew Telford (Imperial College London), using the set-up described in Chapter 2. EL spectra were collected at an injection current density of $200 \mathrm{~mA} / \mathrm{cm}^{2}$. PL spectra were collected using a $473 \mathrm{~nm}$ continuous wave laser as the excitation source, with optical power density of $20-65 \mathrm{mWcm}^{-2}$. Data for both fresh and photoaged $\mathrm{PC}_{61} \mathrm{BM}$ devices were collected on three different pixels (or areas) on two separate devices. The data was analysed by myself.

Electronic structure calculations of the epoxides of $\mathrm{PC}_{61} \mathrm{BM}$ were carried out by Ms. Beth Rice (Imperial College London) using density functional theory and time-dependent density functional theory, as explain in Chapter 2. First, all possible single epoxides (one epoxide defect on one of the 6-6 carbon bonds of $\mathrm{PC}_{61} \mathrm{BM}$ ) were calculated, and then a number of double epoxides, as well as the diols and carbonyl defects, which may be expected to develop from epoxide following the mechanism published by Xiao et al.[8] In each case, the total energy of the optimised defected structures was calculated to find the lowest energy structures. The results of these calculations were interpreted by myself. 


\subsubsection{Part 2: PCDTBT:fullerene}

\section{Film preparation}

PCDTBT:PC ${ }_{61} \mathrm{BM}$ blend films of different blend ratios were made using the donor and acceptor concentrations outlined in Table 4.1 in dichlorobenzene (DCB) and stirred at $90^{\circ} \mathrm{C}$ overnight.

Table 4.1: Concentration of PCDTBT (D) and $\mathrm{PC}_{61} \mathrm{BM}(\mathrm{A})$ in DCB for solutions of different blend wt ratios and the spin speeds employed to make thin films with thicknesses $\approx 70 \mathrm{~nm}$.

\begin{tabular}{||c|c|c|c||}
\hline \hline D:A wt ratio & PCDTBT $(\mathrm{mg} / \mathrm{ml})$ & $\mathrm{PC}_{61} \mathrm{BM}(\mathrm{mg} / \mathrm{ml})$ & spin speed $(\mathrm{rpm})$ \\
\hline $1: 8$ & 5 & 40 & 1000 \\
$1: 4$ & 6 & 24 & 1000 \\
$1: 2$ & 7.5 & 15 & 800 \\
$1: 1$ & 7.5 & 7.5 & 800 \\
$2: 1$ & 8 & 4 & 800 \\
$4: 1$ & 8 & 2 & 800 \\
$8: 1$ & 8 & 1 & 800 \\
\hline \hline
\end{tabular}

To prepare PCDTBT:PC ${ }_{61} \mathrm{BM}$ films of decreasing film thickness, a master solution was first prepared with $6 \mathrm{mg} / \mathrm{ml} \mathrm{PCDTBT}$ and $12 \mathrm{mg} / \mathrm{ml} \mathrm{PC}_{61} \mathrm{BM}$ in $\mathrm{CB}$, i.e. 1:2 wt ratio. Then, the master solution was diluted with $\mathrm{CB}$ to the concentrations given in Table 4.2, keeping the same donor:acceptor wt ratio and stirred at $90^{\circ} \mathrm{C}$ overnight. These solution were spun at $800 \mathrm{rpm}$ at $7500 \mathrm{rpms}^{-1}$ acceleration for 60 seconds to yield the film thicknesses stated in Table 4.2. The films were spincoated on silicon wafers for GIWAXS measurements and quartz substrates for UV-vis and transient absorption spectroscopies. Samples (in powder form) for mass spectroscopy measurements were prepared by spincoating films onto glass substrates, scraping the film off into a vial, before and after photoaging in air.

Table 4.2: PCDTBT:PC ${ }_{61} \mathrm{BM}$ films (1:2 wt\% ratio) with decreasing thicknesses which are controlled by reducing the concentration of PCDTBT and $\mathrm{PC}_{61} \mathrm{BM}$ (in $\mathrm{CB}$ ) in solutions.

\begin{tabular}{||c|c|c||}
\hline \hline PCDTBT $(\mathrm{mg} / \mathrm{ml})$ & $\mathrm{PC}_{61} \mathrm{BM}(\mathrm{mg} / \mathrm{ml})$ & Film thickness $(\mathrm{nm})$ \\
\hline 6 & 12 & 93 \\
5.4 & 10.8 & 78 \\
4.8 & 9.6 & 75 \\
4.2 & 8.4 & 56 \\
3.6 & 7.2 & 45 \\
3 & 6 & 35 \\
\hline
\end{tabular}


Device fabrication Devices were fabricated with an inverted structure of ITO/ $\mathrm{ZnO} /$ PCDTBT: $\mathrm{PC}_{61} \mathrm{BM} / \mathrm{MoO}_{3} / \mathrm{Ag}$ following the method in Chapter 2. All the devices were encapsulated with glass slides and epoxy. The devices with different active layer blend ratios were devices were fabricated and characterised with Dr. Harrison Lee. This included external quantum efficiency (EQE) measurements carried out using a QE X10 system (PV Measurements) in the wavelength range between 300 and $850 \mathrm{~nm}$ in direct current (DC) mode with a light spot of less than $10 \mathrm{~mm}^{2}$. The devices with different active layer thicknesses were fabricated and characterised by myself.

Photoaging Photoaging of the films/devices was performed under $~ 1$ Sun AM1.5G in ambient conditions and temperature using a solar simulator for the aging time specified in the text.

Characterisation GIWAXS measurements were performed with Dr. Andrew Parnell (Sheffield University) using the laboratory beamlined detailed in Chapter 2. Scherrer analysis of the spectra was performed by myself.

The UV-vis absorbance spectra were measured at Swansea University with a Perkin Elmer Lambda 750 spectrophotometer (PerkinElmer, Seer Green, UK), using a quartz substrate as a reference sample for calibration.

Microsecond transient absorption kinetics were measured by Dr. Stoichko Dimitrov (Swansea University) using the set-up at Imperial College London described in Chapter 2. Samples were excited at $560 \mathrm{~nm}$ with pulses $\left(1.67 \mu \mathrm{Jcm}^{-2},<10 \mathrm{~ns}\right.$ and $\left.20 \mathrm{~Hz}\right)$ and probed at $1100 \mathrm{~nm}$. The data was interpreted by myself.

\subsection{Results and discussion}

\subsubsection{Effect of fullerene aggregation on the photostability of fullerene films in air}

\subsubsection{Spectroscopic studies}

UV-vis absorbance measurements were taken as photobleaching is routinely used to probe the degradation of conjugated materials. $[9,10]$ 

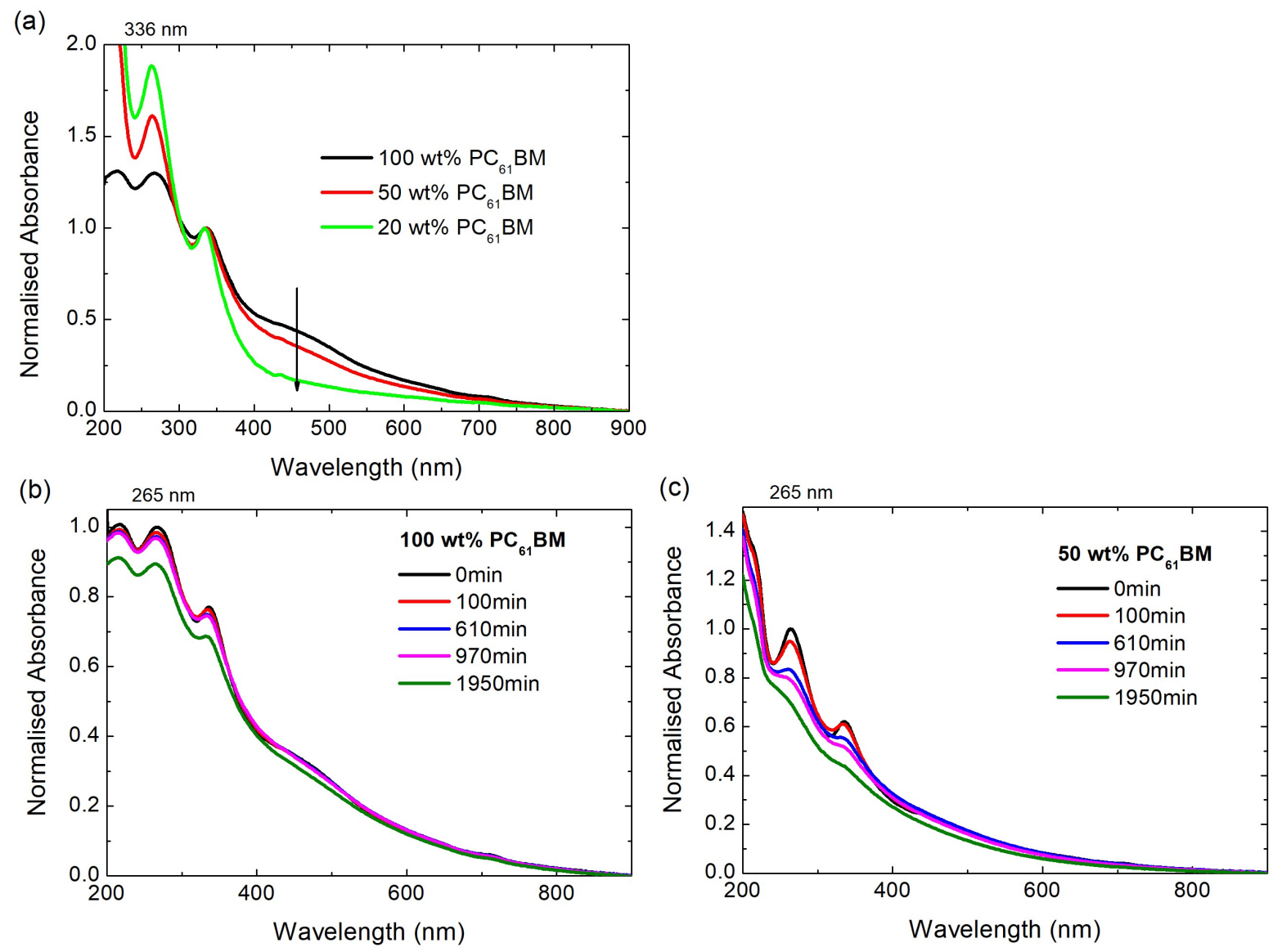

Figure 4.1: UV-Vis absorbance spectra of (a) neat $\mathrm{PC}_{61} \mathrm{BM}$ and $\mathrm{PC}_{61} \mathrm{BM}$ :PS films without photoaging (normalised at $336 \mathrm{~nm}$ ), and of (b) neat and (c) $50 \mathrm{wt} \% \mathrm{PC}_{61} \mathrm{BM}$ films, normalised to $\sim 265 \mathrm{~nm}$, with increasing photoaging time under AM1.5 irradiation in air.

Figure 4.1 (a) shows the $\mathrm{UV}$-vis absorbance spectra for neat $\mathrm{PC}_{61} \mathrm{BM}$ and $\mathrm{PC}_{61} \mathrm{BM}: \mathrm{PS}$ blend films without photoaging, normalised to the $\mathrm{PC}_{61} \mathrm{BM}$ peak at $336 \mathrm{~nm}$. As the wt\% $\mathrm{PC}_{61} \mathrm{BM}$ is increased, the relative absorbance from 350 - $750 \mathrm{~nm}$ increases; consistent with $\mathrm{PC}_{61} \mathrm{BM}$ molecules being more aggregated in the higher wt\% films.[11, 12] The heightened absorbance in the visible range for more aggregated $\mathrm{PC}_{61} \mathrm{BM}$ is assigned to intermolecular interactions resulting in significant CT absorbance. The neat PS film has negligible absorbance in this spectral range (Figure B.1). Figure $4.1 \mathrm{~b}$-c show how the neat and $50 \mathrm{wt} \% \mathrm{PC}_{61} \mathrm{BM}$ films are photobleached in ambient air under AM1.5 irradiation over $1950 \mathrm{~min}(32.5 \mathrm{hr})$. The spectra are normalised to the $\mathrm{PC}_{61} \mathrm{BM}$ peak at $\sim 265 \mathrm{~nm}$. The photobleaching of 80 and $20 \mathrm{wt} \%$ films are shown in Figure B.2. Upon comparing the evolution of these spectra, it can be observed that, while there is minimal photobleaching of the neat $\mathrm{PC}_{61} \mathrm{BM}$ film, the blend $\mathrm{PC}_{61} \mathrm{BM}$ films were significantly photobleached, with the less aggregrated films undergoing more photobleaching. This indicates that the less aggregated the $\mathrm{PC}_{61} \mathrm{BM}$ film, the more susceptible it is to photobleaching. 

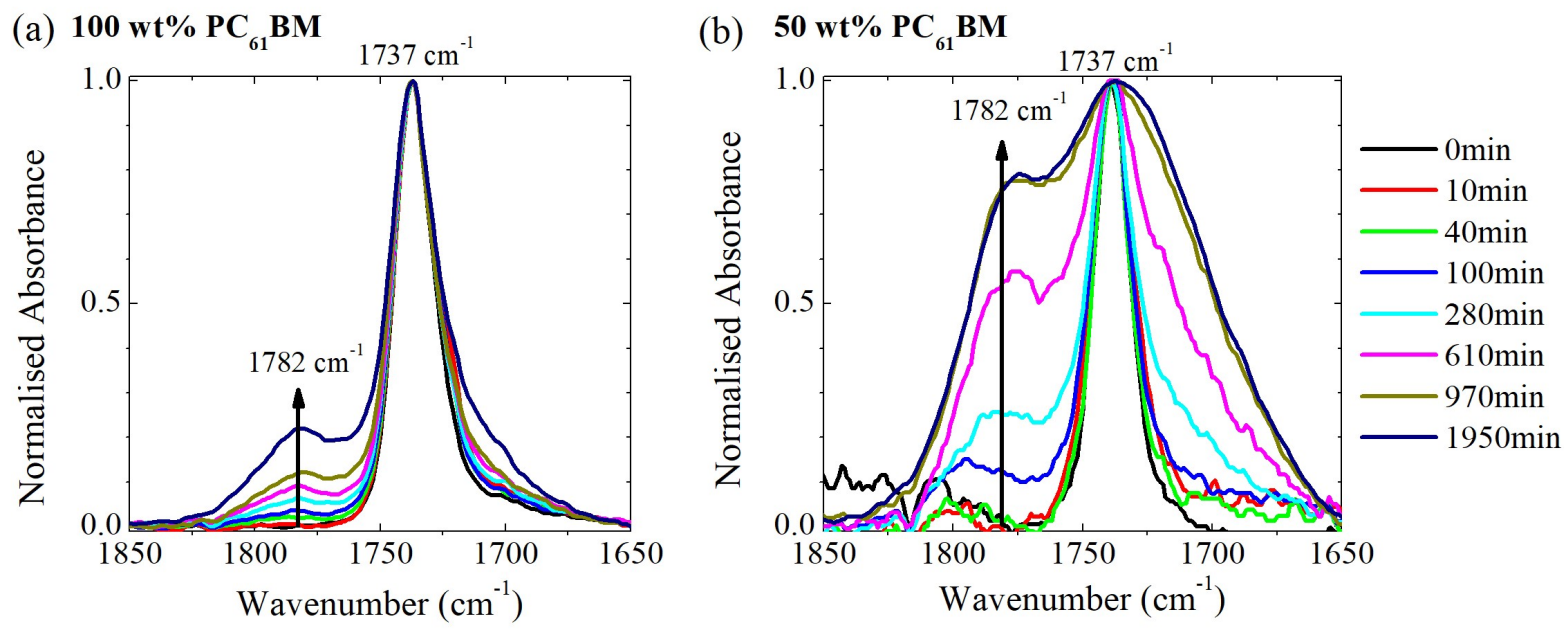

Figure 4.2: Normalised ATR-FTIR absorbance spectra of (a) neat and 50 wt\% $\mathrm{PC}_{61} \mathrm{BM}$ films respectively, with increasing photoaging times.

Next, ATR-FTIR spectroscopy was used to investigate the chemical nature of this photodegradation. Figures 4.2 (a) and (b) compare the normalised ATR-FTIR absorbance spectra of a neat $\mathrm{PC}_{61} \mathrm{BM}$ film and $50 \mathrm{wt} \% \mathrm{PC}_{61} \mathrm{BM}$ film, respectively, as a function of photoaging time. The spectra are probed in the carbonyl region and normalised to the prominent $\mathrm{PC}_{61} \mathrm{BM}$ FTIR absorption peak at $1737 \mathrm{~cm}^{-1}$, assigned to the $\mathrm{C}=\mathrm{O}$ stretch of the ester functional group of the $\mathrm{PC}_{61} \mathrm{BM}$ side chain.[13] The magnitude of a new peak at $1782 \mathrm{~cm}^{-1}$ increased (relative to the $1737 \mathrm{~cm}^{-1}$ peak) with photoaging time (observable from $\sim 100 \mathrm{~min}$ for the neat $\mathrm{PC}_{61} \mathrm{BM}$ film. The peak at $1782 \mathrm{~cm}^{-1}$ has previously been assigned to the formation of carbonyls (the stretching mode of $\mathrm{C}=\mathrm{O}$ ), likely associated with oxidation on the $\mathrm{PC}_{61} \mathrm{BM}$ cage. $[14,15]$ Notably, the relative magnitude of this peak is significantly higher for the $50 \mathrm{wt} \% \mathrm{PC}_{61} \mathrm{BM}$ film compared to the neat film, with the same photoaging time (0.80 vs 0.22 after 1950 min of photoaging). Figure B.3 shows the normalised FTIR absorbance spectra for 95, 90, 80 and 20 wt\% $\mathrm{PC}_{61} \mathrm{BM}$ films with up to 1950 mins of photoaging. These results show that the lower wt\% of $\mathrm{PC}_{61} \mathrm{BM}$ in the film, the higher the relative intensity of the carbonyl peak at $1782 \mathrm{~cm}^{-1}$, implying that the less aggregated $\mathrm{PC}_{61} \mathrm{BM}$ film is significantly easier to photo-oxidise, which coincides with its higher tendency to photobleach. FTIR signals measured from neat and photoaged PS films were negligible for the range investigated (Figure B.4). 

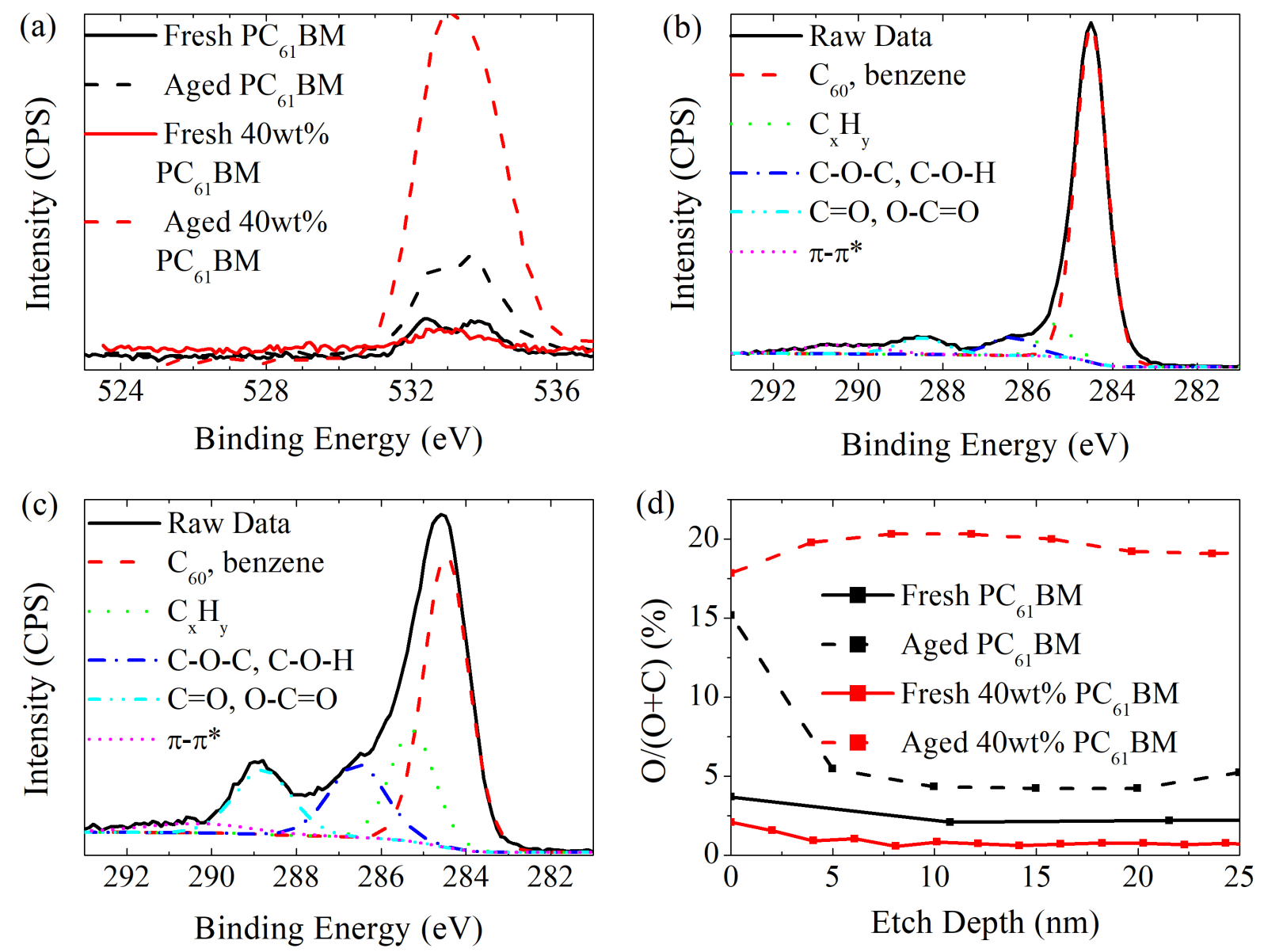

Figure 4.3: (a) XPS spectrum (oxygen 1s envelope) of neat and $40 \mathrm{wt} \% \mathrm{PC}_{61} \mathrm{BM}$ films, before and after 920 minutes of photoaging; XPS spectra (carbon 1s envelope fitted with peaks for different carbon environments) of (b) neat $\mathrm{PC}_{61} \mathrm{BM}$ and (c) 40 wt\% $\mathrm{PC}_{61} \mathrm{BM}$ films, both after 920 minutes of photoaging; (d) atomic percentage of oxygen as a function of depth of neat and $40 \mathrm{wt} \% \mathrm{PC}_{61} \mathrm{BM}$ films, before and after 920 minutes of photoaging, respectively.

Further chemical analysis of this $\mathrm{PC}_{61} \mathrm{BM}$ photo-oxidation was undertaken using XPS, a surface sensitive technique. Figure 4.3a compares the XPS oxygen 1s envelope $(\mathrm{O}(1 \mathrm{~s}))$ of neat and $40 \mathrm{wt} \% \mathrm{PC}_{61} \mathrm{BM}$ films, before and after $920 \mathrm{~min}$ of photoaging. The amount of measured oxygen increases after the photoaging, with significantly more oxygen observed for the photoaged $40 \mathrm{wt} \% \mathrm{PC}_{61} \mathrm{BM}$ film than the neat film. (No $\mathrm{O}(1 \mathrm{~s})$ signal is observed from neat, fresh, or photoaged PS film.[16]) Figures 4.3b-c show the XPS spectra for the carbon $1 \mathrm{~s}$ envelope $(\mathrm{C}(1 \mathrm{~s}))$ of neat and $40 \mathrm{wt} \% \mathrm{PC}_{61} \mathrm{BM}$ films, respectively, both photoaged for $920 \mathrm{~min}$. The $\mathrm{C}(1 \mathrm{~s})$ envelope can be fitted with a number of peaks for different carbon environments (Table B.1). For the photoaged neat $\mathrm{PC}_{61} \mathrm{BM}$ film, there is a minor increase in the magnitude of the peaks at 286.1 and $288.3 \mathrm{eV}$; the increase in these peaks is much larger for the photoaged $40 \mathrm{wt} \% \mathrm{PC}_{61} \mathrm{BM}$ film (the XPS $\mathrm{C}(1 \mathrm{~s}$ ) envelope spectra for fresh films can be found in Figure B.5 and the $\mathrm{O}(1 \mathrm{~s})$ envelope for neat $\mathrm{PC}_{61} \mathrm{BM}$ film before and after $1 \mathrm{hr}$ of photoaging in Figure B.6). The broadened peak at $286.1 \mathrm{eV}$ is consistent with oxidised species such as C-O-C (epoxide), C-O-H, or 
$\mathrm{C}-\mathrm{O}$, while and the peak at $288.3 \mathrm{eV}$ is consistent with carbonyls such as $\mathrm{C}=\mathrm{O}$ or $\mathrm{O}-\mathrm{C}=\mathrm{O}$ (including an expected contribution from the $\mathrm{PC}_{61} \mathrm{BM}$ ester side chain).[17, 18] $\mathrm{C}(1 \mathrm{~S})$ envelopes of fresh and photo-aged PS films do not exhibit significant photo-oxidation as shown in reference [16].

It has been suggested that $\mathrm{PC}_{61} \mathrm{BM}$ can be photo-oxidised to form epoxides, then diols, and then carbonyls under sufficient photoaging (Figure B.7).[8] This data supports this mechanism, and it is therefore likely that in addition to the carbonyl defects observable in the FTIR spectra there is a substantial and probably larger population of epoxide defects. The $\mathrm{C}(1 \mathrm{~s})$ results show that the oxygen was chemically bound to the carbon atoms, and the level of photo-oxidation is much more significant for the less aggregated $40 \mathrm{wt} \% \mathrm{PC}_{61} \mathrm{BM}$ film, consistent with the UV-vis and FTIR photoaging data (Figures 4.1 and 4.2 , respectively).

XPS is able to form a depth profile of samples by etching through films with an argon gas cluster ion source gun and quantifying the chemical composition after each etch. Figure $4.3 \mathrm{~d}$ shows the atomic percentage of oxygen as a function of depth into the neat and $40 \mathrm{wt} \% \mathrm{PC}_{61} \mathrm{BM}$ films, before and after $920 \mathrm{~min}$ of photoaging. A $2.7 \%$ oxygen contribution would be expected from the ester group in neat $\mathrm{PC}_{61} \mathrm{BM}$ films. The lower oxygen percentage in the nonaged blend film is consistent with dilution of $\mathrm{PC}_{61} \mathrm{BM}$ by $\mathrm{PS}$, which contains no oxygen. Interestingly, the photo-oxidation on the surface of the neat $\mathrm{PC}_{61} \mathrm{BM}$ film (oxygen atomic concentration $\sim 15.2 \%$ ) is much higher than in the bulk $(\sim 4.2 \%)$, while significant photo-oxidation continues deep into the bulk for the $40 \mathrm{wt} \%$ $\mathrm{PC}_{61} \mathrm{BM}$ film ( 17.9 - 20.3\%). The evolution of the $\mathrm{C}(1 \mathrm{~s})$ and $\mathrm{O}(1 \mathrm{~S})$ envelopes depth of $\mathrm{PC}_{61} \mathrm{BM}$ and $40 \mathrm{wt} \% \mathrm{PC}_{61} \mathrm{BM}$ films is shown and discussed in detail in reference [16].

The epoxide:carbonyl ratio $(\mathrm{C}-\mathrm{O}: \mathrm{C}=\mathrm{O})$ which was estimated from the $\mathrm{C}(1 \mathrm{~s})$ envelope and the total measured oxygen are plotted as a function of etch depth for the degraded blend film (Figure B.8). The epoxide:carbonyl ratio is quite constant up to $60 \mathrm{~nm}$ below the surface. At a depth of $>60 \mathrm{~nm}$, this ratio increases slightly together with a decrease oxidation, which is consistent with epoxide formation occurring first. It is reasonable to have less photo-oxidation at the deeper part of the film, as it will be relatively more difficult for oxygen to penetrate to these depths. However, as the difference in the epoxide:carbonyl ratio is small, the photo-oxidation should not be dominated by the penetration of oxygen, implying that the significant difference in photo-oxidation between the films should also not be related to the penetration of oxygen. 


\subsubsection{Investigation into the photo-oxidation mechanism}

In order to gain insight on the mechanism of the photo-oxidation, microsecond TAS measurements were performed on the $\mathrm{PC}_{61} \mathrm{BM}$ and $\mathrm{PS}: \mathrm{PC}_{61} \mathrm{BM}$ films under nitrogen and oxygen. The films were selectively excited in the $\mathrm{PC}_{61} \mathrm{BM}$ absorption region (355 $\mathrm{nm}$ ) and probed at their triplet $\mathrm{T}_{1} \rightarrow \mathrm{T}_{\mathrm{n}}$ absorption region (700 nm) (Figure B.9a).[19] The decays were analysed using a biexponential function and lifetimes extracted from the fitted decays. On this time scale, singlet states have undergone intersystem crossing to triplet states as shown in Figure B.9a, and by Chow et al.[20] In addition, the decays measured under oxygen are quenched when compared to those under nitrogen (Figure 4.4a and Table 4.3), consistent with previous observations that triplet states are quenched by triplet ground state oxygen.[21]

(a)

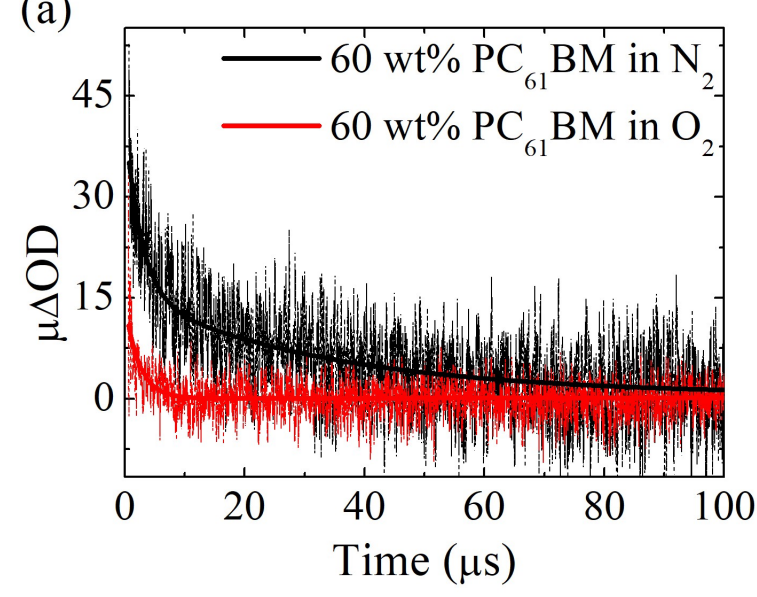

(b)

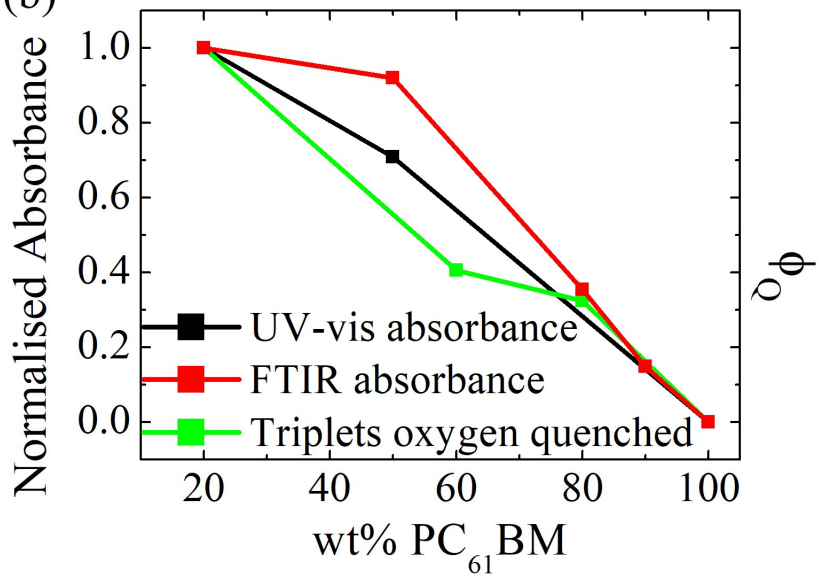

Figure 4.4: (a) Transient absorption decays of $\mathrm{PC}_{61} \mathrm{BM}$ triplet excitons pumped at 355 $\mathrm{nm}$ and probed at $700 \mathrm{~nm}$ of a $60 \mathrm{wt} \% \mathrm{PC}_{61} \mathrm{BM}$ film in nitrogen and oxygen environments, (b) fractional loss of UV-Vis absorbance (at $265 \mathrm{~nm}$ ), relative growth of $\mathrm{C}=\mathrm{O}$ FTIR signal (at $1782 \mathrm{~cm}^{-1}$ ) and relative yield of triplet states quenched by oxygen (calculated using Eq. 3), all normalised and as a function of wt\% $\mathrm{PC}_{61} \mathrm{BM}$, following photoaging of 1950 mins (UV-Vis absorbance), 970 mins (FTIR), and 0 mins (TAS) respectively.

Table 4.3: Properties of the triplet kinetics in blend $\mathrm{PC}_{61} \mathrm{BM}$ films: where $\tau_{\mathrm{N}_{2}}$ and $\tau_{\mathrm{O}_{2}}$ are the triplet lifetimes under nitrogen and oxygen respectively, $Y_{\mathrm{N}_{2}}$ is the relative triplet yield in a nitrogen environment (corrected for photons absorbed), $\Phi_{\mathrm{O}_{2}}$ is the efficiency of oxygen quenching, $k_{\mathrm{O}_{2}}$ is the rate constant of oxygen quenching, and $\Phi_{\mathrm{Q}}$ is the relative fraction of triplets oxygen quenched.

\begin{tabular}{||c|c|c|c|c|c|c||}
\hline \hline $\begin{array}{c}\mathrm{wt} \% \\
\mathrm{PC}_{61} \mathrm{BM}\end{array}$ & $\begin{array}{c}\tau_{\mathrm{N}_{2}} \\
(\mu \mathrm{s})\end{array}$ & $\begin{array}{c}\tau_{\mathrm{O}_{2}} \\
(\mu \mathrm{s})\end{array}$ & $Y_{\mathrm{N}_{2}}$ & $\Phi_{\mathrm{O}_{2}}$ & $\begin{array}{c}k_{\mathrm{O}_{2}} \\
\left(\times 10^{6} \mathrm{~s}^{-1}\right)\end{array}$ & $\Phi_{\mathrm{Q}}$ \\
\hline 80 & $9.1 \pm 0.1$ & $2.2 \pm 0.2$ & 34.0 & $0.76 \pm 0.07$ & $0.35 \pm 0.04$ & $26.0 \pm 3.0$ \\
60 & $6.2 \pm 0.1$ & $2.4 \pm 0.2$ & 53.0 & $0.61 \pm 0.04$ & $0.25 \pm 0.03$ & $32.0 \pm 4.0$ \\
20 & $6.8 \pm 0.1$ & $1.7 \pm 0.1$ & 107.0 & $0.74 \pm 0.03$ & $0.43 \pm 0.02$ & $80.0 \pm 4.0$ \\
\hline \hline
\end{tabular}


In a nitrogen atmosphere, the amplitude of photoinduced $\mathrm{PC}_{61} \mathrm{BM} \mathrm{T}_{1} \rightarrow \mathrm{T}_{\mathrm{n}}$ absorption at $\sim 0.6 \mu$ s (the instrument response time) was observed to increase with decreasing $\mathrm{PC}_{61} \mathrm{BM}$ content, with no signal apparent for the neat $\mathrm{PC}_{61} \mathrm{BM}$ film (Figure B.9b-c) which was also unobservable by Chow et al.[20] In all cases where $T_{1} \rightarrow T_{n}$ absorption was observed, its decay kinetics were accelerated in the presence of oxygen. Figure 4.4b and Table 4.3 show the relative fraction of excited states that were oxygen quenched $\Phi_{\mathrm{Q}}$ as a function of $\mathrm{wt} \% \mathrm{PC}_{61} \mathrm{BM}$ (assuming only triplet states are quenched). As the $\mathrm{wt} \% \mathrm{PC}_{61} \mathrm{BM}$ is reduced, the yield of triplets quenched by oxygen $\Phi_{\mathrm{Q}}$ increases. Since the triplet yield at low wt $\% \mathrm{PC}_{61} \mathrm{BM}$ is significantly enhanced, while the quenchng efficiencies $\left(\Phi_{\mathrm{O}_{2}}\right)$ are comparable (see Table 4.3 ), the increase of relative fraction of excited states being oxygen quenched $\left(\Phi_{\mathrm{Q}}\right)$ can be attributed primarily to the significantly enhanced triplet yield at low wt\%. Besides, the triplet lifetimes remain comparable for all of the $\mathrm{PC}_{61} \mathrm{BM}$ wt\% (see Table 4.3), which further suggests that the relative fraction of excited states quenched by oxygen depends on the triplet yield. Note that the rate constant of oxygen quenching of the triplets $\left(k_{\mathrm{O}_{2}}\right)$ remains comparable for all of the wt\% (see Table 4.3), implying the oxygen diffusion rates are similar for the different compositions. Therefore, the rate of oxygen diffusion into the films should not be the main parameter for the significant difference in photo-oxidation with the different wt $\% \mathrm{PC}_{61} \mathrm{BM}$, consistent with the XPS data (Figure B.8).

Figure $4.4 \mathrm{~b}$ shows a clear correlation between the relative fraction of triplets being quenched by oxygen and the level of photo-oxidation of the $\mathrm{PC}_{61} \mathrm{BM}$ films as a function of $\mathrm{PC}_{61} \mathrm{BM}$ aggregation. The figure shows carbonyl formation (on the $\mathrm{PC}_{61} \mathrm{BM}$ cage, $970 \mathrm{~min}$ ) and loss of UV-vis absorbance (1950 $\mathrm{min}$ ) both correlate well with the relative fraction of oxygen quenched triplet states. The lower wt $\% \mathrm{PC}_{61} \mathrm{BM}$ (less aggregated) films exhibit a higher fraction of triplet states that were oxygen quenched, more carbonyl formation, and a larger loss of UV-vis absorbance. 


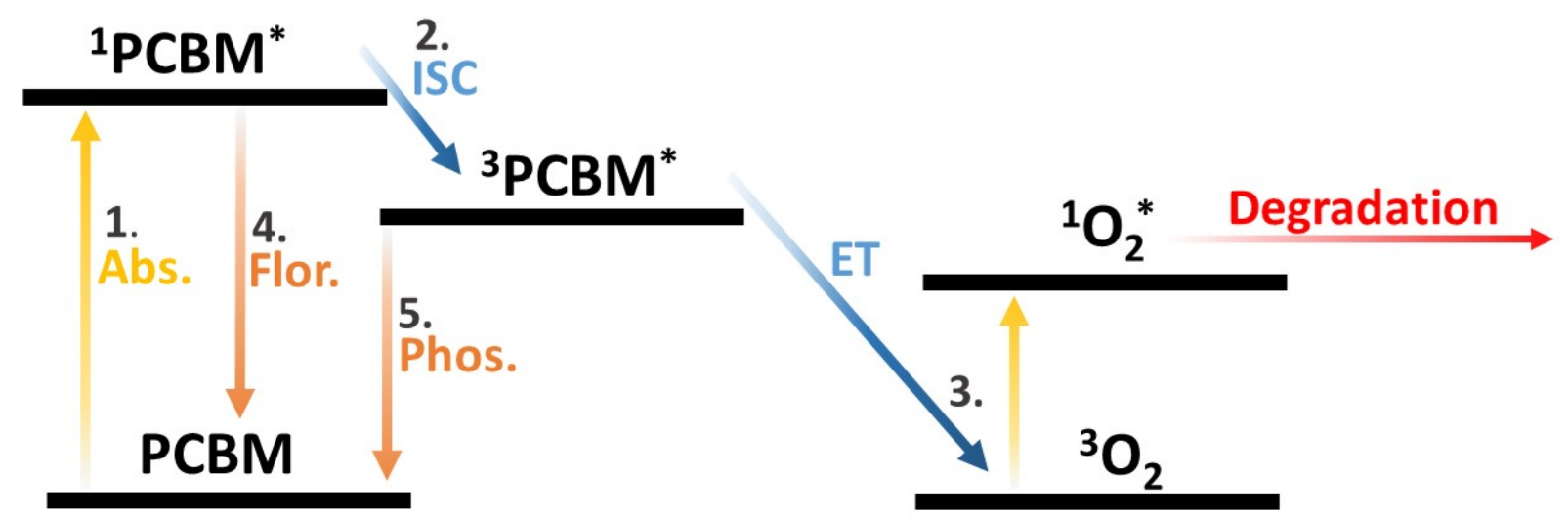

Figure 4.5: Model used to describe the possible degradation mechanism of $\mathrm{PC}_{61} \mathrm{BM}$ via triplet-mediated singlet oxygen generation: 1) Absorption of photons by PCBM, exciting it to its singlet state $\left({ }^{1} \mathrm{PC}_{61} \mathrm{BM}^{*}\right)$. 2) Triplet states $\left({ }^{3} \mathrm{PC}_{61} \mathrm{BM}^{*}\right)$ form via intersystem crossing (ISC). 3) Energy transfer (ET) to molecular oxygen $\left({ }^{3} \mathrm{O}_{2}\right)$ generates singlet oxygen $\left({ }^{1} \mathrm{O}_{2}\right)$. This process competes with 4) florescence (Flor.) and 5) phosphorescence (Phos.).

TAS has been applied to study the photo-oxidation of conjugated polymers, and it has been suggested that photo-oxidation of some donor polymers is triplet-mediated,[9] with polymer triplet lifetimes increasing with lower polymer crystallinity.[10] Here, a strong correlation was found between the yield of triplets being oxygen quenched and the level of photo-oxidation of the $\mathrm{PC}_{61} \mathrm{BM}$ films as a function of $\mathrm{PC}_{61} \mathrm{BM}$ aggregation. This is consistent with triplet-mediated photo-oxidation, similar to that suggested as the cause of photodegradation in some donor polymers.[9] This suggests that the dominant degradation mechanism at play is singlet oxygen generation via the triplet excitons (Figure 4.5), whereby $\mathrm{PC}_{61} \mathrm{BM}$ triplets are formed via intersystem crossing (ISC) from the photoinduced $\mathrm{PC}_{61} \mathrm{BM}$ singlet states. These triplet states can be quenched by molecular oxygen, via energy transfer (ET), to generate highly reactive singlet oxygen, which causes the photo-oxidation of the $\mathrm{PC}_{61} \mathrm{BM}$. The top surface of the neat $\mathrm{PC}_{61} \mathrm{BM}$ film could be less aggregated than the bulk as it is exposed to air on one side and so has fewer neighbouring molecules for aggregation. Therefore, it would be easier to form triplets which lead to the photo-oxidation, which could explain the photo-oxidation observed on the surface of the neat $\mathrm{PC}_{61} \mathrm{BM}$ film (Figure 4.3d) whilst there were no detectable triplet states. TAS may not be sensitive enough to probe this small amount of triplets at the surface, consistent with negligible triplet absorption of the neat $\mathrm{PC}_{61} \mathrm{BM}$ film.

In donor/acceptor blends, the oxygen quenching of polymer triplets generated by the nongeminate recombination of dissociated polarons was shown to be the dominant pathway to photo-oxidation, but there is also an additional contribution from those triplets directly generated from singlet excitons by intersystem crossing. [9] However, the $\mathrm{PC}_{61} \mathrm{BM}$ triplet states were not taken into consideration for these systems. Research efforts to increase polymer stability have been making progress; thieno[3,2-b]thiophene-diketopyrrolo- 
pyrrole (DPP-TT-T) for example was shown to be very stable under white LED light irradiation ( $\left.\sim 80 \mathrm{~mW} \mathrm{~cm}^{-2}\right)$ under a pure oxygen environment for over $250 \mathrm{hr}$.[10] As polymer stability increases, the contribution from these $\mathrm{PC}_{61} \mathrm{BM}$ triplets could well play a bigger role in singlet oxygen generation, and hence photo-oxidation.

In OPV devices, it has been reported that the active layer is composed of a finely intermixed polymer/fullerene phase where the fullerene is dispersed, as well as relatively pure aggregated $\mathrm{PC}_{61} \mathrm{BM}$ domains.[22] The yield of $\mathrm{PC}_{61} \mathrm{BM}$ triplet states is likely to show a complex dependence on this nanomorphology, as well as on blend energetics. It is for example uncertain whether charge recombination in blends can yield $\mathrm{PC}_{61} \mathrm{BM}$ triplets in addition to polymer triplets. [9, 23] The triplet energies of polymers and fullerenes are typically $0.9-1.2$ and $\sim 1.5 \mathrm{eV}$, respectively; [20] therefore, energetically polymer triplets are more favourable to form. However, fullerene triplets have been observed in the blend with photoactive polymers. [24, 25] It should also be noted that the permeability of the polystyrene matrix used in our samples to molecular oxygen is likely to differ from that of typical conjugated polymers. This may affect the rate of degradation in donor: $\mathrm{PC}_{61} \mathrm{BM}$ blends relative to our model system. Nevertheless, our observations herein are that (a) $\mathrm{PC}_{61} \mathrm{BM}$ triplet formation can lead to the generation of oxidising species in the presence of oxygen (most likely singlet oxygen) and (b) $\mathrm{PC}_{61} \mathrm{BM}$ is susceptible to photo-oxidation by these oxidising oxygen species.

\subsubsection{Impact of photo-oxidation on electrical and optical properties of films and devices}

The consequences of the $\mathrm{PC}_{61} \mathrm{BM}$ photo-oxidation on the electronic and optical properties of films were investigated in order to explore the relevance of $\mathrm{PC}_{61} \mathrm{BM}$ photo-oxidation to device function. To study the effect of the photo-oxidation on charge transport, the current density versus electric field of electron-only devices using $\mathrm{PC}_{61} \mathrm{BM}$ as the active layer were measured with different photoaging times (Figure 4.6). Notably, even with < 40 min photoaging, the current density dropped by $4-5$ times. It is reasonable that a small amount of photo-oxidation (undetectable by the optical techniques) is sufficient to cause a significant effect on the electron transport, since it is well-known that a small density of charge traps can cause a significant drop in charge carrier mobility.[26] This rapid loss in mobility is consistent with the XPS data of the $\mathrm{PC}_{61} \mathrm{BM}$ film with $1 \mathrm{hr}$ photoaging which also showed significant photo-oxidation on the surface layer (oxygen concentration $~ 9.6 \%$; Figure B.6). 


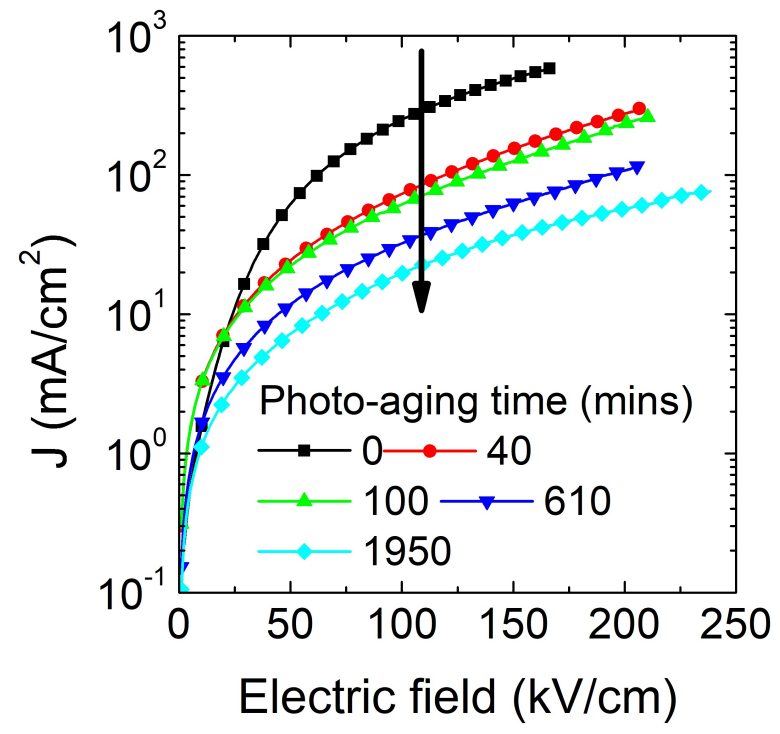

Figure 4.6: Current density versus electric field of electron-only devices using $\mathrm{PC}_{61} \mathrm{BM}$ as the active layer with different photoaging times.

TD-DFT calculations were performed to model the effect of the photo-oxidation on the optical properties of $\mathrm{PC}_{61} \mathrm{BM}$. There was an observed redshift in the $\mathrm{PC}_{61} \mathrm{BM} \mathrm{PL}$ spectra, modelled from a calculated deepening of the LUMO level, when x1 epoxide, x1 diol, or x2 carbonyl defects were added (Figure 4.8b), following the suggested mechanism in Figure B.7. The redshifts observed in the modelled PL spectra of $\mathrm{PC}_{61} \mathrm{BM}$ upon addition of oxygen to form defects are consistent with formation of the electron traps. While the shape and the position of the calculated photo-oxidised peaks differ from experimentally obtained spectra due to a number of assumptions, such as neglect of the vibronic structure, the magnitude of the shift in luminescence peaks in calculated and experimental spectra can be compared. The experimental redshift of approximately $0.2 \mathrm{eV}$ in the luminescence peak compares well with the shift expected (from model) due to the presence of epoxide defects and further supports the argument that these defects are likely to dominate the optoelectronic properties. The clear observation of PL and EL from defect states indicate that radiative recombination via trap states is not forbidden in the degraded $\mathrm{PC}_{61} \mathrm{BM}$ films or devices.

Table 4.4: Calculated effect of different defect types on the LUMO energy of oxidised $\mathrm{PC}_{61} \mathrm{BM}$. Depression in LUMO energies calculated from subtracting average LUMO energies of defects from the LUMO of $\mathrm{PC}_{61} \mathrm{BM}$.

\begin{tabular}{||c|c||}
\hline \hline Defect Type & Average Calculated Depression in LUMO (eV) \\
\hline x1 Epoxide & -0.10 \\
x1 Diol & -0.58 \\
x2 Carbonyls & -0.87 \\
\hline \hline
\end{tabular}




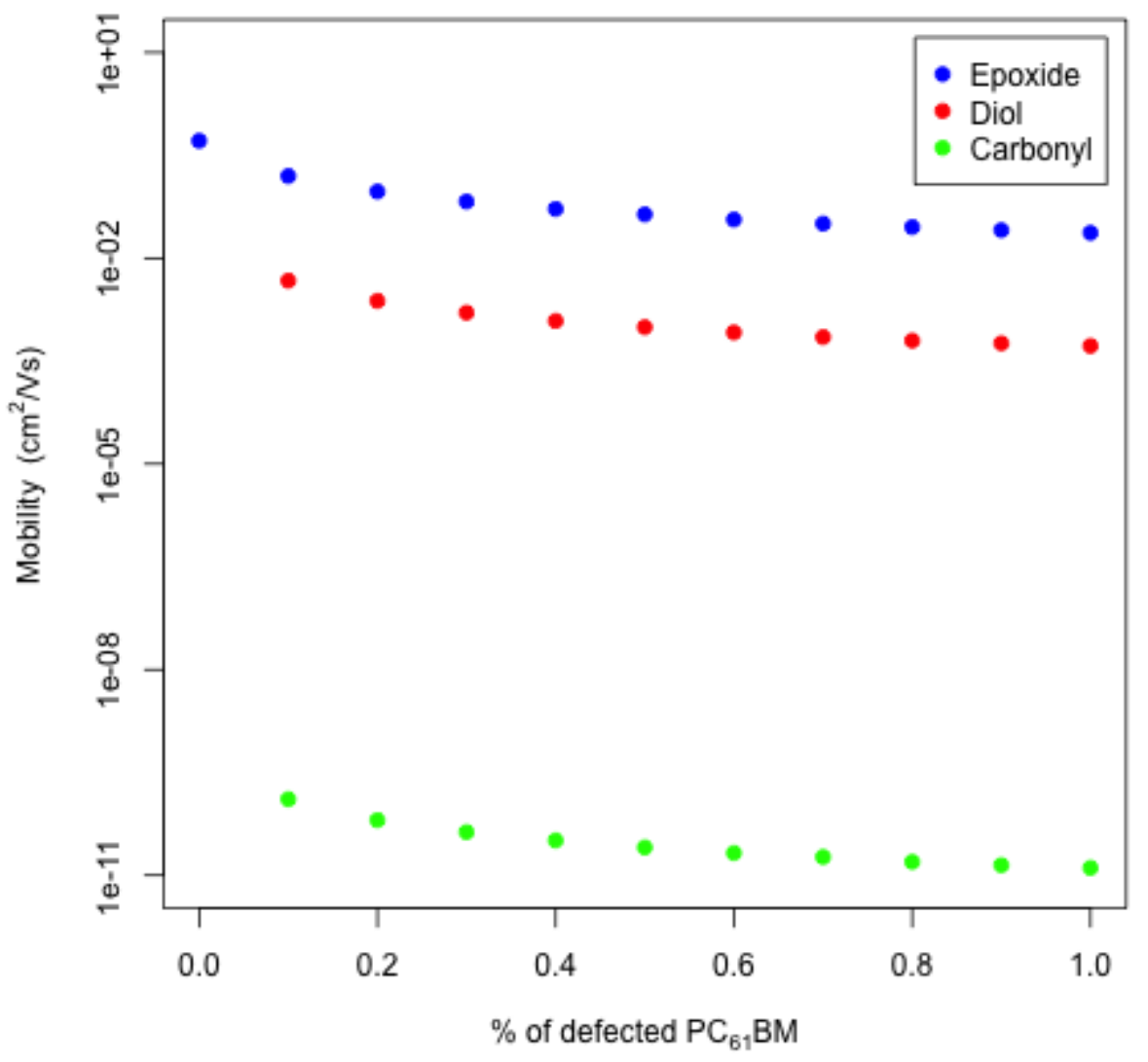

Figure 4.7: Simulated mobility in $\mathrm{PC}_{61} \mathrm{BM}$ doped with different volume fractions of oxidised defects.

TD-DFT calculations were also carried out to determine the effect of photo-oxidation on the LUMO and electron transport of $\mathrm{PC}_{61} \mathrm{BM}$. The average LUMO energy is calculated by taking the average of the LUMO energies for all possible positions of defects (i.e. different photo-oxidation products) of the type specified (epoxide, diol and pair of carbonyls, as indicated in the mechanism in Figure B.7) weighted by the factor exp (-E/kT) where E represents the total energy for the given defect. For each defect structure, the total energy of the structure and HOMO energy of the optimised structure are calculated using DFT (B3LYP/6-31G*) and the LUMO energy is estimated by adding the energy of the first excited state calculated using TD-DFT. The resulting average LUMO energies were subtracted from the LUMO of $\mathrm{PC}_{61} \mathrm{BM}$ to find the depression in LUMO energies listed in Table 4.4. It can be seen as more serious photo-oxidation occurs, the depression of the $\mathrm{PC}_{61} \mathrm{BM}$ LUMO increases. The electron mobility was then simulated using a master equation model for steady state electron transport and treating the defect as a site with energy depressed below the $\mathrm{PC}_{61} \mathrm{BM}$ LUMO by the amount given in Table 4.4. Figure 4.7 shows that the effect on mobility is dominated by the depth of the defect rather than the number of defects. This corresponds well with the significant drop in current density 
of electron only devices with a short amount of photoaging time ( $<40$ mins) (Figure 4.6). The model assumes that defects are normally empty and electron density is low; in practice very deep carbonyl defects are likely to be partly filled. These calculations strongly suggest that relatively shallow defects, such as epoxides, are more likely to be responsible for the mobility loss than deep carbonyl defects.
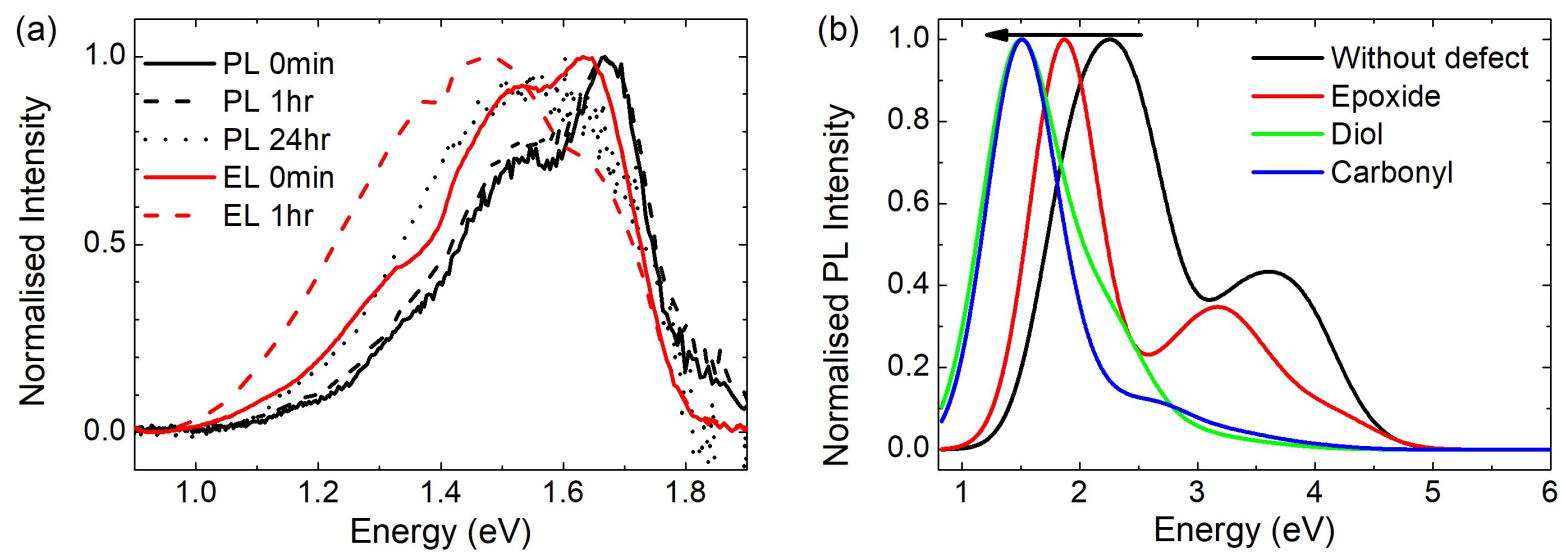

Figure 4.8: (a) Normalised PL and EL spectra of $\mathrm{PC}_{61} \mathrm{BM}$ films and devices, respectively, before and after the photoaging, and (b) calculated PL spectra (normalised) of a $\mathrm{PC}_{61} \mathrm{BM}$ molecule with and without oxidative defects.

The impact of photodegradation on the optical and electronic properties of $\mathrm{PC}_{61} \mathrm{BM}$ was then further probed by photo- and electro-luminescence measurements and correlated with TD-DFT calculations of $\mathrm{PC}_{61} \mathrm{BM}$ with various defects. Figure 4.8 (a) shows PL and EL spectra of $\mathrm{PC}_{61} \mathrm{BM}$ films and devices before and after the photoaging. Both the $\mathrm{PL}$ and EL shift to lower energies after photoaging, indicating the generation of intraband defect states that are radiatively coupled to the ground state. Interestingly, after $1 \mathrm{hr}$ of photoaging, the neat $\mathrm{PC}_{61} \mathrm{BM}$ film does not show a measurable redshift in the $\mathrm{PL}$, while it does have a significant redshift in the EL spectrum (from 1.64 to $1.48 \mathrm{eV}$ ). It is likely that for the EL, the electrons are transported to the lowest lying defect states where the emission occurs. Therefore, the EL is dominated by the emission of the oxidative defects; hence, it has a more pronounced redshift. On the other hand, the PL signal is from all emissive species probed, where mainly the top surface of the degraded $\mathrm{PC}_{61} \mathrm{BM}$ film was photo-oxidised (see XPS section). For the $\mathrm{PC}_{61} \mathrm{BM}$ film photoaged for $24 \mathrm{hr}$, the PL does redshift (from 1.66 to $1.55 \mathrm{eV}$ ) which is consistent with more photo-oxidised defects/traps being formed (see FTIR section). The PL and EL spectra are an average of all the emissive states probed (fresh and defected), whereas the calculations show the shift for where all the $\mathrm{PC}_{61} \mathrm{BM}$ molecules have defects, thus the calculated redshifting is more pronounced than the experimental.

Although UV-vis absorbance is routinely used to probe degradation of conjugated polymers through photobleaching, [27-29] it is apparent from the electron transport data Figure (4.6) that the functional electronic degradation of $\mathrm{PC}_{61} \mathrm{BM}$ films occurs even without 
observable change in optical absorbance. It is likely that epoxide/diol defects were formed prior to the formation of carbonyls; [30] therefore, the $\mathrm{PC}_{61} \mathrm{BM}$ and blend films may have already photo-oxidised before carbonyl formation was observed at $\sim 100$ min by FTIR. This is supported by: the significant drop in electron transport of the $\mathrm{PC}_{61} \mathrm{BM}$ electrononly devices, even after 40 min photoaging; the significant surface oxidation observed via increased signal at the higher binding energies in the XPS $\mathrm{C}(1 \mathrm{~s})$ envelopes of neat $\mathrm{PC}_{61} \mathrm{BM}$ films after $1 \mathrm{hr}$ photoaging (Figure B.6); and the slightly higher epoxide:carbonyl ratio at depth of $>60 \mathrm{~nm}$ ascertained from the XPS depth profile (Figure B.8). Alternatively, it could also be possible that carbonyl defects were formed $<100 \mathrm{~min}$ but the FTIR measurements were not sensitive enough to detect these. It should be noted that even the 90 wt\% $\mathrm{PC}_{61} \mathrm{BM}$ film shows considerably more photo-oxidation than the neat $\mathrm{PC}_{61} \mathrm{BM}$ film (Figure 4.4b), implying that the addition of a small wt\% of PS can have a considerable effect on the photo-oxidation of $\mathrm{PC}_{61} \mathrm{BM}$ films. The drop in electron transport after 40 min shows that a small amount of electron traps can cause a significant drop in electrical transport, and importantly, for the first time, it is shown that neat $\mathrm{PC}_{61} \mathrm{BM}$ films can be degraded very easily under 1 sun conditions, unlike previous studies which use harsh conditions that may not be directly relevant to the illumination conditions for solar cells. [14, 13, 31]

\subsubsection{Control of fullerene aggregation in PCDTBT:PC ${ }_{61} \mathrm{BM}$ films and devices and the effect to their photostability in air}

\subsubsection{Spectroscopic studies on thin films}

Although the previous section provides insight on the effect of $\mathrm{PC}_{61} \mathrm{BM}$ aggregation to its stability, it is with a non-photoactive polymer (PS). To be directly relevant to actual photovoltaic layers, here, PCDTBT:PC ${ }_{61} \mathrm{BM}$ films/devices were studied with different blend ratios, to investigate both the aggregation of the photoactive layer, and if changing the aggregation can control its photo-oxidation. The blend ratio (PCDTBT:PC $\left.{ }_{61} \mathrm{BM}\right)$ was varied from 8:1 to 1:8 by weight. 

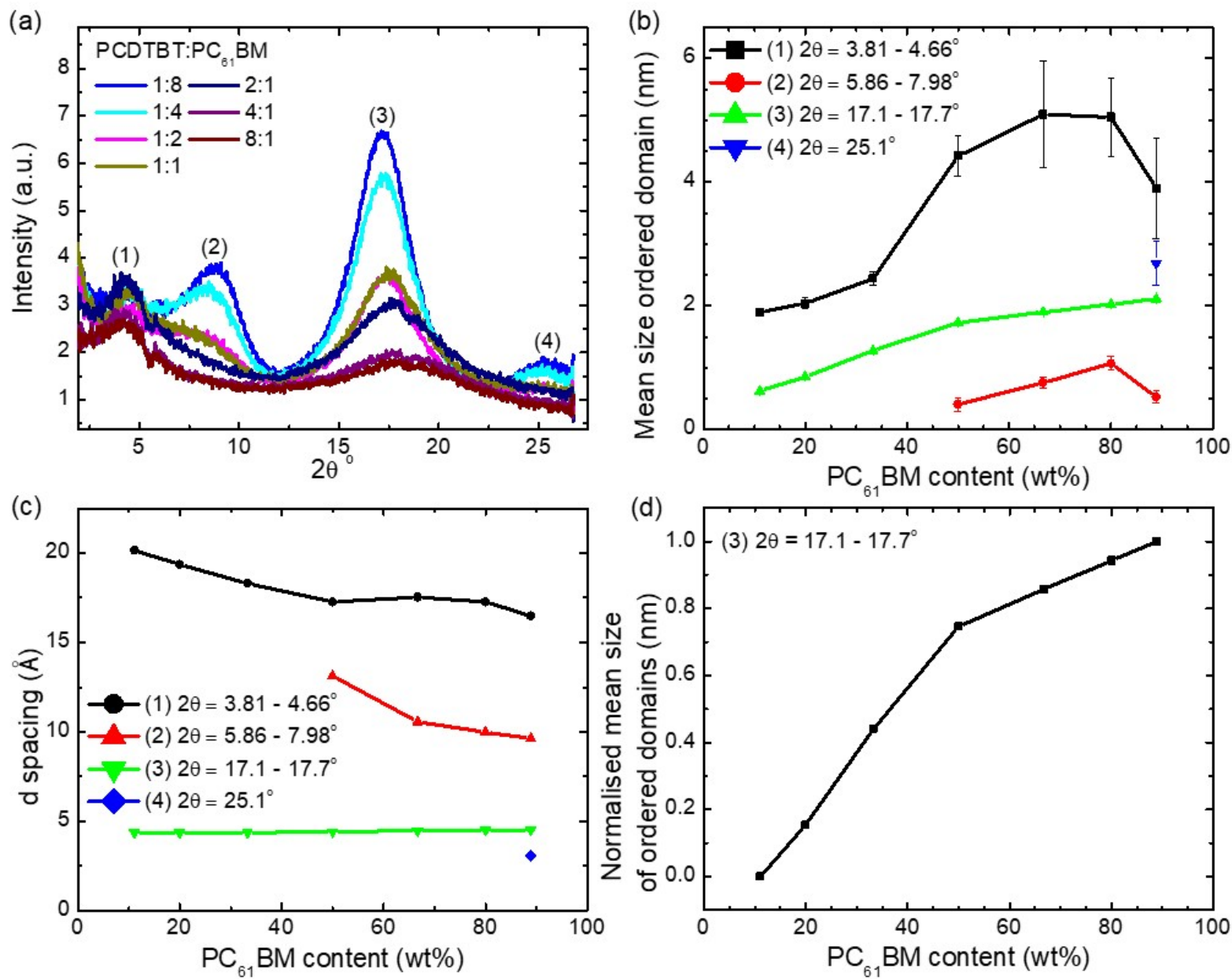

Figure 4.9: (a) GIWAXS spectra for the PCDTBT:PC ${ }_{61} \mathrm{BM}$ films with different blend ratios. (b) Mean size of ordered domains and (c) d-spacings corresponding to the peaks observed as a function of $\mathrm{PC}_{61} \mathrm{BM}$ content. (d) normalised mean size of ordered domains $\left(\mathrm{PC}_{61} \mathrm{BM}\right.$ aggregation) extracted from the peak at $17.1-17.7^{\circ}$ as a function of $\mathrm{PC}_{61} \mathrm{BM}$ content.

Table 4.5: GIWAXS peak positions of the PCDTBT:PC ${ }_{61} \mathrm{BM}$ films with different blend ratios to 3 s.f.

\begin{tabular}{||c|c|c||c|c||}
\hline \hline $\mathrm{D}: \mathrm{A}$ & $2 \theta\left(^{\circ}\right)\left[\mathrm{Q}\left(\AA^{-1}\right)\right]$ & $2 \theta\left(^{\circ}\right)\left[\mathrm{Q}\left(\AA^{-1}\right)\right]$ & $2 \theta\left(^{\circ}\right)\left[\mathrm{Q}\left(\AA^{-1}\right)\right]$ & $2 \theta\left(^{\circ}\right)\left[\mathrm{Q}\left(\AA^{-1}\right)\right]$ \\
\hline $8: 1$ & $3.81[0.312]$ & - & $17.6[1.44]$ & - \\
$4: 1$ & $3.97[0.325]$ & - & $17.7[1.44]$ & - \\
$2: 1$ & $4.20[0.343]$ & - & $17.6[1.44]$ & - \\
$1: 1$ & $4.45[0.364]$ & $5.86[0.497]$ & $17.4[1.42]$ & - \\
$1: 2$ & $4.38[0.358]$ & $7.27[0.594]$ & $17.3[1.41]$ & - \\
$1: 4$ & $4.45[0.364]$ & $7.70[0.629]$ & $17.2[1.40]$ & - \\
$1: 8$ & $4.66[0.381]$ & $7.98[0.653]$ & $17.1[1.40]$ & $25.1[2.04]$ \\
\hline \hline
\end{tabular}

Firstly, GIWAXS measurements were undertaken to confirm that varying the $\mathrm{PC}_{61} \mathrm{BM}$ content of PCDTBT: $\mathrm{PC}_{61} \mathrm{BM}$ films did affect the $\mathrm{PC}_{61} \mathrm{BM}$ aggregation as with PS. Figure 
4.9a shows the GIWAXS spectra for all PCDTBT:PC ${ }_{61} \mathrm{BM}$ films and Table 4.5 shows the position of the extracted peaks. The first peak at $3.81-4.66^{\circ}(\mathrm{d}$ spacing $=20.2$ - 16.5A) coincides with the characteristic lamella spacing of the PCDTBT neat film (d $=20.9 \AA$ ). $[32]$ By applying Scherrer analysis (Figure 4.9b) on this PCDTBT peak, it can be seen that when the $\mathrm{PC}_{61} \mathrm{BM}$ wt\% is increased, the mean size of ordered PCDTBT domains increased and the d-spacing decreased, hence the PCDTBT was more crystalline. The other three peaks coincide with those observed for as-spun neat $\mathrm{PC}_{61} \mathrm{BM}$ films $(\mathrm{Q} \approx$ $0.71 \AA^{-1}, 1.39 \AA^{-1}$, and $\left.2.04 \AA^{-1}\right)$. [33] The peaks at $5.86-7.98^{\circ}(\mathrm{Q}=0.497-0.653 \AA)$ and 17.1 - $17.6^{\circ}\left(\mathrm{Q}=1.40\right.$ - 1.44 $\AA$ ) increase in peak intensity with increasing $\mathrm{PC}_{61} \mathrm{BM}$ wt\%, which is reasonable. Applying Scherrer analysis (Figure $4.9 \mathrm{~b}$ ) to the peak at $17.1-17.6^{\circ}$ reveals an increase in mean ordered domain size and similar d-spacing $(4.38-4.51 \AA)$ with $\mathrm{PC}_{61} \mathrm{BM}$ content, conducive of a larger grain size of similarly packed $\mathrm{PC}_{61} \mathrm{BM}$. Figure $4.9 \mathrm{~d}$ shows the normalised mean size of ordered domains as a function of $\mathrm{PC}_{61} \mathrm{BM}$ content for this peak, which can be used as a indicator of the degree of $\mathrm{PC}_{61} \mathrm{BM}$ aggregation for these films with varying $\mathrm{PC}_{61} \mathrm{BM}$ wt\%. The peak at $5.86-7.98^{\circ}$ only appears for the higher $\mathrm{PC}_{61} \mathrm{BM}$ wt\% films (1:1 - 1:8) and Sherrer analysis reveals the higher the $\mathrm{PC}_{61} \mathrm{BM}$ wt\%, the larger the mean size of ordered domains and the smaller the d-spacing (13.1 - 9.63A) (i.e. larger domains of more closely packed $\left.\mathrm{PC}_{61} \mathrm{BM}\right)$. The peak at $25.1^{\circ}(\mathrm{d}$-spacing $=3.08 \AA)$ only appears at highest $\mathrm{PC}_{61} \mathrm{BM}$ wt\% film. The broad PCDTBT peak due to the $\pi-\pi$ stacking at $\sim 20^{\circ}(\mathrm{d}$-spacing $=4.4 \AA)$ is unobservable in these GIWAXS measurements of blend films, as the signal is dominated by the $\mathrm{PC}_{61} \mathrm{BM}$ peaks.[32] Overall, these measurements show that the higher the $\mathrm{PC}_{61} \mathrm{BM}$ content of the film, the more closely packed and larger the domain sizes (i.e. more aggregated) for both the $\mathrm{PC}_{61} \mathrm{BM}$ and the PCDTBT.

Next, the effect of varying the PCDTBT:PC ${ }_{61} \mathrm{BM}$ wt\% ratio, and hence $\mathrm{PC}_{61} \mathrm{BM}$ aggregation, on the photobleaching of films was undertaken to probe the film photochemical stability. Figure 4.10 a-c show the UV-vis absorbance of 8:1, 1:1, and 1:8 PCDTBT:PC ${ }_{61}$ BM films with up to 120 minutes of photoaging under 1 Sun AM1.5G radiation in air. The UV-vis absorbance of the 4:1, 2:1, 1:2, and 1:4 PCDTBT:PC ${ }_{61} \mathrm{BM}$ are shown in Figure B.10. These results are summarised in Figure B.11 where the fractional loss of absorbance is plotted as a function of degradation time for (a) PCDTBT $(579 \mathrm{~nm})$ and $\mathrm{PC}_{61} \mathrm{BM}(266 \mathrm{~nm})$ for each blend film. Figure 4.10d then summarises this data by showing the fractional loss of absorbance after 120 minutes of degradation of both the PCDTBT $(579 \mathrm{~nm})$ and $\mathrm{PC}_{61} \mathrm{BM}(266 \mathrm{~nm})$ peaks as a function of the wt\% $\mathrm{PC}_{61} \mathrm{BM}$. As the wt\% of $\mathrm{PC}_{61} \mathrm{BM}$ was reduced, the photobleaching of the $\mathrm{PC}_{61} \mathrm{BM}$ peak at $266 \mathrm{~nm}$ increased. This implies that $\mathrm{PC}_{61} \mathrm{BM}$ was more susceptible to photobleaching when it was more dispersed in PCDTBT (i.e. less aggregated, further supported by the GIWAXS data), and is in line with the results with PS:PC ${ }_{61} B M$. Similarly, the PCDTBT peak at $579 \mathrm{~nm}$ also underwent increased photobleaching for lower $\mathrm{PC}_{61} \mathrm{BM}$ wt\% films 
which can be explained with the GIWAXS data which show the less $\mathrm{PC}_{61} \mathrm{BM}$ films have less crystalline PCDTBT. The films at the two extremes of wt\% $\mathrm{PC}_{61} \mathrm{BM}$ are not so consistent with the observed trends (8:1 for $\mathrm{PC}_{61} \mathrm{BM}$ and 1:8 for PCDTBT) which could be explained by solubility issues at these extreme wt\%, leading to morphological differences. By comparing the fractional loss of absorbance of the $\mathrm{PC}_{61} \mathrm{BM}$ and PCDTBT peaks, it is clear that the PCDTBT is prone to more photobleaching than $\mathrm{PC}_{61} \mathrm{BM}$, consistent with other studies where donor polymers are generally photobleached faster than fullerene acceptors.[34, 9] However, this doesn't necessarily indicate that the electronic properties of $\mathrm{PC}_{61} \mathrm{BM}$ degrade less than those of PCDTBT as shown in Figure 4.6; the electron transport properties degrade significantly for the photoaged $\mathrm{PC}_{61} \mathrm{BM}$ film, even where there is very minor photobleaching.

(a)

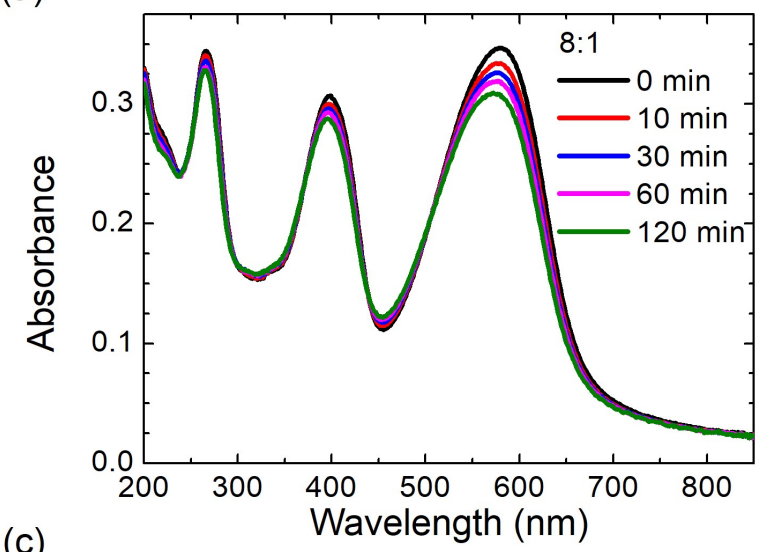

(c)

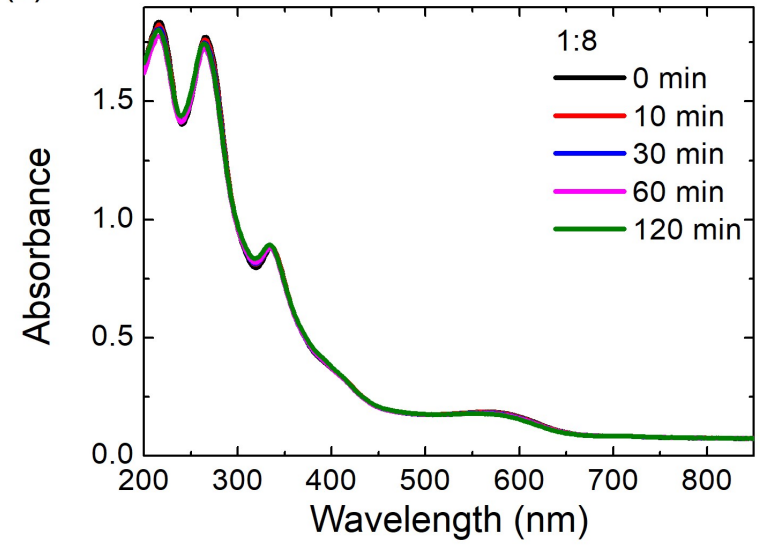

(b)

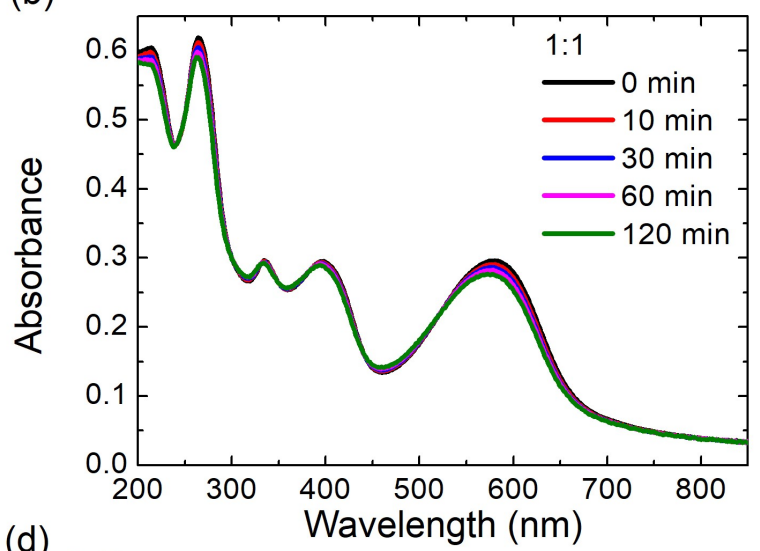

(d)

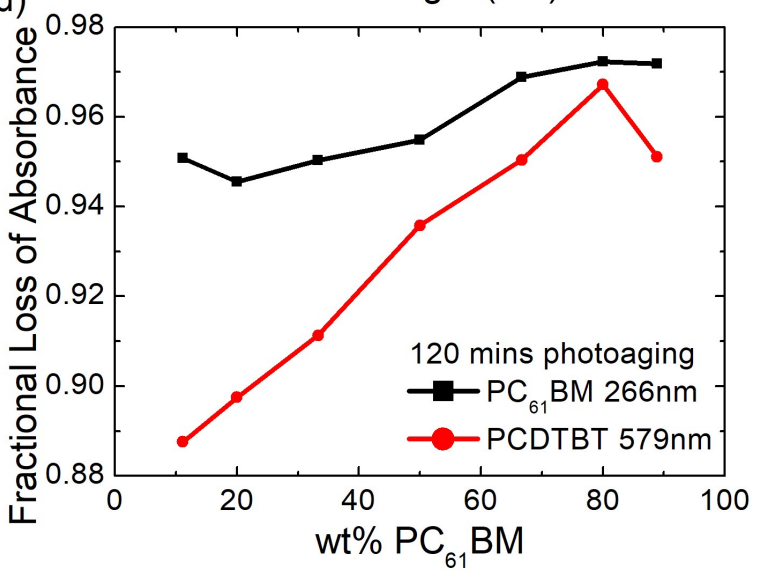

Figure 4.10: Photobleaching of PCDTBT:PC ${ }_{61} \mathrm{BM}$ films with wt rations of (a) 8:1 (b) 1:1 (c) 1:8 films, and (d) fractional loss of absorbance against the wt\% of $\mathrm{PC}_{61} \mathrm{BM}$ in films (photoaged for 120 minutes). 

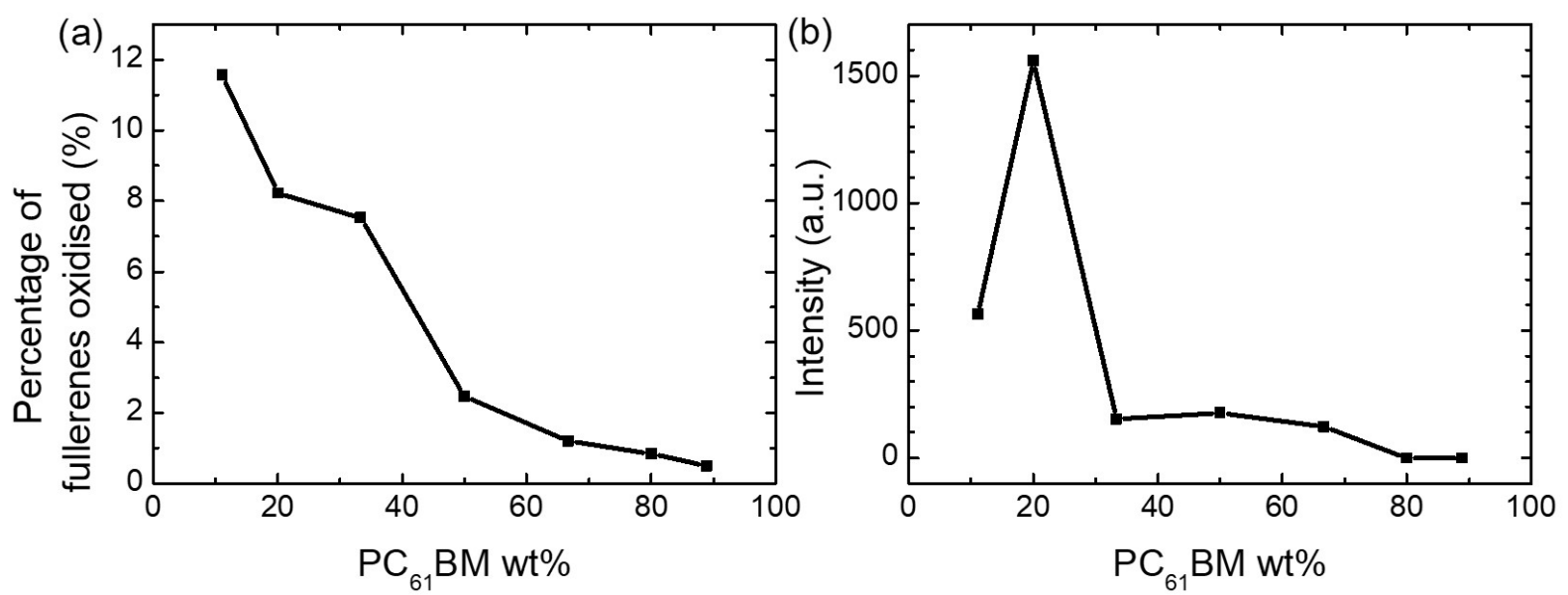

Figure 4.11: (a) Percentage of fullerenes oxidised and (b) intensity of oxidised fullerene peaks as a function of $\mathrm{PC}_{61} \mathrm{BM}$ content within PCDTBT:PC ${ }_{61} \mathrm{BM}$ films after 120 minutes of exposure to AM1.5G solar simulator exposure in air, obtained via MALDI TOF.

To study whether the photobleaching of the blend films could be correlated to photooxidation of $\mathrm{PC}_{61} \mathrm{BM}$, mass spectroscopy measurements were performed on photoaged films which were scratched off and redissolved for the measurements (Figure B.12). Figure 4.11a shows the percentage of fullerenes that were oxidised as a fraction of the total fullerene species as a function of $\mathrm{PC}_{61} \mathrm{BM}$ content. In general, the lower $\mathrm{PC}_{61} \mathrm{BM}$ wt\% in the films (i.e. less aggregated $\mathrm{PC}_{61} \mathrm{BM}$ ), the more photo-oxidation occurs. The film with the most aggregated $\mathrm{PC}_{61} \mathrm{BM}(1: 8)$ underwent the least photo-oxidation after 120 minutes of photoaging, with $0.5 \%$ of the $\mathrm{PC}_{61} \mathrm{BM}$ molecules in the film oxidising. Whereas the film with the least aggregated $\mathrm{PC}_{61} \mathrm{BM}(8: 1)$ underwent $\sim 23$ times as much photo-oxidation, with $11.6 \%$ of the $\mathrm{PC}_{61} \mathrm{BM}$ molecules oxidising. Figure $4.11 \mathrm{~b}$ shows the mass spectrometry intensity for oxidised fullerenes without any normalisation for wt $\%$ of $\mathrm{PC}_{61} \mathrm{BM}$ present in each film and its nonlinearity shows that the percentage of fullerenes that are oxidised does not just depend on the $\mathrm{PC}_{61} \mathrm{BM}$ wt\%. This is limited also by the solubility of oxgen within the organic films. This mass spectroscopy data correlates well with the PS:PC ${ }_{61} \mathrm{BM}$ XPS data which also shows significantly more photo-oxidation when $\mathrm{PC}_{61} \mathrm{BM}$ is blended with $\mathrm{PS}$. The increased photo-oxidation in less aggregated $\mathrm{PC}_{61} \mathrm{BM}$ in PCDTBT is thus, likely the cause of the observed increase in photobleaching.

\subsubsection{Impact of photoaging in air on devices}

To understand how the photo-oxidation (which correlates to aggregation) of $\mathrm{PC}_{61} \mathrm{BM}$ affects the device stability, devices where the PCDTBT:PC ${ }_{61} \mathrm{BM}$ films were photoaged for 0,10 , and 30 minutes before the metal contacts were evaporated. Then, the devices were characterised by measuring their $\mathrm{PCE}, \mathrm{V}_{\mathrm{oc}}, \mathrm{J}_{\mathrm{sc}}$ and $\mathrm{FF}$ as a function of photoaging time of the active layer, and the device parameters are normalised to that of the devices 
with the unaged active layer films for comparison (Figure B.13).

(a)
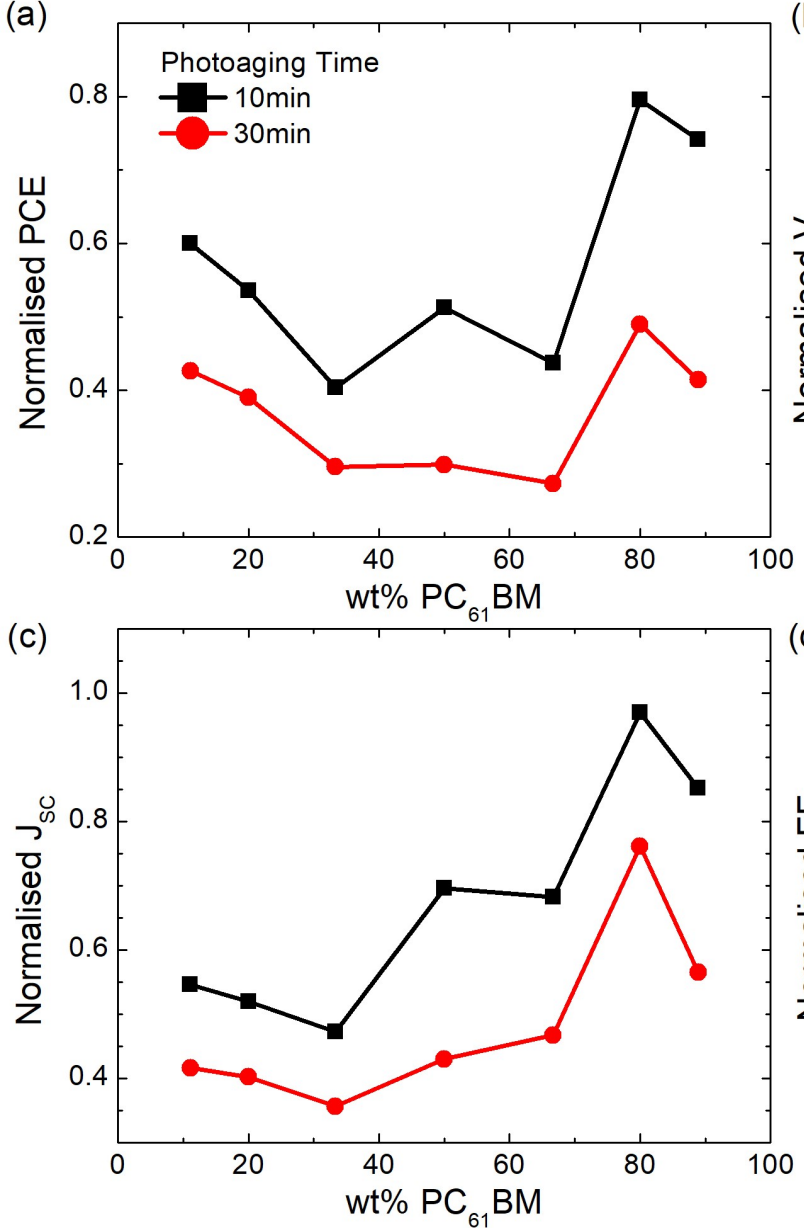

(b)

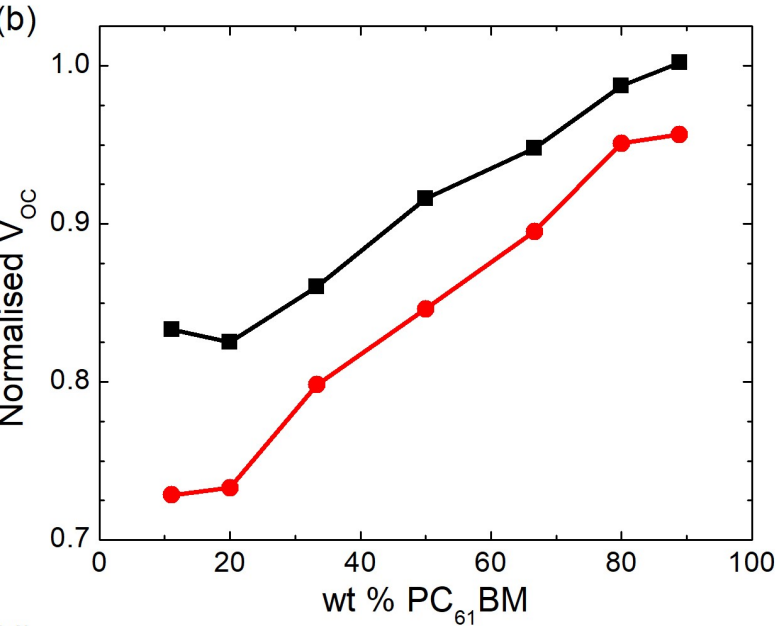

(d)

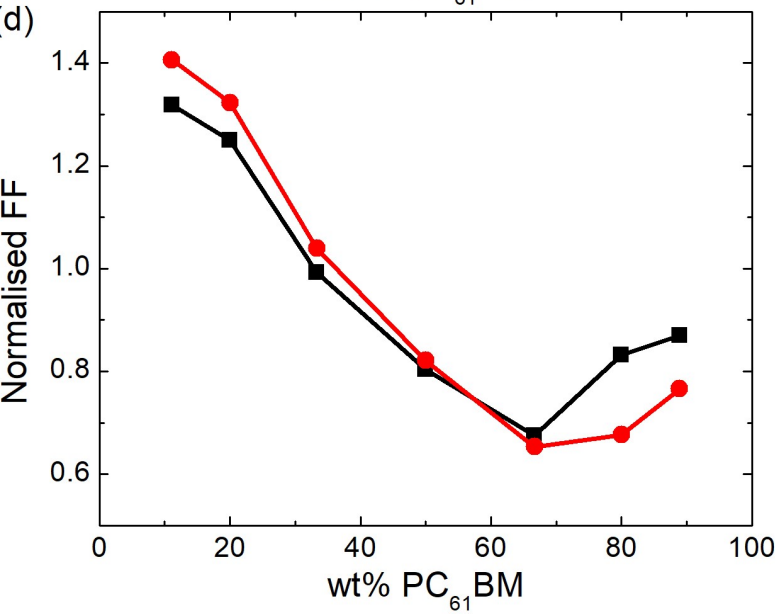

Figure 4.12: Device characteristics (a) PCE (b) $\mathrm{V}_{\text {oc }}$ (c) $\mathrm{J}_{\mathrm{sc}}$ (d) FF of PCDTBT:PC ${ }_{61} \mathrm{BM}$ devices with different blend ratios as a function of the wt\% of $\mathrm{PC}_{61} \mathrm{BM}$ after 10 and 30 minutes of photoaging of the active layer. The device parameters are normalised to that of 0 minutes of photoaging.

Figure 4.12a-d shows the normalised $\mathrm{PCE}, \mathrm{V}_{\mathrm{oc}}, \mathrm{J}_{\mathrm{sc}}$ and $\mathrm{FF}$ as a function of wt $\% \mathrm{PC}_{61} \mathrm{BM}$ after 10 and 30 minutes of photoaging. Figure $4.12 \mathrm{~b}$ shows that the $\mathrm{V}_{\text {oc }}$ drops much less with higher $\mathrm{PC}_{61} \mathrm{BM}$ content. As shown in Chapter 3 , the $\mathrm{V}_{\text {oc }}$ of devices is much more sensitive than the other device characteristics to degradation (photo-oxidation) of $\mathrm{PC}_{61} \mathrm{BM}$ (no significant $\mathrm{V}_{\mathrm{oc}}$ change was observed when the polymer donors were degraded). Therefore, the smaller $\mathrm{V}_{\mathrm{oc}}$ drop for devices with more $\mathrm{PC}_{61} \mathrm{BM}$ aggregation (higher $\mathrm{PC}_{61} \mathrm{BM}$ content) correlates very well with there being less photo-oxidation of $\mathrm{PC}_{61} \mathrm{BM}$ with more $\mathrm{PC}_{61} \mathrm{BM}$ aggregation. Similarly, in general the $\mathrm{J}_{\mathrm{sc}}$ drops much less with higher $\mathrm{PC}_{61} \mathrm{BM}$ content (Figure 1.11c), which can also be correlated to less photooxidation of $\mathrm{PC}_{61} \mathrm{BM}$. It is likely also due to degradation of PCDTBT with higher $\mathrm{PC}_{61} \mathrm{BM}$ contents (see PCDTBT photobleaching in Figure 4.10d) due to the increase in PCDTBT crytallinity (Figure 4.9). The trend of FF is more complicated (see Figure 4.12d), as the $\mathrm{FF}$ does not always drop with increasing $\mathrm{PC}_{61} \mathrm{BM}$ content nor with photoaging. The reduction of FF has been previously been shown can be consistent with trap formation.[29] 
Here, the increase in $\mathrm{FF}$ for devices with between 66 and 11 wt\% $\mathrm{PC}_{61} \mathrm{BM}$ could be explained if the traps formed could continue to form charge transport pathways. This is further supported by the increase in $\mathrm{FF}$ for devices with $11-50 \mathrm{wt} \% \mathrm{PC}_{61} \mathrm{BM}$ with photoaging time. The PCE of devices generally drops with decreasing wt\% $\mathrm{PC}_{61} \mathrm{BM}$ between 89 - $33 \mathrm{wt} \%$, correlating well with the $\mathrm{J}_{\mathrm{sc}}$ and with some contribution from the $\mathrm{V}_{\mathrm{oc}}$. Between $33-11 \mathrm{wt} \% \mathrm{PC}_{61} \mathrm{BM}$, there is less of a drop in PCE, correlating with the high and increasing in FF.
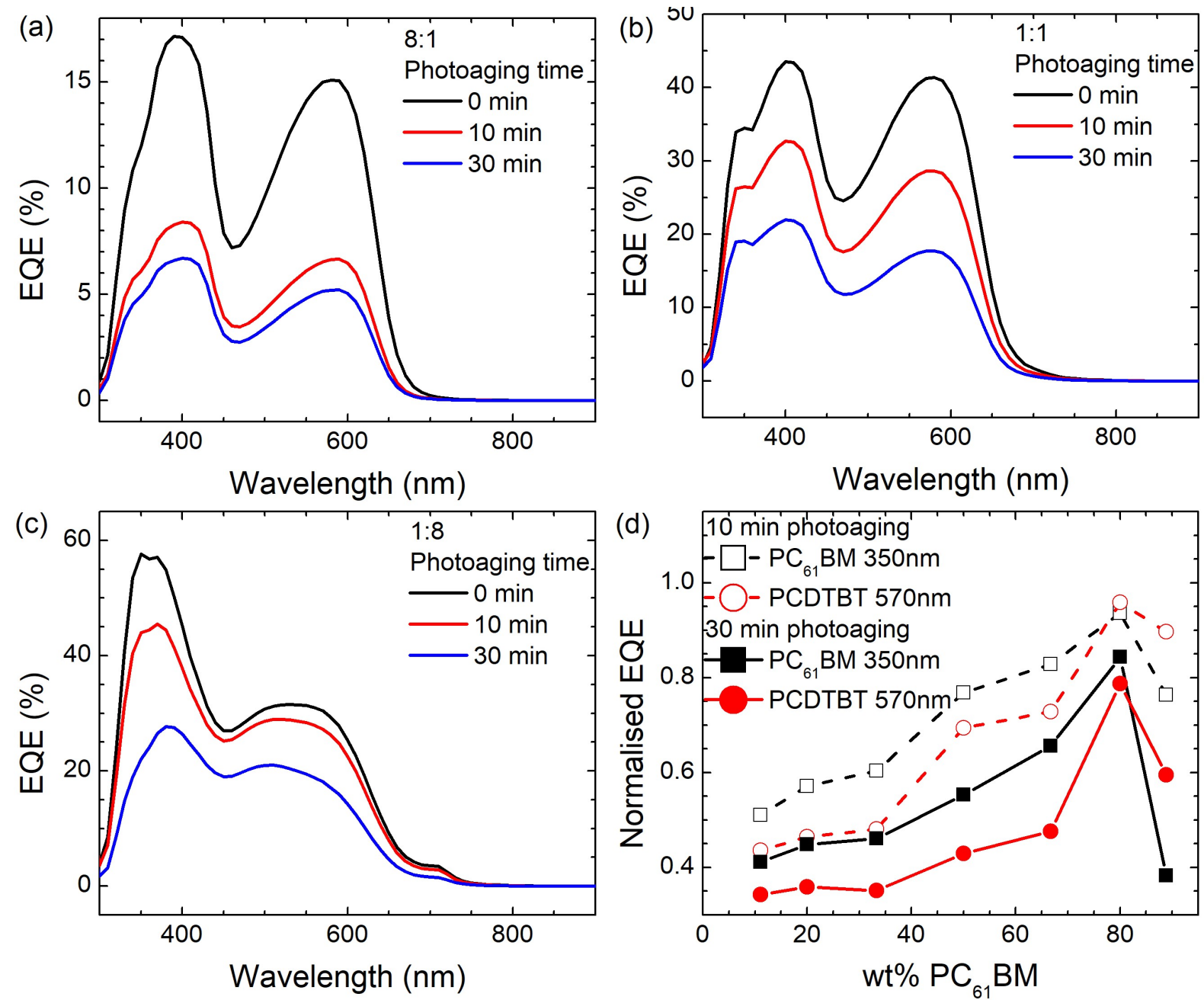

Figure 4.13: EQE spectra of PCDTBT:PC ${ }_{61} \mathrm{BM}$ (a) 8:1, (b) 1:1 (c) 1:8 devices after 0, 10, and 30 minutes of photoaging and $(\mathrm{d})$ normalised EQE at $350 \mathrm{~nm}\left(\mathrm{PC}_{61} \mathrm{BM}\right.$ peak) and $570 \mathrm{~nm}$ (PCDTBT peak) after 10 and 30 minutes of photoaging as a function of $\mathrm{PC}_{61} \mathrm{BM}$ wt\%. The EQE are normalised to that of devices that underwent 0 minutes of photoaging.

To gain insight into the changes in $\mathrm{J}_{\mathrm{sc}}$ with photoaging, the EQE of the same devices was then measured. The EQE spectra of the 8:1, 1:1, and 1:8 devices are shown in Figure 4.13 (a), (b), and (c) respectively, after 0, 10 and 30 minutes of photoaging (those of the 4:1, 2:1, 1:2, and 1:4 PDCTBT:PC ${ }_{61}$ BM devices are shown in Figure B.14 a-d). The change in EQE for these devices (normalised to that of the fresh devices) at excitation wavelength of $570 \mathrm{~nm}(\mathrm{PCDTBT})$ and $350 \mathrm{~nm}\left(\mathrm{PC}_{61} \mathrm{BM}\right)$ as a function of photoaging time is shown 
in Figure B.15. These results are summarised in Figure 4.13d where the normalised EQE of the devices degraded for 10 and 30 minutes are plotted as a function of wt $\% \mathrm{PC}_{61} \mathrm{BM}$. Generally, the EQE at $570 \mathrm{~nm}$ and $350 \mathrm{~nm}$ degrade less for devices with higher wt\% $\mathrm{PC}_{61} \mathrm{BM}$, correlating well with the less photobleaching of PCDTBT and $\mathrm{PC}_{61} \mathrm{BM}$ (Figure $14.10 \mathrm{~d}$ ) and the reduced drop in $\mathrm{J}_{\mathrm{sc}}$ (Figure 4.12c). Note that there is a higher drop of EQE at $570 \mathrm{~nm}$ than that at $350 \mathrm{~nm}$, correlating well with more photobleaching at 570 $\mathrm{nm}$. The EQE of the device with the highest wt\% $\mathrm{PC}_{61} \mathrm{BM}$ does not fit well with this correlation, undergoing more degradation than expected, this could be due to problems with solubility at the wt\% extremities, leading to morphological differences (supported by the mean size of ordered domains in Figure 4.9b). These results imply that the larger drop in $\mathrm{J}_{\mathrm{sc}}$ with lower content of $\mathrm{PC}_{61} \mathrm{BM}$ could be correlated to degradation of both PCDTBT and $\mathrm{PC}_{61} \mathrm{BM}$, with PCDTBT contributing more.

\subsubsection{Investigation into the photo-oxidation mechanism}

Next, transient absorption spectroscopy measurements were undertaken to investigate what photo-excited species were involved in the photo-oxidation/degradation of the blend films in order to identify a degradation mechanism. Figure 4.14 from reference shows the photoinduced absorption bands of neat PCDTBT and 1:2 PCDTBT:PC ${ }_{61}$ BM blend films, with maxima at $\sim 1050 \mathrm{~nm}$ and $\sim 1000 \mathrm{~nm}$ respectively.[35]

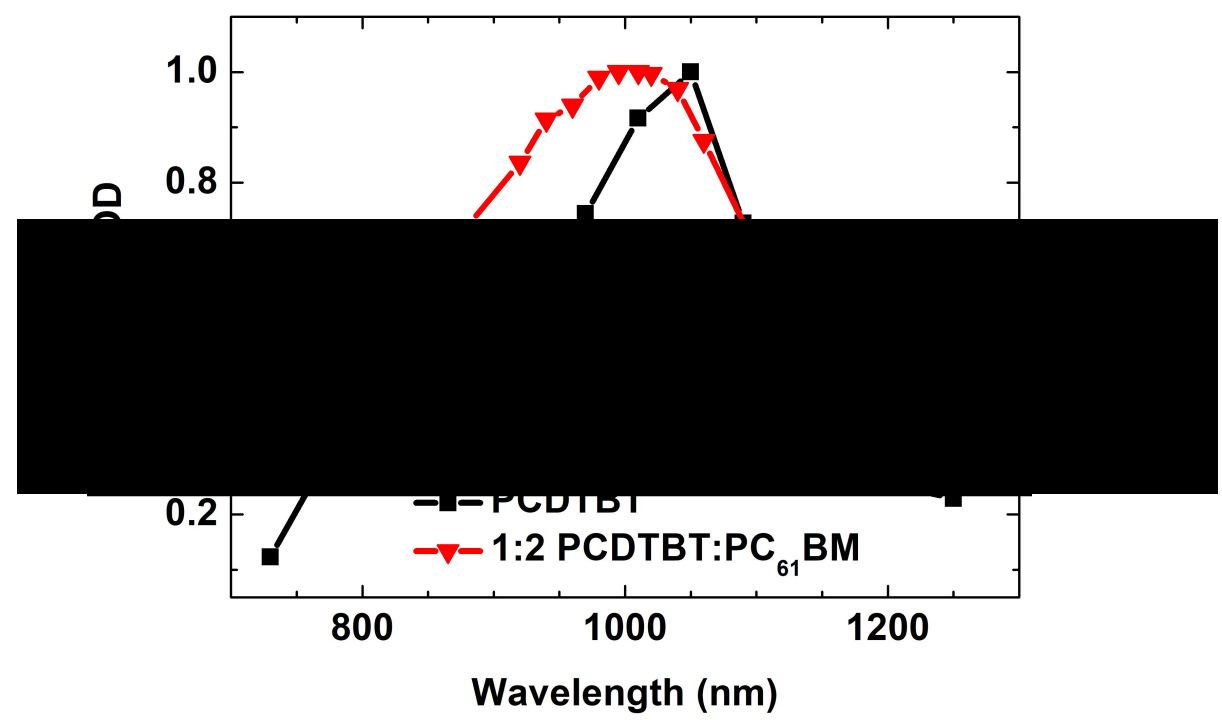

Figure 4.14: Transient absorption spectra of neat PCDTBT film and blend film with 67 wt\% $\mathrm{PC}_{61} \mathrm{BM}$, measured at $1 \mu$ s after laser excitation and pumped at $560 \mathrm{~nm}$ with 3 $\mu \mathrm{Jcm}^{-2}$ excitation density under a nitrogen atmosphere. From reference [35] 

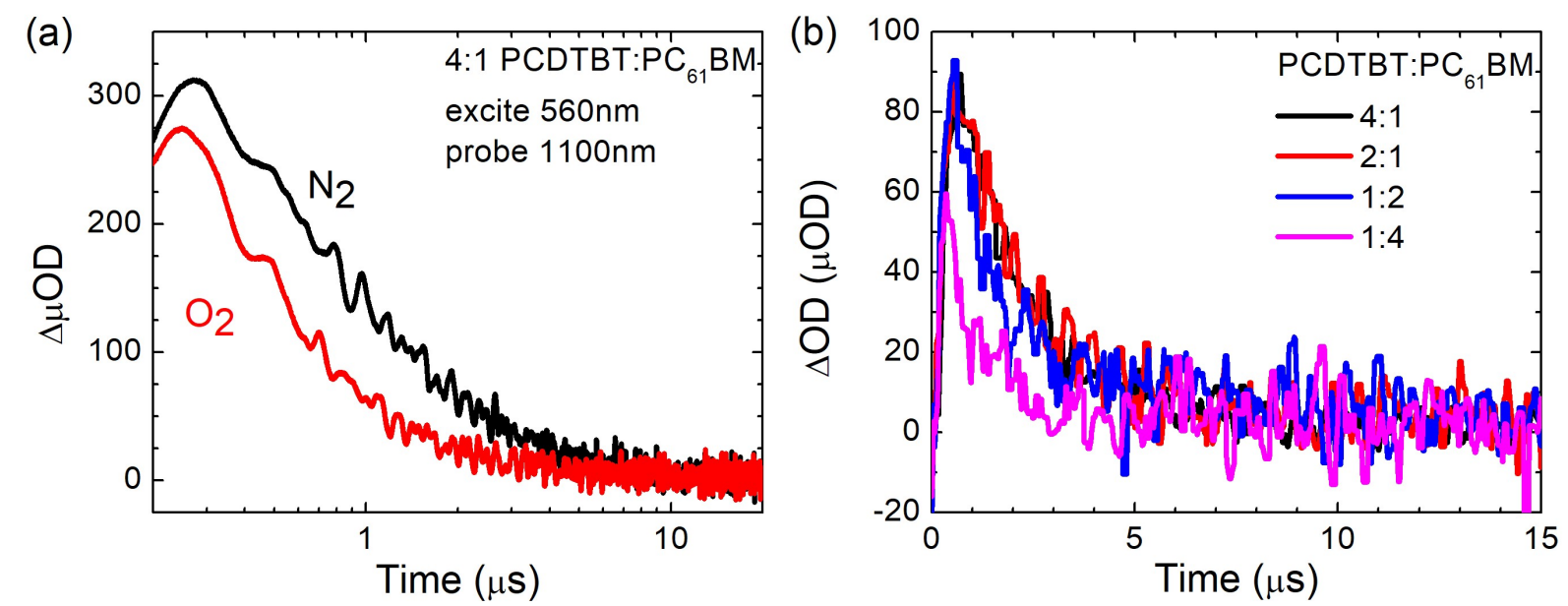

Figure 4.15: TAS measurements: a) Representative TAS decay kinetics of a 4:1 PCDTBT:PC ${ }_{61} \mathrm{BM}$ film, excited at $560 \mathrm{~nm}$ with $1.67 \mu \mathrm{jcm}^{-2}$ and probed at $1100 \mathrm{~nm}$. The kinetic was measured under nitrogen and oxygen environments. b) The change in optical density $(\triangle \mathrm{OD})$ between transient absorption kinetics measured under nitrogen and oxygen for 4:1, 2:1, 1:2 and 1:4 PCDTBT:PC ${ }_{61}$ BM blend films. All TAS kinteics are corrected for the number of photons absorbed.

The films were selectively excited at the polymer absorption region (560 nm) and probed at the blend absorption maximum (1100 nm). Transient absorption (TA) kinetics of a representative film (4:1 PCDTBT: $\left.\mathrm{PC}_{61} \mathrm{BM}\right)$ is shown in Figure 4.15a. The kinetics exhibited exponential decay at early timescales $(<1.0-1.9 \mu$ s followed by power law decays at longer timescales and could be fitted with a combination of the two functions $\left(\mathrm{y}=\mathrm{A}+\mathrm{y}_{0} \mathrm{e}^{\left(-\mathrm{t} / \tau_{1}\right)}+\mathrm{Bt}^{\mathrm{n}}\right)$, indicative of the triplet and polaron states respectively. $\mathrm{TA}$ kinetics of a neat PCDTBT film exhibited the similar exponential behaviour (Figure B.16a); thus, the exponential decays of the TA in blend films can be assigned to that of the polymer triplet state. The TA kinetics of 2:1, 1:2 and 1:4 PCDTBT $\mathrm{PC}_{61} \mathrm{BM}$ films are shown in Figure B.16 (b,c,d). The TA decays were strongly oxygen sensitive, consistent with our study with $\mathrm{PC}_{61} \mathrm{BM}$ :PS blends and previous observations of quenching of triplet states by the triplet ground state of molecular oxygen, forming singlet oxygen.[21] Figure 4.15b shows the subtraction of TA kinetics for films measured under nitrogen from those measured under oxygen, hence giving the yield of polymer triplet states that were quenched by oxygen. It can be seen that the yield of oxygen quenching increases with the decrease of $\mathrm{PC}_{61} \mathrm{BM}$ content; i.e more oxygen quenching of the polymer triplet states with less $\mathrm{PC}_{61} \mathrm{BM}$ aggregation. 


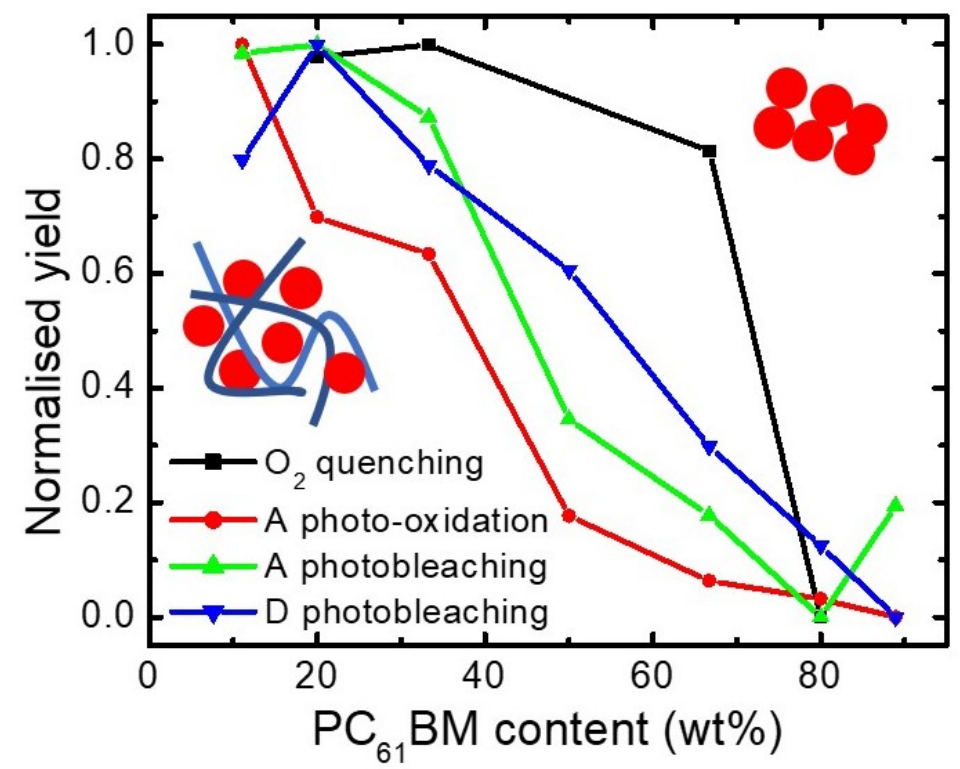

Figure 4.16: Normalised yields of: polymer triplet states quenched by oxygen as calculated from TAS measurements, percentage of $\mathrm{PC}_{61} \mathrm{BM}$ acceptor $(\mathrm{A})$ molecules photo-oxidised after 8 hours of degradation as measured by MALDI-TOF, fractional loss of UV-vis absorbance of $\mathrm{PC}_{61} \mathrm{BM}$ acceptor (A) and PCDTBT donor (D) after 8 hours of photoaging, as a function of $\mathrm{PC}_{61} \mathrm{BM}$ wt\%.

The yield of oxygen quenching was calculated by integrating the difference of the exponential TA decay measured under nitrogen and oxygen (area under the curves in Figure 4.15b. Figure 4.16 shows how this enhancement in oxygen quenching of the polymer triplet state with $\mathrm{PC}_{61} \mathrm{BM}$ wt\% correlates well with the increase in photobleaching of $\mathrm{PC}_{61} \mathrm{BM}$ and PCDTBT, and the increased photo-oxidation of $\mathrm{PC}_{61} \mathrm{BM}$. This trend where the degradation of both $\mathrm{PC}_{61} \mathrm{BM}$ and $\mathrm{PCDTBT}$ is dependent on $\mathrm{PC}_{61} \mathrm{BM}$ aggregation correlates well with the loss of $\mathrm{V}_{\mathrm{oc}}\left(\mathrm{PC}_{61} \mathrm{BM}\right.$ photo-oxidation) and $\mathrm{J}_{\mathrm{sc}}$ (photobleaching of PCDTBT and $\left.\mathrm{PC}_{61} \mathrm{BM}\right)$ with $\mathrm{PC}_{61} \mathrm{BM}$ content.

Here, a strong correlation was found between the yield of triplets being oxygen quenched and the level of photo-degradation of PCDTBT:PC ${ }_{61} \mathrm{BM}$ films and devices as a function of $\mathrm{PC}_{61} \mathrm{BM}$ aggregation. This is consistent with triplet-mediated photo-oxidation, similar to that suggested as the cause of photodegradation in some donor polymers. [9, 21] This suggests that the dominant degradation mechanism for this system is singlet oxygen generation via the polymer triplet excitons (Figure 4.17). Here, electrons are transferred from the photo-excited PCDTBT singlet state $\left(\mathrm{S}_{1}\right)$ to $\mathrm{PC}_{61} \mathrm{BM}$, forming either charge transfer states (CT) or, through charge dissociation (CD), charge separated (CS) states. The CT states intersystem cross (ISC) between the energetically degenerate singlet $\left({ }^{1} \mathrm{CT}\right)$ and triplet $\left({ }^{3} \mathrm{CT}\right)$ states efficiently. Electrons are back charge transferred from interfacial ${ }^{3} \mathrm{CT}$ to populate the PCDTBT triplet $\left(\mathrm{T}_{1}\right)$ state (i.e germinate recombination), which has previously been shown to be possible for SiIDT-DTBT:PC ${ }_{71} B M$ blends.[36] It is also possible to form ${ }^{3} \mathrm{CT}$ states from non-germinate recombination of CS states.[36] 
These $\mathrm{T}_{1}$ states are quenched by molecular oxygen $\left({ }^{3} \mathrm{O}_{2}\right)$, via energy transfer, to form highly reactive singlet oxygen $\left({ }^{1} \mathrm{O}_{2}\right)$ which causes photodegradation in both PCDTBT and $\mathrm{PC}_{61} \mathrm{BM}$, and implications to the device stability When $\mathrm{PC}_{61} \mathrm{BM}$ is less aggregated, the electrons transferred from PCDTBT to $\mathrm{PC}_{61} \mathrm{BM}$ cannot be extracted away efficiently, which enhances the probability of back electron transfer to PCDTBT triplets and singlet oxygen generation, causing more degradation. Besides electron extraction, there are two other processes that compete with this degradation mechanism: germinate recombination from the PCDTBT triplet state $\left(\mathrm{T}_{1}\right)$ to the ground state $\left(\mathrm{S}_{0}\right)$ and non-germinate of the charge separated (CS) electrons back to the PCDTBT ground state $\left(\mathrm{S}_{0}\right)$.

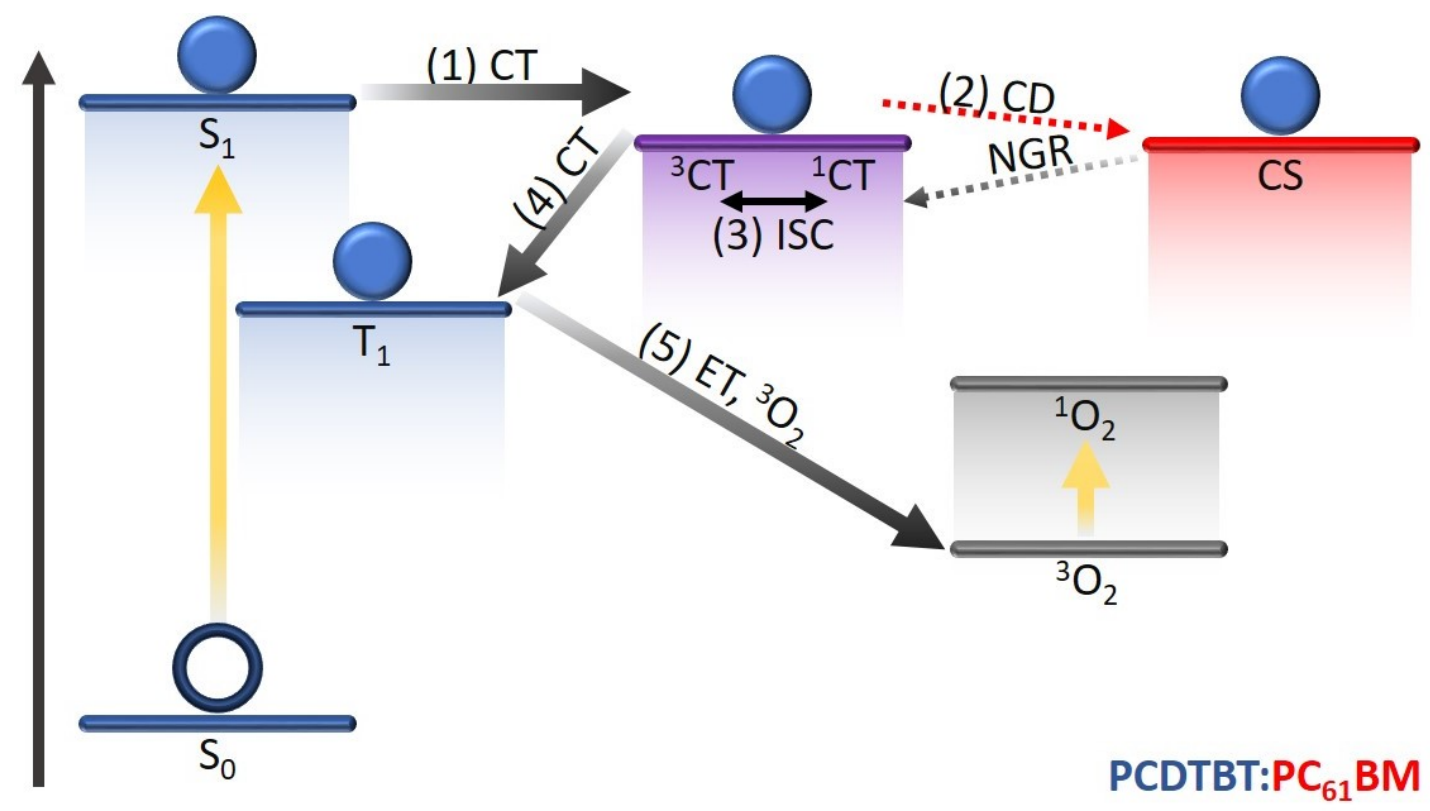

Figure 4.17: Model used to explain photodegradation of PCDTBT:PC ${ }_{61} \mathrm{BM}$ via polymertriplet mediated singlet oxygen generation: (1) Electrons are transferred from the photoinduced PCDTBT singlet state $\left(\mathrm{S}_{1}\right)$ to $\mathrm{PC}_{61} \mathrm{BM}$, forming either charge transfer states (CT) or, (2) through charge dissociation (CD), charge separated (CS) states. (3) The CT states intersystem cross (ISC) between the singlet $\left({ }^{1} \mathrm{CT}\right)$ and triplet $\left({ }^{3} \mathrm{CT}\right)$ states efficiently. (4) Electrons are back charge transferred from ${ }^{3} \mathrm{CT}$ to the PCDTBT triplet $\left(\mathrm{T}_{1}\right)$ state. (5) Energy transfer from $\mathrm{T}_{1}$ to molecular oxygen $\left({ }^{3} \mathrm{O}_{2}\right)$ results in singlet oxygen $\left({ }^{1} \mathrm{O}_{2}\right)$ formation.

It is possible that singlet oxygen could be formed via the quenching of the fullerene triplet states after photo-absorption by the fullerene. This mechanism would correspond well with that shown with PS, which was also dependent on $\mathrm{PC}_{61} \mathrm{BM}$ aggregation. However, the absorption of visible light is greater for PCDTBT than $\mathrm{PC}_{61} \mathrm{BM}$, so it is more likely that the dominant degradation pathway is via the polymer triplets. Additionally, the typical triplet energy of polymers is lower than fullerenes $(0.9-1.2 \mathrm{eV}$ and $\sim 1.5 \mathrm{eV}$ respectively)[20] so polymer triplets are more favourable energetically to form. 


\subsubsection{Thickness dependent aggregation and stability}

An interesting question is: how does film thickness affect the photodegration of PCDTBT: $\mathrm{PC}_{61} \mathrm{BM}$ films that have similar $\mathrm{PC}_{61} \mathrm{BM}$ aggregation? To gain insight to this question, films/devices (inverted) were prepared with the same PCDTBT: $\mathrm{PC}_{61} \mathrm{BM}$ blend ratio (1:2), and therefore similar $\mathrm{PC}_{61} \mathrm{BM}$ aggregation, but with different solution concentrations (therefore, different film thicknesses).

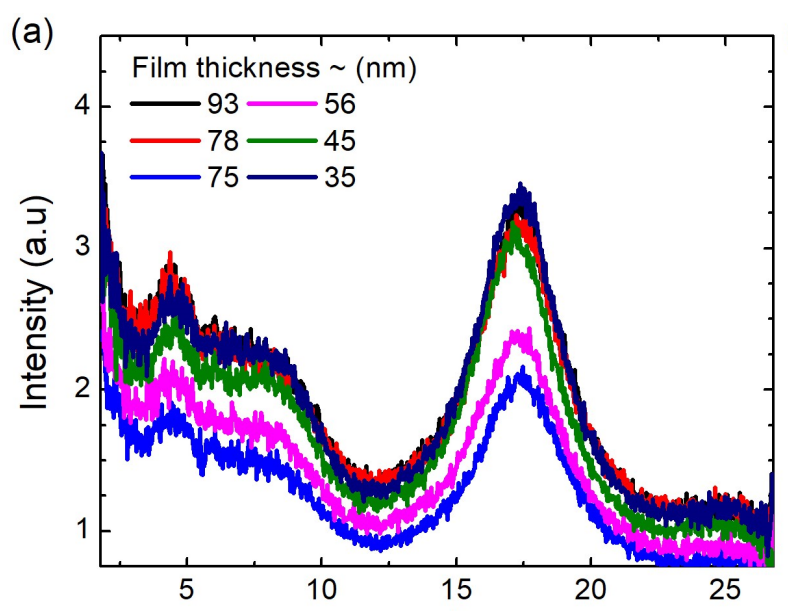

$2 \theta^{\circ}$

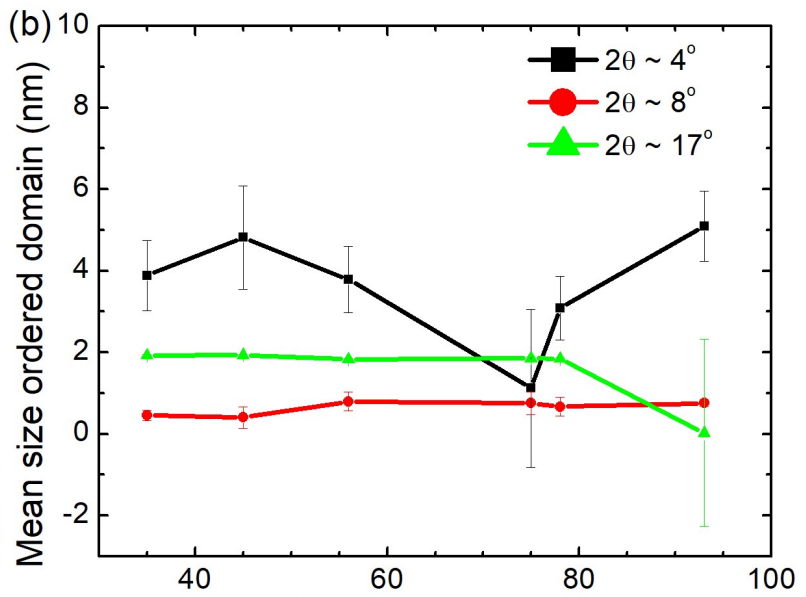

Film thickness $(\mathrm{nm})$

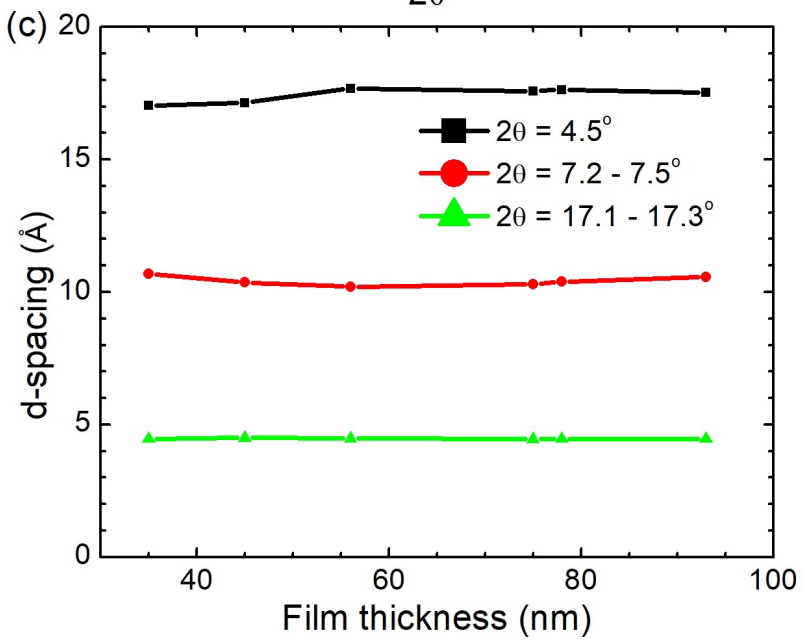

Figure 4.18: (a) GIWAXS spectra for the 1:2 PCDTBT:PC ${ }_{61} \mathrm{BM}$ films with different film thicknesses. (b) Mean size of ordered domains and (c) d-spacings corresponding to the peaks observed as a function of film thickness.

To investigate how different film thickness affects the $\mathrm{PC}_{61} \mathrm{BM}$ aggregation in the blend films, GIWAXS measurements were undertaken. Figure 4.18a shows the GIWAXS spectra for the 1:2 PCDTBT:PC ${ }_{61} \mathrm{BM}$ films with different thicknesses. GIWAXS peaks $\left(2 \theta \sim 4,8\right.$ and $\left.17^{\circ}\right)$ were observed, consistent with the GIWAXS peak positions of 1:2 PCDTBT: $\mathrm{PC}_{61} \mathrm{BM}$ film discussed in previous section. Figure $4.18 \mathrm{~b}$ shows the mean size of ordered domains of the PCDTBT:PC ${ }_{61} \mathrm{BM}$ films as a function of film thickness. The mean size of ordered domains corresponding to the two peaks $\left(2 \theta \sim 8\right.$ and $\left.17^{\circ}\right)$ assigned to $\mathrm{PC}_{61} \mathrm{BM}$ do not change with film thickness, indicating that the aggregation of the 
$\mathrm{PC}_{61} \mathrm{BM}$ in the films is similar. The peak due to the PCDTBT lamella spacing $\left(2 \theta \sim 4^{\circ}\right)$ has more variability, but this is likely due to the fact that a polymer chain is much larger than a fullerene molecule.

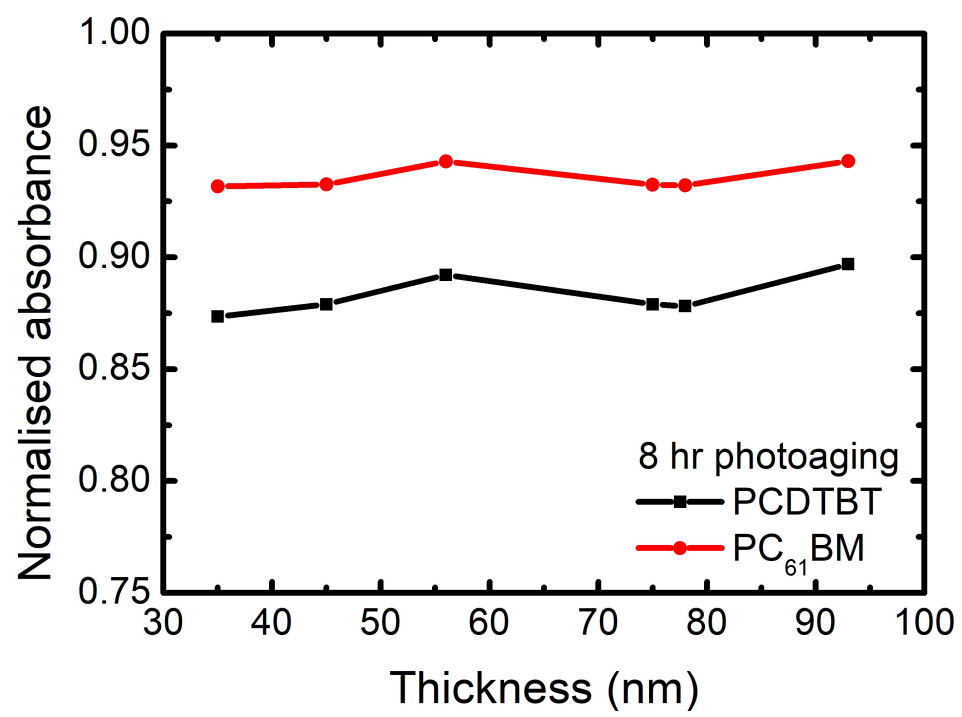

Figure 4.19: Absorbance of PCDTBT (581 nm) and $\mathrm{PC}_{61} \mathrm{BM}(265 \mathrm{~nm})$ peaks in 1:2 PCDTBT:PC ${ }_{61} \mathrm{BM}$ films, after 8 hours of photoaging under 1 sun AM1.5G solar simulator radiation in air, normalised to the unaged spectra, as a function of film thickness.

Photobleaching measurements were undertaken to determine whether these films with similar $\mathrm{PC}_{61} \mathrm{BM}$ aggregation, degraded similarly. The UV-vis absorbance spectra of films of different film thicknesses with increasing photoaging times are shown in Figure B.17 and the rate of degradation of the PCDTBT and $\mathrm{PC}_{61} \mathrm{BM}$ peaks are shown in Figure B.18. Figure 4.19 shows the normalised absorbance of both the PCDTBT and $\mathrm{PC}_{61} \mathrm{BM}$ peaks as a function of film thickness, after 8 hours of photoaging. The PCDTBT and $\mathrm{PC}_{61} \mathrm{BM}$ absorbance peaks dropped to $87.4-89.4 \%$ and $93.2-94.3 \%$, respectively, of that of the fresh films. These results show that the photobleaching of the PCDTBT and $\mathrm{PC}_{61} \mathrm{BM}$ in the blend films (with different thicknesses) is similar, which could be explained by the similar $\mathrm{PC}_{61} \mathrm{BM}$ aggregation (and PCDTBT crystallinity) in the films, and it is not dependent on the film thickness. Similar to the photobleaching of 1:2 PCDTBT:PC ${ }_{61} B M$ film discussed in the previous section, PCDTBT is photobleached more than $\mathrm{PC}_{61} \mathrm{BM}$ in these films. 


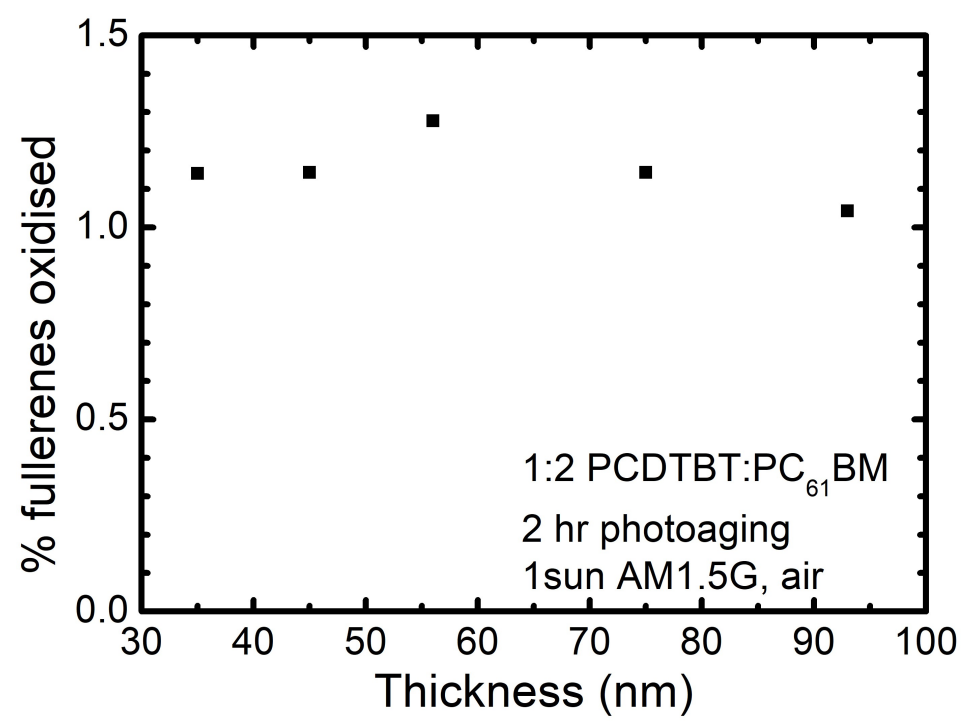

Figure 4.20: Percentage of $\mathrm{PC}_{61} \mathrm{BM}$ photo-oxidised in the films, after 2 hours of photoaging under 1 sun AM1.5G solar simulator radiation in air, as a function of film thickness.

Mass spectrometry was performed to study whether the films (different thicknesses) with similar $\mathrm{PC}_{61} \mathrm{BM}$ aggregation underwent similar amounts of photo-oxidation of $\mathrm{PC}_{61} \mathrm{BM}$ (Figure B.19). Figure 4.20 shows the percentage of $\mathrm{PC}_{61} \mathrm{BM}$ photo-oxidised as a function of the film thickness, for films which were photoaged for 2 hours under 1 sun AM1.5G radiation in air. The percentage of $\mathrm{PC}_{61} \mathrm{BM}$ photo-oxidised in the films is between $1.04 \%-1.28 \%$. Therefore, the percentage of $\mathrm{PC}_{61} \mathrm{BM}$ photo-oxidation is similar for the films with different thicknesses, which correlates well with their similar $\mathrm{PC}_{61} \mathrm{BM}$ aggregation, and similar photobleaching.

To study the effect of film thickness (with similar $\mathrm{PC}_{61} \mathrm{BM}$ aggregation) to the device stability, devices were fabricated where the PCDTBT:PC ${ }_{61} \mathrm{BM}$ films were photoaged prior to electrode deposition. Figure 4.21 shows the normalised device parameters of the devices with varied PCDTBT: ${ }_{61}$ BM thickness as a function of photoaging time (prior to electrode deposition). The absolute values of the device parameters as a function of photoaging time can be found in Figure B.20. Notably, from Figure B.21, there is not much variability in initial device performance $(3.97-4.73 \%)$. There is a drop in PCE and FF for fresh devices thicker than $75 \mathrm{~nm}$, in agreement to previous reports of PCDTBT devices made with both $\mathrm{PC}_{61} \mathrm{BM}$ and $\mathrm{PC}_{71} \mathrm{BM}$, where this was attributed to poor charge transport in these blends, low hole mobilities in particular, leading to significant bimolecular recombination in thicker junctions under 1 sun operating conditions. [37, 38] In general, the PCE dropped less with photoaging for thinner films, which was mainly due to the $\mathrm{J}_{\mathrm{sc}}$ and $\mathrm{FF}$ which also show a similar trend with photoaging time. Interestingly, the $\mathrm{V}_{\mathrm{oc}}$ dropped similarly for all the film thicknesses, correlating very well with the similar $\mathrm{PC}_{61} \mathrm{BM}$ photo-oxidation, as the $\mathrm{PC}_{61} \mathrm{BM}$ aggregation is similar for all the films. 

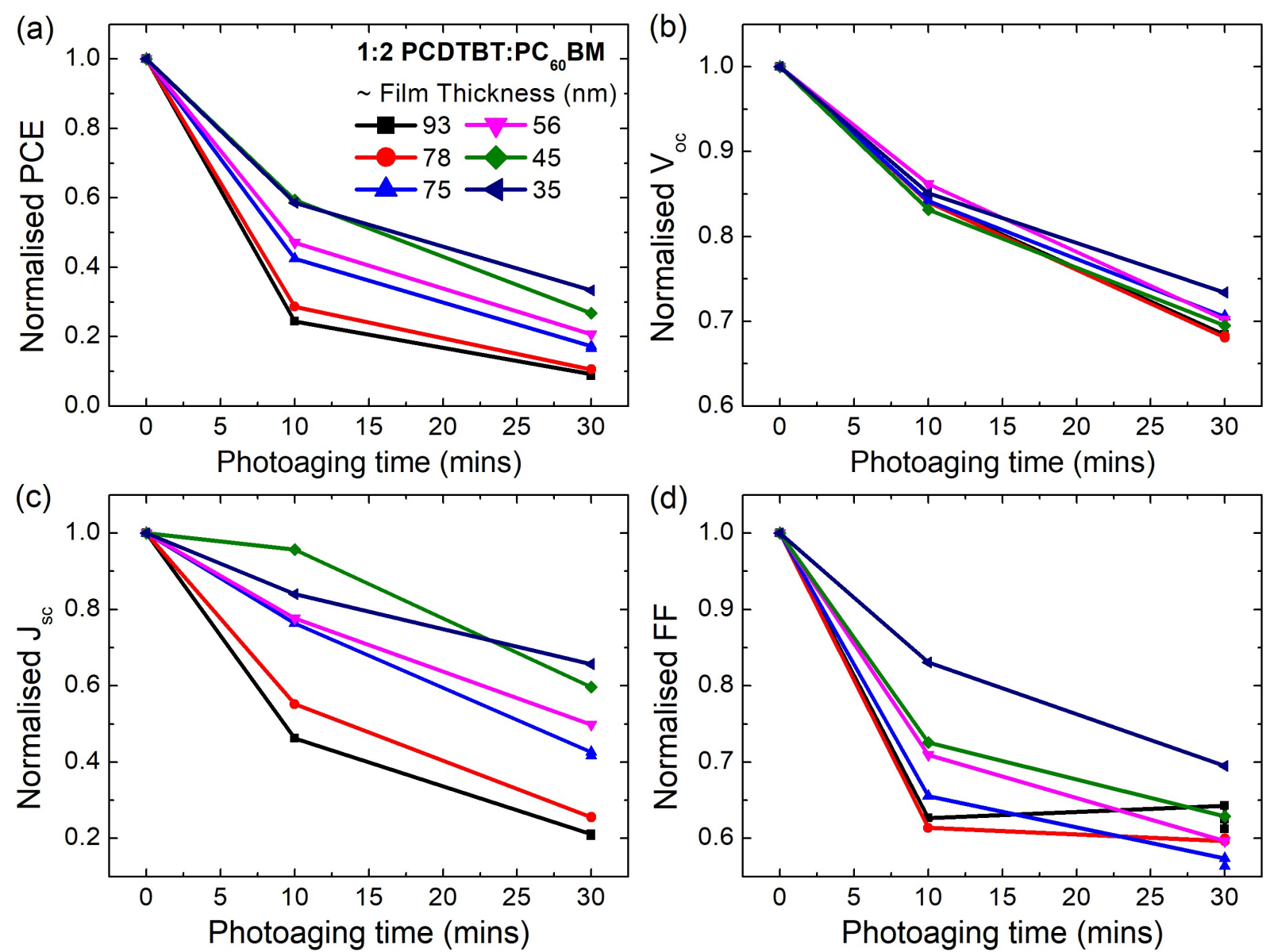

Figure 4.21: Normalised device characteristics (a) PCE (b) $\mathrm{V}_{\mathrm{oc}}$ (c) $\mathrm{J}_{\mathrm{sc}}$ (d) FF PCDTBT:PC ${ }_{61} \mathrm{BM}$ devices with different film thicknesses as a function of photoaging time. The PCDTBT:PC ${ }_{61} \mathrm{BM}$ films were photoaged prior to the electrode deposition. 

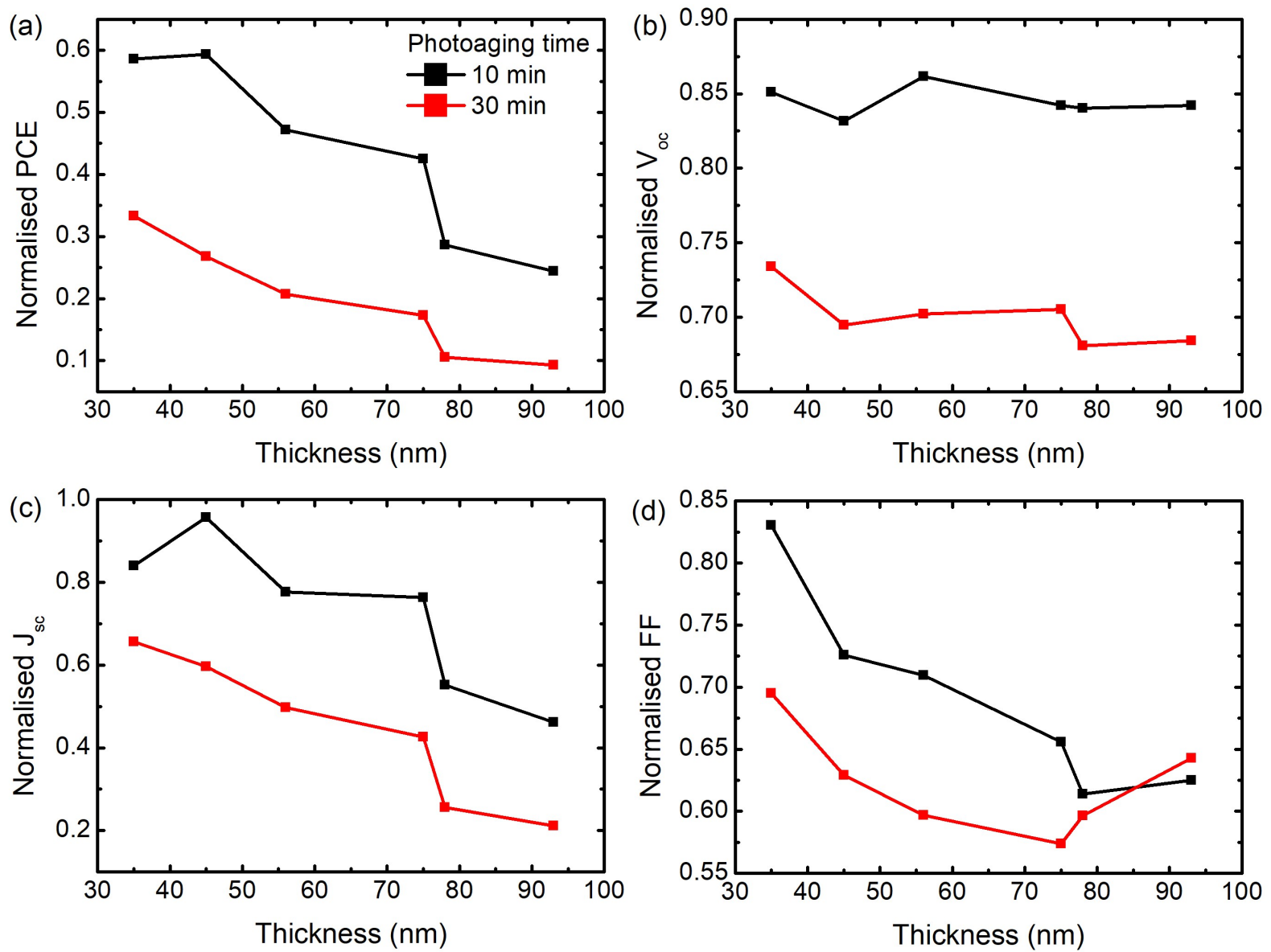

Figure 4.22: Normalised device characteristics (a) PCE (b) $\mathrm{V}_{\text {oc }}$ (c) $\mathrm{J}_{\mathrm{sc}}$ (d) FF of 10 and 30 minutes photoaged PCDTBT:PC ${ }_{61} \mathrm{BM}$ devices as a function of PCDTBT:PC ${ }_{61} \mathrm{BM}$ film thickness. The device parameters are normalised to that of fresh devices

Figure 4.22 shows the device parameters after 10 and 30 minutes of photoaging as a function of film thickness. The device parameters are normalised to that of the unaged devices (the absolute values of the device parameters after the photoaging, as a function of thickness are shown in Figure B.21). It is clear that as the PCDTBT:PC ${ }_{61} \mathrm{BM}$ films get thinner, the PCE drops less, i.e. the more stable the device. Even clearer is the drop in PCE with thicker films is mainly due to the drop in $\mathrm{J}_{\mathrm{sc}}$ and FF. The general drop in $\mathrm{J}_{\mathrm{sc}}$ for all thickness could be correlated to the photobleaching and loss of conjugation of the donor.[29] In general, the FF also shows a smaller drop with thinner films, except for film thickness $>75 \mathrm{~nm}$ after 30 mins photoaging (which does not follow the trend). It is possible that for thicker films with longer photoaging times, the absolute $\mathrm{FF}$ is relative low compare with thinner films or shorter photoaging times, which makes the $\mathrm{FF}$ here not fit the trend. While the similar $\mathrm{V}_{\text {oc }}$ drop for all film thicknesses correlates well to the similar photo-oxidation of $\mathrm{PC}_{61} \mathrm{BM}$, the $\mathrm{J}_{\mathrm{sc}}$ drop does not correlate with the similar photobleaching for all the film thicknesses. Hence, it is proposed here that despite the films degrading similarly, the charges are easier to be transported away from the PCDTBT:PC ${ }_{61} \mathrm{BM}$ interface and extracted for thinner films due to shorter transport length, and thus reducing charge recombination. This proposition can explain why the FF 
increases with degraded thinner films. This finding suggests it could be good to employ thinner films in devices if they have comparable PCE to thicker films, as they can be more photostable in air.

\subsection{Conclusions}

This chapter finds the photochemistry and stability of fullerene films and devices is strongly dependent upon film nanomorphology. The first part of this chapter provides an in-depth study of the photo-oxidation of $\mathrm{PC}_{61} \mathrm{BM}$. We find that the photochemical stability of this fullerene acceptor is strongly dependent upon film morphology: the less aggregated the fullerene (in PS), the faster it is photo-oxidised. This enhanced photooxidation correlates with increased oxygen quenching of the triplet states, suggesting the primary degradation mechanism is triplet-mediated singlet oxygen generation. This photo-oxidation was shown to have a substantial impact on electron mobility, indicative of electron trap formation. This conclusion was supported with an observed red-shift in PL and EL spectra with photoaging and TD-DFT calculations showing red-shifting of PL spectra with photo-oxidised defects due to a suppression of LUMO level.

The second part of this chapter extends the study to explore how $\mathrm{PC}_{61} \mathrm{BM}$ aggregation affects the stability of donor polymer (PCDTBT):fullerene blend films and devices. GIWAXS measurements confirmed that films with a higher $\mathrm{PC}_{61} \mathrm{BM}$ content were more aggregated when blended with PCDTBT (and the PCDTBT was more crystalline). Then $\mathrm{UV}-\mathrm{V}$ is measurements showed that films with less aggregated $\mathrm{PC}_{61} \mathrm{BM}$, underwent more photobleaching of both the $\mathrm{PC}_{61} \mathrm{BM}$ and PCDTBT, which correlated with an increase in the amount of $\mathrm{PC}_{61} \mathrm{BM}$ photo-oxidation as measured by mass spectrometry, and decrease in device stability - particularly correlating well with the significant drop in $\mathrm{V}_{\text {oc }}$. The enhanced photo-oxidation and reduced device stability correlates with an increase in oxygen quenching of the polymer triplet state, suggesting again the dominate degradation pathway is singlet oxygen generation mediated via the polymer triplet states. The correlation between increased stability with increased $\mathrm{PC}_{61} \mathrm{BM}$ aggregation is due to the enhanced electron transport away from the PCDTBT:PC ${ }_{61} \mathrm{BM}$ interface and extraction, reducing the likelihood of back-electron transfer to the polymer triplets which can lead to singlet oxygen formation, and hence degradation of the PCDTBT and $\mathrm{PC}_{61} \mathrm{BM}$.

The photoaging of 1:2 PCDTBT:PC ${ }_{61} \mathrm{BM}$ films/devices as a function of active layer thickness, shows that with similar $\mathrm{PC}_{61} \mathrm{BM}$ aggregation, devices with a thinner active layer are more stable. Since the photobleaching of PCDTBT: $\mathrm{PC}_{61} \mathrm{BM}$ and photo-oxidation of $\mathrm{PC}_{61} \mathrm{BM}$ are similar for all thicknesses, likely due to the similar $\mathrm{PC}_{61} \mathrm{BM}$ aggregation, the 
films should degrade similarly. Therefore, here, it is proposed that the charges in thinner films could be easier to extract for similar levels of degradation (traps), as the charges need to travel a shorter distance to the electrode to be successfully extracted before charge recombination occurs; hence there is less device degradation for thinner films.

As fullerene and fullerene:polymer films are commonly used for organic photovoltaics and a broad range of other optoelectronic devices, the findings in this chapter highlight photo-oxidation of fullerene acceptors can be crucial to the environmental stability of fullerene-based organic electronics. 


\section{REFERENCES}

[1] L. Fernandes, H. Gaspar, and G. Bernardo, "Inhibition of thermal degradation of polystyrene by C60 and PCBM: A comparative study," Polymer Testing, vol. 40, pp. 63-69, 2014.

[2] K. Campbell, B. Gurun, B. G. Sumpter, Y. S. Thio, and D. G. Bucknall, "Role of Conformation in $\pi-\pi$ Interactions and Polymer/Fullerene Miscibility," The Journal of Physical Chemistry B, vol. 115, pp. 8989-8995, 2011.

[3] D. G. Bucknall, G. Bernardo, M. L. Shofner, D. Nabankur, D. Raghavan, B. G. Sumpter, S. Sides, A. Huq, and A. Karim, "Phase Morphology and Molecular Structure Correlations in Model Fullerene-Polymer Nanocomposites," Materials Science Forum, vol. 714, pp. 63-66, 2012.

[4] Y. Huang, W. Wen, S. Mukherjee, H. Ade, E. J. Kramer, and G. C. Bazan, "HighMolecular-Weight Insulating Polymers Can Improve the Performance of Molecular Solar Cells," Advanced Materials, vol. 26, pp. 4168-4172, 2014.

[5] Z. Zhu, Q. Xue, H. He, K. Jiang, Z. Hu, Y. Bai, T. Zhang, S. Xiao, K. Gundogdu, B. R. Gautam, H. Ade, F. Huang, K. S. Wong, H.-L. Yip, S. Yang, and H. Yan, "A PCBM Electron Transport Layer Containing Small Amounts of Dual Polymer Additives that Enables Enhanced Perovskite Solar Cell Performance," Advanced Science, vol. 3, p. 1500353, 2016.

[6] M. H. Richter, D. Friedrich, and D. Schmeißer, "Valence and Conduction Band States of PCBM as Probed by Photoelectron Spectroscopy at Resonant Excitation," BioNanoSci, vol. 2, pp. 59-65, 2012.

[7] J. Díaz, G. Paolicelli, S. Ferrer, and F. Comin, "Separation of the $\mathrm{sp}^{3}$ and $\mathrm{sp}^{2}$ components in the C1s photoemission spectra of amorphous carbon films," Physical Review B, vol. 54, pp. 8064-8069, 1996.

[8] Z. Xiao, J. Yao, D. Yang, F. Wang, S. Huang, L. Gan, Z. Jia, Z. Jiang, X. Yang, B. Zheng, G. Yuan, S. Zhang, and Z. Wang, "Synthesis of [59]Fullerenones through Peroxide-Mediated Stepwise Cleavage of Fullerene Skeleton Bonds and X-ray Structures of Their Water-Encapsulated Open-Cage Complexes," Journal of the American Chemical Society, vol. 129, pp. 16149-16162, 2007.

[9] Y. W. Soon, H. Cho, J. Low, H. Bronstein, I. McCulloch, and J. R. Durrant, "Correlating triplet yield, singlet oxygen generation and photochemical stability in polymer/fullerene blend films.," Chemical communications, vol. 49, pp. 1291-3, 2013. 
[10] Y. W. Soon, S. Shoaee, R. S. Ashraf, H. Bronstein, B. C. Schroeder, W. Zhang, Z. Fei, M. Heeney, I. McCulloch, and J. R. Durrant, "Material Crystallinity as a Determinant of Triplet Dynamics and Oxygen Quenching in Donor Polymers for Organic Photovoltaic Devices," Advanced Functional Materials, vol. 24, pp. 1474$1482,2014$.

[11] S. Cook, H. Ohkita, Y. Kim, J. J. Benson-Smith, D. D. Bradley, and J. R. Durrant, "A photophysical study of PCBM thin films," Chemical Physics Letters, vol. 445, pp. 276-280, 2007.

[12] A. A. Y. Guilbert, M. Schmidt, A. Bruno, J. Yao, S. King, S. M. Tuladhar, T. Kirchartz, M. I. Alonso, A. R. Goñi, N. Stingelin, S. A. Haque, M. Campoy-Quiles, and J. Nelson, "Spectroscopic Evaluation of Mixing and Crystallinity of Fullerenes in Bulk Heterojunctions," Advanced Functional Materials, vol. 24, pp. 6972-6980, 2014 .

[13] S. Chambon, A. Rivaton, J. L. Gardette, and M. Firon, "Photo- and thermal degradation of MDMO-PPV:PCBM blends," Solar Energy Materials and Solar Cells, vol. 91, pp. 394-398, 2007.

[14] H. Neugebauer, C. Brabec, J. Hummelen, and N. Sariciftci, "Stability and photodegradation mechanisms of conjugated polymer/fullerene plastic solar cells," Solar Energy Materials and Solar Cells, vol. 61, pp. 35-42, 2000.

[15] H. Neugebauer, C. Brabec, J. Hummelen, R. Janssen, and N. Sariciftci, "Stability studies and degradation analysis of plastic solar cell materials by FTIR spectroscopy," Synthetic Metals, vol. 102, pp. 1002-1003, 1999.

[16] J. D. McGettrick, E. Speller, Z. Li, W. C. Tsoi, J. R. Durrant, and T. Watson, "Use of gas cluster ion source depth profiling to study the oxidation of fullerene thin films by XPS," Organic Electronics, vol. 49, pp. 85-93, 2017.

[17] J. Kettle, H. Waters, Z. Ding, M. Horie, and G. Smith, "Chemical changes in PCPDTBT:PCBM solar cells using XPS and TOF-SIMS and use of inverted device structure for improving lifetime performance," Solar Energy Materials and Solar Cells, vol. 141, pp. 139-147, 2015.

[18] National Institute of Standards and Technology, "NIST X-ray Photoelectron Spectroscopy Database, Version 4.1," 2012.

[19] J. J. Benson-Smith, H. Ohkita, S. Cook, J. R. Durrant, D. D. C. Bradley, and J. Nelson, "Charge separation and fullerene triplet formation in blend films of polyfluorene polymers with [6,6]-phenyl C61 butyric acid methyl ester.," Dalton Transactions, pp. 10000-5, 2009. 
[20] P. C. Y. Chow, S. Albert-Seifried, S. Gélinas, and R. H. Friend, "Nanosecond intersystem crossing times in fullerene acceptors: implications for organic photovoltaic diodes.," Advanced materials (Deerfield Beach, Fla.), vol. 26, pp. 4851-4, 2014.

[21] A. Distler, P. Kutka, T. Sauermann, H.-J. Egelhaaf, D. M. Guldi, D. Di Nuzzo, S. C. J. Meskers, and R. A. J. Janssen, "Effect of PCBM on the Photodegradation Kinetics of Polymers for Organic Photovoltaics," Chemistry of Materials, vol. 24, pp. 4397-4405, 2012.

[22] F. C. Jamieson, E. B. Domingo, T. McCarthy-Ward, M. Heeney, N. Stingelin, and J. R. Durrant, "Fullerenecrystallisation as a key driver of charge separation in polymer/fullerene bulk heterojunction solar cells," Chemical Science, vol. 3, pp. 485-492, 2012.

[23] H. Ohkita, S. Cook, Y. Astuti, W. Duffy, M. Heeney, S. Tierney, I. McCulloch, D. D. C. Bradley, and J. R. Durrant, "Radical ion pair mediated triplet formation in polymer-fullerene blend films," Chem. Commun., vol. 82, pp. 3939-3941, 2006.

[24] M. A. Faist, T. Kirchartz, W. Gong, R. S. Ashraf, I. McCulloch, J. C. de Mello, N. J. Ekins-Daukes, D. D. C. Bradley, and J. Nelson, "Competition between the Charge Transfer State and the Singlet States of Donor or Acceptor Limiting the Efficiency in Polymer:Fullerene Solar Cells," Journal of the American Chemical Society, vol. 134, pp. 685-692, 2012.

[25] H. Kraus, M. C. Heiber, S. Väth, J. Kern, C. Deibel, A. Sperlich, and V. Dyakonov, "Analysis of Triplet Exciton Loss Pathways in PTB7:PC71BM Bulk Heterojunction Solar Cells," Scientific Reports, vol. 6, p. 29158, 2016.

[26] T. Yasuda and T. Tsutsui, "Organic field-effect transistors based on high electron and ambipolar carrier transport properties of copper-phthalocyanine," Chemical Physics Letters, vol. 402, pp. 395-398, 2005.

[27] M. Jørgensen, K. Norrman, and F. C. Krebs, "Stability/degradation of polymer solar cells," Solar Energy Materials and Solar Cells, vol. 92, pp. 686-714, 2008.

[28] M. Jørgensen, K. Norrman, S. A. Gevorgyan, T. Tromholt, B. Andreasen, and F. C. Krebs, "Stability of polymer solar cells.," Advanced materials, vol. 24, pp. 580-612, 2012.

[29] N. Grossiord, J. M. Kroon, R. Andriessen, and P. W. Blom, "Degradation mechanisms in organic photovoltaic devices," Organic Electronics, vol. 13, pp. 432-456, 2012. 
[30] R. Dattani, K. F. Gibson, S. Few, A. J. Borg, P. A. DiMaggio, J. Nelson, S. G. Kazarian, and J. T. Cabral, "Fullerene oxidation and clustering in solution induced by light," Journal of Colloid and Interface Science, vol. 446, pp. 24-30, 2015.

[31] S. Yamane, J. Mizukado, Y. Suzuki, M. Sakurai, L. Chen, and H. Suda, "MALDITOF MS Study of the Photooxidation of PCBM and Its Suppression by P3HT," Chemistry Letters, vol. 44, pp. 339-341, 2015.

[32] N. Blouin, A. Michaud, D. Gendron, S. Wakim, E. Blair, R. Neagu-Plesu, M. Belletête, G. Durocher, Y. Tao, and M. Leclerc, "Toward a Rational Design of Poly(2,7Carbazole) Derivatives for Solar Cells," Journal of the American Chemical Society, 2007.

[33] E. Verploegen, R. Mondal, C. J. Bettinger, S. Sok, M. F. Toney, and Z. Bao, "Effects of Thermal Annealing Upon the Morphology of Polymer-Fullerene Blends," Advanced Functional Materials, vol. 20, pp. 3519-3529, 2010.

[34] E. T. Hoke, I. T. Sachs-Quintana, M. T. Lloyd, I. Kauvar, W. R. Mateker, A. M. Nardes, C. H. Peters, N. Kopidakis, and M. D. McGehee, "The Role of Electron Affinity in Determining Whether Fullerenes Catalyze or Inhibit Photooxidation of Polymers for Solar Cells," Advanced Energy Materials, vol. 2, pp. 1351-1357, 2012.

[35] Y. W. Soon, Charge Separation, Triplets and Photochemical stability in Polymer/Fullerene Solar Cells. PhD thesis, Imperial College London, 2013.

[36] S. D. Dimitrov, S. Wheeler, D. Niedzialek, B. C. Schroeder, H. Utzat, J. M. Frost, J. Yao, A. Gillett, P. S. Tuladhar, I. McCulloch, J. Nelson, and J. R. Durrant, "Polaron pair mediated triplet generation in polymer/fullerene blends," Nature Communications, vol. 6, p. 6501, 2015.

[37] S. Wakim, S. Beaupré, N. Blouin, B.-R. Aich, S. Rodman, R. Gaudiana, Y. Tao, and M. Leclerc, "Highly efficient organic solar cells based on a poly(2,7-carbazole) derivative," Journal of Materials Chemistry, vol. 19, p. 5351, 2009.

[38] M. Stolterfoht, A. Armin, B. Philippa, R. D. White, P. L. Burn, P. Meredith, G. Juška, and A. Pivrikas, "Photocarrier drift distance in organic solar cells and photodetectors," Scientific Reports, vol. 5, p. 9949, 2015. 
CHAPTER

FIVE

\section{EFFECT OF LUMO ENERGY ON THE PHOTOCHEMICAL STABILITY OF FULLERENE ACCEPTORS IN AIR}

In this chapter, six fullerene acceptors with different LUMO levels were investigated to study how energetics of the LUMO could affect neat film and device photochemical stability. The degree of photobleaching of neat films and photo-oxidation via carbonyl formation on the fullerene cage, correlated well with the fullerene LUMO levels obtained via cyclic voltammetry. Generally, fullerenes with a lower lying LUMO underwent less photo-oxidation. However, Kloc-6 was an exception to this rule, suggesting that besides LUMO level, there are other factors which could also affect the photo-oxidation, such as the chemical structure of the fullerene ball. A good correlation was observed between the level of photo-oxidation in neat films with the photodegradation of devices (PCE and $\mathrm{J}_{\mathrm{sc}}$ ) made with selectively degraded fullerenes, while the reduction in $\mathrm{V}_{\mathrm{oc}}$ correlated well with the fullerene LUMO. Furthermore, the large drop in PCE shows the significance of fullerene photo-oxidation in its role on the stability of OSCs. 


\subsection{Introduction}

This chapter investigates the photochemical stability of a range of fullerene acceptors in air, including: the effect of LUMO energy on the photostability of the fullerene itself (neat films); the effect to the stability of devices; and also includes Kloc-6 (which has a deeper LUMO level than $\left.\mathrm{PC}_{61} \mathrm{BM} / \mathrm{PC}_{71} \mathrm{BM}\right)$. Firstly, cyclic voltammetry was used to measure the LUMO energy of each acceptor. Then, the films were studied before and after photoaging in air including UV-vis absorbance measurements to study the photobleaching of each acceptor and blend film, and FTIR and mass spectroscopy to study chemical degradation of the fullerenes. Then devices were fabricated to study the effect of the photo-oxidation of the different fullerenes to device stability. Finally, polymer:fullerene:fullerene ternary blend devices were fabricated to test whether a fullerene that was more susceptible to photo-oxidation could act as a sacrificial component, improving device stability.

\subsection{Experimental details}

Film preparation: Neat fullerene solutions were made with a concentration of $24 \mathrm{mg} / \mathrm{ml}$ in chloroform and stirred at $60^{\circ} \mathrm{C}$ overnight. Films were then spincoated at $1500 \mathrm{rpm}$ with $2500 \mathrm{rmps}^{-1}$ acceleration for 60 seconds. Quartz (UV-vis spectroscopy and TAS), ITO (cyclic voltammetry) and glass (MALDI-TOF and FTIR for photoaging) substrates respectively were used. Films were photoaged in air under 1 sun AM1.5G illumination for the time stated in the text. For MALDI-TOF and ATR-FTIR, were degraded as a film and scraped off to create a powder.

Device fabrication: Devices were fabricated were fabricated with an inverted structure of ITO/ ZnO/ Active layer/ $\mathrm{MoO}_{3} / \mathrm{Ag}$ as described in Chapter 2. The active layer was formed by dissolving $24 \mathrm{mg}$ P3HT and $24 \mathrm{mg}$ fullerene per $\mathrm{ml}$ of CB overnight on a stirring hotplate at $60^{\circ} \mathrm{C}$ and then spincoating on top of the $\mathrm{ZnO}$ at $2000 \mathrm{rpm}$ with 2500 $\mathrm{rpms}^{-1}$, and annealing for 10 minutes at $130^{\circ} \mathrm{C}$. The films were then photoaged before the electrodes were deposited and the device was encapsulated. Ternary blend devices were also made using the above method but for the active layer film, 1:2 PCDTBT:fullerene 6:12 $\mathrm{mg} \mathrm{m}^{-1}$ in $\mathrm{CB}$ that had been stirred overnight at $90^{\circ} \mathrm{C}$. The fullerene part of the solution was a combination of $\mathrm{PC}_{61} \mathrm{BM}$ and a small percentage of ICBA or ICTA, such that the total fullerene concentration was $12 \mathrm{mg} \mathrm{ml}^{-1}$. The films were spincoated at 800 rpm, with $7500 \mathrm{rpms}^{-1}$ for 60 seconds.

Characterisation: Cyclic voltammetry measurements were performed using the method described in Chapter 2. Measurements were undertaken at Imperial College 
London with the help of Dr. Laia Francàs, Dr. Hyojung Cha and Mr. George Fish (all Imperial College London), data analysis was done by myself. The UV-vis absorbance spectra were measured with a Perkin Elmer Lambda 750 spectrophotometer at Swansea Univeristy. Dr. Mark F. Wyatt carried out the MALDI-TOF measurements and data analysis was performed by myself, both using the method describe in Chapter 2. The ATIR-FTIR spectra were processed from raw data that consisted of 64 scans and $1 \mathrm{~cm}^{-1}$ resolution and the baseline was corrected in the Spectrum 10 software.

\subsection{Measurement of fullerene LUMO levels}

The LUMO level of fullerene acceptors has been reported many times in literature but there is a large variability in values due to a number of factors, such as sample preparation, measuring technique and experimental set-up. A study compiled the data of 37 different $\mathrm{CV}$ experiments on $\mathrm{PC}_{61} \mathrm{BM}$ and found the LUMO to range from -4.38 to -3.62 eV.[1] Therefore, to ensure the CV measurements (which were undertaken to estimate the LUMO energy of the fullerene acceptors used in this chapter) are comparable, the same experimental set-up and conditions were used for each fullerene.

Figure 5.1 shows the cyclic voltammetry scans for different neat fullerene films. The HOMO level was extracted from the oxidation potential of the cyclic voltammetry scans by fitting the first oxidation peak to find the onset. The reduction potential of cyclic voltammetry scans is sensitive to any water (or oxygen) present in the electrolyte. So here, the LUMO instead was calculated by subtracting the bandgap of the fullerenes (1.8 $\mathrm{eV})$ to the HOMO extracted from the scans. The LUMO of $\mathrm{PC}_{61} \mathrm{BM}$ was found to be $-4.11 \mathrm{eV}$ which agrees well with the range cited in literature.[1] $\mathrm{PC}_{71} \mathrm{BM}$ and Kloc-6 were found to have LUMO levels 0.17 and $0.62 \mathrm{eV}$ lower than $\mathrm{PC}_{61} \mathrm{BM}$ (5.1). The LUMO obtained for ICBA and BisPBCM were very similar at 0.24 and $0.29 \mathrm{eV}$ higher than that obtained for $\mathrm{PC}_{61} \mathrm{BM}$ (5.1). ICTA had a much higher LUMO, which was $0.42 \mathrm{eV}$ higher than that obtained for $\mathrm{PC}_{61} \mathrm{BM}$ (5.1). These relative differences in LUMO are similar to those previously reported.[2-5] 
(a)

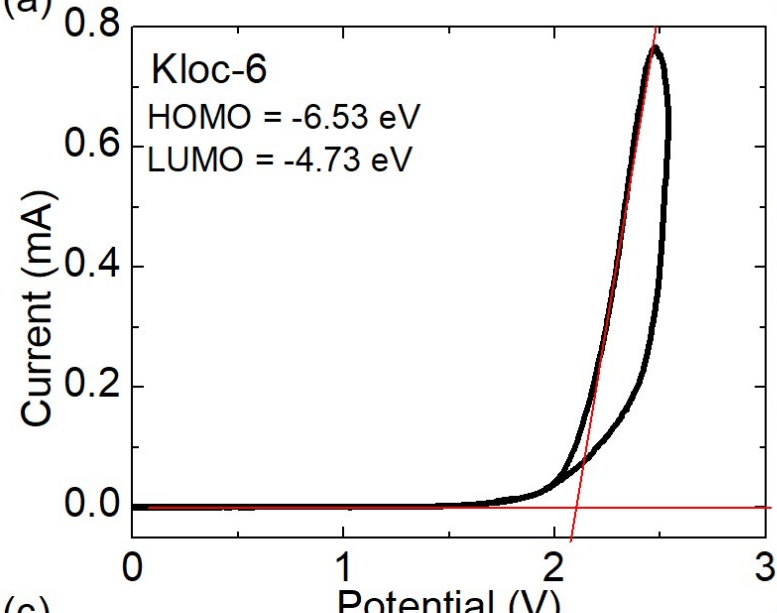

(c) Potential (V)
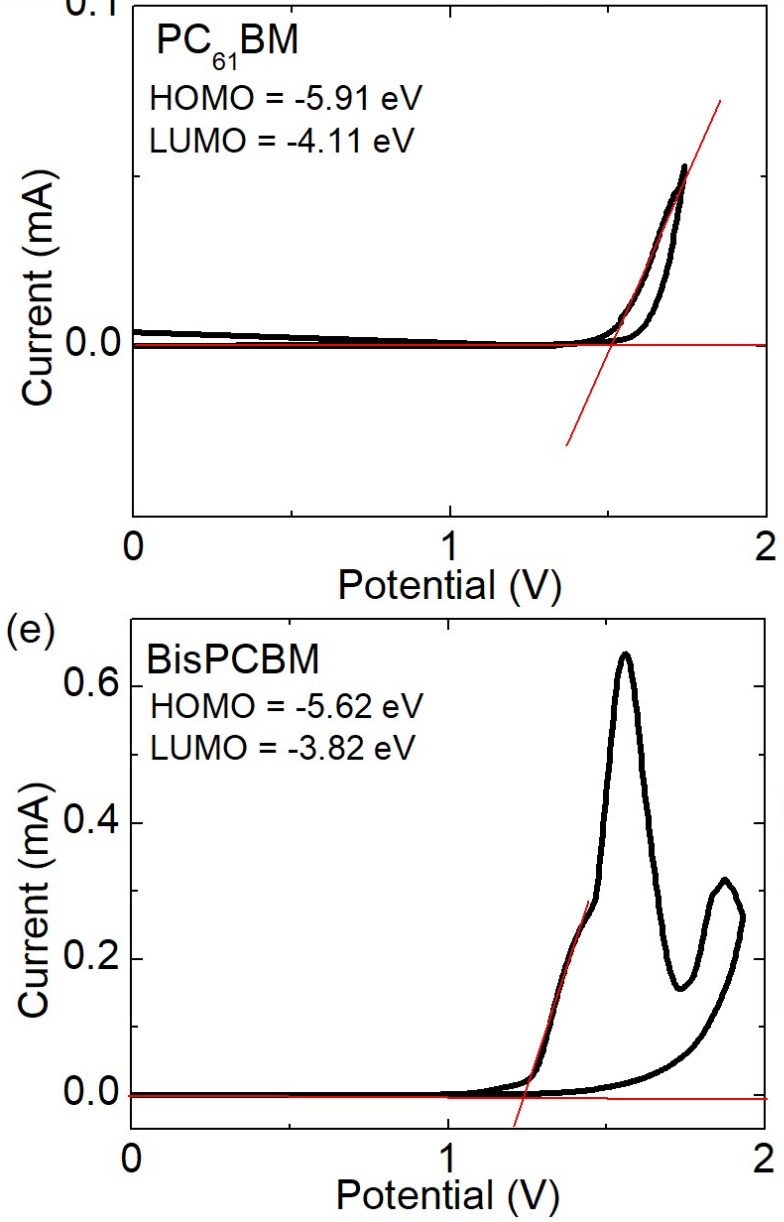

(b)

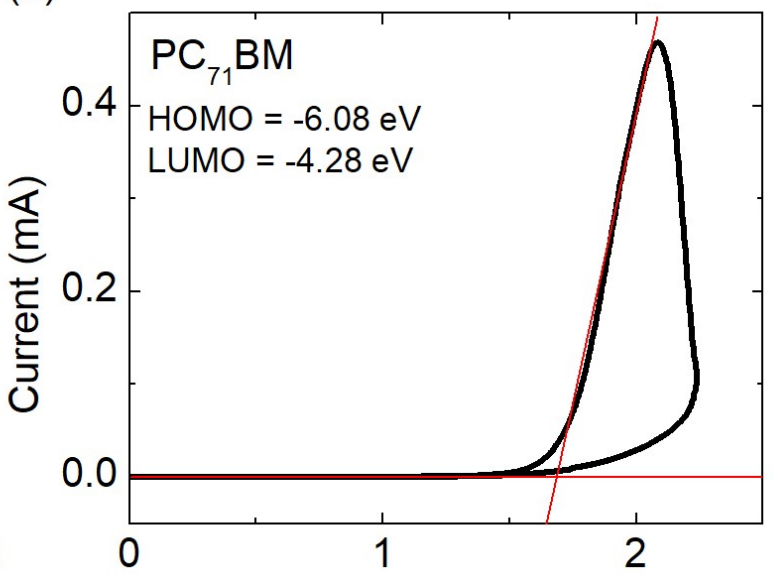

(d)
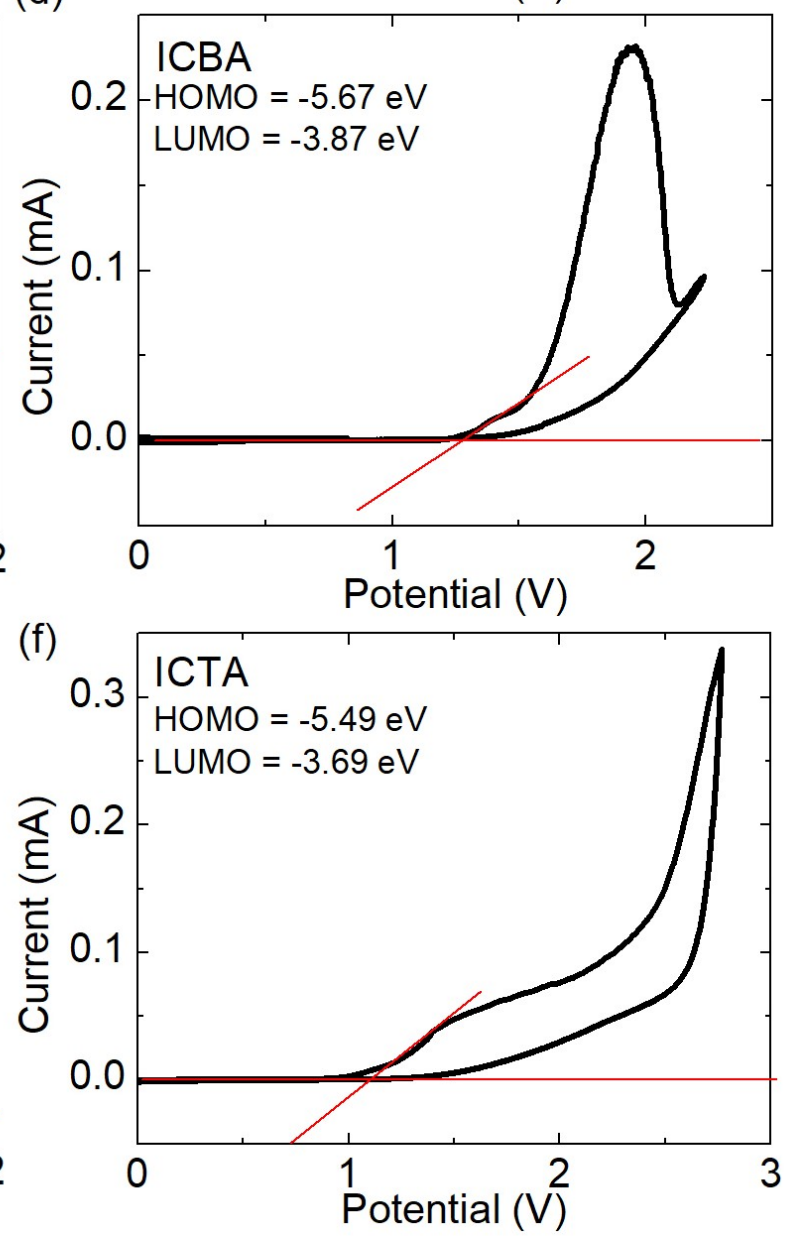

Figure 5.1: Cyclic voltammetry scans for: (a) Kloc-6 (b) $\mathrm{PC}_{71} \mathrm{BM}$ (c) $\mathrm{PC}_{61} \mathrm{BM}$ (d) ICBA (e) BisPCBM (f) ICTA, with the onset of the first oxidation potential fitted. 


\subsection{Spectroscopic studies of LUMO dependence of photo-oxidation in fullerenes}

After the relative LUMO levels for each fullerene acceptor was determined, the photodegradation of the acceptors (in thin films) under 1 sun AM1.5G illumination in air was investigated using UV-vis absorbance spectroscopy.

Figure 5.2 shows the normalised absorbance spectra of the six fullerene acceptors photoaged for up to 32 hours (and the absolute are shown in Figure C.1). It can be observed that the rate of photobleaching varies significantly between the fullerenes, with $\mathrm{PC}_{71} \mathrm{BM}$ being the most stable, followed by $\mathrm{PC}_{61} \mathrm{BM}$ and Kloc-6, while BisPCBM, ICBA, and ICTA are relatively unstable.

Figure 5.3 shows the normalised absorbance of the neat fullerene neat films as a function of photoaging time, which summarises the rates of the photobleaching. It shows the magnitude of photobleaching in the following order (for photoaging times $>16$ hours): ICTA $>$ ICBA $>$ BisPCBM $>$ Kloc- $6>\mathrm{PC}_{61} \mathrm{BM}>\mathrm{PC}_{71} \mathrm{BM}$. With a photoaging time of 32 hours, the absorbance drops $34 \%$ for the least stable fullerene ICTA, which is 6.8 times more than that for the most stable fullerene $\mathrm{PC}_{71} \mathrm{BM}$ which only drop by $5 \%$. Figure 1.3 (b) shows the normalised absorbance after 32 hours of photoaging plotted as a function of the LUMO level obtained via cyclic voltammetry. Here, there is a clear correlation between the photobleaching and LUMO level of fullerene acceptors: the higher lying the LUMO level of the fullerene acceptor, the more photobleaching it undergoes. Interestingly, although Kloc-6 has lowest lying LUMO level, it photobleaches more than $\mathrm{PC}_{61} \mathrm{BM}$ and $\mathrm{PC}_{71} \mathrm{BM}$. This could be explained by its chemical structure being significantly different compared to the other fullerene derivatives: it has different side-chain and the inclusion of oxygen and nitrogen atoms within the fullerene ball, weakening the fullerene cage. In addition to this, it has been suggested that the curvature of fullerene balls determines how easily it is photo-oxidised, whereby the higher the more curved the fullerene cage, the more vulnerable it is to chemical additions. [6-8] This could another factor that contributes to how susceptible a fullerene is to photo-oxidation with the size of fullerene ball and number of sidechains changing the curvature of the ball. 

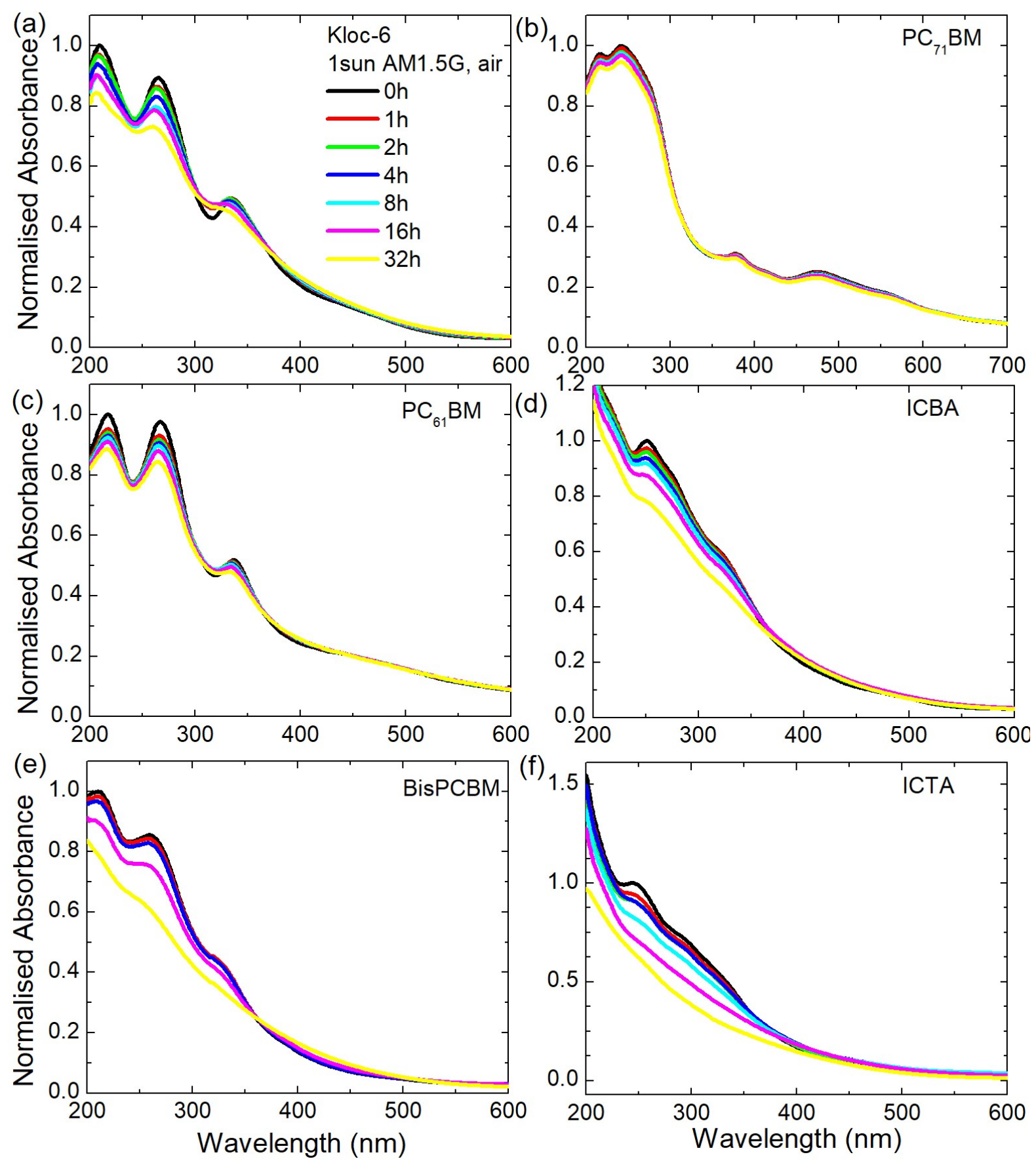

Figure 5.2: Normalised UV-vis absorption spectra of fullerene acceptor neat films under 1 Sun AM1.5G radiation in air, as a function of irradiation time: (a) Kloc-6 (b) $\mathrm{PC}_{71} \mathrm{BM}$ (c) $\mathrm{PC}_{61} \mathrm{BM}$ (d) ICBA (e) BisPCBM (f) ICTA 
(a)

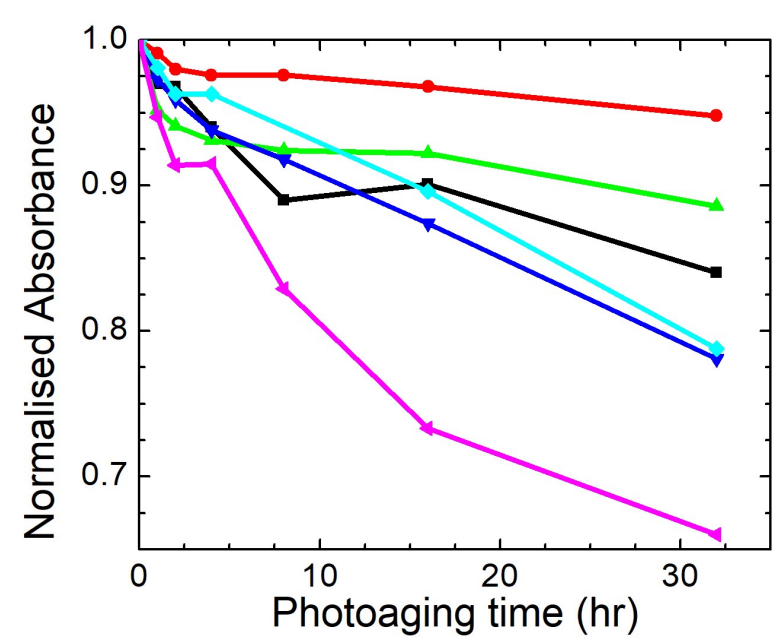

(b)

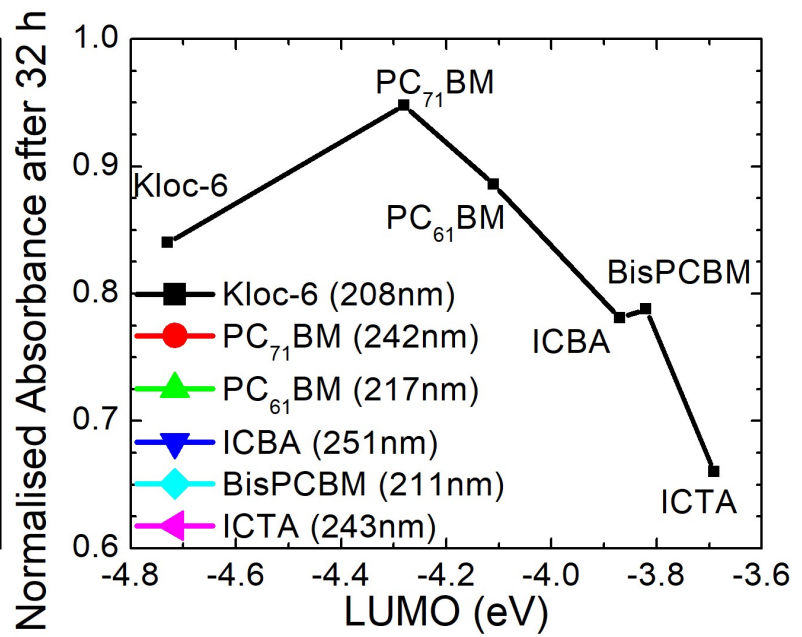

Figure 5.3: Trends in fullerene acceptor photobleaching: (a) normalised UV-vis absorbance as a function of phtoaging time, and (b) normalised absorbance after 32 hours of photoaging as a function of fullerene acceptor LUMO level. (The absorbance is normalised to that of the unaged film.)

(a)

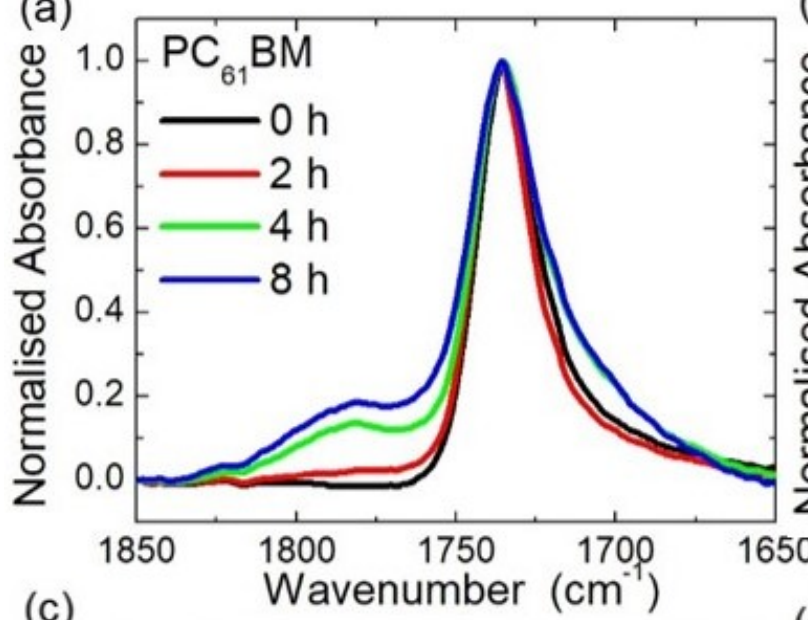

(c)

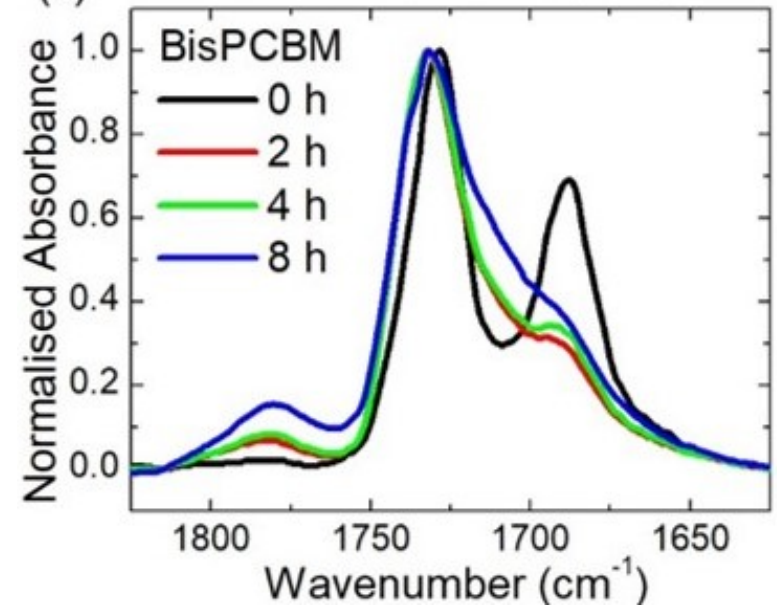

(b)
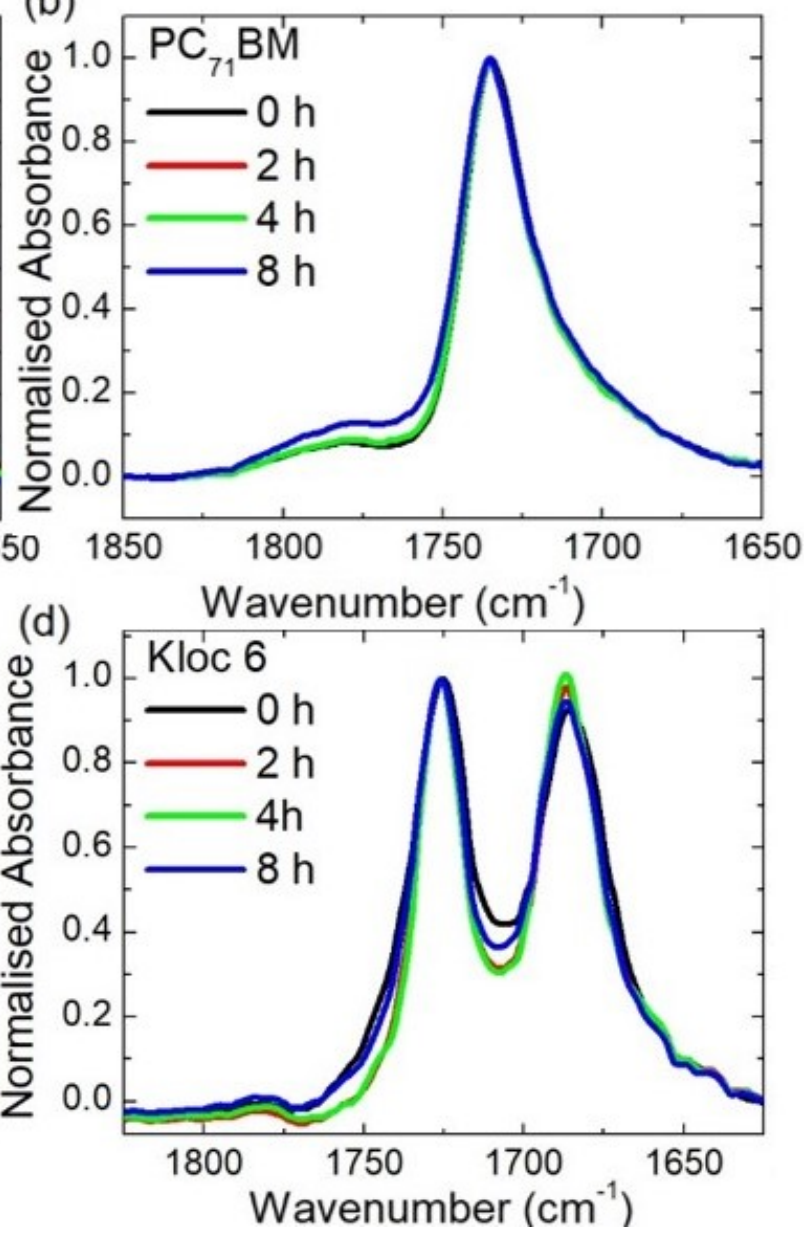

Figure 5.4: Normalised FTIR spectra of: (a) $\mathrm{PC}_{61} \mathrm{BM}$ (b) $\mathrm{PC}_{71} \mathrm{BM}$ (c) Kloc-6 neat films fresh and photoaged degraded for 2, 4 and 8 hours in air under 1 Sun AM1.5G illumination.

As demonstrated in chapter 4, FTIR measurements can be useful to probe oxidation of 
fullerenes, indicated by carbonyl formation on the fullerene cage at $\sim 1780 \mathrm{~cm}^{-1}$. Figure 5.4a shows the FTIR spectra for $\mathrm{PC}_{61} \mathrm{BM}$ (normalised to the ester group on the sidechain $\left(1736 \mathrm{~cm}^{-1}\right)$. It shows the formation and growing of a carbonyl group $\left(1783 \mathrm{~cm}^{-1}\right)$ which can be assigned to the oxidation of the fullerene cage (relative absorbance of 0.18 after 8 hours of photoaging) as observed in Chapter 4 and literature.[9, 10] The formation of the same carbonyl group $\left(1780 \mathrm{~cm}^{-1}\right)$ is observable for $\mathrm{PC}_{71} \mathrm{BM}$ but to less of an extent (relative absorbance of 0.13 after 8 hours of photoaging), implying less photo-oxidation of the fullerene cage compared to $\mathrm{PC}_{61} \mathrm{BM}$. BisPCBM also exhibited the growth of the $\mathrm{C}=\mathrm{O}$ stretch at $1782 \mathrm{~cm}^{-1}$ in addition to a peak at 1688 which can be assigned to an aryl ketone $\mathrm{C}=\mathrm{O}$ stretch which could be caused by the fullerene ball beginning to fragment. However, since there are two sidechains with the ester group on BisPCBM, the relative intensity of this peak is twice as much than for $\mathrm{PC}_{61} \mathrm{BM}$ and $\mathrm{PC}_{71} \mathrm{BM}$, so the level of oxidation is higher. For Kloc- 6 , two strong peaks can be observed for the $\mathrm{C}=\mathrm{O}$ stretches of the amide and ketone groups on the fullerene cage (1686 and $1726 \mathrm{~cm}^{-1}$, respectively). When normalised to the peak from the ketone group, there is minimal carbonyl formation on the cage (0.01) unlike the other fullerenes. This could be caused if only a small amount of photo-oxidation was needed to cause fragmentation. The other fullerenes, ICBA and ICTA, had peaks assigned to the aryl ketone $\mathrm{C}=\mathrm{O}$ stretch (1694 and 1685 $\mathrm{cm}^{-1}$, respectively), the aldehyde $\mathrm{C}=\mathrm{O}$ stretch (1730 and $1739 \mathrm{~cm}^{-1}$, respectively) and the same $\mathrm{C}=\mathrm{O}$ stretch observed for the other fullerenes $\left(1780 \mathrm{~cm}^{-1}\right)$ (peak assignments of the FTIR spectra and absolute spectra shown in Table C.1 and Figure (C.2). The spectra for 0 hours of photoaging include these peaks (which broaden with photoaging time) although the fresh molecules do not contain oxygen, so the samples must have oxidised during processing or in storage, highlighting how easily these two fullerenes are oxidised. The

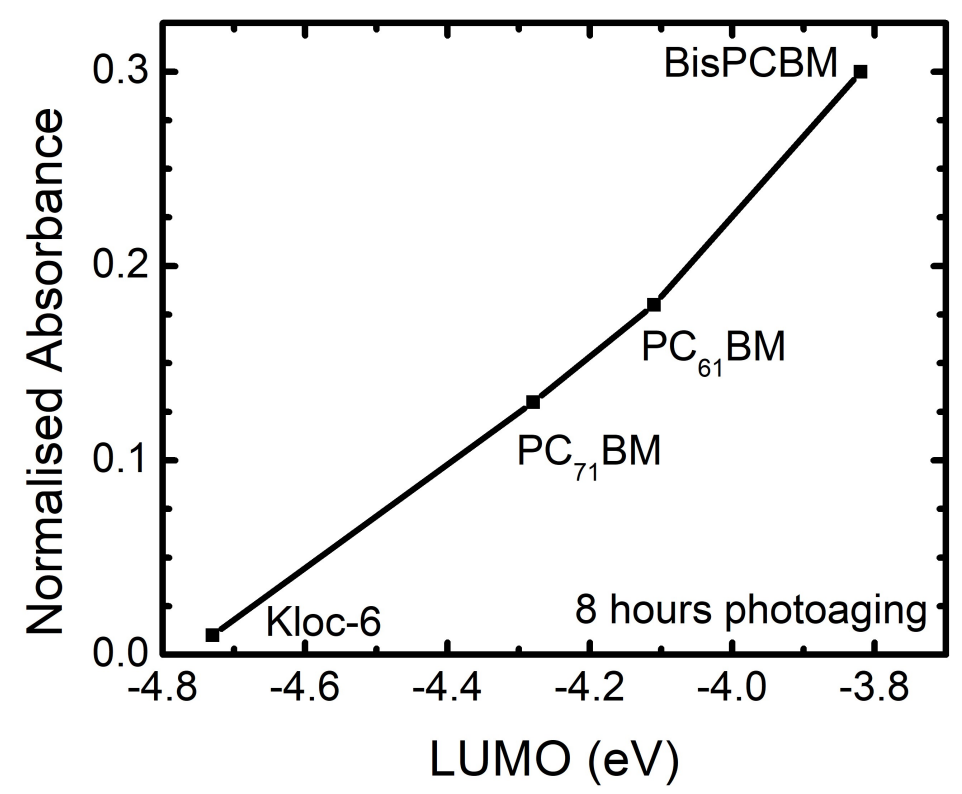

Figure 5.5: Normalised absorbance of the carbonyl peak at $\sim 1780 \mathrm{~cm}^{-1}$ after 8 hours of degradation for different fullerene acceptors as a function of LUMO level. 
Figure 5.5 shows the normalised absorbance of the carbonyl peak $\left(\sim 1780 \mathrm{~cm}^{-1}\right)$ after 8 hours of photoaging for the fullerene acceptors as a function of LUMO level. Interestingly, there is large variation in the relative absorbance with magnitude of the absorbance increases in the following order: Kloc-6 (0.01), $\mathrm{PC}_{71} \mathrm{BM}(0.13), \mathrm{PC}_{61} \mathrm{BM}(0.18)$ and BisPCBM (0.30). There is a good correlation between carbonyl formation and the LUMO energy of the fullerene acceptor. The higher lying the LUMO, the more carbonyls are formed on the fullerene cage. This correlates well with the enhanced photobleaching shown in Figure 5.2b.

The mass spectroscopic spectra of the photoaged films were then measured using MALID- TOF mass spectrometry in order to identify and quantify any photo-oxidation or fragmentation of the fullerenes, as explained in Chapter 2. The samples for MALDI-TOF measurements were prepared by redissolving the photoaged films.

The MALDI-TOF spectra for fullerene films that had been photoaged for 32 hours under 1 sun AM1.5G illumination in air are shown in Figure 5.6, showing a large variation in the photo-oxidation between the fullerenes. $\mathrm{PC}_{71} \mathrm{BM}$ and $\mathrm{PC}_{61} \mathrm{BM}$ photo-oxidised the least, with 3.4 and $9.9 \%$ of the fullerenes being oxidised respectively and up to 8 and 6 additional oxygen atoms binding onto the fullerene molecule respectively. BisPCBM has a smaller amount of fullerenes photo-oxidised (24.4\%) than that of Kloc-6 (34.4 \%) but BisPCBM has more additional oxygen atoms per oxidised fullerene compared to Kloc6 (10 and 6 respectively). This could be explained either by the oxygen and nitrogen atoms on the fullerene cage of Kloc-6 either preventing further photo-oxidation of the same molecule or these atoms causing extra strain on the fullerene cage so that the cage breaks after 6 additional oxygen atoms have been added. ICBA is even less stable with $71.4 \%$ of the fullerene morecules being oxidised and up to ten oxygen atoms being added. The fresh peak for ICTA completely disappears after 32 hours of degradation, suggesting that the molecule has fragmented. 

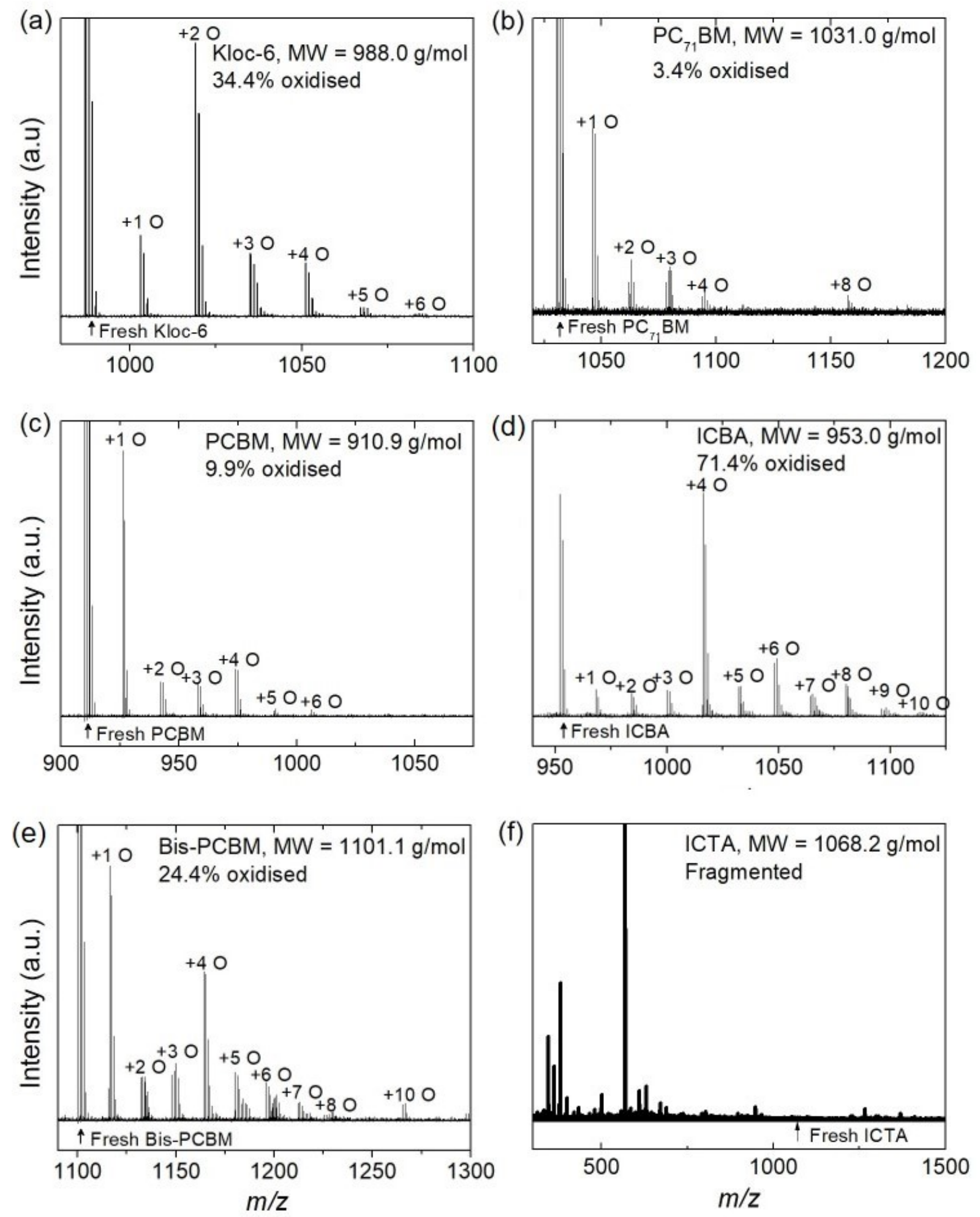

Figure 5.6: MALDI-TOF spectra of: (a) Kloc-6 (b) $\mathrm{PC}_{71} \mathrm{BM}$ (c) $\mathrm{PC}_{61} \mathrm{BM}$ (d) ICBA (e) BisPCBM (f) ICTA neat films degraded for 32 hours in air under 1 Sun AM1.5G illumination. 
(a)

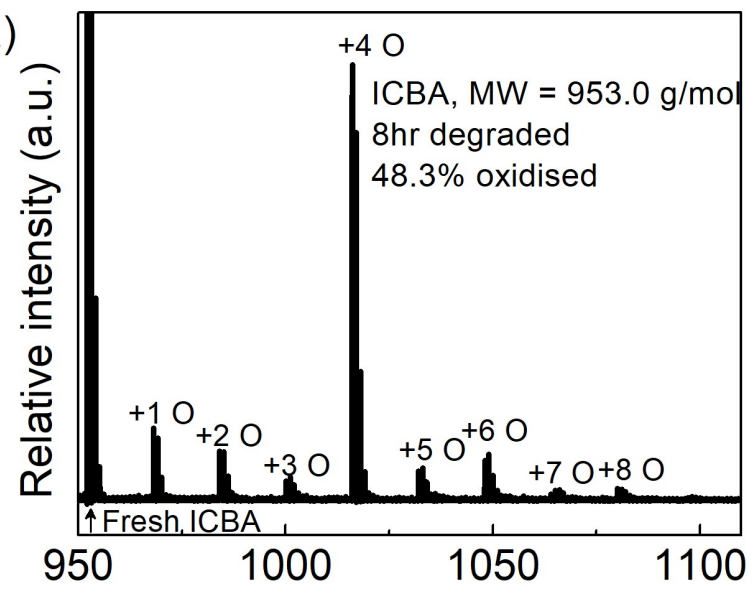

(c)

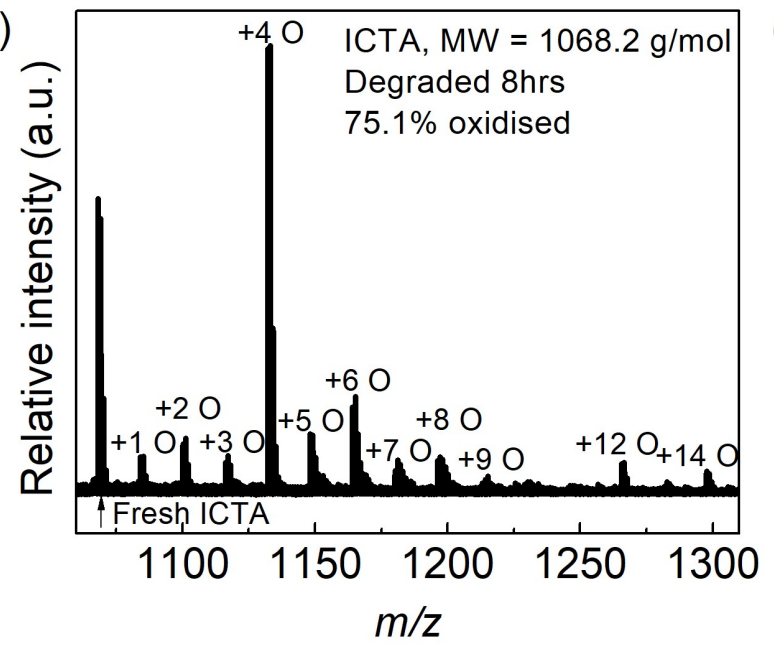

(b)

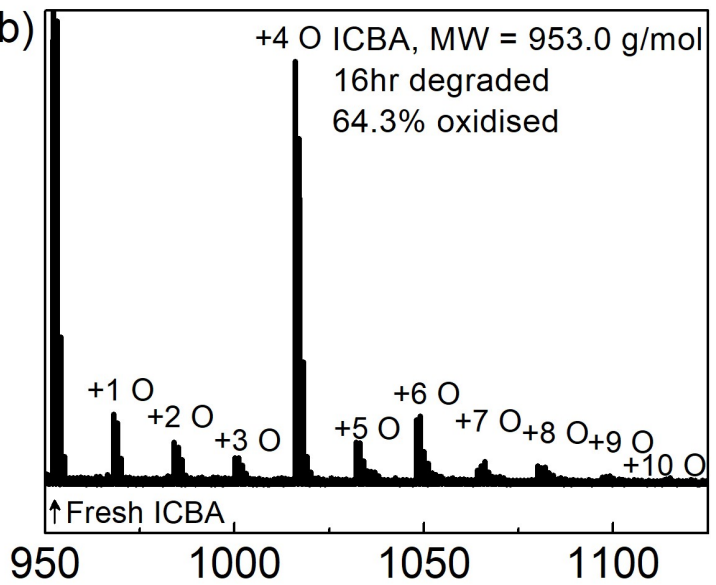

(d)

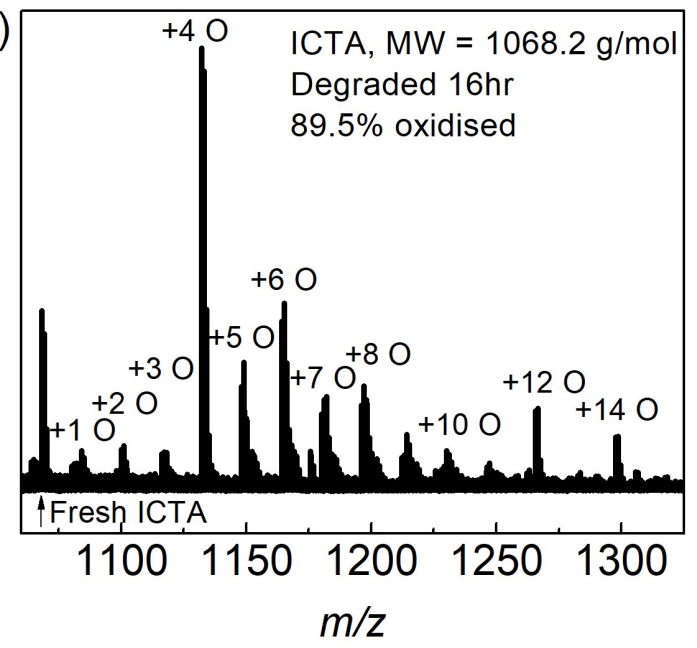

Figure 5.7: MALDI-TOF spectra of: ICBA (a) degraded for 8 hours and (b) 16 hours and ICTA (c) degraded for 8 hours and (d) 16 hours in air under 1 Sun AM1.5G. illumination. 

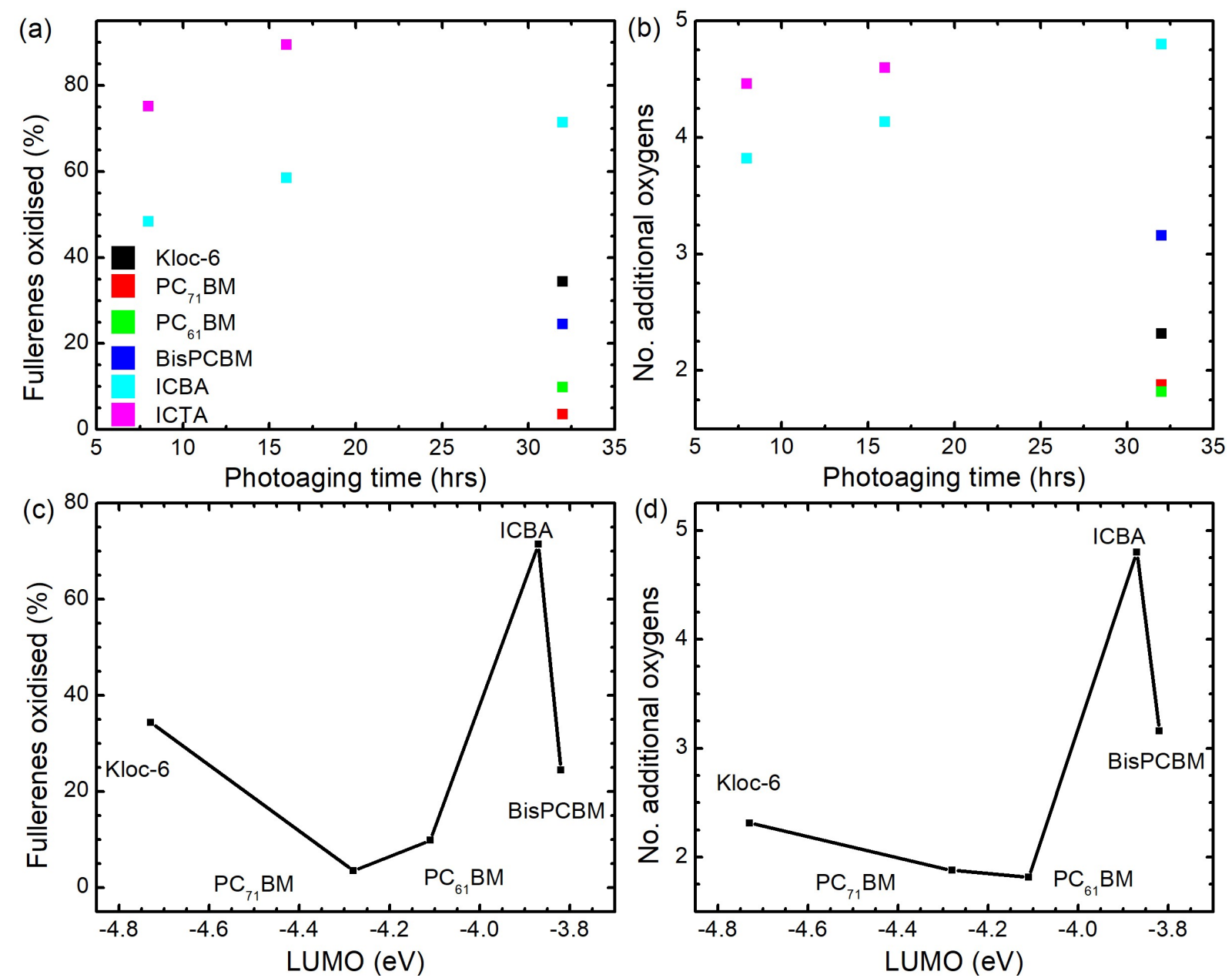

Figure 5.8: Mass spectroscopy (a) percentage of fullerenes photo-oxidised and (b) the number of additional oxygens per molecule as a function of photoaging time for neat fullerene acceptor films after up to 32 hours of degradation to 1 Sun AM1.5G illumination in air. (c) Percentage of fullerenes photo-oxidised and d) the number of additional oxygens per photo-oxidised molecule after 32 hours of photoaging as a function of LUMO level.

MALDI-TOF tests were carried out at shorter timescales for ICBA and ICTA to investigate whether ICTA photo-oxidises before fragmenting. Figures 5.7 a) and c) show the MALDI-TOF spectra for ICBA and ICTA after 8 hours of photoaging. It is shown that 48.3 and $75.1 \%$ of the ICBA and ICTA molecules had photo-oxidised, with up to 8 and 14 extra oxygen atoms bound onto the cage respectively. These levels of photo-oxidation are even more than the other four fullerenes which underwent 32 hours of photo-aging. To gain more insight on the evolution/dynamic of the photo-oxidation of ICBA and ICTA, these fullerenes were further photo-oxidised with longer (16 hours) photo-aging in air. These levels of photo-oxidation are even more than the other four fullerenes after 32 hours of photoaging. Figure 5.7b and d show that ICBA and ICTA have 64.3 and 89.5 $\%$ of the molecules photo-oxidised, respectively, and ICBA has up to 10 and ICTA has $>14$ additional oxygen atoms. These data show that ICTA underwent serious photooxidation, and with sufficiently long photoaging, the large amount of addition of oxygens to the fullerene cage leads to fragmentation of the molecule. 
Figure 5.8a and b show the percentage of photo-oxidised fullerenes and the number of additional oxygens respectively as a function of degradation time. Here, it clearly shows that ICBA and ICTA oxidise considerably more than the other fullerene acceptors. Even after 8 hours of photoaging, ICBA and ICTA photo-oxidised more than the other four fullerenes when photoaged for 32 hours. Additionally, the substantial rate of oxidation of ICTA suggests that after 32 hours ICTA has oxidised so much that the fullerene ball has been pulled apart, fragmenting the molecule. These two fullerene derivatives have the indene side-chain. This side-chain attaches onto the fullerene ball twice per side chain, which would could create extra strain on the fullerene ball, making it easier to fragment.

Figure 5.8c and d show the percentage of fullerenes oxidised and the additional oxygens per molecule after 32 hours of photoaging as a function of fullerene acceptor LUMO level. As ICTA had fragmented after this amount of degradation, (see Figure 5.6f), it is not included in this figure. In general, the higher lying the LUMO level, the more susceptible the fullerene acceptor to photo-oxidation. Here, Kloc-6 undergoes more oxidation than expected according to the observed trend, which could be due to the difference in chemical structure, correlating with the photobleaching. ICBA exhibits a higher much higher level of oxidation compared to the other fullerenes, which can explain the likely fragmentation of the molecule due to high levels of oxidation observed in mass spectrometry measurements after 8 and 16 hours of degradation (Figure 5.8a), likely due to the indene sidechain.

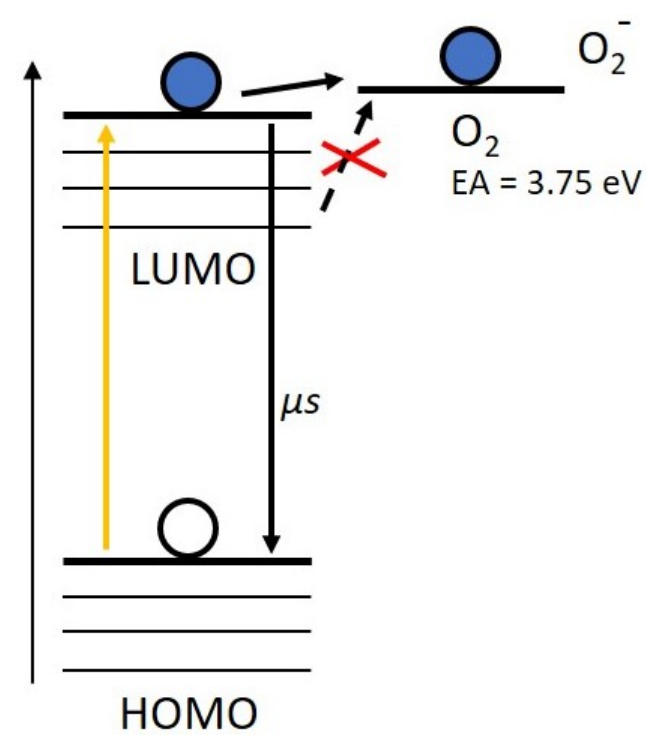

Figure 5.9: Proposed degradation mechanism, namely the photodegradation caused by the formation of superoxide $\left(\mathrm{O}_{2}^{-}\right)$via electron transfer from the LUMO levels of the acceptors to molecular oxygen $\left(\mathrm{O}_{2}\right)$ which has an electron affinity $(\mathrm{EA})$ of $3.75 \mathrm{eV}$.

This overall trend, corresponds well with the photobleaching and carbonyl formation, showing that the higher the LUMO level of the fullerene acceptor, the more susceptible it is to photo-oxidation to form carbonyls, and the more it degrades optically. This LUMO dependence of the photo-oxidation is consistent with degradation via superoxide $\left(\mathrm{O}_{2}^{-}\right)$ 
generation.[11] As illustrated in Figure 5.9 the photoexcited electron in the fullerene is transferred to molecular oxygen, reducing it to the reactive superoxide anion. The deeper the LUMO (from the electron affinity of oxygen $3.75 \mathrm{eV}[11]$ ), the less energetically favourable for electron transfer from the fullerene to molecular oxygen, making superoxides are less likely to form, and hence less photo-oxidation of the fullerene will occur. Aggregation could also affect the photo-oxidation of fullerenes as seen in Chapter 4, which would be affected by the structure of the fullerenes. However, $\mathrm{PC}_{71} \mathrm{BM}$ is expected to be less aggregated than $\mathrm{PC}_{61} \mathrm{BM}$ as it has a larger fullerene cage, so should not be able to pack as tightly, but it is more stable. BisPCBM and ICBA both have two side chains (although different), so are expected to have similar aggregation, but ICBA is undergoes considerably more photo-oxidation. These two cases and the trend of photo-oxidation with LUMO suggest that the LUMO dependent degradation mechanism is dominate.

\subsection{Impact of LUMO dependent photo-oxidation on fullerene based films and devices}

In chapter 3 and 4 , it was seen that photo-oxidation of fullerenes had a detrimental effect on the performance of devices. Here, the effect of photo-oxidation was investigated in blend films and devices to ascertain whether this LUMO dependence of photo-oxidation would also affect device performance.
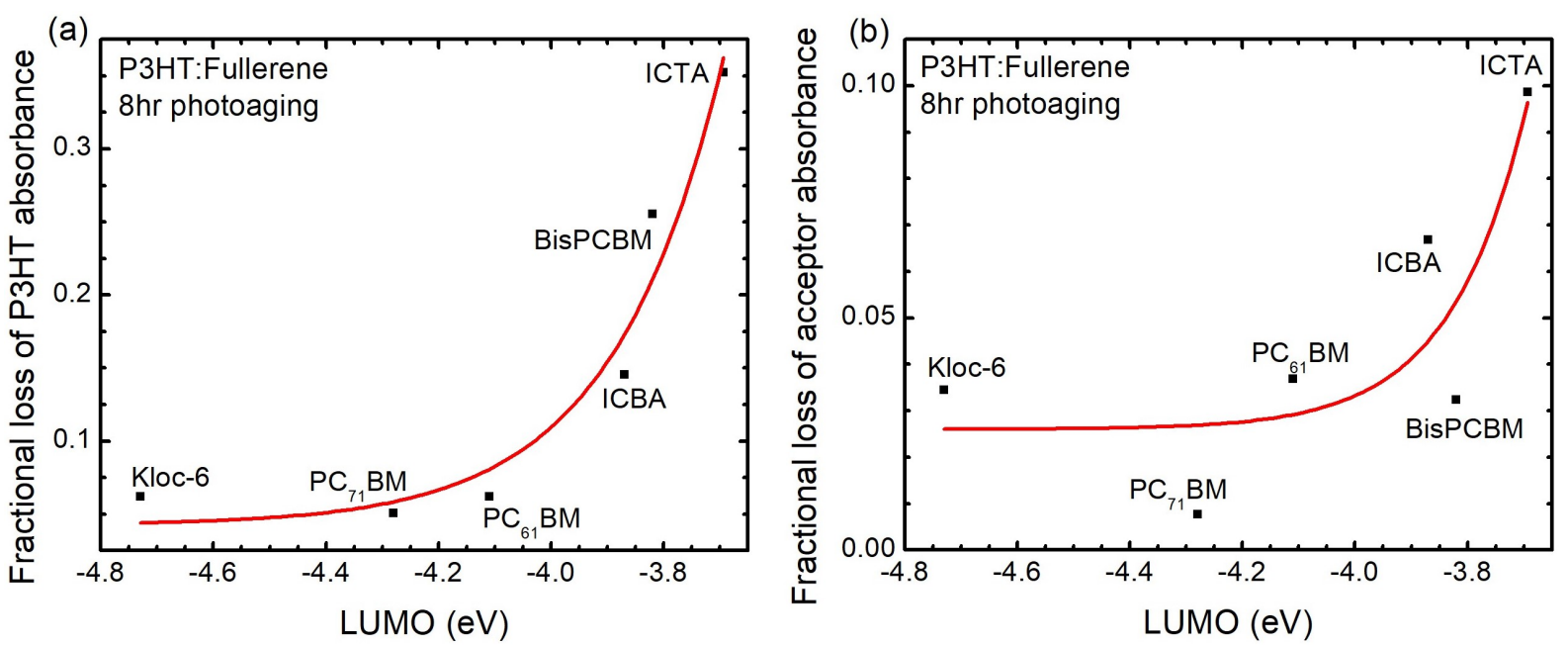

Figure 5.10: Fractional loss of (a) the P3HT and (b) acceptor peaks in P3HT:fullerene blend films after photoaging under 1 Sun irradiation in air for 8 hours as a function of acceptor LUMO level. (At donor (D) and acceptor (A) wavelengths: Kloc-6 $\mathrm{D}=479 \mathrm{~nm}$ $\mathrm{A}=334 \mathrm{~nm}, \mathrm{PC}_{71} \mathrm{BM} \mathrm{D}=470 \mathrm{~nm} \mathrm{~A}=241 \mathrm{~nm}, \mathrm{PC}_{61} \mathrm{BM} \mathrm{D}=477 \mathrm{~nm} \mathrm{~A}=336 \mathrm{~nm}, \mathrm{BisPCBM}$ $\mathrm{D}=487 \mathrm{~nm} \mathrm{~A}=260 \mathrm{~nm}$, ICBA $\mathrm{D}=468 \mathrm{~nm} \mathrm{~A}=250 \mathrm{~nm}$, ICTA $\mathrm{D}=453 \mathrm{~nm} \mathrm{~A}=246 \mathrm{~nm}$.

Firstly P3HT:fullerene blend films photoaged in air for up to 8 hours under 1 sun 
AM1.5G illumination to investigate how blending the fullerenes with a polymer affected the photo-oxidation of both the fullerene and polymer. The UV-vis absorbance spectra for the P3HT:fullerene films are shown in Figure C.3. Figure 5.10a and b show the fractional loss of photobleaching of the P3HT and fullerene acceptor peaks respectively in the blend films after 8 hours of photobleaching as a function of acceptor LUMO level. In general, it is shown that the photobleaching of the P3HT in the blend films correlates well with the LUMO of both the fullerenes, whereby the shallower the LUMO of the acceptor, the more photobleaching there is of the P3HT in the blend film. Analogously, there is a relationship between the LUMO of the acceptor with the photobleaching it undergoes when blended with P3HT, where the acceptors with shallower LUMOs generally undergo more photobleaching in the blend films. These trends of photodegradation with acceptor LUMO match those observed for neat fullerene films well and further supports the hypothesis that degradation occurs via superoxide formation with the LUMO dependence of the P3HT as well as the fullerenes as both would react with the generated superoxide. MALDI-TOF measurements were performed on each degraded solution in order to quantify the amount of photo-oxidaton (Figure 5.11). It was shown that the fullerenes oxidised similarly in solution to when they were photoaged as neat thin films (Figure 5.6), albeit to less of an extent as the photoaging time was reduces. Interestingly, Kloc-6 exhibits the same amount of photo-oxidation in the film photoaged for 32 hours and the solution photoaged for 24 hours, which further supports the concept that Kloc-6 easily fragments after reaching a certain level of photo-oxidation. 

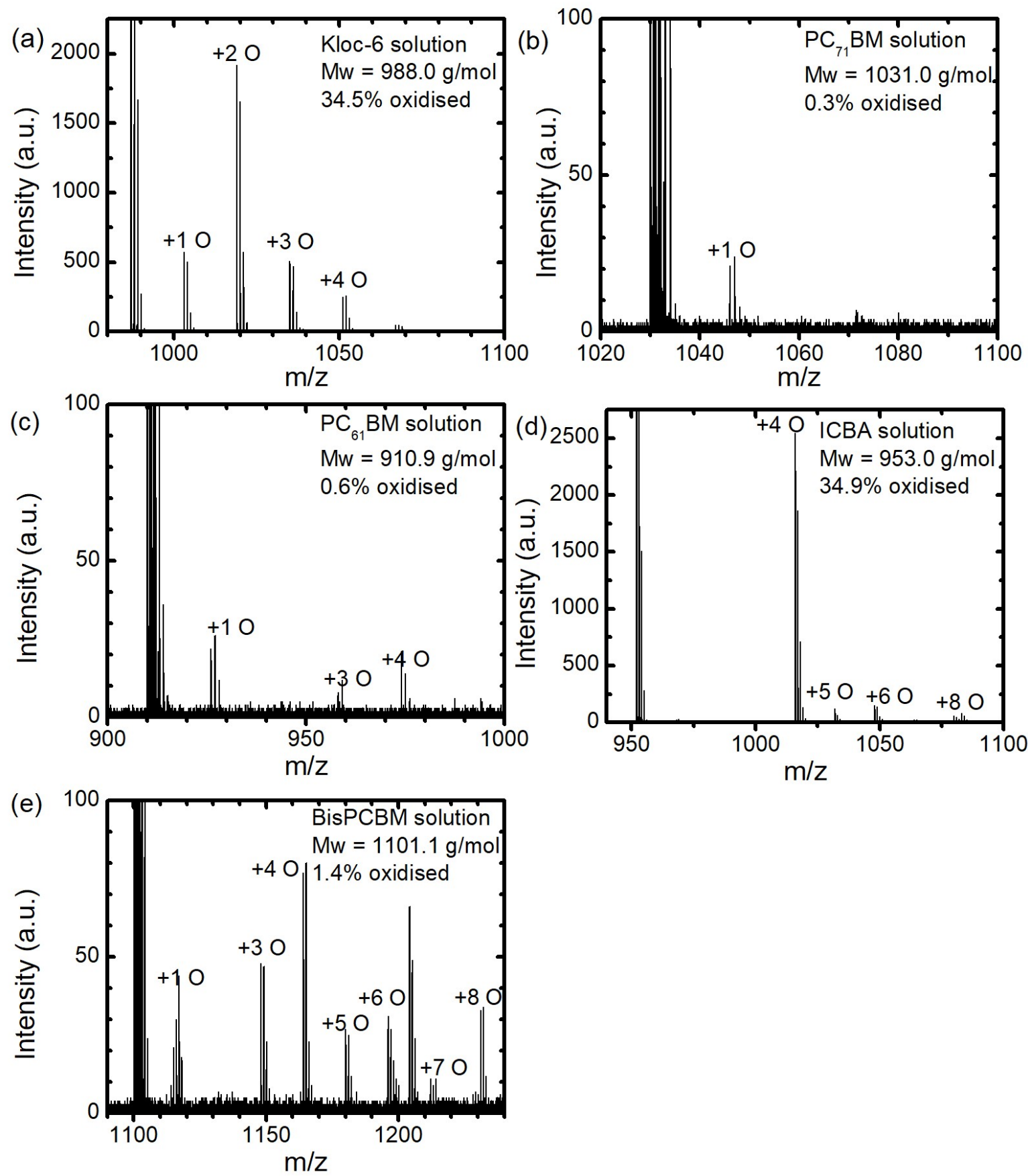

Figure 5.11: MALDI-TOF spectra of: (a) Kloc-6 (b) $\mathrm{PC}_{71} \mathrm{BM}$ (c) $\mathrm{PC}_{61} \mathrm{BM}$ (d) ICBA (e) BisPCBM solutions degraded and stirred for 24 hours in air under 1 Sun AM1.5G illumination.

Next, inverted OPV devices were fabricated using fullerene solution that was photoaged separately for 24 hours (same approach as used in chapter 3), so that the effect of fullerene photo-oxidation could be studied. ICTA was not included in this part of the study due to its poor device performance $(1.56 \%)$.[5] 

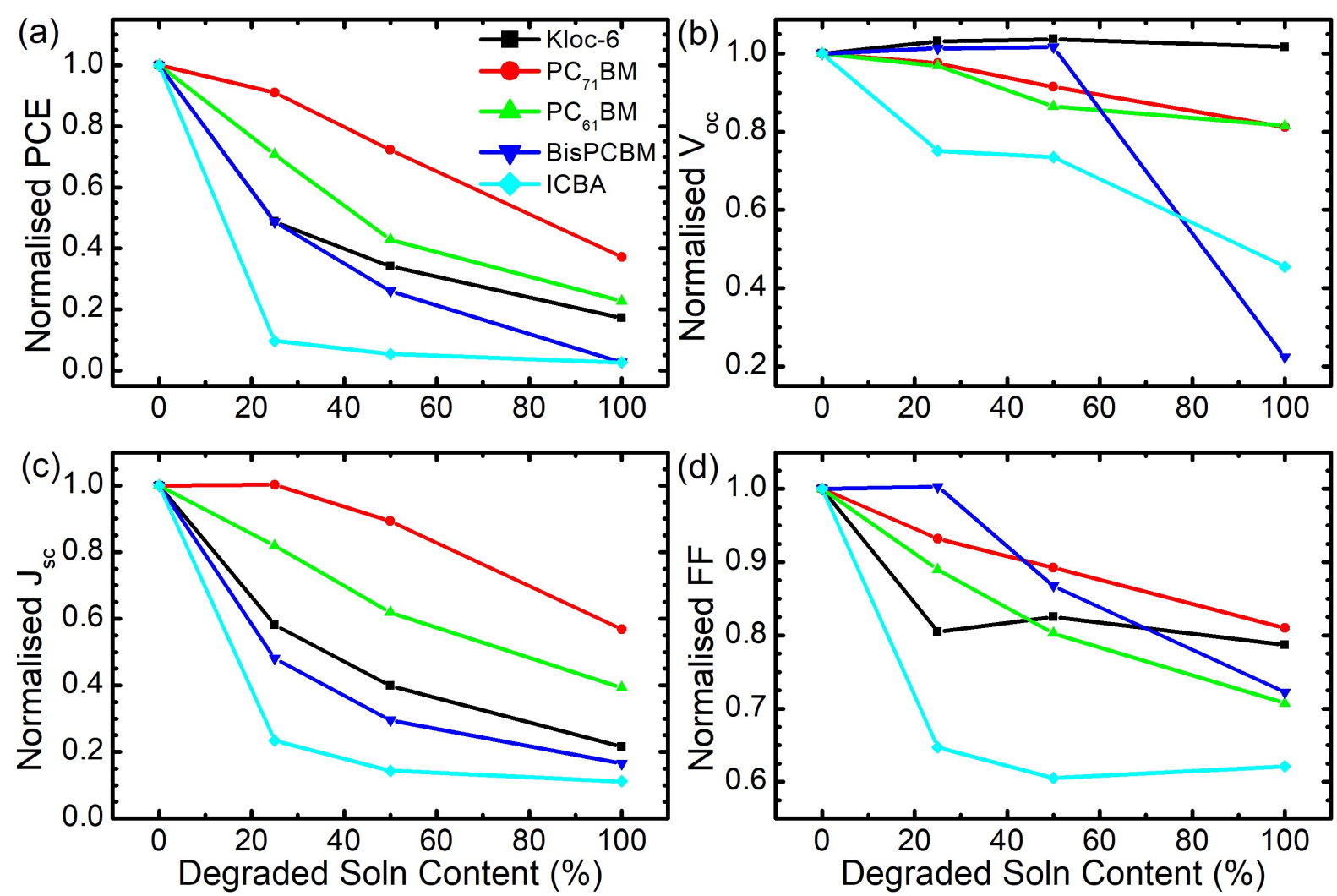

Figure 5.12: Normalised device characteristics of P3HT:fullerene devices (a) PCE, (b) $\mathrm{V}_{\text {oc }}$, (c) $\mathrm{J}_{\mathrm{sc}}$, and (d) FF as a function of degraded fullerene solution content. Characteristics were normalised to that of devices fabricated with fresh fullerene solution.

Figure 5.12 shows the normalised parameters for P3HT:fullerene devices as a function of the percentage of degraded fullerene solution used to prepare the active layer (Figure C.4 shows the absolute values as a function of percentage of degraded solution). $\mathrm{PC}_{71} \mathrm{BM}$ was the most stable fullerene device, maintaining $37 \%$ of initial PCE when $100 \%$ of the degraded fullerene solution was employed. $\mathrm{PC}_{61} \mathrm{BM}$ was the next most stable device (23\% remaining), followed by Kloc-6 (17\% remaining), and then BisPCBM and ICBA which degraded the same amount (3\% remaining). Despite BisPCBM and ICBA devices degrading by the same amount upon using $100 \%$ of the degraded fullerene solution, BisPCBM device degrades considerably less (25\% remaining) than that of ICBA (10 $\%$ remaining) upon using $25 \%$ of the degraded fullerene solution. It is likely that the amount of photo-oxidised fullerene is sufficiently high for the $100 \%$ degraded solution that it causes serious degradation to both BisPCBM and ICBA devices.

$\mathrm{PC}_{71} \mathrm{BM}$ was the most stable fullerene device, degrading to 0.37 normalised PCE with $100 \%$ of the degraded fullerene solution employed. $\mathrm{PC}_{61} 1 \mathrm{BM}$ was the next most stable, followed by Kloc-6, and both BisPCBM and ICBA which degraded the same amount (0.23, 0.17, 0.03, 0.03 respectively). Despite BisPCBM and ICBA degrading by the same amount upon using $100 \%$ of the degraded fullerene solution, it an be seen that ICBA is less stable as it degrades to 0.10 normalised PCE upon using $25 \%$ of the degraded fullerene solution. 
The loss in PCE is caused by predominately a loss of $\mathrm{J}_{\mathrm{sc}}$ and contributions of losses in $\mathrm{V}_{\mathrm{oc}}$ and FF. The loss in $\mathrm{J}_{\mathrm{sc}}$ with degraded fullerene solution content correlates well with the loss of PCE, with the devices keeping the same trend (normalised $\mathrm{J}_{\mathrm{sc}}$ with $100 \%$ degraded solution: $0.57,0.39,0.22,0.17,0.11$ respectively). The $\mathrm{V}_{\text {oc }}$ degraded differently to the PCE. Kloc-6 had a stable $\mathrm{V}_{\text {oc }}$, then $\mathrm{PC}_{71} 1 \mathrm{BM}$ and $\mathrm{PC}_{61} 1 \mathrm{BM}$ degraded similarly, ICBA was less stable, and BisPCBM was stable with up to $50 \%$ degraded fullerene solution, but then the least stable with $100 \%$ degraded fullerene solution (normalised $\mathrm{V}_{\mathrm{oc}}$ with 100\% degraded solution: 1.02, 0.82, 0.81, 0.45, 0.22 respectively). The FF also degraded differently from the from the PCE. $\mathrm{PC}_{71} 1 \mathrm{BM}$ was the most stable, followed by Kloc-6, BisPCBM, $\mathrm{PC}_{61} 1 \mathrm{BM}$ and then ICTA (normalised FF with 100\% degraded solution: 0.81, $0.79,0.72,0.71$ and 0.62 respectively). ICTA degraded rapidly to 0.65 after adding just $25 \%$ of the degraded fullerene solution.
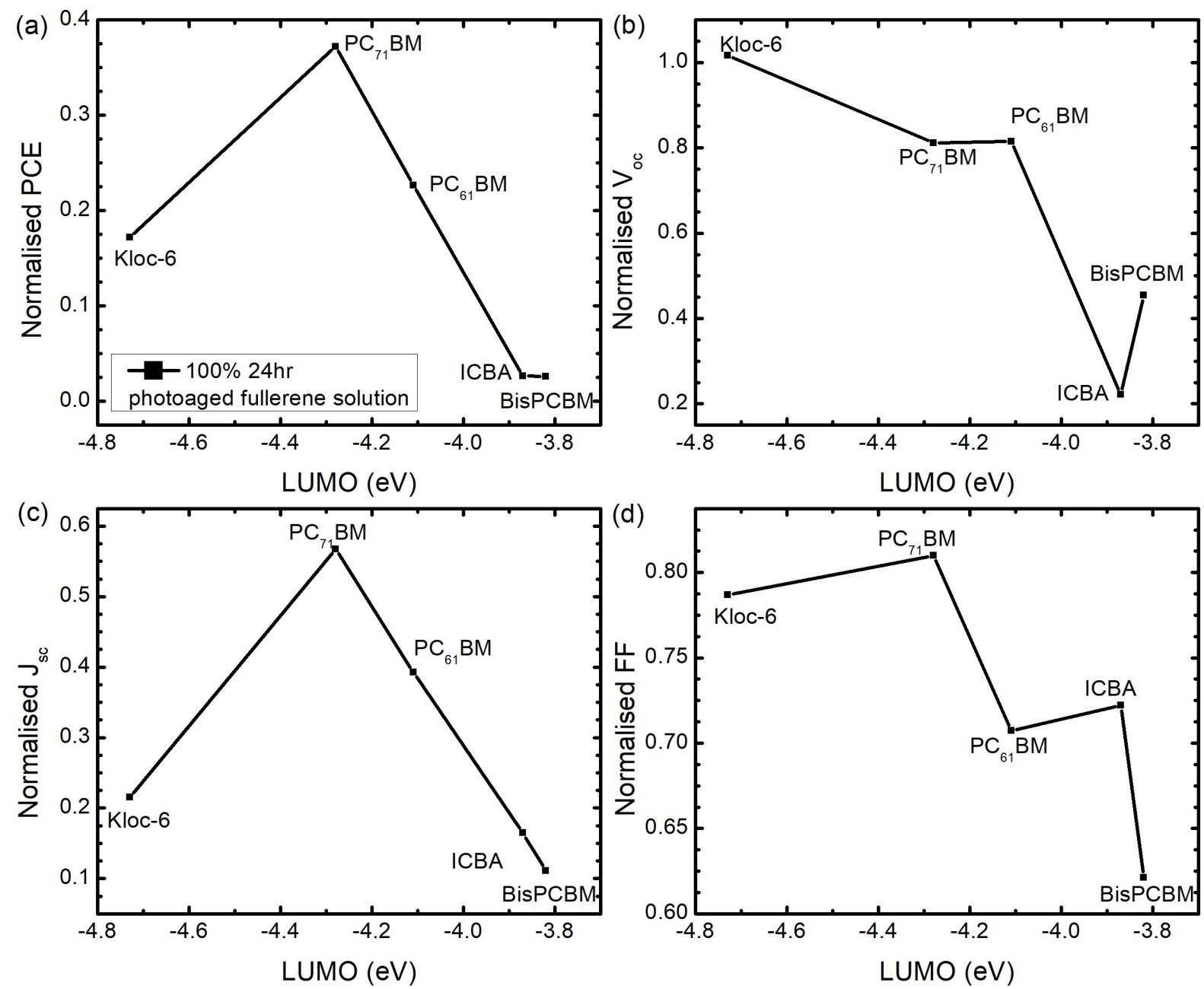

Figure 5.13: Normalised device characteristics (a) PCE, (b) $\mathrm{V}_{\mathrm{oc}}$, (c) $\mathrm{J}_{\mathrm{sc}}$, and (d) FF of solution degraded devices as a function of fullerene acceptor LUMO level. The device characteristics were normalised to those obtained from devices fabricated with fresh fullerene solutions.

Figure 5.13 shows the characteristics of devices fabricated $100 \%$ of the degraded fullerene solution, as a function of fullerene LUMO level, normalised to devices made with fresh 
fullerene solution. In general, the lower lying the LUMO level, the more stable the solution degraded device. However, Kloc-6 did not fit this trend, being less stable than its low lying LUMO would suggest, but corresponding with the spectroscopic studies, likely related to its inclusion of oxygen and nitrogen atoms on the fullerene cage. Interestingly, for Kloc- 6 the $\mathrm{V}_{\mathrm{oc}}$ is very stable and there is only a small loss of $\mathrm{FF}$, perhaps because photo-oxidation has little effect its already low lying LUMO level (stable $V_{\text {oc }}$ ), leading to less (or shallower) charge traps due to oxidised Kloc-6 having a similar LUMO level (relatively low drop in FF). The loss of PCE with increasing LUMO is mainly determined from $\mathrm{J}_{\mathrm{sc}}$ and to a lesser extent $\mathrm{FF}$. The loss of $\mathrm{V}_{\mathrm{oc}}$ has a good correlation with increasing LUMO, apart from ICBA which degraded more than BisPCBM despite having a deeper LUMO but corresponds well with the levels of photo-oxidation of the degraded solution (Figure 5.11). The trends observed correspond well with the spectroscopic studies undertaken previously in this chapter, where fullerenes with higher lying LUMO levels were observed to undergo more photobleaching, carbonyl formation and photo-oxidation.

\subsection{PDCTBT:PC ${ }_{61} \mathrm{BM}$ :fullerene ternary blend devices}

Earlier in this chapter, it was observed that fullerenes with a higher lying LUMO level were more easily photo-oxidised. These higher lying LUMO fullerenes (ICBA and ICTA) could be used as oxygen scavengers, so that they could protect the photo-degradation of the active layer by sacrificing themselves to be photo-oxidised. Therefore, here, ICBA or ICTA was added to the PCDTBT:PC ${ }_{61} \mathrm{BM}$ devices (i.e. to form ternary blend devices), to study if they could enhance the photo-stability of these devices. Inverted ternary blend devices were fabricated with up to $25 \mathrm{wt} \%$ of the acceptor domain being either ICBA or ICTA. Although it is likely that the LUMO level of ICBA and ICTA can get deeper when they are photo-degraded (as $\mathrm{PC}_{61} \mathrm{BM}$ in chapter 3 ), and can cause charge traps if the LUMO suppressed to be deeper than that of $\mathrm{PC}_{61} \mathrm{BM}$, which should only happen when they have deep traps as the difference in LUMO between $\mathrm{PC}_{61} \mathrm{BM}$ and ICBA and ICTA is around $0.24 \mathrm{eV}$ and $0.42 \mathrm{eV}$, respectively.

The ternary blend devices were fabricated where the active layer was photoaged before electrode deposition in order to determine the intrinsic stability of the active layer. PCDTBT:PC ${ }_{61} \mathrm{BM}$ devices were fabricated where $10 \mathrm{wt} \%$ and $25 \mathrm{wt} \%$ ICBA or ICTA were added to the fullerene phase. The addition of ICBA or ICTA did not drastically affect the initial device performance (Figure C.5). However, it can be seen that there was a slight drop in initial PCE between adding $10 \mathrm{wt} \%$ and $25 \mathrm{wt} \%$ of ICBA or ICTA to the fullerene phase $(\sim 5.6 \%$ to $\sim 5.1 \%)$. This may be due to the LUMO-LUMO and HOMO-HOMO offset between the polymer and the ICBA or ICTA not being large 
enough for efficient charge generation and the lower electron mobility of ICBA and ICTA, hence a lower charge carrier collection efficiency.[12] Figure 5.14 shows the normalised device parameters for the ternary blend devices as a function of photoaging time of the active layer. There is no improvement in device stability for devices with an additional higher-lying LUMO fullerene electron acceptor component, as all devices degraded to $<20 \%$ initial PCE after just 10 minutes of photoaging, and completely degraded after 30 minutes of photoaging. Furthermore, the devices with the least sacrificial fullerene component were the most stable.
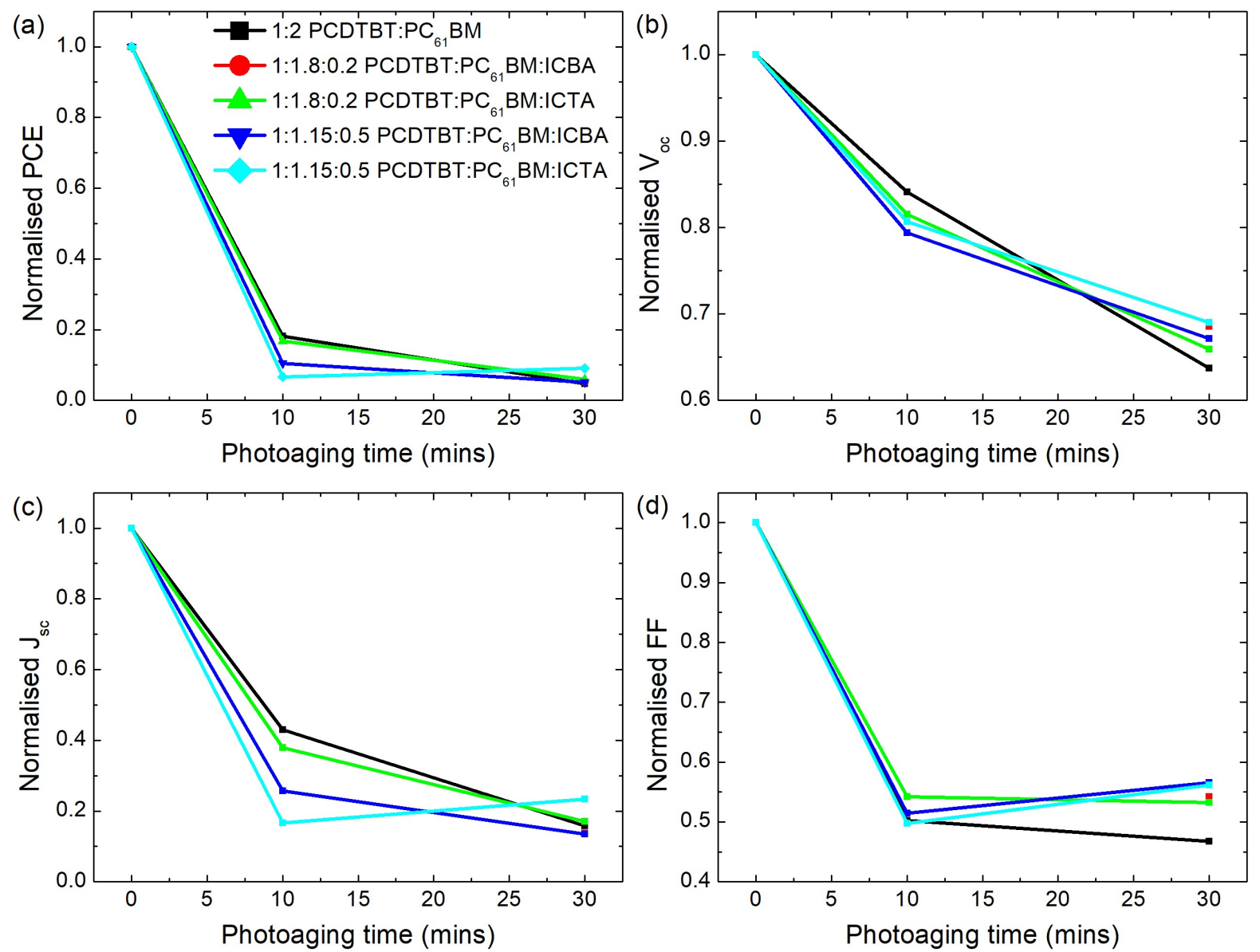

Figure 5.14: Normalised device characteristics (a) PCE, (b) $\mathrm{V}_{\mathrm{oc}}$, (c) $\mathrm{J}_{\mathrm{sc}}$, and (d) FF as a function of BHJ film degradation time.

To explain why the addition of higher LUMO sacrificial fullerene acceptors to OPV devices did not improve device stability, several suggestions are proposed here. i) The ICBA/ICTA could degrade so seriously that very deep traps are formed, reducing the charge transport. ii) The $10 \mathrm{wt} \%$ of ICBA/ICTA may still be high enough that the ICBA/ICTA molecules still act as an electron transport pathway, so that degradation of ICBA/ICTA also degrades the charge transport. iii) There could be an uneven distribution of ICBA/ICTA within the films (phase separation) so that the ICBA/ICTA does not mix well with the $\mathrm{PC}_{61} \mathrm{BM}$ to protect $\mathrm{PC}_{61} \mathrm{BM}$, in addition of ICBA/ICTA degradation. 


\subsection{Conclusion}

In this chapter, six different fullerenes were investigated to study how their different LUMO levels could affect their neat/blend film and device photo-chemical stability in air. It was found that in general the degree of photobleaching of the neat films correlated well with the amount of carbonyl formation and fullerene photo-oxidation, all of which generally correlating with the LUMO levels measured with cyclic voltammetry. Generally, fullerenes with a lower lying LUMO underwent less photo-oxidation, suggesting that the dominant degradation mechanism is superoxide formation via the fullerene LUMO level. Kloc-6 which has the lowest lying LUMO, did not photo-oxidise the least which suggests that besides LUMO level, there are other factors which could also affect the photo-oxidation, such as the chemical structure of the carbon ball. P3HT:fullerene blend films showed the same trend in degradation whereby both the fullerene and P3HT degrades more for fullerenes with a higher lying LUMO level, again indicative that superoxide formation is the dominant degradation pathway. There was also a good correlation between the large drop of PCE and $\mathrm{J}_{\mathrm{sc}}$ of the P3HT:fullerene devices after the photoaging (fullerene solution degraded) with the fullerene LUMO level (PCE and $\mathrm{J}_{\mathrm{sc}}$ drop less with fullerenes with a lower lying LUMO, except Kloc-6). Interestingly, the degree of $\mathrm{J}_{\mathrm{sc}}$ drop for Kloc-6 (compared with other fullerenes) correlates with its degree of photobleaching and photo-oxidation. Notably, the degree of reduction in $\mathrm{V}_{\text {oc }}$ correlated very well with the LUMO level, with lower lying LUMO having less $V_{\text {oc }}$ reduction. The large drop in PCE also shows that the impact of fullerene photo-oxidation plays a significant role in the stability of organic photovoltaics as well as other for organic optoelectronic device applications. These results suggest that designing fullerene electron acceptors with lower lying LUMO should reduce photo-oxidation, but it is also necessary to consider the chemical structure of the fullerene cage. Furthermore, fullerenes with higher lying LUMO levels were added to PCDTBT: $\mathrm{PC}_{61} \mathrm{BM}$ devices to act as oxygen scavengers, however, there was no improvement in the photo-stability and further work may need to understand this. Overall, the results in this chapter suggest that designing fullerene acceptors with a lower lying LUMO should be considered when designing future acceptors with an increased photochemical stability. 


\section{REFERENCES}

[1] B. W. Larson, J. B. Whitaker, X.-B. Wang, A. A. Popov, G. Rumbles, N. Kopidakis, S. H. Strauss, and O. V. Boltalina, "Electron Affinity of Phenyl- $\mathrm{C}_{61}-$ Butyric Acid Methyl Ester (PCBM)," The Journal of Physical Chemistry C, vol. 117, pp. 1495814964, 2013.

[2] F. Zhang, Z. Zhuo, J. Zhang, X. Wang, X. Xu, Z. Wang, Y. Xin, J. Wang, J. Wang, W. Tang, Z. Xu, and Y. Wang, "Influence of $\mathrm{PC}_{60} \mathrm{BM}$ or $\mathrm{PC}_{70} \mathrm{BM}$ as electron acceptor on the performance of polymer solar cells," Solar Energy Materials and Solar Cells, vol. 97, pp. 71-77, 2012.

[3] M. Lenes, G.-J. A. H. Wetzelaer, F. B. Kooistra, S. C. Veenstra, J. C. Hummelen, and P. W. M. Blom, "Fullerene Bisadducts for Enhanced Open-Circuit Voltages and Efficiencies in Polymer Solar Cells," Advanced Materials, vol. 20, pp. 2116-2119, 2008.

[4] Y. He, H.-Y. Chen, J. Hou, and Y. Li, "Indene-C 60 Bisadduct: A New Acceptor for High-Performance Polymer Solar Cells," Journal of the American Chemical Society, vol. 132, pp. 1377-1382, 2010.

[5] H. Kang, C.-H. Cho, H.-H. Cho, T. E. Kang, H. J. Kim, K.-H. Kim, S. C. Yoon, and B. J. Kim, "Controlling Number of Indene Solubilizing Groups in Multiadduct Fullerenes for Tuning Optoelectronic Properties and Open-Circuit Voltage in Organic Solar Cells," ACS Applied Materials \& Interfaces, vol. 4, pp. 110-116, 2012.

[6] H. S. Silva, J. Cresson, A. Rivaton, D. Bégué, and R. C. Hiorns, "Correlating geometry of multidimensional carbon allotropes molecules and stability," Organic Electronics, vol. 26, pp. 395-399, 2015.

[7] M. Wohlers, A. Bauer, T. Rühle, F. Neitzel, H. Werner, and R. Schlögl, "The Dark Reaction of $\mathrm{C}_{60}$ and of $\mathrm{C}_{70}$ with Molecular Oxygen at Atmospheric Pressure and Temperatures between $300 \mathrm{~K}$ and $800 \mathrm{~K}, "$ Fullerene Science and Technology, vol. 5, pp. 49-83, 1997.

[8] V. Blazinic, L. K. Ericsson, S. A. Muntean, and E. Moons, "Photo-degradation in air of spin-coated PC60BM and PC70BM films," Synthetic Metals, vol. 241, pp. 26-30, 2018.

[9] H. Neugebauer, C. Brabec, J. Hummelen, R. Janssen, and N. Sariciftci, "Stability studies and degradation analysis of plastic solar cell materials by FTIR spectroscopy," Synthetic Metals, vol. 102, pp. 1002-1003, 1999. 
[10] S. Chambon, A. Rivaton, J. L. Gardette, and M. Firon, "Photo- and thermal degradation of MDMO-PPV:PCBM blends," Solar Energy Materials and Solar Cells, vol. 91, pp. 394-398, 2007.

[11] E. T. Hoke, I. T. Sachs-Quintana, M. T. Lloyd, I. Kauvar, W. R. Mateker, A. M. Nardes, C. H. Peters, N. Kopidakis, and M. D. McGehee, "The Role of Electron Affinity in Determining Whether Fullerenes Catalyze or Inhibit Photooxidation of Polymers for Solar Cells," Advanced Energy Materials, vol. 2, pp. 1351-1357, 2012.

[12] M. A. Faist, S. Shoaee, S. Tuladhar, G. F. A. Dibb, S. Foster, W. Gong, T. Kirchartz, D. D. C. Bradley, J. R. Durrant, and J. Nelson, "Understanding the Reduced Efficiencies of Organic Solar Cells Employing Fullerene Multiadducts as Acceptors," Advanced Energy Materials, vol. 3, pp. 744-752, 2013. 
CHAPTER

SIX

\section{STABILITY OF NON-FULLERENE ACCEPTORS}

This chapter investigates the photostability of NFAs. Firstly, it is found that Eh-IDTBR and O-IDTBR based devices exhibited superior burn-in free behaviour under light-only exposure compared to $\mathrm{PC}_{71} \mathrm{BM}$ based devices that undergo a strong burn-in and (M-) ITIC based devices undergoes a continuous degradation without stabilising. Then a range of optical and chemical measurements were utilised to investigate the photodegradation (in air) of benchmark fullerene-based and NFA-based OSC blend films, and a critical correlation between the electron acceptor LUMO level and active layer film stability when exposed to light and air was established. More specifically, the lower-lying the LUMO level of the acceptor, the more stable the OSC. This correlation can be explained by a reduction in acceptor LUMO level mediated formation of superoxide. 


\subsection{Introduction}

The previous chapters in this thesis have studied fullerene-based electron acceptors and have showed how these can be easily photo-oxidised, with a detrimental effect to device performance. In this chapter, a new class of electron accepting material was studied: nonfullerene acceptors. The motivation of this chapter is to study the photodegradation of NFA materials and the role they have upon device stability. In the first part, the stability of NFAs was studied under illumination in a dry nitrogen environment and the burn-in behaviour of the two NFA families was investigated in comparison to that of fullerene systems. Then, the stability of IDF-/IDT-type acceptor family under illumination in air is investigated using UV-vis spectroscopy and mass spectroscopies. Finally, based on cyclic voltammetry, transient absorption and fluorescence spectroscopy data, a degradation mechanism is proposed.

\subsection{Experimental details}

Film preparation: Solutions were prepared by dissolving P3HT:NFA (10:10 mg/ml) and P3HT:fullerene acceptor $(24: 24 \mathrm{mg} / \mathrm{ml}$ ) in CB (in line with optimised devices) and were mixed overnight on a hotplate at $60^{\circ} \mathrm{C}$. Films were spincoated to a thickness of $\sim$ $40 \mathrm{~nm}$ for P3HT:IDFBR, 150 P3HT:IDTBR, $\sim 140 \mathrm{~nm} \mathrm{P3HT:Eh-IDTBR} \mathrm{and} \sim 100$ $\mathrm{nm}$ for the P3HT:fullerene films in accordance with optimised devices. All films were annealed on a hotplate in a nitrogen environment at $130^{\circ} \mathrm{C}$ for 10 minutes.

Photoaging: Photoaging of the films/devices was performed under $\sim 1$ Sun AM1.5G or equivalent in ambient conditions and temperature using a solar simulator or white LED light source, with no filters, for the aging time specified in the text. The aging of complete devices took place under 1 sun equivalent white LED illumination as these measurements were of longer duration and LED bulbs have a longer lifetime than solar simulators. Additional tests on films under white LED illumination were undertaken to compare with the ones aged under solar simulator illumination. For photoaging undertaken under nitrogen the environmental chambers were used as shown in Chapter 2.

Characterisation: The UV-vis absorbance spectra were measured with a Perkin Elmer Lambda 750 spectrophotometer at Swansea University.

The spectra and decays of the photoinduced species of the films were measured using microsecond transient absorption spectroscopy as described in Chapter 2, using the wavelengths given in Table 6.1. Measurements were carried out at Imperial College London with Dr. Stoichko Dimitrov and the data analysis was performed by myself. 
Table 6.1: Excitation wavelengths ( $\left.\lambda_{\text {pump }}\right)$, approximately at the ground state absorption maximum, and probe wavelengths $\left(\lambda_{\text {probe }}\right)$, around the maximum of transient absorption spectrum, used in the TAS studies.

\begin{tabular}{||c|c|c||}
\hline \hline Film & $\lambda_{\text {pump }}(\mathrm{nm})$ & $\lambda_{\text {probe }}(\mathrm{nm})$ \\
\hline IDFBR & 520 & 1200 \\
IDTBR & 680 & 1100 \\
P3HT:IDFBR & 520 & 1000 \\
P3HT:IDTBR & 500 & 1100 \\
\hline \hline
\end{tabular}

Fluorescence spectroscopy and cyclic voltammetry measurements were carried out at Imperial College London with the help of Nicholas Aristdou and Dr. Laia Francas, Dr Hyojung Cha and George Fish respectively as described in Chapter 2, data analysis was carried out by myself. Mass spectrometry was carried out by Dr. Mark Wyatt using the MALDI matrix anthracene and data analysis was done by myself following the details in Chapter 2.

\subsection{Light-only stability of non-fullerene acceptors}

\subsubsection{Impact on rhodanine flanked acceptor based devices}

The effect of this photodegradation was investigated by testing encapsulated devices under 1 sun equivalent white LED illumination in a dry nitrogen environment. The white LED light source was used for these long device degradation measurements as it has a longer lifetime than the solar simulator. However, it should be noted that this illumination does not include either UV radiation which contains high energy photons which may degrade organic materials nor IR radiation which the IDTBR absorbs a considerable amount. This spectral mismatch means that the devices do not absorb the same amount of photons as if they were illuminated under an AM1.5 solar simulator, giving different absolute $\mathrm{J}-\mathrm{V}$ characteristics. It was attempted to rectify this by adjusting increasing intensity of the LED light source until it gave the same photocurrent for one of the devices as under AM1.5G solar simulator illumination. Since different donor:acceptor systems have different absorptions, the J-V characteristics still needed some correction to be comparable to those obtained under solar simulator illumination. In addition, this difference in absorption cause the amount of degradation undergone by the organic materials under the white LED light source to differ compared to under solar simulator radiation. Figure D.1 shows the absolute J-V characteristics where the initial device 
performances have been corrected to be the same as those measured under AM1.5G solar simulator radiation.
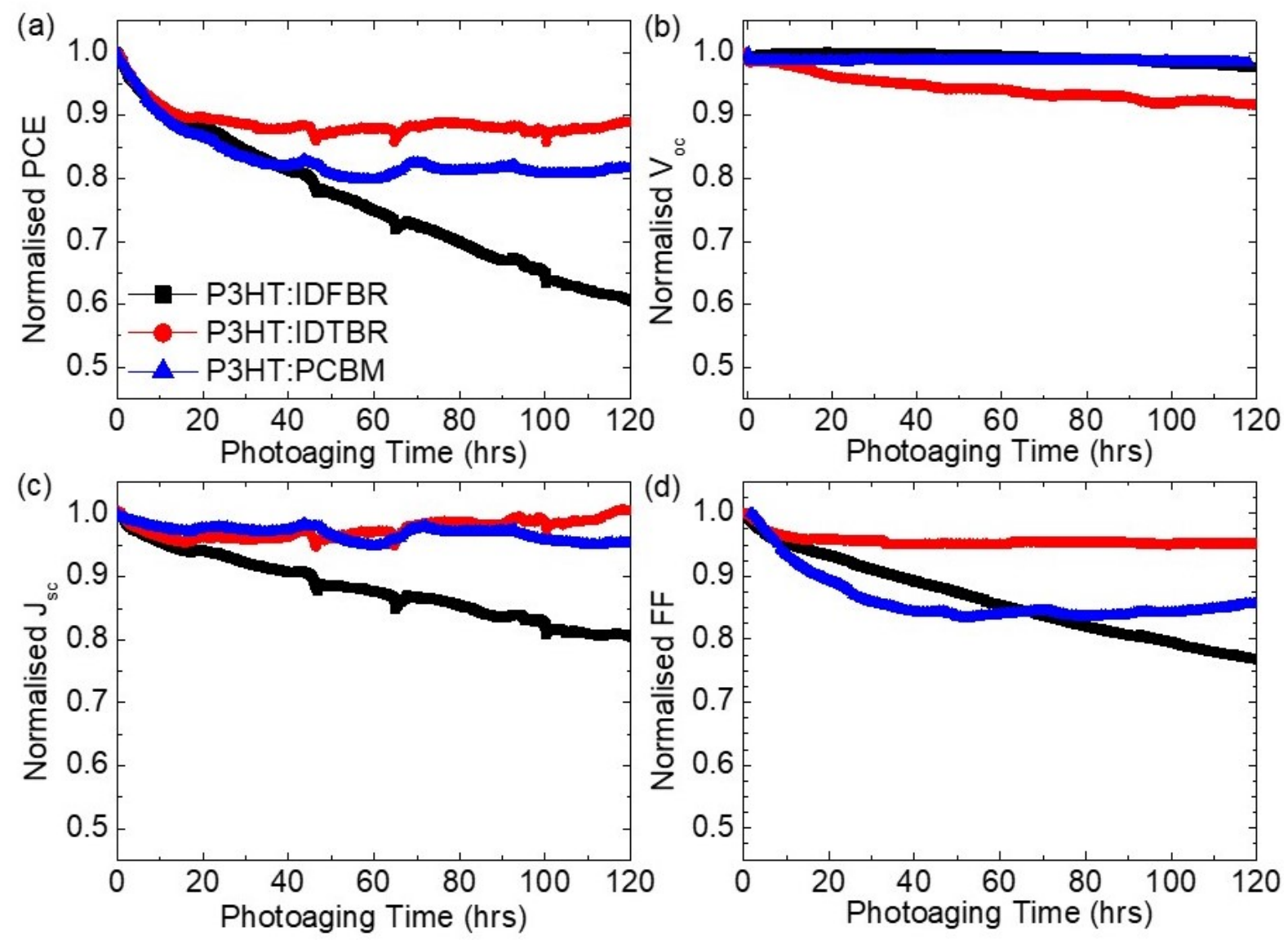

Figure 6.1: Normalised device characteristics (a) PCE, (b) $\mathrm{J}_{\mathrm{sc}}$, (c) $\mathrm{V}_{\mathrm{oc}}$ and (d) FF as a function of device photoaging time under dry nitrogen.

Figure 6.1 shows how the two non-fullerene acceptor based devices (P3HT: IDFBR and P3HT:IDTBR) compare to fullerene based device (P3HT:PC ${ }_{61} \mathrm{BM}$ ) when photoaged under dry nitrogen. A representative pixel from multiple devices were chosen for each material system for this plot. From Figure 6.1(a) the burn-in of the IDTBR and $\mathrm{PC}_{61} \mathrm{BM}$ devices can be observed. It can be observed that the IDTBR device is the most stable under these conditions, exhibiting the smallest burn-in until $\sim 18$ hours of photoaging, before stabilising at $\sim 88 \%$ of its initial PCE for up to 120 hours of irradiation time. The $\mathrm{PC}_{61} \mathrm{BM}$ device exhibited a larger burn-in, stabilising after $\sim 40$ hours at of $\sim 81 \%$ of its initial PCE for up to 120 hours. The IDFBR device is very unstable and had a rapid degradation over the 120 hours reaching $\sim 60.5 \%$ of its initial PCE.

To gain some insight into the burn-in/photodegradation of the devices, the normalised $\mathrm{J}_{\mathrm{sc}}, \mathrm{V}_{\mathrm{oc}}, \mathrm{FF}$ are also plotted in Figure 6.1. The $\mathrm{J}_{\mathrm{sc}}$ of the IDTBR and $\mathrm{PC}_{61} \mathrm{BM}$ devices remain constant throughout the measurement time, but there is a substantial drop of $\sim 20 \%$ for the IDFBR device. The stable $\mathrm{V}_{\text {oc }}$ of $\mathrm{PC}_{61} \mathrm{BM}$ devices is consistent with no considerable photo-oxidation of $\mathrm{PC}_{61} \mathrm{BM}$ occurring during the photaging, as photo- 
oxidation of $\mathrm{PC}_{61} \mathrm{BM}$ will reduce $\mathrm{V}_{\text {oc }}$ (see Chapter 3), which is reasonable due to the lack of environmental oxygen in these photoaging conditions. The FF of the IDTBR device is very stable, but the $\mathrm{FF}$ of the $\mathrm{PC}_{61} \mathrm{BM}$ device is unstable, degrading to $84.3 \%$ before stabilising, while the FF of the IDFBR device decreases at a constant rate to reach 76.7 $\%$ of its initial FF by 120 hours of photoaging. Compared to IDTBR, the main factor for the higher degree of burn-in for the $\mathrm{PC}_{61} \mathrm{BM}$ device is the more severe drop in $\mathrm{FF}$, which overcomes the less severe drop in $\mathrm{V}_{\mathrm{oc}}$. Whereas, for IDFBR, both the $\mathrm{J}_{\mathrm{sc}}$ and FF drop in a faster rate and continuously. The loss in $\mathrm{FF}$ in the IDFBR and $\mathrm{PC}_{61} \mathrm{BM}$ devices could be due to an increase in charge recombination or a formation of space limited charges due to unbalanced charge transport.[1] The loss in $\mathrm{J}_{\mathrm{sc}}$ in the IDFBR device could be due to the chemical degradation of the photoactive layer reducing the photon absorption and/or reducing the percolation paths.[1] The small loss in $\mathrm{V}_{\text {oc }}$ of the IDTBR device can be due to an increase in the energy bandgap so could be a supression in the LUMO of the NFA or an increase in the P3HT HOMO.

\subsubsection{Spectroscopic studies of rhodanine flanked acceptors}

To gain some insight into the burn-in degradation, spectroscopic studies were undertaken to study the photodegradation experienced by the active layer materials used in the devices.
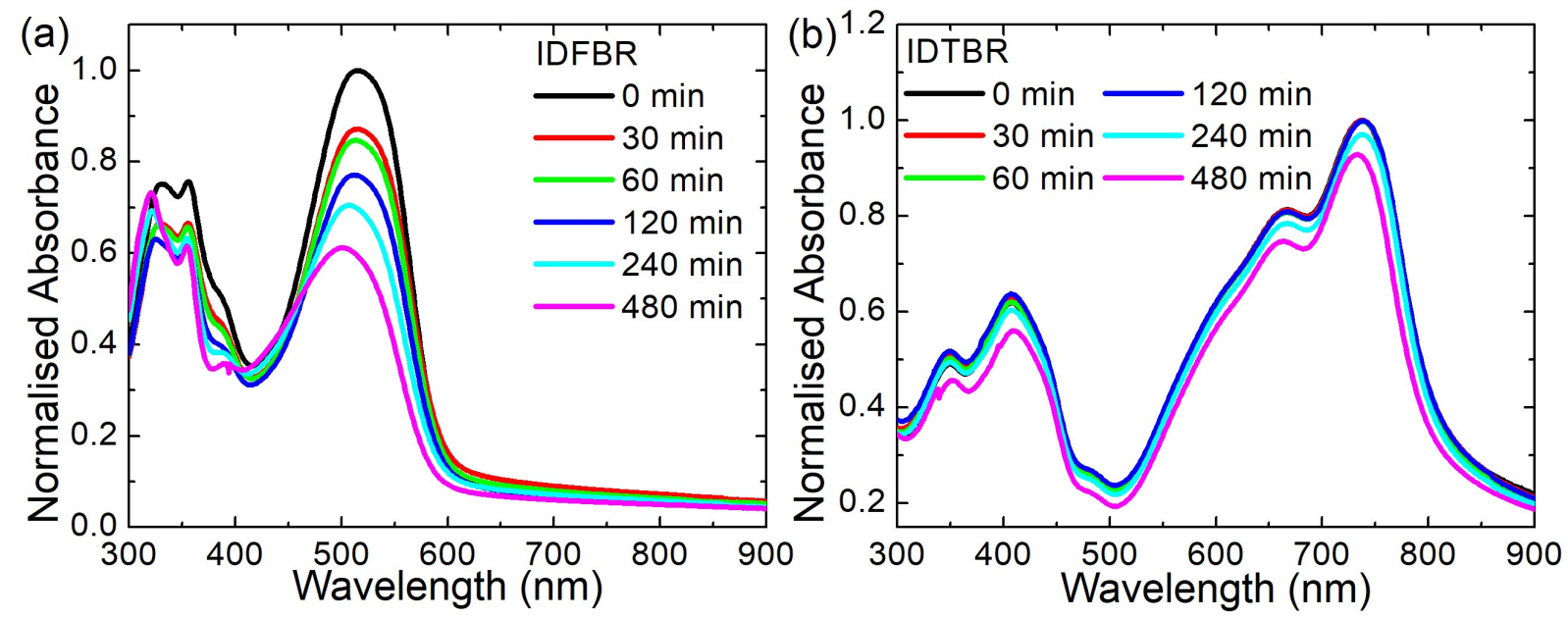

Figure 6.2: Normalised UV-Vis absorbance of (a) IDFBR and (b) IDTBR annealed neat films under 1 sun AM1.5G illumination under dry nitrogen.

Photobleaching measurements were first undertaken to study the photodegradation of these non-fullerene acceptors. The neat annealed IDFBR film shows a substantial amount of photobleaching after 8 hours under 1 sun AM1.5G solar simulator illumination in dry nitrogen, with the peak at $516 \mathrm{~nm}$ reducing to $61.2 \%$ of its initial value and blueshifting to $501 \mathrm{~nm}$ (Figure 6.2a). This shows that it is surprisingly unstable under light in a 
nitrogen environment, and the blueshift of the peak suggests a loss of conjugation. It should also be noted that the absorbance in the region below $350 \mathrm{~nm}$ in the IDFBR spectrum increases with degradation. On the other hand, it can be seen that the neat annealed IDTBR film is relatively stable under dry nitrogen, with the absorbance of the peak at $734 \mathrm{~nm}$ only photobleaching to $92.9 \%$ of its initial value after 8 hours under 1 sun AM1.5G solar simulator illumination in dry nitrogen (Figure 6.2b). These neat NFA films both exhibit degradation under dry nitrogen, with the IDFBR degradation being severe. Photobleaching studies were also undertaken using a UV-cutting filter to show that degradation happened similarly (Figures D.2 IDFBR $29.9 \%$ IDTBR 96.4 \%), showing that UV light was not a critical factor. Organic molecules have been shown to undergo a process called "photodissociation" or "photolysis" whereby the absorption of a photon adds enough energy to exceed the bond dissociation energy, breaking the bond. This is more likely to happen when the molecule is in a highly-excited vibrational state, as energy required to dissociate the bond is less. [2, 3] Photodissociation mechanisms have been suggested for molecules such as cyclobutane and polystyrene. $[4,5]$
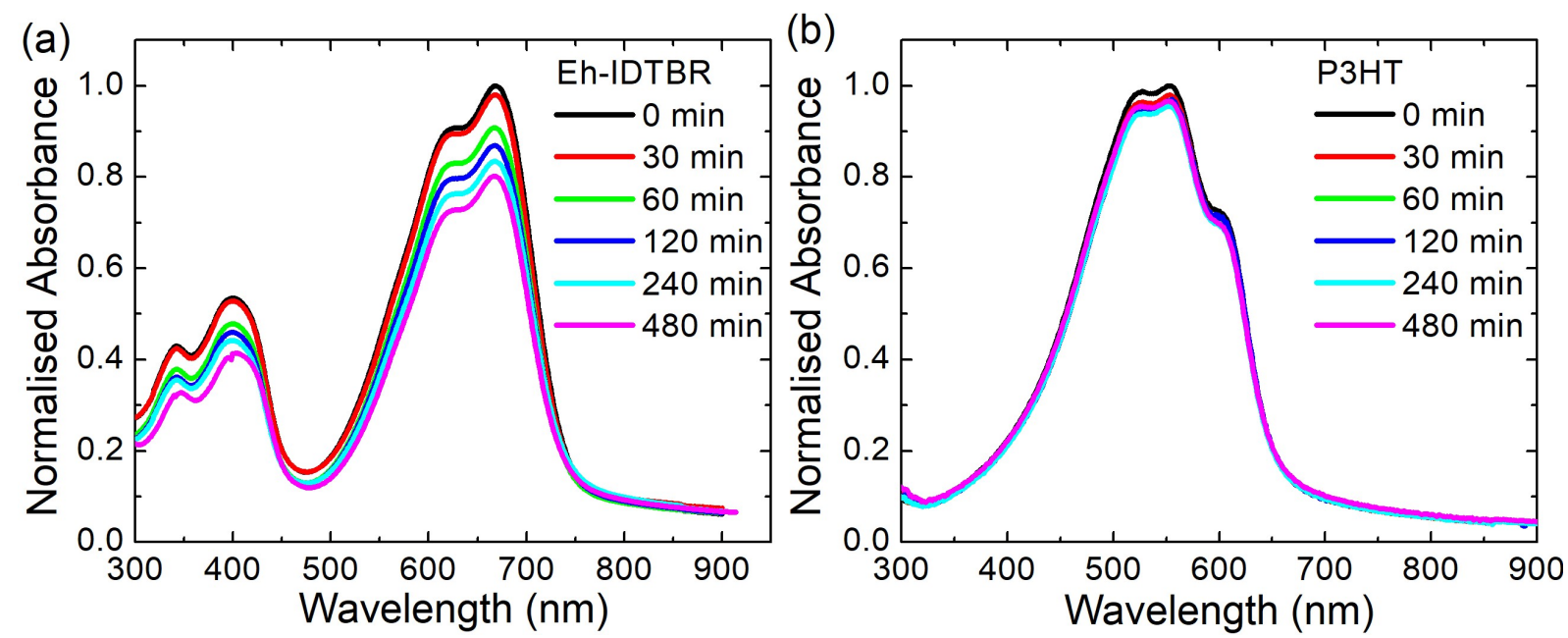

Figure 6.3: Normalised UV-Vis absorbance of (a) Eh-IDTBR and (b) P3HT annealed neat films under 1 sun AM1.5G illumination under dry nitrogen.
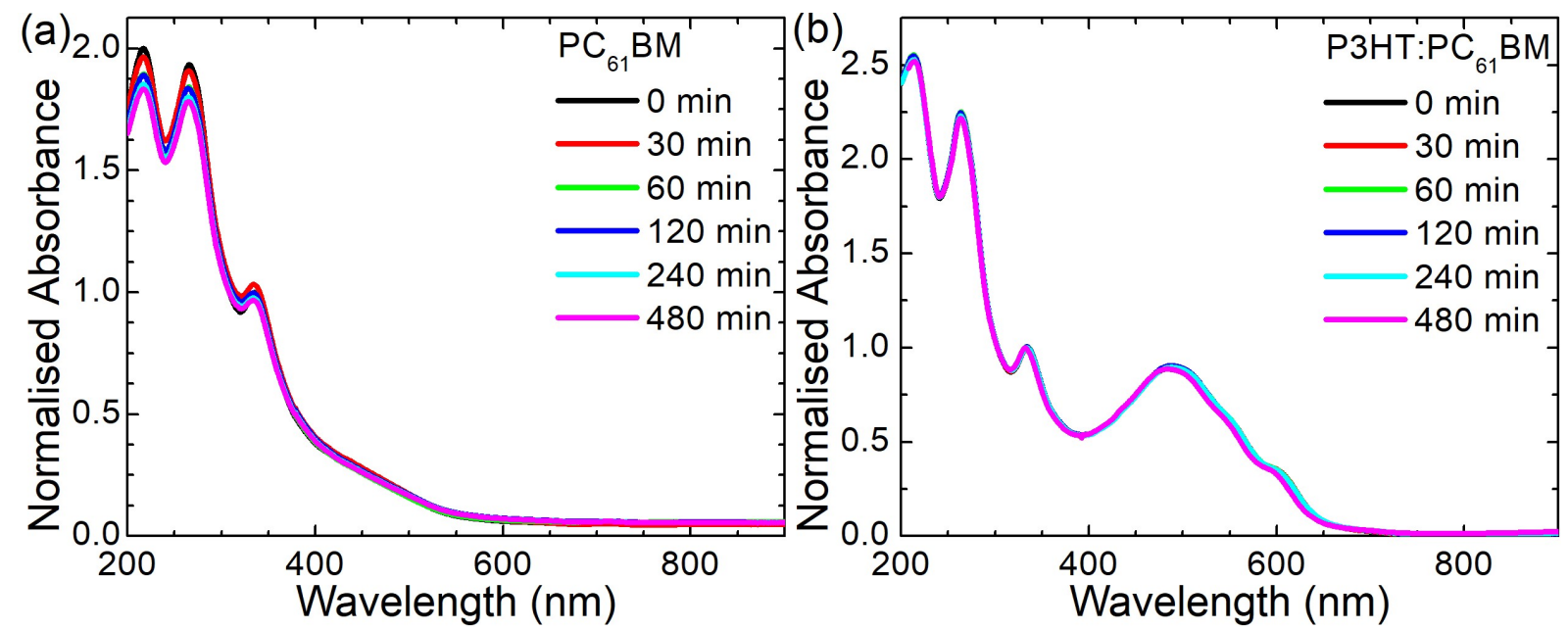

Figure 6.4: Normalised UV-Vis absorbance of (a) $\mathrm{PC}_{61} \mathrm{BM}$ and (b) P3HT:PC 61 BM annealed films under 1 sun AM1.5G illumination under dry nitrogen. 
The photobleaching of some other systems were also measured for comparison. Neat annealed Eh-IDTBR (branched sidechain instead of linear) was found to be more stable than IDFBR and less stable than IDTBR, with its absorbance degrading to $80.2 \%$ of its initial value after 8 hours of photobleaching under AM1.5G illumination in nitrogen (Figure 6.3a). P3HT was very stable under Am1.5G illumination in air for 8 hours where the absorbance decreased to just $96.5 \%$ its initial value. $\mathrm{PC}_{61} \mathrm{BM}$ was found to be similarly stable degrading to $96.8 \%$ of its initial absorbance and the $\mathrm{P} 3 \mathrm{HT}: \mathrm{PC}_{61} \mathrm{BM}$ blend became even more stable with both the $\mathrm{P} 3 \mathrm{HT}$ and $\mathrm{PC}_{61} \mathrm{BM}$ peaks degrading to $99.0 \%$ of their initial value (Figure 6.4a,b).
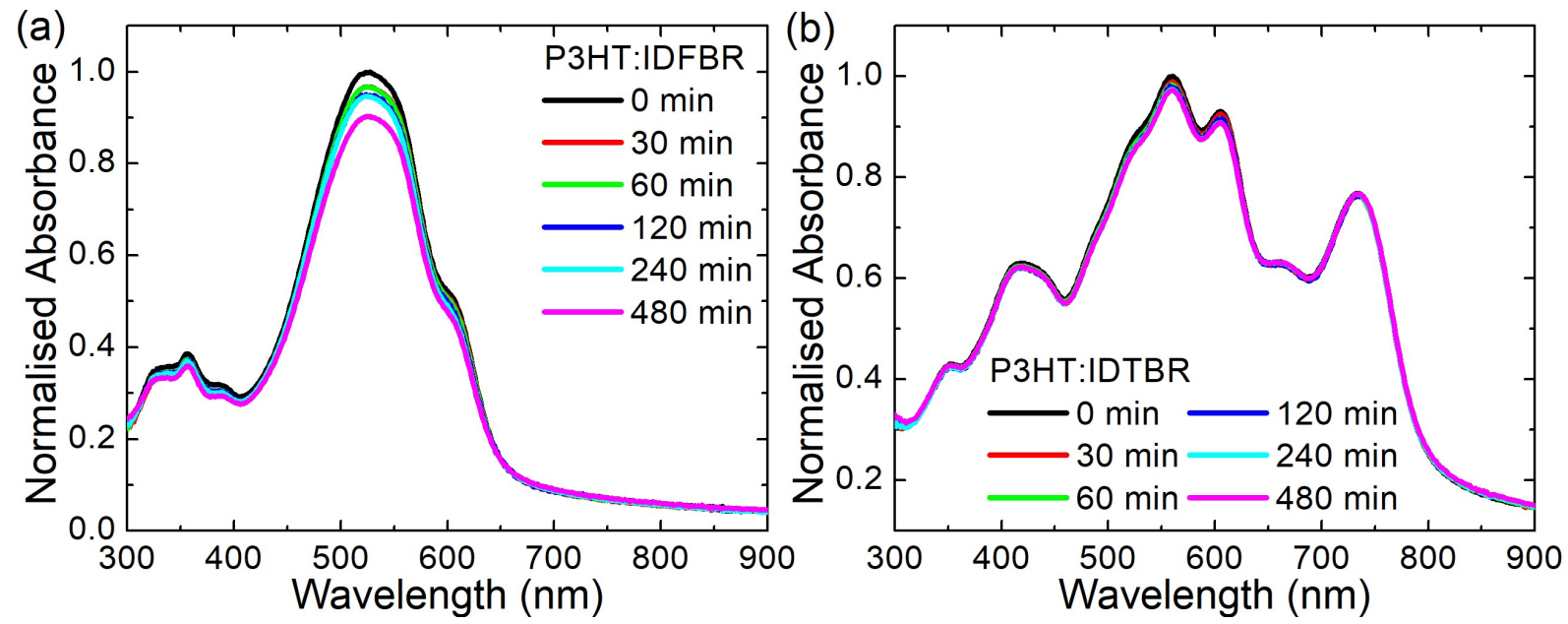

Figure 6.5: Normalised UV-Vis absorbance of (a) P3HT:IDFBR and (b) P3HT:IDTBR annealed films under 1 sun AM1.5G illumination under dry nitrogen.

The photobleaching of NFA blend films was also measured (Figure 6.5a,b). By looking at the P3HT shoulder at $600 \mathrm{~nm}$ of the P3HT:IDFBR data, it can be seen that the P3HT degrades significantly and more than the neat P3HT film under illumination in nitrogen (absorbance reduced to 92.9 and $96.5 \%$ initial value respectively), thus is destabilised upon blending with IDFBR. By looking at the peak at $\sim 350 \mathrm{~nm}$ due to the IDFBR, it can be seen that there is less change, (absorbance reduced to 93.0 and $81.2 \%$ initial value respectively), thus is stabilised upon blending with P3HT. Similarly, by looking at the region where there is low contribution from IDTBR to the P3HT:IDTBR absorbance spectrum, it can be seen that the P3HT degrades similarly in the blend film under nitrogen to the neat P3HT film (absorbance reduced to 97.4 and $96.5 \%$ initial value respectively). The peak at $734 \mathrm{~nm}$ due to the IDTBR, it can be seen that there is a stabilisation upon blending with P3HT when compared to the neat IDTBR film (absorbance reduced to 99.8 and $92.9 \%$ initial value respectively). 

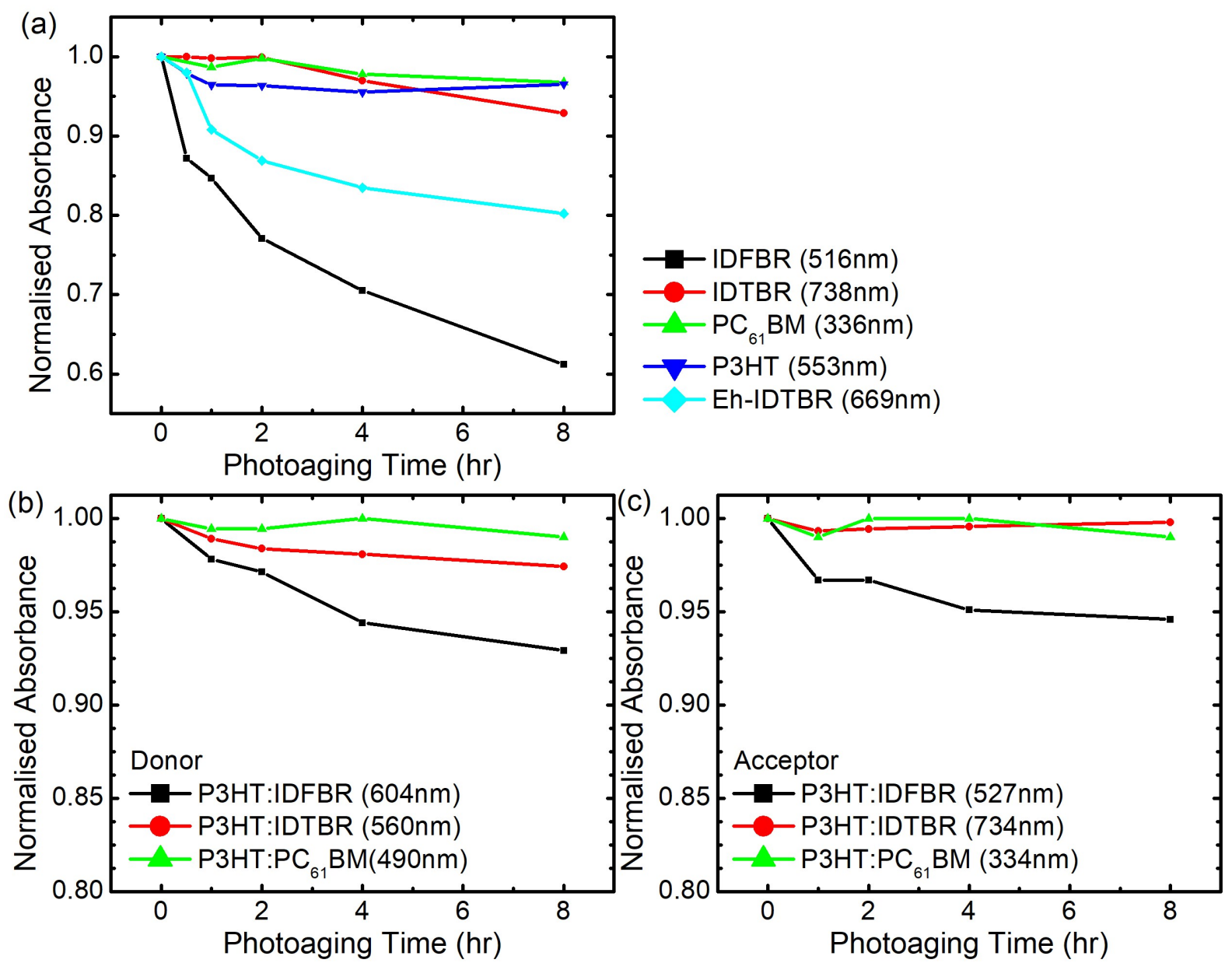

Figure 6.6: Normalised absorbance at specified wavelengths of annealed a) $\mathrm{PC}_{61} \mathrm{BM}$, P3HT and NFA films and b) P3HT:acceptor films (donor contribution) and c) P3HT:acceptor films (acceptor contribution) as a function of photoaging time under AM1.5G illumination in dry nitrogen.

To better compare the above photobleaching measurements, the normalised absorbance at the specified wavelengths of the neat films and blend films are plotted in Figure 6.6a-c, summarising the photobleaching of these neat and blend films under dry air and AM1.5G illumination as a function of time. It can be seen that for neat films (Figure 6.6a), $\mathrm{PC}_{61} \mathrm{BM}$ and P3HT are the most stable neat materials, followed by IDTBR, and then Eh-IDTBR and (especially) IDFBR which are both less stable by comparison. For the blend films, the spectra are deconvoluted to resolve the absorbance from the donor (Figure 6.6b) and acceptors (Figure 6.6c), respectively. Upon comparing Figure 6.17a with Figure 6.6b and $6.6 \mathrm{c}$, it is clear that the P3HT stability in blended is dependent on the acceptor with which it is blended: it is destabilised with IDFBR, remains similar with IDTBR and is stabilised with $\mathrm{PC}_{61} \mathrm{BM}$. However, the acceptors are all stabilised when they are blended with P3HT. 


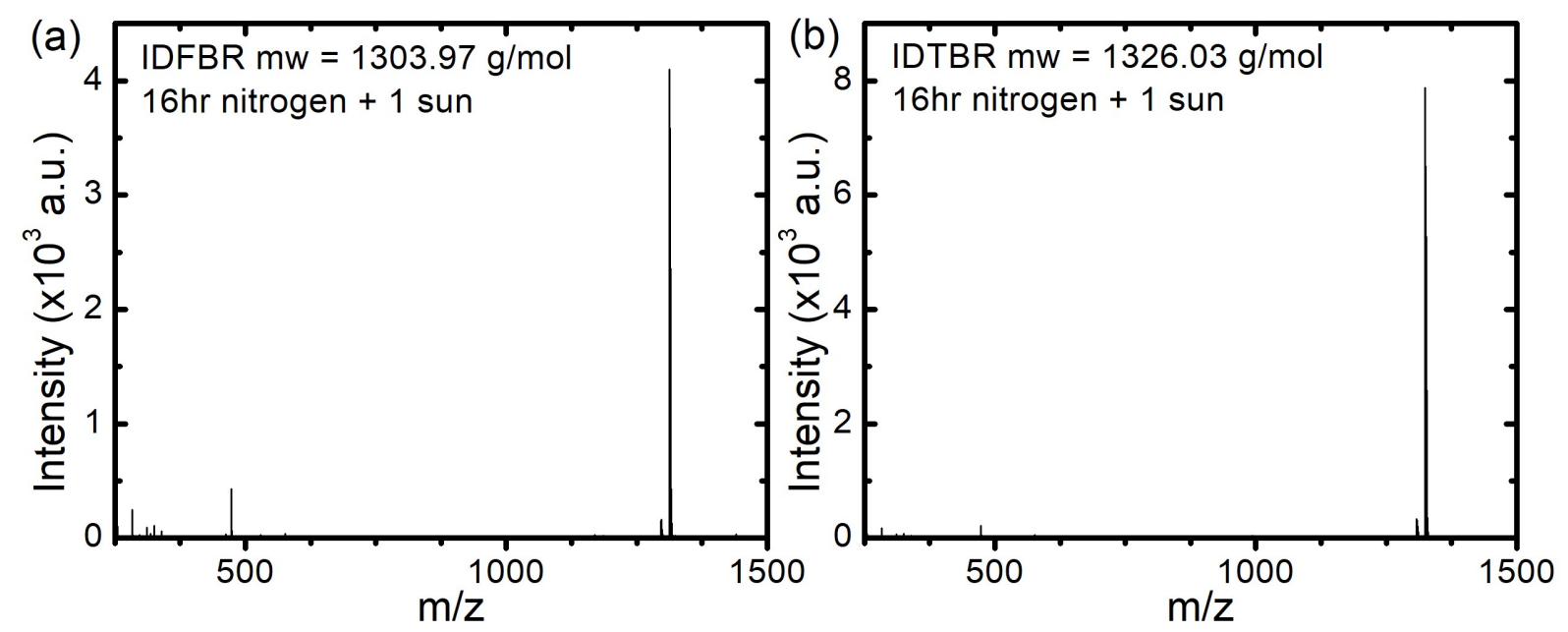

Figure 6.7: Negative ion mode MALDI-TOF measurements of: a) IDTBR and b) IDFBR neat films after 16 hours of photoaging under AM1.5G illumination in nitrogen.

Mass spectroscopy measurements were performed on IDFBR and IDTBR powders which were scratched from neat films. The aim of these measurements was to probe if there is any change in mass of the molecules, indicating a change in chemical structure. Figure 6.7a shows the MALDI-TOF spectrum for IDFBR after 16 hours of photoaging under 1 sun AM1.5G illumination in nitrogen. The peaks at around $1304 \mathrm{~g} / \mathrm{mol}$ correlate well with the $\mathrm{Mw}$ of IDFBR. There is an appearance of some peaks at lower $\mathrm{m} / \mathrm{z}$ values than the mw of IDFBR compared to fresh IDFBR (Figure D.7a), indicating some chemical decomposition upon photoaging in nitrogen. For the IDTBR, there are minimal changes compared to the fresh IDTBR (Figure D.7b). This indicates minimal change in the chemical structure of IDTBR after photoaging in nitrogen. These results correspond well with the observed photobleaching, where the IDTBR film was more stable than the IDFBR neat film under illumination in nitrogen (Figure 6.2). 


\subsubsection{Comparison of burn-in behaviour for different systems}

To determine if a general rule could be ascertained for the burn-in behaviour of devices based on the type of acceptor that was used, IDT-based devices were compared with ITIC and $\mathrm{PC}_{71} \mathrm{BM}$ based devices using a range of benchmark polymers. PCE10 was used as a common polymer for each acceptor and then PCE12 or PCE11 was used depending on which performed best with the acceptor.
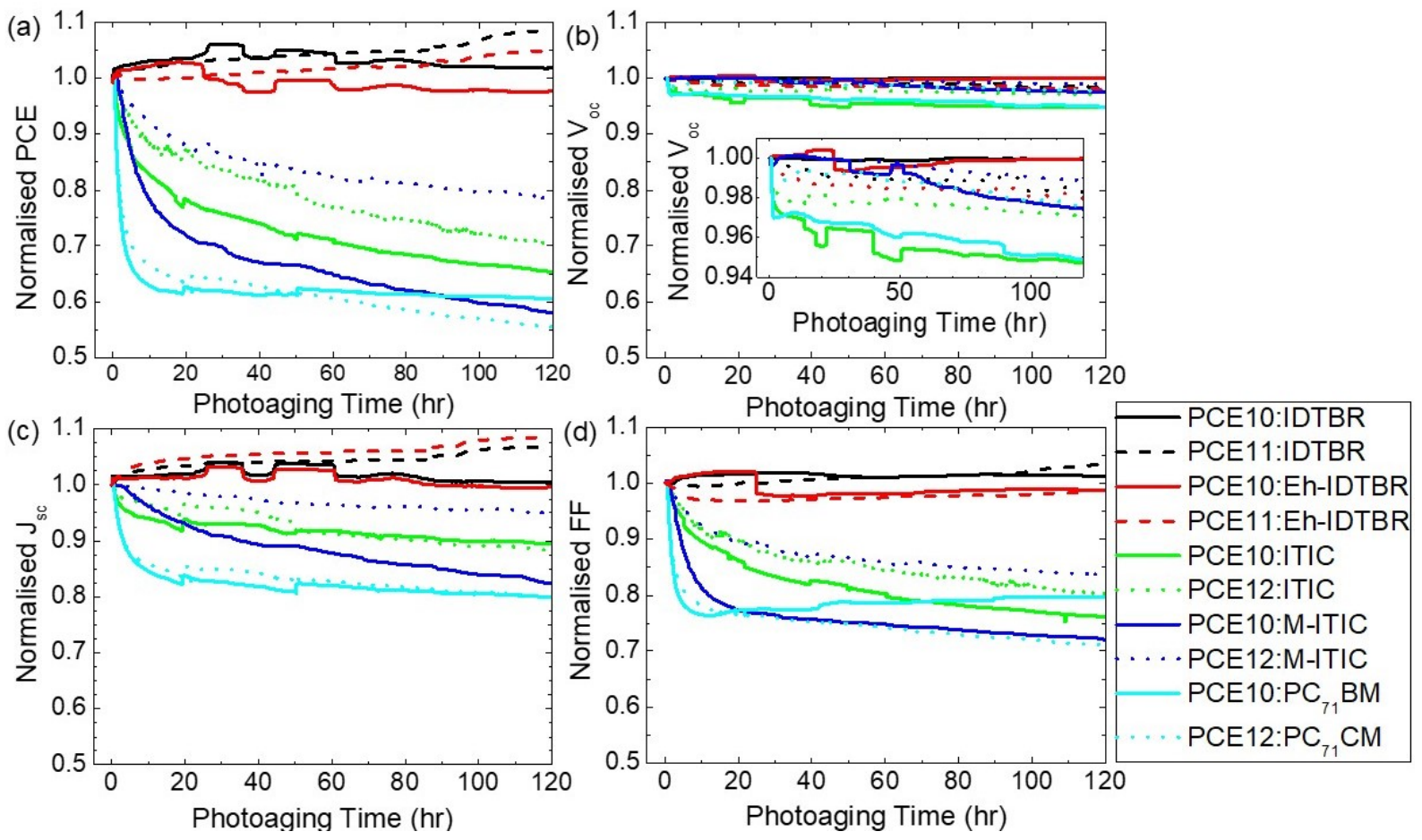

Figure 6.8: Normalised device characteristics (a) PCE, (b) $\mathrm{V}_{\text {ос }}$ which is enlarged in the inset, (c) $\mathrm{J}_{\mathrm{sc}}$ and (d) FF as a function of device photoaging time under 1 Sun equivalent white LED light in dry nitrogen.

Figure 6.8 shows the device characteristics of the solar cells as a function photoaging time under 1 sun equivalent white light (LED) illumination in a dry nitrogen environment. A representative pixel from multiple devices were chosen for each material system for this plot. The $\mathrm{T}_{80}$ lifetimes are shown in Table D.2. Firstly, PCE10:PC ${ }_{71} \mathrm{BM}$ and PCE12: $\mathrm{PC}_{71} \mathrm{BM}$ exhibit a strong burn-in, losing $\sim 40 \%$ of initial device efficiency after just 20 hours of degradation. This strong burn-in was mainly due to a strong loss in $\mathrm{FF}(\sim 23 \%)$ and loss in $\mathrm{J}_{\mathrm{sc}}(15 \%)$, with a small loss in $\mathrm{V}_{\mathrm{oc}}$. The loss in FF can be attributed to shallow trap formation due to a chemical degradation of fullerene acceptor as proposed for PCE11:PC ${ }_{71} \mathrm{BM}$ devices, $[6]$ or a morphological change such as spinodal demixing or dimerisation.[7, 8] However, the $15 \%$ loss in $\mathrm{J}_{\mathrm{sc}}$ was not observed using PCE11.[6] After the burn-in phase, the PCE10:PC 71 BM device stabilises, whereas the $\mathrm{PCE}$ of the PCE12: $\mathrm{PC}_{71} \mathrm{BM}$ device continues to degrade but at a slower rate, due to the continued loss in FF. 
The Eh- and O-IDTBR based devices with donor polymers PCE10 and PCE11 exhibited a burn-in free behaviour consistent with a previous report with PCE11: Eh-IDTBR.[6] Here, all the device characteristics were stable under illumination under dry nitrogen for 120 hours. There is a small increase in PCE and $\mathrm{J}_{\mathrm{sc}}$ for the PCE11 devices, which can be assigned to the activation of the $\mathrm{ZnO}$ interlayer which takes a longer time under white LED light than when UV-light is present. This is in line with previously reported improvement of $\mathrm{J}_{\mathrm{sc}}$ with UV light-soaking of OSCs with $\mathrm{ZnO}$ and other metaloxide electron transport layers whose origin has been suggested to be through shallow trap-filling by the photogenerated charges or a lowering of the metal oxide work function, through desorption of oxygen, improving the electron selectivity at the interface with the active layer. $[9,10]$

The photodegradation behaviour of the ITIC and M-ITIC based devices is different to that of both IDT- and fullerene based devices. They did not stabilise during the 120 hours of testing, so cannot be classed as having a burn-in, rather they were unstable under this light-only degradation. After 120 hours, their PCEs had degraded to PCE12:M-ITIC 78.8 \%, PCE12:ITIC 70.4 \%, PCE10:ITIC 65.4 \% and PCE10:M-ITIC 58.0\% of their initial PCEs. It is worth to note, after $~ 90$ hours, PCE of the PCE10:M-ITIC had degraded more than the fullerene-based devices, as the PCE of the fullerene-based devices stabilised after the burn-in phase. In brief, the Eh-/O-IDTBR-based devices can be burn-in free and hence would have superior stability when encapsulated in dry nitrogen. However, more research is required to understand why they are burn-in free and why there is minor burn-in when P3HT is used as the donor (see Figure 6.1).

\subsection{Stability of rhodanine flanked acceptors under il- lumination and air}

\subsubsection{Device behaviour}

This part will investigate the stability of the rhodanine flanked acceptors under illumination in air (i.e. with the presence of oxygen). 

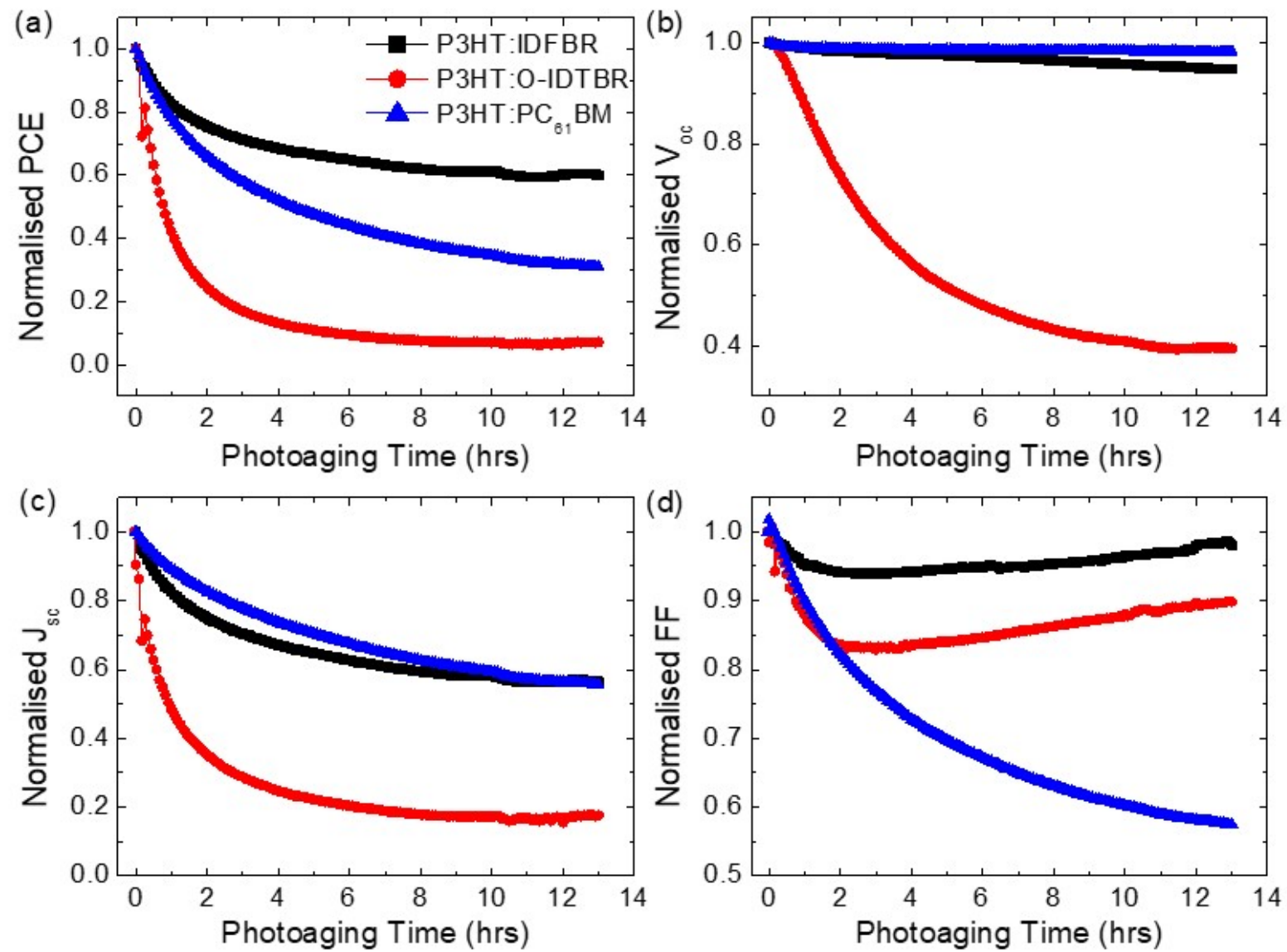

Figure 6.9: Normalised device characteristics (a) PCE (b) $\mathrm{V}_{\mathrm{oc}}$, (c) $\mathrm{J}_{\mathrm{sc}}$, (d) FF as a function of photoaging time under white LED illumination in air. The photoaging was performed on complete devices.

Firstly, the stability of the (completed) devices was tested under 1 sun AM1.5G illumination in air and the results are shown in Figure 6.9, the absolute data in Figure D.8 and the $\mathrm{T}_{80}$ lifetimes in Table D.3. A representative pixel from multiple devices were chosen for each material system for this plot. Surprisingly, it was shown that the P3HT:IDFBR device is actually the most stable under these conditions, degrading to $\sim 50 \%$ of its initial PCE after 13 hours of photoaging.[11] While the P3HT:IDTBR device was the least stable, degrading rapidly to $\sim 13 \%$ of the initial PCE after 4 hours. The cause of the instability of the P3HT:IDTBR device was mainly a loss in $\mathrm{J}_{\mathrm{sc}}$ and $\mathrm{V}_{\mathrm{oc}}$ and a small loss in FF. A loss in $\mathrm{J}_{\mathrm{sc}}$ can be due to a loss in: photon absorption due to photobleaching of the photoactive layer; exciton dissociation efficiency by an increase in the exciton diffusion length; and charge carrier transport and charge extraction efficiency due to charge trap formation or loss of percolation paths possibly due to loss of conjugation in active layer.[1] The loss in $\mathrm{V}_{\mathrm{oc}}$ could be due to a suppression of the IDTBR LUMO, forming electron traps as seen in previous chapters with fullerenes, or a raising of the P3HT HOMO or an increase in recombination. The loss in FF indicates a reduction in the charge extraction of the device, including increase in recombination losses or the formation of space charges due to unbalanced charge transport.[1] The stability of the P3HT:PC ${ }_{61} \mathrm{BM}$ device was in- 
between that of the P3HT:IDFBR and the P3HT:IDTBR, degrading to $\sim 31 \%$ of initial PCE after being illuminated for 13 hours. This degradation was determined by a loss in $\mathrm{J}_{\mathrm{sc}}$ and $\mathrm{FF}$. These results are surprising as it is expected that P3HT:IDFBR device will be the least stable, as under dry nitrogen there is strong photobleaching of neat IDFBR film (Figure 6.2a), mild photobleaching of the P3HT:IDFBR blend film (Figure 6.5) and devices undergo a severe degradation (Figure 6.1). In addition, it should be noted that there was a large device to device and even pixel to pixel variability in device stability of complete devices photoaged in air, likely due to differences in the oxygen diffusion rates through the top electrode so Figure 6.9 should just be used for reference.[12]
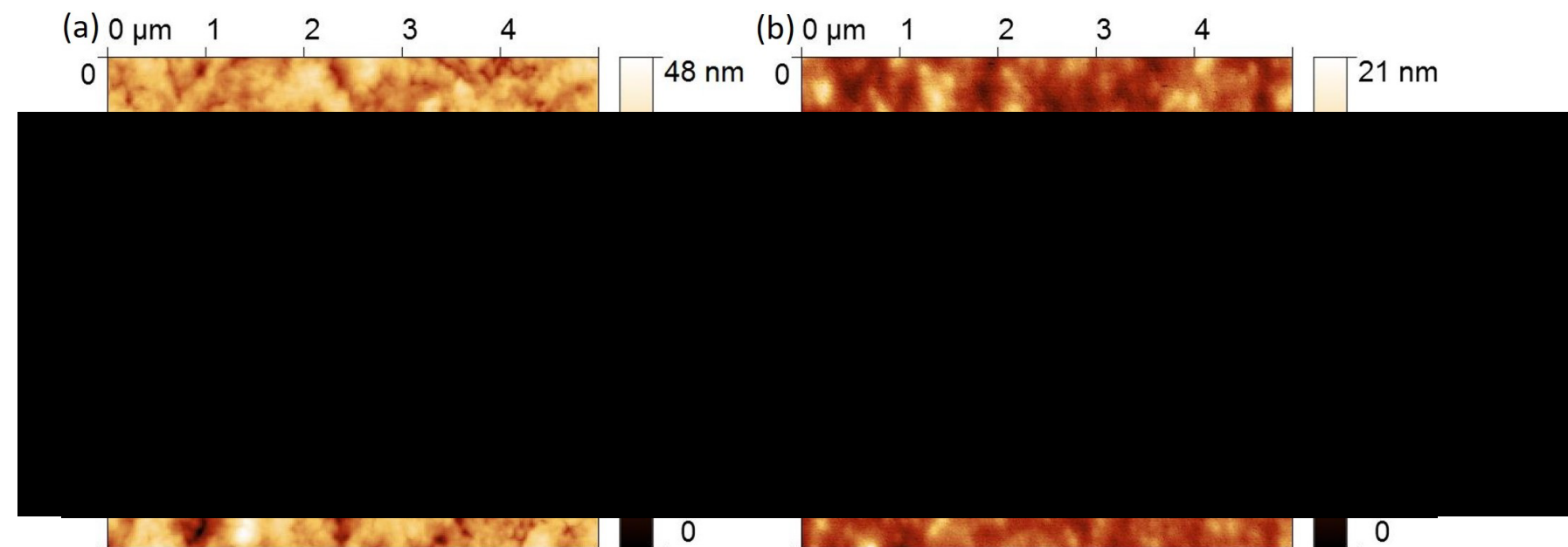

Figure 6.10: Topography images of annealed (a) P3HT:IDTBR and (b) P3HT:IDFBR films measured by Joel Luke at Imperial College London.

The higher stability of the P3HT:IDFBR device may be related to its film morphology, as IDFBR is highly amorphous while O-IDTBR is a highly crystalline material. A rougher surface of the blend films could have adverse effect on device stability by by causing an inhomogeneous deposition of the $\mathrm{MoO}_{3}$ interlayer and the silver electrode. These layers act as a barrier for oxygen entering the active layer, so these pinholes create a pathway for oxygen to diffuse into the active layer at a quicker rate and cause degradation. Therefore, AFM measurements were performed on the annealed P3HT:IDTBR and P3HT:IDFBR films. As calculated from Figure 6.10, the root mean squared roughness of an annealed P3HT:IDTBR films is $5.89 \mathrm{~nm}$ which is much greater than that of an annealed P3HT:IDFBR film $(1.82 \mathrm{~nm})$. Therefore, the AFM data is consistent with the above argument. 

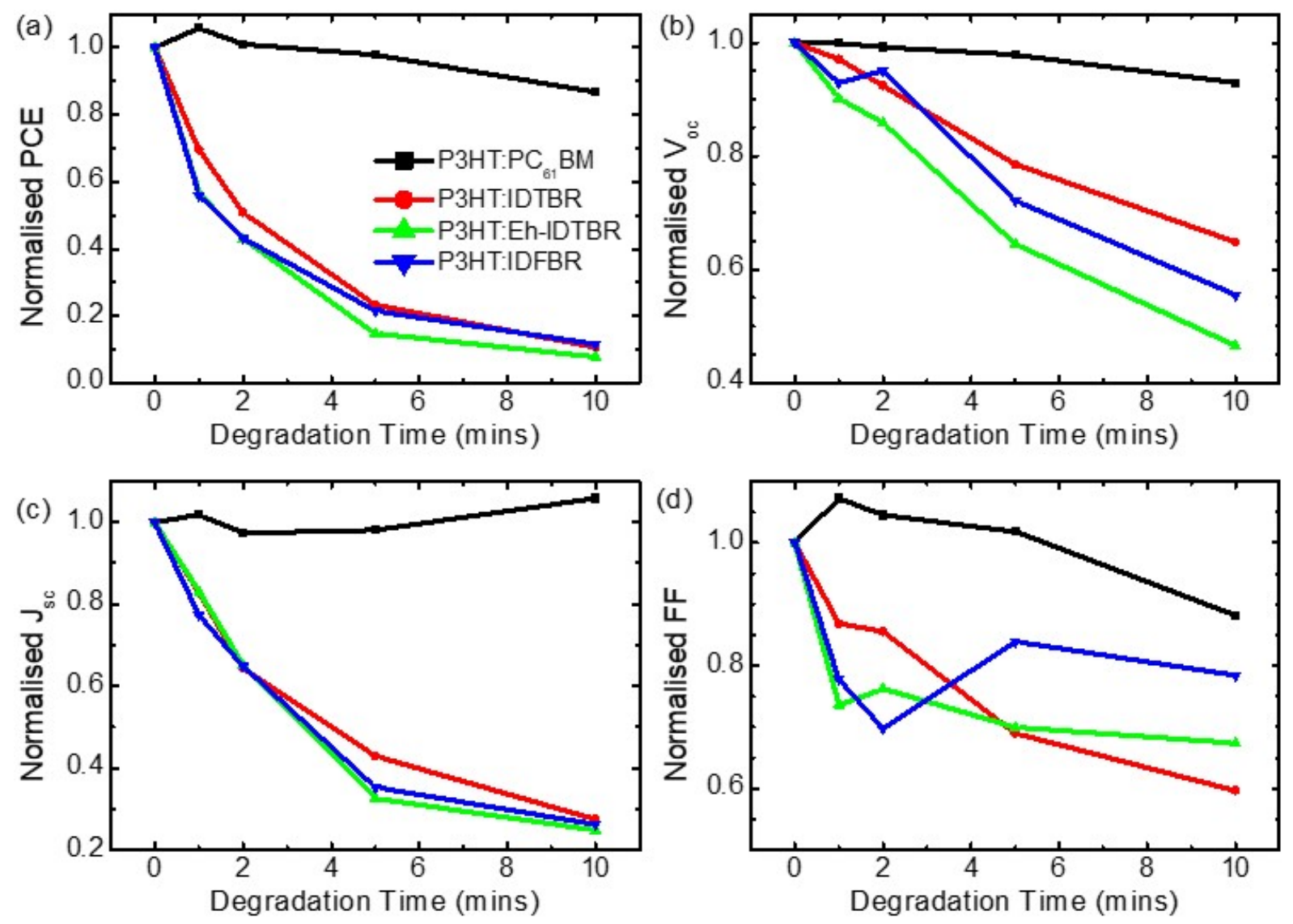

Figure 6.11: Normalised device characteristics (a) PCE (b) $V_{o c}$ (c) $J_{s c}$ (d) FF of P3HT:PC ${ }_{61}$ BM, P3HT:IDTBR, P3HT:Eh-IDTBR and P3HT:IDFBR devices, as a function of active layer photoaging time in air.

To mitigate the complication of oxygen diffusion and degradation of the electrodes, and study the effect of photo-degradation of the organic photovoltaic layer to the device stability in isolation, devices were made where the active layer was photoaged in nitrogen, under a solar simulator, prior to the electrode deposition. A representative pixel from multiple devices were chosen for each material system for this plot. Figure 6.11 shows the device characteristics as a function of photoaging time under 1 Sun AM1.5G illumination in air (active layer photoaged prior to electrode deposition). It is clear that the P3HT:PC ${ }_{61} \mathrm{BM}$ device was the most stable, degrading by only $15 \%$ of initial PCE after 10 minutes photoaging on the active layer. This loss in device performance was due to a loss in both $\mathrm{FF}$ and $\mathrm{V}_{\mathrm{oc}}$, while the $\mathrm{J}_{\mathrm{sc}}$ remained stable. The drop in $\mathrm{V}_{\mathrm{oc}}$ is consistent with photo-oxidation of the $\mathrm{PC}_{61} \mathrm{BM}$, as demonstrated in previous chapters. On the other hand, the NFA-based P3HT devices undergo a rapid degradation to $\sim 10 \%$ of its initial PCE after 10 minutes of photoaging of the active layers in air. This serious degradation is mainly due to a rapid decrease of the $\mathrm{J}_{\mathrm{sc}}$ for all the devices, degrading at a similar rate to $\sim 30 \%$ of their initial $\mathrm{J}_{\mathrm{sc}}$. The $\mathrm{V}_{\text {oc }}$ and FF of the P3HT:NFA devices also degraded significantly but to less of an extent. 


\subsubsection{Spectroscopic studies of photodegradation}

To gain more insight into the photo-degradation of the active layer in dry air, photobleaching of annealed films under 1 sun illumination in air was undertaken and compared to that of films photo-aged under a dry nitrogen environment.

(a)

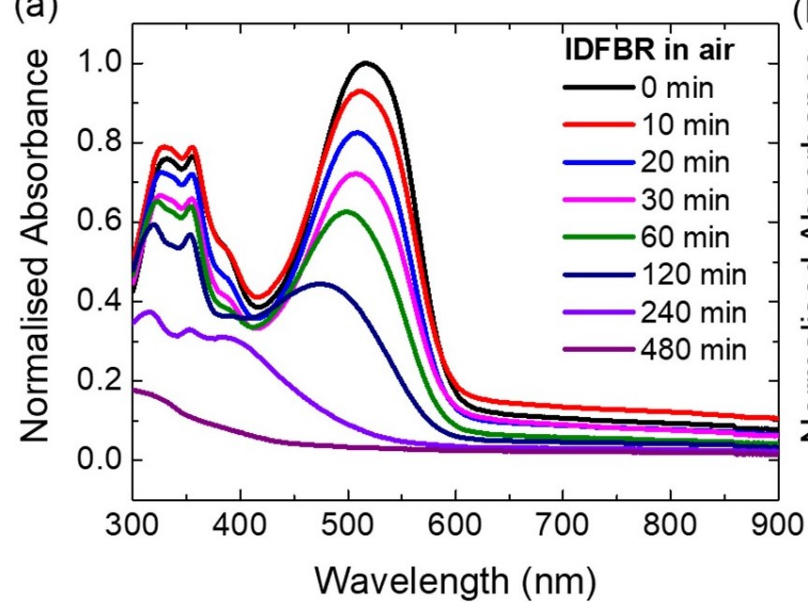

(b)

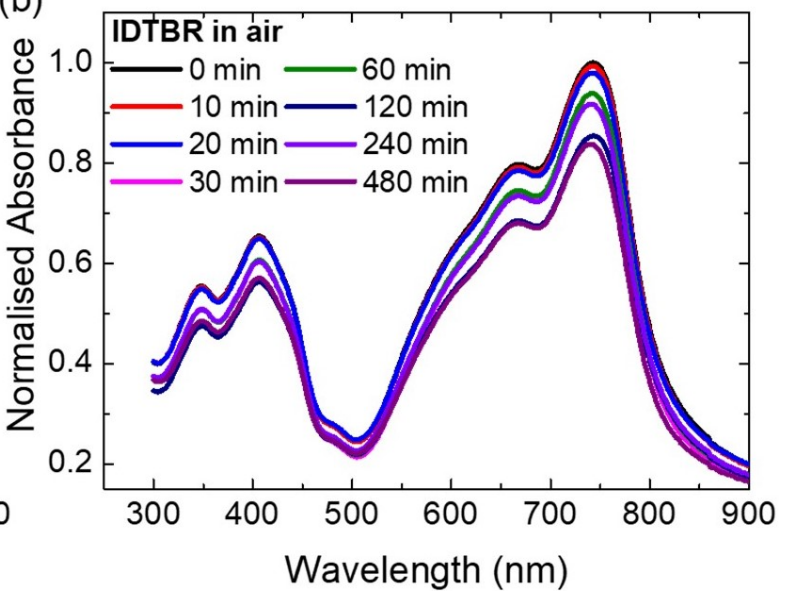

Figure 6.12: Photobleaching of (a) IDFBR and (b) IDTBR neat films under 1 sun illumination in air.

As shown in Figure 6.12a, the neat IDFBR film degrades twice as fast in air than in dry nitrogen (Figure 6.2), with the main absorbance peak at $520 \mathrm{~nm}$ completely photobleached after 8 hours of photoaging. In addition, it can be seen that the main peak of the IDFBR at $515 \mathrm{~nm}$ undergoes a blueshift of $140 \mathrm{~nm}$, indicating a loss of conjugation.[13] The photobleaching of IDTBR film was also accelerated in the presence of air (Figure 6.12b) with the main absorbance peak degrading to $83.8 \%$ of its initial value after 8 hours of illumination. The UV-vis absorbance of the non-annealed films is shown in Figure D.5. The degradation of the neat NFAs is affected by the type of illumination, when the photobleaching of the neat films in Figure 6.12 are compared with that in Figure D.6 where the films are degraded under white LED light at a level tuned so that the $\mathrm{J}_{\mathrm{sc}}$ of an IDTBR device was the same as under AM1.5G illumination, it was clear that the degradation of films of different materials degraded at different rates to how they degraded under 1 sun AM1.5G illumination. Under these white light conditions, IDTBR degraded similarly but the IDFBR degraded nearly twice as fast. This indicates that the degradation is spectrum dependent but the trend is still valid. 

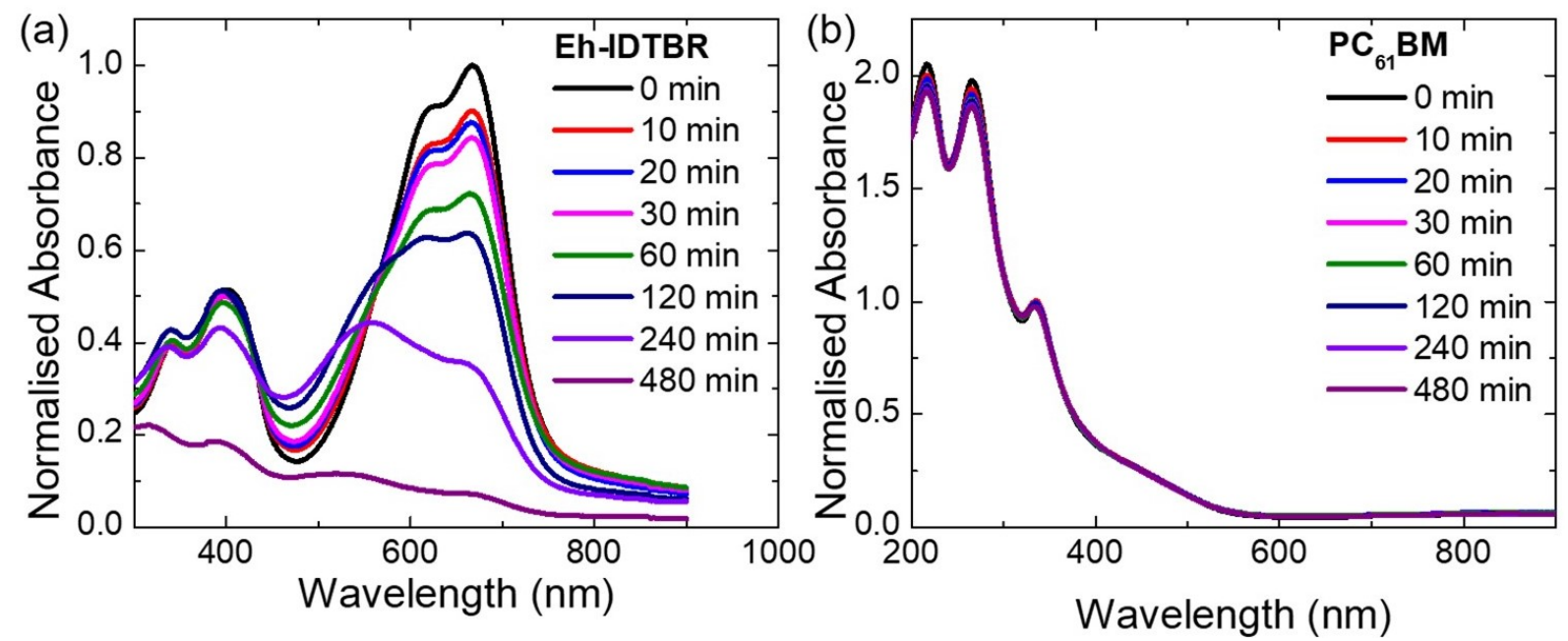

Figure 6.13: Photobleaching of (left) neat annealed $\mathrm{PC}_{61} \mathrm{BM}$ and (right) an annealed P3HT: $\mathrm{PC}_{61} \mathrm{BM}$ blend films under 1 sun illumination in air.

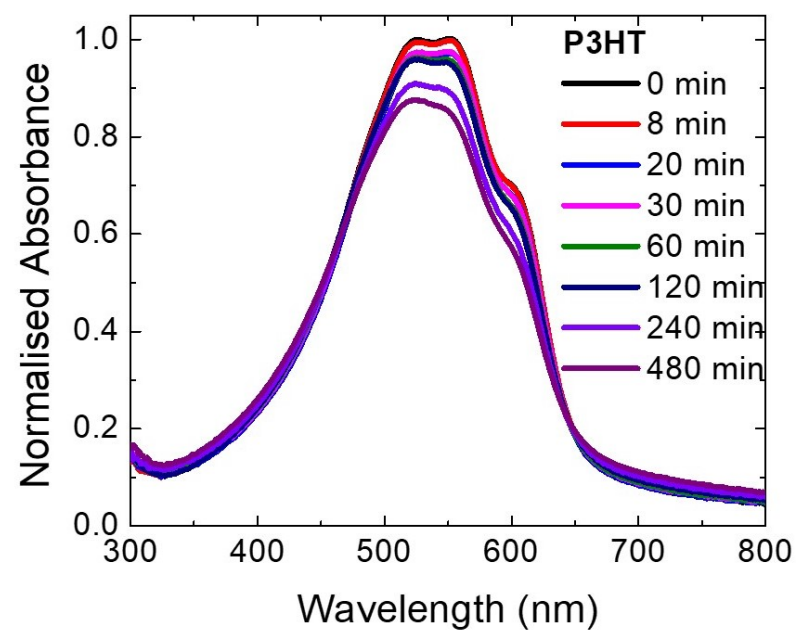

Figure 6.14: Photobleaching of a P3HT neat film under 1 sun illumination in air.

Another member of the rhodanine flanked acceptor family was studied. Eh-IDTBR has the same structure as the (O-) IDTBR which this study focuses upon, but utilises branched sidechains instead of linear. Figure 6.13a shows the photobleaching of an annealed Eh-IDTBR film in air under 1 sun illumination. Neat $\mathrm{PC}_{61} \mathrm{BM}$ was also measured to compare the NFAs against. It can be seen that optically the $\mathrm{PC}_{61} \mathrm{BM}$ is very stable, with the normalised absorbance at $336 \mathrm{~nm}$ dropping to just $97.6 \%$ of its initial value. The photobleaching spectra of neat P3HT film was also measured for reference (Figure 6.14), showing the absorbance peak at $527 \mathrm{~nm}$ dropping to $87.6 \%$ in of its initial value with 8 hours of photoaging in dry air. 
(a)

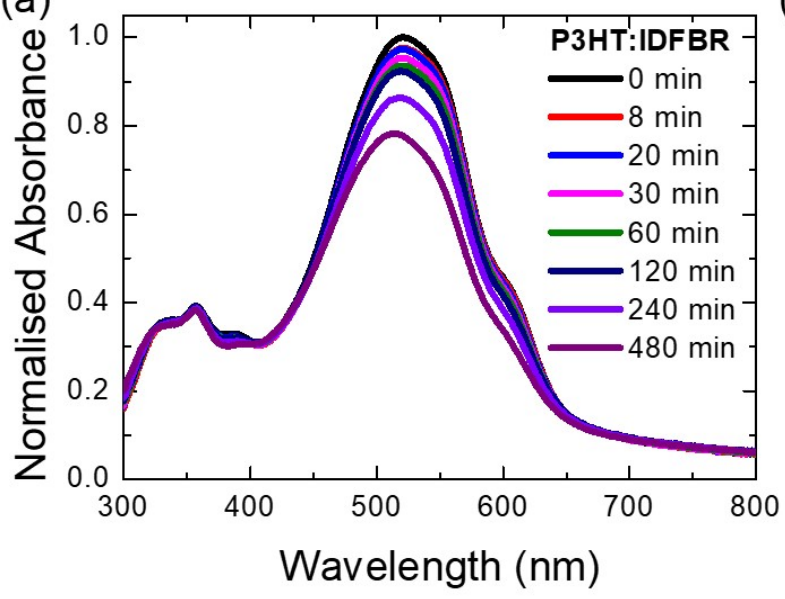

(b)

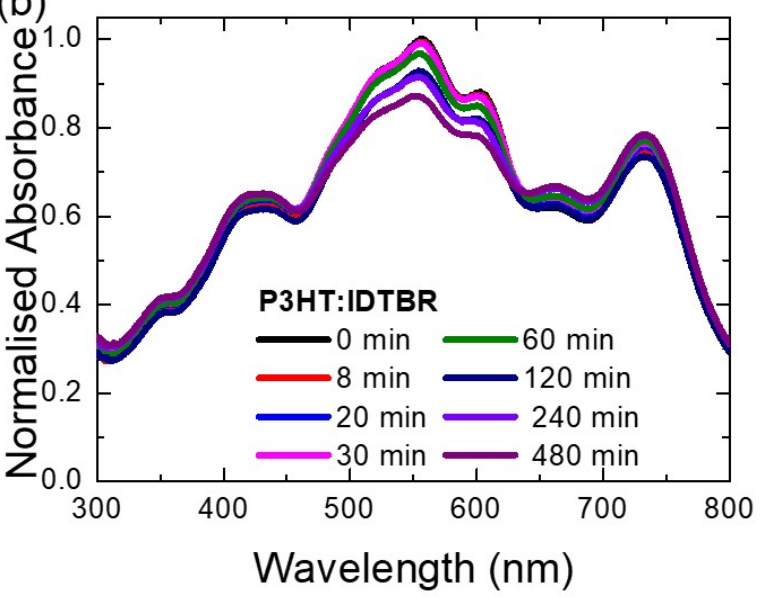

Figure 6.15: Photobleaching of (left) P3HT:IDFBR and (right) P3HT:IDTBR under 1 sun illumination in air.
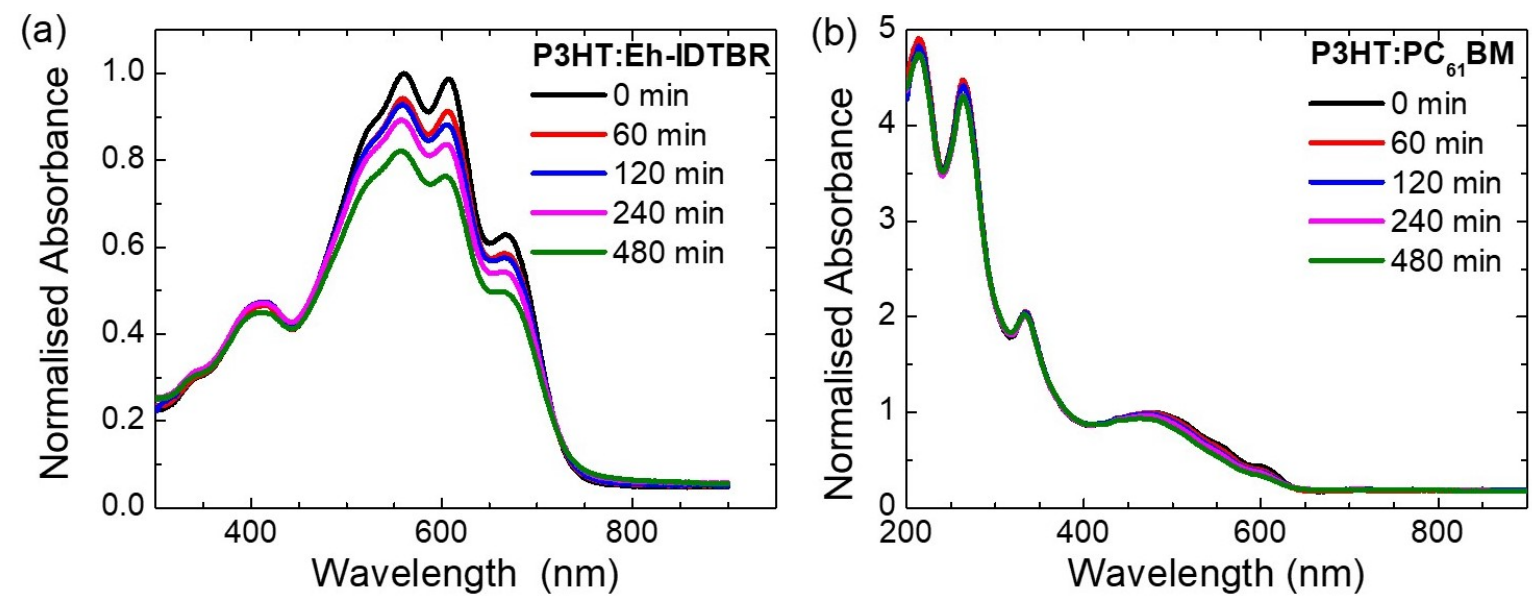

Figure 6.16: Photobleaching of (a) Eh-IDTBR and (b) $\mathrm{PC}_{61} \mathrm{BM}$ neat films under 1 sun illumination in air.

The next step was to study photobleaching of the blend films in air to probe how the polymer donor (P3HT) and the acceptors interact with each other. Figure 6.15a shows that IDFBR is drastically stabilised upon blending with P3HT as the less intense IDFBR absorbance peak at $358 \mathrm{~nm}$ does not decrease significantly (82.7\% remains after 8 hours photoaging). It appears that it is the P3HT in the blend that is degrading the most as the absorbance shoulder of the P3HT at $600 \mathrm{~nm}$ reduces more significantly (remain 74.1 $\%$ after 8 hours photoaging). Figure $6.15 \mathrm{~b}$ shows that the IDTBR is also stabilised when blended with P3HT, as the main absorbance peak at $743 \mathrm{~nm}$ from the IDTBR alone remains stable. Similarly, the photobleaching data indicates that the P3HT is less stable in the blend, as the absorbance at $500-600 \mathrm{~nm}$ which is mainly from P3HT degrades (87.6\% remains after 8 hours photoaging). Again, the blend with IDTBR undergoes less photobleaching than the blend with IDFBR. 

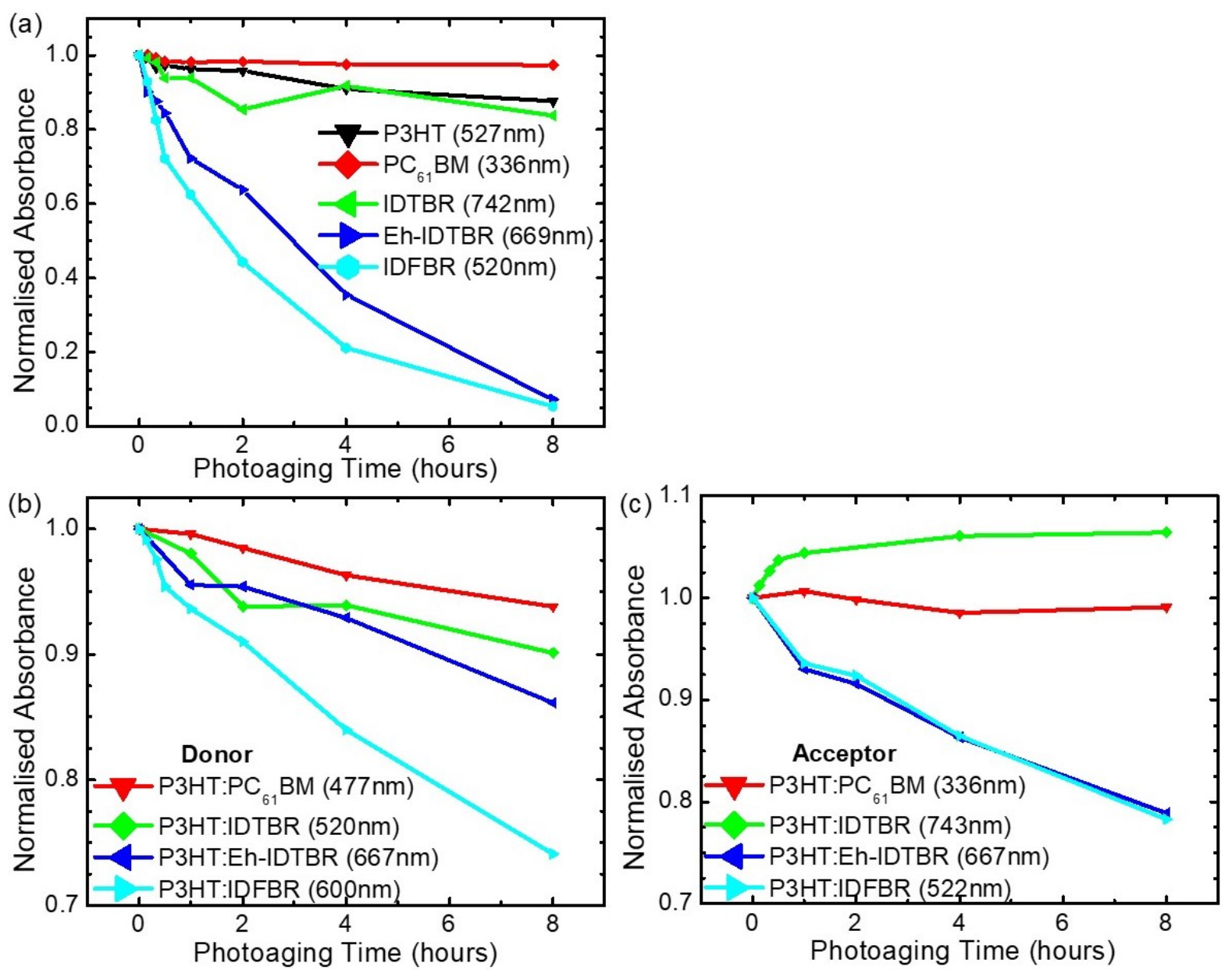

Figure 6.17: Normalised absorbance at specified wavelengths of annealed a) $\mathrm{PC}_{61} \mathrm{BM}$, P3HT and NFA films and b) P3HT:acceptor films (donor contribution) and c) P3HT:acceptor films (acceptor contribution) as a function of photoaging time under AM1.5G illumination and air.

To have more quantitative analysis on the photobleaching, the normalised absorbance at the specified wavelengths of the neat films and blend films are plotted as a function of the photoaging time in Figure 6.17a-c, summarising the photobleaching of these neat and blend films under dry air and AM1.5G illumination as a function of time. It can be seen that for neat films (Figure 6.17a), $\mathrm{PC}_{61} \mathrm{BM}$ is the most stable neat material, followed by P3HT and IDTBR which have similar rates of photobleaching, and then Eh-IDTBR and IDFBR which are both very unstable by comparison. For the blend films, the spectra are deconvoluted to resolve the absorbance from the donor (Figure 6.17b) and acceptors (Figure 6.17c), respectively. Upon comparing Figure 6.17a with Figure 6.17b and 6.17c, it is clear that the NFAs are stabilised once blended with the P3HT, while the P3HT either becomes less stable (with IDFBR) or remains similar (with O- and Eh-IDTBR). For $\mathrm{PC}_{61} \mathrm{BM}$, the same is true, the $\mathrm{P} 3 \mathrm{HT}$ is stabilised whilst the $\mathrm{PC}_{61} \mathrm{BM}$ remains the same. These measurements coincide well with the photobleaching of the neat films as both show IDTBR to be more stable than IDFBR. Comparing with Figure 6.6, it is clear that both neat and blend films undergo more photodegradation when in photoaged in the 
presence of oxygen.
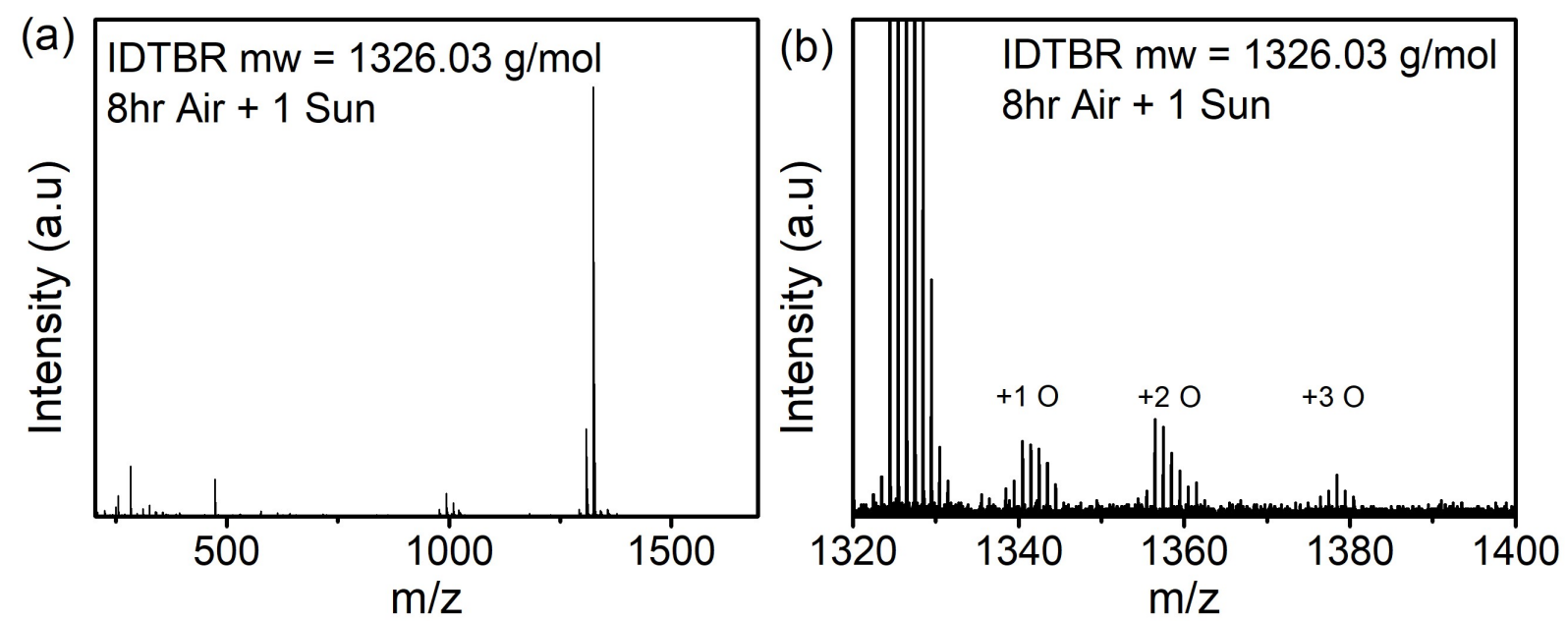

Figure 6.18: Negative ion mode MALDI-TOF measurements of: a) IDTBR after 8 hours of degradation and b) zoomed-in on oxidation.
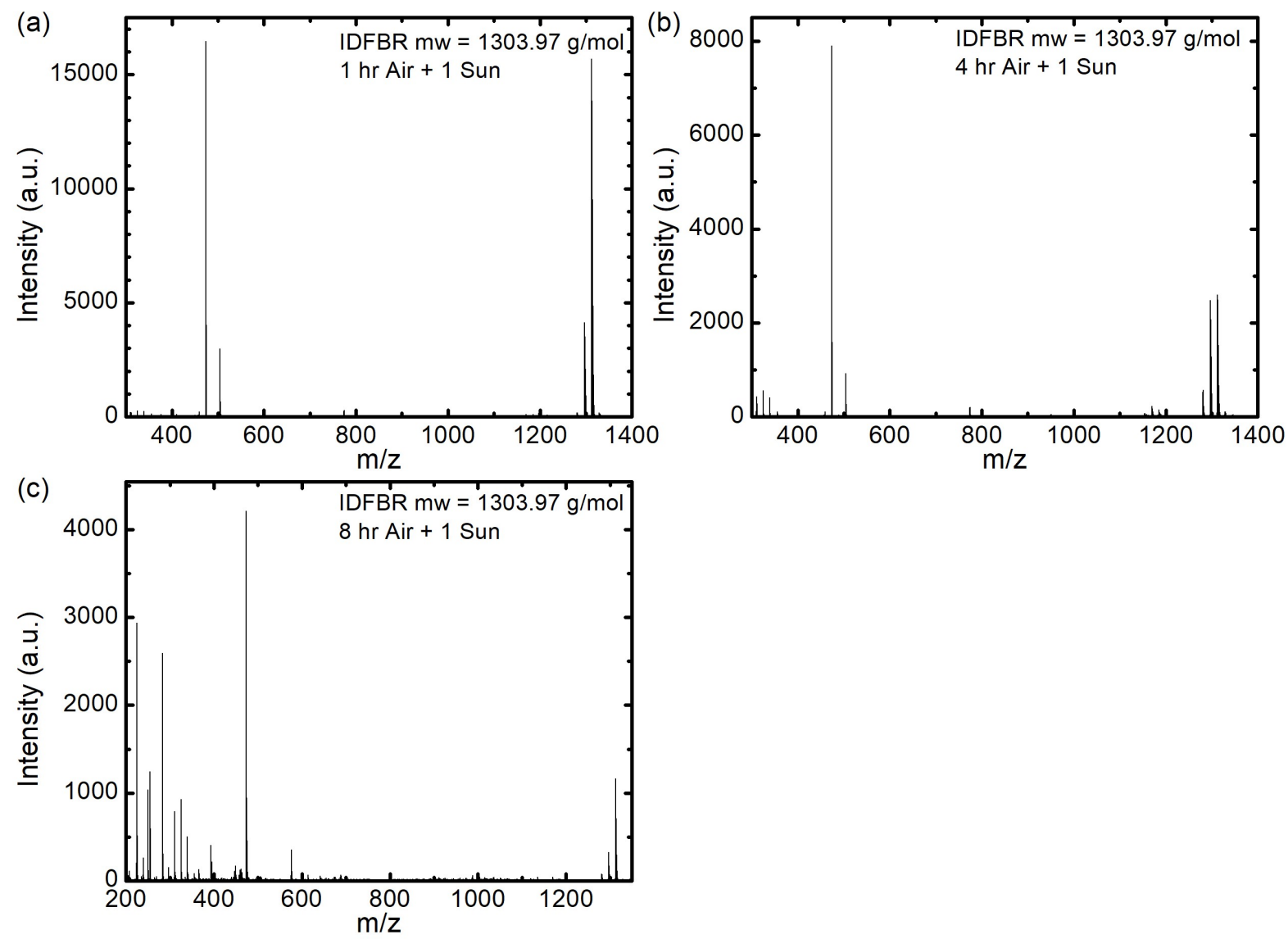

Figure 6.19: Negative ion mode MALDI-TOF measurements of IDFBR photoaged as a thin film under AM1.5G illumination and air for: a) 1 hour, b) 4 hours and c) 8 hours.

Next, mass spectroscopy measurements were performed on neat IDTBR and IDFBR powders which were scratched from neat films. The aim of these measurements was to probe if there is any change in mass of the molecules which can indicate oxidation, decomposition, etc. Figure 6.18 a shows the mass spectroscopy for IDTBR after 8 hours 
of aging under 1 sun AM1.5G illumination in air. The peaks at around $1326 \mathrm{~g} / \mathrm{mol}$ correlate well with the Mw of IDTBR. Interestingly, there are some signals at higher $\mathrm{m} / \mathrm{z}$ values (Figure $6.18 \mathrm{~b}$ ) which are equal to the Mw of IDTBR plus $\mathrm{m} / \mathrm{z}$ of 1,2 , or 3 oxygen atoms, indicating that there is photo-oxidation of the IDTBR with up to 3 oxygen atoms bound onto the IDTBR molecule after 8 hours of photoaging. Further analysis reveals that $4.0 \%$ of the IDTBR molecules had been photo-oxidised. For IDFBR, molecular fragmentation can be observed by the decrease in the peak corresponding with the fresh IDFBR peak at around $1304 \mathrm{~g} / \mathrm{ml}$ with increasing photoaging time (Figure 6.19). Both IDTBR and IDFBR degrade more under illumination in air than in nitrogen (Figure 6.7), with the IDFBR being very severe. These results correlate well with the photobleaching on neat films as both show that IDTBR is more stable than IDFBR when photoaged under AM1.5G illumination and air (Figure 6.12).

\subsubsection{Investigation into the photodegradation mechanism.}

Transient absorption spectroscopy was employed to identify transient species in neat and blend films after photoexcitation, in order to identify possible degradation mechanisms.

Figure 6.20 shows the photoinduced absorption bands of the neat IDFBR and IDTBR films, both with maxima at $\sim 1100 \mathrm{~nm}$. As seen in Figure 6.21a, transient decay kinetics of the neat IDFBR film, probed at the photoinduced maxima (1200 nm), exhibits a biexponential decay function under a nitrogen environment and a monoexponential decay function under oxygen. The exponential decay kinetic has an average lifetime of 0.33 $\mu \mathrm{s}$ under nitrogen $\left(\mathrm{t}_{1}=0.18 \mu \mathrm{s}\right.$ and $\left.\mathrm{t}_{2}=1.50 \mu \mathrm{s}\right)$ which accelerates by almost a factor of 2 to $0.16 \mu \mathrm{s}$ under oxygen. The exponential microsecond decay dynamics of these transient absorption signals, allow this signal to be assigned to triplet exciton $\mathrm{T}_{1} \rightarrow$ $\mathrm{T}_{\mathrm{n}}$ absorption, where the triplets were formed via direct intersystem crossing from the singlet excitons. $[14,15]$ The oxygen sensitivity of this transient absorption signal indicate oxygen quenching of these triplet states, suggesting these triplet excitons quenched by triplet ground state oxygen, forming singlet oxygen, causing more degradation, as seen previously for PS:PC ${ }_{61} \mathrm{BM}$ blends in Chapter 3. 

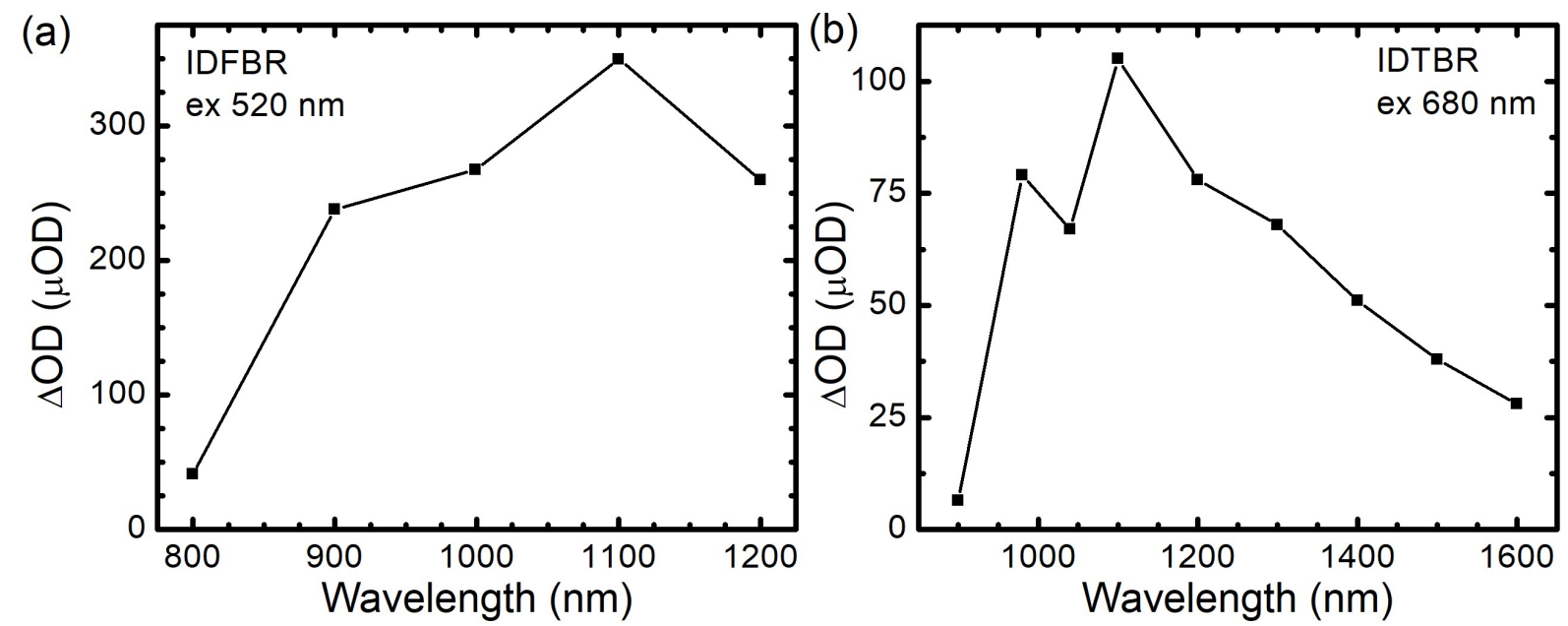

Figure 6.20: Transient absorption spectra under dry nitrogen for (a) IDFBR (ex $520 \mathrm{~nm}$ ) and (b) IDTBR (ex $680 \mathrm{~nm}$ ).
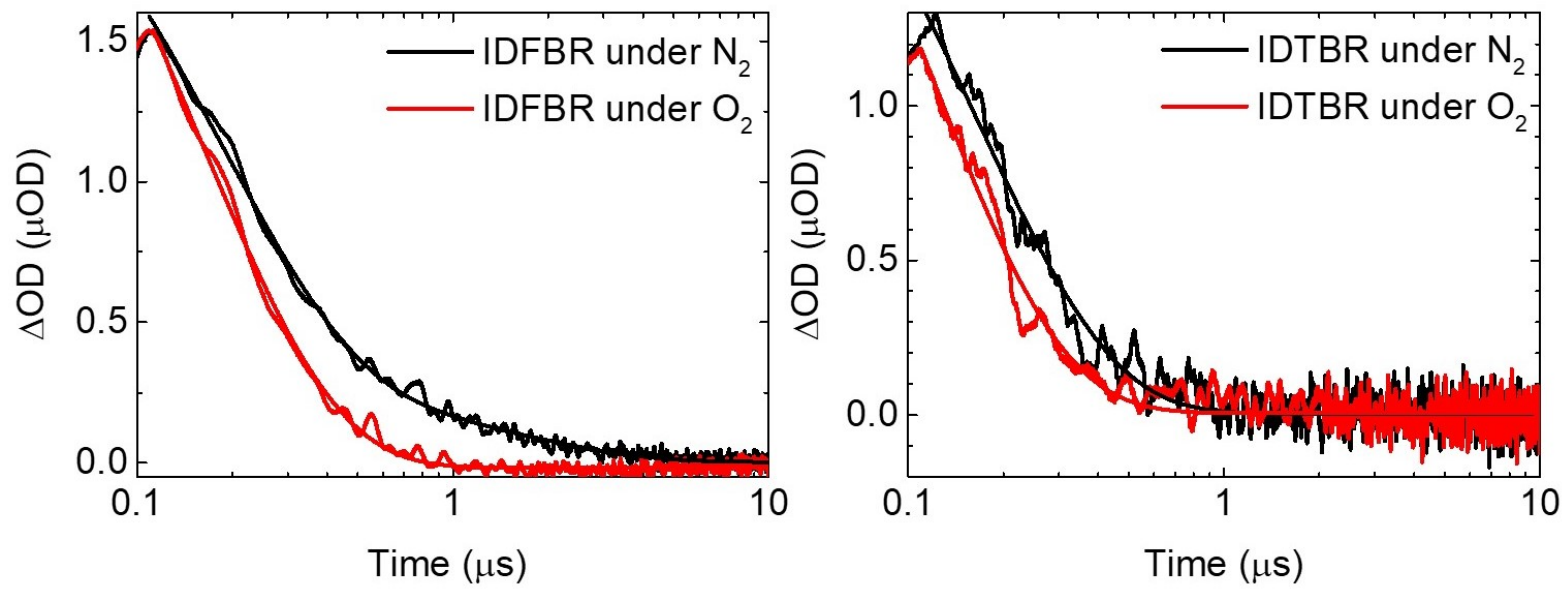

Figure 6.21: Transient decay kinetics under dry nitrogen and oxygen atmospheres for (left) IDFBR (ex. $520 \mathrm{~nm}$, probe $1200 \mathrm{~nm}$ ), (right) IDTBR (ex. $680 \mathrm{~nm}$, probe $1100 \mathrm{~nm}$ ). Values of $\triangle \mathrm{OD}$ have been corrected for the amount of photons absorbed.

Similarly, the photoinduced species from the neat IDTBR film decays mono-exponentially in both nitrogen and oxygen environment (Figure 6.21b). The lifetime of the species is again oxygen sensitive, shorten from $0.17 \mu$ s under nitrogen to $0.12 \mu$ s under oxygen. Again, this is consistent with triplet exciton $\mathrm{T}_{1} \rightarrow \mathrm{T}_{\mathrm{n}}$ absorption being quenched by molecular oxygen. These measurements show that the IDFBR triplets are longer lived than those of IDTBR.

Table 6.2: Properties of triplets and oxygen quenching for neat IDFBR and IDTBR films under nitrogen and oxygen atmospheres. $\tau_{\mathrm{N}_{2}}$ and $\tau_{\mathrm{O}_{2}}$ are the lifetimes under nitrogen and oxygen, $\mathrm{Y}_{\mathrm{N}_{2}}$ is the triplet yield, $\Phi_{\mathrm{O}_{2}}$ is the yield of oxygen quenching, $\mathrm{k}_{\mathrm{O}_{2}}$ is the rate constant and $\Phi_{\mathrm{Q}}$ is the relative fraction of excited states quenched.

\begin{tabular}{||c|c|c|c|c|c|c||}
\hline \hline & $\tau_{\mathrm{N}_{2}}(\mu \mathrm{s})$ & $\tau_{\mathrm{O}_{2}}(\mu \mathrm{s})$ & $\mathrm{Y}_{\mathrm{N}_{2}}$ & $\Phi_{\mathrm{O}_{2}}$ & $\mathrm{k}_{\mathrm{O}_{2}}\left(\mu \mathrm{s}^{-1}\right)$ & $\Phi_{\mathrm{Q}}(\%)$ \\
\hline IDFBR & 0.33 & 0.16 & 1.53 & 0.511 & 3.17 & 78.3 \\
IDTBR & 0.17 & 0.12 & 1.30 & 0.310 & 2.71 & 40.2 \\
\hline \hline
\end{tabular}


The triplet yields were determined from the initial $\mathrm{T}_{1} \rightarrow \mathrm{T}_{\mathrm{n}}$ absorption amplitude under nitrogen and corrected for the relative fraction of light absorbed by the individual film at the excitation wavelength. These show that a greater number of triplets are formed in the IDFBR neat film compared to the IDTBR neat film (Table 6.2: $\mathrm{Y}_{\mathrm{N}_{2}}$ ). Moreover, the rate constant of oxygen quenching of triplets $\left(\mathrm{k}_{\mathrm{O}_{2}}\right)$, yield of oxygen quenching of triplets $\left(\Phi_{\mathrm{O}_{2}}\right)$, and the relative fraction of excited states quenched $\left(\Phi_{\mathrm{Q}}\right)$ can be calculated using the triplet yields and lifetimes $\left(\tau_{\mathrm{N}_{2}}=\right.$ lifetime under nitrogen and $\tau_{\mathrm{O}_{2}}=$ lifetime under oxygen), as previously explained in Chapter 2, and are also listed in Table 6.2. $\Phi_{\mathrm{Q}}$ is $\sim$ doubled upon comparision of IDFBR (78.3\%) with IDTBR (40.2\%), due to its relatively greater triplet yield and rate constant. The increased $\mathrm{k}_{\mathrm{O}_{2}}$ for IDFBR can be explained by the amorphous nature of the film, allowing for a faster oxygen ingress.[11]
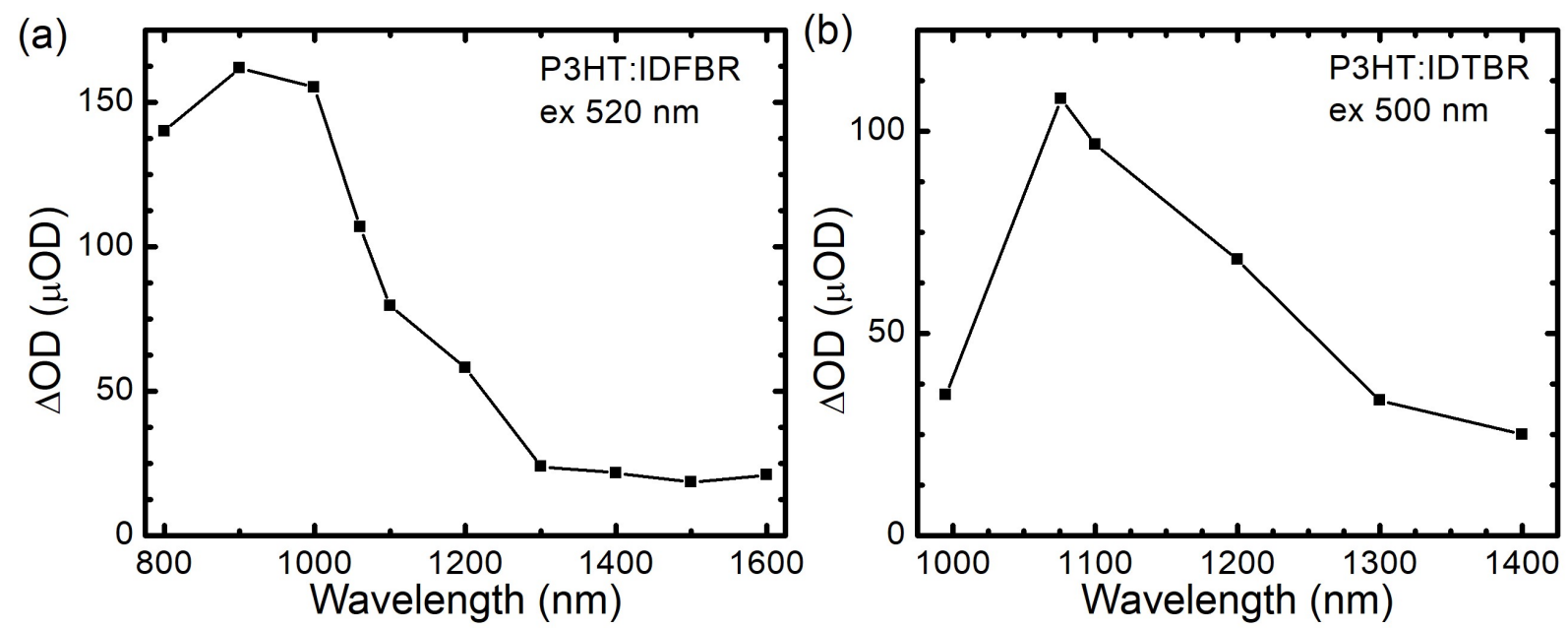

Figure 6.22: Transient absorption spectra under nitrogen for (a) P3HT:IDFBR (ex 520 $\mathrm{nm})$ and (b) P3HT:IDTBR (ex $500 \mathrm{~nm}$ ).
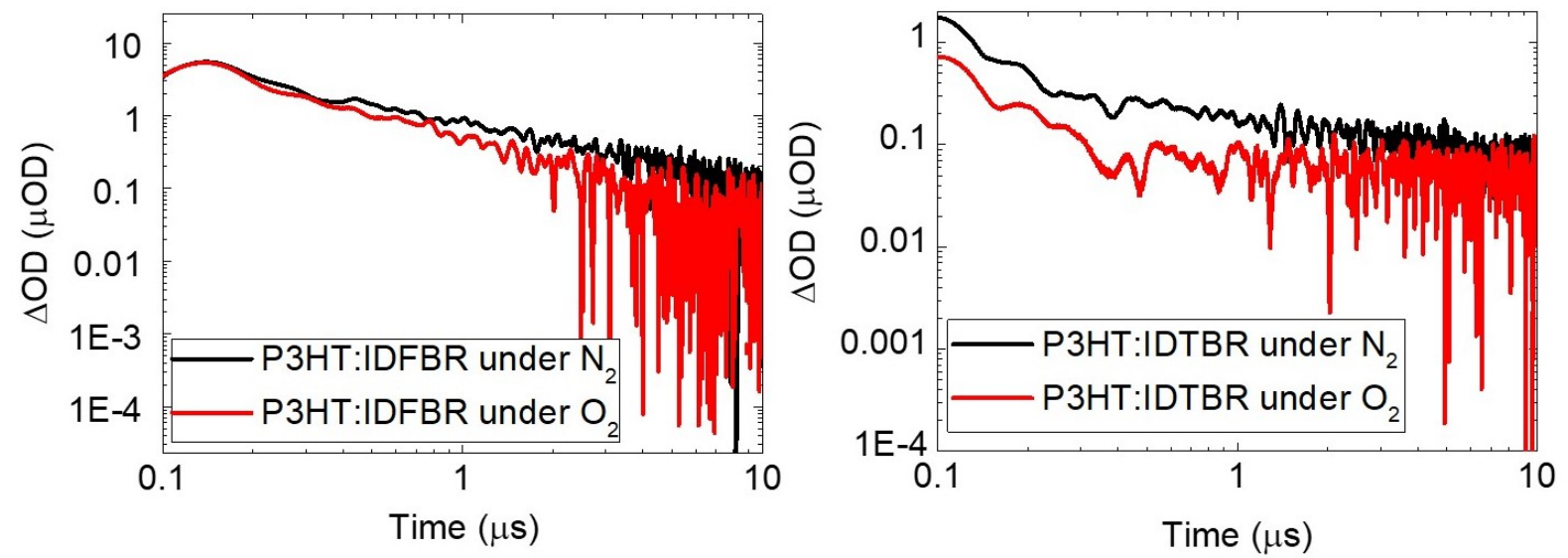

Figure 6.23: Transient decay kinetics under nitrogen and oxygen atmospheres for (a) P3HT:IDFBR (ex $520 \mathrm{~nm}$, probe $1000 \mathrm{~nm}$ ), (b) P3HT:IDTBR (ex $500 \mathrm{~nm}$, probe 1060 $\mathrm{nm}$ ). 

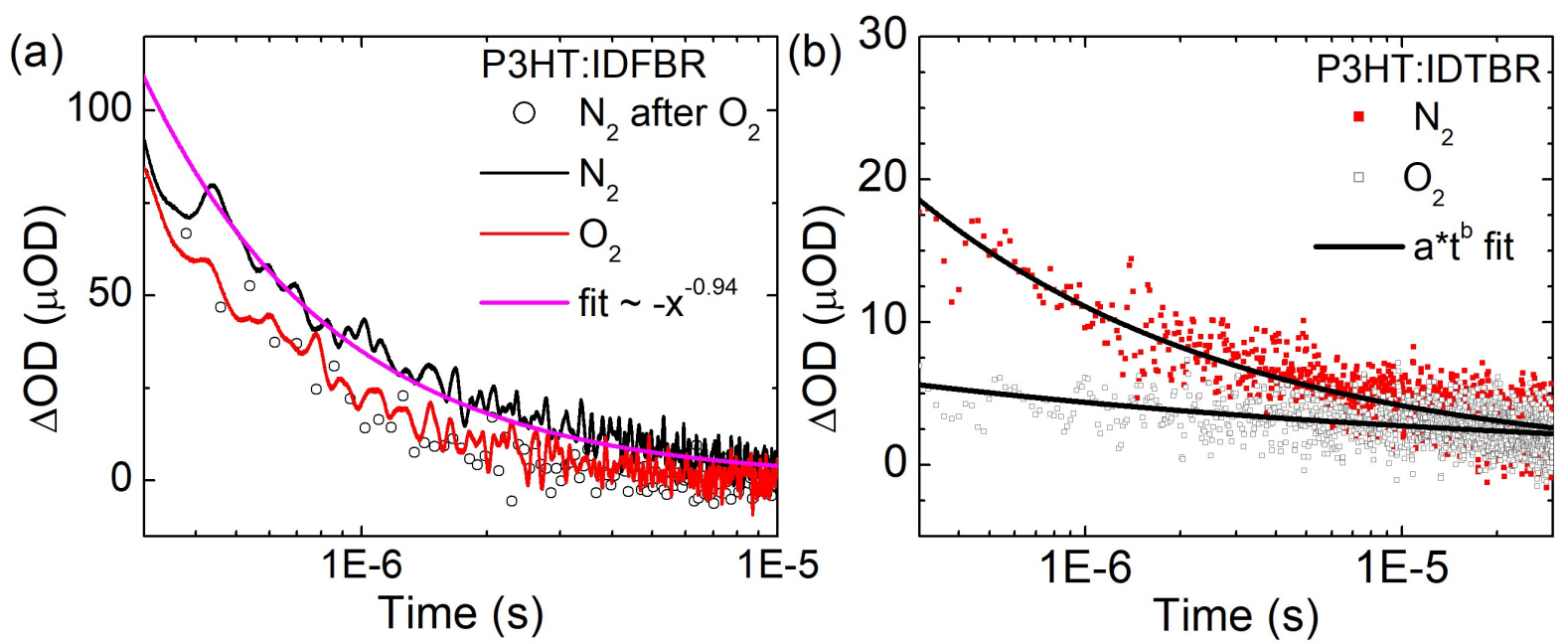

Figure 6.24: Fits of transient decay kinetics under nitrogen and oxygen atmospheres for (a) P3HT:IDFBR (ex $520 \mathrm{~nm}$, probe $1000 \mathrm{~nm}$ ), (b) P3HT:IDTBR (500 nm, probe 1060 $\mathrm{nm})$.

Transient absorption spectroscopy measurements were also taken of blend films. Figure 6.22 shows the transient absorption spectra of P3HT:IDFBR and P3HT: IDTBR blend films, exhibiting maxima at $900 \mathrm{~nm}$ and $1060 \mathrm{~nm}$ respectively. The transient decay kinetics of the photoinduced states in these blend films under dry nitrogen and dry oxygen are shown in Figure 6.23, exhibiting a different decay behaviour to the neat films. The P3HT:IDFBR blend film did not exhibit any exponential decay of the TAS signal which would be indicative of $\mathrm{T}_{1} \rightarrow \mathrm{T}_{\mathrm{n}}$ triplet exciton absorption, within the time resolution of our transient spectrometer $(\sim 100 \mathrm{~ns})$. Instead, it shows a slower decay which can be fitted with a power law decay function $\Delta \mathrm{OD}=\mathrm{At}^{-\alpha}$ (where $\mathrm{A}$ and $\alpha$ are both constants), which is seen as a linear fit on a log-log scale (Figure 6.24a).[14, 16-18] This is characteristic of non-germinate recombination of dissociated charges. This power law decay was observed to be sensitive to oxygen suggesting the dissociated polarons are reacting with molecular oxygen to form radical superoxide anions (Figure 6.23a).

The transient decay kinetics of the P3HT:IDTBR blend film, probed at $1060 \mathrm{~nm}$, decays biphasically under both nitrogen and oxygen (Figure 6.23b). There is a faster decay $(<5$ $\mu \mathrm{s})$ with an exponential lifetime which is oxygen sensitive, consistent with the quenching of triplet states. Following this, there is a slower power law decay phase ( $>5 \mu \mathrm{s})$, indicative of non-germinate recombination of dissociated charges (Figure 6.24b). The oxygen sensitivity of this phase suggests that dissociated polarons react molecular oxygen to form radical superoxide anions. The polaron involved here is likely the charge separated electron from the acceptor as this could react with molecular oxygen to form the negatively charged superoxide, whereas a hole could not.

The transient absorption spectra of regioregular $\mathrm{P} 3 \mathrm{HT}: \mathrm{PC}_{61} \mathrm{BM}$ has previously been measured from 0.5 to $100 \mu$ s after the laser excitation at $400 \mathrm{~nm}$ with a fluence of 30 
$\mu \mathrm{Jcm}^{-2}$. Two domains were observed at 700 and $1000 \mathrm{~nm}$ ascribed to delocalised polarons in crystalline P3HT domains and localised polarons trapped in relatively disordered P3HT domains respectivley, which were not sensitive to oxygen.[19] The lack of oxygen quenching of polarons in the $\mathrm{P} 3 \mathrm{HT}: \mathrm{PC}_{61} \mathrm{BM}$ blend film correlates with well with the low photobleaching observed in the previous section compared to the P3HT:NFA blends.

The TAS data suggests that triplets are quenched by oxygen in the neat NFA films (likely forming singlet oxygen), while polarons are quenched by oxygen in the P3HT:NFA films (likely forming superoxide). This implies that in blend films, molecular oxygen is being reduced by the polarons, forming superoxide anions. To further support the formation of superoxide, sensitisation studies of a fluorescent molecular probe (hydroethidine) were performed at Imperial College London (recorded using an excitation wavelength of $520 \mathrm{~nm}$ and a $10 \mathrm{~mm}$ slit width on a Horiba Jobin-Yvon Fluorolog-3 spectrofluorometer. Illumination was provided by a tungsten halogen lamp with $\sim 1.5 \mathrm{mWcm}^{-2}$ intensity). Hydroethidine was employed as the molecular probe due to its selective reactivity with superoxide anions to form ethidium, a fluorescent compound $\left(\lambda_{\text {excitation }}=520 \mathrm{~nm}\right.$ and $\lambda_{\text {emission }}=610 \mathrm{~nm}$ ), and insensitive to singlet oxygen, hydroxyl radicals, $\mathrm{H}_{2} \mathrm{O}_{2}$ and nitrogen radicals. [20-23] When this molecular probe reacts with superoxide, it reacts to form a fluorescent molecule. The fluorescence of this molecule in the probe solution is measured as a function of illumination time and is a direct measure of the amount superoxide anions generated by each thin film sample (for more detail refer to Chapter 2).

(a)

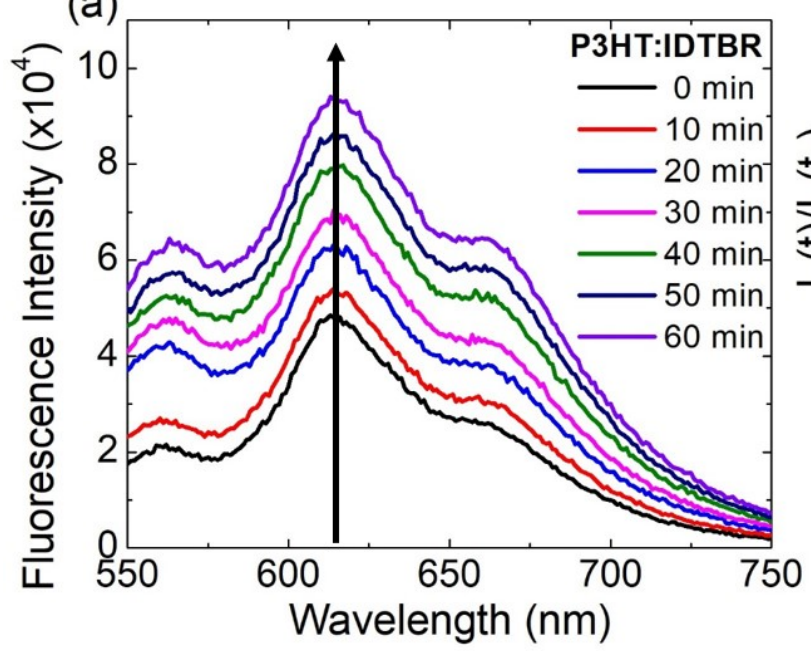

(b)

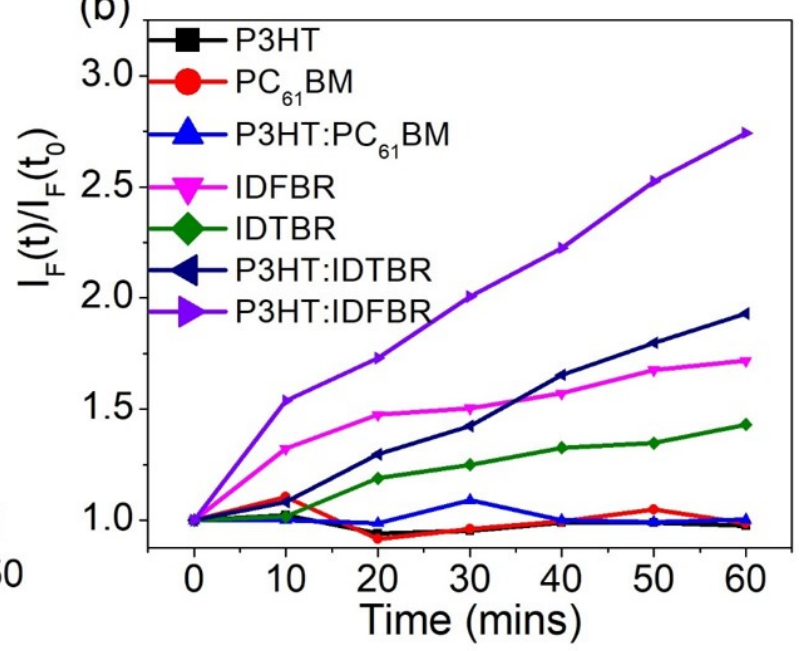

Figure 6.25: (a) fluorescence spectra for P3HT:IDFBR as a function of illumination time (b) normalised fluorescence intensity increase of the HE probe at $610 \mathrm{~nm}$ (excitation at $520 \mathrm{~nm}) . \mathrm{I}_{\mathrm{F}}(\mathrm{t})$ is the fluorescence maximum at time $\mathrm{t}$, while $\mathrm{I}_{\mathrm{F}}(\mathrm{t} 0)$ is the background fluorescence intensity. $\mathrm{I}_{\mathrm{F}}(\mathrm{t}) / \mathrm{I}_{\mathrm{F}}\left(\mathrm{t}_{0}\right)$ ratio corresponds to the yield of superoxide generation for the thin film samples.

Figure 6.25a, as an example, shows the fluorescence spectra for P3HT:IDTBR as a function of illumination time. The increase in the fluorescence signal with longer photo- 
aging time is consistent with more superoxide formation at longer times which increases the fluorescence of the molecular probe. Figure $6.25 \mathrm{~b}$ shows the results obtained from photoluminescence spectra of the films tested in a solution of hydroethidine probe in air as a function of photoaging time. As shown in this figure, the P3HT:IDFBR film produces by far the highest amount of superoxides, 1.6 times more than the neat IDFBR film $(2.74$ and 1.72 respectively). The P3HT:IDTBR film generates 1.4 times more superoxides than the neat IDTBR film (1.93 and 1.43 respectively). These results correlate well with the TAS data which show that the blend films have a greater yield of polarons which can react with oxygens to form the superoxides. Since there was no observable TAS signal from polaron states in the neat NFA film, it suggests that there is another patheway to form superoxide, possibly by a reaction between radicals formed as a product of triplet-mediated photo-oxidation of the NFAs, but this is less efficient than via the polaron states as in the blend films. In contrast, $\mathrm{P} 3 \mathrm{HT}, \mathrm{PC}_{61} \mathrm{BM}$ and $\mathrm{P} 3 \mathrm{HT}: \mathrm{PC}_{61} \mathrm{BM}$ films did not enhance the fluorescence of the molecules, implying no significant formation of superoxides. This is consistent with the polarons formed in the $\mathrm{P} 3 \mathrm{HT}: \mathrm{PC}_{61} \mathrm{BM}$ not being quenched by oxygen, as previously observed.[19]

These results could explain the relative instability of the P3HT:IDFBR and P3HT:IDTBR devices compared to the P3HT:PC ${ }_{61} \mathrm{BM}$ device (Figure 6.11), where the active layers were degraded prior to electrode deposition. As reactive superoxides were observed for the P3HT:NFA films, but not the P3HT:PC ${ }_{61}$ BM films. P3HT:IDFBR and P3HT:IDTBR both are shown to produce superoxide anions, with the P3HT:IDFBR producing more. The P3HT: IDTBR blend film had a biphasic transient decay kinetic, with the first phase being assigned to the absorption of triplet excitons. It can be speculated that these triplet excitons could be quenched to form singlet oxygen which could provide the extra instability observed in the P3HT:IDTBR devices, making them less stable than the P3HT:IDFBR devices.

\subsubsection{Correlation between acceptor energetics and photodegrad- ation}

It is more difficult to form superoxides in the P3HT:PC ${ }_{61} \mathrm{BM}$ film than the P3HT:NFA, likely because $\mathrm{PC}_{61} \mathrm{BM}$ has a LUMO lower than the electron affinity of molecular oxygen $(3.75 \mathrm{eV})$, so it is energetically unfavourable to form superoxides. As shown in the previous chapter, the LUMO level of fullerene neat films correlates well with their photostability in the presence of oxygen (higher lying LUMO, less stable). Therefore, it will be interesting to study if there is any correlation between the LUMO level of the acceptors to the photo-degradation (photobleaching) of the donor (P3HT) which can be destabilised by 
the acceptors as shown in previous section, in addition to the photodegradation of the acceptors.
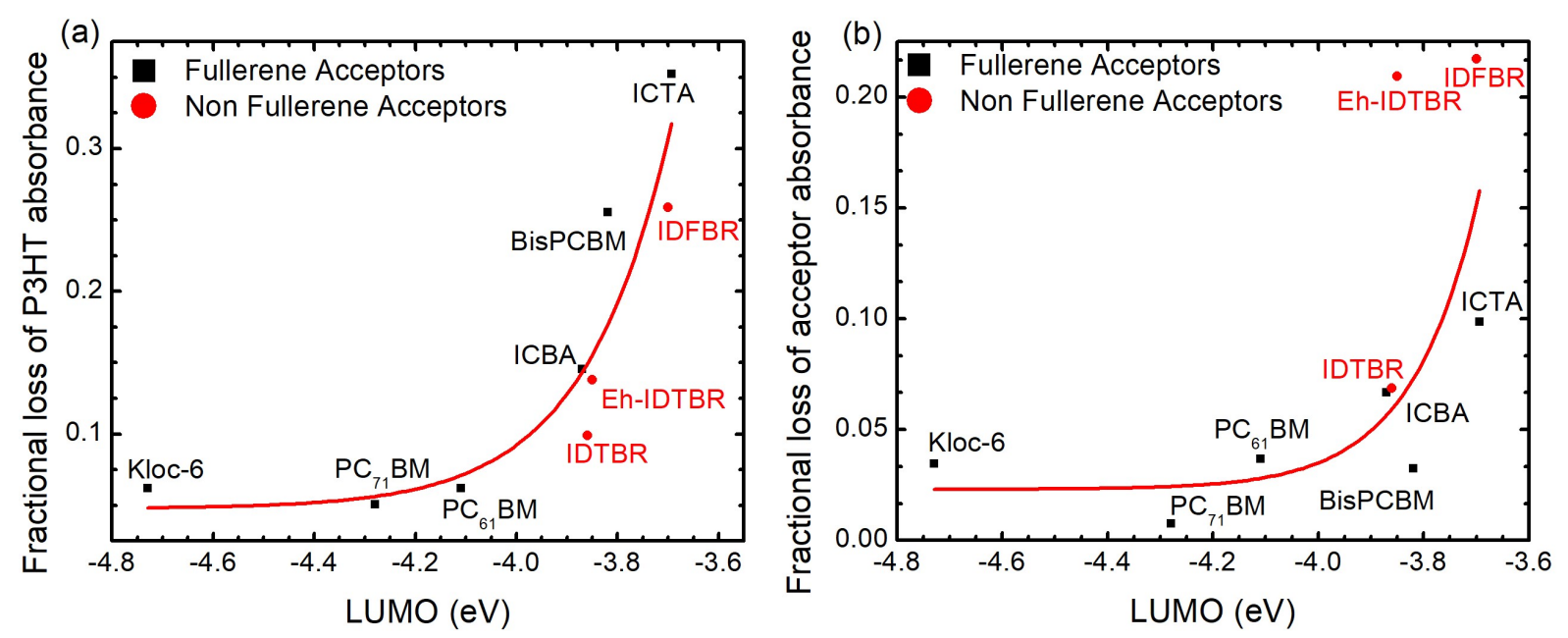

Figure 6.26: Fractional loss of (a) the P3HT and (b) acceptor peaks in P3HT blends with fullerene and non-fullerene acceptors after degrading under 1 Sun irradiation in air for 8 hours as a function of acceptor LUMO level. (At donor (D) and acceptor (A) wavelengths: Kloc-6 $\mathrm{D}=479 \mathrm{~nm} \mathrm{~A}=334 \mathrm{~nm}, \mathrm{PC}_{71} \mathrm{BM} \mathrm{D}=470 \mathrm{~nm} \mathrm{~A}=241 \mathrm{~nm}, \mathrm{PC}_{61} \mathrm{BM}$ $\mathrm{D}=477 \mathrm{~nm} \mathrm{~A}=336 \mathrm{~nm}$, BisPCBM D=487nm A=260nm, ICBA D=468nm A=250nm, ICTA $\mathrm{D}=453 \mathrm{~nm} \mathrm{~A}=246 \mathrm{~nm}$, IDTBR $\mathrm{D}=556 \mathrm{~nm} \mathrm{~A}=733 \mathrm{~nm}$, Eh-IDTBR $\mathrm{D}=560 \mathrm{~nm} \mathrm{~A}=683$, IDFBR $D=600 \mathrm{~nm} A=522 \mathrm{~nm})$. Both plots are fitted with an exponential growth function y $=\mathrm{y}_{0}+\mathrm{Ae}^{((\mathrm{x}-\mathrm{x} 0) / \mathrm{t})}$.

Figure 6.26a shows the photobleaching of the P3HT peak in the blend films after 8 hours of photoaging as a function of the LUMO level of the neat acceptors as measured with cyclic voltammetry (Figure D.9). It shows that the photobleaching of the P3HT in the blend films correlates well with the LUMO of both the fullerenes and NFAs, whereby the shallower the LUMO of the acceptors, the more photobleaching there is of the P3HT in the blend film. As discussed in the previous chapter, Kloc-6 is more unstable than $\mathrm{PC}_{61} \mathrm{BM}$ despite having a lower lying LUMO level. Figure $6.26 \mathrm{~b}$ shows there is an analogous relationship between the LUMO of the acceptor with the photobleaching it undergoes in a blend film with P3HT, where the acceptors with shallower LUMOs generally undergoing more photobleaching in the blend films. IDFBR and Eh-IDTBR degrade $\sim$ twice as much as the other electron acceptors, implying that there are more contributing factors to the photostability of these materials in air which could include their amorphous morphology, oxygen diffusion or molecular conformation. Both plots could be fitted with The data can be fitted with an exponential growth function due to the Boltzmann dependence of the electron transfer to the molecular oxygen ground state being dependent on the LUMO energy of the acceptor. 


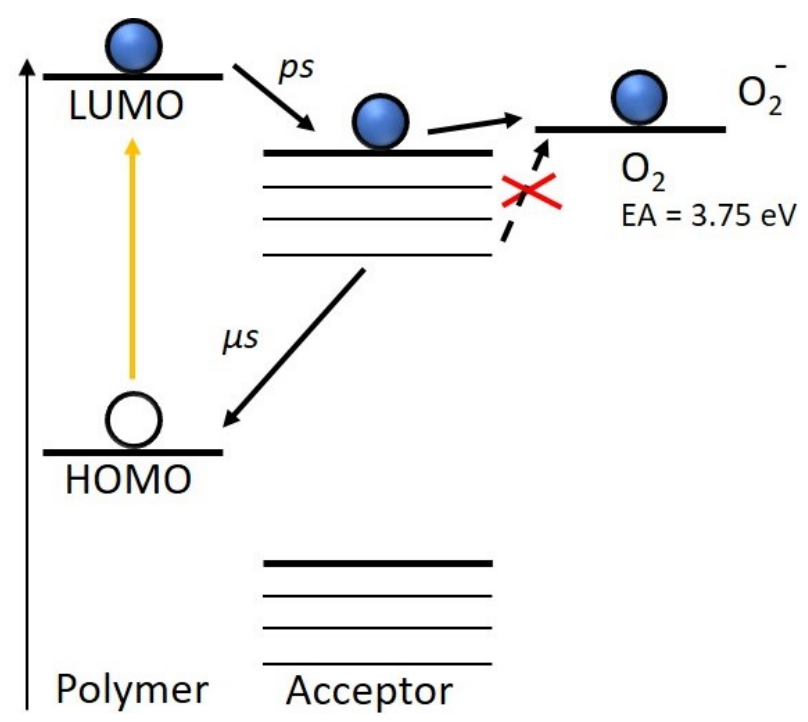

Figure 6.27: Proposed degradation mechanism: (1) electrons excited to the LUMO level of the photo-excited donor can be (2) transferred to the LUMO of the acceptor. Depending on the LUMO level of the acceptor relative to the electron affinity of molecular oxygen $(\mathrm{EA}=3.75 \mathrm{eV}[24])$, (3a) electron transfer to molecular oxygen can occur, reducing oxygen to the radical superoxide anion. The reactive superoxides can then react with the donor (here, P3HT) or acceptor causing the enhanced photobleaching. This process competes with (3b) non-germinate recombination to the HOMO of the donor (and charge extraction in full devices).

The correlation between the photobleaching of the P3HT in the blend films and the LUMO levels of the acceptors also match excellently with the TAS data and fluorescence data (Figure 6.25) which shows that more superoxides are formed in the blend films with acceptors which have shallower LUMO levels. Based on these findings, here, it is proposed that the photo-degradation of the blend films in air could be: as shown in Figure 6.27, after photo-excitation of the donor, electrons are transferred from the LUMO of the donor to the LUMO of the acceptor. If the LUMO level of the acceptor is shallow enough, electron transfer to molecular oxygen will be energetically favourable and superoxide anions are formed. The superoxides are highly reactive and can then attack the donor (P3HT here) or acceptor materials in the blend film, leading to the stronger photobleaching of P3HT or acceptor in the blend films with shallower LUMOs (e.g. P3HT:IDFBR). For electron acceptors with a deeper LUMO level, where it will be less energetically favourable for electron transfer from the acceptor LUMO to molecular oxygen, superoxides are less likely to form, hence, less photobleaching of the donor and acceptors (e.g. P3HT:PC ${ }_{61} \mathrm{BM}$ ) in the blend films will occur. Alternatively, electrons at the LUMO level of the acceptor can also originate from direct photo-excitation of the acceptor which is more likely in the case of the NFAs due to their typically stronger optical absorption. Hoke et al showed that the level of degradation undergone by a polymer in a blend film can be determined by the LUMO level of the fullerene electron acceptor with which it was blended.[24] Moreover, no experimental proof was provided for the generation of superoxide anions. Here, more direct evidence from the TAS and fluorescence data is demonstrated. Furthermore, here, 
it is shown that this correlation can be extended to NFAs.

It should also be noted that currently the mostly widely used electron donors of fullerene-free OSCs are still those originally optimised for fullerenes (e.g. P3HT, PCE10, PCE11 and PCE12), with most high performance NFAs developed to date optimised to match these (or energetically similar) donor polymers.[11, 6, 25, 26] One optimisation strategy has been designing NFAs with a raised LUMO level in order to achieve a higher $\mathrm{V}_{\text {oc }}$ (hence power conversion efficiency) by creating a higher donor HOMO/acceptor LUMO offset. Herein, it has been demonstrated that such strategy may lead to a significant decrease in the photochemical stability of the resulting OSCs by significantly increasing the yields of superoxide formation. These results highlight the complexity in the material design to simultaneously achieve superior efficiency and stability of OSCs. A redesign of the NFAs with deeper LUMO energy levels, as well as their matching donor polymers with deepened HOMO levels to compensate the loss in $\mathrm{V}_{\mathrm{oc}}$, might be a promising route toward the development of both efficient and environmentally stable fullerene-free OSCs.

\subsection{Conclusion}

This chapter focuses on the photostability of NFAs. Firstly, it was found that IDTBR and Eh-IDTBR based devices exhibited superior burn-in free behaviour under light-only exposure compared to $\mathrm{PC}_{71} \mathrm{BM}$ based devices that undergo a strong burn-in and (M-) ITIC based devices undergoes a continuous degradation without stabilising. Then a range of optical and chemical measurements were utilised to investigate the photo-degradation (in air) of benchmark fullerene-based and NFA-based OSCs. A critical correlation between the electron acceptor LUMO level and the photochemical stability of the blend film when exposed to light and air was established. More specifically, lower-lying the acceptor LUMO level, the more stable the blend film. This correlation can be explained by a reduction in superoxide formation which is mediated via the acceptor LUMO level. These results highlight the need of redesigning electron acceptor materials with deepened LUMO energy levels, as well as their donor polymers with deepened HOMO levels to overcome the challenge of simultaneously improving device efficiency and stability, thereby paving the way toward simultaneously achieving the high efficiency and long term environmental stability of OSCs. 


\section{REFERENCES}

[1] N. Grossiord, J. M. Kroon, R. Andriessen, and P. W. Blom, "Degradation mechanisms in organic photovoltaic devices," Organic Electronics, vol. 13, pp. 432-456, 2012.

[2] S. Forget and S. Chenais, Organic solid-state lasers. Springer, 2013.

[3] A. Burrows, J. S. J. S. Holman, A. F. Parsons, G. Pilling, and G. J. Price, Chemistry ${ }^{3}$ : introducing inorganic, organic, and physical chemistry. Oxford University Press, 2017.

[4] Y. Lei, B. Suo, A. Li, Y. Dou, Y. Wang, and Z. Wen, "Involvement of excited triplet state in the photodissociation of cyclobutane," International Journal of Quantum Chemistry, vol. 108, pp. 788-796, 2007.

[5] E. Yousif and R. Haddad, "Photodegradation and photostabilization of polymers, especially polystyrene: review.," SpringerPlus, vol. 2, p. 398, 2013.

[6] H. Cha, J. Wu, A. Wadsworth, J. Nagitta, S. Limbu, S. Pont, Z. Li, J. Searle, M. F. Wyatt, D. Baran, J.-S. Kim, I. McCulloch, and J. R. Durrant, "An Efficient, "Burn in" Free Organic Solar Cell Employing a Nonfullerene Electron Acceptor," Advanced Materials, vol. 29, p. 1701156, 2017.

[7] N. Li, J. D. Perea, T. Kassar, M. Richter, T. Heumueller, G. J. Matt, Y. Hou, N. S. Güldal, H. Chen, S. Chen, S. Langner, M. Berlinghof, T. Unruh, and C. J. Brabec, "Abnormal strong burn-in degradation of highly efficient polymer solar cells caused by spinodal donor-acceptor demixing," Nature Communications, vol. 8, p. 14541, 2017.

[8] T. Heumueller, W. R. Mateker, A. Distler, U. F. Fritze, R. Cheacharoen, W. H. Nguyen, M. Biele, M. Salvador, M. von Delius, H.-J. Egelhaaf, M. D. McGehee, and C. J. Brabec, "Morphological and electrical control of fullerene dimerization determines organic photovoltaic stability," Energy Environ. Sci., vol. 9, pp. 247-256, 2016.

[9] M. R. Lilliedal, A. J. Medford, M. V. Madsen, K. Norrman, and F. C. Krebs, "The effect of post-processing treatments on inflection points in current-voltage curves of roll-to-roll processed polymer photovoltaics," Solar Energy Materials and Solar Cells, vol. 94, pp. 2018-2031, 2010.

[10] S. Trost, K. Zilberberg, A. Behrendt, A. Polywka, P. Görrn, P. Reckers, J. Maibach, T. Mayer, and T. Riedl, "Overcoming the "Light-Soaking" Issue in Inverted Organic 
Solar Cells by the Use of Al:ZnO Electron Extraction Layers," Advanced Energy Materials, vol. 3, pp. 1437-1444, 2013.

[11] D. Baran, R. S. Ashraf, D. A. Hanifi, M. Abdelsamie, N. Gasparini, J. A. Röhr, S. Holliday, A. Wadsworth, S. Lockett, M. Neophytou, C. J. M. Emmott, J. Nelson, C. J. Brabec, A. Amassian, A. Salleo, T. Kirchartz, J. R. Durrant, and I. McCulloch, "Reducing the efficiency-stability-cost gap of organic photovoltaics with highly efficient and stable small molecule acceptor ternary solar cells," Nature Materials, vol. 16, pp. 363-369, 2017.

[12] S. Shoaee and J. R. Durrant, "Oxygen diffusion dynamics in organic semiconductor films," J. Mater. Chem. C, vol. 3, no. 3, pp. 10079-10084, 2015.

[13] H. Hintz, H.-J. Egelhaaf, H. Peisert, and T. Chassé, "Photo-oxidation and ozonization of poly(3-hexylthiophene) thin films as studied by UV/VIS and photoelectron spectroscopy," Polymer Degradation and Stability, vol. 95, pp. 818-825, 2010.

[14] H. Ohkita, S. Cook, Y. Astuti, W. Duffy, S. Tierney, W. Zhang, M. Heeney, I. McCulloch, J. Nelson, D. D. C. Bradley, and J. R. Durrant, "Charge carrier formation in polythiophene/fullerene blend films studied by transient absorption spectroscopy.," Journal of the American Chemical Society, vol. 130, pp. 3030-42, 2008.

[15] F. Etzold, I. A. Howard, N. Forler, D. M. Cho, M. Meister, H. Mangold, J. Shu, M. R. Hansen, K. Müllen, and F. Laquai, "The effect of solvent additives on morphology and excited-state dynamics in PCPDTBT:PCBM photovoltaic blends.," Journal of the American Chemical Society, vol. 134, pp. 10569-83, 2012.

[16] T. Kirchartz, B. E. Pieters, J. Kirkpatrick, U. Rau, and J. Nelson, "Recombination via tail states in polythiophene:fullerene solar cells," Physical Review B, vol. 83, p. 115209, 2011.

[17] A. F. Nogueira, I. Montanari, J. Nelson, J. R. Durrant, C. Winder, N. S. Sariciftci, and C. Brabec, "Charge Recombination in Conjugated Polymer/Fullerene Blended Films Studied by Transient Absorption Spectroscopy," The Journal of Physical Chemistry B, vol. 107, pp. 1567-1573, 2003.

[18] C. G. Shuttle, B. O’Regan, A. M. Ballantyne, J. Nelson, D. D. C. Bradley, and J. R. Durrant, "Bimolecular recombination losses in polythiophene: Fullerene solar cells," Physical Review B, vol. 78, p. 113201, 2008.

[19] J. Guo, H. Ohkita, S. Yokoya, H. Benten, and S. Ito, "Bimodal Polarons and Hole Transport in Poly(3-hexylthiophene):Fullerene Blend Films," Journal of the American Chemistry Society, vol. 132, pp. 9631-9637, 2010. 
[20] A. Gomes, E. Fernandes, and J. L. Lima, "Fluorescence probes used for detection of reactive oxygen species," Journal of Biochemical and Biophysical Methods, vol. 65, pp. 45-80, 2005.

[21] N. Aristidou, I. Sanchez-Molina, T. Chotchuangchutchaval, M. Brown, L. Martinez, T. Rath, and S. A. Haque, "The Role of Oxygen in the Degradation of Methylammonium Lead Trihalide Perovskite Photoactive Layers," Angewandte Chemie International Edition, vol. 54, pp. 8208-8212, 2015.

[22] D. Bryant, N. Aristidou, S. Pont, I. Sanchez-Molina, T. Chotchunangatchaval, S. Wheeler, J. R. Durrant, and S. A. Haque, "Light and oxygen induced degradation limits the operational stability of methylammonium lead triiodide perovskite solar cells," Energy \& Environmental Science, vol. 9, pp. 1655-1660, 2016.

[23] N. Aristidou, C. Eames, I. Sanchez-Molina, X. Bu, J. Kosco, M. S. Islam, and S. A. Haque, "Fast oxygen diffusion and iodide defects mediate oxygen-induced degradation of perovskite solar cells," Nature Communications, vol. 8, p. 15218, 2017.

[24] E. T. Hoke, I. T. Sachs-Quintana, M. T. Lloyd, I. Kauvar, W. R. Mateker, A. M. Nardes, C. H. Peters, N. Kopidakis, and M. D. McGehee, "The Role of Electron Affinity in Determining Whether Fullerenes Catalyze or Inhibit Photooxidation of Polymers for Solar Cells," Advanced Energy Materials, vol. 2, pp. 1351-1357, 2012.

[25] D. Baran, T. Kirchartz, S. Wheeler, S. Dimitrov, M. Abdelsamie, J. Gorman, R. S. Ashraf, S. Holliday, A. Wadsworth, N. Gasparini, P. Kaienburg, H. Yan, A. Amassian, C. J. Brabec, J. R. Durrant, and I. McCulloch, "Reduced voltage losses yield 10\% efficient fullerene free organic solar cells with $>1 \mathrm{~V}$ open circuit voltages," Energy \&6 Environmental Science, vol. 9, pp. 3783-3793, 2016.

[26] W. Zhao, D. Qian, S. Zhang, S. Li, O. Inganäs, F. Gao, and J. Hou, "FullereneFree Polymer Solar Cells with over 11\% Efficiency and Excellent Thermal Stability," Advanced Materials, vol. 28, pp. 4734-4739, 2016. 


\section{CONCLUSIONS AND FURTHER WORK}

OSCs have reached the threshold for commercial viability in terms of device efficiency, but device stability remains a barrier to commercialisation. Research efforts have focussed on the stability of the donor polymer or device electrodes and interlayers, leaving the photochemical stability of the electron acceptors unclear.

Chapter 3 investigates the photodegradation of the fullerene acceptor, $\mathrm{PC}_{61} \mathrm{BM}$, under illumination and air, and its role on the overall stability of OSCs. Donor polymer: $\mathrm{PC}_{61} \mathrm{BM}$ OSCs were shown to undergo a severe photodegradation under 1 sun AM1.5G illumination and air. Device degradation of OSCs made when the active layer was degraded prior to top electrode deposition and when the $\mathrm{PC}_{61} \mathrm{BM}$ was selectively photo-aged before blending with the polymer correlated well, revealing the significance of the role of the fullerene in OSC photochemical stability. Remarkably, if just $1 \%$ of $\mathrm{PC}_{61} \mathrm{BM}$ within the fullerene phase were photo-oxidised, there was a $\sim 65 \%$ loss of device PCE, with $\mathrm{J}_{\mathrm{sc}}, \mathrm{V}_{\mathrm{oc}}$ and FF degrading simultaneously. When devices were made using selectively degraded polymers, there was no significant reduction in $\mathrm{V}_{\text {oc }}$ so we can attribute $\mathrm{V}_{\text {oc }}$ reduction to acceptor degradation. Analogous device degradation was observed for devices employing different benchmark polymers and fullerenes, and both standard and inverted device architectures, where the fullerenes were selectively photo-aged. These results show that the photo-oxidation of fullerenes have a widespread and detrimental effect to the stability of polymer:fullerene OSCs.

Chapter 4 explores how film morphology affects fullerene photo-oxidation and its effect on OSC device performance. It was found that the less aggregated the fullerene (in PS), the faster it was photo-oxidised. This enhanced photo-oxidation correlates with an increase in oxygen quenching of the fullerene triplet states, suggesting the primary degradation mechanism is triplet-mediated singlet oxygen generation. The fullerene photooxidation was shown to impact substantially on electron mobility, indicative of electron trap formation, which was supported with observed red-shifting in PL and EL spectra 
with photo-aging and TD-DFT calculations of photo-oxidised defects with suppressed LUMO levels. GIWAXS measurements showed how higher $\mathrm{PC}_{61} \mathrm{BM}$ content of donor polymer (PCDTBT):fullerene blend films were more aggregated. Films with less aggregated $\mathrm{PC}_{61} \mathrm{BM}$, underwent more photobleaching of both the $\mathrm{PC}_{61} \mathrm{BM}$ and $\mathrm{PCDTBT}$, correlating with an increase in the $\mathrm{PC}_{61} \mathrm{BM}$ photo-oxidation measured by mass spectrometry, and a decrease in device stability, particularly correlating well with the significant drop in $\mathrm{V}_{\mathrm{oc}}$. This correlated with an increase in oxygen quenching of the polymer triplet state, suggesting the dominate degradation pathway is singlet oxygen generation via the polymer triplet state.

In Chapter 5, six fullerenes with differing LUMO levels were investigated to study how energetics could affect neat fullerene film and device photo-chemical stability. The amount of photobleaching of neat films correlated well with observed carbonyl formation and fullerene photo-oxidation, all of which in line with the LUMO levels acquired via cyclic voltammetry. Generally, fullerenes with lower lying LUMO levels underwent less photo-oxidation. There was a good correlation between the large drop of PCE and $\mathrm{J}_{\mathrm{sc}}$ of devices employing selectively degraded fullerenes with the observed fullerene photooxidation. Notably, the degree $\mathrm{V}_{\text {oc }}$ reduction correlated very well with fullerene LUMO level, with lower lying LUMOs having less $\mathrm{V}_{\text {oc }}$ reduction.

Chapter 6 focussed on NFA stability. Firstly, the light-only degradation of NFAs was investigated. It was found that O-/Eh-IDTBR based devices had superior burn-in free behaviour, whereas $\mathrm{PC}_{71} \mathrm{BM}$ devices degraded rapidly to $\sim 60 \%$ of their initial performance before stabilising and M-/ITIC based devices were unstable and continued to degrade without stabilising past the 120 hours of device testing. Then the stability of this superior NFA family was studied under illumination and air. A critical correlation was found that O-IDTBR was much more stable than IDFBR in neat films, but when blended with the relatively stable polymer P3HT, the film became unstable. It was found for non-fullerene acceptors and a range of fullerenes, the lower the LUMO level of the acceptor, the more stable the polymer (P3HT):acceptor film. This relationship was shown to be strongly mediated by the yield of superoxide formation via the acceptor LUMO level.

In our work concerning the energetic origin of photochemical instabilities in Chapters 5 and 6, we found that Kloc-6 did not fit the rule where the lower the LUMO level, the more stable the acceptor/blend film/device due to a lower yield of superoxide anions. We suggest that because of structural differences: the inclusion of oxygen and nitrogen atoms on the fullerene cage of Kloc- 6 and having a different sidechain, both of which could weaken the fullerene cage, causing it to fragment faster upon photo-oxidation. Further work could be undertaken to better understand this process. We suggest TD-DFT calculations could be used to compare the bond strength of the different fullerene cages to see how much 
photo-oxidation is required for fragmentation to occur.

At the end of Chapter 5, polymer: $\mathrm{PC}_{61} \mathrm{BM}$ :fullerene ternary blend devices were fabricated and the photochemical stability was tested. Since the extra fullerene component of these devices (ICBA and ICTA) has a higher lying LUMO level than $\mathrm{PC}_{61} \mathrm{BM}$, it was expected that these would improve the other two components in the active layer by acting as sacrificial oxygen radical scavengers. However, no improvement to device stability was found by including these extra fullerenes and so further investigations could provide reasons for this. For example, SCLC mobility measurements of electron-only devices could provide insight into the charge transport and trapping. The vertical phase separation of the films could also be studied by using XPS depth profiling of fresh films.

In Chapter 6, it was found that the correlation between the electron acceptor LUMO level and the photochemical stability of both the polymer and electron acceptor in blend films was due to the yield of superoxide generation mediated by the acceptor LUMO. In Chapter 5, the photochemical stability of neat fullerene acceptors was found to correlate with their LUMO level. It would be interesting to use transient absorption spectroscopy to probe the photoexcited states which may provide insight to a photodegradation mechanism for neat fullerene acceptors.

The burn-in of OSCs is an area of current research interest. In Chapter 6, we show some preliminary results to show the generality of burn-in: how some NFAs are burnin free (O-/Eh-IDTBR), other NFAs are unstable (M-/ITIC and IDFBR) and fullerene acceptors have a significant burn-in. Further work could be undertaken to provide insight into the origin of burn-in. We suggest optical, structural and transient optoelectronic measurements to investigate chemical changes and the formation of charge traps under the light-only burn-in conditions.

The work presented in this thesis establishes relationships between electron acceptor structural/nanomorphological/electronic properties with material/device stability. Namely more aggregated electron acceptors leads to a lower yield of singlet oxygen and electron acceptors with a lower LUMO level (coupled with donors with a lower HOMO) have a lower yield of superoxide anions, thus increasing the photochemical stability. These are rules for the design of future accepting materials to help overcome the current challenge in OSC stability, thereby paving the way toward achieving long-term environmental stability of low-cost OSCs without or with minimal encapsulation. 

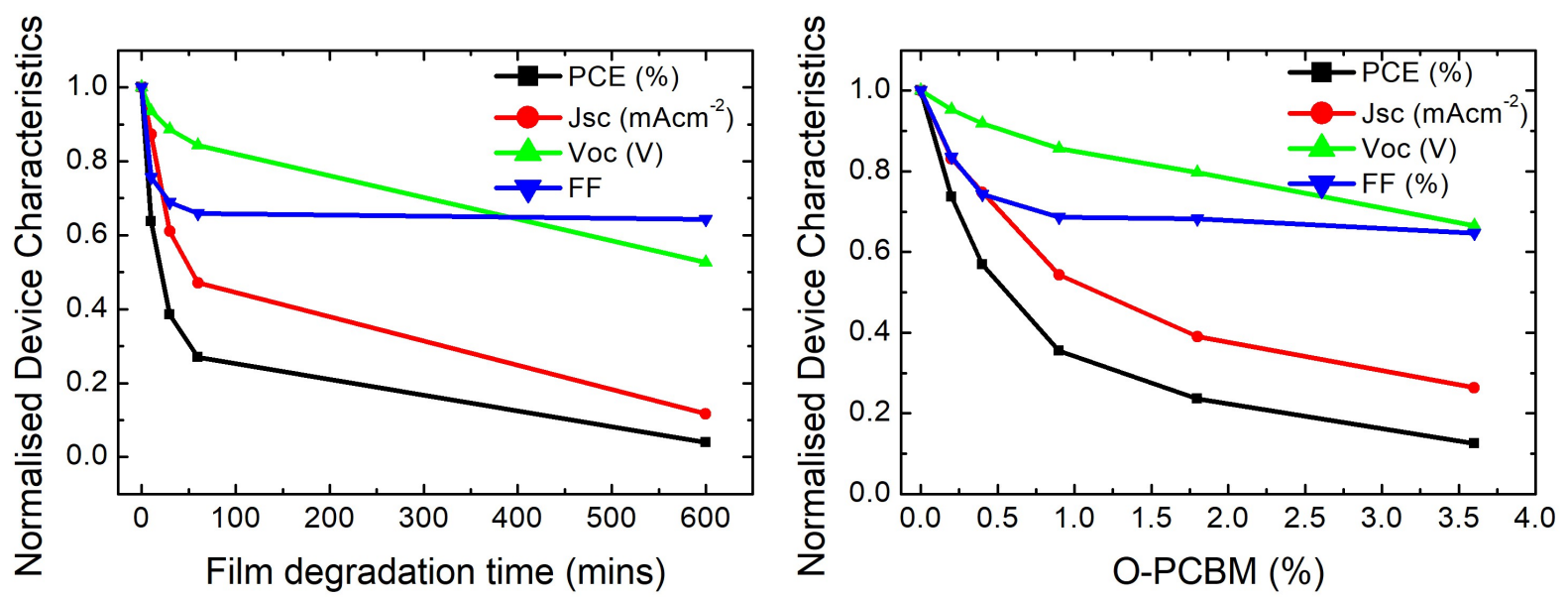

Figure A.1: Summary of normalised device parameters of (a) ITO/PEDOT:PSS/PCDTBT:PC ${ }_{61} \mathrm{BM}(1: 2) / \mathrm{Ca} / \mathrm{Al}$ devices with different degradation times under simulated AM1.5G illumination in air, before top electrode deposition and (b) PCDTBT:PC ${ }_{61} \mathrm{BM}$ blend devices made with different fractions of degraded $\mathrm{PC}_{61} \mathrm{BM}$, the percentages indicating the relative fraction of $\mathrm{O}-\mathrm{PC}_{61} \mathrm{BM}$ in the acceptor phase.

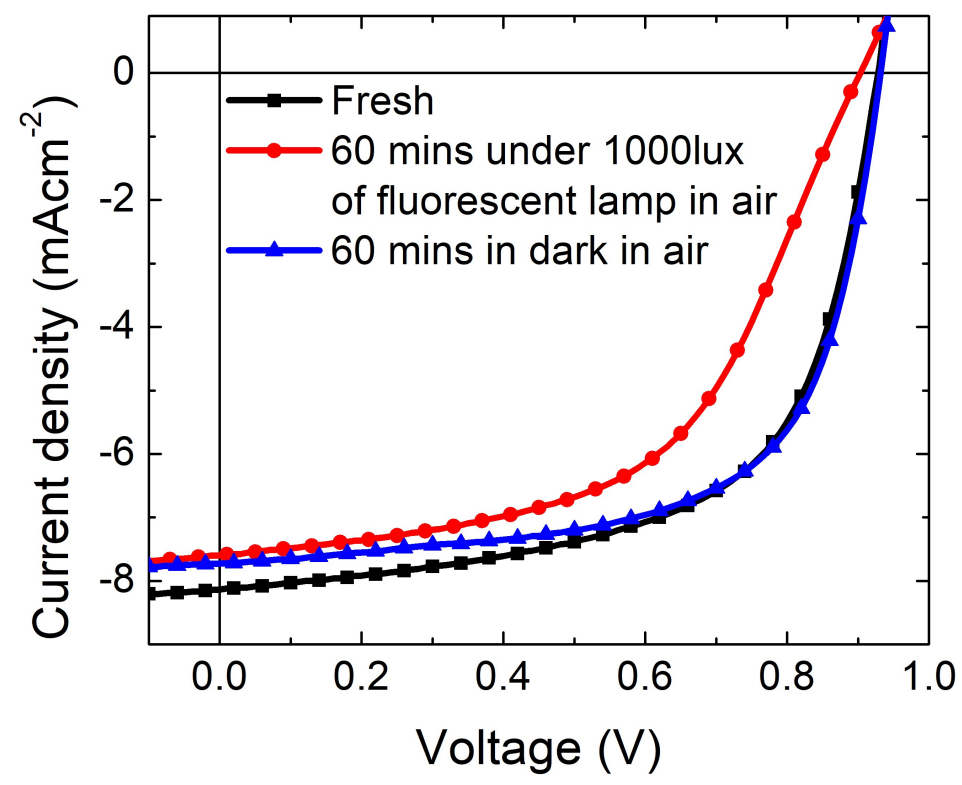

Figure A.2: J-V characteristics of 1:2 PCDTBT:PC ${ }_{61} \mathrm{BM}$ devices exposed to air for 60 minutes in the dark and under 1000 lux of compact fluorescent lamp. 
(a)
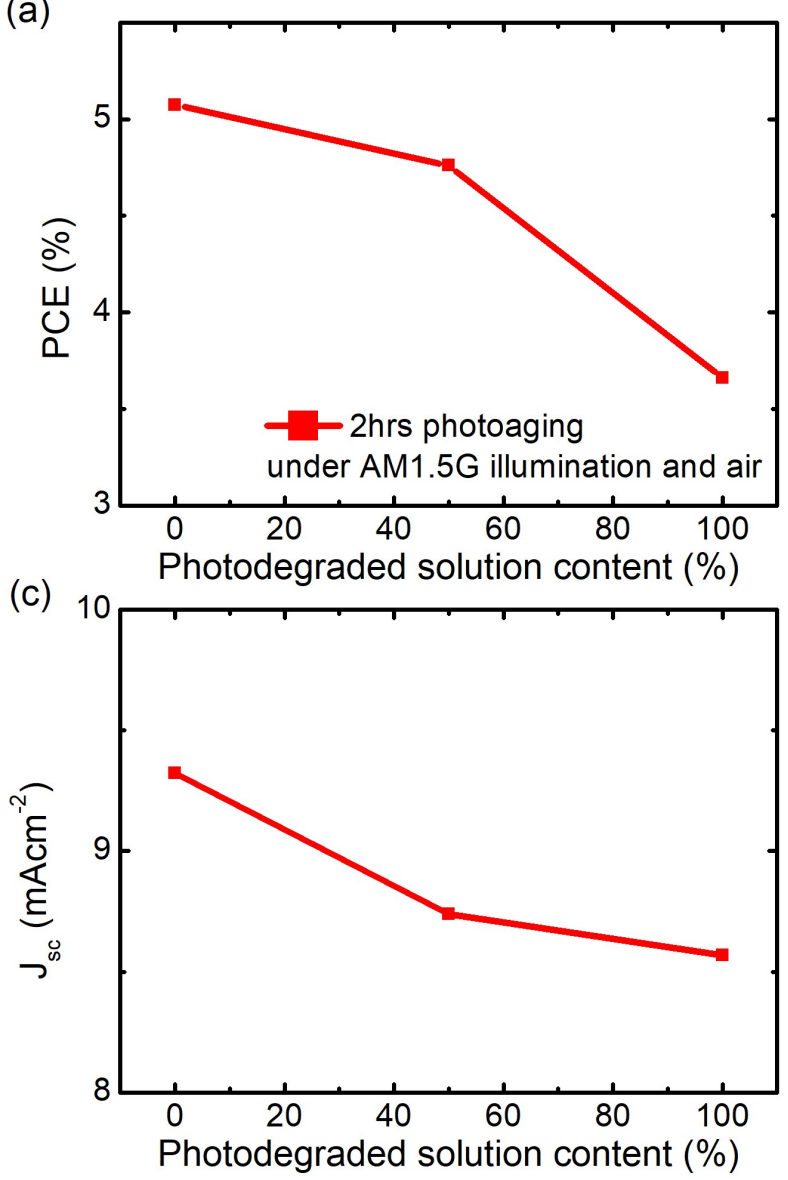

(b)

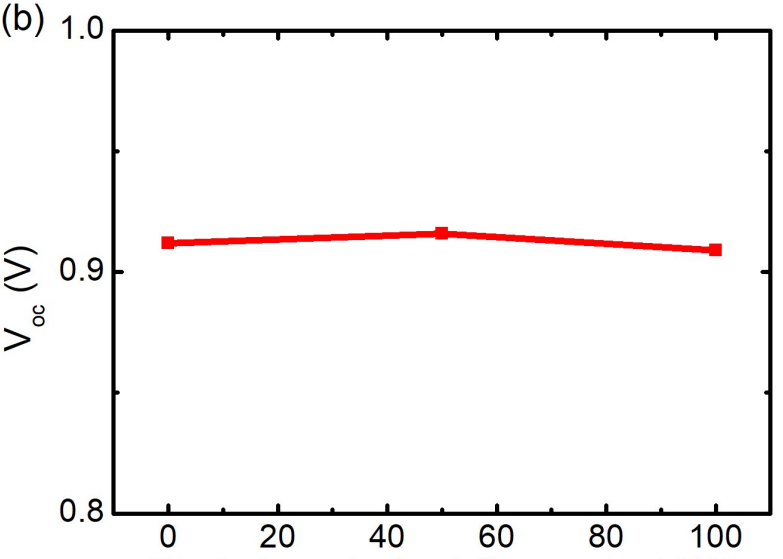

(d) Photodegraded solution content (\%)

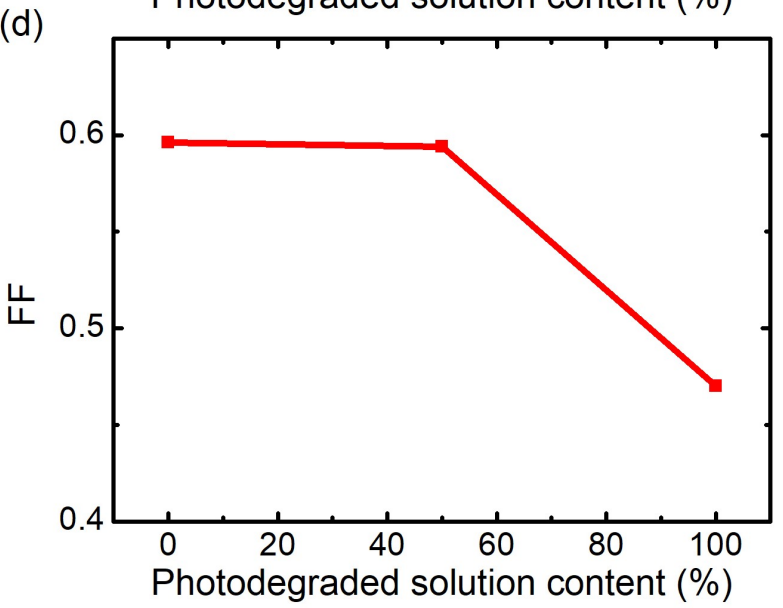

Figure A.3: Device characteristics (a) PCE, (b) $\mathrm{V}_{\text {oc }}$, (c) $\mathrm{J}_{\mathrm{sc}}$, and (d) FF of 1:2 PCDTBT: $\mathrm{PC}_{61} \mathrm{BM}$ devices as a function of the proportion of degraded PCDTBT solution employed to form the active layer.
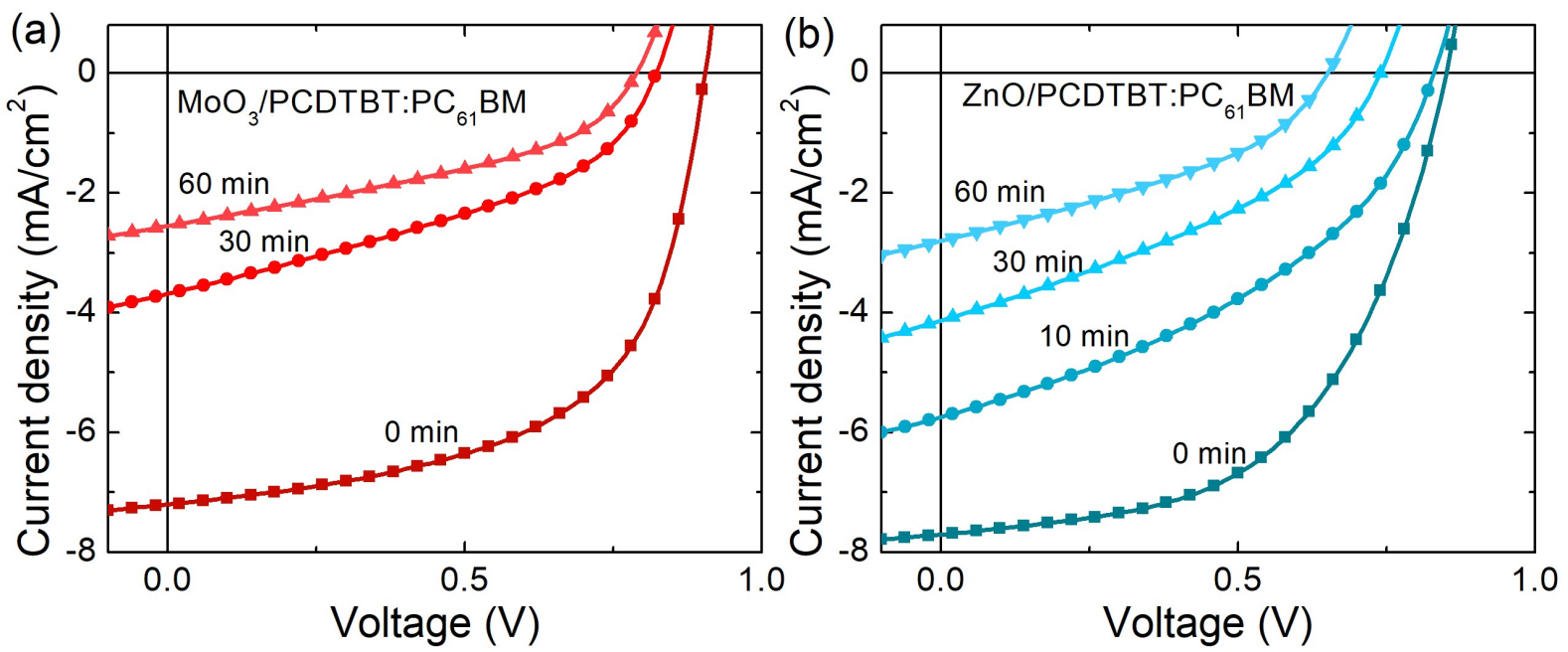

Figure A.4: J-V characteristics of (a) $\mathrm{MoO}_{3}$ and (b) $\mathrm{ZnO}$ based devices where the 1:2 PCDTBT:PC ${ }_{61} \mathrm{BM}$ film was exposed to air and 1sun AM1.5G illuminaition prior to top electrode $\left(\mathrm{Ca} / \mathrm{Al}\right.$ and $\mathrm{MoO}_{3} / \mathrm{Ag}$ respectively) deposition. 


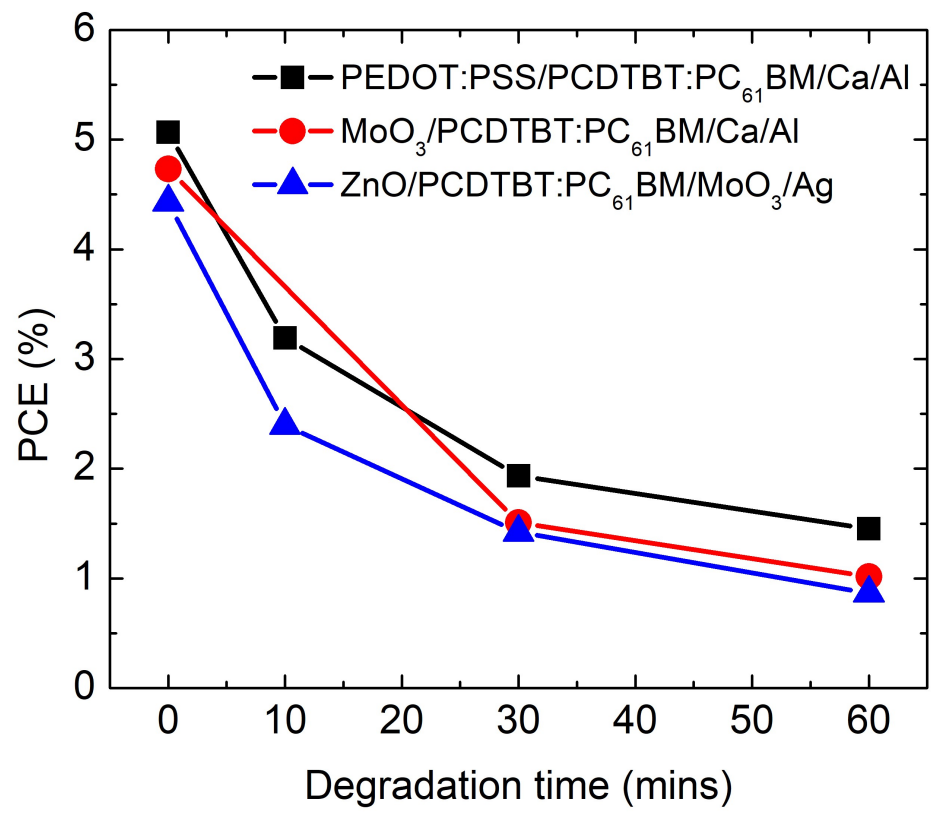

Figure A.5: PCE as a function of BHJ degradation time of 1:2 PCDTBT:PC ${ }_{61} \mathrm{BM}$ devices utilising PEDOT:PSS and $\mathrm{MoO}_{3}$ interlayers with a conventional architecture with $\mathrm{Ca} / \mathrm{Al}$ top electrodes and $\mathrm{ZnO}$ for the inverted architecture devices with $\mathrm{MoO}_{3} / \mathrm{Ag}$ top electrodes. 


\section{B.0.1 Part 1: Fullerene and PS:Fullerene}

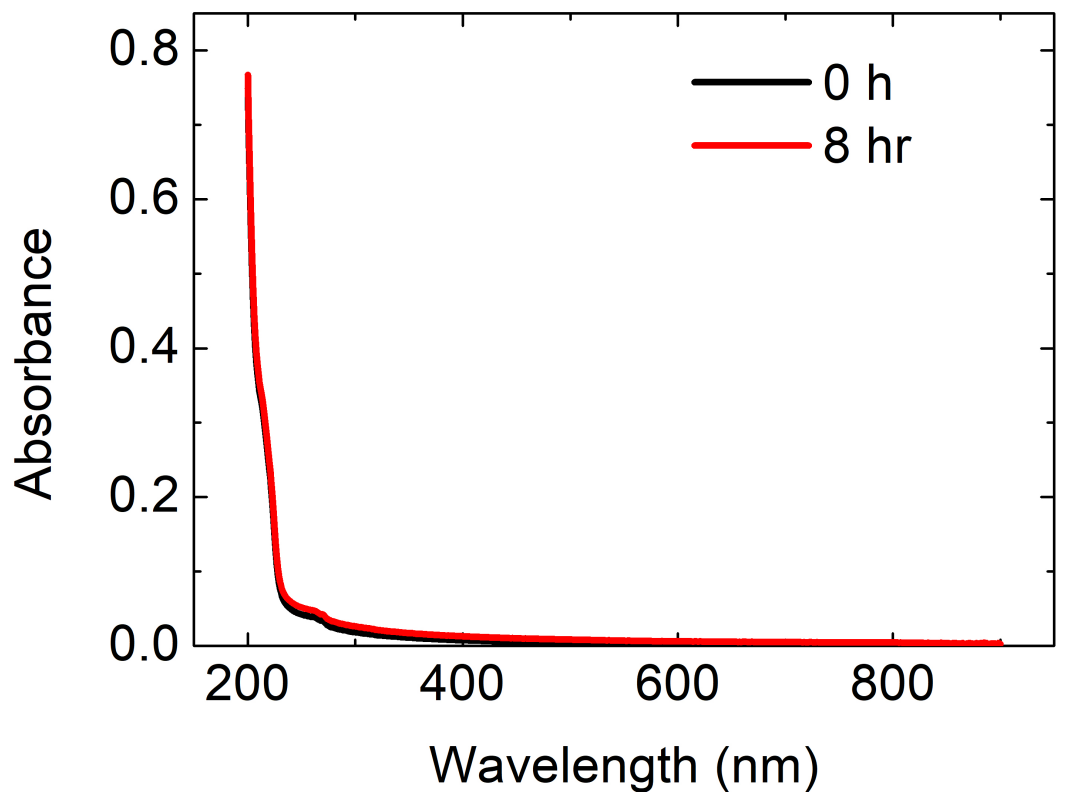

Figure B.1: UV-Vis absorbance spectra of neat PS before and after photoaging under AM1.5 irradiation, in air.
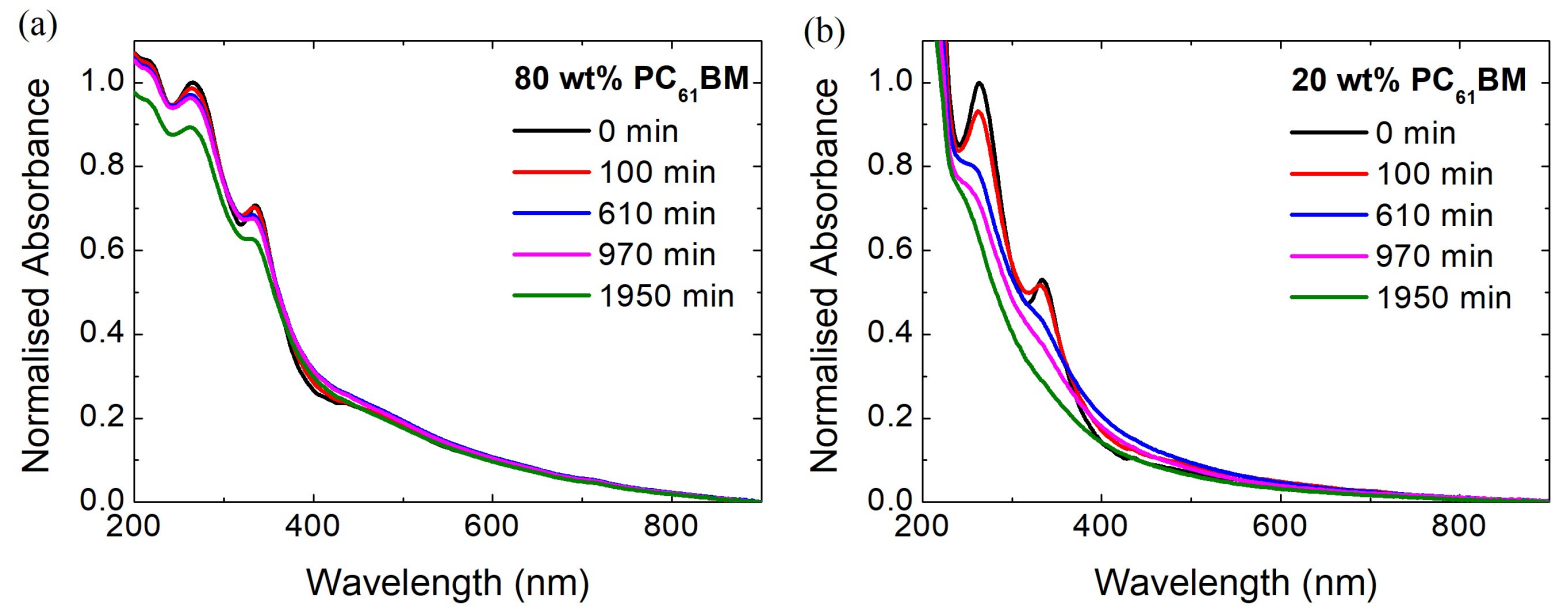

Figure B.2: UV-Vis absorbance spectra of (a) $80 \mathrm{wt} \%$ and (b) $20 \mathrm{wt} \% \mathrm{PC}_{61}$ :PS films (normalised at $265 \mathrm{~nm}$ ), with increasing photoaging time under AM1.5 irradiation and air. 

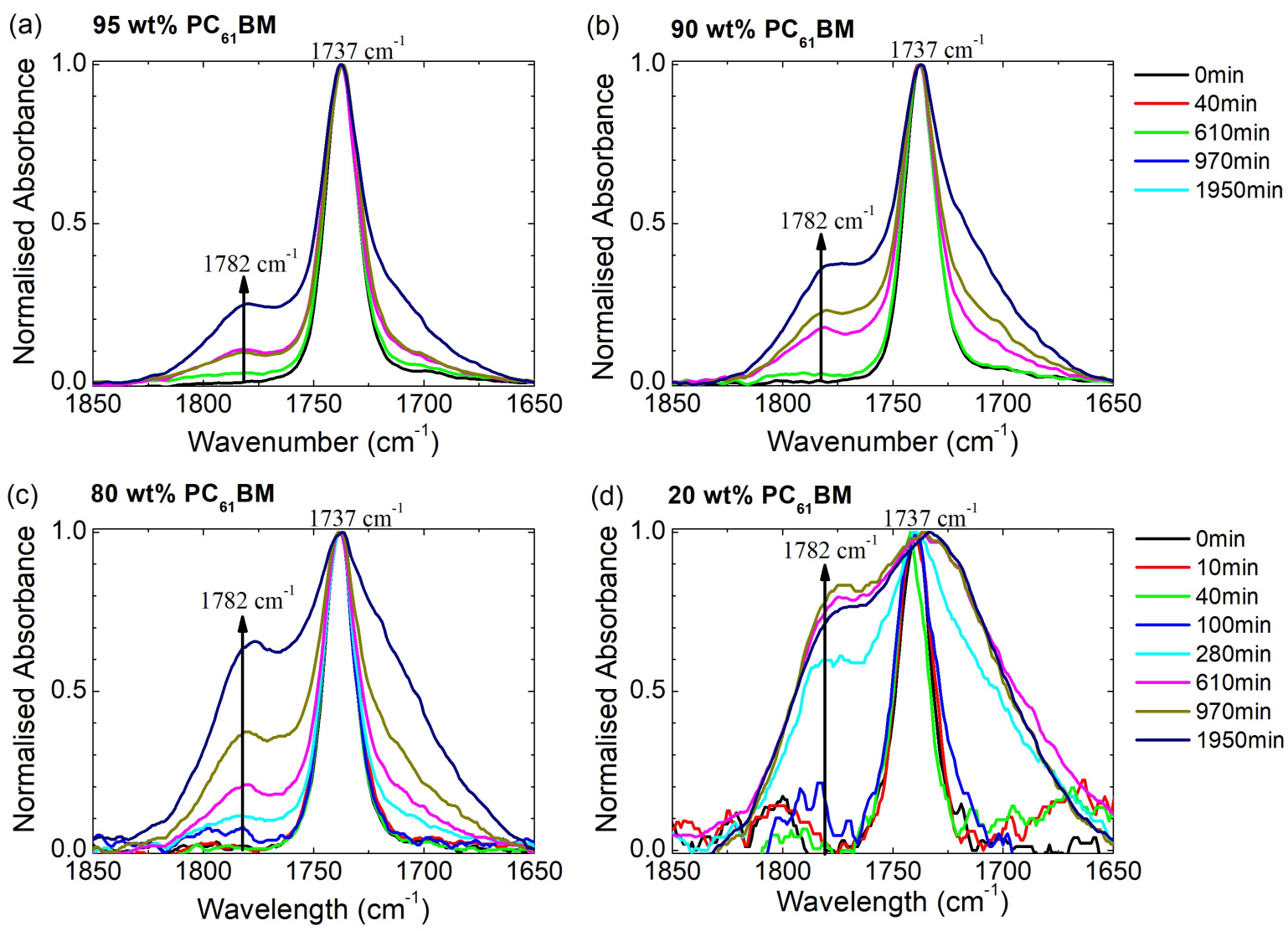

Figure B.3: Normalised ATR-FTIR absorbance spectra of (a) 95 wt\% (b) 90 wt\% (c) 80 wt\% and (d) $20 \mathrm{wt} \% \mathrm{PC}_{61} \mathrm{BM}$ films respectively, with increasing photoaging times.

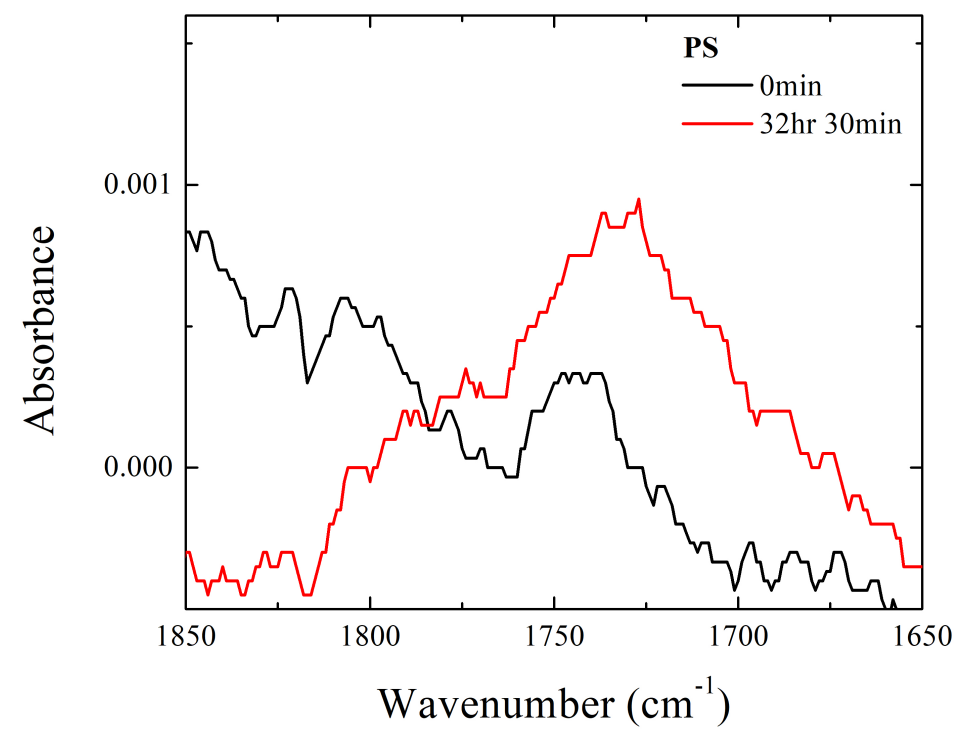

Figure B.4: ATR-FTIR spectra of fresh and photoaged PS. 
Table B.1: XPS peak assignments and typical bulk compositions of neat (N) and $40 \mathrm{wt} \%$ (B) $\mathrm{PC}_{61} \mathrm{BM}$ films, before and after photoaging for 920 minutes.

\begin{tabular}{||c|c|c|c|c|c|c||}
\hline \hline \multirow{2}{*}{ Element } & \multirow{2}{*}{ Position (eV) } & \multirow{2}{*}{ Assignment } & \multicolumn{3}{|c||}{ Typical Bulk Compositions (Atomic \%) } \\
\cline { 4 - 7 } & & Fresh N & Aged N & Fresh B & Aged B \\
\hline $\mathrm{C}(1 \mathrm{~s})$ & 284.0 & C60 & 84.0 & 75.1 & 82.1 & 39.0 \\
$\mathrm{C}(1 \mathrm{~s})$ & 284.8 & $\mathrm{CxHy}$ & 4.8 & 6.1 & 10.2 & 14.8 \\
$\mathrm{C}(1 \mathrm{~s})$ & 286.1 & $\mathrm{C}-\mathrm{O}-\mathrm{C} / \mathrm{C}-\mathrm{O}$ & 1.6 & 4.4 & 1.0 & 12.4 \\
$\mathrm{C}(1 \mathrm{~s})$ & 288.3 & $\mathrm{C}=\mathrm{O} / \mathrm{O}=\mathrm{C}-\mathrm{O}$ & 2.9 & 4.6 & 0.4 & 10.9 \\
$\mathrm{C}(1 \mathrm{~s})$ & 289.9 & $\pi \rightarrow \pi^{*}$ & 4.4 & 5.4 & 4.9 & 3.0 \\
$\mathrm{O}(1 \mathrm{~s})$ & 529.4 & ITO substrate & $\mathrm{N} \backslash \mathrm{A}$ & $\mathrm{N} \backslash \mathrm{A}$ & $\mathrm{N} \backslash \mathrm{A}$ & $\mathrm{N} \backslash \mathrm{A}$ \\
$\mathrm{O}(1 \mathrm{~s})$ & 531.1 & $\mathrm{C}-\mathrm{O}-\mathrm{C}$ & 1.1 & 1.9 & 0.8 & 11.4 \\
$\mathrm{O}(1 \mathrm{~s})$ & 532.6 & $\mathrm{C}=\mathrm{O}$ & 1.1 & 2.4 & 0.6 & 8.5 \\
\hline \hline
\end{tabular}
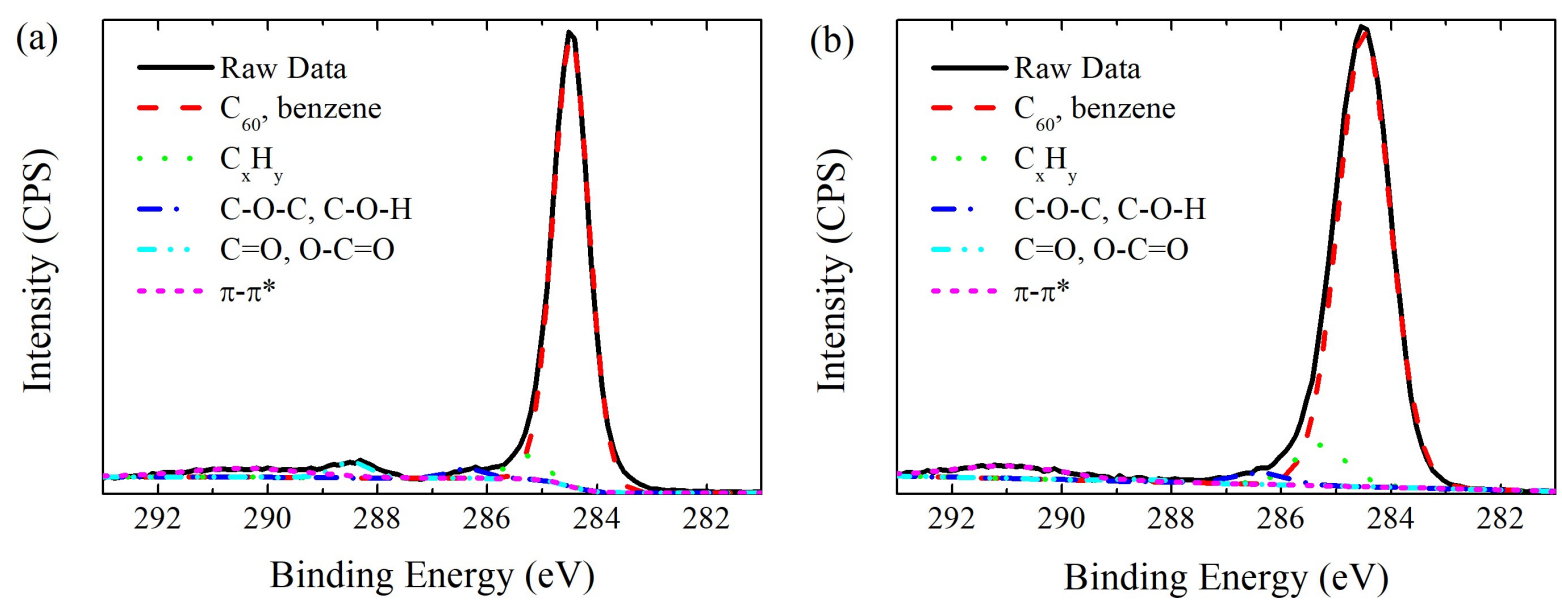

Figure B.5: XPS spectrum (carbon 1s envelope) of (a) neat and (b) 40 wt\% $\mathrm{PC}_{61} \mathrm{BM}$ films before photoaging (in counts per second (CPS)).
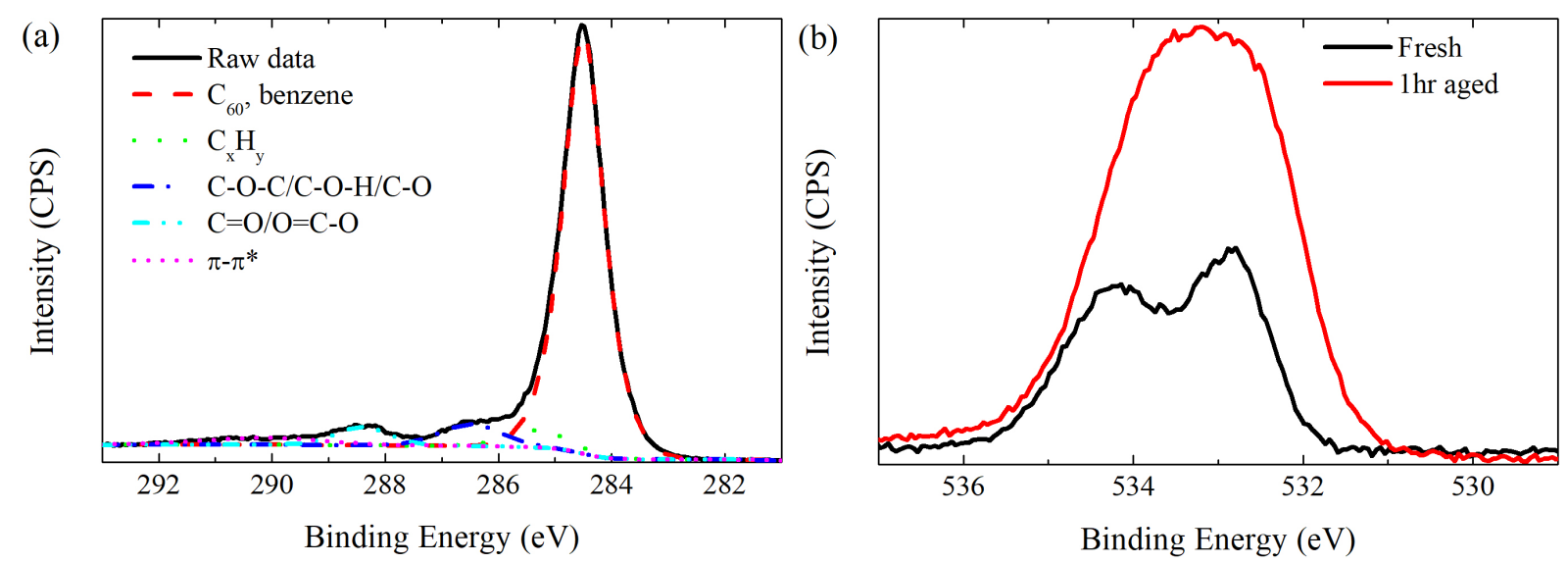

Figure B.6: XPS spectrum (a) (carbon (1s) envelope) of $\mathrm{PC}_{61} \mathrm{BM}$ film after 1 hour of photoaging and (b) (oxygen (1s) envelope) of $\mathrm{PC}_{61} \mathrm{BM}$ films fresh and after 1 hour of photoaging. 


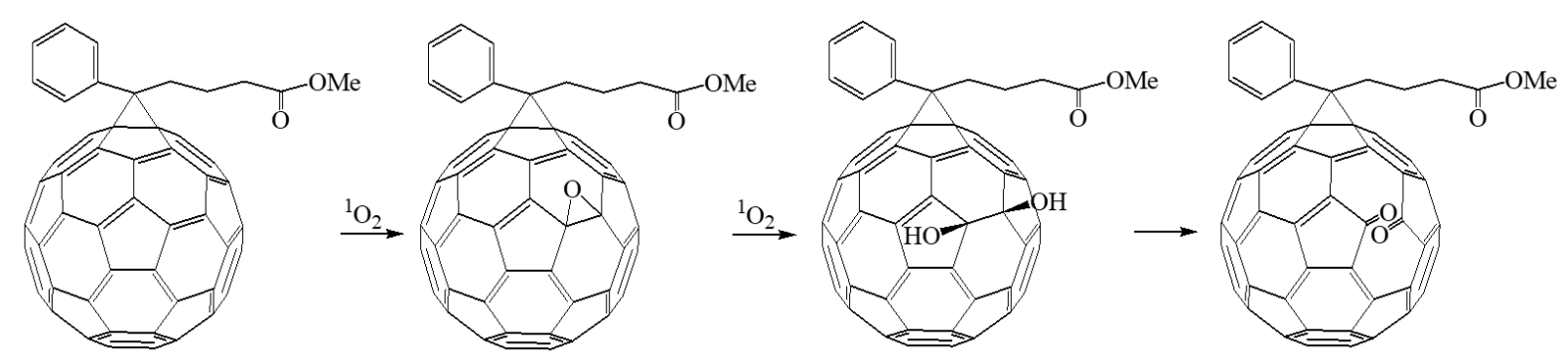

Figure B.7: Possible photo-oxidation mechanism of $\mathrm{PC}_{61} \mathrm{BM}$ : Sequential formation of epoxides, diols and carbonyls.

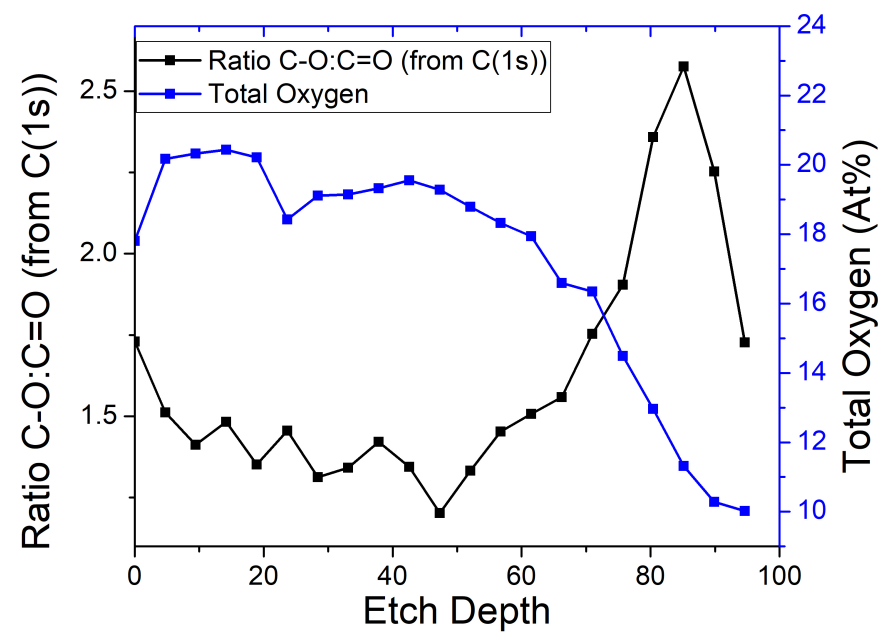

Figure B.8: Epoxide:carbonyl ratio $(\mathrm{C}-\mathrm{O}: \mathrm{C}=\mathrm{O})$ ratio as estimated from the $\mathrm{C}(1 \mathrm{~s})$ envelope and total measured atomic percentage of oxygen as a function of etch depth for photoaged $40 \mathrm{wt} \% \mathrm{PC}_{61} \mathrm{BM}$ film. Note that the data at the surface $(0 \mathrm{~nm})$ is less reliable as it is subject to surface contamination as often observed in the field. 

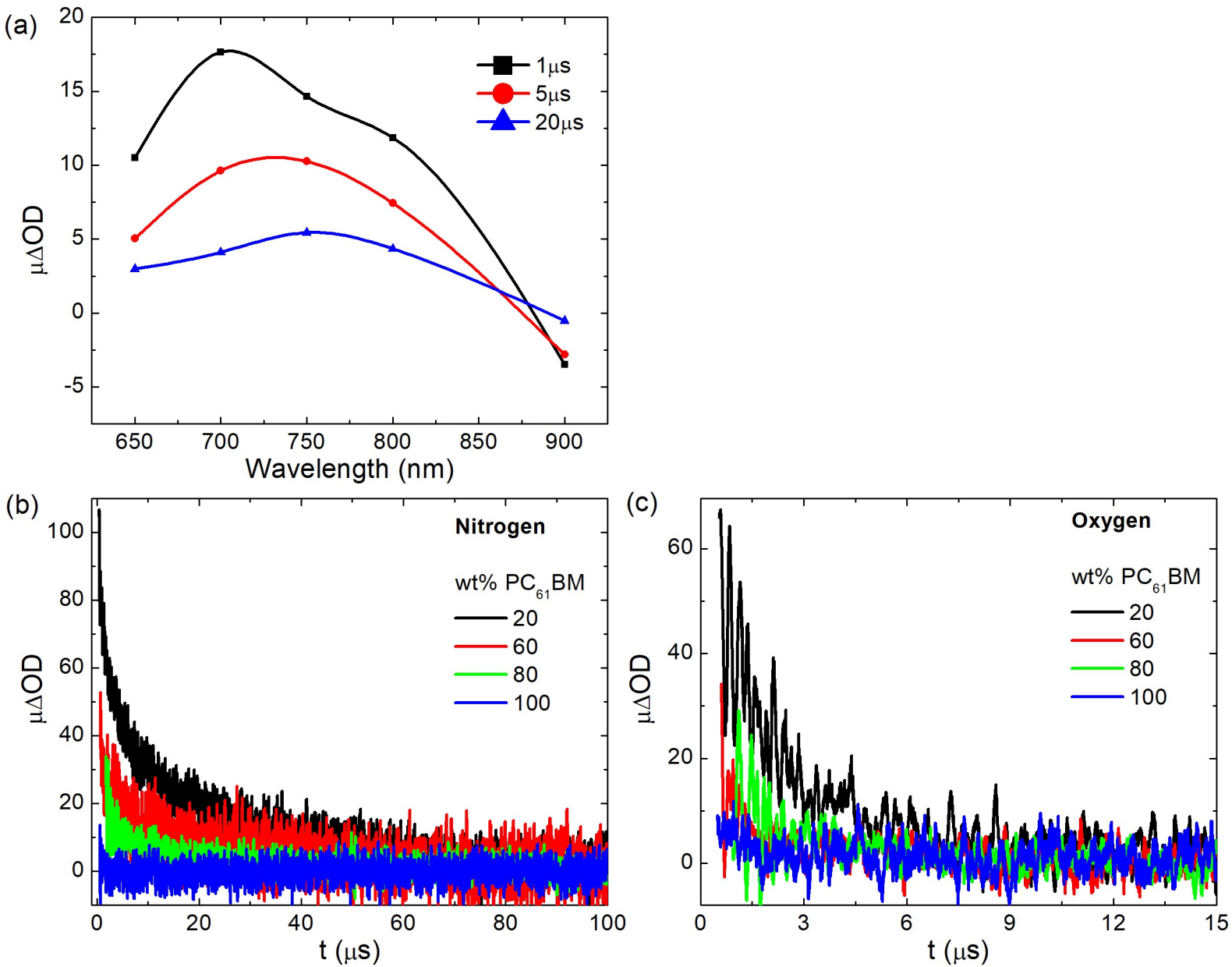

Figure B.9: TAS measurements: (a) Transient absorption spectra (with different time delays) of an $80 \mathrm{wt} \% \mathrm{PC}_{61} \mathrm{BM}: \mathrm{PS}$ film, showing the spectral region where triplet-triplet absorption occurs. TAS decay kinetics (excited at $355 \mathrm{~nm}$ and probed at $700 \mathrm{~nm}$ ) of different wt\% $\mathrm{PC}_{61} \mathrm{BM}$ films under (a) nitrogen and (b) oxygen environments, fitted with biexponential decays. 


\section{B.0.2 Part 2: PCDTBT:Fullerene}
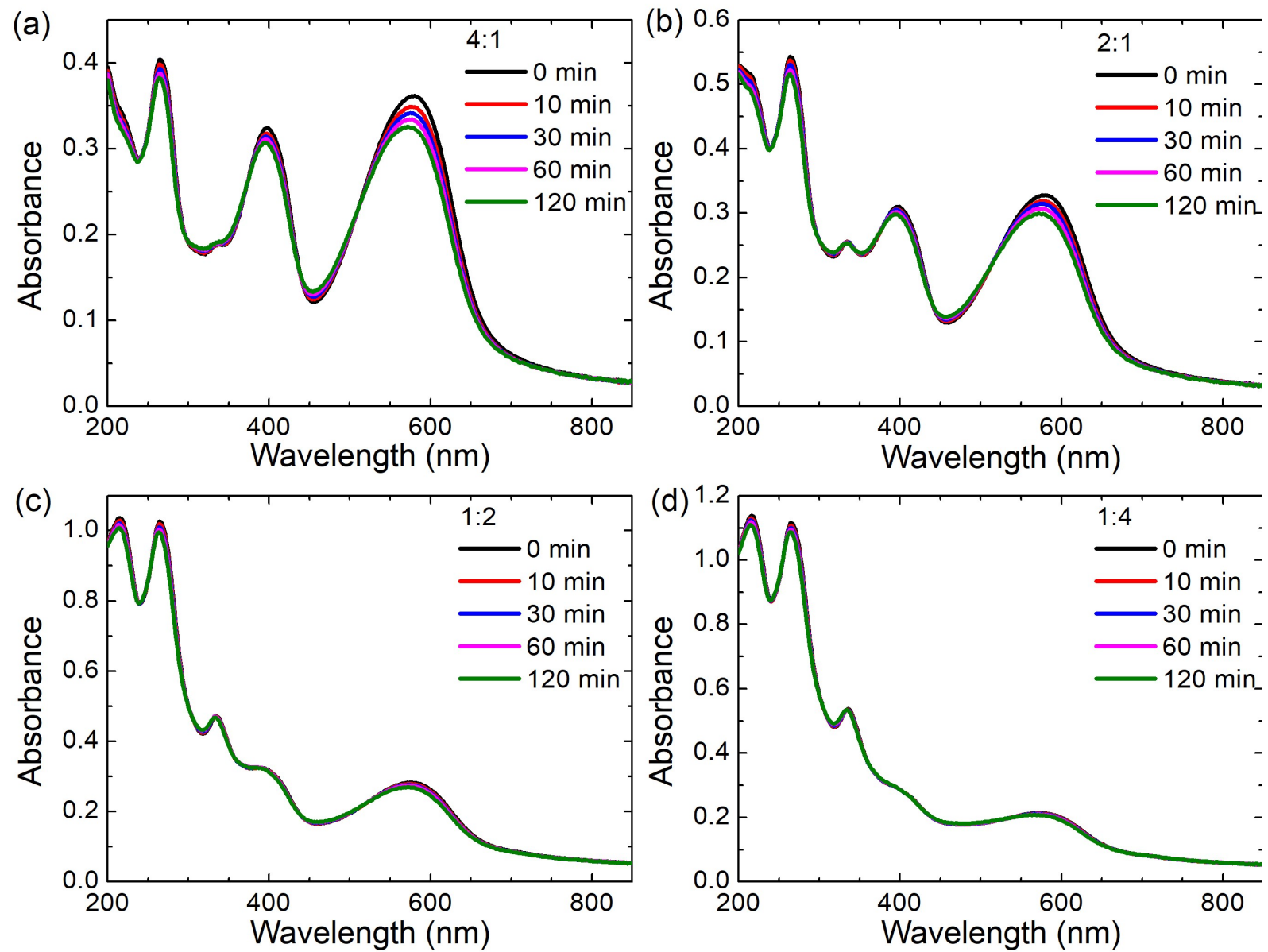

Figure B.10: UV-Vis absorbance spectra of (a) 4:1 $\quad$ (b) 2:1 $\quad$ (c) $1: 2$ (d) 1:4 PCDTBT:PC ${ }_{61} \mathrm{BM}$ films with up to 120 minutes of photoaging.
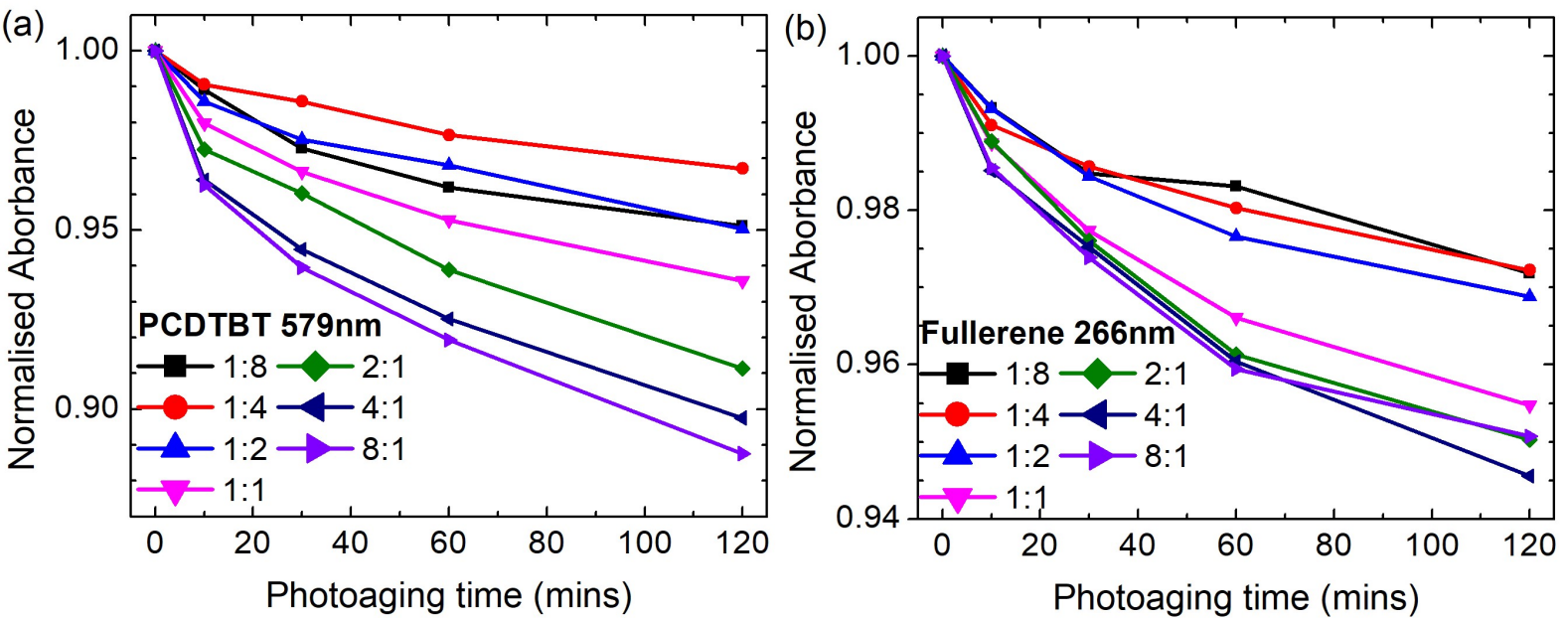

Figure B.11: Normalised absorbance of the (a) PCDTBT (579 nm) and (b) $\mathrm{PC}_{61} \mathrm{BM}$ $(266 \mathrm{~nm})$ peaks of the PCDTBT:PC ${ }_{61} \mathrm{BM}$ films with varying wt\% ratios with up to 120 minutes of photoaging. 

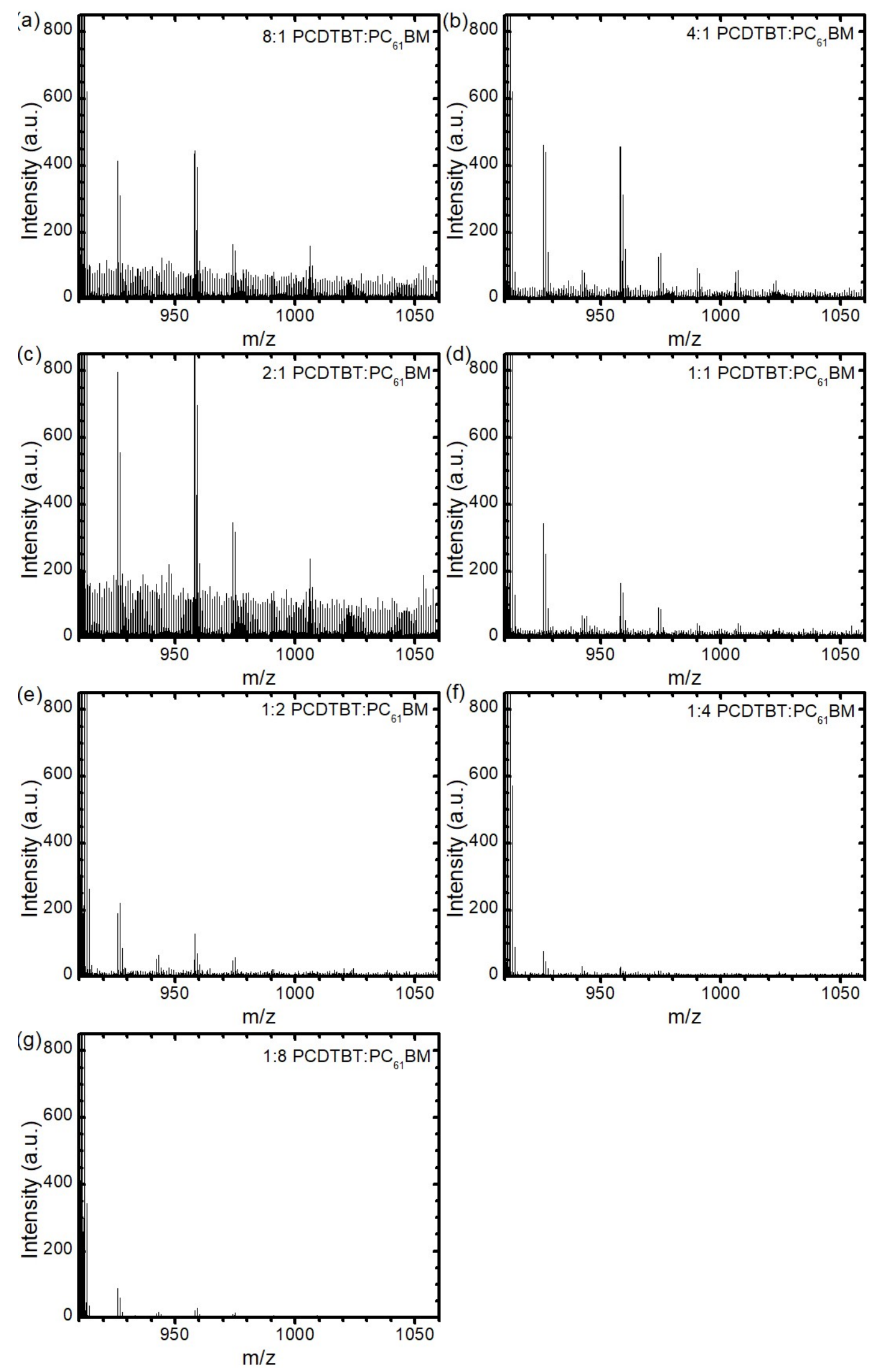

Figure B.12: MALDI-TOF measurements of PCDTBT:PC ${ }_{61} \mathrm{BM}$ blend films with varying wt\% ratios photoaged for 2 hours in air under 1 sun AM1.5G illumination. Mw $\mathrm{PC}_{61} \mathrm{BM}$ $=910.90 \mathrm{gmol}^{-1}$. 

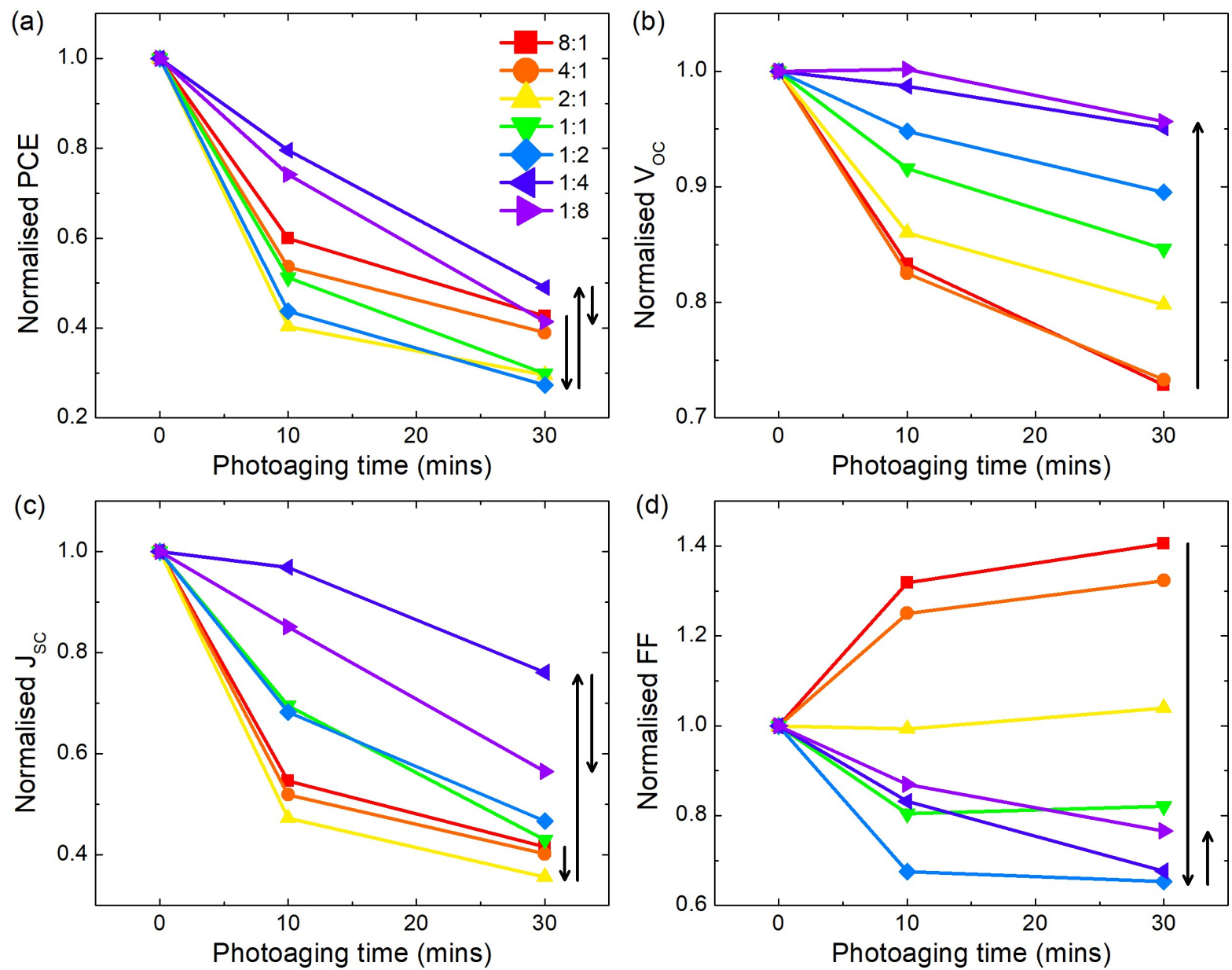

Figure B.13: Device characteristics (a) PCE (b) $\mathrm{V}_{\mathrm{oc}}$ (c) $\mathrm{J}_{\mathrm{sc}}$ (d) FF of PCDTBT:PC 61 BM devices with different wt $\%$ ratios as a function of photoaging time. 

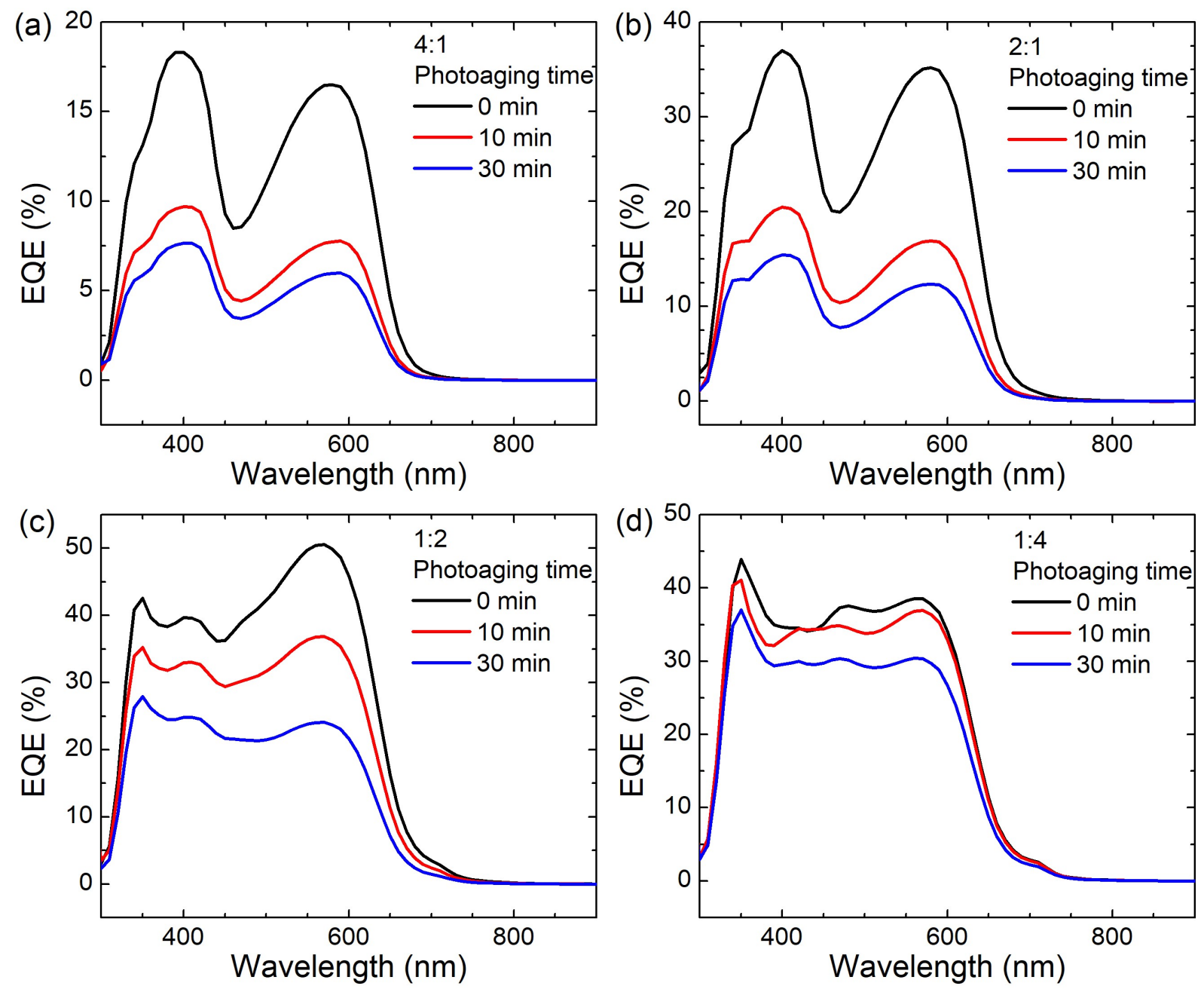

Figure B.14: EQE spectra of (a) 4:1, (b) 2:1 (c) 1:2 and (d) 1:4 PCDTBT:PC ${ }_{61}$ BM devices after 0,10 , and 30 minutes of photoaging.
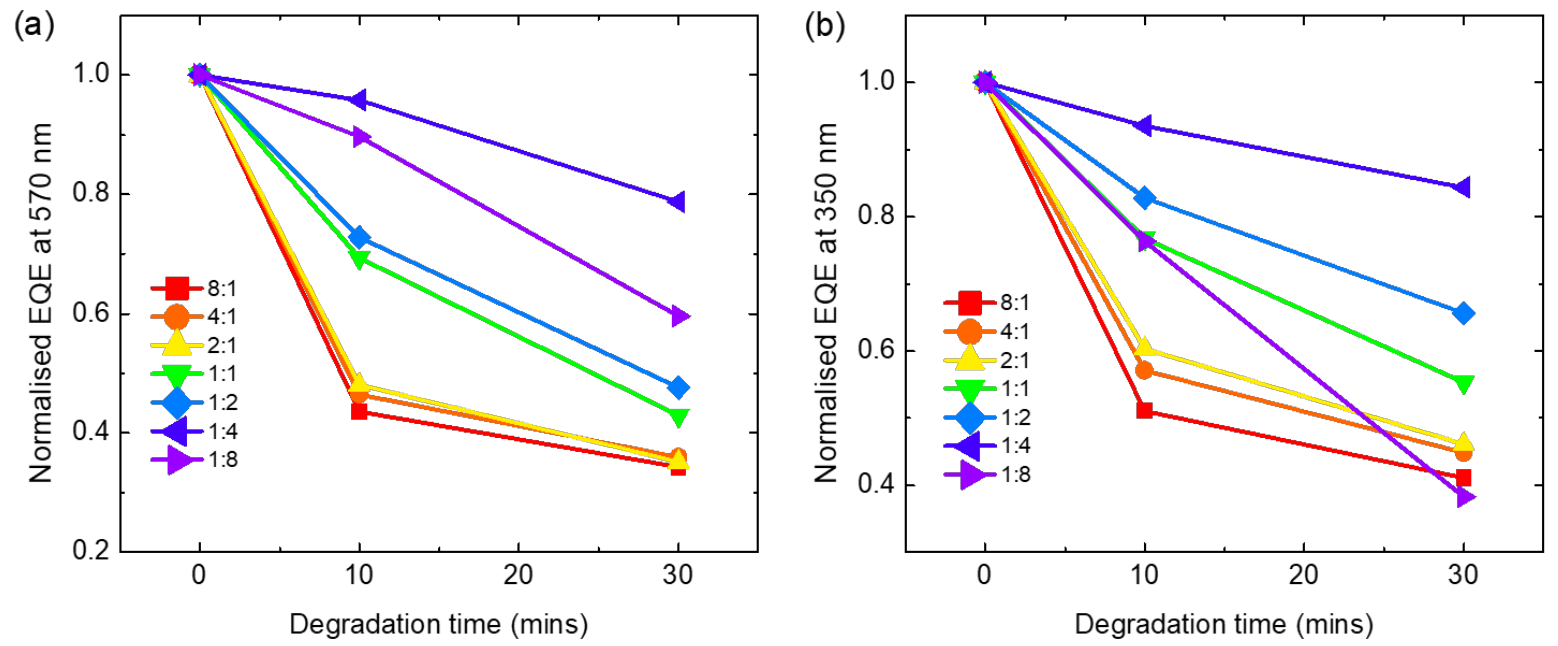

Figure B.15: Normalised EQE at (a) PCDTBT (570 nm) and (b) PC $_{61} \mathrm{BM}(350 \mathrm{~nm})$ of PCDTBT: $\mathrm{PC}_{61} \mathrm{BM}$ devices with different wt $\%$ ratios, as a function of photoaging time. 
(a)
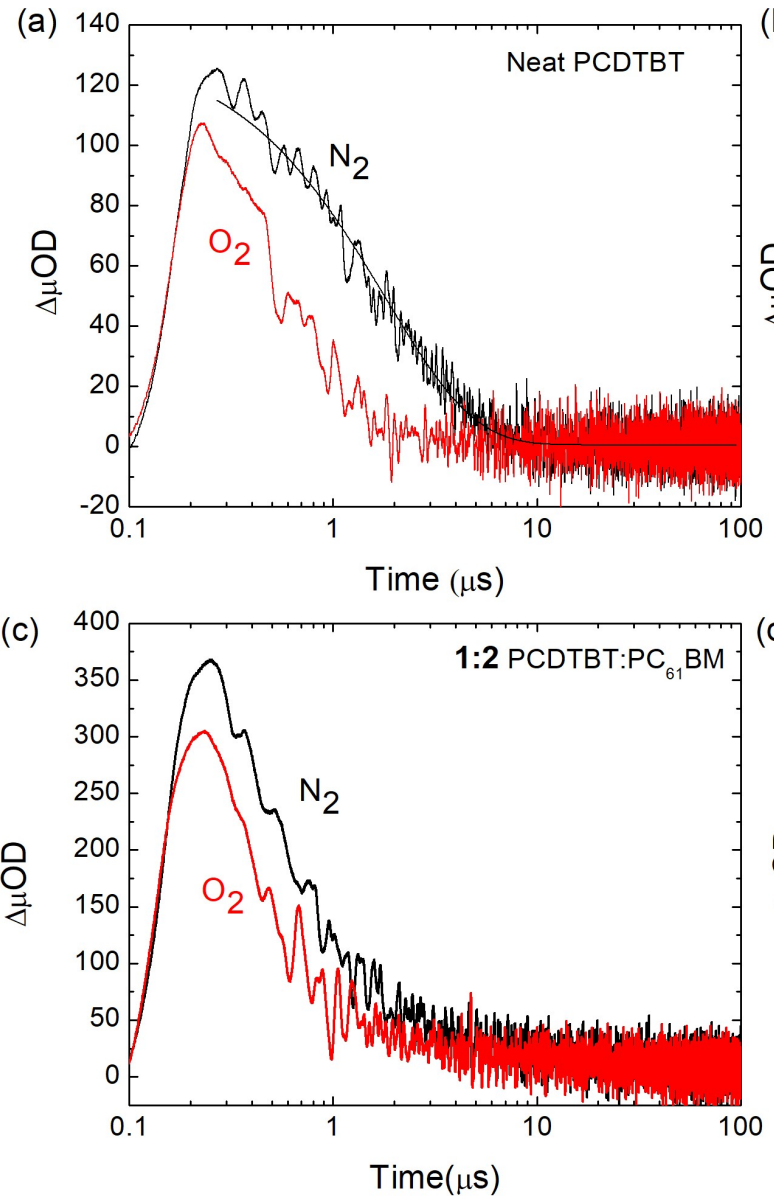

(b)

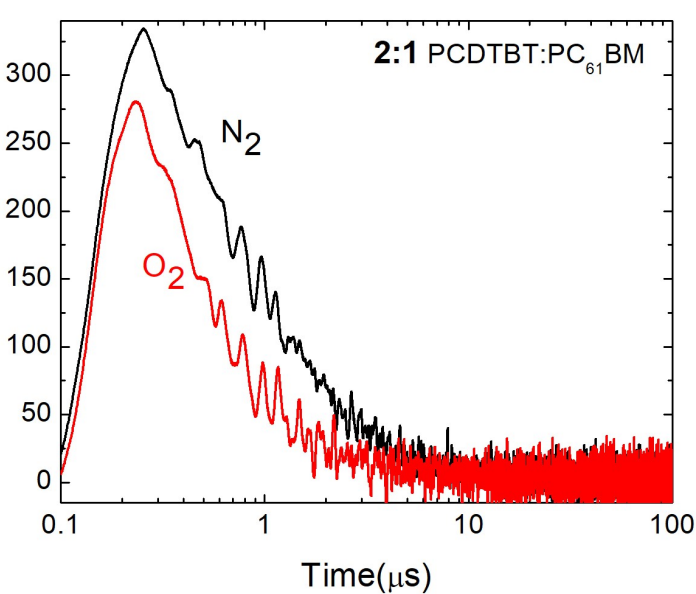

(d)

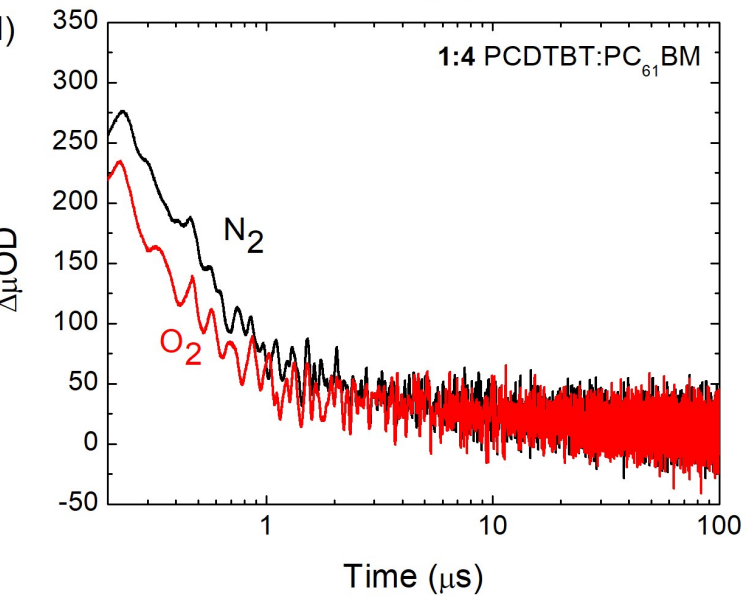

Figure B.16: TAS decay kinetics spectra of a) neat PCDTBT (with exponential fit), b) 2:1, c) 1:2 and d) 1:4 PCDTBT:PC ${ }_{61} B M$ films, excited at $560 \mathrm{~nm}$ with $1.67 \mu \mathrm{jcm}^{-2}$ and probed at $1100 \mathrm{~nm}$. The kinetic was measured under nitrogen and oxygen environments. 

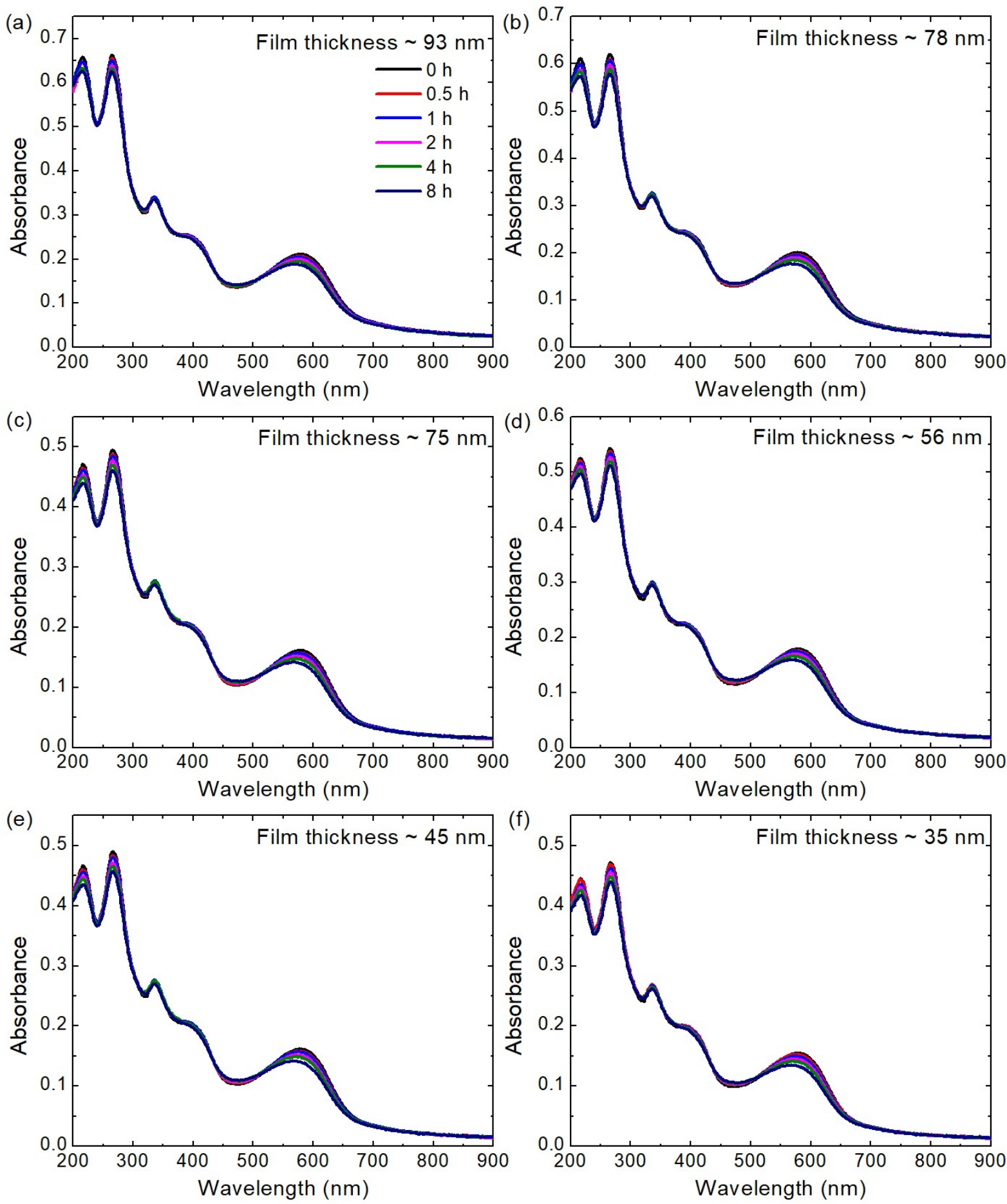

Figure B.17: Photobleaching of 1:2 PCDTBT:PC ${ }_{61} \mathrm{BM}$ films, varying in thickness from $93-35 \mathrm{~nm}$, with up to 8 hours of photoaging under 1 sun AM1.5G illumination in air. 

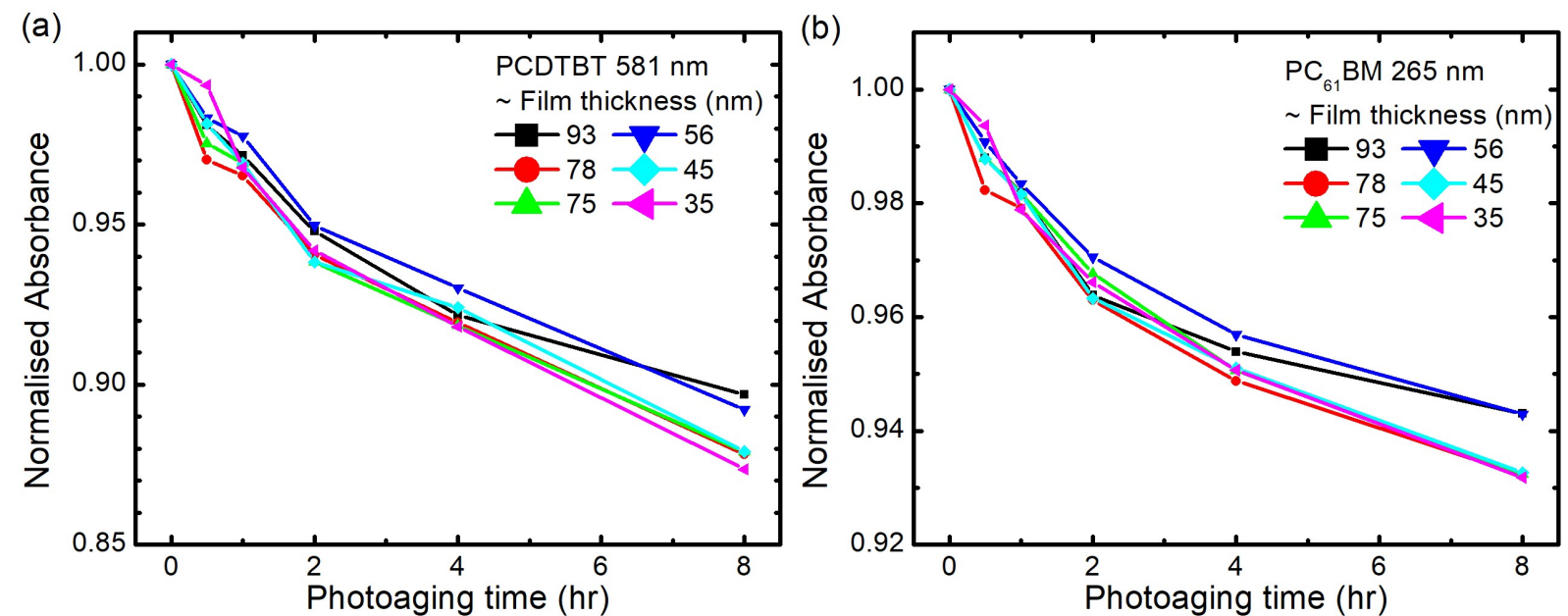

Figure B.18: Normalised UV-Vis absorbance at (a) $581 \mathrm{~nm}$ (PCDTBT) and (b) $265 \mathrm{~nm}$ $\left(\mathrm{PC}_{61} \mathrm{BM}\right)$ of 1:2 PCDTBT: $\mathrm{PC}_{61} \mathrm{BM}$ films with different thicknesses, as a function of photoaging time. 

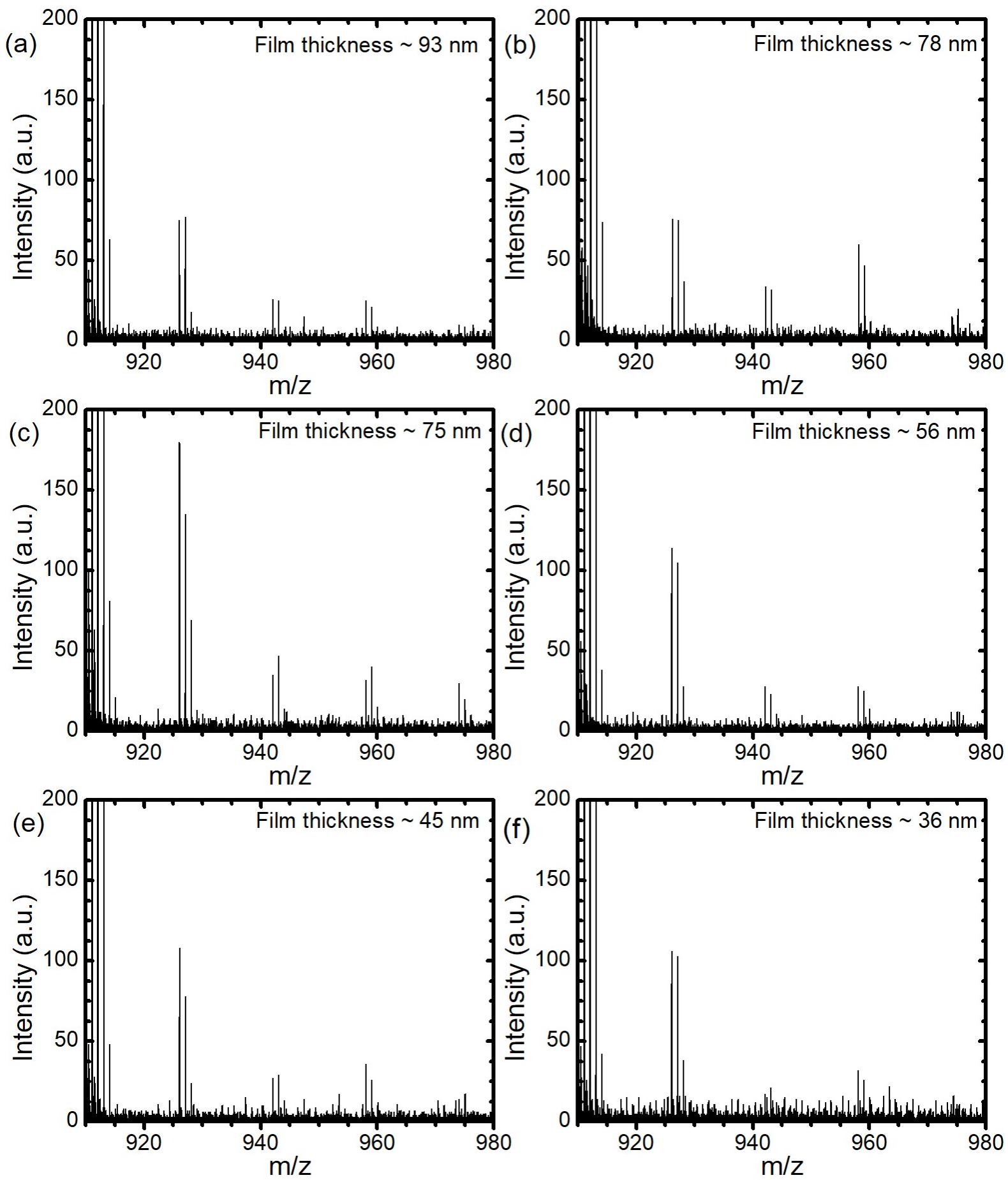

Figure B.19: MALDI-TOF measurements of 1:2 PCDTBT:PC ${ }_{61} \mathrm{BM}$ blend films with different thicknesses, photoaged for 2 hours in air under 1 sun AM1.5G illumination. Mw $\mathrm{PC}_{61} \mathrm{BM}=910.90 \mathrm{gmol}^{-1}$. 


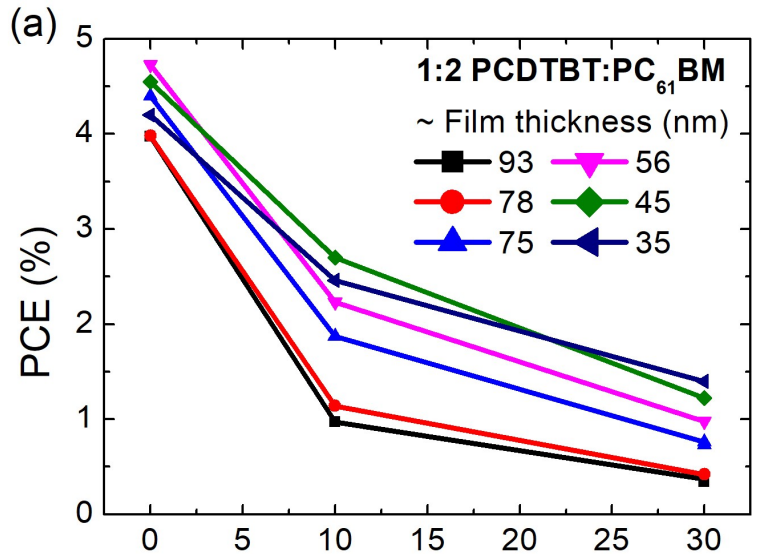

Photoaging time (mins)

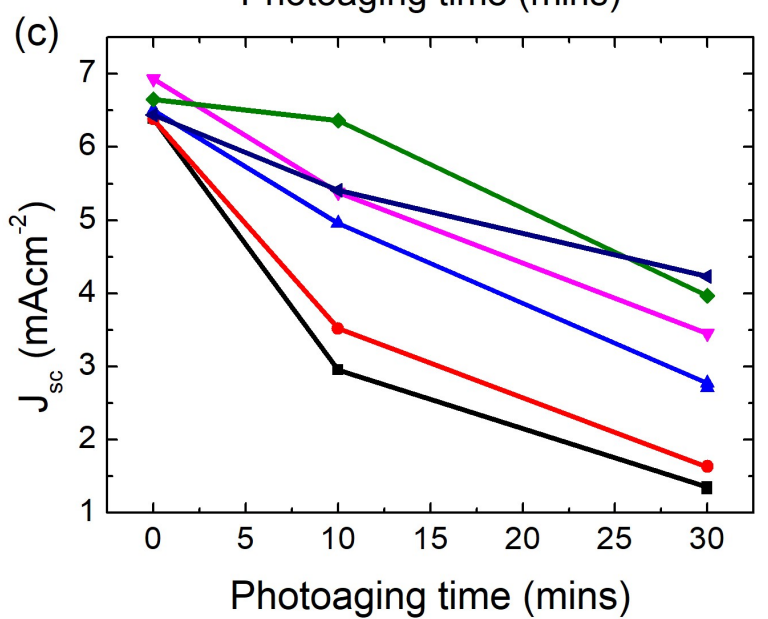

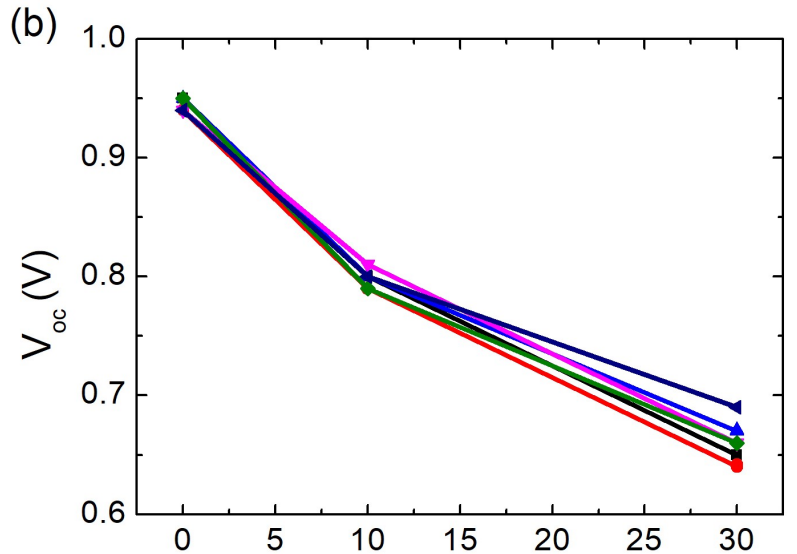

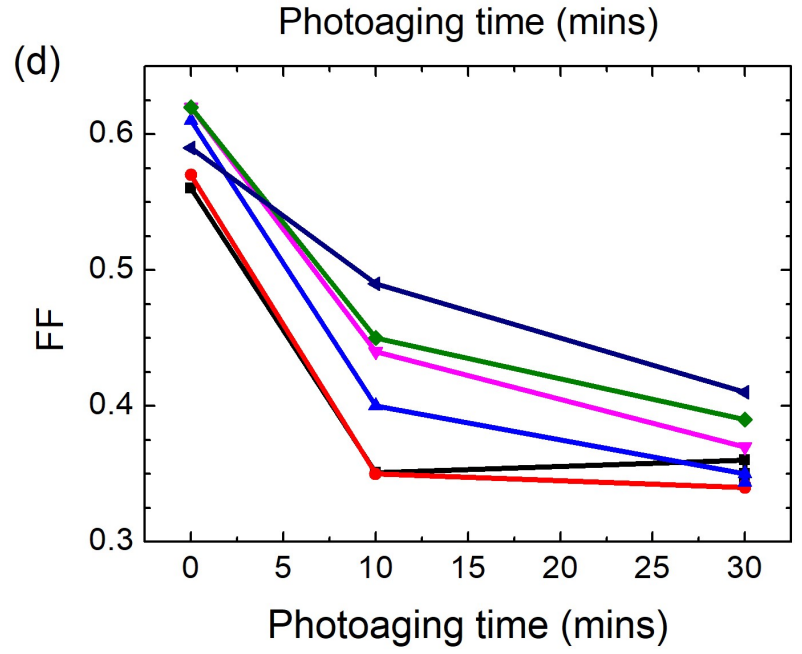

Figure B.20: Device characteristics (a) PCE (b) $\mathrm{V}_{\text {oc }}$ (c) $\mathrm{J}_{\mathrm{sc}}$ (d) FF of PCDTBT:PC ${ }_{61} \mathrm{BM}$ devices with different film thicknesses as a function of degradation time. 

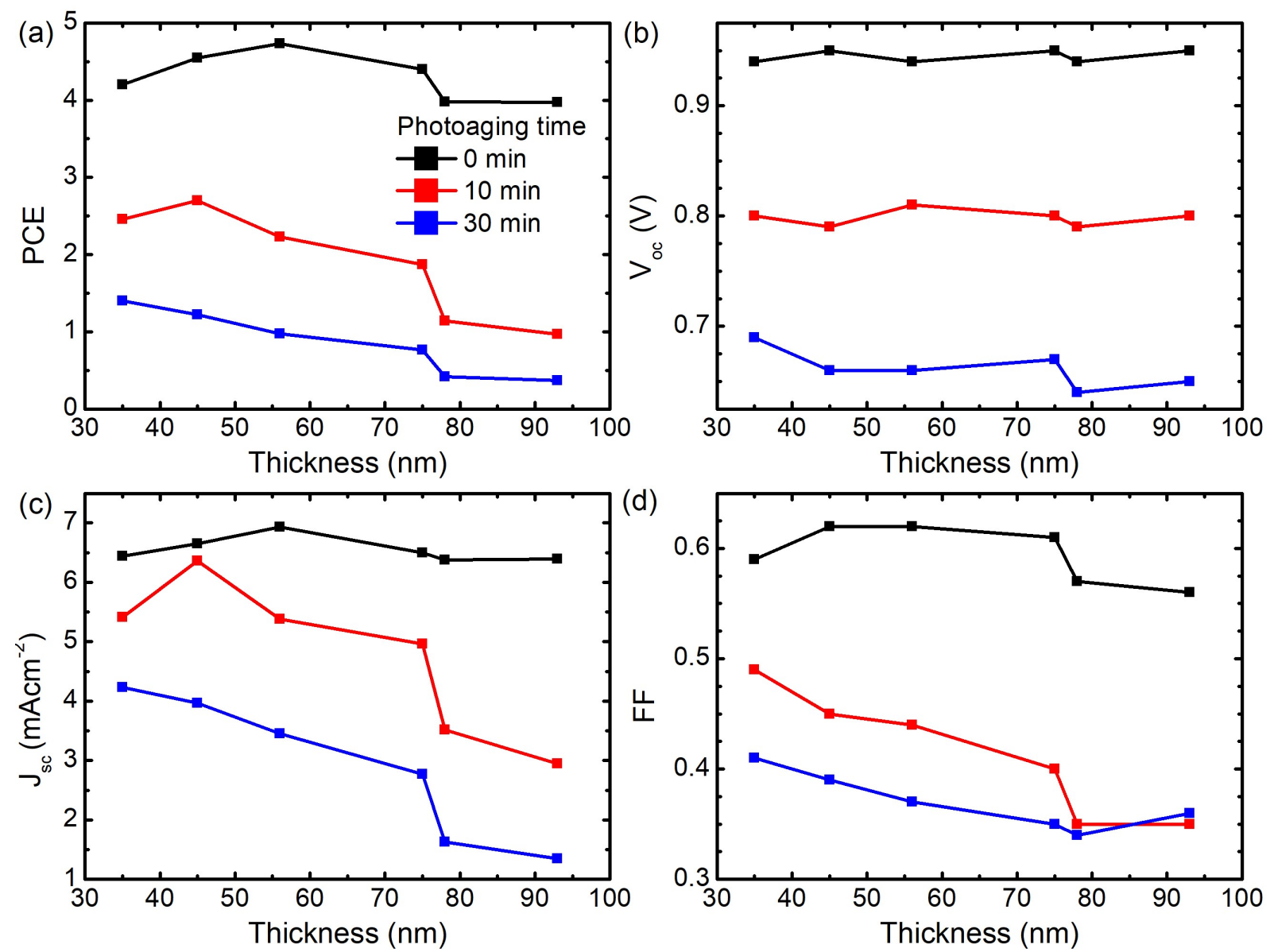

Figure B.21: Device characteristics (a) PCE (b) $\mathrm{V}_{\mathrm{oc}}$ (c) $\mathrm{J}_{\mathrm{sc}}$ (d) FF of $1: 2$ PCDTBT: $\mathrm{PC}_{61} \mathrm{BM}$ devices photaged for 0,10 and 30 minutes, as a function of active layer film thickness. 

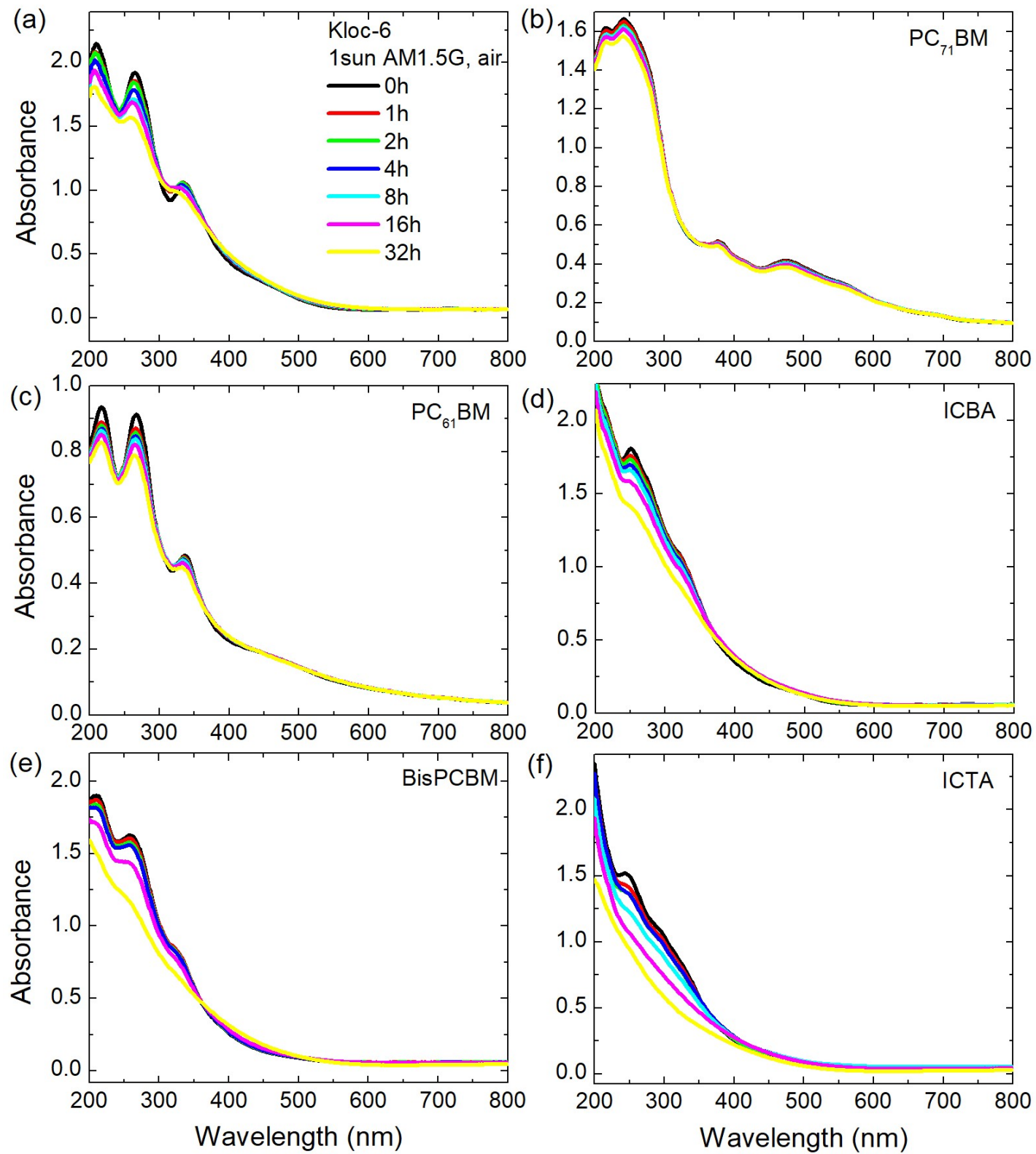

Figure C.1: UV-vis absorption spectra of (a) Kloc-6 (b) $\mathrm{PC}_{71} \mathrm{BM}$ (c) $\mathrm{PC}_{61} \mathrm{BM}$ (d) ICBA (e) BisPCBM (f) ICTA neat films for up to 32 hours under 1 sun AM1.5G illumination in air. 
Table C.1: IR peak position and assignment for photo-oxidised fullerenes.

\begin{tabular}{|c|c|c|}
\hline Fullerene & IR peak position $\left(\mathrm{cm}^{-1}\right)$ & Assignment \\
\hline Kloc-6 & 1686 & $\begin{array}{c}\text { Amide } \mathrm{C}=\mathrm{O} \text { stretch (on cage) } \\
\text { or aryl ketone } \mathrm{C}=\mathrm{O} \text { stretch }\end{array}$ \\
\hline Kloc-6 & 1726 & Ketone $\mathrm{C}=\mathrm{O}$ stretch (on cage) \\
\hline Kloc-6 & 1782 & $\mathrm{C}=\mathrm{O}$ stretch \\
\hline $\mathrm{PC}_{71} \mathrm{BM}$ & 1735 & Ester $\mathrm{C}=\mathrm{O}$ stretch (sidechain) \\
\hline $\mathrm{PC}_{71} \mathrm{BM}$ & 1782 & $\mathrm{C}=\mathrm{O}$ stretch \\
\hline $\mathrm{PC}_{61} \mathrm{BM}$ & 1735 & Ester $\mathrm{C}=\mathrm{O}$ stretch (sidechain) \\
\hline $\mathrm{PC}_{61} \mathrm{BM}$ & 1782 & $\mathrm{C}=\mathrm{O}$ stretch (on cage) \\
\hline ICBA & 1694 & Aryl ketone $\mathrm{C}=\mathrm{O}$ stretch \\
\hline ICBA & 1730 & Aldehyde $\mathrm{C}=\mathrm{O}$ stretch \\
\hline ICBA & 1780 & $\mathrm{C}=\mathrm{O}$ stretch \\
\hline BisPCBM & 1688 & Aryl ketone $\mathrm{C}=\mathrm{O}$ stretch \\
\hline BisPCBM & 1733 & Ester $\mathrm{C}=\mathrm{O}$ stretch (side chain) \\
\hline BisPCBM & 1782 & $\mathrm{C}=\mathrm{O}$ stretch \\
\hline ICTA & 1685 & Aryl ketone $\mathrm{C}=\mathrm{O}$ stretch \\
\hline ICTA & 1739 & Aldehyde $\mathrm{C}=\mathrm{O}$ stretch \\
\hline ICTA & 1780 & $\mathrm{C}=\mathrm{O}$ stretch \\
\hline
\end{tabular}



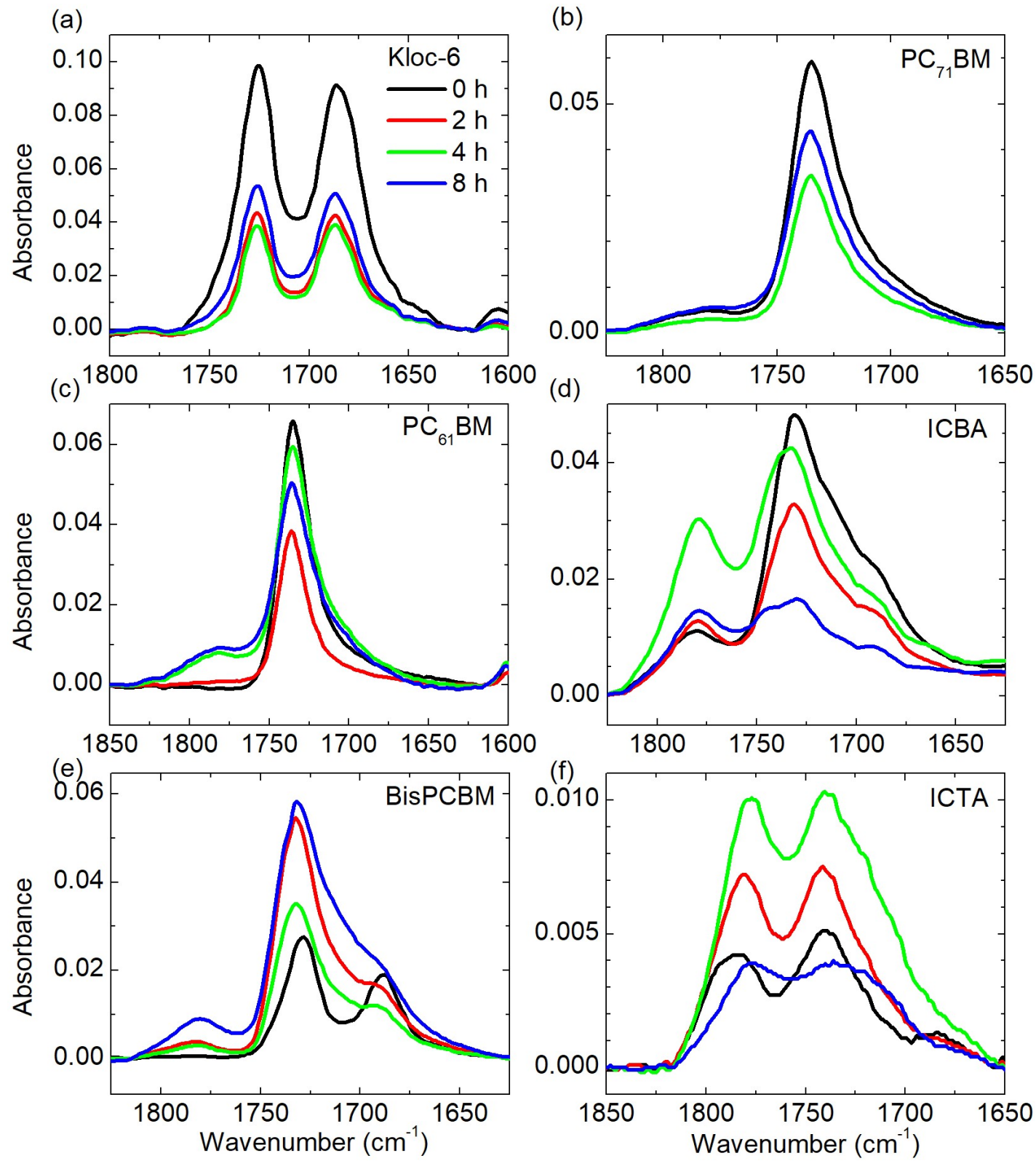

Figure C.2: FTIR spectra of: (a) Kloc-6 (b) $\mathrm{PC}_{71} \mathrm{BM}$ (c) $\mathrm{PC}_{61} \mathrm{BM}$ (d) ICBA (e) BisPCBM (f) ICTA neat films fresh and photoaged degraded for 2, 4 and 8 hours in air under 1 Sun AM1.5G illumination. 

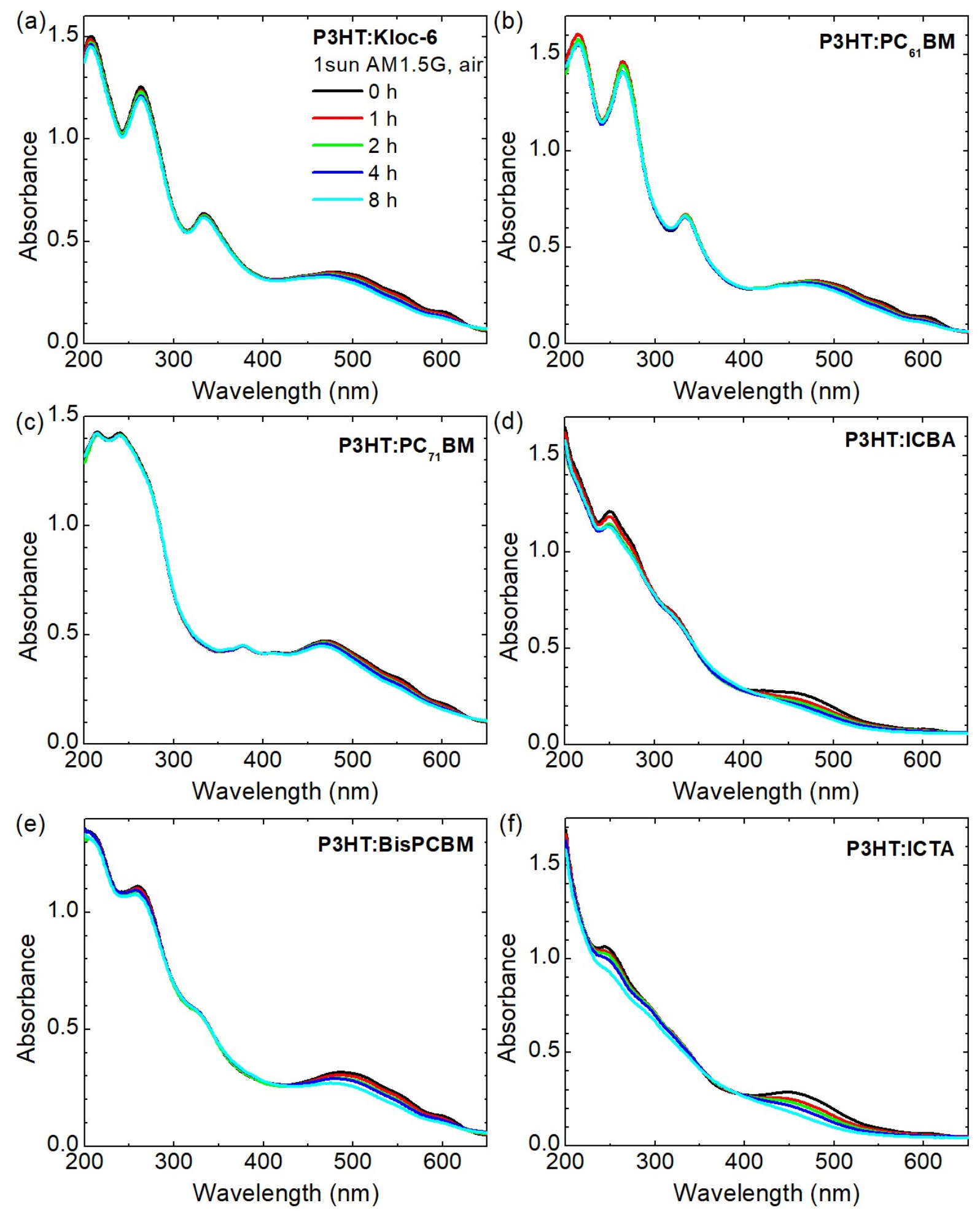

Figure C.3: UV-vis absorption spectra of blend films with up to 8 hours under 1 sun AM1.5G illumination in air. P3HT blended with: (a) Kloc-6 (b) $\mathrm{PC}_{71} \mathrm{BM}$ (c) $\mathrm{PC}_{61} \mathrm{BM}$ (d) ICBA (e) BisPCBM (f) ICTA 

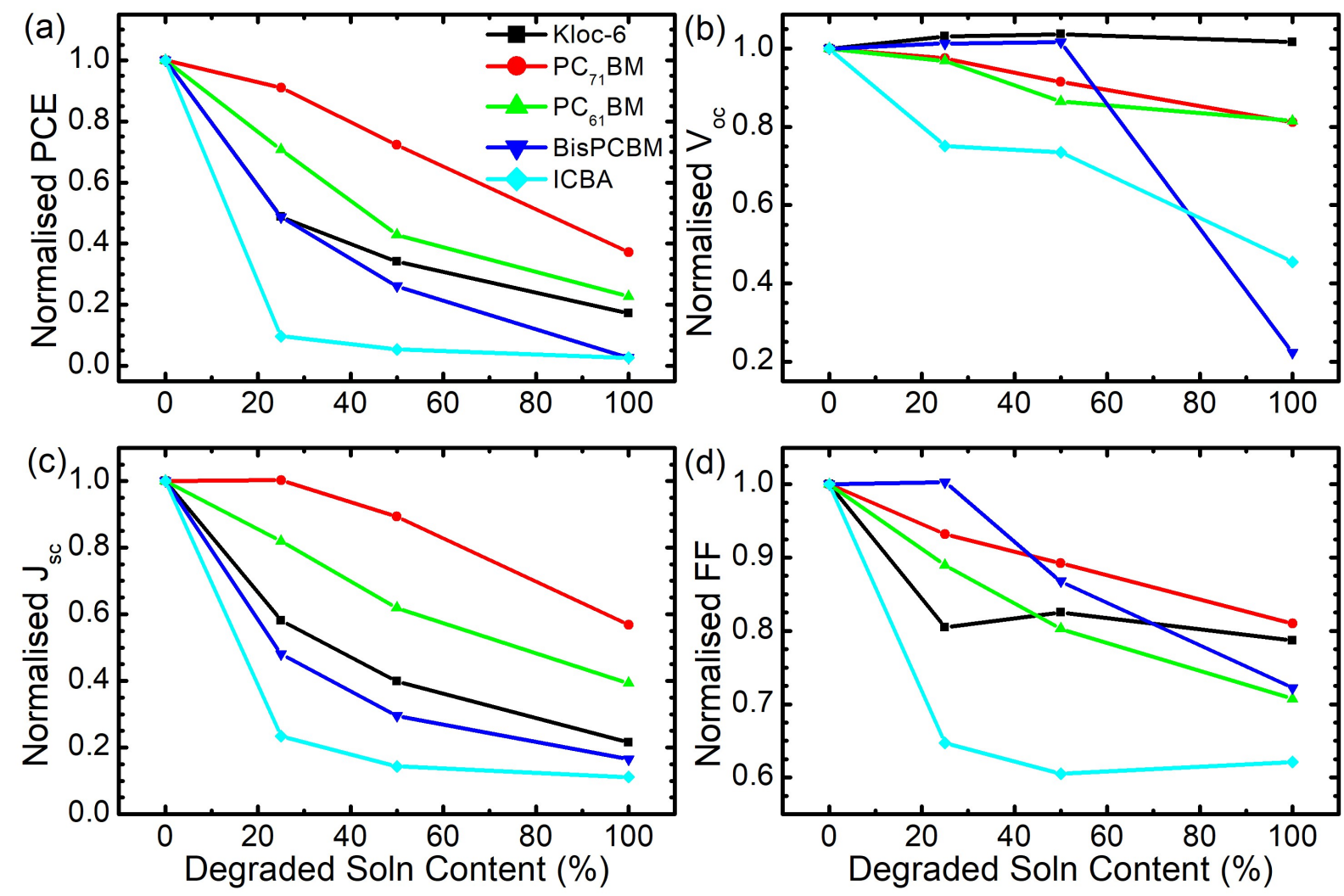

Figure C.4: Device characteristics of P3HT:fullerene devices as a function of degraded solution content: (a) PCE, (b) $\mathrm{V}_{\mathrm{oc}}$, (c) $\mathrm{J}_{\mathrm{sc}}$, and (d) FF.

(a)
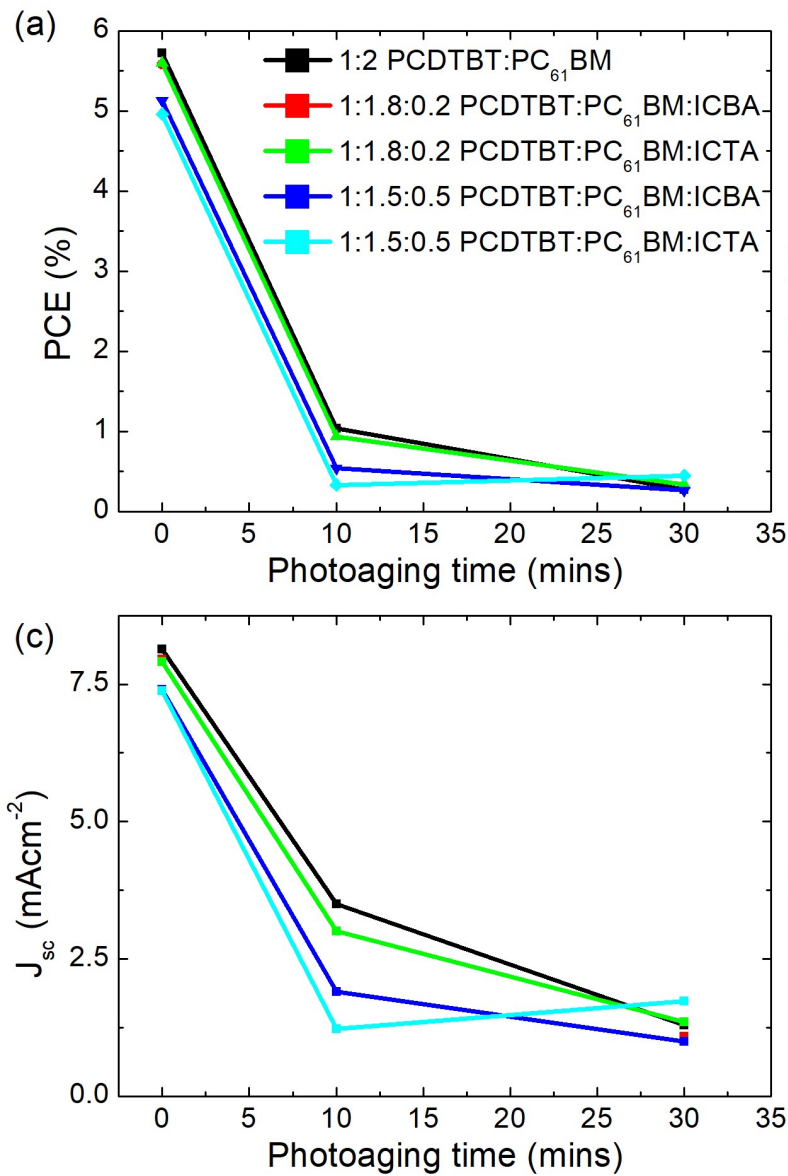

(b)
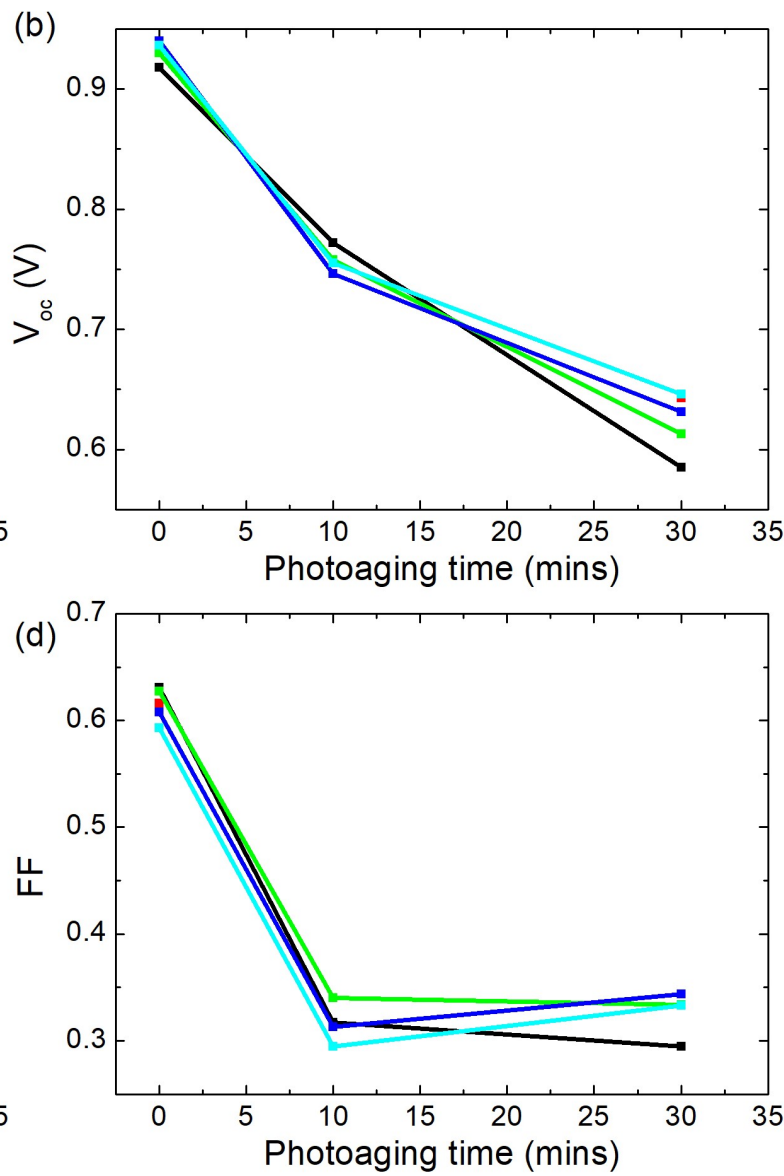

Figure C.5: Device characteristics (a) PCE, (b) $\mathrm{V}_{\mathrm{oc}}$, (c) $\mathrm{J}_{\mathrm{sc}}$, and (d) FF as a function of BHJ film photoaging time. 
FOUR
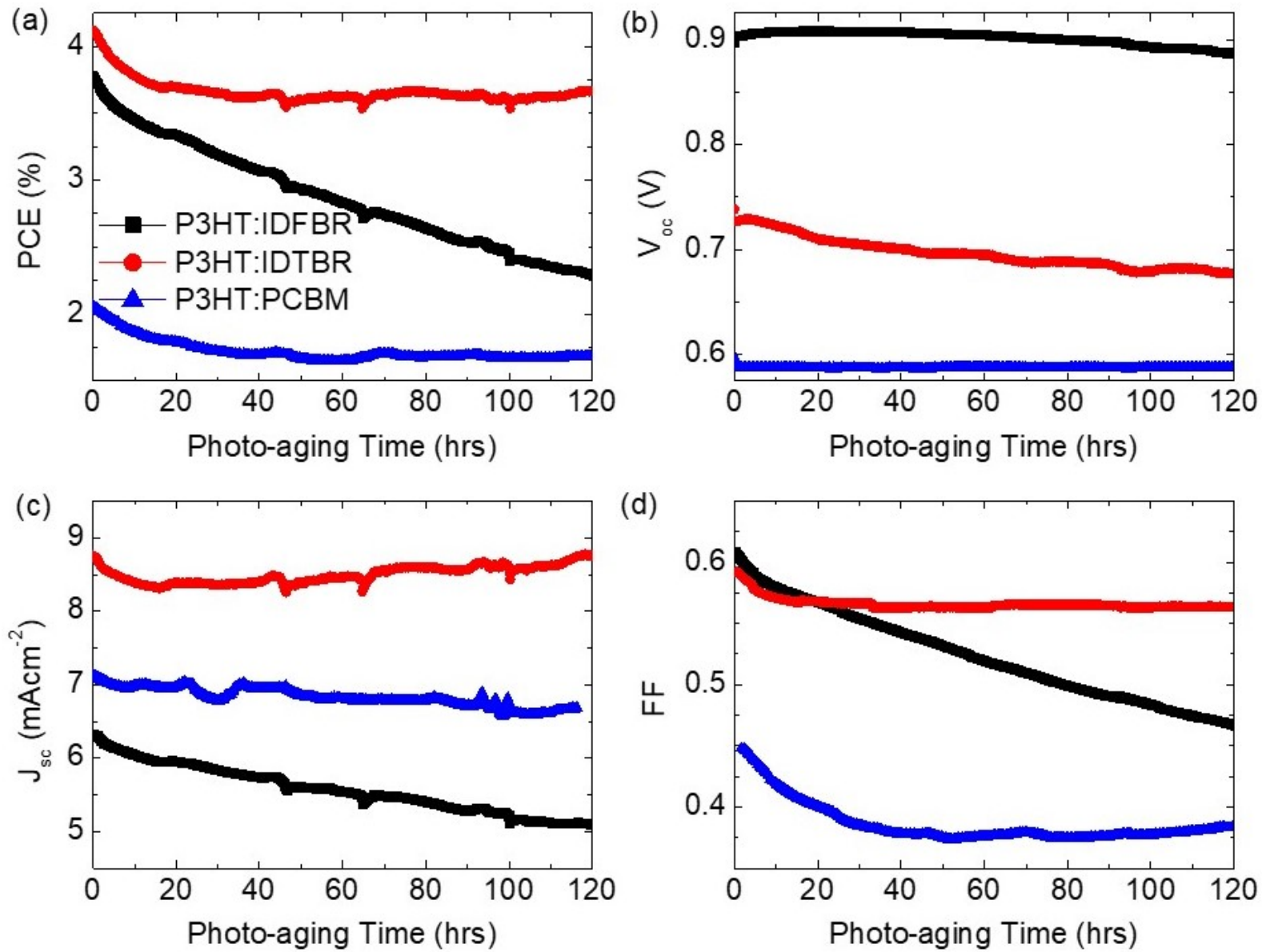

Figure D.1: Device characteristics (a) PCE (b) $\mathrm{V}_{\mathrm{oc}}$, (c) $\mathrm{J}_{\mathrm{sc}}$, (d) FF as a function of device photoaging time under dry nitrogen. 

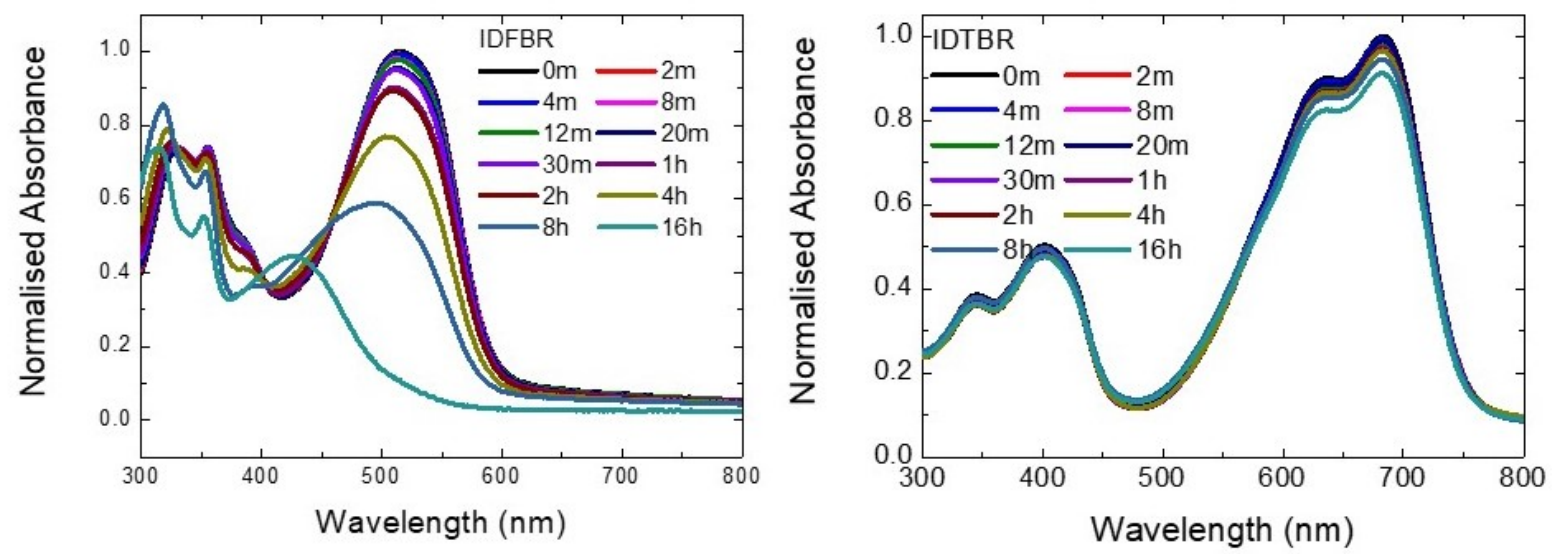

Figure D.2: Photobleaching of (a) IDFBR (b) IDTBR films under 1 sun illumination with a UV-cutting filter, in nitrogen.
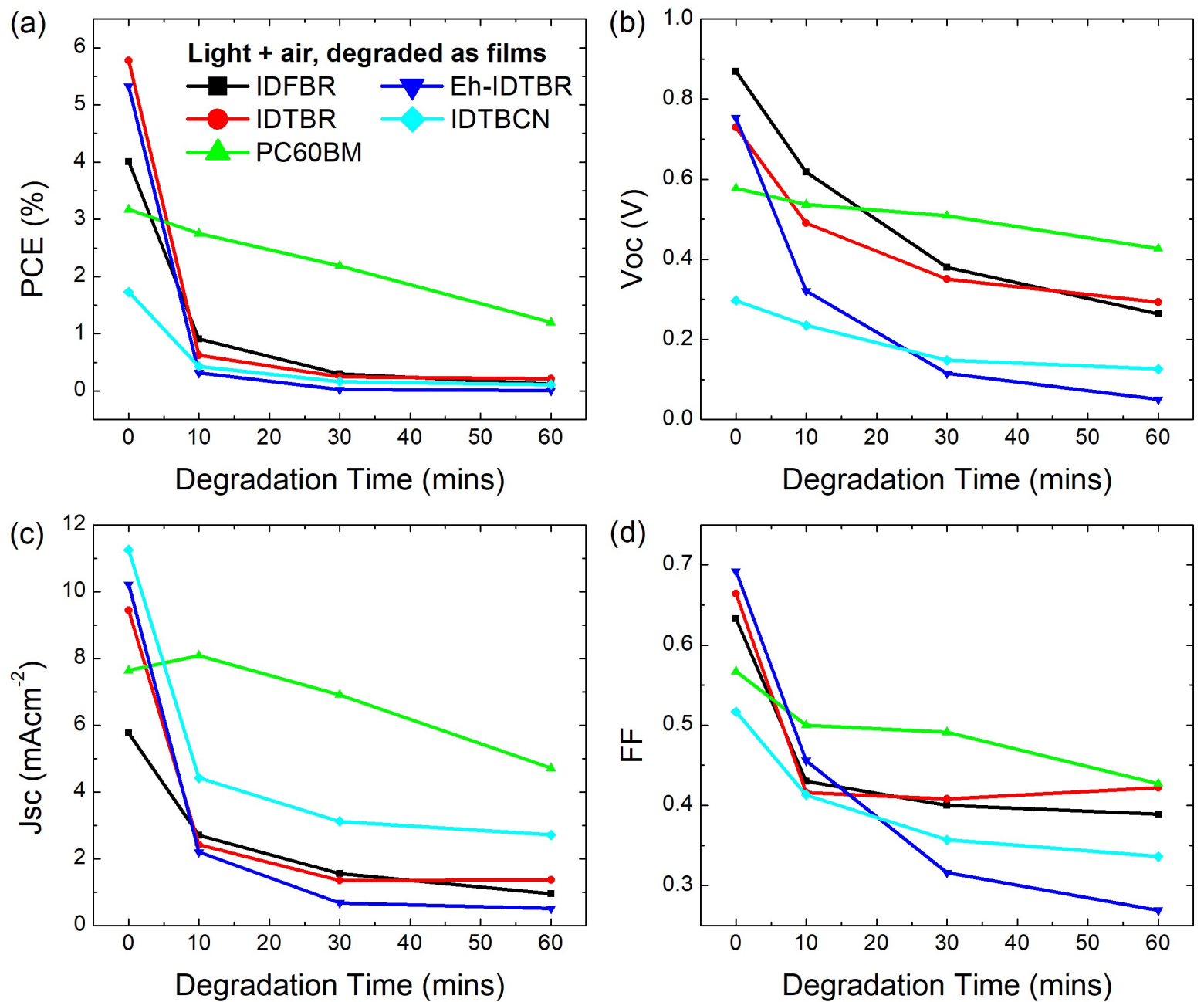

Figure D.3: Device characteristics (a) PCE (b) $\mathrm{V}_{\mathrm{oc}}$ (c) $\mathrm{J}_{\mathrm{sc}}$ (d) FF of P3HT devices blended with different various acceptors, degraded prior to electrode deposition, as a function of degradation time. 
Table D.1: $\mathrm{T}_{80}$ lifetimes of devices aged under white LED illumination in nitrogen. A dash indicates that the device had not reached its $\mathrm{T}_{80}$ in the 120 hours of measuring.

\begin{tabular}{||c|c||}
\hline \hline & $\mathrm{T}_{80}$ (hrs) \\
\hline P3HT:IDFBR & 45 \\
P3HT:IDTBR & - \\
P3HT:PCBM & 49 \\
\hline \hline
\end{tabular}
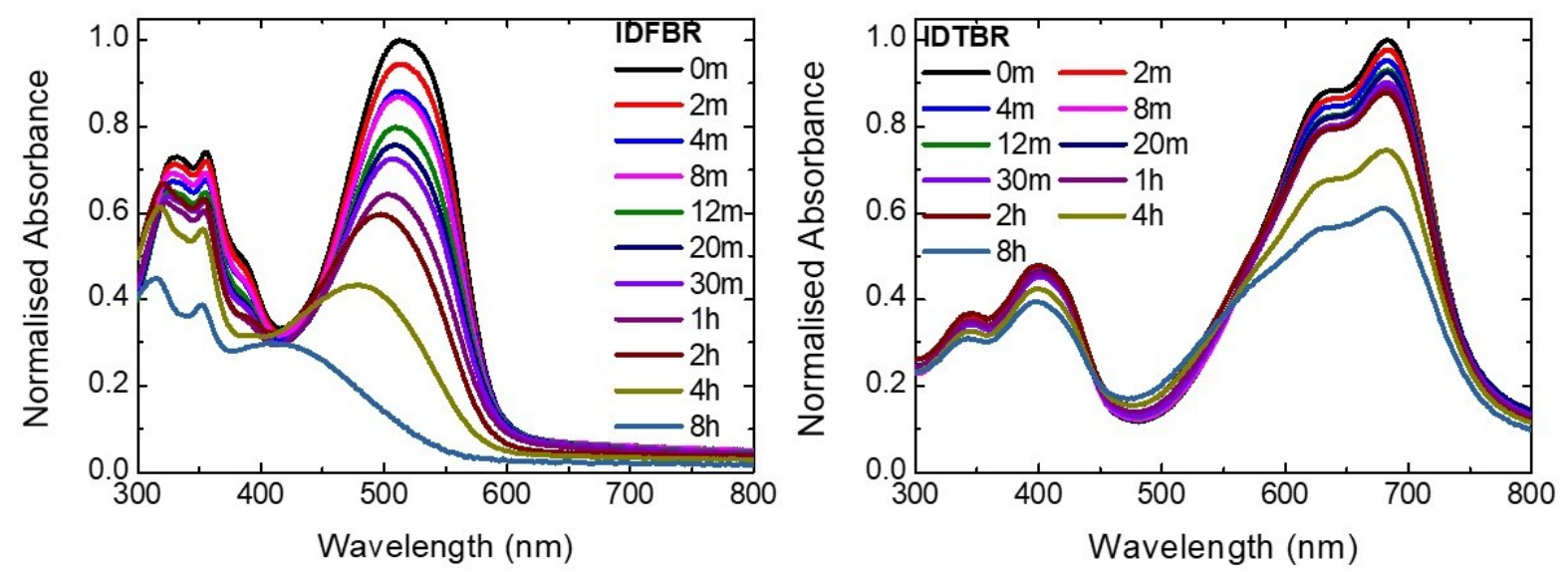

Figure D.4: Photobleaching of (a) IDFBR (b) IDTBR films under 1 sun illumination with a UV-cutting filter, in air.
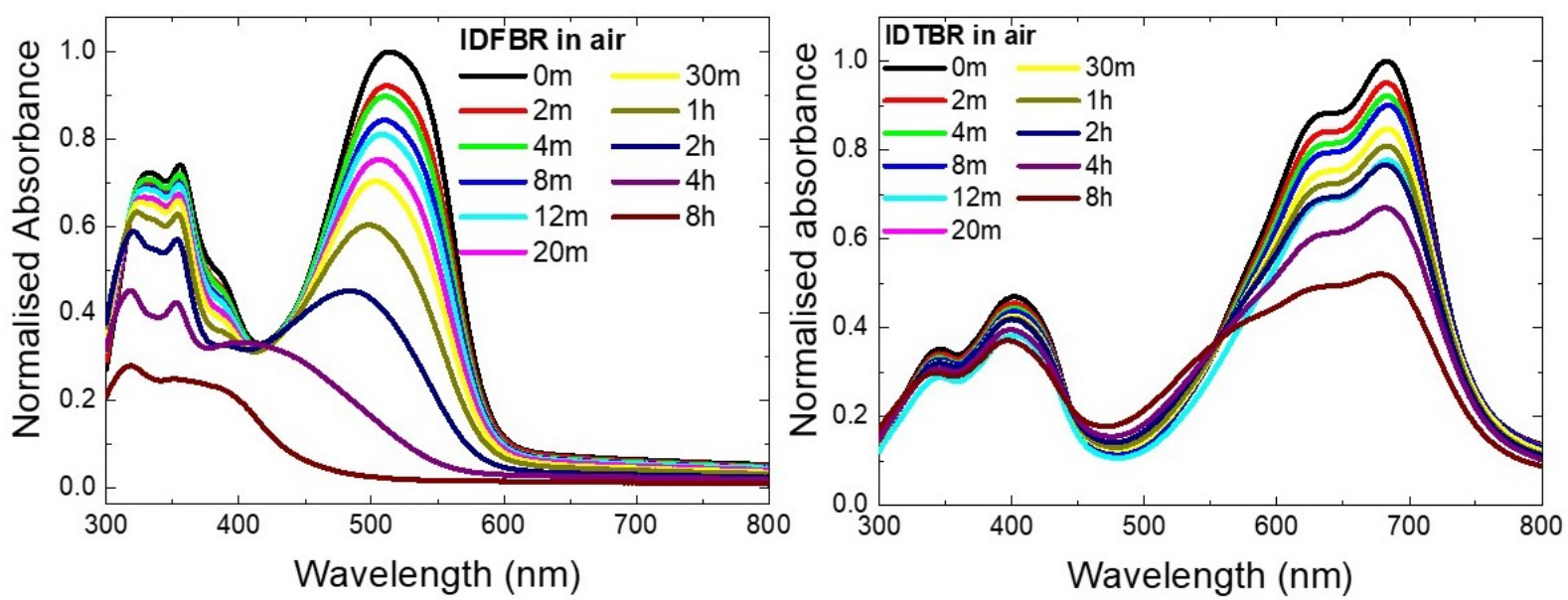

Figure D.5: Photobleaching of unannealed (a) IDFBR and (b) IDTBR neat films under 1 sun illumination in air. 

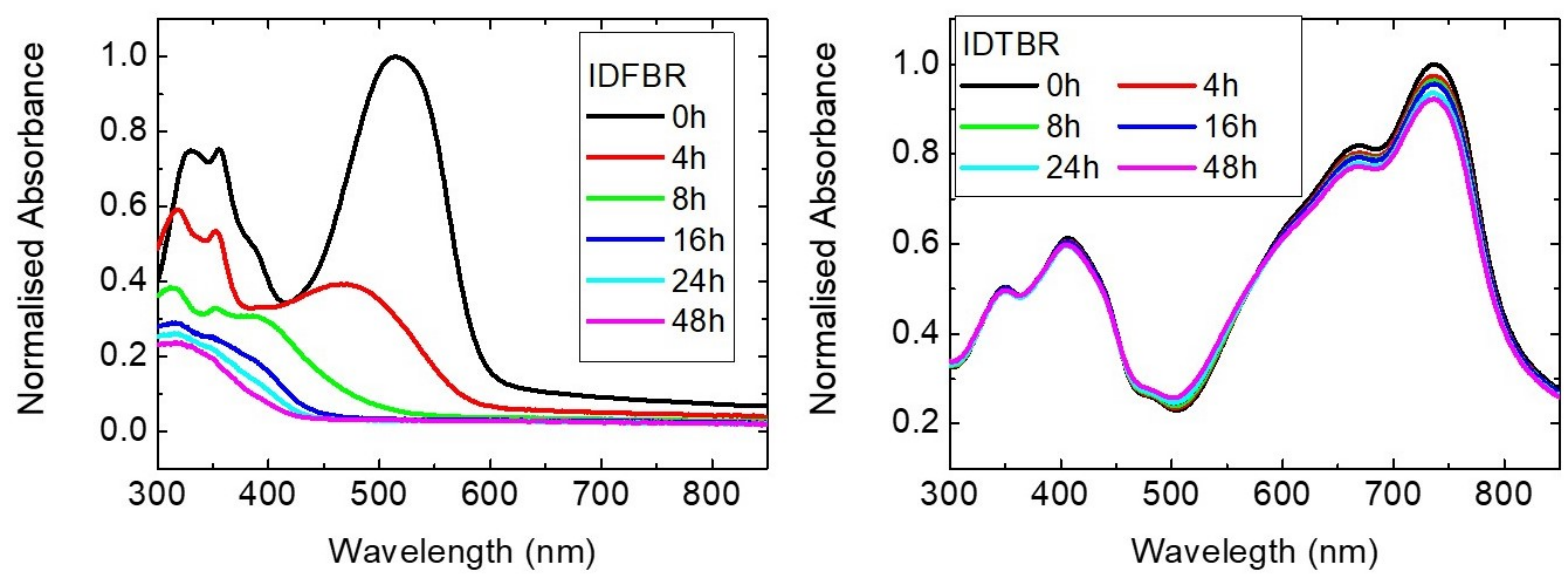

Figure D.6: Photobleaching of (a) IDFBR (b) IDTBR annealed films under 1 sun equivalent white LED illumination in air.

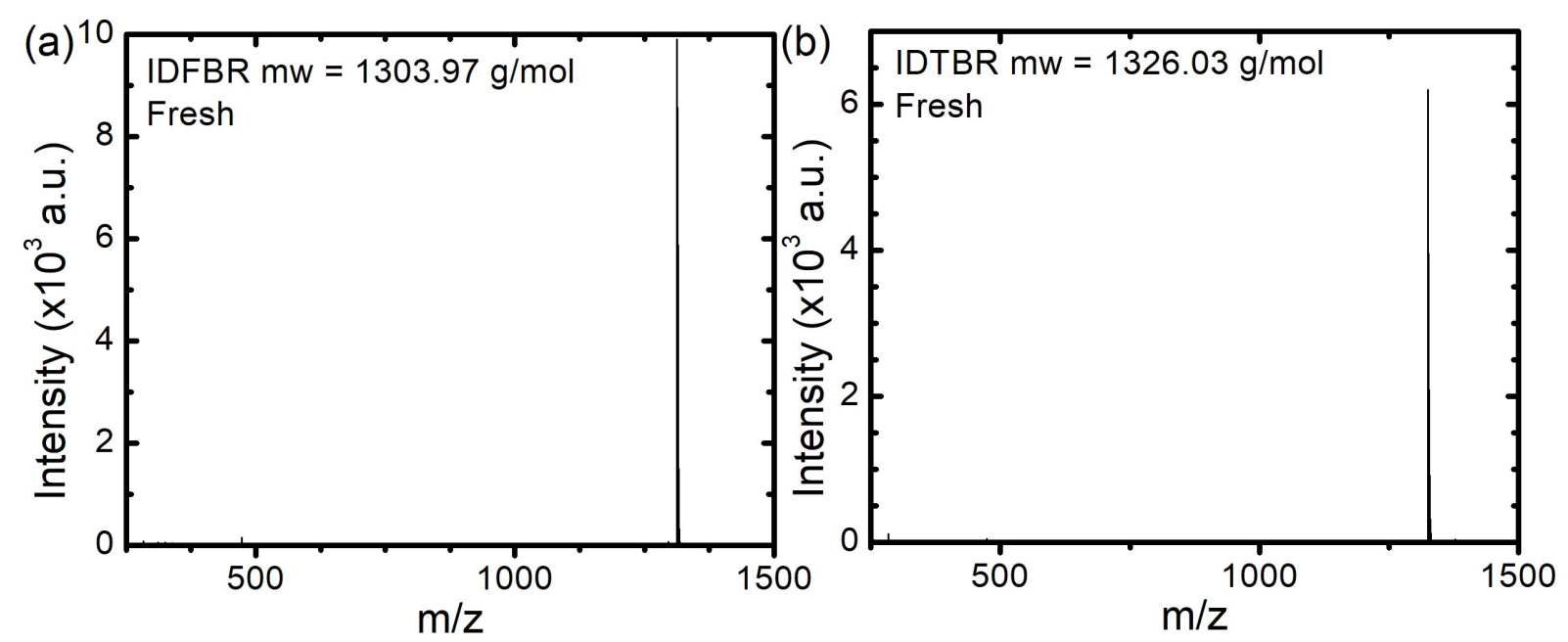

Figure D.7: Negative ion mode MALDI-TOF measurements of: a) IDTBR and b) IDFBR fresh neat films

Table D.2: $\mathrm{T}_{80}$ lifetimes of devices aged under white LED illumination in nitrogen. A dash indicates that the $\mathrm{T}_{80}$ had not yet been reached during the 120 hours of testing.

\begin{tabular}{|c|c|}
\hline & $\mathrm{T}_{80}(\mathrm{hrs})$ \\
\hline PCE10:O-IDTBR & - \\
\hline PCE11:O-IDTBR & - \\
\hline PCE10:Eh-IDTBR & - \\
\hline PCE11:Eh-IDTBR & - \\
\hline PCE10:ITIC & 15.4 \\
\hline PCE11:ITIC & - \\
\hline PCE12:ITIC & 50.3 \\
\hline PCE10:M-ITIC & 8.9 \\
\hline PCE12:M-ITIC & 93.5 \\
\hline PCE10:PC ${ }_{71} \mathrm{BM}$ & 1.6 \\
\hline PCE10:PC ${ }_{71} \mathrm{BM}$ & 1.7 \\
\hline
\end{tabular}



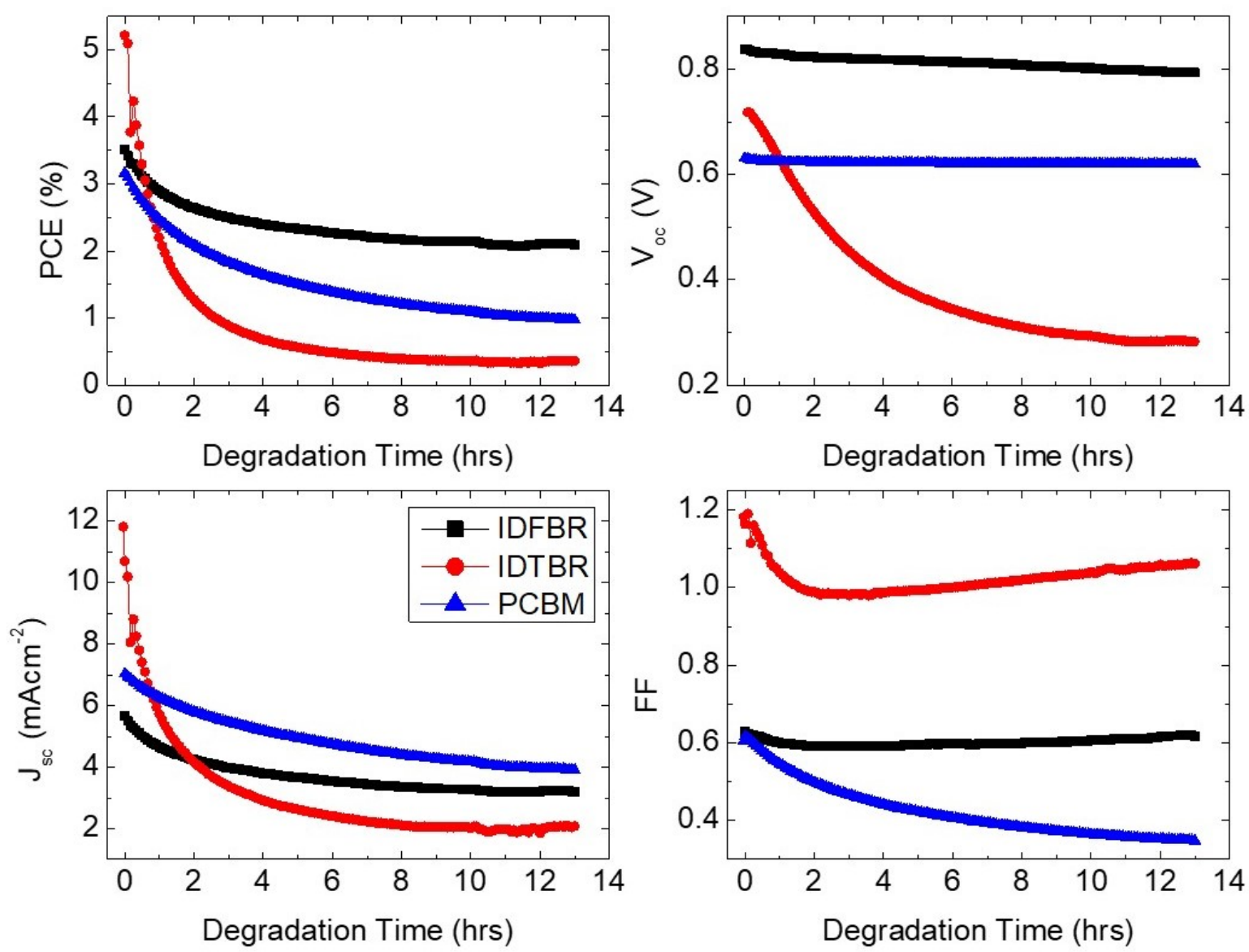

Figure D.8: Device characteristics (a) PCE (b) $\mathrm{J}_{\mathrm{sc}}$, (c) $\mathrm{V}_{\mathrm{oc}}$, (d) FF as a function of device degradation time (as completed devices) in air.

Table D.3: $\mathrm{T}_{80}$ lifetimes of devices aged under white LED illumination in air.

\begin{tabular}{||c|c||}
\hline \hline & $\mathrm{T}_{80}(\mathrm{hrs})$ \\
\hline P3HT:IDFBR & 1.25 \\
P3HT:IDTBR & 0.25 \\
P3HT:PCBM & 0.81 \\
\hline \hline
\end{tabular}



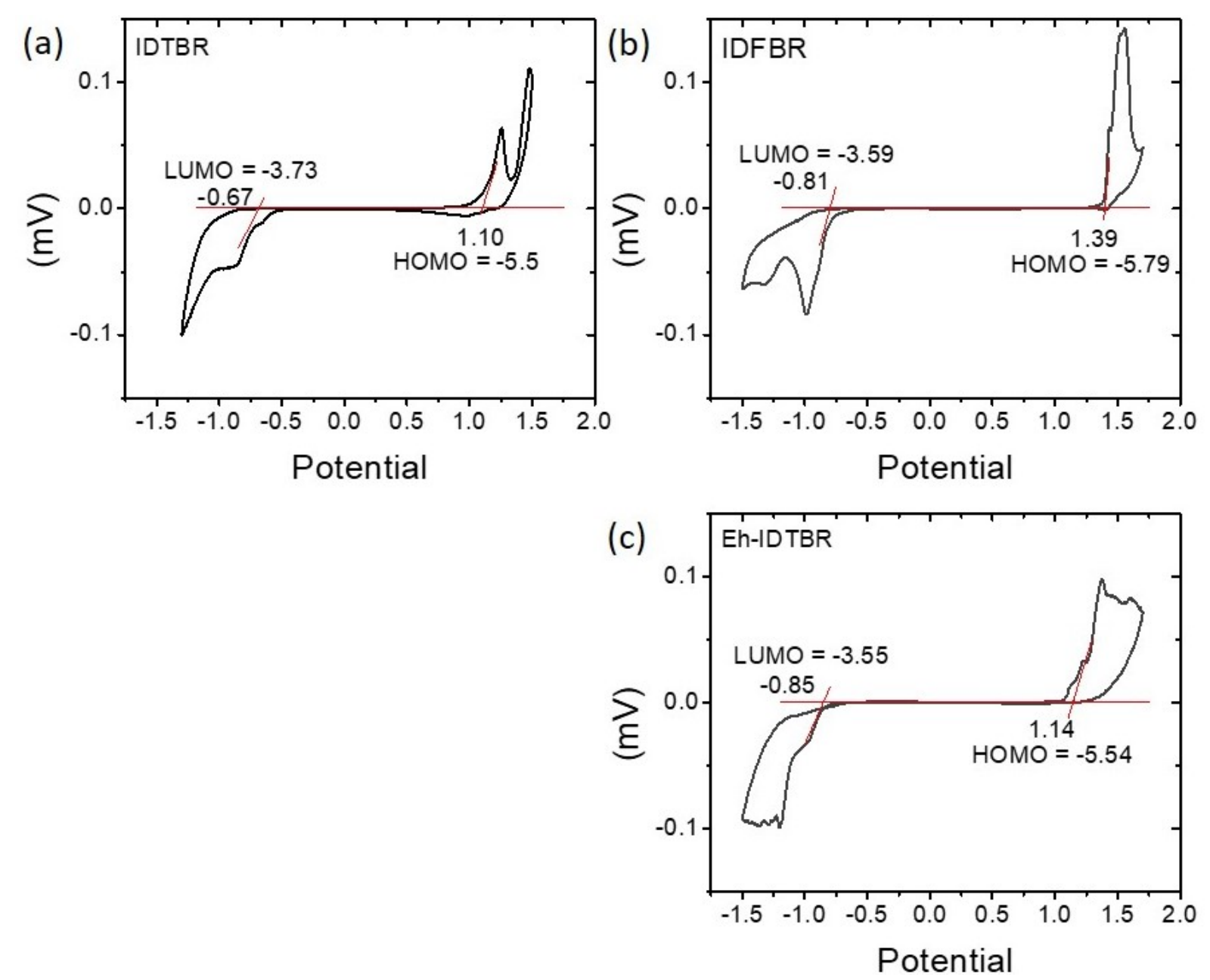

Figure D.9: Cyclic voltammetry scans of neat NFA films. 